\title{
Die Großschmetterlinge (Macrolepidoptera) der Diluviallandschaften um Eberswalde. Vierter Nachtrag, Ergänzungen zur Verbreitung, Biologie und Phänologie seit 2013
}

\author{
Mit 28 Figuren und 27 Tabellen \\ ARNOLD RICHERT \\ ${ }^{1}$ Altenhofer Straße 68, 16227 Eberswalde \\ Published on 2018-12-06 \\ DOI:10.21248/contrib.entomol.68.2.177-346
}

\section{Zusammenfassung}

Die Entwicklung der Großschmetterlingsfauna der Diluviallandschaften um Eberswalde wurde bis zum Jahre 2018 unter Berücksichtigung folgender Schwerpunkte fortgeschrieben:

(1) Veränderungen im Artenbestand: Vier Arten wurden neu nachgewiesen; acht als verschollen gemeldete Arten wurden wieder entdeckt, darunter als besonders bemerkenwerte Wiederfunde die Noctuide Eremobia ochroleuca nach $>60$ Jahren und die Geometride Eustroma reticulata nach 33 Jahren; neun Arten des bisherigen Bestandes werden als verschollen gemeldet. (2) Ergänzungen zur Kenntnis der Biologie, insbesondere der Nahrungsökologie der Tagfalter, sowie der aktuellen Veränderungen der Phänologie. (3) Auswertung älterer Quellen, die dem Verfasser bisher nicht bekannt waren; dadurch konnte das Gesamtbild der Großschmetterlingsfauna abgerundet werden. (4) Abschließende Analyse des Durchforschungrades der einzelnen Naturräume des Gebietes.

\section{Schlüsselwörter}

Tagfalter, Nachtfalter, Phänologie, Ökologie, Zeitreihen, Langzeituntersuchung, Artenzusammensetzung, Naturschutz, Brandenburg

\section{Abstract}

The changes in the fauna of macrolepidoptera of the diluvial landscapes around Eberswalde was described until the year 2018 considering the following major aspects: (1) Changes in species composition: Four species were newly recorded; eight species thought to have disappeared were rediscovered, with two particularly remarkable findings, the Nolidae Eremobia ochroleuca after $>60$ years of absence and the Geometridae Eustroma reticulata after 33 years; eight of the species known for the region could not be confirmed by newer records. (2) Additions are given to the knowledge of the biology, especially of the nutrition ecology of butterflies, as well as recent changes in the phylogeny. (3) Examination of older sources, which have not been known by the author before; these allowed completing the overall picture of the macrolepidoptera. (4) Supplementary information on the level of knowledge on the different sub-regions is given.

\section{Key words}

butterflies, moths, phenology, ecology, time series, long-term research, species composition, nature conservation, Brandenburg 


\section{Inhalt}

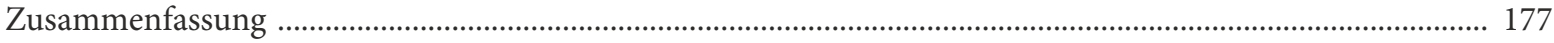

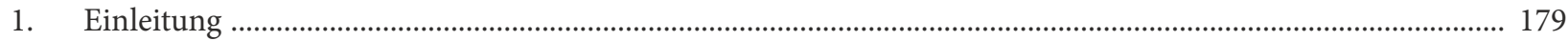

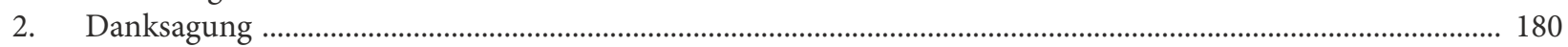

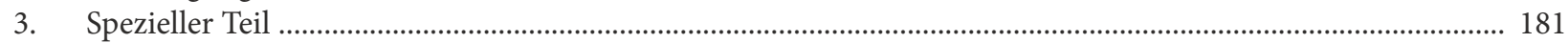

3.1. Erläuterungen zu den Ausführungen im speziellen Teil ................................................................................... 181

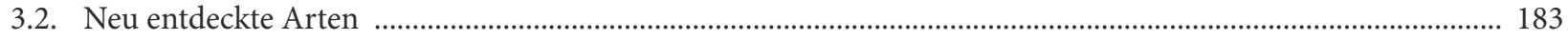

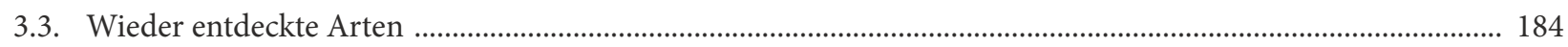

3.4. Ergänzungen zu den Anmerkungen in den Teilen I-IV (Richert 1999-2004) (aktualisierte und neue Fundortangaben sowie Beobachtungen zur Biologie, Ökologie und Phänologie) ....................................... 186

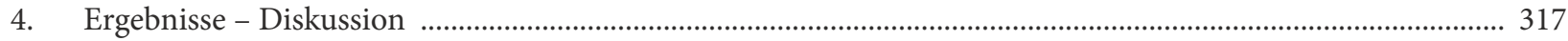

4.1. Phänologische Veränderungen im Untersuchungszeitraum 2014-2018 …………………………………….... 317

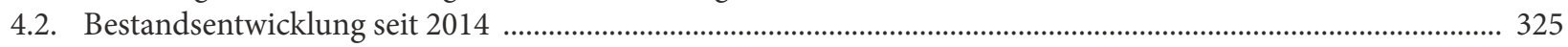

4.3. Analyse des Durchforschungsgrades der Naturräume des Gebietes ................................................................. 326

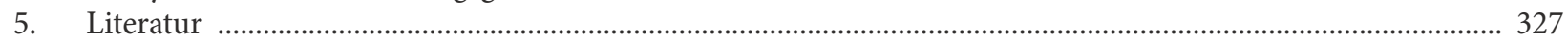

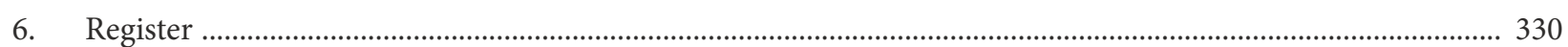

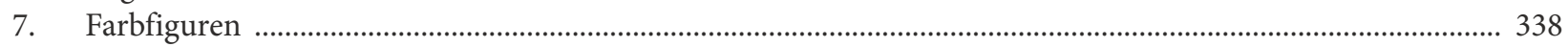

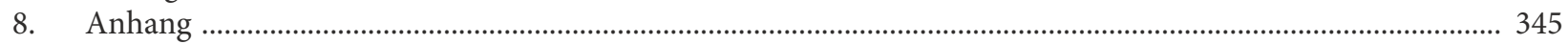

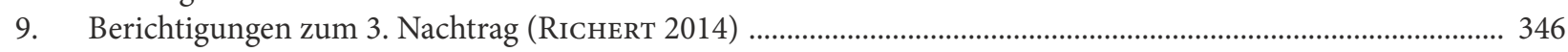




\section{Einleitung}

Im Jahr 1961 gründeten Entomologen und weitere an der Sache interessierte Personen aus Eberswalde und Umgebung eine „Fachgruppe Entomologie“ im Kulturbund der DDR, unter dessen Schirmherrschaft damals eine derartige Vereinstätigkeit möglich war. Auf der Gründungsveranstaltung hielt der von uns allen verehrte Mentor ganzer Generationen von Schmetterlingskundlern (nicht nur aus Brandenburg), Dr. Ernst Urbahn aus Zehdenick, den einführenden Vortrag. Darin bezeichnete er die faunistische Forschung im Raum Eberswalde als besonders dankbare Aufgabe für die neu gegründete Fachgruppe. Das Gebiet war damals in dieser Hinsicht noch wenig bearbeitet.

Seither sind fast sechs Jahrzehnte kontinuierlicher Freizeitforschung vergangen, in denen der Verfasser und viele Mitarbeiter und Gewährsleute, die heute $z$. T. nicht mehr unter uns weilen, ein umfangreiches Datenmaterial zur Großschmetterlingsfauna der Diluviallandschaften um Eberswalde zusammengetragen haben. Ihre Tätigkeit wurde zunächst vom Eberswalder Forstinstitut, später vom Deutschen Entomologischen Institut unterstützt.

Im Ergebnis und als Auswertung des Datenmaterials liegt ein umfangreiches Werk über die Großschmetterlinge unseres Gebietes vor, das nicht nur den Artenbestand, sondern auch seine fortwährende Veränderung dokumentiert. Der 4. Nachtrag zu diesem Werk wird voraussichtlich die letzte Darstellung über den gesamten Artenbestand der Großschmetterlinge und dessen Veränderungen sein. Diese Ankündigung ist nicht etwa als Abschied von der stets mit Freude und Passion betriebenen faunistischen Freizeitforschung zu werten, im Gegenteil! Die noch verbleibende Zeit soll in ein noch offenes reizvolles Thema investiert werden: Aus unserem Gebiet liegen viele (historische und aktuelle) Daten über Kleinschmetterlinge vor, die einer zusammenfassenden Darstellung harren.

Der 4. Nachtrag zur Eberswalder Großschmetterlingsfauna hat folgende Schwerpunkte:

\section{Fortschreibung der aktuellen Faunenent- wicklung}

Seit Redaktionsschluss des 3. Nachtrages im Februar 2014 (RICHERT 2014) sind 5 Jahre vergangen, in denen die Entwicklung der Schmetterlingsfauna vom Verfasser in bewährter Zusammenarbeit mit langjährigen Mitarbeitern und Gewährsleuten (siehe Danksagung) weiter beobachtet wurde (siehe auch RICHERT 2015, 2017). Die eigenen faunistischen Untersuchungen erfolgten vorrangig in den stillgelegten Steingruben bei Groß Ziethen, in einem Moor am Rande der Finowfließniederung im Forst Biesenthal, im ehemaligen Kiesgrubengelände nördlich von Stecherschleuse und auf Trockenhängen bei Stolpe a.O. Die Schmetterlingsfauna des Geistberges in Oderberg wurde über mehrere
Jahre von meinem langjährigen Mitarbeiter K. Schwabe intensiver untersucht. Seine Ergebnisse werden auch im 4. Nachtrag mitgeteilt.

Die Beobachtungen im Untersuchungszeitraum erweitern und vertiefen die Kenntnis über Verbreitung der Arten im Gebiet, über ihre Biologie und Phänologie. Insbesondere die Kenntnisse zur Nahrungsbiologie der Imagines vor allem von Tagfaltern wurden umfangreich ergänzt (siehe dazu auch RICHERT \& BRAUnER i. präp.). Allen seit 2014 festgestellten phänologischen Veränderungen galt meine besondere Aufmerksamkeit. Sie werden bei den betroffenen Arten benannt und im Abschnitt Diskussion besonders ausgewertet.

\section{Auswertung dem Verfasser bisher nicht be- kannter älterer Quellen, die das Gesamtbild der Großschmetterlingsfauna abrunden}

- Unter dem Titel „Die Großschmetterlinge des Biosphärenreservats Schorfheide-Chorin" fassten U. Barthelme \& G. Rinnhofer die Ergebnisse ihrer Sammeltätigkeit im Eberswalder Raum von 1965 bis 1994 in Form einer unveröffentlichten kommentierten Artenliste (Schreibmaschinen-Manuskript) zusammen. Dieses Manuskript befand sich in der Landesanstalt für Großschutzgebiete Land Brandenburg. Günter Rinnhofer war als Präparator im DEI in Eberswalde tätig und hat oft gemeinsam mit Uwe Barthelme im Eberswalder Gebiet gesammelt. Beide haben eine sichere Artenkenntnis und sind als Mitarbeiter und Gewährsleute für die Erarbeitung der Eberswalder Fauna bereits aufgeführt (RICHERT 1999: 8). Die in ihrem Manuskript mitgeteilten Daten standen für die Publikation der Eberswalder Schmetterlingsfauna leider nur teilweise zur Verfügung. Sie stammen aus Lebensräumen unseres Gebietes, die dem Verfasser überwiegend bezüglich ihres Artenspektrums bekannt sind, aber nicht regelmäßig von ihm selbst kontrolliert werden konnten. Alle im Manuskript vermerkten Daten wurden kritisch bewertet. Sie bieten eine willkommene Ergänzung und ermöglichen in Einzelfällen eine genauere Datierung für das Erlöschen von Populationen im Gebiet.

- In den Jahren 1994-1995 wurde im Rahmen des Gewässerrandstreifenprogramms Unteres Odertal auch die Großschmetterlingsfauna des Gebietes von Dr. Alfred Borkowski im Auftrag der Umwelt-Service $\mathrm{GmbH}$ (AG Unteres Odertal) untersucht. Auf 12 Probeflächen wurde sowohl die Tag- als auch die Nachtfalterfauna erfasst. Die Ergebnisse legte Borkowski in einer umfangreichen Studie (Borkowski 1996; 84 S., 12 GeländeKarten) dar. Daten der faunistischen Untersuchungen von Borkowski wurden außerdem als einfache Artenliste (ohne nähere Erläuterungen und Fundortangaben) in einem umfangreichen Werk über das Untere Odertal publiziert (VössıNG 1998).

Vier der Probeflächen (PF) von Borkowski liegen in unserem Untersuchungsgebiet. Da das nordöstliche Grenzgebiet des Eberswalder Raumes bisher nur lückenhaft 
untersucht wurde, werden faunistisch bedeutsame Funde von Borkowski an diesen Plätzen nach kritischer Bewertung im 4. Nachtrag ergänzend mitgeteilt. Es handelt sich um folgende Flächen:

PF 3 Lunower Hölzchen (176 nachgewiesene Arten): Fundortkennzeichnung im 4. Nachtrag: Naturraum g, MTB 3050/IV Lunow Umg./Gebiet Lunower Hölzchen;

PF 5 Trockenpolder Stolzenhagen (97 nachgewiesene Arten):

Fundortkennzeichnung im 4. Nachtrag: Naturraum h: MTB 3050/II Stolzenhagen Umg./Odertal: Trockenpolder nördlich Pomeranzengraben;

PF 17 Gellmersdorfer Forst (200 nachgewiesene Arten):

Fundortkennzeichnung im 4. Nachtrag: Naturraum g: 3050/II Gellmersdorf Umg./Gellmersdorfer Forst: Mühlenberggebiet;

PF 12 Stolper Strom (76 nachgewiesene Arten):

Fundortkennzeichnung im 4. Nachtrag: Naturraum h: 3050/II Stolpe Umg./Odertal: Gebiet zwischen Stolper Strom und Fernitz-Felch.

Neben einer ausführlichen Darstellung der Untersuchungsergebnisse auf den Probeflächen werden in der Arbeit auch einige faunistisch interessante Funde außerhalb derselben genannt, u. a. aus dem NSG Krähen- und Jungfernberge (vgl. Richert 1979, 1982, 1982a), welches nicht in die Untersuchungen Borkowski's einbezogen war. Auch diese Beobachtungen werden im 4. Nachtrag mitgeteilt, weil sie für einige Arten Rückschlüsse auf die Kontinuität des Bestandes in diesem Gebiet ermöglichen.

- Nachlassauswertung der Sammlung Kittel.

Im Jahre 2015 verstarb Fred Kittel, mein langjähriger Mitarbeiter bei der Bearbeitung der Eberswalder Schmetterlingsfauna (RICHERT 2016). Seine Sammlung enthielt größere Serien heimischer Falter. Faunistisch bedeutsames Belegmaterial aus dem Gebiet wurde inzwischen in die Sammlung des Verfassers integriert (seit 2009 dem Deutschen Entomologischen Institut übereignet). Die Sammlung Kittel wurde von der Hochschule für Nachhaltige Entwicklung (HNE) Eberswalde, Fachbereich Landschaftsnutzung und Naturschutz, übernommen.

Es zeigte sich, dass die Daten von Belegen der Sammlung Kittel nicht lückenlos für die Publikationen über die Eberswalder Schmetterlingsfauna erfasst worden waren. Solche Daten werden ergänzend mitgeteilt.

- Im Spätsommer und Herbst des Jahres 2013, also bereits im Bearbeitungszeitraum des 3. Nachtrages der Eberswalder Schmetterlingsfauna (RICHERT 2014), wurden im Rahmen einer Bachelorabeit der Hochschule für Nachhaltige Entwicklung HNE Eberswalde vergleichende Untersuchungen zur Nachtfalterfauna auf Windwurflächen im Choriner Endmoränenbogen durchgeführt (Müller 2015). Die Ergebnisse konnten 2015 vom Verfasser faunistisch ausgewertet werden. Die Falterbelege aus diesen Untersuchungen lagen mir zur Überprüfung vor. Die kontinuierlichen Fangreihen an den Untersuchungsorten über einen längeren Zeitraum lieferten wertvolle Ergänzungen unserer Kenntnisse über die Verbreitung und über die Phänologie einzelner Arten.

Infolge der neu erschlossenen Daten wurden für einige Arten Korrekturen von Angaben der absoluten Flugzeit bis zum Jahre 2013 notwendig (bei den betroffenen Arten vermerkt).

\section{Danksagung}

Die Zusammenstellung der umfangreichen Daten der vorliegenden Arbeit war nur möglich durch Zuarbeit von Mitarbeitern und Gewährsleuten, denen mein besonderer Dank gilt:

Herrn U. Barthelme, Eberswalde, für die Mitteilung aktueller Daten vom ehemaligen TÜP Trampe und aus dem ehemaligen Kiesgrubengelände zwischen Oder-HavelKanal, Stecherschleuse und Niederfinow;

Herrn O. Brauner, Eberswalde, für die Überlassung umfangreicher Daten aus dem Gebiet (u. a. auch über Blütenbesuche von Tagfaltern; er ermöglichte mir außerdem die Auswertung des Monitorings Solaranlagen Flugplatz Finow von 2014-2017 sowie seiner Kartierungsergebnisse der FFH-Art Großer Feuerfalter im Rahmen der B 167 Ortsumgehung Eberswalde aus dem Jahre 2014;

Herrn R. Busse, Zerpenschleuse, für die Mitteilung von Daten aus dem Ortsgebiet und der näheren Umgebung von Zerpenschleuse sowie vom ehemaligen TÜP Trampe;

Herrn F. Clemens, Schmachtenhagen, für die Übermittlung von Daten über Beobachtungen im NSG Buckowseerinne bei Lichterfelde;

Herrn K. Dörbandt, Berlin, für die Überlassung von Daten aus dem Eberswalder Raum;

Herrn M. Falkenberg, Karlsruhe, für seine Hilfe bei der Determination von Lithosiina;

Herrn Dr. J. Gelbrecht, Königs Wusterhausen, für sachdienliche, stets anregende Diskussionen, Determinationshilfen und Datenübermittlung aus dem Gebiet;

Herrn F. Gottwald, Parlow, für die Überlassung von Daten aus FFH-Gebieten des Biosphärenreservats SchorfheideChorin und umfangreiche Mitteilungen von Daten zum Blütenbesuch von Tagfaltern;

Herrn H. Haase, Groß Schönebeck, für die Überlassung seiner Daten vorwiegend von Groß Schönebeck und Umgebung und für Mitteilungen über seine Zuchterfahrungen;

Herrn M. Just, Liepe, für die Dedikation seines Videos „Die Lieper Tierwelt“ mit Sequenzen über Funde von Arctia villica;

Herrn M. Kleße, Wriezen, für Mitteilungen von Daten aus dem südlichen Nachbargebiet, von Gabow und Hohensaaten;

Herrn Dr. H. Kretschmer, Neuenhagen, für die Überlassung von Daten aus dem Eberswalder Gebiet sowie 
sachdienlichen Gedankenaustausch zu Problemen der Faunenentwicklung;

Herrn Dr. J. Möller, Eberwalde, für Mitteilungen von Nektarsaugpflanzen;

Herrn F. Ockruck, Basdorf, für die Überlassung von Daten aus dem Eberswalder Gebiet;

Frau Dr. Rieger, Steinigtwolmsdorf (ehemals Eberswalde), für die Mitteilung von Falterfunden aus dem Gebiet;

Herrn K. Schwabe, Eberswalde, für die Überlassung seiner Daten und eine umfangreiche Fotobelegsammlung aus dem Eberswalder Gebiet sowie für hilfreiche Begleitung bei nächtlichen Exkursionen;

Herrn I. Seidel, Werneuchen, für die Mitteilung von Funddaten und Überlassung von Fotodokumenten aus unserem Gebiet;

Herrn Franz Theimer, Berlin, für die Überlassung von Daten aus dem Gebiet, inbesondere von seinem A. lepigone-Fund (einschließlich Foto) und für die Genehmigung zur Publikation dieses Nachweises;

Herrn Dr. A. Vössing, Berlin, (Nationalparkstiftung Unteres Odertal), für die Überlassung der Studie über Untersuchungsergebnisse zur Großschmetterlingsfauna im Nationalpark Unteres Odertal und für die Genehmigung zur Verwendung dieser Daten sowie für die Dedikation seiner Publikation „Der Internationalpark Unteres Odertal“"

Dem Landesamt für Umwelt, Gesundheit und Verbraucherschutz gilt mein Dank für die Erteilung arten- und flächenschutzrechtlicher Ausnahmegenehmigungen zur Erfassung der Schmetterlinge im Gebiet;

Dem Senckenberg Deutsches Entomologisches Institut, Müncheberg, danke ich für die Herausgeberschaft, insbesondere Frau C. Grunow für den Satz und ihr Entgegenkommen bei meinen Ergänzungswünschen zum Manuskript und Herrn C. Kutzscher für die Falteraufnahmen.

Ohne diese umfangreiche Zuarbeit und Unterstützung wäre der 4. Nachtrag nicht entstanden.

Herzlichen Dank.

\section{Spezieller Teil}

\subsection{Erläuterungen zu den Ausführungen im speziellen Teil}

Nomenklatur und Systematik: Um die Kontinuität zu wahren, wird die Nomenklatur und Systematik nach Karsholt \& Razowski 1996 in Gaedike \& Heinicke 1999 verwendet, abweichend davon wird bei den Rhopaloceren und Hesperiiden wie in Teil I der Fauna der Nomenklatur bei NÄssig (1995) und ReINHARDT (1995) gefolgt (siehe dazu RicherT 1999).

Pflanzennamen werden nach "SCHMEIL-FitsCHEN" (SEybold 2009) genannt; für Garten- bzw. Zierpflanzen, welche in l.c. nicht berücksichtigt sind, wurde zusätzlich „ROTHMALER“ 5 (JÄGER et al. 2008) genutzt.

Nummerierung und Reihenfolge der Arten entspricht der Darstellung in RicherT 1999-2004; außerdem werden bei allen Arten die Nummern der Europaliste (KARSHOLT \& RAzOWSKi 1996) angegeben, die auch in der Deutschlandliste (GAedike \& Heinicke 1999, GAEDIKE et al. 2017) verwendet wurden.

Schutz- und Gefährdungsstatus: Bei jeder Art, die einen besonderen Schutz- bzw. Gefährdungsstatus besitzt, wurde dieser den Ausführungen vorangestellt (FFHArten, besonders/streng geschützte Arten nach der „Verordnung zur Neufassung der BArtschV“ von 2005, Rote-Liste-Arten entsprechend Rote Liste Deutschland nach Bundesamt Für NATURschutz (Hrsg.) 2011, Rote Liste Land Brandenburg nach GELBRECHT et al. 2001).

Phänologie/Voltinismus: Um festzustellen, inwieweit sich bereits beobachtete Tendenzen der letzten Jahrzehnte fortsetzen, galt meine besondere Aumerksamkeit auch im neuen Beobachtungszeitraum den Veränderungen der Phänologie und des Voltinismus. Als messbare Größe wurde die absoluten Flugzeit genutzt (als absolute Flugzeit gilt der Zeitraum zwischen dem frühesten Auftreten der Imagines - absoluter Flugzeitanfang - und ihrem spätesten Auftreten - absolutes Flugzeitende im Freiland des untersuchten Gebietes; bei Arten mit mehreren Generationen beschränken sich diese Angaben in der Regel auf die erste und letzte Generation, da sich die einzelnen Generation bei vielen Arten nicht immer scharf abgrenzen lassen). Alle Abweichungen von bereits publizierten phänologischen Daten bis 2013 (vgl. Richert 2014, Redaktionsschluss Februar 2014) wurden für jedes Jahr des neuen Beobachtungszeitraumes 2014-2018 für die betroffenen Arten dokumentiert und am Ende des Nachtrages tabellarisch dargestellt und ausgewertet.

Fundortangaben erfolgen in der schon früher von mir genutzten Form:

Fundortangaben von Gewährsleuten sind mit deren Namen nach der Jahreszahl des Fundes versehen, 
Fundortangaben aus der Literatur sind mit Autor / Jahr gekennzeichnet; alle nicht derartig gekennzeichneten Jahresangaben beruhen auf eigenen Beobachtungen. Für schwer bestimmbare Arten lagen mir Belegexemplare zur Begutachtung / Bestimmung vor. Wenn eine Überprüfung nicht möglich war, wird darauf hingewiesen.

Die Fundortangaben werden einerseits den einzelnen Naturräumen unserer Landschaft $(\mathrm{a}-\mathrm{h})$ zugeordnet, andererseits dem betreffenden Messtischblatt und dessen Quadranten. Dadurch wird eine Auswertung für die Messtischblatt-(MTB)- Rasterkartierung möglich.

Der in Texten gebrauchte Ausdruck „im Gebiet“ bezieht sich auf den in der Gebietsabgrenzung angegebenen geographischen Raum (RICHERT 1999: 6 bzw. RICHERT 2014: 255).

Neue Nachweise von bekannten Fundorten: Unter dieser Rubrik werden Daten von bereits publizierten Fundorten mitgeteilt (vgl. RICHERT 1999-2004, 2010, 2014).

Fundorte bzw. Fundorte (Ergänzung): Unter der Rubrik Fundorte bzw. Fundorte (Ergänzung) werden im Gebiet neu entdeckte sowie bisher nicht publizierte Fundorte (u. a. aus bisher nicht bekannten Quellen, siehe Einleitung) aufgelistet.

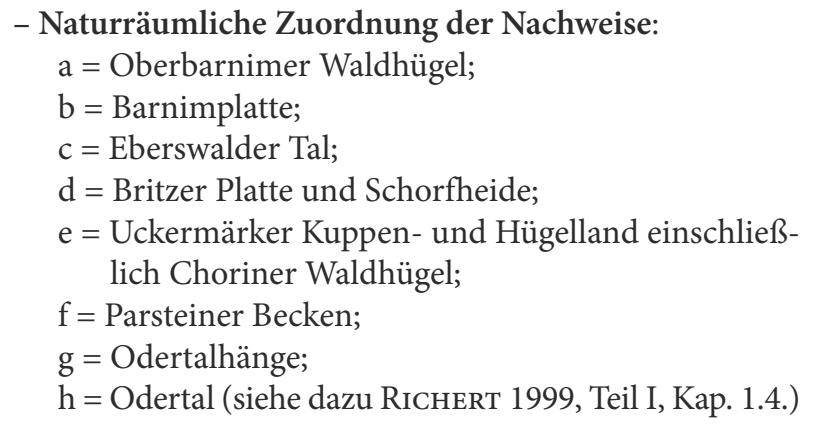

- MTB-Nr. mit Quadrantenbezeichnung in nachstehender Reihenfolge: I / II ; III / IV

\section{Abkürzungen:}

A $\quad=$ Anfang (in Verbindung mit der römischen Ziffer des jeweiligen Monats, die erste Dekade umfassend);

$$
\begin{array}{ll}
\text { a.K. } & =\text { am Köder; } \\
\text { aFA } & =\text { absoluter Flugzeitanfang; } \\
\text { aFE } & =\text { absolutes Flugzeitende; } \\
\text { a.L. } & =\text { am Licht; }
\end{array}
$$$$
\text { BArtschV = Bundesartenschutzverordnung; }
$$$$
\mathrm{d}=\text { diës }=\operatorname{Tag}(\mathrm{e}) \text { (in Verbindung mit Zahlen- }
$$
angaben zur Vordatierung der absoluten Flugzeit);

E = Ende (in Verbindung mit der römischen Ziffer des jeweiligen Monats, die dritte Dekade umfassend);

Ebw. = Eberswalde;

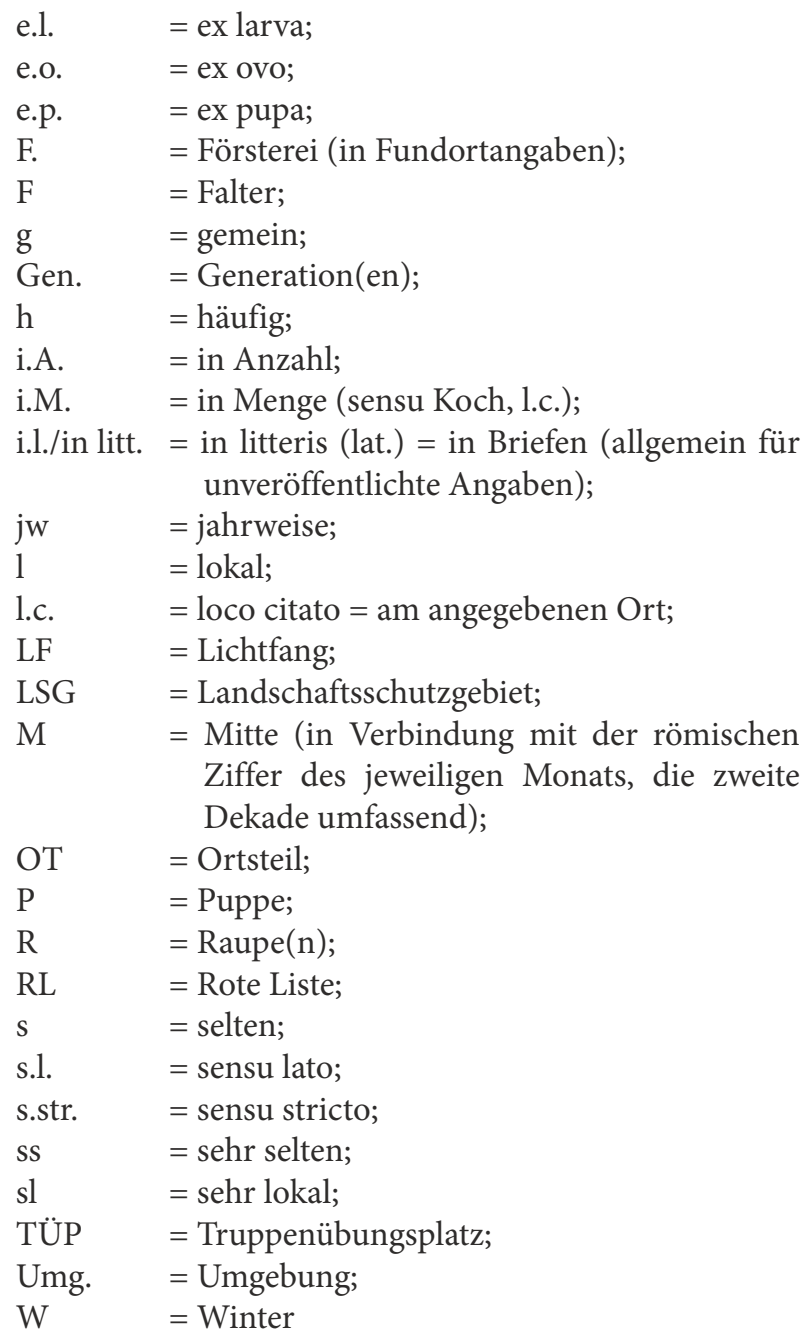

Kürzel für Biotoptypen (nach GeLBRECHT et al. 2001):

BW = Buchen- und Buchenmischwälder auf basischen Böden;

$\mathrm{CB}=$ Calluna und Besenginsterheiden (offen und leicht verbuscht);

EB = Eichengebüsch auf sandigen, trockenwarmen, offenen Standorten bzw. an Waldrändern;

EW = Eichen- und Eichenmischwälder;

FA = extensiv genutzte Flussauen und deren Randbereiche;

FM = flechtenreiche Mauern;

FW = flechtenreiche Eichen-, Laub- und Mischwälder;

GL $=$ Gartenlandschaft;

HE $\quad=$ Hecken mit einheimischen Gehölzen (z. B. Schlehe, Rose, Weißdorn);

HF = Hochstaudenfluren;

HW = Kiefern- und Eichenwälder mit Heidelbeeren;

$\mathrm{KW}=$ blütenreiche Kiefernwälder;

LL $\quad=$ lindenreiche Laubwälder;

LW = unterholzreiche Laubwälder;

MB = Moor- und Bruchwälder;

MW = Mähwiesen auf Niedermoor; 


\begin{tabular}{|c|c|}
\hline NM & $\begin{array}{l}=\text { offene Niedermoore einschließlich Seggen- } \\
\text { und Schilfröhrichte ohne Nutzung mit } \\
\text { angrenzenden Gewässerrandstrukturen; }\end{array}$ \\
\hline NW & $\begin{array}{l}=\text { moorige Nadelwälder mit Birken und } \\
\text { Heidelbeere; }\end{array}$ \\
\hline SM & $\begin{aligned}= & \text { oligotroph-saure Moore einschließlich Ledo- } \\
& \text { Pinetum; }\end{aligned}$ \\
\hline SO & = Sonderstandorte (Sand-, Kiesgruben etc.); \\
\hline TR & $\begin{aligned}= & \text { Trockenrasen (Dünen, Silbergrasfluren, } \\
& \text { halbkontinentale Halbtrockenrasen, Schaf- } \\
& \text { schwingelfluren, ältere Ackerbrachen); }\end{aligned}$ \\
\hline UW & $\begin{array}{l}=\text { ulmenreiche Wälder und Waldrandstru } \\
\text { turen; }\end{array}$ \\
\hline & = Weicholzauen; \\
\hline & $=$ innere und äußere Waldsäume \\
\hline
\end{tabular}

\subsection{Neu entdeckte Arten}

Nymphalidae (Edelfalter); (Zu Teil I: Allgemeiner Teil - Tagfalter, RICHERT 1999)

\section{1a (07259) Nymphalis xanthomelas (ESPER, 1781)}

- Verordnung zur Neufassung der BArtschV von 2005: Streng geschützte Art.

- RL Land Brandenburg: Kategorie 0 (ausgestorben oder verschollen);

- RL Deutschland (2011): D (Daten unzureichend).

Seit der Jahrtausendwende wurden gelegentliche Wiederfunde in Deutschland bekannt (vgl. REINHARDT et al. 2007; ReInHARDT \& Bolz 2011). Im Jahre 2013 wurde ein F in unserem Nachbargebiet bei Gartz a.O. entdeckt (Fotobeleg J. Müller, Kretschmer i.l.). Die Art, deren westliche Arealgrenze östlich unseres Gebietes liegt, unternimmt neuerdings wieder Vorstöße nach Westen und erreichte inzwischen auch unseren Raum: Am 19.III.2015 beobachtete Brauner im Stadtgebiet in Finow einen $\mathrm{F}$ (Belegfoto lag mir vor), der sich an einem Fensterrahmen sonnte und danach in einen blühenden Salweidenbusch (Salix caprea) flog und dort saugte. Damit liegt ein erster Nachweis aus unserem Gebiet vor (vgl. Richert 1999).

\section{Fundorte:}

c 3148/III Ebw. OT Finow/Stadtgebiet (2015: Brauner)

Lasiocampidae (Wollraupenspinner, Glucken); (Zu Teil II: Spinner und Schwärmer, RICHERT 2001)

\section{9a (6745) Malacosoma franconica (DENIs \& SCHIFFERMÜLLER, 1775)}

- Neufassung BArtschV 2005: Streng geschützte Art.

- RL Land Brandenburg: Kategorie 1 (vom Aussterben bedroht): TR;
- RL BR Deutschland (2011): Kategorie 1 (vom Aussterben bedroht).

Noch um die Jahrtausendwende verwiesen GeLbRECHT \& Kallies (2001) auf die dringende Notwendigkeit der Unterschutzstellung der Lebensräume der letzten mitteleuropäischen Populationen dieser bundesweit vom Aussterben bedrohten Art in Nordwestbrandenburg und in Mecklenburg-Vorpommern und forderten ein wissenschaftlich begründetes Pflegemanagement. Seither kam es zu einer unerwarteten süd- und südostwärts gerichteten Arealerweiterung im Norden Brandenburgs (Gelbrecht, Göritz \& Ockruck, 2011; Ockruck \& GeLBRECHT, 2011), begünstigt durch Stilllegung vormals landwirtschaftlich genutzter Flächen auf armen Böden, auf denen sich Sandtrockenrasen entwickelt hatten. Diese Entwicklung hat sich bis in unser Gebiet fortgesetzt: Im Frühjahr 2015 fand Haase (pers. Mitt.) bei Groß Schönebeck in Ortsnähe am Liebenthaler Weg 5 Häutungsnester der Art. Weitere Vorkommen wurden bei Böhmerheide entdeckt (Ockruck, pers. Mitt.; Haase, pers. Mitt.). Ein Männchen, e.l. 09.VI.2016 vom Weißen See bei Böhmerheide, leg. et coll. Haase, lag mir vor. Somit hat die Art inzwischen die Westgrenze unseres Gebietes erreicht. Im Jahre 2016 wurde die Art an diesen Plätzen vergeblich gesucht.

Im Jahre 2017 fand Haase Raupengesellschaften nördlich von Groß Schönebeck im Revier Rehluch auf einer gänzlich von Wald umgebenen Lichtung/Brache westlich der Priesterberge. Zur Absicherung des Artnachweises eingetragenen Raupen ergaben ein Männchen 20.VI.2017 e.l., ein Weibchen 23.VI.2017 e.l.

Dieses Vorkommen konnte auch 2018 bestätigt werden. Es wurden zahlreiche Raupen beobachtet. Als Beleg wurden am 15.V.2018 mehrere Raupen eingetragen, welche die F vom 04.-17.VI.2018 ergaben.

Fundorte:

d 3047/III Groß Schönebeck Umg./Liebenthaler Weg (2015: Haase); Schorfheide: Revier Rehluch, Abt. 38 und 61 westlich der Priesterberge (2017, 2018: Haase)

3146/II Böhmerheide Umg. (2015: Ockruck)/nördlich des Weißen Sees (2015: Haase) (westlich angrenzendes Nachbargebiet)

Noctuidae (Zu Teil III: Die Eulenfalter (Noctuidae et Pantheidae), Richert 2003)

348a (9078) Thysanoplusia orichalcea (FABRICIUS, 1775) = aurifera HüBNER, 1813

T. orichalcea ist eine palaeosubtropisch verbreitete Art (Segerer \& Hausmann 2011; Steiner in Ebert 1997), die infolge seltener Zuwanderung bzw. passiver Einschleppung auch in Mitteleuropa gefunden wurde (STEINER et al. 2014).

Am 17.II.2015 entdeckte meine Frau in der Küche unserer Wohnung in Ebw. OT Finow einen frisch geschlüpften F, dessen Herkunft zunächst völlig unklar blieb. In einem 
eingefrischten Bund frischer Petersilie aus dem Supermarkt fanden wir schließlich gut versteckt ein weißliches Gespinst mit der P-Exuvie. Nachfragen ergaben, dass die Petersilie aus Italien importiert worden war. Somit liegt eindeutig ein Fall von Verschleppung vor. Es handelt sich um einen Erstfund in Brandenburg (RICHERT 2015).

\section{Fundorte:}

c 3148/III Ebw. OT Finow/Stadtgebiet: Altenhofer Straße, im Hause (2015)

\section{5a (9479) Athetis lepigone (MösCHLER, 1860)}

A. lepigone ist eine östlich-kontinentale Art, die seit 2007 (als Arealerweiterer oder Irrgast?) auch in Deutschland gefunden wurde (GAEDIKE et al. 2017, WEISBACH \& GELBRECHT 2013). Im Jahre 2017 wurde sie mehrfach auch im Land Brandenburg, u. a. auch in unserem Gebiet nachgewiesen: Theimer hatte am 15.VIII.2017 einen $\mathrm{F}$ an der südlichen Grenze des Gebietes bei Biesenthal a.L. Auf diese unscheinbare Art ist zukünftig besonders zu achten.

Fundorte:

b 3247/II Biesenthal (2017: Theimer)

\subsection{Wieder entdeckte Arten}

\section{Papilionidae (Zu Teil I: Allgemeiner Teil - Tagfalter, RICHERT 1999)}

\section{3a (06957) Iphiclides podalirius (LinNaEus, 1758)}

- Verordnung zur Neufassung der BArtschV von 2005: Besonders geschützte Art.

- RL Land Brandenburg: Kategorie 2 (stark gefährdet);

- RL Deutschland (2011): Kategorie 2 (stark gefährdet).

Die Zuordnung dieser Art zu den Wiederentdeckungen (oder Neuentdeckungen?) ist insofern problematisch, als in der Vergangenheit außer der Sichtung eines Falters im Jahre 1975 weder Nachweise bekannt waren noch Belege aus dem Gebiet vorlagen. I. podalirius wurde deshalb seinerzeit nicht in die Liste der bei uns nachgewiesenen Schmetterlingsarten aufgenommen (RICHERT 1999).

Nachdem der Segelfalter seit den 1990er Jahren den Südosten Brandenburgs nahezu geschlossen besiedelt hat, wurde 2015/2016 eine nordwärts gerichtete Ausbreitung entlang des Odertals bis Lebus (Nachbargebiet) beobachtet (GELBRECHT et al. 2016).

Diese Entwicklung hat sich seither fortgesetzt. Inzwischen liegt auch eine neue Beobachtung aus unserem Gebiet vor: Ende Juli wurden zwei F auf dem Teufelsberg bei Oderberg gesichtet (Kretschmer, pers. Mitt.). Ob es sich um migrierende Falter handelte oder ob eine bleibende Ansiedlung im Gebiet erfolgt ist, bleibt zukünftigen Untersuchungen vorbehalten.
Umgehend erfolgten gezielte weitere Suchen auf den Oderhängen bei Falkenberg, Oderberg, Gabow, Stolzenhagen und Stolpe blieben zunächst erfolglos (Richert, Schwabe)

\section{Noctuidae (Zu Teil III: Die Eulenfalter (Noctuidae et Pantheidae, Richert 2003)}

\section{1 (9358) Schinia scutosa (DENIs \& SCHIFFER- MÜLLER, 1775)}

„Irrgast“ im Gebiet.

Am 15.IX.2014 wurde ein abgeflogener F auf dem Krugberg bei Brodowin a.L. gefangen. Am 17.III.2015 hatte ich einen sehr abgeflogenen $\mathrm{F}$ in meinem Garten in Finow a.L. Damit liegen nach 72 Jahren nun aktuelle Nachweise der in den Steppen Südosteuropas beheimateten Art aus unserem Gebiet vor.

\author{
Fundorte (Ergänzung): \\ c 3148/III Ebw. OT Finow/Stadtgebiet: Altenhofer Straße, im \\ Garten (2015) \\ f 3049/IV Brodowin/Krugberg (2014)
}

\section{5 (9797) Eremobia ochroleuca (DENIS \& SCHIF- FERMÜLLER, 1775)}

- RL Land Brandenburg: Kategorie 1 (vom Aussterben bedroht): TR;

- RL Deutschland (2011): Kategorie 2 (stark gefährdet).

Nachdem die Art seit mehr als 60 Jahren im Gebiet vermisst wurde (vgl. RicherT 2003), konnte sie nun erneut bei uns nachgewiesen werden: M VII 2014 wurde ein Vorkommen in der Uckermark bei Mürow bekannt (Gelbrecht, pers. Mitt.). Es handelte sich um ein unmittelbar nordöstlich angrenzendes Nachbargebiet. Die Vermutung lag nahe, dass die Art, die sich offenbar seit Jahren südwärts ausbreitet, inzwischen auch unser Gebiet erreicht hat. Eine Überprüfung der Vermutung im Parsteiner Becken, einer Exklave der Uckermärker Lehmplatte, war erfolgreich: Am 14.VII.2014 fand ich zwei F bei Neu Künkendorf an der Chaussee nach Parstein in der Agrarlandschaft am Straßenrand. Fünf weitere F fand ich am gleichen Tag auf dem Bunkerberg bei Herzsprung an der Straße nach Bölkendorf. Alle F saßen innerhalb von Glatthafer-Beständen auf Blüten von SkabiosenFlockenblumen (Centaurea scabiosa), woran sie z. T. auch saugten. Während die Männchen mehr oder weniger abgeflogen waren, waren die Weibchen überwiegend frisch.

Nachsuchen am gleichen Tag auf ähnlichen Standorten bei Klein Ziethen (Debritzberg) blieben erfolglos.

Das Vorkommen bei Herzsprung konnte im Jahre 2015 (15. und 17.VII. >4 F) und 2016 (01.VII. 1 F) bestätigt werden.

Inzwischen scheint die Art im Gebiet weiter nach SW vorgedrungen zu sein. Am 17.VII.2015 wurden $4 \mathrm{~F}$, am 
04.VIII.2015 3 F auf dem Debritzberg bei Klein Ziethen beobachtet. Damit liegt ein weiterer Fundort vor. Dieses Vorkommen konnte am 17.VII.2017 bestätigt werden (1 F).

Fast alle Falter saßen wieder auf/an/unter Blüten der Skabiosen-Flockenblume (Centaurea scabiosa), woran sie z. T. ruhten, z. T. saugten. Sie werden dabei mitunter Opfer von Spinnen (Belegfoto Schwabe).

2016 entdeckte Schwabe ein neues Vorkommen am Krummen See bei Bölkendorf. Dort fand er einen Falter, der im Blütenstand von Dost (Origanum vulgare) ruhte. Im Jahre 2017 wurde am 17.VII. ein F in der Feldflur bei Gellmersdorf an der Straße nach Lüdersdorf an einer Centaurea scabiosa-Blüte gefunden.

Die Suche in weiter südlich gelegenen potenziellen Habitaten (Drumlins bei Brodowin, Sonnenberge bei Parstein, Oderhänge bei Oderberg und Gabow, TÜP Trampe) verlief bisher ergebnislos.

Phänologie/Voltinismus: Univoltin. Die wenigen Funddaten $(\mathrm{n}=10)$ reichen für eine Kennzeichnung der Flugzeit im Gebiet nicht aus: 01.VII.(2016) bis 04.VIII. (2015).

Fundorte (Ergänzung):

e 3050/I Gellmersdorf Umg./an der Straße nach Lüdersdorf (2017: Richert)

f 3049/II Klein Ziethen Umg./Debritzberg (2015, 2017: Richert)

3050/I Neu Künkendorf Umg./Chaussee nach Parstein (2014); Herzprung Umg./Bunkerberg an der Straße nach Bölkendorf (2014: Richert; 2015: Richert, Schwabe); Bölkendorf Umg./Krummer See (2016: Schwabe)

\section{4 (9841) Gortyna flavago (DENIS \& SCHIFFER- MÜLLER, 1775)}

Die Art wurde lange im Gebiet vermisst. Die letzten mir bekannten Funde im Gebiet stammten aus dem Jahr 1998 (RicherT 2014). Nun liegt nach 17 Jahren ein neuer Fund aus dem Gebiet vor: Am 13.IX.2015 hatte Busse einen $\mathrm{F}$ im Ortsgebiet von Zerpenschleuse in seiner Lichtfalle. Wahrscheinlich kam G. flavago auch in der Zwischenzeit bei uns vor, aber nur in sehr geringer Bestandsdichte.

Ältere Funde (Belege in coll. Kittel) werden ergänzend nachgetragen.

Neue Nachweise von bekannten Fundorten:

c 3147/I Zerpenschleuse/Ortsgebiet: Berliner Straße, im Garten (2015)

3147/II Finowfurt/Hirtenweg - Besters Fließ (1985: Kittel)

Fundorte (Ergänzung):

c 3148/IV Ebw.-Westend/Unterheide: Wildparkstraße (1974, 1976: Kittel)
521 (9928) Hecatera bicolorata (HufNAGEL, 1766) = serena DENIS \& SCHIFFERMÜLLER, 1775

Am 27.VI.2016 hatte Schwabe an den Oderhängen südwestlich des Schiffshebewerks bei Niederfinow einen F a.L. Damit liegt ein Wiederfund nach 26 Jahren vor, in denen die Art im Gebiet als verschollen galt (RICHERT 2010).

Ein weiterer Falter wurde 2018 in Oderberg auf dem Geistberg a.L. beobachtet (Schwabe).

Phänologie/Voltinismus: 03.VI.(2018) aFA, phänologische Verfrühung 14d gegenüber bis 2013.

Fundorte (Ergänzung):

g 3149/4 Niederfinow/Oderhänge - ehemalige Kiesgruben südwestlich des Schiffshebewerks (2016: Schwabe)

3150/I Oderberg/Geistberg (2018: Schwabe)

\section{4 (10171) Graphiphora augur (FABRICIUS, 1775)}

- RL Land Brandenburg: Vorwarnliste: FA, GL, WS. Nachdem die Art seit 1997 im Gebiet vermisst wurde, kann nunmehr ein neuer Nachweis gemeldet werden: Am 07.VII.2014 hatte ich einen abgeflogenen Falter in den stillgelegten Steingruben bei Groß Ziethen a.L. Wahrscheinlich kam G. augur auch zwischenzeitlich bei uns vor, allerdings in sehr geringer Bestandsdichte.

Fundorte (Ergänzung):

e 3049/I Groß Ziethen Umg./Steingruben an den Ihlowbergen (2014, Einzelfund)

\section{Geometridae (Zu Teil IV: Die Spanner (Geome- tridae), RICHERT 2004)}

\section{0 (7606) Plagodis pulveraria (Linnaeus, 1758)}

- RL Land Brandenburg: Kategorie 2 (stark gefährdet): LW.

Für die im Gebiet seit 1992 als verschollen gemeldete Art (Richert 2010: 160) liegt ein neuer Nachweis vor: Am 08.VI.2014 hatte Ockruck einen F im NSG Biesenthaler Becken am Langerönner Weg a.L. Das ist zugleich ein Erstfund im Naturraum b (Barnimplatte, vgl. RicHERT 2004).

Fundorte (Ergänzung):

b 3247/II Biesenthal Umg./NSG Biesenthaler Becken: Langerönner Weg (2014: Ockruck)

\section{9 (8366) Eustroma reticulata (DENIS \& SCHIF- FERMÜLLER, 1775)}

- RL Land Brandenburg: Kategorie 2 (stark gefährdet): $\mathrm{MB}$;

- RL Deutschland: Vorwarnliste.

Seit 1985 im Gebiet verschollen (Richert 2010). Am 16.VII.2018 hatte Schwabe im NSG Plagefenn einen F 
am Licht. Damit liegt ein Wiederfund nach 33 Jahren vor. Offenbar ist die Art im Gebiet bodenständig, jedoch so selten, dass sie kaum gefunden wird. Bei mehrjährigen faunistischen Untersuchungen im NSG Plagefenn (RICHERT 2005) konnte sie dort nicht nachgewiesen werden.

\section{Fundorte (Ergänzung):}

e 3149/II Liepe Umg./Forst Chorin: NSG Plagefenn - LedumMoor am Fuß der Plageberge (2018: Schwabe \& Richert)

\subsection{Ergänzungen zu den Anmerkungen} in den Teilen I - IV (RICHERT 1999 - 2004) (aktualisierte und neue Fundortangaben sowie Beobachtungen zur Biologie, Ökologie und Phänologie)

Neben aktuellen Nachweisen werden bisher nicht berücksichtigte ältere Funde (nach Belegen in coll. Kittel, nach Untersuchungsergebnissen 1994-1995 von Borkowski aus dem Unteren Odertal, nach Mitteilungen von Barthelme und Rinnhofer aus der 2. Hälfte des vorigen Jahrhunderts aus dem Eberswalder Raum sowie nach Psychidae-Belegen/Aufsammlungen von F. Theimer aus den 1990er Jahren bei Oderberg) ergänzend mitgeteilt.

Angesichts des dramatischen Insektenschwundes (vgl. SEgERER 2017) werden bei weiteren, ehemals als allgemein verbreitet geltenden Arten, welche neuerdings aber vielfach unregelmäßg und nicht mehr häufig beobachtet werden, alle mir bekannten Fundorte angegeben. Die Auswahl ist subjektiv.

\section{Zu Teil I: Tagfalter (RICHERT 1999)}

\section{Hesperiidae (Dickkopffalter)}

\section{1 (6901) Pyrgus malvae (Linnaeus, 1758)}

- Verordnung zur Neufassung der BArtschV von 2005: Besonders geschützte Art.

- RL Land Brandenburg: Kategorie 3 (gefährdet): TR, MW, WS;

- RL Deutschland (2011): Vorwarnliste.

Neue Nachweise von bekannten Fundorten:

c 3149/III Stecherschleuse Umg./ehemaliges Kiesgrubengelände nördlich des Ortes (2017: Richert)

d 3148/I Lichterfelde Umg./NSG Buckowseerinne: Trockenhänge südwestlich Margaretenhof (2014)

g 3150/III Gabow/Granitberggebiet (2014)

Fundorte (Ergänzung):

g 3150/I Oderberg/Geistberg (2018: Schwabe)

\section{4 (6882) Carcharodus alceae (ESPER, 1780)}

- Verordnung zur Neufassung der BArtschV von 2005: Besonders geschützte Art.

- RL Land Brandenburg: Kategorie 3 (gefährdet): TR, GL.

Weitere im Gebiet festgetellte Nektarsaugpflanze: Hydrangea macrophylla, Garten- (Bauern-) Hortensie. Phänologie/Voltinismus: 08.IX.(2018) aFE 2. Gen., phänologische Verspätung 16d gegenüber bis 2013.

\section{Neue Nachweise von bekannten Fundorten: \\ b 3149/III Sommerfelde Umg./ehemaliger TÜP Panzerbahn (2016: Seidel) \\ 3248/II Trampe/ehemaliger TÜP (2016: Seidel) \\ c 3148/III Ebw. OT Finow/Stadtgebiet: Altenhofer Straße, im Garten (2015, 2016, 2017, 2018: Richert) \\ d 3148/I Lichterfelde/NSG Buckowseerinne: Streuobstwiesen südöstlich vom Gr. Buckowsee (2018) \\ g 3050/II Stolzenhagen/NSG Krähen- und Jungfernberge (2018: Richert \& Schwabe) \\ 3149/II Trockenweide Liepe (2017: Brauner) \\ 3149/IV Falkenberg (Hohenfinow) Umg./NSG Schäfergrund (2016: Seidel)}

Fundorte (Ergänzung):

c 3148/I Finowfurt OT Steinfurt/Oder-Havel-Kanal an der Straßenbrücke (2017)

d 3147/I Groß Schönebeck Umg./Mülldeponie am Weg nach Klandorf östlich der Bahnlinie (2018: Haase)

\section{5 (6879) Erynnis tages (LinNAEUs, 1758)}

- RL Land Brandenburg: Kategorie 3 (gefährdet): TR, MW.

Bereits Mitte der 1990er Jahre fand Borkowski die Art im Gellmersdorfer Forst. Demnach war sie nach Jahrzehnte langem Fehlen bereits früher als bisher bekannt im Gebiet erneut aufgetaucht (vgl. RICHERT 2010).

Auf dem ehemaligen TÜP Trampe war die 1. Gen. 2014 häufig: Ich zählte insgesamt 19 F. 2017 beobachtete ich im Gelände der ehemaligen Kiesgruben bei Stecherschleuse ca. $30 \mathrm{~F}$.

Weitere im Gebiet festgestellte Nekarsaugpflanzen (vgl. RICHERT 2010 und 2014):

Cerastium arvense, Acker-Hornkraut; Glechoma hederacea, Gundermann; Origanum vulgare, Dost; Senecio vernalis, Frühlings-Greiskraut; Syringa vulgaris, Gewöhnlicher Flieder (O. Brauner).

Phänologie/Voltinismus:

1. Gen.: 22.IV.2014, phänologische Verfrühung 3d, 21.IV. (2015) aFA, erneute phänologische Verfrühungen 1d, insgesamt 4d gegenüber bis 2013;

2. Gen.: 23.VIII.(2015) aFE, phänologische Verspätung $18 \mathrm{~d}$ gegenüber bis 2013 .

Im Jahre 2016 wurde am 31.VIII. ein frischer F auf dem TÜP Trampe beobachtet, weitere 3 abgeflogene F am 
03.IX.: aFE, erneute phänologische Verspätung von 11d, insgesamt also um 29 d gegenüber bis 2013.

Unklar ist, ob es sich hier um F einer partiellen 3. Gen. oder um Nachzügler der 2. Gen. handelt. Die 1. Gen wurde in diesem Jahr E (22.) IV bis E (24.) V beobachtet, die 2. Gen. ab M (20.)VII. Somit ist die Ausbildung einer partiellen 3. Gen. nicht auszuschließen. Angesichts des wechselhaften Wetters im August könnte es sich bei den späten Faltern um Nachzügler oder um eine langgestreckte 2. Gen. handeln.

Neue Nachweise von bekannten Fundorten:

b 3248/I Trampe/ehemaliger TÜP (2014, 2018: Richert, 2016: Richert, Seidel; 2017: Seidel)

c 3149/IV Stecherschleuse Umg./am Rande des ehemaligen Kiesgrubengeländes nordöstlich des Ortes (2015: Schwabe; 2017: Richert)

g 3050/II Stolzenhagen/NSG Krähen- und Jungfernberge (2015, 2016: Richert, 2018: Richert \& Schwabe)

3149/II Trockenweide Liepe (2017: Brauner)

3149/IV Falkenberg (Hohenfinow) Umg./NSG Schäfergrund (2016: Seidel; 2017: Brauner)

3150/I Oderberg/NSG Pimpinellenberg (2014, 2015, 2016, 2017, 2018: Brauner); Geistberg (2015, 2016, 2018: Schwabe)

3150/III Gabow/Granitberggebiet (2014: Richert)

3150/IV Altglietzen Umg./Gabower Berge (2017: Brauner)

Fundorte (Ergänzung):

c 3149/I Niederfinow Umg./Oder-Havel-Kanal an der Klosterbrücke (2016: Richert)/in Höhe Pipelinetrasse (2017: Brauner)

3149/III Ebw./Finowbruch am Eichwerder (2018: Schwabe); Stecherschleuse Umg./ehemaliges Kiesgrubengelände nördlich des Ortes (2017: Richert), Hochfläche südwestlich der Waldstraße (2017: Richert)

g 3050/II Gellmersdorf Umg./Gellmersdorfer Forst: Mühlenberggebiet (1994/1995: Borkowski); Stolpe/Trockenhänge östlich der Straße nach Crussow und am Stadtweg (2018)

\section{6 (6917) Heteropterus morpheus (PALlas, 1771)}

- RL Land Brandenburg: Kategorie 3 (gefährdet): NM, MB.

Auf dem ehemaligen TÜP Trampe wurde der F A VII 2016 lokal „ungewöhnlich häufig“beobachtet (pers. Mitt. I. Seidel).

Weitere im Gebiet festgestellte Nektarsaugpflanzen (vgl. RICHERT 1999 u. 2010):

Echium vulgare, Natterkopf; Eupatorium cannabium, Wasserdost; Jasione montana, Berg-Sandglöckchen; Medicago x varia, Luzerne; Origanum vulgare, Dost; Stachys palustris, Sumpf-Ziest.

Außerdem wurde Saugen an feuchter Moor-Erde beobachtet.

Neue Nachweise von bekannten Fundorten:

b 3248/I Trampe/ehemaliger TÜP (2016: Richert, Seidel; 2018: Richert)
Fundorte (Ergänzung):

b 3148/IV Ebw. Umg./Oberheide: Waldschneise am Leuenberger Graben zwischen Möllergrab (am WiebeckeDamm) und Leuenberger Wiesen (2014: Schwabe)

3249/I Feldsoll östlich Trampe (2017: Brauner)

c 3147/IV Finowfurt Umg./Finowfließniederung nördlich des Lehns-Sees (2015)

3148/III Ebw. OT Finow Umg./Forst Finowtal: Biesenthaler Straße nordöstlich Flugplatz (2015: Brauner), Fotovoltaikanlage Flugplatz Finow (2014: Brauner)

d 3047/IV Groß Schönebeck/Waldinnensaum bei Meelake (2018: Brauner)

e 3149/I Chorin Umg./Waldmoor östlich Kloster Chorin (2018: Brauner)

f 3049/IV Brodowin Umg./Parsteiner See - Westufer (2015)

3050/I Bölkendorf Umg./Krummer See (2016: Schwabe)

\section{6a (06919) Carterocephalus palaemon (PALlas, 1771)}

- RL Land Brandenburg: Vorwarnliste: WS, MB.

Barthelme legte sechs Sammlungsexemplare vor, die er im ehemaligen Kiesgrubengelände zwischen OderHavel-Kanal und dem Ort Stecherschleuse in den Jahren 2013, 2015 und 2016 „auf Massenbeständen von Vogelwicke" gefunden hatte. Offenbar handelt es sich bei der Angabe der Nektarsaugpflanze um Vicia tenuifolia, Feinblättrige Wicke, eine Art der Artengruppe Vogel-Wicke, Vicia cracca agg., die am angegebenen Fundort Massenbestände ausgebildet hat. Brauner beobachtete den F beim Saugen an Rubus caesius, Kratzbeere.

Damit liegt ein erster Nachweis im Naturraum c (Eberswalder Tal, vgl. Richert 1999) vor. Intensive Nachsuchen in diesem Gelände blieben in den Jahren 2017 und 2018 erfolglos. Auch im NSG Krähen- und Jungfernberge bei Stolzenhagen suchte ich 2016 vergeblich nach der Art. Phänologie/Voltinismus: Univoltin; die neuen Funde erfolgten im Zeitraum 30.V.(2013) - 16.VI.(2016).

Fundorte (Ergänzung):

c 3149/III Stecherschleuse Umg./ehemaliges Kiesgrubengelände nördlich des Ortes (2013, 2015, 2016: Barthelme)

g 3050/II Alt Galow, südlich des Ortes (2018: Brauner) (nördlich angrenzendes Nachbargebiet)

\section{7 (06920) Carterocephalus silvicola (MEIGEN,} 1829)

- RL Land Brandenburg: Kategorie 1 (vom Aussterben bedroht), MB, LW;

- RL Deutschland (2011): Kategorie 2 (stark gefährdet). Ein bisher unbekannter älterer Fund wird nachgetragen: Barthelme \& Rinnhofer fanden die Art „1991 letztmalig am Eichwerder" bei Eberswalde.

Weitere im Gebiet festgestellte Nektarsaugpflanze (vgl. RICHERT 1999):

Scutellaria galericulata, Sumpf-Helmkraut; Symphytum officinale, Gewöhnlicher Beinwell (Gottwald). 
Fundorte (Ergänzung):

c 3149/III Eberswalde/Eichwerder (1991: Barthelme \& Rinnhofer)

\section{8 (6924) Thymelicus sylvestris (PoDA, 1761)} = thaumas HUFNAGEL, 1766

Weitere im Gebiet festgestellte Nektarsaugpflanzen (vgl. RICHERT 2014):

Achillea millefolium, Gemeine Schafgarbe; Anchusa officinalis, Gewöhnliche Ochsenzunge; Ballota nigra, Schwarznessel; Carduus crispus, Krause Distel; Centaurea scabiosa, Skabiosen-Flockenblume; Dianthus carthusianorum, Karthäuser-Nelke; Jasione montana, Berg-Sandglöckchen; Knautia arvensis, Acker-Witwenblume; Medicago x varia, Luzerne; Petrorhaga prolifera, Sprossende Felsennelke; Scabiosa columbaria, TaubenSkabiose; Symphytum offinalis, Gewöhnlicher Beinwell; Trifolium medium, Mittlerer Klee; Trifolium pratense, Rot- (Wiesen-)Klee; Vicia cracca, Vogel-Wicke; Vicia villosa, Zottige Wicke.

Phänologie/Voltinismus: 05.VI.(2018) aFA F i.A. auf Trockenhängen bei Stolpe (Oderhänge!); phänologische Verfrühung 2d gegenüber bis 2013 .

\section{9 (6923) Thymelicus lineola (OCHSENHEIMER, 1808)}

Weitere im Gebiet festgestellte Nektarsaugpflanzen (vgl. RICHERT 2014):

Arctium tomentosum, Filzige Klette; Anchusa officinalis, Gewöhnliche Ochsenzunge; Armeria maritima, Gewöhnliche Grasnelke; Ballota nigra, Schwarznessel; Centaurea jacea, Gemeine (Wiesen-) Flockenblume; Centaurea stoebe, Rispen-Flockenblume; Cirsium palustre, Sumpf-Kratzdistel; Cirsium vulgare, Gemeine Distel; Convolvulus arvensis, Acker-Winde; Epilobium hirsutum, Zottiges Weidenröschen; Gentiana cruciata, KreuzEnzian; Helichrysum arenarium, Sand-Strohblume; Jasione montana, Berg-Sandglöckchen; Matricaria spec., Kamille; Medicago x varia, Luzerne; Onopordum acanthium, Eselsdistel; Petrorhaga prolifera, Sprossende Felsennelke; Prunella vulgaris, Gemeine Braunelle; Rubus fruticosus agg., Brombeere; Scabiosa columbaria, Tauben-Skabiose; Trifolium pratense, Wiesen-(Rot-) Klee; Vicia villosa, Zottige Wicke.

\section{0 (6925) Thymelicus acteon (ROTTEMBURG, 1775)}

- RL Land Brandenburg: Kategorie 2 (stark gefährdet), TR;

- RL Deutschland (2011): Kategorie 3 (gefährdet).

Barthelme \& Rinnhofer führen aus der 2. Hälfte des letzten Jahrhunderts das NSG Pimpinellenberg bei Oderberg als Fundort auf. Damit wird die Angabe von Ebert \& Kruel („Oderberg/Trockenhänge“, RicherT 1999) konkretisiert. Außerdem wird der Eichwerder bei Eberswalde genannt. Dieser Fundort fiel Siedlungsfolgen zum Opfer: Dort türmt sich jetzt eine riesige, abgedeckte Müllhalde auf.

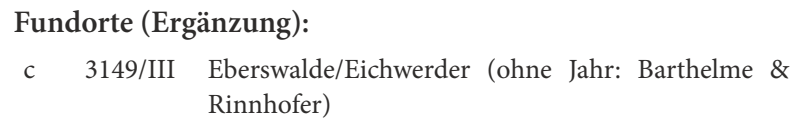

\section{1 (6928) Hesperia comma (LiNNAEUs, 1758)}

- RL Land Brandenburg: Kategorie 2 (stark gefährdet), $\mathrm{CB}, \mathrm{TR}$;

- RL Deutschland (2011): Kategorie 3 (gefährdet).

Ein mir bisher nicht bekannter Fund aus älterer Zeit in der Eberswalder Unterheide (Beleg in coll. Kittel) wird nachgetragen.

Weitere im Gebiet festgestellte Nektarsaugpflanzen (vgl. RICHERT 2010):

Buddleja davidii, Chinesischer Sommerflieder; Tanacetum vulgare, Rainfarn (Brauner).

Diese Beobachtung an Sommerflieder erfolgte in meinem Garten im August 2015, der überdurchschnittlich heiß und trocken war, sodass in den ca. zwei Kilometer entfernten angestammten Lebensräumen kaum Nektarsaugpflanzen zur Verfügung standen. Auch Brauner beobachtete am 18.VIII.2018 den Blütenbesuch an Buddleja davidii sowie an Lobelia erinus (Blaue Lobelie) in einem Garten in Finow. Deshalb ist ein gelegentliches Ausweichen der F in blütenreiche Gärten zu vermuten.

Neue Nachweise von bekannten Fundorten:

c 3148/III Ebw. OT Finow/Stadtgebiet, Altenhofer Straße, im Garten (2015) und Biesenthaler Straße, im Garten (2018: O. Brauner) und Umg./Forst Finowtal: Photovoltaikanlage auf dem Flugplatz (2014-2017: Brauner)

3149/IV Stecherschleuse Umg./am Rande des ehemaligen Kiesgrubengeländes nordöstlich des Ortes (2017)

g 3050/II Gellmersdorf Umg./Gellmersdorfer Forst: Mühlenberggebiet (1994/1995: Borkowski)

3150/III Gabow Umg./Gabower Berge östlich Granitberg (2018: Richert, Schwabe)

Fundorte (Ergänzung):

c 3148/IV Eberswalde-Westend/Unterheide: Drehnitzwiesen (1969: Kittel)

3050/IV Lunow Umg./Bereich Lunower Hölzchen (1994/ 1995: Borkowski)

\section{2 (6930) Ochlodes sylvanus (ESPER, 1777) = venatus $($ BREMER \& GREY, 1853)}

Weitere im Gebiet festgestellte Nektarsaugpflanzen (vgl. RICHERT 2014):

Armeria maritima, Gewöhnliche Grasnelke; Anchusa officinalis, Gewöhnliche Ochsenzunge; Hieracium pilosella, Kleines Habichtskraut; Iberis umbellata, Doldige Schleifenblume; Iris pseudacorus, Wasser-Schwertlilie; 
Trifolium medium, Mittlerer Klee; Vicia tenuifolia, Schmalblättrige Wicke.

Phänologie/Voltinismus: Am 01.IX.2018 fing Haase bei Groß Schönebeck ein frisches Männchen. Damit liegt ein weiterer Nachweis für die Ausbildung einer partiellen 2. Gen. in günstigen Jahren im Gebiet vor (vgl. RICHERT 2004 1. Nachtrag).

\section{Papilionidae (Schwalbenschwänze)}

\section{3 (6960) Papilio machaon (Linnaeus, 1758)}

- Verordnung zur Neufassung der BArtschV von 2005: Besonders geschützte Art.

- RL Land Brandenburg: Vorwarnliste: TR, GL, WS.

In meinem Garten beobachtete ich E VIII 2014 auf einem Weg eine auffällige Ansammlung zahlreicher Feuerwanzen (Pyrrhocoris apterus), welche am Kadaver einer P. machaon-R saugten.

Bei Stolpe a.O. sah ich die Falter am 30.IV.2018 in bemerkenswerter Anzahl (insgesamt $10 \mathrm{~F}$ ), wo sie auf den Trockenhängen an der Straße nach Crussow in rastlosem Flug patrouillierten.

Weitere im Gebiet dokumentierte Nektarsaugpflanzen (vgl. Richert 2014):

Centaurea cyanus, Kornblume; Cosmos bipinnatus, Kosmee; Dianthus carthusianorum, Karthäuser-Nelke; Trifolium pratense, Wiesenklee.

Als weitere Raupennahrungspflanze nutzt die Art im Gebiet Foeniculum vulgaris, Echten Fenchel.

Phänologie und Voltinismus:

Am 05.IX.2016 fand ich $2 \mathrm{R}$ an Dill in meinem Garten, die Verpuppung erfolgte am 12/13.IX.2016. Bemerkenwert sind die Schlupfdaten der F: 04.VI.2017 $1 \mathrm{~F}$ e.l., 04.VII.2017 1 F e.l. Demnach gehören im Juli zu beobachtende F nicht zwangsläufig zu einer 2. Gen.

Das Jahr 2018 fiel in Nordostbrandenburg durch einen deutlich zu warmen April (4,7 über langjährigem Mittel) und Mai (3,7 über langjährigem Mittel) auf. Extreme Hitze und Trockenheit kennzeichneten auch den Sommer. Die Auswirkung auf den Voltinismus im Gebiet zeigen folgende Daten: Die 1. Gen. flog bereits ab April. Am 06.V.2018 trug Haase bei Groß Schönebeck frisch an Peucedanum oreoselinum abgelegte Eier ein. Nach ca. einer Woche schlüpften die Raupen; Verpuppung ab 29.V. Bereits ab 16.VI. schlüften die F der 2. Gen. (gesamte Entwicklungsdauer vom Ei bis zum Falter 41 Tage).

Aus am 05.VII.2018 von Haase eingetragenen Eiern schlüpften die R ab 13.VII., die Verpuppung erfolgte ab 28.VII., ab 07.VIII. schlüpften die Falter der 3. Gen. Die gesamte Entwicklung vom Ei bis zur Imago vollzog sich also in nur 33 Tagen.

Der Falterflug dauerte bis M IX.

Neue Nachweise von bekannten Fundorten:

b 3149/III Sommerfelde Umg./ehemaliger TÜP Panzerbahn (2016: Seidel)

3248/II Trampe/ehemaliger TÜP (2014: Richert, 2016: Seidel) c 3147/I Zerpenschleuse/Ortsgebiet: Berliner Straße, im Garten (2018: Busse)

3148/II Ebw.-Nordend - Siedlungweg an den Buchen, im Garten (2018: Prietzschk) und Umg./Oder-HavelKanal nördlich des Ortes (2015: Schwabe)

3148/III Finowfurt/Wiesen am Finowkanal westlich der Schleuse (2014); Ebw. OT Finow/Stadtgebiet: Altenhofer Straße, im Garten (2014, 2015, 2016, 2017 2018)

3148/IV Ebw./Stadtgebiet: Kleingartenanlage im Schwärzetal nördlich Forstbotanischer Garten (2015: Schwabe)

3149/III Stecherschleuse Umg./ehemaliges Kiesgrubengelände nördlich des Ortes (2017: Richert)

3149/IV Stecherschleuse Umg./am Rande des ehemaligen Kiesgrubengeländes nordöstlich des Ortes (2017: Richert)

d 3047/III Groß Schönebeck/Ortsgebiet: Friedenstraße, im Garten (2018: Haase)

3148/I Lichterfelde Umg./NSG Buckowseerinne: Streuobstwiesen - Moospfuhlgebiet südöstlich des Gr. Buckowsees (2014); Triften südlich Margaretenhof (2015)

e 3049/I Groß Ziethen Umg./Steingruben an den Ihlowbergen (2014)

f 3049/IV Brodowin Umg./Gr. Rummelsberg (2015)

g 3050/II Stolpe a.O./Trockenhänge östlich der Straße nach Crussow und am Stadtweg (2018: Richert); Stolzenhagen/NSG Krähen- und Jungfernberge (2015, 2016 : Richert);

3149/II Liepe/Trockenweide (2017: Brauner)

3150/I Oderberg/NSG Pimpinellenberg (2014, 2015, 2016, 2017, 2018: Brauner)

3150/IV Altglietzen Umg./Gabower Berge (2017: Brauner)

Fundorte (Ergänzung):

b 3247/I Prenden/Ortsgebiet (2017: R. M. Siegel).

c 3148/III Ebw. OT Finow Umg./Forst Finowtal: Fotovoltaikanlage Flugplatz Finow (2014, 2017: Brauner)

3149/III Sommerfelde Umg./Finowbruch nördlich des Ortes, am Bahnübergang (2018: Schwabe)

e 3049/II Klein Ziethen Umg./Debritzberg $(2014,2015)$

g 3050/II Gellmersdorf Umg./Gellmersdorfer Forst: Mühlenberggebiet (1994/1995: Borkowski);

3050/IV Lunow Umg./Bereich Lunower Hölzchen (1994/ 1995: Borkowski)

\section{Pieridae (Weißlinge)}

\section{Der Leptidea sinapis-reali-juvernica-Komplex}

Leptidea sinapis und Leptidea reali waren von FreEse \& FIEDLER (2002) als echte Biospecies beschrieben worden (siehe dazu Richert 2010: 12).

Leptidea juvernica wurde „erst in jüngster Zeit als eigenständige Art erkannt (DINCĂ et al. 2011). Sie lässt sich von L. reali REISSINGER, 1989, durch verschiedene, voneinander unabhängige molekulare Merkmale abgrenzen, allerdings nicht morphologisch. L. reali scheint auf Südwest- und Südeuropa beschränkt zu sein und kommt nach bisheriger Kenntnis in Deutschland nicht vor" (SEgerer \& Hausmann 2011: 58)

Demnach sind die Angaben zum Taxon Leptidea reali in Richert (2010) und (2014) dem Taxon Leptidea juver- 
nica zuzuordnen. Allerdings sind molekulargenetische Untersuchungen an Funden aus dem Gebiet nicht erfolgt. Da L. sinapis und reali nach äußeren Merkmalen nicht sicher zu unterscheiden sind, können Freilandbeobachtungen ohne anschließende genitalmorphologische Untersuchung der beobachteten Falter nur für den Artenkomplex ausgewertet werden: Die Eiablage von Faltern des sinapis-juvernica-Komplexes beobachtete Brauner an Vicia hirsuta, Behaarte Wicke, an Lotus corniculatus, Gewöhnlicher Hornklee und an Securigera varia, Bunte Kronwicke.

F dieses Art-Komplexes wurden 1994/1995 von Borkowski bei Gellmersdorf im Gellmersdorfer Forst (Mühlenberggebiet) und bei Lunow im Gebiet des Lunower Hölzchens nachgewiesen.

Die folgenden Beobachtungsdaten zu den beiden Arten sind durch Genitalpräparation gesichert.

\section{4 (6966) Leptidea sinapis (LINNAEUs, 1758)}

- RL Land Brandenburg: Vorwarnliste: WS, MW;

- RL Deutschland (2011): Kategorie D (Daten unzureichend).

Auf den Trockenhängen bei Stolpe a.O. kommt die Art sympatrisch mit L. juvernica vor.

Als Nektarsaugpflanze wurde im Gebiet Echium vulgare, Gewöhnlicher Natternkopf, festgestellt. Die Angabe ist durch Genitalpräparation des betreffenden Falters gesichert.

Ich beobachtete die Eiablage am 03.VII. auf Magerrasen an Hasen-Klee (Trifolium arvense). Nach langem Suchflug in der Vegetation (lockerer Calamagrostis-Bestand) heftete das Weibchen ein $\mathrm{Ei}$ an der Unterseite eines Blattes an. Das Weibchen wurde nachträglich zur sicheren Bestimmung durch Genitalpräparation gefangen. Weiterhin beobachtete ich die Eiablage an Feinblättriger Wicke, Vicia tenuifolia.

\section{Neue Nachweise von bekannten Fundorten: \\ c 3149/III Stecherschleuse Umg./ehemaliges Kiesgrubenge- lände nördlich des Ortes (2017, det. genit. Richert)}

\section{Fundorte (Ergänzung):}

c 3148/III Ebw. OT Finow Umg./Forst Finowtal: Biesenthaler Straße nahe Samithsee (2017, det. genit. Richert); Stellweg nördlich Einflugschneise Flugplatz (2018, det. genit Richert)

e 3049/I Groß Ziethen Umg./stillgelegte Steingruben an den Ihlowbergen (2014, det. genit. Richert)

g 3050/II Stolpe a.O./Trockenhänge am Stadtweg (2018, det. genit. Richert)

\section{4a (6967) Leptidea reali (REISSINGER, 1989) - Leptidea juvernica Williams, 1946}

- RL Land Brandenburg: Nicht aufgeführt;

- RL Deutschland (2011): Kategorie D (Daten unzureichend).
Die Art wurde im Naturraum e (Uckermärker Kuppenund Hügelland, vgl. Richert 1999) neu nachgewiesen: Sie kommt in den Steingruben bei Groß Ziethen und auf Trockenhängen bei Stolpe a.O. sympatrisch mit L. sinapis vor.

Weitere im Gebiet festgestellte Nektarsaugpflanzen (vgl. RICHERT 2014):

Cirsium arvense, Acker-Kratzdistel; Epilobium parviflorum, Kleinblütiges Weidenröschen.

\section{Neue Nachweise von bekannten Fundorten: \\ b 3248/II Trampe/ehemaliger TÜP (2014, det. genit.; 2016, det. genit. Richert) \\ Fundorte (Ergänzung): \\ c 3147/IV Finowfurt Umg./Finowfließniederung nördlich des Lehns-Sees (2015, det. genit. Richert) \\ e 3049/I Groß Ziethen Umg./stillgelegte Steingruben an den Ihlowbergen (2014, det. genit. Richert) \\ g 3050/II Stolpe Umg./Trockenhänge an der Straße nach Crus- sow (2017, det. genit. Richert), Trockenhänge am Stadtweg (2018, det. genit. Richert; Stolzenhagen/ NSG Krähen- und Jungfernberge (2016, det. genit. Richert)}

\section{5 (7021) Colias hyale (Linnaeus, 1758)}

- Verordnung zur Neufassung der BArtschV von 2005: Besonders geschützte Art.

Weitere im Gebiet festgestellte Nektarsaugpflanzen (vgl. RICHERT 2014):

Aster linosyris, Gold-Aster; Aster novi-belgii, Neubelgische Herbstaster; Capsella bursa-pastoris, Gewöhnliches Hirtentäschelkraut; Centaurea jacea, Gemeine (Wiesen-) Flockenblume; Cirsium oleraceum, Kohl-Kratzdistel; Cirsium vulgare, Gemeine Kratzdistel; Dianthus cartusianorum, Karthäuser-Nelke; Epilobium angustifolium, Schmalblättriges Weidenröschen; Hyopchoeris radicata, Gemeines Ferkelkraut; Jasione montana, Berg-Sandglöckchen; Knautia arvensis, Acker-Witwenblume; Leontodon autumnalis, Herbst-Löwenzahn; Origanum vulgare, Dost; Picris hieracioides, Gemeines Bitterkraut; Scabiosa columbaria, Tauben-Skabiose; Tagetes patula, Studentenblume; Zinnia violacea (= elegans), Garten-Zinnie.

\section{6 (7015) Colias crocea (FourCRoy, 1785)}

- Verordnung zur Neufassung der BArtschV von 2005: Besonders geschützte Art.

Barthelme \& Rinnhofer nennen mit der Bemerkung „letztmal(ig) 1970“ zwei mir bisher nicht bekannte Falterfunde aus dem Gebiet.

\footnotetext{
Fundorte (Ergänzung):

c 3149/III Ebw./Eichwerder (im Zeitraum 1965 bis 1970: Barthelme \& Rinnhofer)

d 3148/I Werbellin Umg./Kl. Buckowsee (im Zeitraum 1965 bis 1970: Barthelme \& Rinnhofer)
} 


\section{7 (7024) Gonepteryx rhamni (LinNAEus, 1758)}

Die Nutzung weiterer Nektarsaugpflanzen wurden im Gebiet beobachtet (vgl. Richert 2014): Aesculus hippocastanum, Gewöhnliche Rosskastanie; Anchusa (=Lycopsis) arvensis, Acker-Krummhals; Anthemis tinctoria, Färber-Hundskamille; Arctium tomentosum, Filzige Klette; Aster novae-angliae, NeuenglandHerbstaster, Raublatt-Aster; Aster novi-belgii, Neubelgien-Herbstaster; Ballota nigra, Schwarznessel; Cardaminopsis arenosa, Sand-Schaumkresse; Centaurea scabiosa, Skabiosen-Flockenblume; Centaurea stoebe, Rispen-Flockenblume; Dipsacus sylvestris, Wilde Karde; Doronicum pardalianches, Kriechende Gemswurz; Iberis umbellata, Doldige Schleifenblume; Lathyrus latifolius, Breitblättrige Platterbse; Lotus corniculatus, Gewöhnlicher Hornklee; Matricaria spec., Kamille; Medicago x varia, Luzerne; Narcissus pseudonarcissus, Osterglocke; Primula acaulis, Stengellose Schlüsselblume; Primula veris, Wiesen-Schlüsselblume; Prunella vulgaris, Gewöhnliche Braunelle; Prunus domestica, GartenPflaume; Salix cinerea, Grau-Weide; Scabiosa columbaria, Tauben-Skabiose; Stachys palustre, Sumpf-Ziest; Taraxacum offinalis, Gemeiner Löwenzahn; Vicia sativa ssp. nigra (= angustifolia), Saat-Wicke; Vicia villosa, Zottige Wicke.

Phänologie/Voltinismus: Eine bemerkenswerte Beobachtung gelang im Wintermonat Januar:

Am 07.I.2014 hatten $2 \mathrm{~F}$ ihr Winterquartier verlassen und krochen bei milden Temperaturen und Sonnenschein $\left(+10^{\circ} \mathrm{C} /\right.$ Schatten $)$ in der Bodenschicht im Wald am Stadtsee bei Ebw. umher (K. Hielscher, pers. Mitt.).

\section{8 (6993) Aporia crataegi (LinNAEus, 1758)}

Weitere im Gebiet festgestellte Nektarsaugpflanzen (vgl. Richert 1999, 2010, 2014):

Echium vulgare, Natterkopf; Lychnis flos-cuculi, KuckucksLichtnelke.

Neue Nachweise von bekannten Fundorten:

b 3248/II Trampe/ehemaliger TÜP (2014: Richert, 2017: Seidel)

c 3149/III Stecherschleuse Umg./ehemaliges Kiesgrubengelände nördlich des Ortes $(2014,2017)$

e 3049/I Groß Ziethen Umg./Steingruben an den Ihlowbergen (2014)

f 3049/IV Brodowin Umg./Kl. Rummelsberg (2014)

Fundorte (Ergänzung):

g 3050/IV Lunow Umg./Bereich Lunower Hölzchen (1994/ 1995: Borkowski)

\section{9 (6995) Pieris brassicae (Linnaeus, 1758)}

Weitere im Gebiet festgestellte Nektarsaugpflanzen (vgl. RicherT 2014):

Aster novi-anglicae, Raublatt-Aster; Ballota nigra, Schwarznessel; Calystegia sepium, Gewöhnliche Zaun- winde; Centaurea scabiosa, Skabiosen-Flockenblume; Cirsium oleraceum, Kohl-Kratzdistel; Chondrilla juncea, Knopel-Lattich; Dianthus carthusianorum, KarthäuserNelke; Doronicum pardalianches, Kriechende Gemswurz; Heliopsis helianthoides, Garten-Sonnenauge; Knautia arvensis, Acker-Witwenblume; Matthiola incana, Garten-Levkojen; Narcissus pseudonarcissus, Osterglocken, Primula acaulis = vulgaris, Stengellose Schlüsselblume; Salvia officinalis, Wiesen-Salbei; Scabiosa columbaria, Tauben-Skabiose; Syringa vulgaris, Gemeiner Flieder; Taraxacum officinale, Gewöhnlicher Löwenzahn; Tilia tomentosa, Silberlinde.

Weitere im Gebiet festgestellte Raupenfutterpflanzen: Lunaria annua, Judas-Silberblatt; Kahlfraß E IX/A X durch R im L4/L5 Stadium in meinem Garten; Armoracia rusticana, Meerrettich: einige R im L5-Stadium waren auf die ca. $5 \mathrm{~m}$ entfernt vom Judas-Silberblattbestand stehenden Pflanzen übergewechselt.

Phänologie/Voltinismus: 1. Gen.: 30.III.(2014), aFA, $1 \mathrm{~F}$ in meinem Garten in Finow, phänologische Verfrühung 13d gegenüber bis 2013;

3. (4.?) Gen.: 15.X.(2017) aFE, 1 Männchen, an RaublattHerbstaster in meinem Garten in Finow saugend, phänologische Verspätung 5d gegenüber bis 2013.

\section{0 (6998) Pieris rapae (Linnaeus, 1758)}

Weitere im Gebiet festgestellte Nektarsaugpflanzen (vgl. Richert 2014):

Anchusa officinalis, Gemeine Ochsenzunge; Aster novaeangliae, Raublatt-Aster; Armeria maritima, Gewöhnliche Grasnelke; Ballota nigra, Schwarznessel; Brassica oleracea, Gemüse-Kohl (Broccoli, ausgeschossen und in Blüte); Centaurea scabiosa, Skabiosen-Flockenblume; Centaurea stoebe, Rispen-Flockenblume; Cerastium arvense, Acker-Hornkraut; Chondrilla juncea, Großer Knorpellattich; Cirsium oleraceum, Kohl-Kratzdistel; Convolvulus arvensis, Ackerwinde; Cosmos bipinnatus, Garten-Kosmee; Dianthus carthusianorum, KarthäuserNelke; Epilobium hirsutum, Zottiges Weidenröschen; Geranium palustre, Sumpf-Storchschnabel; Hypericum perforatum, Tüpfel-Johanniskraut; Hypochoeris radicata, Gemeines Ferkelkraut; Knautia arvensis, AckerWitwenblume; Lamium spec., Taubnessel; Lapsana communis, Rainkohl; Ligustrum vulgare, Gemeiner Liguster; Lotus corniculatus, Gewöhnlicher Hornklee; Lunaria annua, Judas-Silberblatt; Lycopsis (= Anchusa) arvensis, Acker-Krummhals; Matricaria spec., Kamille; Origanum vulgare, Dost; Picris echioides, Wurmlattich, Natterkopf-Bitterkraut; Picris hieracioides, Gemeines Bitterkraut; Potentilla reptans, Kriechendes Fingerkraut; Ranunculus spec., Hahnenfuß; Raphanus raphanistrum, Hederich; Scabiosa columbaria, Tauben-Skabiose; Sedum telephium, Purpur-Fetthenne; Sinapis spec., Senf; Stachys palustris, Sumpfziest; Solidago canadensis, Kanadische Goldrute; Symphytum officinale, Gewöhnlicher Beinwell; Tagetes patula, Studentenblume; Trifolium medium, Mittlerer Klee; Trifolium repens, Weiß-Klee; 
Vicia cracca, Vogel-Wicke; Vicia villosa, Zottige Wicke; Viola arvensis, Acker-Stiefmütterchen; Zinnia violacea, Garten-Zinnie.

Phänologie/Voltinismus: 31.III.(2017), aFA, $1 \mathrm{~F}$ in meinem Garten an Scilla sibirica saugend., phänologische Verfrühung 3d gegenüber bis 2013.

3./4 (?) Gen.: 18.X.(2014), aFE, ein frischer F in meinem Garten in Finow; phänologische Verspätung 5d gegenüber bis 2013 .

\section{1 (7000) Pieris napi (Linnaeus, 1758)}

Weitere im Gebiet festgestellte Nektarsaugpflanzen (vgl. RICHERT 2014):

Achillea millefolium, Gemeine Schafgarbe; Anchusa officinalis, Gewöhnliche Ochsenzunge; Arctium tomentosum, Filzige Klette; Armeria maritima, Gewöhnliche Grasnelke; Aster linosyris, Goldhaar-Aster; Aster novianglicae, Raublatt-Aster; Ballota nigra, Schwarznessel; Bidens spec., Zweizahn, Calamintha acinos (= Acinos arvensis), Feld-Steinquendel; Capsella bursa-pastoris, Gewöhnliches Hirtentäschelkraut; Cardaminopsis arenosa, Sand-Schaumkresse; Centaurea scabiosa, Skabiosen-Flockenblume; Chondrilla juncea, Großer Knorpellattich; Cirsium oleraceum, Kohl-Kratzdistel; Convolvulus arvensis, Acker-Winde; Crepis biennis, Wiesen-Pippau; Daucus carota, Wilde Möhre; Diplotaxis tenuifolia, Schmalblättriger Doppelsame (Gewürzrauke); Echium vulgare, Natterkopf; Epilobium hirsutum, Zottiges Weidenröschen; Hieracium pilosella, Kleines Habichtskraut; Hypericum perforatum, Tüpfel-Johanniskraut; Knautia arvensis, Acker-Witwenblume; Leontodon autumnalis, Herbst-Löwenzahn; Ligustrum vulgare, Gemeiner Liguster; Lycopsis (= Anchusa) arvensis, Acker-Krummhals; Matricaria spec., Kamille; Medicago $\mathrm{x}$ varia, Luzerne; Picris hieracioides, Gewöhnliches Bitterkraut; Polygala vulgaris, Gewöhnliche Kreuzblume; Ranunculus bulbosus, Knolliger Hahnenfuß; Raphanus raphanistrum, Hederich; Rubus caesius, Kratzbeere; Salix cinerea, Grau-Weide, nur an blühenden Zweigen dicht über dem Boden (ca. $20 \mathrm{~cm}$ ); Salvia pratensis, Wiesen-Salbei; Scabiosa columbaria, Tauben-Skabiose; Senecio jacobaea, Jakobs-Greiskraut; Sinapis spec., Senf; Thlaspi arvensis, Acker-Hellerkraut; Tilia cordata, Winterlinde; Trifolium campestre, Feld-Klee; Trifolium pratense, Wiesen- (Rot-)Klee; Trifolium repens, Weiß-Klee; Valeriana officinalis, Arznei-Baldrian; Vicia villosa, Zottige Wicke; Viola arvensis, Acker-Stiefmütterchen; Viola odorata, März-Veilchen; Viola tricolor, Gewöhnliches Stiefmütterchen.

Als weitere Raupennahrungspflanze im Gebiet (vgl. Richert 2014) wurde Alliaria petiolata, Knoblauchrauke, festgestellt.

Phänologie/Voltinismus: 1. Gen.: 31.III.(2014), aFA, F i.M., frisch und abgeflogen; phänologische Verfrühung 2 d gegenüber bis 2013 .

\section{2 (7005) Pontia edusa (FAbricius, 1777)}

Weitere im Gebiet festgestellte Nektarsaugpflanzen (vgl. Richert 2014):

Armeria maritima, Gewöhnliche Grasnelke; Ballota nigra, Schwarznessel; Calamintha (=Acinos) arvensis, Feld-Steinquendel; Centaurea jacea, Gemeine/WiesenFlockenblume; Centaurea scabiosa, Skabiosen-Flockenblume; Chondrilla juncea, Binsen-Knorpellattich; Dianthus carthusianorum, Karthäuser-Nelke; Jasione montana, Berg-Sandglöckchen; Knautia arvensis, AckerWitwenblume; Leontodon autumnalis, Herbst-Löwenzahn; Origanum vulgare, Dost; Scabiosa columbaria, Tauben-Skabiose; Senecio jacobaea, Jakobs-Greiskraut; Sinapis spec., Senf; Trifolium pratense, Wiesen (Rot-) Klee.

Neue Nachweise von bekannten Fundorten:

b 3248/II Trampe/ehemaliger TÜP (2016: Richert, Seidel)

c 3148/III Ebw. OT Finow Umg./Forst Finowtal: Biesenthaler Straße am Flugplatz (2017), Einflugschneise Flugplatz (2018), Fotovoltaikanlage Flugplatz Finow (2015-2017: Brauner)

3148/II Ebw.-Nordend Umg./Oder-Havel-Kanal nördlich des Ortes (2015: Schwabe)

g 3050/II Stolzenhagen/NSG Krähen- und Jungfernberge (2016: Richert, 2018: Richert, Schwabe)

3150/IV Altglietzen/Gabower Berge (2018)

Fundorte (Ergänzung):

c 3149/III Sommerfelde Umg./Finowbruch nördlich des Ortes, am Bahnübergang (2018: Schwabe)

f 3050/III Bölkendorf Umg./NSG Tiefer See (2016)

g 3050/II Stolpe a.O./Trockenhänge am Stadtweg (2018); Gellmersdorf Umg./Gellmersdorfer Forst: Mühlenberggebiet (1994, 1995: Borkowski);

3050/IV Lunow Umg./Bereich Lunower Hölzchen (1994, 1995: Borkowski)

h 3050/II Stolpe Umg./Unteres Odertal: Im Bereich östlich Stolper Strom bis Fernitz-Felch (1994, 1995: Borkowki)

\section{3 (6973) Anthocharis cardamines (LinNaEus,} 1758)

Weitere im Gebiet festgestellte Nektarsaugpflanzen (vgl. Richert 2010 und 2014):

Cardamine pratensis, Wiesen-Schaumkraut; Glechoma hederacea, Gundermann; Lychnis flos-cuculi, KuckucksLichtnelke; Sinapis spec., Senf; Stellaria holostea, Große Sternmiere; Syringa vulgaris, Gewöhnlicher Flieder (O. Brauner 2018); Vicia tenuifolia, Feinblättrige Wicke; Viola arvensis, Gewöhnliches Acker-Stiefmütterchen.

Phänologie/Voltinismus: 30.III.(2014), aFA, 1 F (Pimpinellenberg - Oderhänge); phänologische Verfrühung 11d gegenüber bis 2013 .

Neue Nachweise von bekannten Fundorten:

b 3248/II Trampe/ehemaliger TÜP $(2014,2015)$ 
c 3148/III Ebw. OT Finow/Stadtgebiet: Altenhofer Straße, im Garten (2014, 2015, 2016, 2017, 2018) und Umg./ Menningsfließgebiet westlich des Ortes (2015: Richert), Forst Finowtal: Biesenthaler Straße nahe Samithsee (2017)

3149/III Stecherschleuse Umg./ehemaliges Kiesgrubengelände nördlich des Ortes (2017)

g 3050/II Stolzenhagen/NSG Krähen- und Jungfernberge (2015, 2016: Richert); Stolpe a.O./am Stolper Turm (2017)

3150/I Oderberg/NSG Pimpinellenberg (2014: Brauner)

3150/III Gabow Umg./Granitberggebiet (2014)

Fundorte (Ergänzung):

b 3149/III Ebw.-Südende Umg./Hohenfinower Wald: Östliche Leuenberger Wiese (2014)

c 3147/IV Finowfurt Umg./Finowfließniederung südwestlich der Aalkastenbrücke sowie auf Blößen im angrenzenden Wald (2016)

e 3049/I Groß Ziethen Umg./Steingruben an den Ihlowbergen (2015)

f 3049/IV Brodowin Umg./Biodeponie an der Straße nach Pehlitz nordwestlich des Kl. Rummelsberges (2015)

g 3050/II Stolpe a.O./Trockenhänge am Stadtweg und östlich der Straße nach Crussow (2018); Gellmersdorf Umg./Gellmersdorfer Forst: Mühlenberggebiet (1994-1995: Borkowski; 2017: Richert)

3050/IV Lunow Umg./Bereich Lunower Hölzchen (1994/ 1995: Borkowski)

3149/IV Niederfinow/Oderhänge - ehemalige Kiesgruben südwestlich des Schiffshebewerks (2016)

3150/I Oderberg/Geistberg (2018: Schwabe)

\section{Lycaenidae (Bläulinge)}

\section{4 (7036) Lycaena dispar (HAWORTH, 1802)}

- FFH-Art

- Verordnung zur Neufassung der BArtschV von 2005: Besonders geschützte Art.

- RL Land Brandenburg: Kategorie 2 (stark gefährdet): NM, FA;

- RL Deutschland (2011): Kategorie 3 (gefährdet).

Barthelme \& Rinnhofer führen als weiteren Fundort die Umgebung von Neuehütte an.

Aktuelle Funde bestätigen sowohl das Vorkommen bei Neuehütte als auch im NSG Krähen- und Jungfernberge bei Stolzenhagen

Im Rahmen der B 167 Ortsumgehung Eberswalde wurde im Jahr 2014 innerhalb eines Korridors entlang der geplanten Trasse das Vorkommen des Großen Feuerfalters als Art des Anhangs II der FFH-Richtlinie durch den Nachweis von Eiern und Raupen an den Wirtspflanzen erfasst (vgl. BraUner 2014). Neben Moorbereichen wurde dabei u. a. auch der Oder-Havel-Kanal vollständig eingebunden. Dabei erfolgten an 27 verschiedenen, nicht zu intensiv gepflegten Uferabschnitten des Kanals zahlreiche Ei- und Raupenfunde an dort zum Teil im Blockschutt wachsenden Exemplaren der Raupennahrungspflanzen Rumex hydrolapathum (Fluss-Ampfer) und stellenweise auch an der dort selteneren Art R. aqua- ticus (Wasser-Ampfer). Diese Funde weisen auf eine höhere regionale Bedeutung extensiv gepflegter Uferbereiche des Oder-Havel-Kanals für den Großen Feuerfalter hin.

Dieser Lebensraum wurde offenbar erst in neuerer Zeit von der Art besetzt. In den 1950er/1960er Jahren war der Oder-Havel-Kanal bevorzugtes Exkursionziel. Aus dieser Zeit liegen keine Beobachtungen der Art von dort vor. Sie nutzt „... gegenwärtig ein wesentlich breiteres Spektrum ökologischer Nischen“" (GELBRECHT et al. 2017: 104).

Weitere im Gebiet besuchte Nektarsaugpflanzen (vgl. RicherT 1999, 2010, 2014):

Centaurea jacea, Gemeine (Wiesen-) Flockenblume; Geranium palustre, Sumpf-Storchschnabel; Lathyrus pratensis, Wiesen-Platterbse; Lotus uliginosus, SumpfHornklee; Matricaria spec., Kamille; Tanacetum vulgare, Rainfarn; Trifolium campestre, Feld-Klee; Vicia cracca, Vogel-Wicke.

Phänologie/Voltinismus: 24.V.(2016), aFA, ein Männchen im NSG Krähen- und Jungfernberge, phänologische Verfrühung von 5 d gegenüber bis 2013 .

Auch die 2. Generation wurde im Beobachtungszeitraum im Gebiet beobachtet: 14.IX.2015, 23.VIII.2016, 28.VIII.2017 je ein Weibchen.

Neue Nachweise von bekannten Fundorten:

b 3248/II Trampe/ehemaliger TÜP (2014: Seidel; 2015, 2017: Richert; 2018: Barthelme)

g 3150/I Oderberg/Geistberg (2014: Schwabe)

Fundorte (Ergänzung):

d 3047/III Gr. Schönebeck/Bahndamm am Bahnhof (2014: Haase)

c 3147/II Finowfurt Umg./Oder-Havel-Kanal westlich und östlich der Autobahnbrücke (2014: Brauner)

3148/I Ebw. OT Finow Umg./Oder-Havel-Kanal im gesamten Bereich des MTB-Quadranten (2014: Brauner)

3148/II Ebw.-Nordend Umg./Oder-Havel-Kanal östlich Dusterwinkelbrücke (2014: Brauner), im Bereich der Eisenbahnunterführung (2016: Schwabe), westlich der Straßenbrücke B2 (2014: Brauner)

3149/I Neuehütte Umg. (2. Hälfte des 20. Jahrh.: Barthelme \& Rinnhofer)/ Ragöser Fließ Höhe Ragöser Mühle (2015: Brauner; Eifunde)

f 3049/IV Brodowin Umg./Brodowinsee - Südufer (2014: Brauner)

g 3050/II Stolzenhagen/NSG Krähen- und Jungfernberge (1994/1995: Borkowski; 2016: Richert)

h 3050/II Stolpe Umg./Odervorland bei Altwasser „Stolper Loch“, Höhe Eiswachhaus (2017: Brauner; Eifunde)

3050/IV Lunow Umg./Odervorland Höhe Wupla östlich Lunow (2017: Brauner; Eifunde)

\section{5 (7037) Lycaena virgaureae (LINNAEus, 1758)}

- Verordnung zur Neufassung der BArtschV von 2005: Besonders geschützte Art.

- RL Land Brandenburg: Kategorie 3 (gefährdet): TR, WS, MW;

- RL Deutschland (2011): Vorwarnliste. 
Weitere im Gebiet festgestellte Nektarsaugpflanzen (vgl. Richert 1999, 2010, 2014):

Pimpinella saxifraga, Kleine Bibernelle; Sedum acre, Scharfer Mauerpfeffer.

\section{Neue Nachweise von bekannten Fundorten:}

b 3149/III Sommerfelde Umg./ehemaliger TÜP Panzerbahn (2016: Seidel); Tornow Umg./Barnimhänge nördlich des Ortes: Bachtal am Wiesenweg (2017: Richert)

3248/II Trampe/ehemaliger TÜP (2014, 2017, 2018: Richert, 2016: Richert, Seidel)

c 3147/IV Finowfurt Umg./Finowfließ-Niederung nördlich des Lehns-Sees (2015)

3148/III Ebw. OT Finow/Stadtgebiet: Altenhofer Straße, im Garten (2014, 2018) und Umg./Forst Finowtal: Schönholzer Weg bei Kalkofenbrück (2014), Biesenthaler Straße am Flugplatz (2015, 2016); Einflugschneise Flugplatz (2017: Richert), Fotovoltaikanlage Flugplatz Finow (2014-2017: Brauner); Menningsfließgebiet (2014)

3148/IV Ebw./Stadtgebiet: Kleingartenanlage im Schwärzetal nördlich des Forstbotanischen Gartens (2015: Schwabe); Ebw. OT Finow Umg./Forst Finowtal: Stromtrasse südlich des Ortes $(2014,2018)$; Stecherschleuse Umg./am Rande des ehemaligen Kiesgrubengeländes nordöstlich des Ortes (2016: Schwabe)

3149/III Ebw. Umg./Finowbruch östlich des Eichwerder (2015: Schwabe); Sommerfelde Umg./Finowbruch nördlich des Ortes: Am Bahnübergang (2016, 2018: Schwabe); Stecherschleuse Umg./ehem. Kiesgrubengelände nördlich des Ortes (2017, 2018: Richert)

3149/IV Stecherschleuse Umg./am Rande des ehemaligen Kiesgrubengeländes nordöstlich des Ortes an der Schulstraße (2017: Richert)

g 3050/I Oderberg/NSG Pimpinellenberg (2016), Geistberg (2018: Richert \& Schwabe)

3150/III Gabow/Granitberggebiet (2015)

3150/IV Altglietzen Umg./Gabower Berge (2017, 2018: Schwabe)

Fundorte (Ergänzung):

b 3248/I Melchow Umg./Barnimer Heide: Weg nach F. Melchow, an der Bahnlinie (2016)

g 3050/II Stolpe a.O./Trockenhänge am Stadtweg (2018); Gellmersdorf Umg./Gellmersdorfer Forst: Mühlenberggebiet (1994/1995: Borkowski); Stolpe Umg./ Trockenhänge nordwestlich des Ortes an der Straße nach Crussow (2017: Richert)

3050/IV Lunow Umg./Gebiet Lunower Hölzchen (1994/1995: Borkowski)

\section{6 (7034) Lycaena phlaeas (LinNAEUs, 1761)}

- Verordnung zur Neufassung der BArtschV von 2005: Besonders geschützte Art.

Weitere im Gebiet festgestellte Nektarsaugpflanzen (vgl. Richert 2014):

Achillea millefolium, Gemeine Schafgarbe; Armeria maritima, Gewöhnliche Grasnelke; Daucus carota, Wilde Möhre; Euphorbia cyparissias, Zypressen-Wolfsmilch; Euphorbia esula, Esels-Wolfsmilch; Knautia arvensis, Acker-Witwenblume; Lychnis flos-cuculi,
Kuckucks-Lichtnelke; Medicago x varia, Luzerne; Potentilla argentea, Silber-Fingerkraut; Ranunculus spec., Hahnenfuß; Scabiosa columbaria, Tauben-Skabiose; Sedum maximum, Große Fetthenne; Valeriana officinalis, Arznei-Baldrian.

\section{7 (7039) Lycaena tityrus (PoDA, 1761) = dorilis HufNAgel, 1766}

- Verordnung zur Neufassung der BArtschV von 2005: Besonders geschützte Art.

Weitere im Gebiet festgestellte Nektarsaugpflanzen (vgl. Richert 2014):

Achillea millefolium, Gemeine Schafgarbe; Agrimonia eupatoria, Gewöhnlicher Odermennig; Anthemis arvensis, Acker-Hundskamille; Anthemis tinctoria, Färber-Hundskamille; Anthriscus sylvestris, Wiesen-Kerbel; Centaurea jacea, Gemeine (Wiesen-)Flockenblume; Daucus carota, Wilde Möhre; Euphorbia cyparissias, Zypressen-Wolfsmilch; Knautia arvensis, Acker-Witwenblume; Lychnis flos-cuculi, Kuckucks-Lichtnelke; Matricaria spec., Kamille; Medicago x varia, Luzerne; Potentilla argentea Silber-Fingerkraut; Ranunculus sardous, Sardischer Hahnenfuß; Scabiosa columbaria, Tauben-Skabiose; Senecio vernalis, Frühlings-Greiskraut; Trifolium campestre, FeldKlee; Trifolium repens, Weiß-Klee.

Phänologie/Voltinismus: 1. Gen.: 22.IV.(2014), aFA; phänologische Verfrühung 6d gegenüber bis 2013 .

\section{8 (7040) Lycaena alciphron (ROTTEMBURG,} 1775)

- Verordnung zur Neufassung der BArtschV von 2005: Besonders geschützte Art.

- RL Land Brandenburg: Kategorie 2 (stark gefährdet): TR, MW;

- RL Deutschland (2011): Kategorie 2 (stark gefährdet).

Im den Jahren 2017 und 2018 trat die Art ungewöhnlich häufig im ehemaligen, inzwischen weiträumig bewaldeten Kiesgrubengelände zwischen Oder-Havel-Kanal, Stecherschleuse und Niederfinow auf Lichtungen und Blößen mit Trockenrasen auf. Ich zählte z. B. vom 02.VI. bis 08.VII.2017 insgesamt >35 F. Ähnliche Beobachtungen liegen für 2018 vor (Barthelme).

Mir bisher nicht bekannte Fundorte im Gebiet werden ergänzend nachgetragen (z. T. nach Belegen in coll. Kittel).

Weitere im Gebiet festgestellte Nektarsaugpflanzen (vgl. Richert 2010 und 2014): Allium moly, GoldLauch; Anchusa officinalis, Gemeine Ochsenzunge; Anthemis tinctoria, Färber-Hundskamille; Centaurea scabiosa, Skabiosen-Flockenblume; Cirsium palustre, Sumpf-Kratzdistel; Coreopsis lanceolata, Lanzettblättriges Mädchenauge; Dianthus plumarius, Feder-Nelke; Doronicum pardalianches, Kriechende Gemswurz; Euphorbia esula, Esels-Wolfsmilch. 


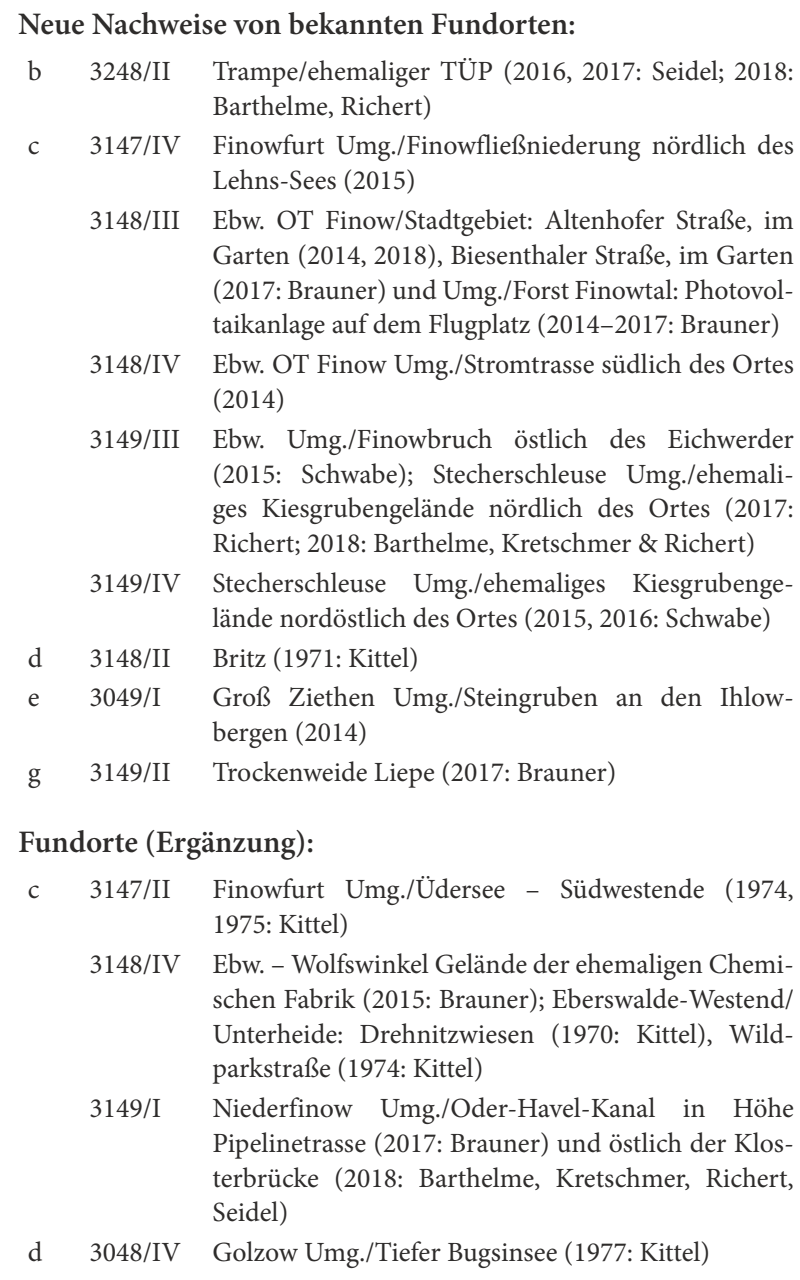

\section{9 (7041) Lycaena hippothoe (LinNAEus, 1761)}

- Verordnung zur Neufassung der BArtschV von 2005: Besonders geschützte Art.

- RL Land Brandenburg: Kategorie 1 (vom Aussterben bedroht): MW;

- RL Deutschland (2011): Kategorie 3 (gefährdet).

Ein mir bisher unbekannter älterer Fund von einem neuen Fundort wird mitgeteilt: In coll. Kittel fand ich zwei Männchen und zwei Weibchen von den Drehnitzwiesen in der Eberswalder Unterheide. Der Fund belegt einmal mehr die ehemals weite Verbreitung der Art im Gebiet.

Barthelme \& Rinnhofer konnten die Art auf dem ehemaligen Fundort Eichwerder bei Eberswalde letztmalig 1983 nachweisen (ein F).

Die aktuell in Brandenburg letzte Population auf dem ehemaligen TÜP Trampe geht weiter zurück: 2017 suchte Seidel vergeblich nach der Art. Kretschmar fand zwar noch einzelne Falter, jedoch mit nicht normal entwickelten Flügeln. Seidel beobachtete verstärkte Austrocknung des Habitats im Frühjahr dieses Jahres und vermutet darin eine mögliche Ursache des derzeitigen auffälligen Rückganges. 2018 wurden zwei $\mathrm{F}$ beobachtet (O. Schmidt, U. Barthelme).
Weitere im Gebiet festgestellte Nektarsaugpflanze (vgl. Richert 2004, 2010):

Cirsium palustre, Sumpf-Kratzdistel.

Neue Nachweise von bekannten Fundorten:

b 3248/II Trampe/ehemaliger TÜP (2014: Richert, Seidel; 2016: Seidel; 2017: Kretschmer; 2018: Barthelme, Schmidt)

Fundorte (Ergänzung):

c 3148/IV Eberswalde-Westend/Unterheide: Drehnitzwiesen (1969, 1974: Kittel)

\section{0(7035) Lycaena helle (DeNis \& SCHIFFER- MÜLLER, 1775) = amphidamas ESPER, 1779}

- Verordnung zur Neufassung der BArtschV von 2005: Streng geschützte Art.

- RL Land Brandenburg: Kategorie 0 (ausgestorben oder verschollen): MW;

- RL Deutschland (2011): Kategorie 2 (stark gefährdet).

Ein historischer Fund wird nachgetragen: Barthelme \& Rinnhofer nennen 4 Falternachweise vom Fundort Eichwerder bei Eberswalde aus dem Zeitraum 19631969. Das Finowbruch östlich von Eberswalde war damals offenbar weiträumiger von der Art besiedelt als bisher bekannt.

Fundorte (Ergänzung):

c 3149/III Eberswalde/Finowbruch am Eichwerder (19631969: Barthelme)

\section{1 (7047) Thecla betulae (LinnaEus, 1758)}

- RL Land Brandenburg: Kategorie 3 (gefährdet): HE, GL.

Der F flog wiederholt beim nächtlichen LF in Gärten im Stadtgebiet in Finow an das Licht.

Ich beobachtete einen F morgens beim Saugen an nächtlichem Tau in meinem Garten.

Bisher unberücksichtigte Funde von Finowfurt (Beleg in coll. Kittel) und aus dem Gellmersdorfer Forst (leg. Borkowski) werden nachgetragen.

\section{Neue Nachweise von bekannten Fundorten:}

b 3149/III Sommerfelde Umg./Barnimhänge nördlich des Ortes: Bachtal („am Wiesengraben“) 2016: Schwabe)

3248/II Trampe/ehemaliger TÜP (2015: Seidel)

c 3147/II Finowfurt (1971: Kittel)

3148/III Ebw. OT Finow/Stadtgebiet: Altenhofer Straße, im Garten (2014, 2015, 2016, 2017, 2018), Biesenthaler Straße, im Garten (2017: Brauner) und Umg./Forst Finowtal: Fotovoltaikanlage Flugplatz Finow (2017: Brauner)

3149/IV Stecherschleuse Umg./am Rande des ehemaligen Kiesgrubengeländes nordöstlich des Ortes (2014 Schwabe) 
d 3047/III Groß Schönebeck/am Bahndamm (2015: Haase)

3148/I Lichterfelde Umg./NSG Buckowseerinne: Moospfuhlgebiet (2014)

Fundorte (Ergänzung):

g 3050/II Gellmersdorf Umg./Gellmersdorfer Forst: Mühlenberggebiet (1994/1995: Borkowski)

3150/I Oderberg/Geistberg (2016: Schwabe)

\section{2 (7049) Neozephyrus quercus (LinNaEus, 1758)}

Phänologie/Voltinismus: 16.VI.(2018) aFA, phänologische Verfrühung 6d gegenüber bis 2013.

Fundorte (Ergänzung):

c 3147/II Finowfurt (1973: Kittel)

3148/IV Eberswalde (1968: Kittel), Ebw./Stadtgebiet: Kleingartenanlage im Schwärzetal nördlich Forstbotanischer Garten (2018: Schwabe)

d 3047/III Groß Schönebeck Umg./Liebenthaler Weg (2015, 2018: Haase)

g 3050/II Gellmersdorf Umg./Gellmersdorfer Forst: Mühlenberggebiet (1994/1995: Borkowski)

3050/IV Lunow Umg./Lunower Hölzchen-Gebiet (1994/1995: Borkowski)

\section{4 (7062) Satyrium w-album (КNOCH, 1782)}

- RL Land Brandenburg: Kategorie 2 (stark gefährdet): HA, UW.

Barthelme \& Rinnhofer führen die Art aus dem Jahre 1991 (2 F) vom Eichwerder bei Eberswalde auf.

Weitere im Gebiet festgestellte Nektarsaugpflanzen: Achillea spec., Schafgarbe; Melilotus albus, Weißer Steinklee; Tripleurospermum perforatum, Duflose Kamille.

Neue Nachweise von bekannten Fundorten:

c 3148/III Ebw. OT Finow/Stadtgebiet: Altenhofer Straße, im Garten (2014)

g 3150/I Oderberg/NSG Pimpinellenberg (2015: Brauner)

Fundorte (Ergänzung):

c 3148/IV Eberswalde-Nordend/Gehörlosenhilfsschule im Gelände der Landesklinik (1983: Kittel)

3149/III Eberswalde/Eichwerder (1991: Barthelme \& Rinnhofer)

d 3047/III Groß Schönebeck/Bahndamm am Bahnhof (2014: Haase)

3048/IV Golzow Umg./Tiefer Bugsinsee (1979: Kittel)

g 3149/IV Niederfinow/Oderhänge - ehemalige Kiesgruben südwestlich des Schiffshebewerks (2016: Schwabe)

\section{5 (7064) Satyrium spini (DENIS \& SCHIFFER- MÜLLER, 1775)}

- RL Land Brandenburg: Kategorie 1 (vom Aussterben bedroht): TR-WS;

- RL Deutschland (2011): Kategorie 3 (gefährdet).
Borkowski konnte das Vorkommen im NSG Krähenund Jungfernberge bei Stolzenhagen 1994/1995 bestätigen. Außer an den Jungfernbergen bei Stolzenhagen fand er den Falter an einer „Feldhecke bei Crussow“. Diese Fundortangabe lässt keine sichere MTB-Zuordnung zu: Der Ort liegt im Grenzbereich der MTB 3050 und 2950 .

Brauner beobachtete den F im Jahre 2017 im NSG Schäfergrund „häufiger als in anderen Jahren“.

Weitere im Gebiet festgestellte Nektarsaugpflanzen (vgl. RICHERT 1999):

Achillea millefolium, Gemeine Schafgarbe; Anthemis tinctoria, Färber-Hundskamille; Centaurea scabiosa, Skabiosen-Flockenblume; Knautia arvensis, AckerWitwenblume; Melilotus albus, Weißer Steinklee; Senecio spec., Greiskraut.

Phänologie/Voltinismus: 12.VI.2018, aFA, phänologische Verfrühung 7d gegenüber bis 2013 .

Neue Nachweise von bekannten Fundorten:

c 3149/III Stecherschleuse Umg./ehemaliges Kiesgrubengelände nördlich des Ortes (2018: Richert \& Kretschmer)

g 3149/IV Falkenberg (Hohenfinow) Umg./NSG Schäfergrund (2017: Brauner); Niederfinow Umg./Oderhänge - ehemalige Kiesgruben südwestlich von Schiffshebewerk (2014: Schwabe); Stecherschleuse Umg./ ehemaliges Kiesgrubengelände nordöstlich des Ortes (2015: Schwabe)

3050/II Stolpe Umg./Trockenhänge am Stadtweg (2017: Richert); Stolzenhagen/NSG Krähen- und Jungfernberge (1994/1995: Borkowski)

Fundorte (Ergänzung):

e ? Crussow Umg. (1994/1995: Borkowski)

3150/I Oderberg Umg./Feldflur nördlich NSG Pimpinellenberg (2016: Richert)

g 3150/I Oderberg/Geistberg (2016: Schwabe);

\section{6 (7063) Satyrium pruni (LinNAEUs, 1758)}

- RL Land Brandenburg: Kategorie 3 (gefährdet): WS, HE.

Borkowski konnte das Vorkommen im NSG Krähenund Jungfernberge bei Stolzenhagen1994/1995 bestätigen.

Schwabe fand die an einem Zweig festgesponnene P E (23.) V in einer Schlehenhecke.

Weitere im Gebiet festgestellte Nektarsaugpflanze (vgl. RICHERT 1999, 2004):

Ligustrum vulgare, Gemeiner Liguster.

Außerdem wurde ein Falter beobachtet, der bei trockenem Wetter in einer Weißdornhecke an einem Blatt saugte, wahrscheinlich an Honigtau.

Phänologie/Voltinismus: Im Jahre 2018 beobachtete Barthelme den F im Gebiet bereits am 20.V.(2018), aFA, phänologische Verfrühung 9d gegenüber bis 2013 . 
Neue Nachweise von bekannten Fundorten:

b 3149/III Sommerfelde Umg./ehemaliger TÜP Panzerbahn (2018: Seidel)

3248/II Trampe/ehemaliger TÜP (2018: Barthelme)

d 3148/I Werbellin Umg./NSG Buckowseerinne (2014 Clemens)

e 3049/I Groß Ziethen Umg./Steingruben an den Ihlowbergen (2014)

f 3049/IV Brodowin Umg./Biodeponie nördlich der Straße nach Pehlitz nordwestlich des Kl. Rummelsberges (2014)

g 3050/II Stolzenhagen/NSG Krähen- und Jungfernberge (1994/1995: Borkowski)

3150/I Oderberg/Geistberg (2015: Schwabe)

Fundorte (Ergänzung):

c 3149/III Eberswalde Umg./Eichwerder (2. Hälfte des 20. Jahrh.: Barthelme \& Rinnhofer), Finowbruch östlich des Eichwerder an der Bahnlinie (2015: Richert, Schwabe); Stecherschleuse Umg./ehemaliges Kiesgrubengelände nördlich des Ortes (2018: Barthelme)

d 3047/III Groß Schönebeck/Ortsgebiet: Friedenstraße, im Garten (2017: Haase)

e 3149/I Kloster Chorin (2. Hälfte des 20. Jahrh.: Barthelme \& Rinnhofer)

\section{7 (7058) Callophrys rubi (LinNaEus, 1758)}

- RL Land Brandenburg: Vorwarnliste. HW, SM, CB;

- RL Deutschland (2011): Vorwarnliste.

Neue Nachweise von bekannten Fundorten:

c 3148/IV Eberswalde-Westend/Unterheide: Wildparkstraße (1976: Kittel); Drehnitzwiesen (1999: Brauner)

3149/III Stecherschleuse/ehemaliges Kiesgrubengelände nördlich des Ortes (2017: Richert)

d 3047/III Groß Schönebeck Umg./Liebenthaler Weg (2018: Haase), Revier Rehluch (2018: Haase)

Fundorte (Ergänzung):

g 3050/II Gellmerdorf Umg./Gellmersdorfer Forst: Mühlenberggebiet (1994/1995: Borkowski)

3050/IV Lunow Umg./Lunower Hölzchen-Gebiet (1994/1995: Borkowski)

\section{9 (7093) Cupido argiades (PALlas, 1771)}

- RL Land Brandenburg: Kategorie 1 (vom Aussterben bedroht);

- RL Deutschland (2011): Vorwarnliste.

Nachdem die Art im Jahre 2013 im Gebiet nach fast einem Jahrhundert wieder gefunden worden war (RICHERT 2014), ist im Jahre 2014 bei uns vergeblich nach ihr gesucht worden. Nun können neue Funde gemeldet werden: Am 20.VII.2015 wurde ein (abgeflogenes) Männchen auf einer Feuchtwiese mit Beständen von Sumpf-Kratzdisteln nördlich des Lehns-Sees in der Finowfließniederung gefunden.

Im Jahre 2016 wurden F i.A. vom 17.VIII. bis 03.IX. auf dem TÜP Trampe beobachtet, wo inzwischen offenbar eine stabile Population existiert (I. Seidel, persönl. Mitt., Fotos).

2017 wurde die Art im Gebiet weiter verbreitet gefunden: Außer vom bekannten Fundort TÜP Trampe wurde der Falter nun auch aus dem Biesenthaler Becken, aus dem östlichen Eberswalder Tal bei Stecherschleuse, aus dem Uckermärker Kuppen- und Hügelland bei Groß Ziethen und aus der Schorfheide bei Groß Schönebeck gemeldet. Haase beobachtete die Eiablage an Feld-Klee, Trifolium campestre.

2018 erfolgte A IX auch der Nachweis auf den Odertalhängen (Schwabe, persönl. Mitt.). Ich beobachtete einen frischen Falter am 01.VIII. in meinem Garten in Finow.

Folgende Nektarsaugpflanzen wurden bisher im Gebiet festgestellt:

Knautia arvensis, Acker-Witwenblume; Lotus corniculatus, Gewöhnlicher Hornklee; Lotus uliginosus, SumpfHornklee.

Phänologie/Voltinismus: Bivoltin; nach dem Zustand des Falters zu urteilen, ist der Fund vom 20.VII.2015 offenbar der 1. Gen. zuzuordnen. Die F-Funde von A (01.)VIII bis A (04.)IX gehören zur 2. Gen., deren Falter 2018 besonders früh auftraten.

\section{Neue Nachweise von bekannten Fundorten:}

b 3248/II Trampe/ehemaliger TÜP (2016: Richert, Seidel; 2017: Richert)

\section{Fundorte (Ergänzung):}

b 3247/II Biesenthal Umg./NSG Biesenthaler Becken (2017: Theimer)

c 3147/IV Finowfurt Umg./Finowfließniederung nördlich Lehns-See (2015: Richert)

3148/III Ebw. OT Finow/Stadtgebiet: Altenhofer Straße, im Garten (2018: Richert)

3149/IV Stecherschleuse Umg./am Rande des ehemaligen Kiesgrubengeländes nordöstlich des Ortes (2017: Schwabe)

e 3049/I Groß Ziethen Umg./Steingruben an den Ihlowbergen (2017: Richert)

d 3147/I Groß Schönebeck Umg./Müllkippe am Weg nach Klandorf östlich der Bahnlinie (2017, 2018: Haase)

g 3150/IV Altglietzen Umg./Gabower Berge (2018: Schwabe)

\section{0 (7097) Celastrina argiolus (LinNAEus, 1758)}

Weitere im Gebiet festgestellte Nektarsaugpflanzen (vgl. Richert 2014):

Anchusa officinalis, Gewöhnliche Ochsenzunge; Anethum graveolans, Dill; Lavandula angustifolia, Echter Lavendel; Matricaria spec., Kamille; Medicago x varia, Luzerne; Melilotus albus, Weißer Steinklee; Origanum vulgare, Dost; Prunus spinosa, Schlehe; Trifolium repens, Weiß-Klee; Vicia cracca, Vogelwicke.

Die Raupe trug ich mit einem Strauß blühender Calluna vulgaris, Heidekraut, ein. 


\section{6 (7128) Plebeius idas (Linnaeus, 1761)}

- Verordnung zur Neufassung der BArtschV von 2005: Besonders geschützte Art.

- RL Land Brandenburg: Kategorie 2 (stark gefährdet): $\mathrm{CB}$;

- RL Deutschland (2011): Kategorie 3 (gefährdet).

Die Vorkommen an bekannten Fundplätzen konnten bestätigt werden. Die stabile Population am Flugplatz Finow fiel 2018 durch eine hohe Populationsdichte auf: Ich zählte E Juni $>80 \mathrm{~F}$.

Auch auf dem altbekannten Fundort auf der Stromtrasse im Forst Finowtal wurde die Art erneut beobachtet, nachdem dort jahrelang vergeblich nach ihr gesucht worden war (RICHERT 2014).

Weitere im Gebiet genutzte Nektarsaugplanzen: Berteroa incana, Graukresse; Sedum sexangulare, Milder Mauerpfeffer.

Die Eiablage beobachtete Haase 2014 in der Schorfheide von A bis E VIII an Besenheide (Calluna vulgaris). Dabei fiel auf, dass die Weibchen oft lange an der Heide sitzen, ehe sie ihre Eier ablegen. Dazu kriechen sie an der Pflanze abwärts und fixieren das Ei einzeln an Verzweigungen der Sprosse. Erwachsene R wurden 2014 vom 04.VI. bis A VII. gefunden, stets in Gesellschaft von Ameisen, welche bereits bei Eiablagebeobachtungen für den Beobachter aufdringlich wurden (Haase, persönl. Mitt.). Die Zucht erwies sich als schwierig. Aus eingetragenen Eiern schlüpften die Eiraupen E IV - A V. Eine e.o.-Zucht ist bisher nicht gelungen, die $\mathrm{R}$ gingen stets ein. Bei aus dem Freiland eingetragenen $R$ fielen geringe Futteraufnahme, verzögertes Wachstum und unterschiedliche Entwicklung auf. Die Puppenruhe dauerte nur relativ kurze Zeit: 11 - 17 Tage, im Durchschnitt 14 Tage. Zwei Männchen schlüpften am 06. und 12.VII.(2015), ein Weibchen (Zwergform) schlüpfte am 26.VII. Bei einer anderen Zucht schlüpfte ein Weibchen am 27.VII.2016.

Haase legte ein aberratives Exemplar aus der Schorfheide vor, bei dem die schwarzen Flecke innerhalb der OcellenReihe auf der Vfl-Us. vergrößert und wurzelwärts streifenförmig verlängert sind.

Phänologie/Voltinismus: Die Zuchten bestätigten, dass die Art ist in unserem Gebiet univoltin ist.

Im Jahre 2018 wurde bei Groß Schönebeck der erste Falter ungewöhnlich früh beobachtet: 07.VI.2018 aFA, phänologische Verfrühung 10d gegenüber bis 2013. Am 19.VI. wurden zugleich neben weiteren Faltern auch noch Raupen am Heidekraut gefunden. Die Flugzeit endete dort früher als in anderen Jahren bereits M VIII (Haase, pers. Mitt.).

\section{Neue Nachweise von bekannten Fundorten:}

c 3148/III Eberwalde OT Finow Umg./Forst Finowtal: Einflugschneise Flugplatz $(2015,2016,2017,2018$ : Richert, 2016: Seidel)

3148/IV Eberswalde OT Finow Umg./Forst Finowtal: Stromtrasse südlich des Ortes (2018: Richert) d 3047/III Groß Schönebeck Umg./Schorfheide: Forst Gr. Schönebeck, Revier Rehluch, Abt. 35/36 (2014, 2015, 2016, 2017, 2018: Haase)

\section{8 (7145) Polyommatus agestis (DenIs \& SCHIF- FERMÜLLER, 1775) = astrarche BERGSTRÄSSER part.}

- RL Land Brandenburg: Vorwarnliste: TR.

Weitere im Gebiet festgestellte Nektarsaugpflanzen (vgl. Richert 2004, 2010 und 2014):

Achillea milefolium, Gemeine Schafgarbe; Aster linosyris, Goldaster; Crepis tectorum, Dach-Pippau; Erodium cicutarium, Reiherschnabel; Jasione montana, Berg-Sandglöckchen; Knautia arvensis, Acker-Witwenblume; Matricaria spec., Kamille; Medicago falcata, Sichelmöhre; Medicago x varia, Luzerne; Petrorhagia prolifera, Sprossende Felsennelke; Potentilla argentea, Silber-Fingerkraut; Origanum vulgare, Dost; Tanacetum vulgare, Rainfarn; Viola arvensis, Acker-Stiefmütterchen.

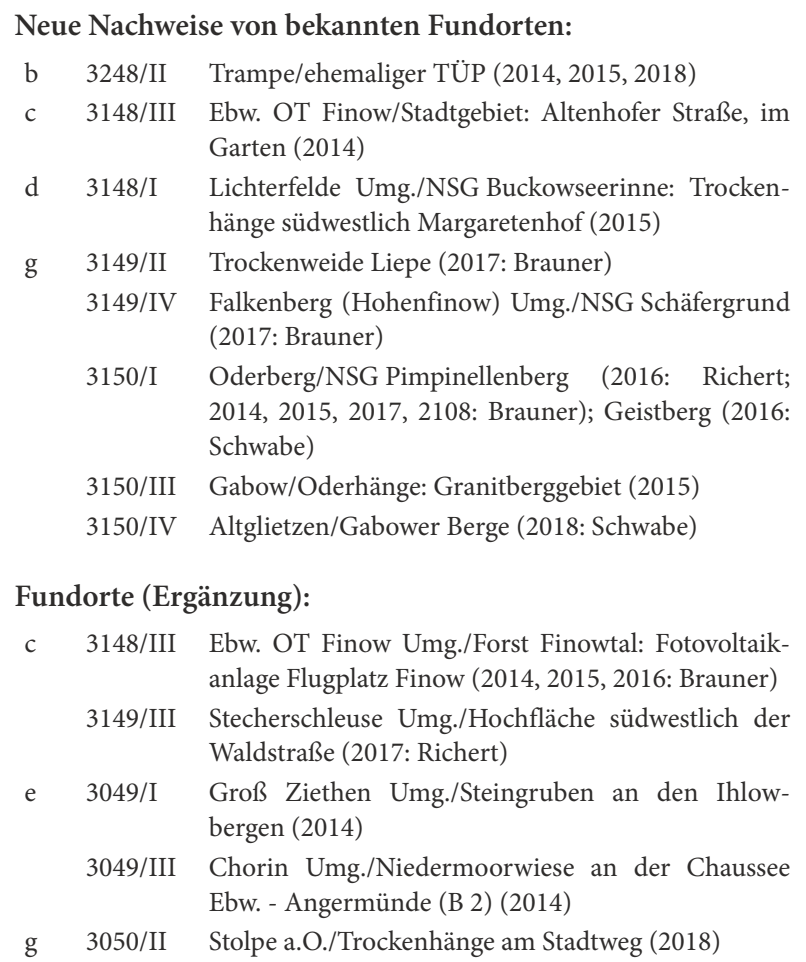

\section{9 (7152) Polyommatus semiargus (RоTTEM-} BURG, 1775)

- Verordnung zur Neufassung der BArtschV von 2005: Besonders geschützte Art.

- RL Land Brandenburg: Kategorie 3 (gefährdet): WS, TR, MW.

Weitere im Gebiet festgestellte Nektarsaugpflanzen (vgl. Richert 2010 und 2014):

Anchusa officinalis, Gewöhnliche Ochsenzunge; Symphytum officinale, Gewöhnlicher Beinwell (nach Beobachtungen von $\mathrm{H}$. Kretschmer bevorzugt bei Trockenheit 
genutzt; die Nektaraufnahme erfolgt durch von Hummeln erzeugte seitliche Löcher in der Blüte); Anthyllis vulneraria, Wundklee; Campanula patula, Wiesen-Glockenblume; Medicago x varia, Bastard-Luzerne; Trifolium campestre, Feld-Klee; Thymus serpyllum, Sand-Thymian.

Neue Nachweise von bekannten Fundorten:

b 3248/II Trampe/ehemaliger TÜP (2016, 2017: Richert, Seidel; 2018: Barthelme, Richert)

c 3149/III Stecherschleuse Umg./ehemaliges Kiesgrubengelände nördlich des Ortes (2017: Richert; 2018: Barthelme)

g 3050/II Stolpe a.O./Trockenhänge an der Straße nach Crussow nordwestlich des Ortes (2017), am Stadtweg (2018)

3150/IV Altglietzen Umg./Gabower Berge (2017: Schwabe; 2017: Brauner)

Fundorte (Ergänzung):

c 3148/III Ebw. OT Finow Umg./Forst Finowtal: Fotovoltaikanlage Flugplatz Finow (2014-2017: Brauner)

3149/III Ebw. Umg./Finowbruch östlich des Eichwerder (2015)

d 3047/III Groß Schönebeck Umg./am Bahnhof (2014, 2017, 2018: Haase), Liebenthaler Weg (2014: Haase)

g 3050/IV Lunow Umg./Gebiet Lunower Hölzchen (1994/1995: Borkowski)

\section{0 (7163) Polyommatus coridon (PoDA, 1761)}

- Verordnung zur Neufassung der BArtschV von 2005: Besonders geschützte Art.

- RL Land Brandenburg: Kategorie 3 (gefährdet): TR.

Die Art wurde zum Insekt des Jahres 2015 für Deutschland, Österreich und Schweiz gekürt.

Die Diluviallandschaften um Eberswalde gehören zum Verbreitungsschwerpunkt der Art in Ostbrandenburg. Eine umfassende Darstellung über die postglaziale Besiedlung Brandenburgs, aktuelle Verbreitung und Gefährdung in Brandenburg gaben Gelbrecht et al. (2015).

Im NSG Krähen- und Jungfernberge bei Stolzenhagen wurden E (27.)VII $2016>100$ F beobachtet. Bei Stolpe a.O. werden die Trockenhänge beiderseits der Straße nach Crussow von einer großen Population besiedelt. Ich sah den F M VII 2017 und 2018 dort i.M., vielfach an Dost (Origanum vulgare) saugend. Größere Vorkommen wurden zudem im Jahr 2017 von Brauner bei TagfalterUntersuchungen auf drei Trockenrasen im Rahmen der Ökosystemaren Umweltbeobachtung (ÖUB) festgestellt (Gabower Hänge/Altglietzen: 27.VII >500 F, NSG Schäfergrund: 30.VII >100 F, Trockenweide Liepe: 01.VIII $>100 \mathrm{~F})$.

Weitere im Gebiet festgestellte Nektarsaugpflanzen (vgl. RicherT 1999 und 2014): Anchusa officinalis, Gewöhnliche Ochsenzunge; Ballota nigra, Schwarznessel; Carlina vulgaris, Gewöhnliche Golddistel; Chondrilla juncea, Großer Knorpellattich; Cirsium vulgare, Gemeine Kratzdistel; Dianthus carthusianorum, KarthäuserNelke; Heliopsis helianthoides, Garten-Sonnenauge;
Hieracium echioides, Natternkopf-Habichtskraut; Hieracium pilosella, Kleines Habichtskraut; Jasione montana, Berg-Sandglöckchen; Lythrum salicaria, Blutweiderich; Knautia arvensis, Acker-Witwenblume; Medicago x varia, Luzerne; Melampyrum arvense, Acker-Wachtelweizen; Picris hieracioides, Gemeines Bitterkraut; Scabiosa columbaria, Tauben-Skabiose; Thymus serpyllum, SandThymian; Veronica spicata, Ähriger Ehrenpreis.

Weitere im Gebiet festgestellte Saugmedien: Eselskot. Ein bei Richert \& Brauner (in Vorber.) publiziertes Belegfoto vom 24.VII.2016 aus dem NSG Krähen- und Jungfernberge bei Stolzenhagen von S. Fuchs zeigt 17 an Eselslosung saugende Falter.

\section{Neue Nachweise von bekannten Fundorten:}

b 3149/III Sommerfelde Umg./ehemaliger TÜP Panzerbahn (2016: Seidel)

3248/II Trampe/ehemaliger TÜP (2016: Richert, Seidel; 2017: Richert)

c 3148/II Ebw.-Nordend Umg./Oder-Havel-Kanal nördlich des Ortes (2015, 2017: Schwabe)

3149/III Stecherschleuse Umg./ehemaliges Kiesgrubengelände nördlich des Ortes (2017: Richert), Hänge zum Finowbruch westlich des Ortes (2017: Schwabe)

3149/IV Stecherschleuse Umg./am Rande des ehemaligen Kiesgrubengeländs nordöstlich des Ortes (2014, 2017: Schwabe; 2017: Richert)

d 3148/I Lichterfelde Umg./NSG Buckowseerinne: Streuobstwiesen südöstlich Gr. Buckowsee - Moospfuhlgebiet (2018)

f 3149/II Brodowin Umg./Karpaten nordwestlich des Rosinsees (2015)

g 3050/II Stolpe a.O. Umg./Trockenhänge östlich Straße nach Crussow und am Stadtweg $(2017,2018)$; Stolzenhagen/NSG Krähen- und Jungfernberge (2015, 2016, 2017: Richert; 2016: Fuchs; 2018: Richert \& Schwabe)

3149/II Trockenweide Liepe (2017: Brauner)

3149/IV Niederfinow Umg./Oderhänge - ehemalige Kiesgruben südwestlich des Schiffshebewerks (2014 Schwabe), Hang am Schiffshebewerk (2014: Haase); Falkenberg (Hohenfinow) Umg./NSG Schäfergund (2016: Seidel; 2017: Brauner; 2018: Richert)

3150/I Oderberg/NSG Pimpinellenberg (2014, 2015, 2016, 2017, 2018: Brauner; 2016: Richert), Geistberg (2016: Schwabe; 2018: Richert, Schwabe)

3150/III Gabow/Granitberggebiet (2014, 2015: Richert; 2016: Schwabe)

3150/IV Altglietzen Umg./Gabower Berge (2017: Brauner; 2018: Richert, Schwabe)

Fundorte (Ergänzung):

c 3148/III Ebw. OT Finow Umg./Forst Finowtal: Fotovoltaikanlage Flugplatz Finow (2014, 2017: Brauner)

3148/IV Ebw.-Wolfswinkel/Gelände der ehemaligen Chemischen Fabrik (2015: Brauner)

b 3149/III Sommerfelde Umg./ehemaliger TÜP Panzerbahn (2016: Seidel)

3247/II Biesenthal Umg./NSG Biesenthaler Becken (2017: Theimer) 


\section{1 (7160) Polyommatus amandus (SCHNEIDER,} 1792)

- Verordnung zur Neufassung der BArtschV von 2005: Besonders geschützte Art.

Weitere im Gebiet festgestellte Nektarsaugpflanzen (vgl. Richert 2014):

Anchusa officinalis, Gewöhnliche Ochsenzunge Symphytum officinale, Gewöhnlicher Beinwell (nach Beobachtungen von $\mathrm{H}$. Kretschmer bevorzugt bei Trockenheit genutzt; die Nektaraufnahme erfolgt durch von Hummeln erzeugte seitliche Löcher in der Blüte); Centaurea scabiosa, Skabiosen-Flockenblume; Lychnis flos-cuculi, Kuckucks-Lichtnelke; Lythrum salicaria, Blut-Weiderich; Symphytum officinale, Gewöhnlicher Beinwell; Vicia tenuifolia, Feinblättrige Wicke.

\section{Neue Nachweise von bekannten Fundorten:}

b 3248/II Trampe/ehemaliger TÜP (2014, 2016: Richert, Seidel; 2017: Seidel; 2018: Barthelme, Richert)

c 3149/III Ebw. Umg./Finowbruch östlich des Eichwerder (2015, 2017: Schwabe); Stecherschleuse Umg./ ehemaliges Kiesgrubengelände nördlich des Ortes (2017: Richert; 2018: Barthelme)

3149/IV Stecherschleuse Umg./ehemaliges Kiesgrubengelände nordöstlich des Ortes (2015: Schwabe)

d 3049/III Groß Schönebeck/am Bahnhof (2014, 2017: Haase), Apfelallee (1999: Haase)

e 3049/I Groß Ziethen Umg./Töpferberge: Magerwiese am nordwestlichen Ortsausgang (2014)

g 3149IV Niederfinow/Oderhänge - ehemalige Kiesgruben südwestlich des Ortes (2016: Schwabe)

Fundorte (Ergänzung):

b 3149/III Sommerfelde Umg./Barnimhänge nördlich des Ortes („am Wiesengraben“) (2017: Schwabe)

c 3148/III Ebw. OT Finow Umg./Forst Finowtal: Fotovoltaikanlage Flugplatz Finow (2014, 2015, 2017: Brauner)

g 3050/IV Lunow Umg./Gebiet Lunower Hölzchen (1994/1995: Borkowski)

\section{2 (7163) Polyommatus icarus (RotTemburg,} 1775)

- Verordnung zur Neufassung der BArtschV von 2005: Besonders geschützte Art.

Weitere im Gebiet festgestellte Nektarsaugpflanzen (vgl. RicherT 2014):

Achillea millefolium, Gemeine Schafgarbe; Anchusa officinalis, Gemeine Ochsenzunge; Anthemis tinctoria, Färber-Hundskamille; Anthyllis vulneraria, Gewöhnlicher Wundklee; Arenaria serpyllifolia, Quendelblättriges Sandkraut; Armeria maritima, Gewöhnliche Grasnelke; Arnoseris minima, Lämmersalat; Cardaminopsis arenosa, Sand-Schaumkresse; Carduus crispus, Rauhe Distel; Centaurea jacea, Gemeine (Wiesen-) Flockenblume; Centaurea scabiosa, Skabiosen-Flockenblume; Cerastium fontanum ssp. vulgare (= holosteoides), Gewöhnliches Hornkraut; Convolvulus arvensis, Acker-Winde; Dianthus carthusianorum, Karthäuser-Nelke; Diplotaxis tenuifolia,
Schmalblättriger Doppelsame (Gewürzrauke); Erigoron (= Conyza) canadensis, Kanadisches Berufskraut; Euphorbia esula, Esels-Wolfsmilch; Hieracium pilosella, Kleines Habichtskraut; Hypochoeris radicata, Gemeines Ferkelkraut; Knautia arvensis, Acker-Witwenblume; Lathyrus pratensis, Wiesen-Platterbse; Leontodon hispidus, Rauher Löwenzahn; Lotus uliginosus, Sumpf-Hornklee; Lychnis flos-cuculi, Kuckucks-Lichtnelke; Lythrum salicaria, Blutweiderich; Matricaria spec., Kamille; Medicago falcata, Sichelklee; Medicago lupulina, Hopfenklee; Medicago x varia, Luzerne; Mentha spec., Minze; Myosotis arvensis, Acker-Vergissmeinnicht; Origanum vulgare, Dost; Petrorhagia prolifera, Sprossende Felsennelke; Phacelia tanacetifolia, Rainfarn-Büschelschön (Borstiger Bienenfreund); Picris echioides, Natterkopf-Bitterkraut (Wurmlattich); Polygonum bistorta, Schlangen-Knöterich; Potentilla argentea, Silber-Fingerkraut; Prunella vulgaris, Gemeine Braunelle; Ranunculus spec., Hahnenfuß; Saxifraga granulata, Knöllchen-Steinbrech; Scabiosa columbaria, Tauben-Skabiose; Senecio vernalis, Frühlings-Greiskraut; Sinapis spec., Senf; Solidago canadensis, Kanadische Goldrute; Symphyotrichum novae-angliae, NeuenglandHerbstaster (Raublatt-Aster); Trifolium arvense, HasenKlee; Trifolium campestre, Feld-Klee; Trifolium dubium, Zwerg-Klee; Vicia cracca, Vogel-Wicke; Vicia villosa, Zottige Wicke; Vicia tenuifolia, Feinblättrige Wicke; Viola arvensis, Acker-Stiefmütterchen.

Als weitere Eiablagepflanze wurde Trifolium pratense, Wiesen- oder Rot-Klee, im Gebiet dokumentiert (GotTWALD 2010).

\section{Nymphalidae (Edelfalter): Heliconiinae}

\section{4 (7202) Argynnis paphia (Linnaeus, 1758)}

- Verordnung zur Neufassung der BArtschV von 2005: Besonders geschützte Art.

Weitere von der Art im Gebiet genutzte Nektarsaugpflanzen (vgl. Richert 2010):

Arctium tomentosum, FilzigeKlette; Ballotanigra,Schwarznessel; Centaurea scabiosa, Skabiosen-Flockenblume; Cirsium oleraceum, Kohl-Kratzdistel; Cirsium vulgare, Gemeine Kratzdistel; Cosmos bipinnatus, GartenKosmee; Echium vulgare, Gewöhnlicher Natternkopf; Helianthus annuus, Gemeine Sonnenblume; Hypericum perforatum, Tüpfel-Johanniskraut; Knautia arvensis, Acker-Witwenblume; Matricaria spec., Kamille; Medicago x varia, Bastard-Luzerne; Pimpinella saxifraga, Kleine Bibernelle; Sambucus nigra, Schwarzer Holunder; Trifolium pratense, Wiesen (Rot-) Klee; Trifolium repens, Weiß-Klee; Vicia villosa, Zottige Wicke.

\section{Fundorte (Ergänzung):}

Es werden bei dieser inzwischen im Gebiet wieder allgemein verbreiteten Art nur bisher nicht bekannte Fundorte genannt.

c 3148/II Ebw.-Nordend Umg./Oder-Havel-Kanal an der Bahnunterführung (2016: Schwabe) 
d 3047/IV Groß Schönebeck/Schorfheide: Waldinnensaum bei Meelake (2018: Brauner)

g 3050/II Stolpe a.O./Trockenhänge am Stadtweg (2017: Richert); Gellmersdorf Umg./Gellmersdorfer Forst: Mühlenberggebiet (1994/1995: Borkowski)

\section{5 (7204) Argynnis aglaja (Linnaeus, 1758)}

- Verordnung zur Neufassung der BArtschV von 2005: Besonders geschützte Art.

- RL Land Brandenburg: Kategorie 2 (stark gefährdet): WS, MW, HF;

- RL Deutschland (2011): Vorwarnliste.

Über die für einen längeren Zeitraum (1975-2004) im Gebiet als verschollen gemeldete Art (RICHERT 1999 und 2010) berichten Barthelme \& Rinnhofer: „letztm.(malig) 1989 am Eichwerder“ bei Eberswalde. Borkowski führt die Art aus dem Gellmersdorfer Forst für den Zeitraum 1994-1995 auf. Demnach trat sie sehr lokal auch zwischenzeitlich im Gebiet auf. Aktuell liegen mir nur vom ehemaligen TÜP Trampe regelmäßige Beobachtungen vor, in anderen Gebietsteilen wird die Art selten gefunden oder scheint zu fehlen.

Weitere im Gebiet genutzte Nektarsaugpflanzen (vgl. Richert 2010): Cirsium palustre, Sumpf-Kratzdistel; Echium vulgare, Natterkopf.

Neue Nachweise von bekannten Fundorten:

b 3248/II Trampe/ehemaliger TÜP (2016: Seidel; 2018: Kretschmer)

Fundorte (Ergänzung):

b 3148/IV Spechthausen Umg./Barnimer Heide: Stromtrasse südwestlich des Ortes (2018)

c 3148/III Ebw. OT Finow Umg./Forst Finowtal: Schönholzer Weg südlich des Ortes (2014)

g 3050/II Gellmersdorf Umg./Gellmersdorfer Forst: Mühlenberggebiet (1994/1995: Borkowski)

\section{6 (7205) Argynnis adippe (DENIS \& SCHIFFER-} MÜLLER, 1775)

- Verordnung zur Neufassung der BArtschV von 2005: Besonders geschützte Art.

- RL Land Brandenburg: Kategorie 2 (stark gefährdet): WS, MW;

- RL Deutschland (2011): Kategorie 3 (gefährdet).

Weitere von der Art im Gebiet genutzte Nektarsaugpflanzen (vgl. Richert 1999, 2010, 2014): Centaurea jacea, Gemeine (Wiesen-) Flockenblume; Echium vulgare, Gewöhnlicher Natternkopf; Origanum vulgare, Dost.

Neue Nachweise von bekannten Fundorten:

b 3248/II Trampe/ehemaliger TÜP (2016: Richert, Seidel; 2017, 2018: Richert)

c 3148/III Ebw. OT Finow/Stadtgebiet: Altenhofer Straße, im Garten $(2014,2018)$ und Umg./Forst Finowtal: Einflugschneise Flugplatz (2016: Seidel), Gestellweg nördlich der Einflugschneise (2018)
3148/IV Ebw. OT Finow Umg./Forst Finowtal: Stromtrasse südlich des Ortes $(2014,2018)$

3149/III Sommerfelde Umg./Finowbruch nördlich des Ortes, am Bahnübergang (2018: Schwabe); Stecherschleuse Umg./ehemaliges Kiesgrubenglände nördlich des Ortes $(2017,2018)$

d 3148/I Lichterfelde Umg./NSG Buckowseerinne: Streuobstwiesen südöstlich Gr. Buckowsee - Moospfuhlgebiet (2018)

g 3149/IV Falkenberg (Hohenfinow) Umg./NSG Schäfergrund (2016: Seidel; 2017: Brauner)

g 3150/I Oderberg/NSG Pimpinellenberg (2014, 2015, 2016, 2017, 2018: Brauner)

Fundorte (Ergänzung):

c 3147/IV Finowfurt Umg./Finowfließniederung nördlich des Lehns-Sees (2015)

3148/III Ebw. OT Finow Umg./Forst Finowtal: Fotovoltaikanlage Flugplatz Finow (2014, 2015: Brauner)

3148/IV Ebw./Stadtgebiet: Kleingartenanlage im Schwärzetal nördlich Forstbotanischder Garten (2018: Schwabe)

d 3047/III Groß Schönebeck Umg./Apfelallee (2016: Haase), Eichhorster Straße (2018: Haase)

3047/IV Groß Schönebeck/Schorfheide: Meelake (2014, 2018 : Brauner)

e 3150/I Oderberg/Feldflur nördlich NSG Pimpinellenberg (2016)

g 3050/II Gellmersdorf Umg./Gellmersdorfer Forst: Mühlenberggebiet (1994/1995: Borkowski)

3150/I Oderberg/Geistberg (2015: Schwabe)

\section{7 (7206) Argynnis niobe (LinNaEus, 1758)}

- Verordnung zur Neufassung der BArtschV von 2005: Besonders geschützte Art.

- RL Land Brandenburg: Kategorie 1 (vom Aussterben bedroht): TR, WS;

- RL Deutschland (2011): Kategorie 2 (stark gefährdet).

„Letztmalig 1991 i.(m) G.(ebiet) bei Liepe“: Dieser Einzelfund durch Barthelme \& Rinnhofer erfolgte noch später als bei Richert 1999 („seit 1982 im Gebiet verschollen") angegeben.

Aktuelle Funde im Gebiet sind mir nicht bekannt.

Neue Nachweise von bekannten Fundorten:

g 3149/II Liepe Umg. (1991: Barthelme \& Rinnhofer)

\section{8 (7210) Issoria lathonia (LinNAEUs, 1758)}

Weitere von der Art im Gebiet genutzte Nektarsaugpflanzen (vgl. RICHERT 2014):

Achillea millefolium, Gemeine Schafgarbe; Armeria maritima, Gewöhnliche Grasnelke; Aster linosyris, Goldaster; Berteroa incana, Graukresse; Calamintha acinos (= Acinos tomentosa), Steinquendel; Carduus crispus, Rauhe Distel; Centaurea scabiosa, Skabiosen-Flockenblume; Centaure stoebe, Rispen-Flockenblume; Chondrilla juncea, Binsenoder Großer Knorpellattich; Chrysanthemum (= Tanacetum vulgare), Rainfarn; Clematis vitalva, Gewöhnliche Waldrebe; Convolvulus arvensis, Acker-Winde; Crepis 
capillaris, Grüner Pippau; Dianthus carthusianorum, Karthäuser-Nelke; Helichrysum arenarium, SandStrohblume; Hieracium echioides, Natterkopfblättriges Habichtskraut; Hypericum perforatum, Tüpfel-Johanniskraut; Iberis umbellata, Doldige Schleifenblume; Knautia arvensis, Acker-Witwenblume; Lythrum salicaria, Blutweiderich; Matricaria spec., Kamille; Picris echioides, Natterkopf-Bitterkraut (Wurmlattich); Prunella vulgaris, Gemeine Braunelle; Ranunculus sardous, Sardischer Hahnenfuß; Rubus spec., Brombeere; Scabiosa columbaria, Tauben-Skabiose; Scabiosa canescens, Graue Skabiose; Sinapis spec., Senf; Trifolium repens, Weiß-Klee; Thymus pulegioides, Feld-Thymian; Thymus serpyllum, SandThymian; Veronica persica, Persischer Ehrenpreis; Vicia cracca, Vogel-Wicke; Vicia villosa, Zottige Wicke; Viola arvensis, Acker-Stiefmütterchen.

Außerdem wurde Saugen an feuchter Erde beobachtet. Die Eiablage beobachtete ich M (15.) VI in meinem Garten in Finow an Garten-Stiefmütterchen, Viola x wittrockiana (einzeln an der Blattunterseite innerhalb des Pflanzenbüschels). Auf einer Photovoltaikanlage bei Finow beobachtete Brauner am 16.VII.2015 mehrfach die Eiablage an Viola arvensis.

\section{9 (7214) Brenthis daphne (DENIS \& SCHIFFER- MÜLLER, 1775)}

- Verordnung zur Neufassung der BArtschV von 2005: Streng geschützte Art.

- RL Land Brandenburg: Kategorie 0 (verschollen bzw. ausgestorben): WS;

- RL Deutschland (2011): Kategorie D (Daten unzureichend).

Im Gebiet verschollen seit 1979 (RICHERT 2010: 160). „Barthelme konnte 1969 den F(und)O(rt) Groß Schönebeck von Friese (vgl. Richert 1999; Anm. Verf.) bestätig. (en). 2 Falter am 14. + 15.7. Danach gelang es uns nicht, daphne erneut nachzuweisen“. Nach Angaben im Manuskript von Barthelme \& Rinnhofer waren die F am Treptow-See gefunden worden.

Die präzisierte Fundortangabe wird nachgetragen.

Neue Nachweise von bekannten Fundorten:

d 3046/IV - 3047/III Groß Schönebeck Umg./Treptowsee (1969: Barthelme)

\section{0 (7213) Brenthis ino (RotTemburg, 1775)}

- RL Land Brandenburg: Kategorie 2 (stark gefährdet): HF.

Über das ehemalige Vorkommen der Art am Eichwerder bei Eberswalde (vgl. RICHERT 1999) berichten Barthelme \& Rinnhofer: „Wir fanden sie ... von 6 (Juni) - 7.(Juli) 1966-71 zu Hundert.(en) auf Blüten. Mit dem Verschwind.(en) der intakten Sumpfwies.(en) setzte ein spürb.(arer) Rückgang der Art ein. Nicht mehr in jed. (em) Jahr und meist vereinzelt. Nur 1988 konnten wir ... ino lokal häufig beobachten. ... Seither liegen uns keine aktuellen Nachweise mehr vor."

Borkowski fand die Art 1994/1995 außerhalb seiner Probeflächen „vereinzelt bis selten auf feuchten Wiesen in den Jungfernbergen bei Stolzenhagen ....

Diese älteren Fundortangaben werden neben aktuellen ergänzend nachgetragen:

\section{Neue Nachweise von bekannten Fundorten: \\ b 3248/II Trampe/ehemaliger TÜP (2014: Richert; 2016: Seidel; 2018: Barthelme, Richert) \\ g 3050/II Stolzenhagen/NSG Krähen und Jungfernberge (1994/1995: Borkowski)}

Fundorte (Ergänzung):

b 3148/IV Ebw. Umg./Oberheide: Waldschneise am Leuenberger Graben zwischen Möllergrab und Leuenberger Wiesen (2014: Schwabe)

c 3149/I Sandkrug/Gr. Heiliger See (vor 1988: Barthelme \& Rinnhofer); Neuehütte Umg./Bachsee (vor 1988: Barthelme \& Rinnhofer)

e 3048/III Althüttendorf Umg./Bugsinseegebiet (vor 1988 Barthelme \& Rinnhofer)

062 (7218) Boloria eunomia (EsPER, 1799) = aphirape HüBNER, 1799

- Verordnung zur Neufassung der BArtschV von 2005: Besonders geschützte Art.

- RL Land Brandenburg: Kategorie 0 (verschollen bzw. ausgestorben): MW;

- RL Deutschland (2011): Kategorie 2 (stark gefährdet). Im Gebiet verschollen seit 1988 (korrigiert):

Barthelme \& Rinnhofer berichten: „Wir fanden eunomia nur noch bei Sandkrug/Umg. Heilig.(er) See 1986 i.A. (15 Falter), letztmalig 1988“. Somit kam die Art im Gebiet noch etwas später vor, als bisher angenommen (vgl. Richert 2010: 160; im Gebiet verschollen seit 1982). Der mir bisher nicht bekannte Fundort für diese Art wird nachgetragen. Spätere Nachsuchen an diesem Platz waren ergebnislos.

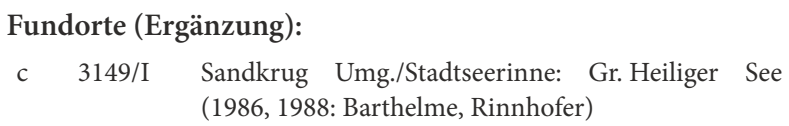

\section{3 (7222) Boloria selene (DENIS \& SCHIFFER- MÜLLER, 1775)}

- Verordnung zur Neufassung der BArtschV von 2005: Besonders geschützte Art.

- RL Land Brandenburg: Kategorie 2 (stark gefährdet): WS, NM, MW;

- RL Deutschland (2011): Vorwarnliste.

Bisher nicht publizierte ältere Funde werden ergänzend nachgetragen:

Barthelme \& Rinnhofer fanden 3 F 1991 bei Neuehütte und bezeichneten die Art danach als verschollen. 
Borkowski wies die Art Mitte der 1990er Jahre im Gellmersdorfer Forst nach.

In coll. Kittel befand sich aus älterer Zeit ein Belegexemplar aus der Eberswalder Unterheide.

Aktuelle Funde aus dem Untersuchungszeitraum liegen mir nicht vor.

Fundorte (Ergänzung):

c 3148/IV Ebw.-Westend/Unterheide: Wildparkstraße (1969: Kittel)

3149/I Neuehütte Umg. (1986, 1988: Barthelme, Rinnhofer)

g 3050/II Gellmersdorf Umg./Gellmersdorfer Forst: Mühlenberggebiet (1994/1995: Borkowski)

\section{4 (7220) Boloria euphrosyne (LinNaEus, 1758)}

- Verordnung zur Neufassung der BArtschV von 2005: Besonders geschützte Art.

- RL Land Brandenburg: Kategorie 0 (ausgestorben oder verschollen): WS;

- RL Deutschland (2011): Kategorie 2 (stark gefährdet).

Im Gebiet verschollen/ausgestorben seit 1973 (RICHERT 2010: 160).

Über den Fundort Neuehütte (vgl. Richert 1999: 32) berichten Barthelme \& Rinnhofer, „Barthelme konnte von 1966-1970 allein bei Neuehütte (Kleinstareal) ca. 150 F nachw.(eisen). Ab 1971 erlosch das Vorkommen schlagartig“. Die präzisierte Fundortangabe wird nachgetragen.

Fundorte (Ergänzung):

c 3149/I Neuehütte Umg./Bachsee (1966-1970: Barthelme)

\section{5 (7228) Boloria dia (LinnAeus, 1767)}

- Verordnung zur Neufassung der BArtschV von 2005: Besonders geschützte Art.

- RL Land Brandenburg: Kategorie 2 (stark gefährdet): TR.

Im Jahr 2018, das sich im Gebiet durch extreme Trockenheit und heiße Tage im Sommer auszeichnete, beobachtete ich die Art erstmalig wiederholt im August in meinem Garten, wo die Falter das dort noch vorhandene Blütenangebot nutzten.

Weitere im Gebiet von der Art genutzte Nektarsaugpflanzen (vgl. Richert 2010 und 2014):

Achillea millefolium, GemeineSchafgarbe; Berteroaincana, Graukresse; Calendula officinalis, Garten-Ringelblume; Centaurea cyanus, Kornblume; Centaurea scabiosa, Skabiosen-Flockenblume; Crepis capillaris, Grüner Pippau; Hieracium echioides, Natterkopfblättriges Habichtskraut; Hieracium pilosella, Kleines Habichtskraut; Hypericum perforatum, Tüpfel-Johanniskraut; Knautia arvensis, Acker-Witwenblume; Matricaria spec., Kamille; Myosotis arvensis, Acker-Vergissmeinnicht; Origanum vulgare, Dost; Picris hieracioides, Gewöhnliches (Gemeines) Bitterkraut; Scabiosa columbaria, Tauben-Skabiose; Trifo- lium pratense, Wiesen- oder Rot-Klee; Trifolium repens, Weiß-Klee; Viola arvensis, Acker-Stiefmütterchen.

Neue Nachweise von bekannten Fundorten:

b 3248/II Trampe/ehemaliger TÜP (2016: Richert, Seidel; 2017: Seidel; 2018: Kretschmer, Richert)

c 3148/III Ebw.-OT Finow Umg./Forst Finowtal: Biesenthaler Straße am Flugplatz (2016)

3149/III Stecherschleuse Umg./ehemaliges Kiesgrubengelände nördlich des Ortes (2017)

3149/IV Stecherschleuse Umg./am Rande des ehemaligen Kiesgrubengeländes nordöstlich des Ortes an der Schulstraße (2017)

d 3047/III Groß Schönebeck Umg./Schorfheide: Revier Rehluch, Abt. 61 (2017: Haase)

g 3050/II Stolzenhagen/NSG Krähen- und Jungfernberge (1994/1995: Borkowski; 2018: Richert \& Schwabe)

3150/I Oderberg/Geistberg (2016, 2018: Schwabe), Feldflur nördlich NSG Pimpinellenberg (2016)

3149/II Liepe/Trockenweide (2017: Brauner)

3149/IV Falkenberg (Hohenfinow) Umg./NSG Schäfergrund (2017: Brauner)

3150/I Oderberg/NSG Pimpinellenberg (2014, 2015, 2016, 2017, 2018: Brauner)

3150/IV Altglietzen Umg./Gabower Berge (2017: Brauner; 2017, 2018: Schwabe)

Fundorte (Ergänzung):

c 3148/III Ebw. OT Finow/Stadtgebiet: Altenhofer Straße, im Garten (2018) und Umg./Forst Finowtal: Fotovoltaikanlage Flugplatz Finow (2014-2017: Brauner)

b 3148/IV Ebw./Oberheide: Waldschneise am Leuenberger Graben zwischen Möllergrab (am Wiebecke-Damm) und Leuenberger Wiesen (2014: Schwabe); Spechthausen Umg./Barnimer Heide: Stromtrasse südwestlich des Ortes (2017: Schwabe)

3149/III Ebw.-Südende Umg./Hohenfinower Wald: Östliche Leuenberger Wiese (2014)

d 3147/I Groß Schönebeck Umg./Müllkippengelände südöstlich des Ortes (2014: Haase)

\section{Nymphalidae (Edelfalter): Nymphalinae}

\section{6 (7243) Vanessa atalanta (LinNAEUs, 1758)}

Weitere im Gebiet von der Art genutzte Nektarsaugpflanzen (vgl. RicheRT 2014):

Centaurea scabiosa, Skabiosen-Flockenblume; Dianthus barbatus, Bart-Nelke; Iberis umbellata, Doldige Schleifenblume; Heliopsis helianthoides, Garten-Sonnenauge; Knautia arvensis, Acker-Witwenblume; Rudbeckia fulgida, Gewöhnlicher oder Leuchtender Sonnenhut; Aster novae-angliae, Neuengland-Herbstaster (Raublatt-Aster); Tagetes patula, Studentenblume; Trifolium pratense, Wiesen- (Rot-) Klee. Als weiteres Saugmedium im Gebiet wurden im Oktober reife Weintrauben dokumentiert.

Haase beobachtete bei Groß Schönebeck im Nordwesten des Gebietes die Eiablage von offensichtlich überwinterten Faltern bereits im Zeitraum vom 01.IV.(2017) bis 21.IV(2015). Die anschließende e.o.-Zucht und die Zucht von $\mathrm{A} \mathrm{V}$ bis $\mathrm{A}$ VI eingetragenen $\mathrm{R}$ ergab die $\mathrm{F} \mathrm{M}$ bis $\mathrm{E}$ (16.-27.)VI. 
Im „Sonnensommer“ 2018 verlief eine e.o.-Zucht extrem schnell: Eiablage 20.VII.; nach 9 Tagen schlüpften die R; Raupenzeit und Puppenruhe insgesamt nur 20 Tage; ab. 18.VIII. schlüpften die F. Die gesamte Entwicklungdauer vom Ei bis zur Imago betrug nur 29 Tage (Haase persönl. Mitt.).

Phänologie/Voltinismus: 30.III.(2014), phänologische Verfrühung 11d gegenüber bis 2013: 6 F bei Groß Schönebeck (Haase); 25.III.(2015), erneute phänologische Verfrühung 5d: $1 \mathrm{~F}$ bei Groß Schönebeck (Haase); 24.III. (2017), aFA, erneute phänologische Verfrühung 1d, insgesamt also $17 \mathrm{~d}$ gegenüber bis 2013. Offensichtlich handelte es sich bei all diesen Beobachtungen um Überwinterer im Gebiet nach relativ milden Wintern (vgl. RicherT 2014).

\section{7 (7245) Vanessa cardui (LINNAEUs, 1758)}

Weitere im Gebiet von der Art genutzte Nektarsaugpflanzen (vgl. Richert 2014):

Arctium tomentosum, Filzige Klette; Armeria maritima, Gewöhnliche Grasnelke; Centaurea scabiosa, SkabiosenFlockenblume; Centaurea stoebe, Rispen-Flockenblume; Cosmos bipinnatus, Garten-Kosmee; Iberis umbellata, Doldige Schleifenblume; Matricaria spec., Kamille; Origanum vulgare, Dost; Raphanus raphanistrum, Hederich; Scabiosa columbaria, Tauben-Skabiose; Senecio jacobaea, Jakobs-Greiskraut.

Haase registrierte den Einflug im Jahre 2016 A VI und beobachtete die Eiablage an Urtica dioica, Große Brennnessel; die anschließende e.o.-Zucht dauerte 44 Tage.

Phänologie/Voltinismus: 11.V.(2015), phänologische Verfrühung 6d gegenüber bis 2013.; 17.IV.(2018), aFA, erneute phänologische Verfrühung (30d gegenüber bis 2013).

\section{8 (7248) Inachis io (LINNAEUs, 1758)}

Weitere im Gebiet von der Art genutzte Nektarsaugpflanzen (vgl. RICHERT 2014):

Anthriscus sylvetris, Wiesen-Kerbel; Armeria maritima, Gewöhnliche Grasnelke; Aster linosyris, Goldaster; Aster novae-angliae, Neuengland-Herbstaster (Raublatt-Aster); Aster novi-belgii, Neubelgien-Herbstaster; Ballota nigra, Schwarznessel; Calendula officinalis, Garten-Ringelblume; Carduus crispus, Krause Distel; Cirsium oleraceum, Kohl-Kratzdistel; Cosmos bipinnatus, Garten-Kosmee; Echinacea purpurea, Purpur-Sonnenhut; Erysium cheiri, Goldlack; Iberis umbellata, Doldige Schleifenblume; Knautia arvensis, Acker-Witwenblume; Mahonia aquifolium, Mahonie; Malva moschata, Moschus-Malve; Matricaria spec., Kamille; Origanum vulgare, Dost; Prunus avium ssp. avium,Vogelkirsche; Prunus cerasus, Sauerkirsche; Prunus domestica, Pflaume; Rudbeckia fulgida, Gewöhnlicher oder Leuchtender Sonnenhut; Scabiosa columbaria, Tauben-Skabiose; Sedum telephium, PurpurFetthenne; Trifolium pratense, Wiesen- (Rot-) Klee; Viola $\mathrm{x}$ wittrockiana, Garten-Stiefmütterchen; Zinnia violacia, Garten-Zinnie.
Mehrfach beobachtete ich an abgefallenen überreifen bzw. angefaulten Birnen saugende Falter.

Phänologie/Voltinismus: R der 1. Gen. des Jahres 2017 wurden im Gebiet im VI gefunden. Frische F traten zunehmend häufig ab M VII auf. A VIII beobachtete ich in meinem Garten ein schlagartes Ende des intensiven Blütenbesuchs von $\mathrm{F}$ der 1. Gen. (nicht nur am bevorzugten Sommerflieder). Ich fand wenig später einige $\mathrm{F}$ dann in dunklen Vestecken im Schuppen, unter Brettern und an anderen dunklen Orten. Offenbar handelte es sich dabei nicht um eine vorübergehende "Sommerruhe“. Darauf weisen auch kontinuierliche Beobachtungen an Faltern von Aglais urticae und Inachis io hin, die M VIII bzw. M IX ihre Winterruheplätze in Gebäuden auf meinem Grundstück aufgesucht hatten und diese wider Erwarten selbst bei Schönwetterperioden im Herbst nicht wieder vor dem nächsten Frühjahr verließen.

E VIII beobachtete ich R-Gesellschaften einer 2. Gen. in unterschiedlichen Entwicklungsstadien. Zur Zucht eingetragene R ergaben die F E IX bis A X. Im Freiland traten frische F ab A IX auf. Dabei handelte es sich offenbar (ausschließlich?) um F der 2. Gen., die in diesem Jahr auffallend individuenreich war. Die fast ausnahmslos frischen F nutzten ab M IX bis M X i.M. das Blütenangebot in diesem Zeitraum (z. B. in Gärten vor allem Sommerflieder und Herbstastern, auf einer Streuobstwiese Wiesen-Flockenblumen, die nach Mahd im Sommer neu ausgetrieben hatten und wieder blühten).

Mir fiel auf, dass F der 1. Gen. A VIII 2017 in meinem Garten von den Blüten des Sommerflieders gegen $20 \mathrm{~h}$ MESZ trotz weiterer Besonnung derselben verschwanden (Aufsuchen von „Schlafplätzen“?), während gleichzeitig daran saugende Admirale die Nektarquelle weiter nutzten, bis der Strauch bei sinkender Sonne völlig im Schatten lag.

\section{9 (7257) Nymphalis antiopa (LinnAEUs, 1758)}

- Verordnung zur Neufassung der BArtschV von 2005: Besonders geschützte Art.

- RL Deutschland (2011): Vorwarnliste.

Haase beobachtete den F regelmäßig im Nordwestteil des Gebietes, jedoch meist vereinzelt (insgesamt $17 \mathrm{~F}$ ) und überwiegend nach der Überwinterung. Nur eine seiner Beobachtungen erfolgte M IX 2015 vor der Überwinterung.

Am 12.VI.2018 wurde eine ausgewachsene L5-Raupe auf einem Grasplatz vor dem aufgeforsteten Kiesgrubengelände am Oder-Havel-Kanal bei Niederfinow gefunden (auf dem Weg zu einem Verpuppungsort?).

Ein frischer $F$ der neuen Jahresgeneration wurde in diesem Jahr bereits sehr früh am 02.VII. beobachtet (Revier Rehluch bei Groß Schönebeck; Haase, persönl. Mitt.). 
Phänologie/Voltinismus: 12.VI.2014, aFE, phänologische Verspätung 9d gegenüber bis 2013; Haase beobachtete an diesem späten Zeitpunkt noch ein abgeflogenes Exemplar. Auch im Jahre 2017 registrierte er einen F noch am 6.VI. Die Flugzeit kann im Gebiet also länger dauern als bisher bekannt.

\section{Neue Nachweise von bekannten Fundorten:}

b 3148/IV Ebw. Umg./Oberheide: Waldcampus der Hochschule für nachhaltige Entwicklung HNE (entspricht der für ältere Daten verwendete Fundortangabe „Institut für Waldschutz") (2014: Schwabe), Wiebecke-Damm am Möllergrab (2014: Schwabe)

3248/II Trampe/ehemaliger TÜP $(2014,2015)$

c 3147/I Zerpenschleuse/Ortsgebiet: Berliner Straße (2017: Busse)

3148/III Ebw. OT Finow/Stadtgebiet: Altenhofer Straße, im Garten $(2015,2016,2017)$ und Umgeb./Forst Finowtal: Einflugschneise Flugplatz $(2016,2018)$

Fundorte (Ergänzung):

c 3148/III Ebw. OT Finow Umg./Forst Finowtal: Fotovoltikanlage Flugplatz Finow (2016: Brauner)

3149/I Niederfinow Umg./Oder-Havel-Kanal östlich der Klosterbrücke (2018: Kretschmer, Seidel, Richert)

d 3047/III Groß Schönebeck/Ortsgebiet: Friedenstraße, im Garten (2014, 2016: Haase) Umg./Liebenthaler Weg (2014, 2017: Haase), am Treptowsee (2014), Alte Döllner Straße (2015: Haase), Revier Rehluch, Abt. 35 (2017: Haase), Abt. 62 (2018: Haase)

g 3050/II Gellmersdorf Umg./Gellmersdorfer Forst: Mühlenberggebiet (1994/1995: Borkowski)

3050/IV Lunow Umg./Gebiet Lunower Hölzchen (1994/1995: Borkowski)

g 3149/II Liepe/Trockenweide (2017: Brauner)

3150/I Oderberg/NSG Pimpinellenberg (2016)

\section{0 (7250) Nymphalis urticae (LinnaEus, 1758)}

Weitere im Gebiet von der Art genutzte Nektarsaugpflanzen (vgl. RicHERT 2014):

Achillea millefolium, Gemeine Schafgarbe; Aster novaeangliae, Neuengland-Herbstaster (Raublatt-Aster); Berteroa incana, Graukresse; Centaurea jacea, Gemeine (Wiesen-) Flockenblume; Cirsium vulgare, Gemeine Kratzdistel; Helianthus annuus, Sonnenblume; Jasione montana, Berg-Sandglöckchen; Hedera helix, Efeu; Iberis umbellata, Doldige Schleifenblume; Ligustrum vulgare, Gemeiner Liguster; Malus domestica, Kultur-Apfel; Matricaria spec., Kamille; Medicago x varia, BastardLuzerne; Prunus spinosa, Schlehe; Echinacea purpurea, Roter Scheinsonnenhut; Scabiosa columbaria, TaubenSkabiose; Sinapis spec., Senf; Solidago canadensis, Kanadische Goldrute; Torilis japonica, Gewöhnlicher Klettenkerbel; Zinnia violacea, Garten-Zinnie.

Die ehemals bei uns überall häufige Art wird neuerdings nur noch vereinzelt beobachtet: 2017 insgesamt nur 14 Beobachtungen im gesamten Gebiet. Diese Entwicklung setzte sich 2018 fort (nur zwei Einzelbeobachtung östlich des Eichwerder bei Eberswalde, persönl. Mitt. Schwabe, und bei Groß Schönebeck, pers. Mitt. Haase).
Phänologie/Voltinismus: Im Jahre 2015 beobachtete ich am 20.VIII. zwei F, die in einem Stallgebäude auf meinem Grundstück in Finow an der Decke ruhten. Wiederholte Kontrollen bis A XII ergaben, dass die F nicht nochmals aktiv wurden, sondern dort überwinterten: Die Diapause dauerte von E VIII 2015 bis 17. bzw. 26.III.2016.

Eine ähnliche Beobachtung erfolgte im Jahre 2016: Bereits am 30.VII. bezog ein Falter an einer dunklen Stelle im Treppenflur meines Hauses sein „Winterquartier“, das er trotz sommerlicher Tage im August und September nicht mehr verließ, während Artgenossen noch bis M IX beim Blütenbesuch in meinem Garten beobachtet wurden. $\mathrm{Ob}$ diese Blütenbesucher ausschließlich Tiere der 2. Gen. waren? Dazu sind weitere Beobachtungen nötig.

\section{1 (7258) Nymphalis polychloros (LinnaEUs,} 1758)

- Verordnung zur Neufassung der BArtschV von 2005: Besonders geschützte Art.

- RL Land Brandenburg: Kategorie 2 (stark gefährdet);

- RL Deutschland (2011): Vorwarnliste.

Neue Nachweise von bekannten Fundorten:

b 3248/II Trampe/ehemaliger TÜP (2014: Kretschmer, M. \& R. Fiddicke; 2015: Kretschmer)

Fundorte (Ergänzung):

g 3150/I Oderberg/NSG Pimpinellenberg (2014: Brauner)

071 a (07259) Nymphalis xanthomelas (ESPER, 1781)

Siehe Kapitel 3.2. Neu entdeckte Arten.

072 (7252) Nymphalis c-album (LINNAEUs, 1758)

Weitere im Gebiet von der Art genutzte Nektarsaugpflanzen (vgl. Richert 2014):

Armeria maritima, Gewöhnliche Grasnelke; Aster novaeangliae. Neuengland-Herbstaster (Raublatt-Aster); Aster novi-belgii, Neubelgien-Herbstaster; Astilbe spec., Astilbe (Ardensii-Hybride); Berteroa incana, Graukresse; Cirsium oleraceum, Kohl-Kratzdistel; Lavandula angustifolia, Echter Lavendel; Prunus avium ssp. avium, Vogelkirsche (Blüten); Prunus domestica, Pflaume (Blüten); Rudbeckia fulgida, Gewöhnlicher oder Leuchtender Sonnenhut; Syringa vulgaris, Gemeiner Flieder.

\section{3 (7255) Araschnia levana (Linnaeus, 1758)}

Weitere im Gebiet festgestellte Nektarsaugpflanzen (vgl. Richert 2014):

Achillea millefolium, Gemeine Schafgarbe; Brassica napus ssp. napus, Raps; Cirsium oleraceum, Kohl-Kratzdistel; Cotoneaster spec., Zwergmispel; Dianthus carthusianorum, Karthäuser-Nelke; Falcaria vulgaris, Sichelmöhre; Matricaria spec., Kamille; Ranunculus acris, Scharfer 
Hahnenfuß; Scabiosa columbaria, Tauben-Skabiose; Senecio jacobaea, Jakobs-Greiskraut; Senecio vernalis, Frühlings-Greiskraut; Sinapis spec., Senf; Solidago canadensis, Kanadische Goldrute; Torilis japonica, Gewöhnlicher Klettenkerbel; Trifolium repens, Weiß-Klee.

Phänologie/Voltinismus: Es liegen folgende Beobachtungen der erst in neuerer Zeit im Gebiet beobachteten 3. Generation vor: Haase fand bei Groß Schönebeck Anfang November 2014 und am 14.X.2017 Raupen (Nachkommen der 3. Generation). Die Verpuppung erfolgte vom 05.-17.XI.

21.VIII.2015 1 F, Groß Schönebeck (Haase); 19.VIII.-07. IX.2016 3 F, Groß Schönebeck (Haase); 31.VIII.2016 Trampe, ein frischer F (Foto Seidel); 31.VIII.-02.IX.2017 3 F, Groß Schönebeck (Haase).

Im Jahre 2018 wurde die Sommergeneration bereits in der 2. Junidekade beobachtet:

19.VI.2018, aFA 2. Gen., phänologische Verfrühung 6d gegenüber bis 2013 .

Die 3. Gen. flog in diesem Jahr ab M VIII.

\section{4 (7268) Euphydryas aurinia (ROTTEMBURG, 1775)}

- Verordnung zur Neufassung der BArtschV von 2005: Besonders geschützte Art.

- RL Land Brandenburg: 0 (ausgestorben oder verschollen): MW;

- RL Deutschland (2011): Kategorie 2 (stark gefährdet).

Barthelme \& Rinnhofer berichten über ihre Beobachtungen an dem bekannten ehemaligen Fundort im Finowbruch am Eichwerder bei Eberswalde: „Barthelme zählte auf einer Sumpfwiese ... von 1966-70 jährlich 250-350 aurinia-Falter von Anf.(ang)6 - Anf.(ang) 7 auf Blüten. Letztmal.(ig) 1971. ...Mit dem Bau der neuen Klärgruben bzw. der Erweiterung der Mülldeponie Anfang der 1970er Jahre verschwanden fast vollständig die oben aufg.(eführten) Pflanzenarten (Polygonum, Eriophorum, Trollius, Menyanthes). Mit ihnen nicht nur E. aurinia, sondern auch andere gefährdete Arten“.

Somit existierte ein Vorkommen im Gebiet noch 4 Jahre später als in Richert 2010: 160, („letzter Nachweis

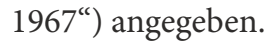

\section{5 (7270) Melitaea cinxia (Linnaeus, 1758)}

- RL Land Brandenburg: Kategorie 2 (stark gefährdet): TR;

- RL Deutschland (2011): Kategorie 3 (gefährdet).

Barthelme \& Rinnhofer berichten über den Fundort Eichwerder bei Eberswalde: „... häufig auf Moor-wies. (en) und Trockenhäng.(en) des Eichwerder. Letztmalig i.A. fand sie Barthelme 1988/89 ... . Danach liegt uns kein aktueller Nachw.(eis) mehr vor."

Die Art gehört zum aktuellen Faunenbestand des Gebietes. Weitere hier festgestellte Nektarsaugpflanzen (vgl. Richert 2014): Berteroa incana, Graukresse;
Hieracium pilosella, Kleines Habichtskraut; Knautia arvensis, Acker-Witwenblume.

Neue Nachweise von bekannten Fundorten:

b: 3248/II Trampe Umg./ehemaliger TÜP (2014, 2017: Richert; 2018: Barthelme)

Fundorte (Ergänzung):

c 3148/IV Ebw. OT Finow Umg./Forst Finowtal: Stromtrasse südlich des Ortes (2015)

3149/III Stecherschleuse Umg./Hochfläche südwestlich der Waldstraße (2017), ehemaliges Kiesgrubengelände nördlich des Ortes (2018: Barthelme)

d 3047/III Groß Schönebeck Umg./Liebenthaler Weg (2017: Haase)

3148/II Britz (1972: Kittel)

\section{7 (7276) Melitaea diamina (LANG, 1789)} = dictynna ESPER, 1794

- RL Land Brandenburg: Kategorie 1 (vom Aussterben bedroht): NM, HF;

- RL Deutschland (2011): Kategorie 3 (gefährdet).

Barthelme \& Rinnhofer führten u. a. einige mir bisher nicht bekannte (ehemalige) Fundorte im Gebiet auf und nennen für ihren letzten Nachweis im Gebiet das Jahr 1991 (Siehe Fundorte/Ergänzung).

Borkowski bestätigte 1994/1995 das Vorkommen im NSG Krähen- und Jungfernberge bei Stolzenhagen: „Nur noch sehr lokal in den Jungfernbergen ... außerhalb der Probeflächen. ... Inzwischen ist der Populationsbestand stark im Rückgang begriffen."

Ein aktuelles Vorkommen entdeckte ich 2016 in der Finowfließniederung nördlich des Lehnssees.

Neue Nachweise von bekannten Fundorten:

g 3050/II Stolzenhagen/NSG Krähen- und Jungfernberge (1994/1995: Borkowski)

Fundorte (Ergänzung):

c 3147/IV Finowfurt Umg./Finowfließniederung nördlich des Lehnssees $(2016,2017)$

3149/I Neuehütte Umg./Bachsee (vor 1991: Barthelme \& Rinnhofer)

d 3047/IV Wildau am Werbellinsee/Fliegner Teiche (vor 1991: Barthelme \& Rinnhofer)

e 3048/II Althüttendorf/Grimnitzsee (vor 1991: Barthelme \& Rinnhofer)

f 3149/II Liepe Umg./Forst Chorin: NSG Plagefenn - Plagesee (vor 1991: Barthelme \& Rinnhofer)

\section{8 (7283) Melitaea athalia (Rottemburg, 1775)}

- RL Land Brandenburg: Vorwarnliste: WS, KW, NM. Die Art hat an vielen Plätzen im Gebiet wieder stabile Populationen. Besonders hohe Populationsdichten wurden in den letzten Jahren im ehemaligen, seit längerer Zeit aufgeforsteten Kiesgrubengelände zwischen Oder-Havel-Kanal, Stecherschleuse und Niederfinow beobachtet. 
Weitere im Gebiet festgestellte Nektarsaugpflanzen (vgl. Richert 1999 und 2014):

Achillea millefolium, Gemeine Schafgarbe; Anthemis tinctoria, Färber-Hundskamille; Armeria maritima, Gewöhnliche Grasnelke; Chrysanthemum leucanthemum, Margerite; Epilobium angustifolium, Schmalblättriges Weidenröschen; Eupatorium cannabium, Wasserdost; Ligustrum vulgare, Gemeiner Liguster; Origanum vulgare, Dost; Tripleurospermum inodorum, Geruchlose Kamille; Valeriana officinalis, Arznei-Baldrian.

Außerdem beobachtete ich Saugen an menschlichem Schweiß und an feuchten Wegstellen.

Phänologie/Voltinismus: Im Jahre 2018 wurde die Art im Gebiet wieder ungewöhnlich früh ab E (28.)V beobachtet (Haase). Am 13.VI.2015 beobachtete Brauner auf dem Gelände der ehemaligen VEB Chemischen Fabrik Finowtal in Eberswalde eine erwachsene Raupe an Plantago lanceolata (Spitzwegerich) fressend.

Neue Nachweise von bekannten Fundorten:

b 3248/II Trampe Umg./ehemaliger TÜP (2014, 2016, 2017: Richert; 2018: Barthelme, Richert)

c 3148/II Ebw.-Nordend Umg./Oder-Havel-Kanal westlich der Wassertorbrücke (2017: Schwabe)

3148/III Ebw. OT Finow/Stadtgebiet: Altenhofer Straße, im Garten $(2014,2017,2018)$ und Umg./Forst Finowtal: Biesenthaler Straße (2015, 2016, 2018), Einflugschneise Flugplatz (2018), am Schönholzer Weg (2016: Walter); Menningsfließgebiet westlich des Ortes (2014)

3148/IV Ebw. OT Finow Umg./Forst Finowtal: Stromtrasse südlich des Ortes $(2014,2018)$

3149/III Stechercheuse Umg./ehemaliges Kiesgrubengelände nördlich des Ortes (2017: Richert; 2018: Barthelme, Kretschmer, Richert); am Rande der Hochfläche südwestlich der Waldstraße (2017)

3149/IV Stecherschleuse Umg./am Rande des ehemaligen Kiesgrubengeländes nordöstlich des Ortes (2016: Schwabe; 2018: Barthelme)

d 3148/I Lichterfelde Umg./NSG Buckowseerinne: Hänge südwestlich Margaretenhof (2015)

e 3049/I Groß Ziethen Umg./Steingruben an den Ihlowbergen (2014)

g 3149/IV Falkenberg (Hohenfinow) Umg./NSG Schäfergrund (2017: Brauner)

3150/I Oderberg/NSG Pimpinellenberg (2018: Brauner)

Fundorte (Ergänzung):

b 3148/I Spechthausen Umg./Barnimer Heide: Stromtrasse südwestlich des Ortes (2018)

c 3148/III Ebw. OT Finow Umg./Forst Finowtal: Fotovoltaikanlage Flugplatz Finow (2015, 2016: Brauner)

3148/IV Ebw.-Wolfswinkel/Gelände der ehemaligen Chemischen Fabrik (2015: Brauner)

3149/I Niederfinow Umg./Oder-Havel-Kanal östlich der Klosterbrücke (2018: Barthelme, Kretschmer, Richert, Seidel)

d 3047/III Groß Schönebeck Umg./Liebenthaler Weg (2018: Haase)

g 3149/IV Niederfinow/Oderhänge - ehemalige Kiesgruben südwestlich des Schiffshebewerks (2016: Schwabe) h 3150/IV Altglietzen Umg./Gabower Berge (2017: Brauner, Schwabe)

\section{9 (7281) Melitaea britomartis Assmann, 1847}

- RL Land Brandenburg: 0 (ausgestorben oder verschollen): WS;

- RL Deutschland (2011): Vorwarnliste.

Barthelme \& Rinnhofer „fanden den F bei Eichhorst OT Wildau letztmal.(ig) 1988 sehr vereinzelt“. Diese Angabe ist unsicher, da die Determination offenbar nur nach dem Habitus erfolgte, aber nicht durch Genitalpräparation abgesichert worden ist. Nach Auskunft von Rinnhofer haben weder er noch Barthelme derartige Untersuchungen bei dieser Art durchgeführt.

\section{0 (7280) Melitaea aurelia NICKERL, 1850}

- RL Land Brandenburg: 0 (ausgestorben oder verschollen);

- RL Deutschland (2011): Vorwarnliste.

Die Beobachtungen von Barthelme \& Rinnhofer werden ergänzend aufgeführt: „I.(m) G.(ebiet) fanden wir aurelia nur am Eichwerder b.(ei) Ebw., von 1966-1970 8 F. Ab 1970 ... verschwunden“. Dadurch wird der historische Fund von Huhst (07.6.1931, Eberswalde, RicherT 1999) konkretisiert und aktualisiert. Der Zeitpunkt des Verschwindens (vgl. RICHERT 2010: 161; „im Gebiet verschollen seit $1966^{\prime \prime)}$ muss korrigiert werden.

Fundorte (Ergänzung):

c 3149/III Ebw. Umg./Eichwerder (1966-1970: Barthelme \& Rinnhofer)

\section{Nymphalidae: Limenitinae (Eisvögel)}

\section{1 (7286) Limenitis populi (LINNAEUs, 1758)}

- Verordnung zur Neufassung der BArtschV von 2005: Besonders geschützte Art.

- RL Land Brandenburg: Kategorie R (extrem seltene Art bzw. Art mit geografischer Restriktion): WS

- RL Deutschland (2011): Kategorie 2 (stark gefährdet).

Barthelme \& Rinnhofer führen Nachweise vom Eichwerder bei Eberswalde auf, die nachfolgend ergänzt werden: 3 F E VI bis A VII 1968, 1969 und 1970 je 1 F; letztmalig dort am 1.VII.1983 2 F, leg. Barthelme und Rinnhofer. Damit liegen nunmehr auch ältere Nachweise aus dem Naturraum c (Eberswalder Tal, RICHERT 1999) vor. Aktuelle Nachweise aus dem Beobachtungszeitraum sind mir nicht bekannt.

Fundorte (Ergänzung):

c 3149/III Ebw./Eichwerder (1968, 1969, 1970, 1983: Barthelme \& Rinnhofer) 


\section{2 (7287) Limenitis camilla (LINNAEUs, 1758)}

- Verordnung zur Neufassung der BArtschV von 2005: Besonders geschützte Art.

- RL Land Brandenburg: Kategorie 2 (stark gefährdet): WS, GL;

- RL Deutschland (2011): Vorwarnliste.

Im Gebiet verschollen seit 1976 (RICHERT 2010: 160).

Barthelme \& Rinnhofer führen Nachweise aus der Zeit vor 1976 an, die nachfolgend ergänzt werden.

\section{Fundorte (Ergänzung):}

c 3149/I Neuehütte (vor 1976: Barthelme \& Riegel)

g 3150/I Oderberg/NSG Pimpinellenberg (vor 1976: Barthelme)

\section{Nymphalidae: Apaturinae (Schillerfalter)}

\section{3 (7299) Apatura iris (LinnaEus, 1758)}

- Verordnung zur Neufassung der BArtschV von 2005: Besonders geschützte Art.

- RL Land Brandenburg: Kategorie 2 (stark gefährdet): WS;

- RL Deutschland (1998) und (2011): Vorwarnliste.

Neben aktuellen Nachweisen wird nach Barthelme \& Rinnhofer ein Fund aus dem Jahre 1991 vom Eichwerder bei Eberswalde ( $5 \mathrm{~F}$ ) ergänzend aufgeführt.

\section{Neue Nachweise von bekannten Fundorten:}

\begin{tabular}{|c|c|c|}
\hline & $3148 / \mathrm{IV}$ & $\begin{array}{l}\text { Ebw./Oberheide: Waldschneise am Leuenberger } \\
\text { Graben zwischen Möllergrab (am Wiebecke-Damm) } \\
\text { und Leuenberger Wiesen (2014: Schwabe) }\end{array}$ \\
\hline 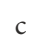 & 3149/III & Ebw./Eichwerder (1991: Barthelme \& Rinnhofer) \\
\hline \multicolumn{3}{|c|}{ Fundorte (Ergänzung): } \\
\hline & 3049/I & $\begin{array}{l}\text { Groß Ziethen Umg./Steingruben an den Ihlowber- } \\
\text { gen (2014) }\end{array}$ \\
\hline & 2949/III & Altkünkendorf Umg./Louisenhof (2014: M. Loll) \\
\hline
\end{tabular}

084 (7298) Apatura ilia (DENIS \& SCHIFFERMÜLLER, 1775)

- Verordnung zur Neufassung der BArtschV von 2005: Besonders geschützte Art.

- RL Land Brandenburg: Vorwarnliste: WS;

- RL Deutschland (2011): Vorwarnliste.

Borkowski fand die Art im Juli 1994 (außerhalb seiner Probeflächen) bei Stolpe a.O.

\section{Neue Nachweise von bekannten Fundorten:}

b 3248/II Trampe/ehemaliger TÜP (2018: Barthelme)

c 3148/III Ebw. OT Finow/Stadtgebiet: Altenhofer Straße, im Garten (2014)

3149/III Ebw. Umg./Finowbruch östlich des Eichwerder (2016); Stecherschleuse Umg./ehemaliges Kiesgrubengelände nördlich des Ortes 2017: Richert; 2018: Barthelme)
3149/IV Stecherschleuse Umg./am Rande des ehemaligen Kiesgrubengeländes nordöstlich des Ortes an der Schulstraße (2017)

Fundorte (Ergänzung):

g 3050/II Stolpe a.O. Umg./am Weg nach Alt Galow (1994: Borkowski)

\section{Nymphalidae: Satyrinae (Augenfalter)}

085 (7307) Pararge aegeria (Linnaeus, 1758)

Weitere im Gebiet festgestellte Nektarsaugpflanze (vgl. RICHERT 2014):

Eupatorium cannabium, Wasserdost. Am 04.IX.2018 beobachtete Brauner am Rand der Meelake (Schorfheide) einen F, der an überreifen Brombeer-Früchten saugte. Phänologie/Voltinismus: In der Regel bisher bivoltin; 3. Gen. jw, zunehmend regelmäßiger: 03.X.(2014), aFE, $3 \mathrm{~F}$ abgeflogen, erneute phänologische Verspätung $5 \mathrm{~d}$ gegenüber bis 2013 .

\section{6 (7309) Lasiommata megera (LiNNAEUs, 1758)}

Im Jahre 2015 wurde der F am Debritzberg bei Klein Ziethen und im Stadtgebiet von Eberswalde beobachtet. Von diesen Plätzen lagen seit Jahrzehnten keine Beobachtungen vor. Die Hoffnung auf eine erneute Ausbreitung im gesamten Gebiet hat sich bisher nicht erfüllt.

Neue Nachweise von bekannten Fundorten:

c 3148/IV Ebw./Stadtgebiet: Kleingartenanlage im Schwärzetal nördlich Forstbotanischer Garten (2015: Schwabe)

f $\quad 3049 / \mathrm{II} \quad$ Kl. Ziethen Umg./Debritzberg (2015)

Fundorte (Ergänzung):

g 3050/II Gellemersdorf Umg./Gellmersdorfer Forst: Mühlenberggebiet (1994/1995: Borkowski)

\section{9 (7334) Coenonympha pamphilus (Linnaeus,} 1758)

- Verordnung zur Neufassung der BArtschV von 2005: Besonders geschützte Art.

Weitere im Gebiet festgestellte Nektarsaugpflanzen (vgl. RICHERT 2014):

Achillea millefolium, Gemeine Schafgarbe; Achillea ptarmica, Sumpf-Schafgarbe; Armeria maritima, Gewöhnliche Grasnelke; Berteroa incana, Graukresse; Centaurea cyanea, Kornblume; Centaurea scabiosa, Skabiosen-Flockenblume; Centaurea stoebe, Rispen-Flockenblume; Cerstium arvense, Acker-Hornkraut; Cirsium arvense, Acker-Kratzdistel; Crepis biennis, Wiesen-Pippau; Daucus carota, Wilde Möhre; Helichrysum arenarium, Sand-Strohblume; Hieracium lachenalii, Gewöhnliches Habichtskraut; Knautia arvensis, Acker-Witwenblume; Leontodom hispidus, Rauher Löwenzahn; Leucanthemum vulgare, Wiesen-Margerite; Matricaria spec., Kamille; Medicago x 
varia, Luzerne; Picris echioides, Natterkopf-Bitterkraut / Wurmlattich; Pimpinella major, Große Bibernelle; Potentilla argentea, Silber-Fingerkraut; Scabiosa columbaria, Tauben-Skabiose; Sedum acre, Scharfer Mauerpfeffer; Taraxacum spec., Löwenzahn; Thlaspi arvense, AckerHellerkraut.

Phänologie Voltinismus: 22.IV.(2014), aFA, phänologische Verfrühung 1d bis gegenüber 2013.

\section{0 (7321) Coenonympha tullia (O. F. MüLLER, 1764) = typhon ROTTEMBURG 1775}

- Verordnung zur Neufassung der BArtschV von 2005: Besonders geschützte Art.

- RL Land Brandenburg: Kategorie 2 (stark gefährdet): SM, NM;

- RL Deutschland (1998) und (2011): Kategorie 2 (stark gefährdet).

Im Gebiet verschollen seit 1990 (korrigiert).

Barthelme \& Rinnhofer führen einen (letztmaligen) Fund (2 F) von 1990 aus dem Eberswalder Tal am Eichwerder auf, der ergänzend nachgetragen wird. Demnach ist die Art seit 1990, also später als bisher angenommen, im Gebiet verschollen (vgl. RicherT 1999 und 2010, im Gebiet verschollen seit 1983).

Neue Nachweise von bekannten Fundorten:

c 3149/III Ebw. Umg./Finowbruch am Eichwerder (1990: Barthelme \& Rinnhofer)

\section{1 (7325) Coenonympha arcania (LinnaEus, 1761)}

- Verordnung zur Neufassung der BArtschV von 2005: Besonders geschützte Art.

- RL Land Brandenburg: Kategorie 2 (stark gefährdet): WS.

Auf den Trockenhängen am Stadtweg bei Stolpe a.O. wurde die Art 2018 ungewöhnlich häufig beobachtet: Ich zählte im Saumbereich und innerhalb lichter Baumbestände $>42$ Falter. Dagegen war die Populationsdichte am bekannten Fundort Klandorf in der Schorfheide im Vergleich zu anderen Jahren unverändert: Der Falter wurde dort nur in geringer Zahl beobachtet (Haase, pers. Mitt.).

Phänologie/Voltinismus: 05.VI.2018: aFA, phänologische Verfrühung 2d gegenüber bis 2013.

Neue Nachweise von bekannten Fundorten:

d 3147/II Klandorf Umg./Forst Pechteich: Revier Eichhorst, Abt. 3118 (2000，2014，2015，2016，2017，2018: Haase)

g 3050/II Stolpe a.O./Trockenhänge am Stadtweg (2018); Gellmersdorf Umg./Gellmersdorfer Forst: Mühlenberggebiet (1994/1995: Borkowski)

3150/I Oderberg/NSG Pimpinellenberg (2015, 2017: Brauner)
Fundorte (Ergänzung):

d 3047/IV Groß Schönebeck/Waldinnensaum bei Meelake (2018: Brauner)

g 3050/IV Lunow Umg./Gebiet Lunower Hölzchen (1994/1995: Borkowski)

092 (7326) Coenonympha glycerion (BORKHAUSEN, 1788) = iphis DENIS \& SCHIFFERMÜLLER, 1775

- Verordnung zur Neufassung der BArtschV von 2005: Besonders geschützte Art.

- RL Deutschland (2011): Vorwarnliste.

Weitere im Gebiet festgestellte Nektarsaugpflanzen (RICHERT 2010 und 2014):

Anthemis tinctoria, Färber-Hundkamille; Centaurea scabiosa, Skabiosen-Flockenblume; Centaurea stoebe, Rispen-Flockenblume; Dianthus carthusianorum, Karthäuser-Nelke; Ligustrum vulgare, Gemeiner Liguster; Origanum vulgare, Dost; Rubus fruticosus agg., Brombeere; Sedum acre, Scharfer Mauerpfeffer; Thymus serpyllum, Sand-Thymian.

Phänologie/Voltinismus: Ein ungewöhnlich später Fund durch Gottwald wurde mir erst neuerdings bekannt: Am 20.IX.2003 registrierte er bei Brodowin vier Falter. „Diese Exemplare gehören mit hoher Wahrscheinlichkeit einer früher nie beobachteten sehr unvollständigen 2. Generation an. Auch in der Oberlausitz wurden vom 27.VIII. bis 5.IX.2012 4 Falter einer sehr unvollständigen 2. Generation beobachtet" (GelBrecht et al. 2017). Das Jahr 2003 fiel durch überdurchschnittliche monatliche Temperaturmittel vom Mai bis September auf, die in unserem Gebiet besonders im Juni und August erheblich über denen des Zeitraumes von 1991-2012 lagen (RICHERT 2014).

Neue Nachweise von bekannten Fundorten:

b: 3248/II Trampe Umg./ehemaliger TÜP (2014, 2016: Richert; 2018: Barthelme, Richert)

c 3148/III Ebw. OT Finow Umg./Forst Finowtal: Biesenthaler Straße $(2015,2016)$

3148/IV Ebw. OT Finow Umg./Forst Finowtal: Stromtrasse südlich des Ortes (2014)

3149/IV Stecherschleuse Umg./ehemaliges Kiesgrubengelände nordöstlich des Ortes (2014, 2015: Schwabe; 2018: Barthelme)

d 3147/I Klandorf/Bergstraße (2016: Seidel)

e 3049/I Groß Ziethen Umg. Steingruben an den Ihlowbergen (2014, 2016); Töpferberge: Nordwestlicher Ortsausgang (2014)

g 3050/II Stolpe/Trockenhänge am Stadtweg (2018)

3149/IV Falkenberg (Hohenfinow) Umg./NSG Schäfergrund (2017: Brauner)

3150/I Oderberg/NSG Pimpinellenberg (2015, 2016, 2017, 2108: Brauner), Geistberg (2016: Schwabe)

3150/IV Altglietzen Umg./Gabower Berge (2017: Brauner)

Fundorte (Ergänzung):

c $3147 / \mathrm{IV}$ Finowfurt Umg./Finowfließniederung nördlich des Lehnssees (2016) 
3148/III Ebw. OT Finow/Stadtgebiet: Altenhofer Straße, im Garten (2016, 2018) und Umg./Forst Finowtal: Flugplatz Finow - Fotovoltaikanlage (2014-2017: Brauner)

3149/I Niederfinow Umg./Oder-Havel-Kanal östlich der Klosterbrücke (2018: Barthelme, Kretschmer, Richert, Seidel)

d 3047/IV Eichhorst Umg./Üderheide: Waldsaum südlich Werbellinsee (2015: Brauner)

f 3049/II Klein Ziethen Umg./Debritzberg (2015)

3050/I Herzsprung Umg./Bunkerberg an der Straße nach Bölkendorf (2015); Bölkendorf Umg./NSG Tiefer See (2016)

g 3149/IV Niederfinow/Oderhänge - ehemalige Kiesgruben südwestlich des Schiffshebewerks (2016: Schwabe)

\section{3 (7344) Aphantopus hyperantus (Linnaeus,} 1758)

Weitere im Gebiet festgestellte Nektarsaugpflanzen (vgl. RICHERT 2014):

Achillea millefolium, Gemeine Schafgarbe; Centaurea scabiosa, Skabiosen-Flockenblume; Centaurea steobe, Rispen-Flockenblume; Chrysanthemum (= Tanacetum) vulgare, Rainfarn; Dianthus carthusianorum, Karthäuser-Nelke; Helichrysum arenarium, Sand-Strohblume; Heliopsis helianthoides, Garten-Sonnenauge; Hieracium lachenalii, Gewöhnliches Habichtskraut; Hieracium pilosella, Kleines Habichtskraut; Matricaria spec., Kamille; Medicago x varia, Luzerne; Mentha aquatica, WasserMinze; Ranunculus spec., Hahnenfuß; Scabiosa columbaria, Tauben-Skabiose; Solidago canadensis, Kanadische Goldrute; Torilis japonica, Gewöhnlicher Klettenkerbel; Trifolium pratense, Wiesen- (Rot-) Klee; Valeriana officinalis, Arznei-Baldrian; Vicia cracca, Vogel-Wicke.

\section{4 (7350) Maniola jurtina (LinNAEus, 1758)}

Weitere im Gebiet festgestellte Nektarsaugpflanzen (vgl. RICHERT 2014):

Achillea millefolium, Gemeine Schafgarbe; Armeria maritima, Gewöhnliche Grasnelke; Ballota nigra, Schwarznessel; Berteroa incana, Graukresse; Campanula trachelium, Nesselblättrige Glockenblume; Carduus crispus, Krause Distel; Centaurea jacea, Gewöhnliche (Wiesen-) Flockenblume; Centaurea scabiosa, Skabiosen-Flockenblume; Chondrilla juncea, Knorpellattich; Chrysanthemum (= Tanacetum) vulgare, Rainfarn; Cirsium oleraceum, Kohl-Kratzdistel; Cosmos bipinnatus, Garten-Kosmee; Crepis biennis, Wiesen-Pippau; Crepis capillaris, Grüner Pippau; Daucus carota, Wilde Möhre; Diplotaxis tenuifolia, Schmalblättriger Doppelsame (Gewürzrauke); Hypochoeris radicata, Gemeines Ferkelkraut; Leontodon autumnalis, Herbst-Löwenzahn; Ligustrum vulgare, Gemeiner Liguster; Matricaria spec., Kamille; Mentha aquatica, Wasser-Minze; Picris echioides, Natterkopfblättriges Bitterkraut (Wurmlattich); Picris hieracioides, Gewöhnliches Bitterkraut; Potentilla argentea, Silber-Fingerkraut; Scabiosa columbaria, Tauben-Skabiose; Torilis japonica, Gewöhnlicher Klettenkerbel; Trifolium medium,
Mittlerer Klee; Trifolium pratense, Wiesen- (Rot-) Klee; Valeriana officinalis, Arznei-Baldrian.

Die Eiablage beobachtete Brauner an Dactylis glomerata, Knäuelgras.

\section{5 (7353) Hyponephele lycaon (RotTEMBURG,} 1775)

- RL Land Brandenburg: Kategorie 2 (stark gefährdet): TR;

- RL Deutschland (2011): Kategorie 2 (stark gefährdet).

Neue Nachweise von bekannten Fundorten:

c 3148/III Ebw. OT Finow Umg./Forst Finowtal: Flugplatzgelände an der Photovoltaikanlage (2014-2017: Brauner), Einflugschneise Flugplatz (2016: Seidel; 2018: Richert)

3148/IV Ebw. OT Finow Umg./Forst Finowtal: Stromtrasse südlich des Ortes (2014)

\section{- (7340) Pyronia tithonus (Linnaeus, 1767)}

- RL Land Brandenburg: Kategorie 3 (gefährdet): WS-TR.

In der kommentierten Artenliste von Barthelme \& Rinnhofer heißt es: „Am 3.8.1969 fand (Barthelme) 2 Expl. im Geb.(iet) Eichwerder am Rand einer Sumpfwiese beim Blütenbesuch. $\mathrm{Ob}$ es sich dabei um verschleppte bzw. zugewanderte Tiere hand.(elte,) kann nicht eindeutig gesagt werden. Trotz intensiv.(er) Suche fand ich die Art nie wieder i.(m) G.(ebiet)“. Belege lagen mir nicht vor. Die Arealgrenze verläuft heute südlich von Berlin (REINHARDT \& Thust 1993).

Angesichts der Tatsache, dass die Art früher aus nordund südöstlichen Nachbargebieten bekannt war (FrIESE 1956, Herrman 1904, vgl. Richert 1999) und „auch in der jüngeren Vergangenheit" von Funden einzelner Falter nördlich dieser Arealgrenze berichtet wird (Gelbrecht et al. 2016: 288), ist die Angabe nicht völlig abwegig. Die Art gehört nicht zum Faunenbestand des Gebietes.

097 (7430) Hipparchia hermione (LinNAEus, 1764) = alcyone (DENIS \& SCHIFFERMÜLLER, 1775)

- Verordnung zur Neufassung der BArtschV von 2005: Streng geschützte Art.

- RL Land Brandenburg: Kategorie 1 (vom Aussterben bedroht): WS-TR;

- RL Deutschland (2011): Kategorie 2 (stark gefährdet).

Im Gebiet verschollen seit 1970 (korrigiert).

Barthelme \& Rinnhofer datieren ihren letztmaligen Fund mit „24.VII.1970, 1 Expl. leg. Barthelme“. Demnach kam die Art noch länger im Gebiet vor als in RICHERT 1999 und 2010: 161 aufgeführt („verschollen seit 1965“). 
Fundorte (Ergänzung):

c 3149/I Neuehütte (im Zeitraum 1965-1970: Barthelme \& Rinnhofer

\section{8 (7436) Hipparchia semele (LinnaEus, 1758)}

- RL Land Brandenburg: Vorwarnliste: TR, CB;

- RL Deutschland (2011): Kategorie 3 (gefährdet).

Weitere im Gebiet festgestellte Nektarsaugpflanzen (vgl. RICHERT 2014):

Centaurea jacea, Gemeine (Wiesen-) Flockenblume; Berteroa incana, Graukresse; Helichrysum arenarium, SandStrohblume.

Phänologie/Voltnismus: aFE 19.IX.2018, 1 F auf der Einflugschneise Flugplatz Finow (Brauner); phänologische Verspätung 10d gegenüber bis 2013.

Neue Nachweise von bekannten Fundorten:

c 3148/III Ebw. OT Finow Umg./Forst Finowtal: Einflugschneise Flugplatz (2014, 2016, 2017, 2018: Richert; 2018: Brauner; 2016: Seidel), am Nordrand des Flugplatzes (2017); Fotovoltaikanlage Flugplatz Finow (2014-2017: Brauner)

3148/IV Ebw. OT Finow Umg./Forst Finowtal: Stromtrasse südlich des Ortes (2014)

Fundorte (Ergänzung):

c 3148/II Ebw.-Nordend Umg./Oder-Havel-Kanal an der Bahnunterführung (2016: Schwabe)

\section{1 (7415) Melanargia galathea (LinnAeus, 1758)}

Weitere im Gebiet festgestellte Nektarsaugpflanzen (vgl. RICHERT 2014):

Armeria maritima, Gewöhnliche Grasnelke; Buddleja davidii, Chinesicher Sommerflieder; Carduus crispus, Krause Distel; Centaurea jacea, Gemeine (Wiesen-) Flockenblume; Chondrilla juncea, Binsen- oder Großer Knorpellattich; Cirsium vulgare, Gemeine Kratzdistel; Dianthus cathusianorum, Karthäuser-Nelke; Scabiosa columbaria, Tauben-Skabiose.

Phänologie/Voltinismus: 05.VI.2018, aFA, phänologische Verfrühung 2d gegenüber bis 2013 .

\section{Zu Teil II: Spinner und Schwärmer (RICHERT 2001)}

\section{Hepialidae (Wurzelbohrer)}

\section{2 (0063) Triodia sylvina (Linnaeus, 1761)}

Die Art wurde inzwischen auch im Parsteiner Becken (Naturraum f; vgl. Richert 2001) nachgewiesen. Damit liegen aus allen Naturräumen unseres Gebietes Nachweise vor.

Phänologie /Voltnismus: Univoltin; 12.IX.(2013), aFE, phänologische Verspätung 2d gegenüber bis 2013 (korrigiert nach MüLler 2015; in RICHERT 2014 war der 10.IX(1999) als aFE angegeben).
Neue Nachweise von bekannten Fundorten:

b 3248/II Trampe/ehemaliger TÜP (2016: Busse \& Müller)

c 3147/I Zerpenschleuse/Ortsgebiet: Berliner Straße, im Garten (2014, 2015, 2016: Busse)

3147/I Marienwerder Umg./Oder-Havel-Kanal nordwestlich des Ortes (2014, 2015: Busse)

3148/III Ebw. OT Finow/Stadtgebiet: Altenhofer Straße, im Garten $(2014,2015,2016,2018)$

Fundorte (Ergänzung):

d 3047/III Groß Schönebeck/Ortsgebiet: Friedenstraße, im Garten (2014: Haase)

c $3147 / \mathrm{IV} \quad$ Finowfurt Umg./Kesselmoor an der Finowfließniederung nordöstlich des Lehnssees (2016: Richert, Schwabe)

3148/IV Ebw.-Westend/Unterheide: Wildparkstraße 1973, 1976: Kittel); Ebw./Stadtgebiet: Kleingartenanlage im Schwärzetal nördlich Forstbotanischer Garten (2018: Schwabe)

e 3049/IV Chorin Umg./Forst Chorin: Teerbrennerberge, $1 \mathrm{~F}$ auf Windwurffläche am Olberg (2013: MüLleR 2015)

f 3049/IV Brodowin/Karpatenweg östlich des Krugbergs (2015)

g 3050/II Gellmersdorf Umg./Gellmersdorfer Forst: Mühlenberggebiet (1994/1995: Borkowski)

3150/I Oderberg/am Friedhof (2016: Schwabe)

\section{3 (0069) Pharmacis fusconebulosa (DE GEER, 1778)}

- RL Deutschland (2011): Vorwarnliste.

Fundorte (Ergänzung):

c $3147 / \mathrm{IV}$ Finowfurt Umg./Kesselmoor an der Finowfließniederung nordöstlich des Lehnssees $(2016,2017)$

\section{4 (0078) Phymatopus hecta (Linnaeus, 1758)}

Neue Nachweise von bekannten Fundorten:

e 3149/II Liepe Umg./Forst Chorin: NSG Plagefenn (2018 Richert, Schwabe)

Fundorte (Ergänzung):

c: 3146/II Zerpenschleuse Umg./Hammerbruch (1999: Haase) (westlich angrenzendes Nachbargebiet)

3147/IV Finowfurt Umg./Kesselmoor an der Finowfließniederung nordöstlich des Lehnssees (2017)

3148/IV Ebw.-Westend/Unterheide: Wildparkstraße (1973: Kittel)

105 (0080) Hepialus humuli (LinNAEus, 1758)

Fundorte (Ergänzung):

c 3147/IV Finowfurt Umg./Kesselmoor an der Finowfließniederung nordöstlich des Lehnssees (2017) 


\section{Psychidae (Sackträger)}

Psychinae

\section{7 (0751) Narycia duplicella (GoEzE, 1783)}

Ein älterer, mir bisher nicht bekannter Fund wird nachgetragen.

Nachweise von bekannten Fundorten:

g 3150/I Oderberg Umg./Gebiet Pimpinellenberg-Teufelsberg (Juni 1994: Theimer)

\section{8 (0762) Dahlica triquetrella (HüBNER, 1813)}

Ein älterer, mir bisher nicht bekannter Fund wird nachgetragen.

Nachweise von bekannten Fundorten:

g 3150/I Oderberg Umg./Gebiet Pimpinellenberg-Teufelsberg (April 1995: Theimer)

109 (0766) Dahlica lazuri (ClerCK, 1759) = fumosella (HEINEMANN, 1870)

Ein älterer, mir bisher nicht bekannter Fund wird nachgetragen.

Nachweise von bekannten Fundorten:

g 3150/I Oderberg Umg./Gebiet Pimpinellenberg-Teufelsberg (April 1999: Theimer)

\section{3 (0866) Bacotia claustrella (BRUAND, 1845) = sepium SPEYER, 1846}

Ein älterer, mir bisher nicht bekannter Fund von Oderberg wird nachgetragen.

Nachweise von bekannten Fundorten:

g 3150/I Oderberg Umg./Gebiet Pimpinellenberg-Teufelsberg (Juni 1994: Theimer)

\section{4 (0868) Proutia betulina (Zeller, 1839)}

Die Art kommt in allen Naturräumen des Gebietes vor. Die Beobachtungslücke im Naturraum h (Odertal) wurde geschlossen (vgl. RicherT 2001).

Neue Nachweise von bekannten Fundorten:

d 3148/I Lichterfelde Umg./NSG Buckowseerinne: Streuobstwiesen - Waldrand südöstlich des Gr. Buckowsees (2015)

f 3049/IV Brodowin Umg./Kl. Rummelsberg (2015)

Fundorte (Ergänzung):

b 3149/III Ebw.-Südend Umg./Hohenfinower Wald: Nordöstlich der Leuenberger Wiesen (2014)

h 3150/II Hohensaaten/Oderniederung an der Alten Schleuse (2014)

\section{6 (0878) Psyche crassiorella (BRUAND, 1851)}

Ein älterer, mir bisher nicht bekannter Fund wird nachgetragen. Damit ist die Art auch auf den Odertalhängen nachgewiesen (Naturraum g; vgl. RicheRT 2001).

Nachweise von bekannten Fundorten:

g 3150/I Oderberg Umg./Gebiet Pimpinellenberg-Teufelsberg (1994: Theimer)

\section{7 (0936) Epichnopteryx plumella (DeNis \&} SCHIFFERMÜLLER, 1775) = pulla ESPER, 1785

Schwabe fand ein frisches Männchen auf einer Stromtrasse mit Calluna-Heide bei Spechthausen. Ein älterer, mir bisher nicht bekannter Fund wird nachgetragen.

Fundorte (Ergänzung):

b 3148/IV Spechthausen Umg./Barnimer Heide: Stromtrasse südwestlich des Ortes (2015: Schwabe)

g 3150/I Oderberg Umg./Gebiet Pimpinellenberg-Teufelsberg (1994: Theimer)

\section{9 (0961) Canephora hirsuta (PoDA, 1761) \\ = unicolor (HUFNAGEL, 1766)}

Neue Nachweise von bekannten Fundorten:

c 3148/IV Ebw. OT Finow/Forst Finowtal: Stromtrasse südlich des Ortes (2015)

3149/IV Stecherschleuse Umg./ehemaliges Kiesgrubengelände nordöstlich des Ortes (2015: Schwabe)

g 3150/I Oderberg Umg./Gebiet Pimpinellenberg-Teufelsberg (1994: Theimer)

Fundorte (Ergänzung):

d 3047/III Groß Schönebeck Umg./Liebenthaler Weg (2017, 2018: Haase)

3148/II Lichterfelde Umg./Waldgebiet nördlich des NSG Buckowseerinne zwischen Gut Blütenberg und Fortsetzung der Rüsternallee (2015)

g 3050/II Stolpe a.O./Trockenhänge am Stadtweg (2018)

3150/I Oderberg/Geistberg (2017: Schwabe)

120 (0963) Pachythelia villosella (OCHSENHEIMER, 1811)

Ein älterer, mit bisher nicht bekannter Fund wird nachgetragen. Damit liegt ein erster Nachweis von den Odertalhängen vor (Naturraum g; vgl. RiCHERT 2001).

Nachweise von bekannten Fundorten:

g 3150/I Oderberg Umg./Gebiet Pimpinellenberg-Teufelsberg (1993: Theimer) 
123 (1012) Sterrhopteryx fusca (HAWORTH, 1809) = hirsutella HüBNER, 1793

Fundorte (Ergänzung):

b 3148/IV Spechthausen Umg./Fischteiche nördlich des Ortes (2016: Schwabe)

c 3147/I Zerpenschleuse/Ortsgebiet: Berliner Straße, im Garten (2014: Busse) (Beleg in coll. Zool. Staatss. München, BARCOD ZSM Lep 90050)

3147/IV Marienwerder Umg./Kiesgrube südwestlich des Sportplatzes (2015)

3148/III Ebw. OT Finow Umg./Forst Finowtal: Einflugschneise Flugplatz (2016)

3148/IV Ebw./Stadtgebiet: Kleingartenanlage im Schwärzetal nördlich Forstbotanischer Garten (2016: Schwabe)

\section{Limacodidae (Schneckenspinner)}

126 (3912) Heterogenea asella (DENIs \& SCHIFFERMÜLLER, 1775)

- RL Deutschland (2011): Vorwarnliste.

Die Art wird zwar vereinzelt, aber mit zunehmender Verbreitung im Gebiet gefunden.

Phänologie/Voltinismus: 07.VI.(2018) aFA, phänologische Verfrühung 3d gegenüber bis 2013.

Neue Nachweise von bekannten Fundorten:

e 3149/II Liepe Umg./Forst Chorin: NSG Plagefenn (2018: Richert \& Schwabe)

Fundorte (Ergänzung):

c 3147/I Zerpenschleuse/Ortsgebiet: Berliner Straße, im Garten (2013, 2015: Busse)

3147/II Finowfurt/Besters Fließ - Hirtenweg (1995: Kittel)

3147/IV Finowfurt Umg./Kesselmoor an der Finowfließniederung nordöstlich des Lehnssees (2016: Richert, Schwabe)

3148/IV Ebw./Stadtgebiet: Kleingartenanlage im Schwärzetal nördlich Forstbotanischer Garten (2016: Schwabe)

e 3049/I Groß Ziethen Umg./Steingruben an den Ihlowbergen bei Sperlingsherberge (2014)

\section{Zygaenidae (Widderchen)}

\section{7 (3924) Rhagades pruni (DENIS \& SCHIFFER- MÜLLER, 1775)}

- Verordnung zur Neufassung der BArtschV von 2005: Besonders geschützte Art.

- RL Land Brandenburg: Kategorie 3 (gefährdet); CB, $\mathrm{HE}$;

- RL Deutschland (2011): Kategorie 3 (gefährdet).

Neue Nachweise von bekannten Fundorten:

c 3148/IV Ebw. OT Finow/Forst Finowtal: Stromtrasse südlich des Ortes (2015)

d 3047/III Groß Schönebeck Umg./Schorfheide: Revier Rehluch, Abt. 35 (2015, 2016: Haase)
Fundorte (Ergänzung):

b 3148/IV Spechthausen Umg./Barnimer Heide: Stromtrasse südwestlich des Ortes (2015)

3248/II Schönholz Umg./Barnimer Heide: Stromtrasse nordwestlich des Ortes (2015)

\section{9 (3945) Adscita statices (LinNAEus, 1758)}

- Verordnung zur Neufassung der BArtschV von 2005: Besonders geschützte Art.

- RL Land Brandenburg: Vorwarnliste: TR, MW;

- RL Deutschland (2011): Vorwarnliste.

Am 29.V.2017 beobachtete Brauner um die Mittagszeit zwischen den Modulreihen der Photovoltaikanlage Finow II drei erwachsene Raupen. Ein Exemplar fraß an einem Blatt von Rumex thyrsiflorus (Rispen-Sauerampfer), zwei weitere ruhten bzw. sonnten sich an benachbarten krautigen Pflanzen.

Neue Nachweise von bekannten Fundorten:

b 3149/III Sommerfelde Umg./ehemaliger TÜP Panzerbahn (2016: Seidel)

3248/II Trampe/ehemaliger TÜP (2016: Richert, Seidel)

c 3148/IV Ebw. OT Finow Umg./Forst Finowtal: Stromtrasse südlich des Ortes (2014)

3149/III Stecherschleuse Umg./ehemaliges Kiesgrubengelände nördlich des Ortes (2017)

3149/IV Stecherschleuse Umg./am Rande des ehemaligen Kiesgrubengeländes nordöstlich des Ortes (2014 Schwabe)

d 3047/III Groß Schönebeck/Ortsgebiet (2015: Haase)

e 2949/III Altkünkendorf Umg./Forst Grumsin: Waldwiesenkomplex nördlich des Gr. Grumsin-Sees (2015)

g 3050/II Gellmerdorf Umg./Gellmersdorfer Forst: Mühlenberggebiet (1994/1995: Borkowski)

3149/IV Falkenberg (Hohenfinow) Umg./NSG Schäfergrund (2016: Seidel)

3150/I Oderberg/NSG Pimpinellenberg (2017: Brauner)

3150/III Gabow/Oderhänge: Granitbergebiet (2015)

Fundorte (Ergänzung):

c 3148/III Ebw. OT Finow Umg./Forst Finowtal: Fotovoltaikanlage Flugplatz Finow (2014-2017: Brauner)

3148/II Ebw.-Nordend Umg./Oder-Havel-Kanal nördlich des Ortes (2015: Schwabe)

g 3050/II Stolpe a.O./Trockenhänge am Stadtweg (2018)

3050/IV Lunow Umg./Gebiet Lunower Hölzchen (1994/1995: Borkowski)

3150/I Oderberg/Geistberg (2016: Schwabe)

130 (3973) Zygaena minos (DENIS \& SCHIFFERMÜLLER, 1775) = pimpinellae (GUHN, 1932)

- Verordnung zur Neufassung der BArtschV von 2005: Besonders geschützte Art.

- RL Land Brandenburg: Kategorie 2 (stark gefährdet): TR;

- RL Deutschland (2011): Kategorie 3 (gefährdet). 
Ältere Funde (Belege in coll. Kittel) werden nach sicherer Bestimmung durch Genitalpräparation ergänzend mitgeteilt.

Auf den Trockenhängen am Stadtweg bei Stolpe a.O. fand ich eine stabile Population mit hoher Individuendichte.

Weitere von der Art im Gebiet genutzte Nektarsaugpflanzen (vgl. Richert 2001 und 2014):

Armeria maritima, Gewöhnliche Grasnelke, Centaurea stoebe, Rispen-Flockenblume; Consolida regalis, FeldRittersporn; Thymus serpyllum, Sand-Thymian.

Phänologie/Voltinismus: 05.VI.2018, aFA, phänologische Verfrühung 1d gegenüber bis 2013.

Neue Nachweise von bekannten Fundorten:

\begin{tabular}{|c|c|c|}
\hline & 3149/III & $\begin{array}{l}\text { Stecherschleuse Umg./ehemaliges Kiesgrubenge- } \\
\text { lände nördlich des Ortes (2017) }\end{array}$ \\
\hline & $3148 / \mathrm{I}$ & $\begin{array}{l}\text { Werbellin Umg./Autobahnanschlussstelle (1963: leg. } \\
\text { Duckert, det. genit. Richert; 1972: leg. Kittel, det. } \\
\text { genit. Richert); Lichterfelde Umg./Moospfuhlgebiet } \\
\text { südlich des Gr. Buckowsees (1957: leg. Duckert, det. } \\
\text { genit. Richert) }\end{array}$ \\
\hline & $3049 / \mathrm{I}$ & $\begin{array}{l}\text { Groß Ziethen Umg./Steingruben an den Ihlow- } \\
\text { bergen }(2014 \text {, det. genit.; } 2016)\end{array}$ \\
\hline & 3049/IV & Brodowin Umg./Schiefer Berg (2015: Brauner) \\
\hline & $3149 / \mathrm{IV}$ & $\begin{array}{l}\text { Niederfinow Umg./Oderhänge - ehemalige Kies- } \\
\text { gruben südwestlich des Schiffshebewerks (2014, } \\
\text { 2016: Schwabe); Falkenberg (Hohenfinow) Umg./ } \\
\text { NSG Schäfergrund (2017: Brauner) }\end{array}$ \\
\hline & $3150 / \mathrm{I}$ & Oderberg/NSG Pimpinellenberg (2015: Brauner) \\
\hline
\end{tabular}

Fundorte (Ergänzung):

f 3050/I Herzsprung Umg./Bunkerberg an der Straße nach Bölkendorf (2015: det. genit.)

g 3050/II Stolzenhagen (1971: leg. Kittel, det. genit. Richert); Stolpe a.O./Trockenhänge am Stadtweg (2017 det. genit. Richert, 2018)

3150/I Oderberg/Teufelsberg (1976: leg. Kittel, det. genit. Richert)

\section{1 (3974) Zygaena purpuralis (BRÜNNICH, 1763)}

- Verordnung zur Neufassung der BArtschV von 2005: Besonders geschützte Art.

- RL Land Brandenburg: Kategorie 1 (vom Aussterben bedroht): TR;

- RL Deutschland (2011): Vorwarnliste.

Ältere Funde (Belege in coll. Kittel) werden nach sicherer Bestimmung durch Genitalpräparation ergänzend mitgeteilt.

Neue Nachweise von bekannten Fundorten:

c 3148/I Ebw. OT Finow Umg./Oder-Havel-Kanal nördlich des Ortes (1966, 1969, 1970: leg. Duckert, det. genit. Richert)

Fundorte (Ergänzung):

c 3147/II Finowfurt Umg./Üdersee - Südwestende (1971: leg. Kittel, det. genit. Richert

3148/III Ebw. OT Finow Umg./Forst Finowtal: Kalkofenbrück im Schwärzetal (1954: leg. Duckert, det. genit. Richert)

\section{3 (3983) Zygaena loti (DeNis \& SCHIFFER-} MÜLLER, 1775) $=$ achilleae $($ ESPER, 1870)

- Verordnung zur Neufassung der BArtschV von 2005: Besonders geschützte Art.

- RL Land Brandenburg: Kategorie 3 (gefährdet): TR. Weitere im Gebiet nachgewiesene Nektarsaugpflanze (vgl. Richert 2001): Ballota nigra, Schwarznessel. Phänologie/Voltinismus: 12.VI.(2018), aFA, phänologische Verfrühung 7d gegenüber bis 2013.

Neue Nachweise von bekannten Fundorten:

c 3148/III Ebw. OT Finow Umg./Forst Finowtal: Stromtrasse südlich des Ortes am Rande des Schwärzetals bei Kalkofenbrück (2014)

3149/I Ebw.-Nordend Umg./Oder-Havel-Kanal östlich der Wassertorbrücke Richtung Ragöser Damm (2015: Schwabe); Niederfinow Umg./Oder-HavelKanal östlich der Klosterbrücke (2018: Kretschmer, Richert, Seidel)

3149/III Stecherschleuse Umg./ehemaliges Kiesgrubengelände nördlich des Ortes (2017)

3149/IV Stecherschleuse Umg./am Rande des ehemaligen Kiesgrubengeländes nordöstlich des Ortes (2014, 2015, 2016: Schwabe)

3149/III Ebw. Umg./Finowbruch östlich des Eichwerder (2016: Schwabe)

f 3049/IV Brodowin Umg./Schiefer Berg (2015: Brauner)

3149/IV Falkenberg (Hohenfinow) Umg./NSG Schäfergrund (2017: Brauner)

g 3050/II Stolpe a.O./Trockenhänge am Stadtweg $(2017,2018$; Stolzenhagen/NSG Krähen- und Jungfernberge (2015)

3150/I Oderberg/NSG Pimpinellenberg (2015, 2017, 2018: Brauner)

3150/III Gabow/Oderhänge: Granitberggebiet (2015: Richert; 2018: Kleße)

3150/IV Altglietzen/Gabower Berge (2017: Schwabe)

Fundorte (Ergänzung):

f 3050/III Parstein Umg./Sonnenberge (2015)

\section{4 (3992) Zygaena viciae (DeNIS \& SCHIFFER- MÜLLER, 1775) $=$ meliloti $($ ESPER, 1789)}

- Verordnung zur Neufassung der BArtschV von 2005: Besonders geschützte Art.

- RL Land Brandenburg: Vorwarnliste: TR, WS.

Als weitere von der Art im Gebiet genutzte Nektarsaugpflanze wurde Thymus serpyllum, Sand-Thymian, festgestellt, als weitere Raupennahrungspflanze Vicia tenuifolia, Feinblättrige Wicke (vgl. RicherT 2001 und 2014).

\section{Neue Nachweise von bekannten Fundorten:}

b 3248/II Trampe/ehemaliger TÜP (2016: Richert, Seidel; 2018: Richert)

c 3148/IV Ebw. OT Finow Umg./Forst Finowtal: Stromtrasse südlich des Ortes am Randes des Schwärzetals bei Kalkofenbrück (2014)

3149/III Stecherschleuse Umg./ehemaliges Kiesgrubengelände nördlich des Ortes $(2017,2018)$ 
d 3047/III Groß Schönebeck/am Bahnhof (2014, 2015, 2016: Haase)

e 3049/I Groß Ziethen Umg./Steingruben an den Ihlowbergen $(2014,2016)$

f 3049/IV Brodowin Umg./Schiefer Berg (2015: Brauner)

g 3050/II Stolzenhagen (1971: Kittel); Gellmersdorf Umg./ Gellmersdorfer Forst: Mühlenberggebiet (1994/1995: Borkowski)

3149/IV Falkenberg (Hohenfinow) Umg./NSG Schäfergrund (2017: Brauner)

3150/III Gabow/Oderhänge: Granitberggebiet (2018: Kleße)

Fundorte (Ergänzung):

c 3148/III Ebw. OT Finow Umg./Forst Finowtal: Fotovoltaianlage Flugplatz Finow (2014, 2015: Brauner)

3148/IV Ebw.-Westend/Unterheide: Drehnitzwiesen (1974: Kittel)

d 3047/III Groß Schönebeck Umg./Liebenthaler Weg (2017: Haase)

g 3050/II Stolpe a.O./Trockenhänge am Stadtweg (2018)

3150/I Oderberg/Geistberg (2014, 2015: Schwabe)

\section{5 (3995) Zygaena ephialtes (LinNAEUs, 1767)}

- Verordnung zur Neufassung der BArtschV von 2005: Besonders geschützte Art.

- RL Land Brandenburg: Kategorie 3 (gefährdet): TR.

Der F wurde im Stadtgebiet von Finow am 10.VII.2018 morgens über mindestens eine Stunde beim Saugen an Buddleja davidii, Chinesischer Sommerflieder, beobachtet nachdem er zuvor dort an einem Blütenstand wohl auch übernachtet hatte (Brauner, Fotobeleg). Am 27.VII.2017 beobachtete Brauner auf den Gabower Bergen mehrere Eiablagen an Securigera varia, Bunte Kronwicke.

\section{Neue Nachweise von bekannten Fundorten:}

b 3149/III Sommerfelde Umg./ehemaliger TÜP (2016: Seidel)

3247/II Biesenthal Umg./Finowfließniederung am Schwarzen See (1956; Duckert)

c 3148/III Ebw. OT Finow/Stadtgebiet: Biesenthaler Straße, im Garten (2018)

3149/III Ebw. Umg./Finowbruch östlich des Eichwerder (2015: Schwabe); Stecherschleuse Umg./ehemaliges Kiesgrubengelände nördlich des Ortes (2017: Richert), Hänge zum Finowbruch westlich des Ortes (2017: Schwabe)

3149/IV Stecherschleuse Umg./am Rande des ehemaligen Kiesgrubengelände nordöstlich des Ortes an der Schulstraße (2015: Schwabe)

g 3050/II Stolpe a.O. Umg./Trockenhänge am Stadtweg (2017, 2018); Stolzenhagen/NSG Krähen- und Jungfernberge (2015)

3149/IV Niederfinow Umg./Oderhänge - ehemalige Kiesgruben südwestlich des Schiffshebewerks (2014: Schwabe)

3150/I Oderberg/Teufelsberg (1975: Kittel)

136 (3998) Zygaena filipendulae (LinnaEus, 1758)

- Verordnung zur Neufassung der BArtschV von 2005: Besonders geschützte Art.
Als weitere von der Art im Gebiet genutzte Nektarsaugpflanzen wurden Cirsium vulgare, Gewöhnliche Distel, und Picris hieracioides, Gewöhnliches Bitterkraut, festgestellt (vgl. RicheRT 2001 und 2014).

Phänologie/Voltinismus: 14.VI.(2018), aFA, phänologische Verfrühung 3d gegenüber bis 2013.

\section{Neue Nachweise von bekannten Fundorten:}

b 3248/II Trampe/ehemaliger TÜP (2016: Richert, Seidel; 2017, 2018: Richert)

d 3148/I Werbellin (1957: Duckert); Lichterfelde Umg./ NSG Buckowseerinne: Hänge südwestlich Margaretenhof (2015)

e 3049/I Groß Ziethen Umg./Steingruben an den Ihlowbergen (2014)

f 3049/IV Brodowin Umg./Gr. Rummelsberg (2016), Kl. Rummelsberg (2014: Brauner)

g 3050/II Stolpe a.O. Umg./Trockenhänge am Stadtweg (2017, 2018); Stolzenhagen/NSG Krähen- und Jungfernberge (2016, 2018: Richert, Schwabe)

3149/IV Falkenberg (oder Hohenfinow) Umg./NSG Schäfergrund (2017: Brauner; 2018: Richert)

3150/I Oderberg/NSG Pimpinellenberg (2014, 2015, 2017, 2018: Brauner)

3150/III Gabow Umg./Granitberggebiet (2014)

3150/IV Altglietzen Umg./Gabower Berge (2017: Brauner)

Fundorte (Ergänzung):

e 3149/II Liepe Umgeb./Frischweide östlich Krugsee (2014: Brauner)

g 3150/I Oderberg/Geistberg (2018: Richert, Schwabe)

\section{7 (3999) Zygaena lonicerae (SCHEVEN, 1777)}

- Verordnung zur Neufassung der BArtschV von 2005: Besonders geschützte Art.

- RL Land Brandenburg: Kategorie 2 (stark gefährdet): WS;

- RL Deutschland (2011): Vorwarnliste.

Phänologie/Voltinismus: 18.VI.(2018), aFA, phänologische Verfrühung 6d gegenüber bis 2013.

Neue Nachweise von bekannten Fundorten:

b 3248/II Trampe/ehemaliger TÜP (2016: Richert, Seidel, 2018: Richert)

d 3148/I Werbellin Umg./Autobahnanschlussstelle (1961: Duckert; Beleg in coll. Kittel)

Fundorte (Ergänzung):

c 3148/I Ebw. OT Finow Umg./Mäckersee (1961: Duckert; Beleg in coll. Kittel)

\section{8 (4000) Zygaena trifolii (ESPER, 1793)}

- Verordnung zur Neufassung der BArtschV von 2005: Besonders geschützte Art.

- RL Land Brandenburg: Kategorie 2 (stark gefährdet): MW, NM;

- RL Deutschland (2011): Kategorie 3 (gefährdet). 
Neben einem neuen Fundort werden ältere Funde (Belege in coll. Kittel) ergänzend mitgeteilt.

Neue Nachweise von bekannten Fundorten:

c 3148/III Ebw. OT Finow Umg./Forst Finowtal: Samithseemoor (1956: Duckert; Beleg in coll. Kittel)

3148/IV Ebw.-Westend/Unterheide: Drehnitzwiesen (1970, 1980: Kittel)

Fundorte (Ergänzung):

c 3147/IV Finowfurt Umg./Finowfließniederung nördlich des Lehnssees $(2015,2016)$

\section{Sesiidae (Glasflügler)}

142 (4044) Synanthedon scoliaeformis (BORKHAUSEN, 1789)

- RL Deutschland (2011): Vorwarnliste.

Neue Nachweise von bekannten Fundorten:

d 3148/I Lichterfelde Umg./NSG Buckowseerinne: Am Rande der Streuobstwiesen südöstlich des Gr. Buckowsees (2014, alte Schlupflöcher)

Nachweise (Ergänzung):

b 3149/III Ebw.-Südend Umg./Hohenfinower Wald: Am Rande der östlichen Leuenberger Wiesen (2014, alte Schlupflöcher)

\section{Cossidae (Bohrer)}

\section{3 (4151) Cossus cossus (Linnaeus, 1758)}

Phänologie/Voltinismus: 30.V.(2018), aFA, phänologische Verfrühung 3d gegenüber bis 2013.

\section{4 (4176) Zeuzera pyrina (LinNAEUs, 1761)}

Die Beobachtungslücke im Naturraum e (Uckermärker Kuppen- und Hügelland einschließlich Choriner Waldhügel, Richert 2001) konnte inzwischen geschlossen werden.

Phänologie/Voltinismus: 03.VI.(2018) aFA, phänologische Verfrühung 1d gegenüber bis 2013.

\section{Neue Nachweise von bekannten Fundorten:}

\footnotetext{
c 3147I Zerpenschleuse/Ortsgebiet: Berliner Straße, im Garten (2014, 2015, 2016, 2017, 2018: Busse)

3148/III Ebw. OT Finow/Stadtgebiet: Altenhofer Straße, im Garten (2014, 2015, 2016, 2017, 2018)

d 3047/III Groß Schönebeck/Ortsgebiet: Friedenstraße, im Garten (2017: Haase)

\section{Nachweise (Ergänzung)}

b 3247/II Biesenthal Umg./NSG Biesenthaler Becken: Nördlich der Pfauenwiesen (2014: Ockruck)

e 3049/I Groß Ziethen Umg./Steingruben an den Ihlow-

bergen (2014)

g 3150/I Oderberg/Geistberg (2018: Schwabe)
}

155 (4178) Phragmataecia castaneae (HüBNER, 1790)

Phänologie/Voltinismus: 02.VIII.(2015), aFE, phänologische Verspätung 12d gegenüber bis 2013.

Neue Nachweise von bekannten Fundorten:

b 3248/II Trampe/ehemaliger TÜP (2016: Busse \& Müller)

c 3147/I Zerpenschleuse/Ortsgebiet: Berliner Staße, im Garten (2014: Busse);

3147/IV Marienwerder Umg./Steinlaake (2015: Richert, Schwabe)

3148/III Ebw. OT Finow/Stadtgebiet: Altenhofer Straße, im Garten (2014, 2015, 2018)

g 3150/III Gabow/Oderhänge: Granitberggebiet (2015: Richert, Schwabe)

3150/IV Altglietzen Umg./Gabower Berge (2015)

Nachweise (Ergänzung)

c 3147/I Marienwerder Umg./Oder-Havel-Kanal nordwestlich des Ortes (2015: Busse)

3147/IV Finowfurt Umg./Kesselmoor an Finowfließniederung nordöstlich des Lehnssees (2016: Richert, 2017: Schwabe)

3148/III Ebw OT Finow Umg./Forst Finowtal: Einflugschneise Flugplatz (2016)

3149/I Britz Umg./F. Britz (1971: Kittel)

e 3049/I Groß Ziethen Umg./Steingruben an den Ihlowbergen (2014)

h 3050/II Stolzenhagen Umg./Trockenpolder nördlich Pomeranzengraben (1994/1995: Borkowski)

\section{Lasiocampidae (Glucken)}

\section{6 (6728) Poecilocampa populi (Linnaeus, 1758)}

Phänologie: 01.XII.(1975), aFE, (korrigiert nach Beleg in coll. Kittel; in RicheRt 2014 war der 16.XI.(2009) als aFE angegeben).

Neue Nachweise von bekannten Fundorten:

c 3147/I Zerpenschleuse/Ortsgebiet: Berliner Straße, im Garten (2014: Busse)

Fundorte (Ergänzung):

d 3047/III Groß Schönebeck/Ortsgebiet: Bahnhof (2016 Haase)

\section{8 (6743) Malacosoma neustria (LinNAeus, 1758)}

Phänologie/Voltinismus: 05.VI.(2018), aFA, phänologische Verfrühung 9d gegenüber bis 2013.

Neue Nachweise von bekannten Fundorten:

c 3147/I Zerpenschleuse/Ortsgebiet: Berliner Straße, im Garten (2014, 2015, 2017, 2018: Busse)

3148/III Ebw. OT Finow/Stadtgebiet: Altenhofer Straße, im Garten (2014)

e 3049/I Groß Ziethen Umg./Steingruben an den Ihlowbergen (2014) 
Fundorte (Ergänzung):

d 3047/III Groß Schönebeck/Ortsgebiet: Friedenstraße, im Garten (2015, 2018: Haase)

g 3050/II Gellmersdorf Umg./Gellmersdorfer Forst: Mühlenberggebiet (1995/1995: Borkowski)

3050/IV Lunow Umg./Gebiet Lunower Hölzchen (1994/1995: Borkowski)

\section{9a (6745) Malacosoma franconica (DENIs \& SCHIFFERMÜLLER, 1775)}

Siehe 3.2. Neu entdeckte Arten.

\section{0 (6749) Lasiocampa trifolii (DENIS \& SCHIF- FERMÜLLER, 1775)}

Neue Nachweise von bekannten Fundorten:

$\begin{array}{ccl}\text { c 3147/I } & \begin{array}{l}\text { Zerpenschleuse/Ortsgebiet: Berliner Straße, im } \\ \text { Garten (2014: Busse) }\end{array} \\ & \text { 3148/III } & \begin{array}{l}\text { Ebw. OT Finow Umg./Forst Finowtal: Einflug- } \\ \text { schneise Flugplatz (2017: Richert, Schwabe) }\end{array} \\ & 3149 / \mathrm{IV} & \begin{array}{l}\text { Stecherschleuse Umg./ehemaliges Kiesgrubenge- } \\ \text { lände nordöstlich des Ortes (2015: Schwabe) }\end{array} \\ \text { g } \quad 3149 / \mathrm{II} & \text { Liepe/Oderhänge (1975: Kittel) } \\ & 3150 / \mathrm{IV} & \text { Altglietzen Umg./Oderhänge: Gabower Berge (2015) }\end{array}$

Fundorte (Ergänzung):

d 3048/? Joachimsthal (1976, 1979: Kittel)

3148/II Britz (1973: Kittel)

\section{4 (6773) Phyllodesma tremulifolia (HüBNER,} 1810)

- Verordnung zur Neufassung der BArtschV von 2005: Besonders geschützte Art.

- RL Land Brandenburg: Kategorie 3 (gefährdet): WS, EW;

- RL Deutschland (2011): Kategorie 3 (gefährdet).

Neue Nachweise von bekannten Fundorten:

c 3147/I Zerpenschleuse/Ortsgebiet: Berliner Straße, im Garten (2014, 2015: Busse)

Fundorte (Ergänzung):

c 3148/IV Ebw./Stadtgebiet: Kleingartenalage im Schwärzetal nördlich Forstbotanischer Garten (2018: Schwabe)

165 (6777) Gastropacha quercifolia (LinNaEus, 1758)

- RL Land Brandenburg: Kategorie 3 (gefährdet): EW, GL, MB;

- RL Deutschland (2011): Kategorie 3 (gefährdet).

In den Steingruben bei Groß Ziethen existiert offenbar eine stabile Population. Dort hatte ich im Jahre 2014 den Fi.A. a.L.
Fundorte (Ergänzung):

e 3049/I Groß Ziethen Umg./Steingruben an den Ihlowbergen (2014)

g 3050/II Gellmersdorf Umg./Gellmersdorfer Forst: Mühlenberggebiet (1994/1995: Borkowski)

h 3150/II Hohensaaten/Deponie an der Straße nach der Siedlung (2014: Kleße)

\section{7 (6780) Odonestis pruni (LinNAEUs, 1758)}

- RL Land Brandenburg: Kategorie 3 (gefährdet): EW, GL, WS;

- RL Deutschland (2011): Kategorie 2 (stark gefährdet).

Phänologie/Voltinismus: 03.VI.(2018) aFA, phänologische Verfrühung 17d gegenüber bis 2013 .

Neue Nachweise von bekannten Fundorten:

c 3147/I Zerpenschleuse/Ortsgebiet: Berliner Straße, im Garten (2014: Busse)

3148/III Ebw. OT Finow/Stadtgebiet: Altenhofer Straße, im Garten (2017: Richert)

g 3150/I Oderberg/Geistberg (2018: Schwabe)

\section{Endromidae (Scheckflügler)}

\section{8 (6784) Endromis versicolora (LINNAEUs, 1758)}

Die Beobachtungslücke im Naturraum e (Uckermärker Kuppen- und Hügelland einschließlich Choriner Waldhügel; vgl. Richert 2001) wurde geschlossen.

Neue Nachweise von bekannten Fundorten:

b 3248/II Trampe/ehemaliger TÜP (2015)

c 3147/I Zerpenschleuse/Ortsgebiet: Berliner Straße, im Garten (2014, 2015, 2018: Busse)

3148/III Ebw. OT Finow Umg./Forst Finowtal: Biesenthaler Straße am Heuweg (2016)

Fundorte (Ergänzung):

c 3146/IV Kreuzbruch Umg./Römerwegbrücke am OderHavel-Kanal östlich des Ortes (Busse 2014) (westlich angrenzendes Nachbargebiet)

3148/IV Ebw./Stadtgebiet: Kleingartenanlage im Schwärzetal nördlich Forstbotanischer Garten (2017, 2018: Schwabe)

d 2947/IV Gollin Umg./Schorfheide, 1 Weibchen, fot. Ebert; nördlich angrenzendes Nachbargebiet)

e 3149/II Liepe Umg./Forst Chorin: NSG Plagefenn südlich Gr. Plagesee (2018: Richert, Schwabe)

\section{Saturniidae (Augenspinner)}

169 (6788) Aglia tau (Linnaeus, 1758)

Phänologie/Voltinismus: 17.IV.(2018), aFA, phänologische Verfrühung 2d gegenüber bis 2013. 
Neue Nachweise von bekannten Fundorten:

c 3147/I Zerpenschleuse/Ortsgebiet: Berliner Straße, im Garten (2018: Busse)

Fundorte (Ergänzung):

b 3148/IV Ebw./Stadtgebiet: Kleingartenanlage im Schwärzetal nördlich Forstbotanischer Garten (2015: Schwabe) und Umgebung: Oberheide südlich der SchillerTreppe (2014)

3248/II Trampe/ehemaliger TÜP (2014)

c $3147 / \mathrm{IV}$ Finowfurt Umg./Waldgebiet zwischen Finowfließniederung und der Waldstraße nach Biesenthal (2016)

3148/III Ebw. OT Finow/Stadtgebiet: Poststraße (2016) und Umg./Forst Finowtal: Biesenthaler Straße östlich des Samithsees (2017)

3148/IV Ebw./Stadtgebiet: Kleingartenanlage im Schwärzetal nördlich Forstbotanischer Garten (2016: Schwabe)

d 3047/III Groß Schönebeck Umg./Liebenthaler Weg (2016: Haase)

3048/IV Golzow Umg./Tiefer Bugsinsee (1979: Kittel)

e 3149/II Liepe Umg./Forst Chorin: NSG Plagefenn: Plageberge (2018: Richert, Schwabe), südlich Gr. Plagesee (2018: Theimer)

\section{0 (6794) Saturnia pavonia (LinNAEUs, 1758)}

- RL Land Brandenburg: Kategorie 3 (gefährdet): CB.

Neue Nachweise von bekannten Fundorten:

d 3047/III Groß Schönebeck Umg./Schorfheide: Revier Rehluch Abt. 35 (2015: Haase)

Fundorte (Ergänzung):

b 3148/III Spechthausen Umg./Barnimer Heide: Stromtrasse südwestlich des Ortes (2015: Schwabe)

\section{Sphingidae (Schwärmer)}

\section{2 (6819) Mimas tiliae (LinnAeus, 1758)}

Phänologie/Voltinismus: 19.IV.(2014), aFA, phänologische Verfrühung 4d gegenüber bis 2013.

\section{3 (6822) Smerinthus ocellata (LiNNAEUs, 1758)}

Phänologie/Voltinismus: 16.VIII.(2018), aFE, phänologische Verspätung 4d gegenüber bis 2013. Bei diesem Fund handelte es sich um ein frisches Exemplar, also um einen F einer partiellen 2. Gen.

\section{5 (6828) Agrius convolvuli (LinNAeUs, 1758)}

„Im Ortskern von Lunow beobachtete ich am 02.09.1994 in der Dämmerung 2 Exemplare, im Schwebeflug an Petunienblüten saugend" (BoRKOwsKI, 1996).

Der "klassische Wanderfalter" (Emigrant, Saisonwanderer 1. Ordnung, Eitschberger \& Steiniger, 1991) erreichte in den Jahren 2015 und 2018 (wie auch andere „Wanderfalter" in diesen witterungsmäßig extremen Jahren) erneut E VIII/A IX unser Gebiet.
Neue Nachweise von bekannten Fundorten:

c 3147/I Zerpenschleuse/Ortsgebiet: Berliner Straße, im Garten (2018: Busse)

3148/III Ebw. OT Finow/Stadtgebiet: Altenhofer Straße, im Garten (2015)

Fundorte (Ergänzung):

d 3047/III Groß Schönebeck/Ortsgebiet: Friedenstraße, im Garten (2015, 2018: Haase)

g 3050/IV Lunow/Ortsgebiet (1994: Borkowski)

\section{7 (6832) Sphinx ligustri (Linnaeus, 1758)}

Die Art wird neuerdings im Gebiet wieder regelmäßiger und verbreiteter, aber meist vereinzelt beobachtet.

Phänologie/Voltinismus: Im Jahre 2016 trat der F ungewöhnlich früh auf: 08.V.(2016), aFA, phänologische Verfrühung $15 \mathrm{~d}$ gegenüber bis 2013.

Neue Nachweise von bekannten Fundorten:

c 3147/I Zerpenschleuse/Ortsgebiet: Berliner Straße, im Garten (2014, 2015, 2016, 2018: Busse)

3148/III Ebw. OT Finow/Stadtgebiet: Altenhofer Straße, im Garten $(2014,2015,2016,2017,2018)$

g 3050/II Stolpe a.O./Trockenhänge am Stadtweg (2018)

Fundorte (Ergänzung):

b 3148/IV Ebw./Stadtgebiet (2016: Schwuchow; 2017: Burow): Kleingartenanlage im Schwärzetal nördlich des Forstbotanischen Gartens (2014, 2016, 2017, 2018: Schwabe)

d 3047/III Groß Schönebeck/Ortsgebiet: Liebenwalder Straße, im Garten (2016, 2018: Haase)

g 3150/I Oderberg/Geistberg (2017, 2018: Schwabe)

\section{8 (6834) Hyloicus pinastri (LiNNAEUs, 1758)}

Der F kommt nicht nur an das Licht, wo er regelmäßig zu beobachten ist, sondern wird gelegentlich auch vom Köder angezogen: Am 15.VIII.2017 beobachtete ich einen F, der in der Dämmerung im Schwebeflug vor dem Rotweinköder am Kiefernstamm schwirrte.

Phänologie/Voltinismus: In der Regel univoltin; 2014 frühzeitiger Flugzeitbeginn E (25.)IV und Ausbildung einer partiellen 2. Gen A (02.)IX.

\section{0 (6840) Hemaris fuciformis (LinNaEus, 1758)}

- Verordnung zur Neufassung der BArtschV von 2005: Besonders geschützte Art.

- RL Land Brandenburg: Kategorie 3 (gefährdet).

Im Jahre 2015 wurde erneut das Auftreten von F einer 2. Gen. im Gebiet festgestellt: Ich beobachtete vom 03.-11.VIII. in meinem Garten in Finow drei F beim Saugen an Buddleja davidii, Chinesischer Sommerflieder. Haase beobachtete den F in seinem Garten in Groß Schönebeck beim Saugen an Phlox paniculata, Stauden-Phlox. 
Schwabe fand die Puppe im Frühjahr beim Gartenputz: Beim Abharken einer Rabatte fiel sie aus dem vorjährigen Laub; F e.p. 08.V.2015. Die Verpuppung musste also an der Erdoberfläche ohne besondere Schutzmechanismen (Gespinst) erfolgt sein.

Neue Nachweise von bekannten Fundorten:

c 3148/III Ebw. OT Finow/Stadtgebiet: Altenhofer Straße, im Garten (2015: Richert), Biesenthaler Straße, im Garten (2018: Brauner)

Fundorte (Ergänzung):

c 3148/IV Ebw./Stadtgebiet: Kleingartenanlage im Schwärzetal nördlich vom Forstbotanischen Garten (2015: Schwabe)

3149/III Stecherschleuse Umg./ehemaliges Kiesgrubengelände nördlich des Ortes (2018: Barthelme)

d 3047/III Groß Schönebeck/Ortsgebiet: Friedenstraße, im Garten (2017: Haase)

h 3149/IV Falkenberg/Ortsgebiet: Kleingartenanlage am Rande des Nieder-Oderbruchs (2015: Schwabe)

\section{1 (6843) Macroglossum stellatarum (SCOPOLI, 1777)}

„Die Falter beobachtete ich mehrfach von Juni bis September (Zeitraum 1994/1995; Anm. Verf.) in Gärten und an Blumenfenstern in ... Lunow, wo sie in der Mittagssonne im Schwebflug an Blüten verschiedener Zierpflanzen saugten, besonders an Phlox und Petunien“" (Borkowski 1996).

Emigrant/Binnenwanderer (EITSCHBERGER \& STEINIGER 1991), der bei uns alljährlich von 2015 bis 2018 erneut beobachtet wurde; 2018 verbreitet und lokal auch in Anzahl (Haase, persönl. Mitt.).

Neue Nachweise von bekannten Fundorten:

c 3147/I Zerpenschleuse/Ortsgebiet: Berliner Straße, im Garten (2015: Busse)

3148/III Ebw. OT Finow/Stadtgebiet (2018: Adams): Altenhofer Straße, im Garten (2015)

d 3047/III Groß Schönebeck/Ortsgebiet: Friedenstraße, im Garten (2018: Haase)

Fundorte (Ergänzung):

b 3148/IV Ebw.-Ostende/Breite Straße nahe Waldfriedhof (2016: Schwabe)

c 3148/I Ebw. OT Finow/Clara-Zetkin-Siedlung: Am OderHavel-Kanal (2018: Schifter)

3149/III Stecherschleuse Umg./ehemaliges Kiesgrubengelände nördlich des Ortes (2017)

f 3049/IV Brodowin/Ortsgebiet (2018: Thiede)

h 3150/IV Lunow/Ortsgebiet (1994/1995: Borkowski; 2018: Thiede)

182 (6849) Proserpinus proserpina (PALLAS, 1772)

FFH-Art.

- RL Land Brandenburg: Vorwarnliste.
Haase beobachtete am 28.VI.2017 einen F am Tag in seinem Garten in Groß Schönebeck beim Saugen an Rhododendron spec., Rhododendron-Blüten.

Neue Nachweise von bekannten Fundorten:

d 3047/III Groß Schönebeck/Ortsgebiet: Friedenstraße, im Garten (2017: Haase)

\section{3 (6853) Hyles euphorbiae (LiNNAEUs, 1758)}

- Verordnung zur Neufassung der BArtschV von 2005: Besonders geschützte Art.

- RL Land Brandenburg: Vorwarnliste: TR;

- RL Deutschland (2011): Kategorie 3 (gefährdet).

Brauner fand erwachsene R M (15.) X 2017 auf dem Flugplatz Finow. Demnach hat die Art in diesem Jahr eine (partielle) 2. Gen. ausgebildet.

Neue Nachweise von bekannten Fundorten:

b 3248/II Trampe/ehemaliger TÜP (2016: Busse \& Müller)

c 3147/I Zerpenschleuse/Ortsgebiet: Berliner Straße, im Garten (2014, 2015, 2016, 2017, 2018: Busse)

3148/III Ebw. OT Finow/Stadtgebiet: Altenhofer Straße, im Garten $(2015,2018)$

Fundorte (Ergänzung):

c 3148/III Ebw. OT Finow Umg./Forst Finowtal: Flugplatzgelände (2017: Brauner)

3149/I Niederfinow Umg./Oder-Havel-Kanal östlich der Klosterbrücke (2018)

g 3050/II Gellmersdorf Umg./Gellmersdorfer Forst: Mühlenberggebiet (1994/1995: Borkowski)

184 (6855) Hyles gallii (RotTEMBURG, 1775) $=$ galii (DENIS \& SCHIFFERMüLLER, 1775)

- Verordnung zur Neufassung der BArtschV von 2005: Besonders geschützte Art.

- RL Land Brandenburg: Vorwarnliste: TR.

Die Beobachtungslücke im Naturraum e (Uckermärker Kuppen- und Hügelland konnte inzwischen geschlossen werden (vgl. RicherT 2001).

Weitere im Gebiet genutzte Nektarsaugpflanzen (vgl. Richert l.c.): Phlox paniculata, Stauden-Phlox; Vicia tenuifolia, Feinblättrige Wicke.

Phänologie/Voltinismus: Univoltin, jw. bivoltin 20.IX.(2014), aFE, phänologische Verspätung 15d gegenüber bis 2013 .

Von der Aussage im 3. Nachtrag, dass nur Raupenfunde M VII aus dem Gebiet bekannt sind, weichen folgende Beobachtungen ab: Am 01.VII.2016 fand Schwabe eine bereits erwachsene R an Galium spec. bei Eberswalde. A (06.)X 2010 beobachtete O. Brauner $2 \mathrm{R}$ in unterschiedlichen Stadien, offensichtlich Nachkommen der 2. Generation. Dieses bisher nicht berücksichtigte Funddatum wird ergänzend nachgetragen. 
Neue Nachweise von bekannten Fundorten:

c 3147/I Zerpenschleuse/Ortsgebiet: Berliner Straße, im Garten (2014: Busse); Marienwerder Umg./OderHavel-Kanal nordwestlich des Ortes (2014: Busse)

3148/III Ebw. OT Finow/Stadtgebiet: Altenhofer Straße, im Garten $(2014,2015,2018)$

Fundorte (Ergänzung):

c 3149/III Ebw. Umg./Finowbruch östlich des Eichwerder (2016); Stecherschleuse Umg./ehemaliges Kiesgrubengelände nördlich des Ortes (2018: Barthelme)

d 3047/III Groß Schönebeck/Ortsgebiet: Friedenstraße, im Garten (2017: Haase) und Umg./Liebenthaler Weg (2013: Haase)

e 2949/III Altkünkendorf Umg./Louisenhof (2014: H. Loll)

g 3050/II Stolpe/Trockenhänge am Stadtweg (2018)

3150/I Oderberg/Geistberg (2018: Richert \& Schwabe)

3150/III Gabow/Granitberggebiet: Osthang (2015)

3150/IV Altglietzen/Gabower Berge (2015)

\section{5 (6862) Deilephila elpenor (LinNAEus, 1758)}

Als weitere R-Fraßpflanze (vgl. Richert 2014) nutzt die Art im Gebiet Parthenocissus quinquefolia, Gewöhnliche Jungfernrebe.

\section{Drepanidae: Thyatirinae (Eulenspinner)}

\section{7 (7481) Thyatira batis (LinNAEus, 1758)}

Ein bemerkenswertes Verhalten wurde im Mai 2015 im Gebiet per Handy-Foto dokumentiert: Ein F war bei der Zerlegung von gejagtem Wild in der Wildkammer am Schwappachweg in Eberswalde angelockt worden und saugte "gierig“ am verspritzten Blut. Als das Blut in den Abfluss gespült wurde, kroch der F selbst dort noch hinein (Beobachter: P. Ebert).

Phänologie/Voltinismus: 04.V.(2015), aFA; phänologische Verfrühung 1d gegenüber bis 2013.

14.IX.(2014) aFE; phänologische Verspätung 8d gegenüber bis 2013 .

In den Jahren 2014 und 2016 wurden noch bis A IX späte F der seit der Jahrtausendwende zunehmend regelmäßig im Gebiet beobachteten 2. Gen. a.L. registriert.

\section{8 (7483) Habrosyne pyritoides (HufNAGEL, 1766) = derasa LinNAEus, 1767}

Phänologie/Voltinismus: In den Jahren 2014, 2015, 2016 und 2018 wurden erneut im IX einzelne F der seit der Jahrtausendwende zunehmend regelmäßig im Gebiet beobachteten 2. Gen. registriert.

\section{9 (7485) Tethea ocularis (LinnaEus, 1767)}

Die Beobachtungslücke im Naturraum f (Parsteiner Becken, vgl. RicherT 2001) wurde inzwischen geschlossen.
Phänologie/Voltinismus: Bivoltin; 1. Gen.: 13.V.(2018), aFA, phänologische Verfrühung 4d gegenüber bis 2013; 2. (partielle) Gen.: 15.IX.(2014) aFE, ein abgeflogener F a.L., phänologische Verspätung 26d gegenüber bis 2013.

Neue Nachweise von bekannten Fundorten:

c 3147/I Zerpenschleuse/Ortsgebiet: Berliner Straße, im Garten (2014, 2018: Busse)

3148/III Ebw. OT Finow/Stadtgebiet: Altenhofer Straße, im Garten (2014, 2016, 2017, 2018)

d 3047/III Groß Schönebeck/Ortsgebiet: Friedenstraße, im Garten (2016: Haase)

g 3150/III Gabow Umg./Granitberggebiet (2015)

Fundorte (Ergänzung):

c 3147/IV Marienwerder Umg./Kiesgrube südwestlich des Sportplatzes (2015)

f 3049/IV Brodowin/Krugberg (2014)

g 3150/I Oderberg/Geistberg (2018: Schwabe)

3150/IV Altglietzen Umg./Gabower Berge (2015)

\section{1 (7499) Theteella fluctuosa (HüBNER, 1803)}

- RL Land Brandenburg: Vorwarnliste: MB.

Neue Nachweise von bekannten Fundorten:

c 3147/I Zerpenschleuse/Ortsgebiet: Berliner Straße, im Garten (2014: Busse)

3148/III Ebw. OT Finow/Stadtgebiet: Altenhofer Straße, im Garten $(2014,2017)$ und Umg./Forst Finowtal: Einflugschneise Flugplatz $(2016,2017)$

d 3047/III Groß Schönebeck/Ortsgebiet: Friedenstraße, im Garten (2014: Haase)

e 3149/II Liepe Umg./Forst Chorin: NSG Plagefenn (2018: Richert \& Schwabe)

Fundorte (Ergänzung):

b 3249/I Gersdorf Umg./Gamengrund südlich Teufelssee (2017: Schwabe)

c 3147/I Marienwerder Umg./Oder-Havel-Kanal nördlich des Ortes (2015: Busse)

3147/IV Finowfurt Umg./Finowfließniederung an der Neuen Brücke (Biesenthaler Straße) (2014), Kesselmoor an der Finowfließniederung nordöstlich des Lehnssees (2016, 2017: Richert, Schwabe)

g 3149/IV Niederfinow Umg./Oderhänge - ehemalige Kiesgruben südwestlich des Schiffshebewerks (2015: Schwabe)

\section{2 (7490) Ochropacha duplaris (Linnaeus, 1761)}

Neue Nachweise von bekannten Fundorten:

c 3147/I Zerpenschleuse/Ortsgebiet: Berliner Straße, im Garten (2014: Busse)

3148/III Ebw. OT Finow/Stadtgebiet: Altenhofer Straße, im Garten $(2014,2015)$

Fundorte (Ergänzung):

b 3247/II Biesenthal Umg./NSG Biesenthaler Becken: Weg nördlich der Pfauenwiesen (2014: Ockruck) 
c 3148/III Ebw. OT Finow Umg./Forst Finowtal: Einflugschneise Flugplatz (2017)

d 3047/III Groß Schönebeck/Ortsgebiet: Friedenstraße, im Garten (1999, 2015: Haase)

\section{3 (7492) Cymatophorina diluta (DENIS \& SCHIFFERMÜLLER, 1775)}

- RL Land Brandenburg: Vorwarnliste: EW.

Neue Nachweise von bekannten Fundorten:

c 3147/II Marienwerder Umg./Oder-Havel-Kanal nördlich des Ortes (2014, 2016: Busse)

3148/III Ebw. OT Finow/Stadtgebiet: Altenhofer Straße, im Garten (2014)

Fundorte (Ergänzung):

c 3147/II Finowfurt/Hirtenweg - Besters Fließ (1993: Kittel)

e 3049/III Chorin Umg./Forst Chorin: Südöstlich des Pferdebergs (2013: MÜLLER 2015)

3049/IV Chorin Umg./Forst Chorin: Teerbrennerberge Windwurffläche Am Olberg (2013: Müller 2015); Kreuzung Denglerweg - Olbergstraße, Windwurffläche östlich der Kreuzung (2013: MüLler 2015)

f 3049/IV Brodowin/Krugberg (2014)

\section{4 (7494) Polyploca ridens (FABRICIUs, 1787)}

Neue Nachweise von bekannten Fundorten:

c 3147/I Zerpenschleuse/Ortsgebiet: Berliner Straße, im Garten (2014: Busse)

Fundorte (Ergänzung):

e 3149/II Liepe Umg./Forst Chorin: NSG Plagefenn südlich Gr. Plagesee (2018: Richert, Schwabe \& Theimer)

\section{Drepanidae: Drepaninae (Sichelflügler)}

\section{7 (7503) Watsonalla binaria (HufNAgeL, 1767)}

Neue Nachweise von bekannten Fundorten:

c 3147/I Zerpenschleuse/Ortsgebiet: Berliner Straße, im Garten (2014, 2016, 2017, 2018: Busse); Marienwerder Umg./Oder-Havel-Kanal nordwestlich des Ortes (2013, 2015, 2016: Busse)

3147/IV Marienwerder/Steinlaake (2015), Kiesgrube südwestlich des Sportplatzes (2015)

3148/III Ebw. OT Finow/Stadtgebiet: Altenhofer Straße, im Garten $(2014,2015,2016,2017,2018)$

Fundorte (Ergänzung):

b 3247/II Biesenthal Umg./NSG Biesenthaler Becken: Langerönner Weg (2014: Ockruck), nördlich der Pfauenwiesen (2015: Ockruck)

c $3147 / \mathrm{IV} \quad$ Finowfurt Umg./Kesselmoor an der Finowfließniederung nordöstlich des Lehnssees (2016)

3148/IV Ebw./Stadtgebiet: Kleingartenanlage im Schwärzetal nördlich des Forstbotanischen Gartens (2014, 2017 , 2018: Schwabe)

e 3049/I Groß Ziethen Umg./Steingruben an den Ihlowbergen (2014) f 3049/IV Brodowin Umg./Karpatenweg östlich des Krugbergs (2015)

g 3050/II Gellmersdorf Umg./Gellmersdorfer Forst: Mühlenberggebiet (1994/1995: Borkowski)

3149/IV Niederfinow/Oderhänge - ehemalige Kiesgruben südwestlich des Schiffshebewerks (2016: Schwabe)

3150/I Oderberg/am Friedhof (2016: Schwabe)

h 3150/II Hohensaaten/Deponie an der Straße nach der Siedlung (2014: Kleße)

\section{9 (7507) Drepana curvatula (BORKHAUSEN, 1790)}

- RL Land Brandenburg: Vorwarnliste: MB.

Neue Nachweise von bekannten Fundorten:

b 3247/II Biesenthal Umg./NSG Biesenthaler Becken: Weg nördlich der Pfauenwiesen (2014: Ockruck), nördlich der Pfauenwiesen (2015: Ockruck)

c 3147/I Zerpenschleuse/Ortsgebiet: Berliner Straße, im Garten (2014, 2015, 2018: Busse), Marienwerder Umg./Oder-Havel-Kanal nördlich des Ortes (2016: Busse)

3148/III Ebw. OT Finow/Stadtgebiet: Altenhofer Straße, im Garten $(2014,2015,2017)$

e 3149/II Liepe Umg./Forst Chorin: NSG Plagefenn - LedumMoor am Fuß der Plageberge (2018: Richert \& Schwabe)

Fundorte (Ergänzung):

b 3247/II Biesenthal Umg./NSG Biesenthaler Becken: Langerönner Weg (2014: Ockruck)

c 3147/IV Finowfurt Umg./Finowfließniederung an der Neuen Brücke (Biesenthaler Straße) (2014), Kesselmoor an der Finowfließniederung nordöstlich des Lehnssees (2016, 2017: Richert, Schwabe)

3147/I Marienwerder Umg./Oder-Havel-Kanal nordwestlich des Ortes (2014, 2015: Busse)

3148/III Ebw. OT Finow Umg./Forst Finowtal: Einflugschneise Flugplatz (2017)

3148/IV Ebw./Stadtgebiet: Kleingartenanlage im Schwärzetal nördlich des Forstbotanischen Gartens (2014, 2015 , 2016, 2017, 2018: Schwabe)

d 3149/I Britz Umg./F. Britz (1973: Kittel)

g 3050/II Gellmersdorf Umg./Gellmersdorfer Forst: Mühlenberggebiet (1994/1995: Borkowski)

3050/IV Lunow Umg./Gebiet Lunower Hölzchen (1994/1995 Borkowski)

\section{1 (7510) Sabra harpagula (ESPER, 1786)}

- RL Land Brandenburg: Kategorie 1 (vom Aussterben bedroht): LL;

- RL Deutschland (2011): Vorwarnliste.

Neue Nachweise von bekannten Fundorten:

c: 3148/III Ebw. OT Finow/Stadtgebiet: Altenhofer Straße, im Garten (2014)

Fundorte (Ergänzung):

e 3049/I Groß Ziethen Umg./Steingruben an den Ihlowbergen bei Sperlingsherberge (2014) 
g 3050/IV Lunow Umg./Gebiet Lunower Hölzchen (1994/1995: Borkowski)

\section{2 (7512) Cilix glaucata (SCOPOLI, 1763)}

Neue Nachweise von bekannten Fundorten:

c 3148/III Ebw. OT Finow/Stadtgebiet: Altenhofer Straße, im Garten (2016)

g 3150/III Gabow/Granitberggebiet - Ostseite (2015: Schwabe)

3150/IV Altglietzen Umg./Gabower Berge (2015, Richert, Schwabe; 2018: Schwabe)

Fundorte (Ergänzung):

c 3147/I Zerpenschleuse/Ortgebiet: Berliner Straße, im Garten (2015, 2017: Busse)

3147/II Finowfurt/Hirtenweg - Besters Fließ (1993, 1995: Kittel)

3149/IV Niederfinow Umg./Oderhänge - ehemalige Kiesgruben südwestlich des Schiffshebewerks (2015: Schwabe)

g 3050/II Gellmerdorf Umg./Gellmersdorfer Forst: Mühlenberggebiet (1994/1995: Borkowski)

3150/I Oderberg/Geistberg (2016, 2017: Schwabe)

\section{Notodontidae (Zahnspinner)}

\section{Thaumetopoeinae}

\section{3 (8689) Thaumetopoea processionea (LINNAEUS,} 1758)

Die Art wurde inzwischen auch in den Naturräumen b (Barnimplatte), d (Britzer Platte und Schorfheide) und e (Uckermärker Kuppen und Hügelland einschließlich Choriner Waldhügel) nachgewiesen (vgl. RICHERT 2001, 2014).

Im Gebiet zeigt sich zurzeit ein deutliches West-Ostgefälle in der Häufigkeit der Art: Während im äußersten Westen in Zerpenschleuse vom 16.VII. - 22.VIII.2014 $241 \mathrm{~F}$ a.L. registriert wurden, wurden in Ebw. nur vereinzelte F a.L. beobachtet. Ähnlich lagen die Verhältnisse im Jahre 2015, allerdings wurden deutlich weniger F (insgesamt 46) registriert.

Aus dem Jahre 2018 liegen mir aus dem Gebiet auffallend wenig Nachweise vor.

Phänologie/Voltinismus: 16.VII.(2014, 2018), aFA, phänologische Verfrühung 1d gegenüber bis 2013.

Neue Nachweise von bekannten Fundorten:

c 3147/I Zerpenschleuse/Ortsgebiet: Berliner Straße, im Garten $(2014,2015,2016)$

3148/III Ebw. OT Finow/Stadtgebiet: Altenhofer Straße, im Garten (2014, 2015, 2016, 2017)

Fundorte (Ergänzung):

b 3247/II Biesenthal Umg./NSG Biesenthaler Becken: Langerönner Weg (2014: Ockruck), nördlich der Pfauenwiesen (2015: Ockruck) c $3147 / \mathrm{IV} \quad$ Finowfurt Umg./Finowfließniederung an der Neuen Brücke (Biesenthaler Straße) (2014)

3148/III Ebw. OT Finow Umg./Forst Finowtal: Einflugschneise Flugplatz (2017)

3148/IV Ebw./Stadtgebiet: Kleingartenanlage im Schwärzetal nördlich des Forstbotanischen Gartens (2014, 2017 Schwabe)

d 3047/III Groß Schönebeck/Ortsgebiet: Friedenstraße, im Garten (2014: Haase)

e 3149/II Liepe Umg./Forst Chorin: NSG Plagefenn - LedumMoor, (2018: Richert \& Schwabe)

\section{4 (8692) Thaumetopoea pinivora (TREITSCHKE, 1834)}

Neue Nachweise von bekannten Fundorten:

c 3148/III Ebw. OT Finow/Stadtgebiet: Altenhofer Straße, im Garten (2015)

\section{Pygaerinae}

\section{5 (8698) Clostera curtula (LINNAEUs, 1798)}

Neue Nachweise von bekannten Fundorten:

c 3147/I Zerpenschleuse/Ortsgebiet: Berliner Straße, im Garten (2014, 2015, 2017: Busse)

3148/III Ebw. OT Finow/Stadtgebiet: Altenhofer Straße, im Garten (2016)

Fundorte (Ergänzung):

c 3148/IV Ebw.-Westend/Unterheide: Wildparkstraße (1974: Kittel)

d 3047/III Groß Schönebeck/Ortsgebiet: Friedenstraße, im Garten (2017: Haase)

h 3050/II Stolzenhagen Umg./Trockenpolder nördlich Pomeranzengraben (1994/1995: Borkowski)

\section{6 (8699) Clostera pigra (HufNAgEL, 1766)}

Phänologie: 25.IX.(1987), aFE, (korrigiert nach Beleg in coll. Kittel; in RICHERT 2014 war der 15.VIII.(1974/2005) als aFE angegeben). Offensichtlich handelt es sich hier um einen Nachzügler nach dem kühlen Sommer des Jahres 1987.

\section{Fundorte (Ergänzung):}

c $3147 / \mathrm{IV}$ Finowfurt Umg./Kesselmoor an der Finowfließniederung nordöstlich des Lehnssees (2016: Schwabe)

3148/IV Ebw.-Westend/Unterheide: Drehnitzwiesen (1974: Kittel), Ebw./Stadtgebiet: Kleingartenanlage im Schwärzetal nördlich des Forstbotanischen Gartens (2014: Schwabe)

d 3047/III Groß Schönebeck/Ortsgebiet: Friedenstraße, im Garten (1999: Haase)

3148/II Britz (1973: Kittel)

h 3150/II Hohensaaten/Deponie an der Staße nach der Siedlung (2014: Kleße) 


\section{7 (8700) Clostera anachoreta (DENIS \& SCHIF- FERMÜLLER, 1775)}

- RL Land Brandenburg: Vorwarnliste: FA, NM, MB;

- RL Deutschland (2011): Kategorie 3 (gefährdet).

Phänologie/Voltinismus: Bivoltin; 2. Gen.: 26.VIII (2014), aFE; phänologische Verspätung 4d gegenüber bis 2013 .

Neue Nachweise von bekannten Fundorten:

c 3147/I Zerpenschleuse/Ortsgebiet: Berliner Straße, im Garten (2014, 2017, 2018: Busse)

3147/II Finowfurt/Besters Fließ: Hirtenweg (1993: Kittel)

3148/III Ebw. OT Finow/Stadtgebiet: Altenhofer Straße, im Garten $(2014,2016,2017,2018)$

Fundorte (Ergänzung):

c 3148/IV Ebw./Stadtgebiet: Kleingartenanlage im Schwärzetal nördlich des Forstbotanischen Gartens (2014: Schwabe)

g 3150/I Oderberg/Geistberg (2017: Schwabe)

\section{Notodontinae}

\section{9 (8704) Cerura vinula (Linnaeus, 1758)}

Ältere Funde (Belege in coll. Kittel) werden ergänzend nachgetragen.

Fundorte (Ergänzung):

c 3148/IV Ebw.-Westend/Unterheide: Wildparkstraße (1973, 1975: Kittel)

\section{0 (8706) Dicranura erminea (ESPER, 1783)}

Neue Nachweise von bekannten Fundorten:

b 3248/II Trampe/ehemaliger TÜP (2016: Busse \& Müller)

c 3147/I Zerpenschleuse/Ortsgebiet: Berliner Straße, im Garten (2014, 2015, 2017: Busse)

3148/III Ebw. OT Finow/Stadtgebiet: Altenhofer Straße, im Garten $(2017,2018)$

g 3150/III Gabow/Granitberggebiet (2015: Schwabe)

3150/IV Altglietzen/Gabower Berge (2015)

Fundorte (Ergänzung):

c 3148/IV Ebw.-Westend/Unterheide: Wildparkstraße (1973, 1975: Kittel)

\section{1 (8708) Furcula furcula (CLERCK, 1759)}

Neue Nachweise von bekannten Fundorten:

c 3147/I Zerpenschleuse/Ortsgebiet: Berliner Straße, im Garten (2014, 2018: Busse)

3148/III Ebw. OT Finow/Stadtgebiet: Altenhofer Straße, im Garten $(2015,2016)$ und Umg./Forst Finowtal: Einflugschneise Flugplatz (2017: Schwabe)

d 3047/III Groß Schönebeck/Ortsgebiet: Friedenstraße, im Garten (2014: Haase)
Fundorte (Ergänzung):

c 3147/IV Finowfurt Umg./Kesselmoor an der Finowfließniederung nordöstlich des Lehnssees (2017: Schwabe)

3148/IV Ebw./Stadtgebiet: Kleingartenanlage im Schwärzetal nördlich des Forstbotanischen Gartens (2014: Schwabe)

g 3050/II Gellmersdorf Umg./Gellmersdorfer Forst: Mühlenberggebiet (1994/1995: Borkowski)

3050/IV Lunow Umg./Gebiet Lunower Hölzchen (1994/1995: Borkowski)

h 3050/II Stolzenhagen Umg./Trockenpolder nördlich Pomeranzengraben (1994/1995: Borkowski)

\section{2 (8709) Furcula bicuspis (BORKHAUSEN, 1790)}

Neue Nachweise von bekannten Fundorten:

b 3247/II Biesenthal Umg./NSG Biesenthaler Becken: Langerönner Weg (2014: Ockruck)

3248/II Trampe/ehemaliger TÜP (2016: Busse \& Müller)

c 3147/I Zerpenschleuse/Ortsgebiet: Berliner Straße, im Garten (2014, 2015, 2016, 2017, 2018: Busse)

3148/III Ebw. OT Finow/Stadtgebiet: Altenhofer Straße, im Garten $(2014,2015,2016,2017,2018)$

Fundorte (Ergänzung):

c $3147 / \mathrm{IV}$ Finowfurt Umg./Kesselmoor an der Finowfließniederung nordöstlich des Lehnssees (2016)

g 3150/I Oderberg/Geistberg (2017: Schwabe)

\section{3 (8710) Furcula bifida (BRAнM, 1787)}

Phänologie: 2. Gen.: 16.VIII.(1974, 2014), aFE, (korrigiert nach Beleg in coll. Kittel, bestätigt durch einen aktuellen Fund; in Richert 2014 war der 15.VIII.(1964, 2005) als aFE angegeben).

Neue Nachweise von bekannten Fundorten:

b 3148/IV Ebw. Umg./Oberheide: Waldschneise vom Möllergrab am Wiebecke-Damm ostwärts (2016: Schwabe)

c 3147/I Zerpenschleuse/Ortsgebiet: Berliner Straße, im Garten (2014, 2015, 2018: Busse)

3148/III Ebw. OT Finow/Stadtgebiet: Altenhofer Straße, im Garten $(2014,2018)$

Fundorte (Ergänzung):

b 3247/II Biesenthal Umg./NSG Biesenthaler Becken: Langerönner Weg (2014: Ockruck)

214 (8716) Notodonta dromedarius (LinNAEUs, 1758)

Phänologie/Voltinismus: 1. Gen.: 02.IV.(2014), aFA, phänologische Verfrühung 18d gegenüber bis 2013.

2. Gen.: 08.IX.(2014), aFE, pänologische Verspätung $6 \mathrm{~d}$ gegenüber bis 2013 .

215 (8718) Notodonta tritophus (DeNIS \& SCHIFFERMÜLLER, 1775) = phoebe SIEBERT, 1790

- RL Deutschland (2011): Vorwarnliste. 


\author{
Neue Nachweise von bekannten Fundorten: \\ b 3247/II Biesenthal Umg./NSG Biesenthaler Becken: Nörd- \\ lich der Pfauenwiesen (2015: Ockruck) \\ c 3147/I Zerpenschleuse/Ortsgebiet: Berliner Straße, im \\ Garten (2014, 2015, 2018: Busse) \\ 3148/III Ebw. OT Finow/Stadtgebiet: Altenhofer Straße, im \\ Garten $(2014,2015,2016,2017)$
}

Fundorte (Ergänzung):

c 3148/IV Ebw.-Westend/Unterheide: Wildparkstraße (1971: Kittel)

d 3047/III Groß Schönebeck/Ortsgebiet: Friedenstraße, im Garten (1999: Haase)

e 3049/I Groß Ziethen Umg./Steingruben an den Ihlowbergen (2014)

g 3050/IV Lunow Umg./Gebiet Lunower Hölzchen (1994: Borkowski)

\section{6 (8719) Notodonta ziczac (LinNAeus, 1758)}

Phänologie/Voltinismus: Bivoltin; 2.(3.?) Gen.: 15.IX. (2014), aFE, phänologische Verspätung $12 \mathrm{~d}$ gegenüber bis 2013 .

Im Jahre 2014 zeichneten sich drei Flugperioden ab: E IV bis E V; A VII bis E VII; A bis M IX. Ob es sich bei den Septemberfunden um eine partielle 3. Gen. oder um Nachzügler handelt, bleibt offen. Wahrscheinlich trifft letzteres zu: Der August war kühler als das langjährige Mittel.

\section{7 (8721) Drymonia dodonaea (DENIS \& SCHIF- FERMÜLLER, 1775) = trimacula ESPER, 1785}

Neue Nachweise von bekannten Fundorten:

e 3149/II Liepe Umg./Forst Chorin: NSG Plagefenn - LedumMoor (2018: Richert \& Schwabe)

Fundorte (Ergänzung):

c 3147/IV Marienwerder Umg./Kiesgrube südwestlich des Sportplatzes (2015)

g 3050/II Gellmersdorf Umg./Gellmersdorfer Forst: Mühlenberggebiet (1994/1995: Borkowski)

3050/IV Lunow Umg./Gebiet Lunower Hölzchen (1994/1995: Borkowski)

\section{8 (8722) Drymonia ruficornis (HuFNAGEL,} 1766) = chaonia DENIS \& SCHIFFERMÜLLER, 1775

Phänologie/Voltinismus: Univoltin; 05.IV.(2014), aFA, phänologische Verfrühung 5d gegenüber bis 2013.

\section{Neue Nachweise von bekannten Fundorten:}

c 3146/IV Kreuzbruch Umg./ehemalige Römerwegbrücke am Oder-Havel-Kanal östlich des Ortes (westlich angrenzendes Nachbargebiet; 2014: Busse)

3147/I Zerpenschleuse/Ortsgebiet: Berliner Straße, im Garten (2014, 2015: Busse)

3148/III Ebw. OT Finow/Stadtgebiet: Altenhofer Straße, im Garten $(2017,2018)$
Fundorte (Ergänzung):

b 3148/IV Spechthausen Umg./Barnimer Heide: Stromtrasse südwestlich des Ortes (2015: Richert, Schwabe)

c 3148/IV Ebw./Stadtgebiet: Kleingartenanlage im Schwärzetal nördlich Forstbotanischer Garten (2015, 2016, 2018: Schwabe)

e 3149/II Liepe Umg./Forst Chorin: NSG Plagefenn südlich Gr. Plagesee (2018: Richert, Schwabe, Theimer)

\section{0 (8724) Drymonia querna (DeNIs \& SCHIFFER- MÜLLER, 1775)}

- RL Deutschland (2011): Vorwarnliste.

Phänologie/Voltinismus: Im Jahre 2014 wurde eine durchgängige Flugzeit vom 05.VII. bis 22.VII. beobachtet. Nur ein Fund am 10.VI.2014 steht relativ isoliert. 25.VIII.(2018) aFE, phänologische Verspätung 23d gegenüber bis 2013 .

Neue Nachweise von bekannten Fundorten:

c 3147/I Zerpenschleuse/Ortsgebiet: Berliner Straße, im Garten (2014, 2015, 2016, 2018: Busse)

3148/III Ebw. OT Finow/Stadtgebiet: Altenhofer Straße, im Garten $(2014,2015,2016,2017,2018)$

d 3047/III Groß Schönebeck/Ortsgebiet: Friedenstraße, im Garten (2014, 2018: Haase)

Fundorte (Ergänzung):

b 3148/IV Ebw.-Ostende/Breite Straße Nähe Waldfriedhof (2018: Schwabe)

c 3148/IV Ebw./Stadtgebiet: Kleingartenanlage im Schwärzetal nördlich Forstbotanischer Garten (2017: Schwabe)

e 3149/II Liepe Umg./Forst Chorin: NSG Plagefenn - LedumMoor (2018: Richert \& Schwabe)

\section{1 (8725) Drymonia velitaris (HufNAGEL, 1766)}

- RL Deutschland (2011): Vorwarnliste.

Ein R im L5-Stadium fand ich am 01.VIII.2014 in meinem Garten an einem Stiel-Eichenbusch (Quercus robur), südexponiert ca. $30 \mathrm{~cm}$ über dem Boden.

Phänologie/Voltinismus: 2. partielle Gen.: 09.X.(2014), aFE, $1 \mathrm{~F}$ a.L. leg. Busse in Zerpenschleuse; phänologische Verspätung 21d gegenüber bis 2013.

Neue Nachweise von bekannten Fundorten:

c 3146/IV Kreuzbruch Umg./ehemalige Römerwegbrücke am Oder-Havel-Kanal östlich des Ortes (2014: Busse)

3147/I Zerpenschleuse/Ortsgebiet: Berliner Straße, im Garten (2014, 2018: Busse)

3148/III Ebw. OT Finow/Stadtgebiet: Altenhofer Straße, im Garten $(2014,2016,2017)$ und Umg./Forst Finowtal: Einflugschneise Flugplatz (2016)

3148/IV Ebw./Stadtgebiet: Kleingartenanlage im Schwärzetal nördlich des Forstbotanischen Gartens (2014: Schwabe)

d 3047/III Groß Schönebeck/Ortsgebiet: Friedenstraße, im Garten (2017: Haase)

e 3149/II Liepe Umg./Forst Chorin: NSG Plagefenn - LedumMoor (2018: Richert \& Schwabe) 
Fundorte (Ergänzung):

c 3147/IV Finowfurt Umg./Kesselmoor an der Finowfließniederung nordöstlich des Lehnssees (2016, 2017: Richert, Schwabe)

\section{2 (8727) Pheosia tremula (Clerck, 1759)}

Phänologie/Voltinismus: Bivoltin; 1. Gen.: 20.IV.(2014), aFA, phänologische Verfrühung $6 \mathrm{~d}$ gegenüber bis 2013 . 2. Gen.: 04.IX.(2014), aFE, phänologische Verspätung 7d gegenüber bis 2013 .

\section{3 (8728) Pheosia gnoma (FABricius, 1776) = dictaeoides ESPER, 1789}

Phänologie/Voltinismus: Bivoltin; 2. Gen.: 18.IX.(2015) aFE, phänologische Verspätung 12d gegenüber bis 2013.

\section{4 (8732) Pterostoma palpina (CLERCK, 1759)}

Phänologie/Voltinismus: Bivoltin; 1. Gen.: 11.IV.(2015) aFA, phänologische Verfrühung 7d gegenüber bis 2013 .

\section{6 (8736) Leucodonta bicoloria (DENIS \& SCHIF-} FERMÜLLER, 1775)

Neue Nachweise von bekannten Fundorten:

c 3146/IV Kreuzbruch Umg./ehemalige Römerwegbrücke am Oder-Havel-Kanal östlich des Ortes (2014: Busse)

e 3149/II Liepe Umg./Forst Chorin: NSG Plagefenn - LedumMoor (2018: Richert \& Schwabe)

Fundorte (Ergänzung):

b 3247/II Biesenthal Umg./NSG Biesenthaler Becken: Langerönner Weg (2014: Ockruck), Weg nördlich der Pfauenwiesen (2014: Ockruck)

c 3147/IV Marienwerder Umg./Kiesgrube südwestlich des Sportplatzes (2015); Finowfurt Umg./Kesselmoor an der Finowfließniederung nordöstlich des Lehnssees (2016, 2017: Richert, Schwabe)

\section{8 (8739) Ptilodon cucullina (DENIS \& ScHIF-} FERMÜLLER, 1775) = cuculla ESPER, 1786

- RL Land Brandenburg: Vorwarnliste: WS.

Neue Nachweise von bekannten Fundorten:

b 3247/II Biesenthal Umg./NSG Biesenthaler Becken: Langerönner Weg (2014: Ockruck), Weg nördlich der Pfauenwiesen (2014: Ockruck)

c 3148/III Ebw. OT Finow/Stadtgebiet: Altenhofer Straße, im Garten $(2014,2015,2016,2017,2018)$

d 3047/III Groß Schönebeck/Ortsgebiet: Friedenstraße, im Garten (1999, 2014, 2015, 2017: Haase)

Fundorte (Ergänzung):

g 3050/II Gellmersdorf Umg./Gellmersdorfer Forst: Mühlenberggebiet (1994/1995: Borkowski)

3050/IV Lunow Umg./Gebiet Lunower Hölzchen (1994/1995: Borkowski)

\section{9 (8741) Odontosia carmelita (ESPER, 1785)}

- RL Deutschland (2011): Vorwarnliste.

Neue Nachweise von bekannten Fundorten:

c 3146/IV Kreuzbruch Umg./ehemalige Römerwegbrücke am Oder-Havel-Kanal (2014: Busse) (westlich angrenzendes Nachbargebiet)

3147/I Zerpenschleuse/Ortsgebiet: Berliner Straße, im Garten (2015: Busse)

3148/III Ebw. OT Finow/Stadtgebiet: Im Garten (2015)

Fundorte (Ergänzung):

b 3148/III Spechthausen Umg./Barnimer Heide: Stromtrasse südwestlich des Ortes (2015)

\section{0 (8747) Gluphisia crenata (ESPER, 1785)}

Phänologie/Voltinismus: Im Jahre 2015 wurde erneut eine partielle 2. Gen. M (13.)VIII im Gebiet beobachtet.

\section{2 (8754) Peridea anceps (Goeze, 1781)}

Neue Nachweise von bekannten Fundorten:

c 3147/I Zerpenschleuse/Ortsgebiet: Berliner Straße, im Garten (2014, 2015, 2016, 2017, 2018: Busse)

3148/III Ebw. OT Finow/Stadtgebiet: Altenhofer Straße, im Garten (2018)

d 3047/III Groß Schönebeck/Ortsgebiet (2015: Haase)

Fundorte (Ergänzung):

c 3146/IV Kreuzbruch Umg./ehemalige Römerwegbrücke am Oder-Havel-Kanal (2014: Busse) (westlich angrenzendes Nachbargebiet)

g 3050/II Gellmersdorf Umg./Gellmersdorfer Forst: Mühlenberggebiet (1994/1995: Borkowski)

3050/IV Lunow Umg./Gebiet Lunower Hölzchen (1994/1995: Borkowski)

\section{4 (8760) Harpyia milhauseri (FABRICIUs, 1775)}

Neue Nachweise von bekannten Fundorten:

c 3147/I Zerpenschleuse/Ortsgebiet: Berliner Straße, im Garten (2014, 2017: Busse)

3147/IV Marienwerder/Steinlaake (2015)

3148/III Ebw. OT Finow/Stadtgebiet: Altenhofer Straße, im Garten $(2014,2017,2018)$

Fundorte (Ergänzung):

b 3247/II Biesenthal Umg./NSG Biesenthaler Becken: Langerönner Weg (2014: Ockruck)

c 3146/IV Kreuzbruch Umg./ehemalige Römerwegbrücke am Oder-Havel-Kanal (2014: Busse) (westlich angrenzendes Nachbargebiet)

3147/I Marienwerder Umg./Oder-Havel-Kanal nordwestlich des Ortes (2015: Busse)

3148/III Ebw. OT Finow Umg./Forst Finowtal: Einflugschneise Flugplatz (2016)

3148/IV Ebw.-Westend/Unterhdeide: Wildparkstraße (1973: Kittel); Ebw./Stadtgebiet: Kleingartenanlage im Schwärzetal nördlich Forstbotanischer Garten (2016 2017, 2018: Schwabe) 
d 3047/III Groß Schönebeck/Ortsgebiet: Friedenstraße, im Garten (2017: Haase)

g 3150/IV Altglietzen/Gabower Berge (2015)

3050/II Gellmersdorf Umg./Gellmersdorfer Forst: Mühlenberggebiet (1994/1995: Borkowski)

3050/IV Lunow Umg./Gebiet Lunower Hölzchen (1994/1995: Borkowski)

\section{Lymantriidae (Schadspinner)}

\section{6 (10375) Lymantria monacha (LINNAEus, 1758)}

Phänologie/Voltinismus: Im Jahre 2018 wurde der Falter sehr zeitig bereits am 20.VI. a.L. beobachtet (Haase, pers. Mitt.).

\section{9 (10392) Dicallomera fascelina (LinNAEus, 1758)}

- RL Land Brandenburg: Kategorie 2 (stark gefährdet): $\mathrm{CB}$;

- RL Deutschland (2011): Kategorie 2 (stark gefährdet).

Die Art wird als im Gebiet verschollen gemeldet (seit 2000 keine Funde; vgl. Richert 2014).

\section{2 (10406) Euproctis similis (FuESSLY, 1775)}

Als weitere Raupennahrungspflanze im Gebiet (vgl. Richert 2014) wurde Rhamnus cathartica, Echter Kreuzdorn, festgestellt (Foto Schwabe).

Phänologie/Voltinismus: Seit der Jahrtausendwende zunehmend regelmäßig bivoltin; so auch im Jahre 2014 .

\section{4 (10414) Leucoma salicis (LiNNAEUs, 1758)}

- RL Deutschland (2011): Vorwarnliste.

Auf den Oderhängen bei Altglietzen hatte ich den F E (26.)V ungewöhnlich h (>28 F) a.L., und zwar überwiegend frische Weibchen.

Haase machte bei einer Eizucht (Eier von einem beim LF eingetragenen Weibchen) folgende bemerkenswerte Beobachtungen: Die R schlüpften aus den Eiern bereits ab 15.VI.(2016). Die Eiraupen fraßen unter freilandnahen Zuchtbedingungen aber kaum, stellten die Nahrungsaufnahme schließlich ein, überdauerten so den Spätsommer und Herbst und überwinterten schließlich „in einer Art Winterkokon“ (Haase); mit einer Ausnahme: Eine R fraß weiter, entwickelte sich rasch (Verpuppung am 20.VII.) und ergab am 02.VIII.2016 einen Falter (Männchen) einer partiellen 2. Gen., welcher kleiner war als F der 1. Gen. Auch bei anderen Zuchten beobachtete Haase Raupenüberwinterung.

Die Beobachtungen sind insofern bemerkenswert, als in der Literatur abweichende Aussagen über das Überwinterungsstadium (Eier bzw. Raupen) zu finden sind. Teils wird ausschließlich oder in der Regel Überwinterung der Eier angegeben, z. B. Bergmann, 1953; КосH 1984; ECKSTEIN 1915; FORSTER WOHLFAHRT 1984); dagegen überwintert nach STEINER et al. 2014 die Raupe.

Eine 2. Generation wird von de Freina \& WitT (1987) für südliche Gebiete angegeben, konnte aber für BadenWürttemberg nicht sicher bestätigt werden (EBERT 1994). Sie wurde bei uns im Freiland noch nicht beobachtet.

Neue Nachweise von bekannten Fundorten:

b 3248/II Trampe/ehemaliger TÜP (2016: Busse \& Müller)

c 3147/I Zerpenschleuse/Ortsgebiet: Berliner Straße, im Garten (2014, 2015, 2016, 2018: Busse)

3148/III Ebw. OT Finow/Stadtgebiet: Altenhofer Straße, im Garten $(2014,2015,2016,2018)$

d 3047/III Groß Schönebeck/Ortsgebiet: Friedenstraße, im Garten (2014, 2016: Haase)

g 3150/III Gabow/Oderhänge: Granitberggebiet (2015: Richert, Schwabe)

3150/IV Altglietzen/Oderhänge: Gabower Berge (2015)

Fundorte (Ergänzung):

c 3147/I Marienwerder Umg./Oder-Havel-Kanal nordwestlich des Ortes (2014, 2016: Busse)

3147/IV Finowfurt Umg./Kesselmoor an der Finowfließniederung nordöstlich des Lehnssees (2017)

3148/III Ebw. OT Finow Umg./Forst Finowtal: Einflugschneise Flugplatz (2016), Nordrand Flugplatz (2017)

3148/IV Ebw./Stadtgebiet: Kleingartenanlage im Schwärzetal nördlich Forstbotanischer Garten (2016: Schwabe)

e 3049/I Groß Ziethen Umg./Steingruben an den Ihlowbergen (2014)

3149/II Liepe Umg./Forst Chorin: NSG Plagefenn - LedumMoor (2018: Richert \& Schwabe)

g 3050/II Gellmersdorf Umg./Gellmersdorfer Forst: Mühlenberggebiet (1994/1995: Borkowski)

3050/IV Lunow Umg./Gebiet Lunower Hölzchen (1994/1995: Borkowski)

3050/II Stolzenhagen Umg./Trockenpolder nördlich Pomeranzengraben (1994/1995: Borkowski)

\section{5 (10416) Arctornis l-nigrum (O. F. MüLLER, 1764)}

Phänologie/Voltinismus: 07.VI.(2018) aFA, phänologische Verfrühung 1d gegenüber bis 2013.

Neue Nachweise von bekannten Fundorten:

c 3147/I Zerpenschleuse/Ortsgebiet: Berliner Straße, im Garten (2014: Busse)

3147/IV Finowfurt Umg./Kesselmoor an der Finowfließniederung nordöstlich des Lehnssees (2017)

3148/III Ebw. OT Finow/Stadtgebiet: Altenhofer Straße, im Garten (2014)

d 3047/III Groß Schönebeck/Ortsgebiet: Friedenstraße, im Garten (2017: Haase)

e 3149/II Liepe Umg./Forst Chorin: NSG Plagefenn - LedumMoor (2018: Richert \& Schwabe)

g 3050/II Gellmersdorf Umg./Gellmersdorfer Forst: Mühlenberggebiet (1994/1995: Borkowski)

3050/IV Lunow Umg./Gebiet Lunower Hölzchen (1994/1995: Borkowski) 


\section{Nolidae (Kleinbären)}

\section{Nolinae}

246 (10423) Meganola strigula (DENIS \& SCHIFFERMÜLLER, 1775)

Neue Nachweise von bekannten Fundorten:

c 3147/I Zerpenschleuse/Ortsgebiet: Berliner Straße, im Garten (2018: Busse)

\section{7 (10425) Meganola albula (DENIS \& SCHIFFER-} MÜLLER, 1775)

- Verordnung zur Neufassung der BArtschV von 2005: Besonders geschützte Art.

Phänologie/Voltinismus: 07.X.(2014), aFE, phänologische Verspätung 41d gegenüber bis 2013; ein weiterer Spätfund am 02.X.2014 $1 \mathrm{~F}$ a.L. leg. Busse und 4.IX. (2018), $1 \mathrm{~F}$ a.L. leg. Schwabe a.L. Handelt es sich dabei um eine partielle 2. Gen.? Die Dauer der phänologischen Verspätung legt die Vermutung nahe. In der Literatur gilt die Art als univoltin (EcKstein 1923, КосH 1984, Forster \& Wohlfahrt 1984, Freina \& Witt 1987. Auch Steiner 1994 hält einen Spätfund (20.IX.1981) in Baden-Württemberg für einen Nachzügler und nennt außerdem ohne Kommentar einen Spätfund (20.X.1953) in der Pfalz.

Neue Nachweise von bekannten Fundorten:

b 3248/II Trampe/ehemaliger TÜP (2016: Busse \& Müller)

c 3147/I Zerpenschleuse/Ortsgebiet: Berliner Straße, im Garten (2014: Busse)

3148/III Ebw. OT Finow/Stadtgebiet: Altenhofer Straße, im Garten $(2014,2015,2017,2018)$

e 3049/I Groß Ziethen Umg./Steingruben an den Ihlowbergen (2014)

g 3050/II Stolpe a.O./Trockenhänge am Stadtweg (2018)

3150/IV Altglietzen/Oderhänge: Gabower Berge (2015: Richert, 2018: Schwabe)

Fundorte (Ergänzung):

b 3247/II Biesenthal Umg./NSG Biesenthaler Becken: Weg nördlich der Pfauenwiesen (2014: Ockruck)

c 3147/IV Finowfurt Umg./Kesselmoor an der Finowfließniederung nordöstlich des Lehnssees $(2016,2017)$

3148/IV Ebw./Stadtgebiet: Kleingartenanlage im Schwärzetal nördlich des Fortbotanischen Gartens (2014: Schwabe)

d 3047/III Groß Schönebeck/Ortsgebiet: Friedenstraße, im Garten (2018: Haase)

h 3150/II Hohensaaten/Deponiegelände am Weg zur Siedlung (2014: Kleße)

\section{8 (10427) Nola cucullatella (LinNAEUs, 1758)}

Neue Nachweise von bekannten Fundorten:

c 3147/I Zerpenschleuse/Ortsgebiet: Berliner Straße, im Garten (2014, 2015: Busse)
3148/III Ebw. OT Finow/Stadtgebiet: Altenhofer Straße, im Garten (2014)

Fundorte (Ergänzung):

c 3147/II Finowfurt/Hirtenweg - Besters Fließ (1992, 1994, 1995, 1999: Kittel)

d 3047/III Groß Schönebeck/Ortsgebiet: Friedenstraße, im Garten (2015: Haase)

\section{9 (10429) Nola confusalis (HERRICH-SCHÄFFER,} 1847)

Phänologie/Voltinismus: 02.VI.(2015), aFE, phänologische Verspätung 4d gegenüber bis 2013.

Neue Nachweise von bekannten Fundorten:

c 3147/IV Marienwerder Umg./Kiesgrube südwestlich des Sportplatzes (2015)

Fundorte (Ergänzung):

c 3148/IV Ebw./Stadtgebiet: Kleingartenanlage im Schwärzetal nördlich Forstbotanischer Garten (2018: Schwabe)

e 3149/II Liepe Umg./Forst Chorin: NSG Plagefenn südlich Gr. Plagesee (2018: Richert, Schwabe \& Theimer)

g 3150/I Oderberg/Geistberg (2016: Schwabe)

\section{0 (10431) Nola aerugula (HüBNER, 1793) \\ = centonalis HüBNER, 1796}

- Verordnung zur Neufassung der BartschV von 2005: Besonders geschützte Art.

- RL Land Brandenburg: Kategorie 3 (gefährdet): NM, $\mathrm{SM}, \mathrm{MB}$;

- RL Deutschland 2011: Vorwarnliste.

Phänologie/Voltinismus: 10.VI.(2018) aFA, phänologische Verfrühung 8d gegenüber bis 2013.

Neue Nachweise von bekannten Fundorten:

c 3148/III Ebw. OT Finow/Stadtgebiet: Altenhofer Straße, im Garten (2018)

\section{Chloephorinae}

\section{1 (10441) Nycteola revayana (Scopoli, 1772)}

Neue Nachweise von bekannten Fundorten:

c 3147/I Zerpenschleuse/Ortsgebiet: Berliner Straße, im Garten (2014: Busse)

3148/III Ebw. OT Finow/Stadtgebiet: Altenhofer Straße, im Garten $(2014,2016)$

g 3150/I Oderberg/Teufelsberg (1973: Kittel)

Fundorte (Ergänzung):

b 3148/IV Spechthausen Umg./Barnimer Heide: Stromtrasse südwestlich des Ortes (2015)

c 3148/IV Ebw.-Westend/Unterheide: Wildparkstraße (1976: Kittel); Ebw./Stadtgebiet: Kleingartenanlage im Schwärzetal nördlich Forstbotanischer Garten (2016: Schwabe) 


$\begin{array}{lll}\text { e 3149/II } & \begin{array}{l}\text { Liepe Umg./Forst Chorin: NSG Plagefenn - Ledum- } \\ \text { Moor (2018: Richert \& Schwabe) }\end{array} \\ \text { g 3050/II } & \begin{array}{l}\text { Gellmersdorf Umg./NSG Gellmersdorfer Forst: } \\ \text { Mühlenberggebiet (1994/1995: Borkowski) }\end{array} \\ & \text { 3150/IV } & \text { Altglietzen/Oderhänge: Gabower Berge (2015) }\end{array}$

\section{3 (10449) Bena bicolorana (Fuessly, 1775)}

Phänologie/Voltinismus: Univoltin; 20.IX. und 02.X. (2014), aFE, je ein frischer F; phänologische Verspätung 33d gegenüber bis 2013. Offensichtlich handelt es sich hierbei um eine partielle 2. Gen. Die Belegexemplare (1 F in coll. Busse, $1 \mathrm{~F}$ in coll. Richert) sind kleiner als $\mathrm{F}$ der 1. Gen., ein Phänomen, das vielfach bei zusätzlichen Gen. zu beobachten ist. Nach EcKsTeIn 1923, КосH 1984 univoltin. Lussi (1997) führt für Baden-Württemberg die spätesten Funde von A (bis 8.) IX an. Nach Forster \& WOHLFahrt (1980) „... in den Südalpen und in Ungarn eine 2. Generation von Mitte August bis Mitte September“ (1.c.: 263).

Neue Nachweise von bekannten Fundorten:

$$
\begin{aligned}
\text { c 3147/I } & \text { Zerpenschleuse/Ortgebiet: Berliner Straße, im } \\
& \text { Garten (2018: Busse) } \\
3148 / \mathrm{III} & \text { Ebw. OT Finow/Stadtgebiet: Altenhofer Straße, im } \\
& \text { Garten (2014) }
\end{aligned}
$$

Fundorte (Ergänzung):

c 3147/I Marienwerder Umg./Oder-Havel-Kanal nordwestlich des Ortes (2015: Busse)

\section{4 (10451) Pseudoips prasinana (Linnaeus, 1758)}

Phänologie/Voltinismus: 10.V.(2016), aFA, $1 \mathrm{~F}$ a.L., leg. et fot. Schwabe, erneute phänologische Verfrühung, insgesamt 9d gegenüber bis 2013. Bereits 2015 erfolgte der erste F-Fund am 11.V. (phänologische Verfrühung 8d gegenüber bis 2013). Zieht man die Vorverlagerung des absoluten Flugzeitbeginns in den letzten Jahren in Betracht, dürften die frischen F ab M VIII $(2015,2016)$ bzw. E VIII (2017) einer 2. Gen. zuzurechnen sein. Dies trifft mit Sicherheit auf einen F-Fund am 20.IX.2014 zu.

\section{6 (10459) Earias vernana (FABRICIUS, 1787)}

\section{Neue Nachweise von bekannten Fundorten:}

c 3148/III Ebw. OT Finow/Stadtgebiet: Altenhofer Straße, im Garten $(2015,2018)$

Fundorte (Ergänzung):

c 3147/I Marienwerder Umg./Oder-Havel-Kanal nordwestlich des Ortes (2016: Busse)

3148/IV Ebw.-Westend/Unterheide: Wildparkstraße (1970, 1973: Kittel)

\section{Arctiidae (Bärenspinner)}

Lithosiinae

\section{7 (10466) Thumata senex (HüBNER, 1808)}

- RL Land Brandenburg: Vorwarnliste: NM.

Neue Nachweise von bekannten Fundorten:

b 3248/II Trampe/ehemaliger TÜP (2016: Busse \& Müller)

c 3147/I Zerpenschleuse/Ortsgebiet: Berliner Straße, im Garten (2015, 2016: Busse)

e $\quad 3149 /$ II Liepe Umg./Forst Chorin: NSG Plagefenn - LedumMoor (2018: Richert \& Schwabe)

Fundorte (Ergänzung):

b 3247/II Biesenthal Umg./NSG Biesenthaler Becken: Weg nördlich der Pfauenwiesen (2014: Ockruck)

3148/IV Ebw./Stadtgebiet: Kleingartenanlage im Schwärzetal nördlich des Forstbotanischen Gartens (2014: Schwabe)

e 3049/I Groß Ziethen Umg./Steingruben an den Ihlowber gen (2014)

\section{8 (10475) Miltochrista miniata (FoRSTER, 1771)}

- RL Land Brandenburg: Vorwarnliste: MB, SM, NM. Ich beobachtete den F nachts beim Saugen an Blüten von Buddleja davidii, Chinesischer Sommerflieder.

Phänologie/Voltinismus: 01.VI.(2018) aFA, phänologische Verfrühung 5d gegenüber bis 2013.

Eine partielle 2. Gen. wurde 2014 erneut beobachtet. Dabei fiel auf, dass es sich nicht nur um Einzeltiere handelte, der F trat i.A. auf: 17.IX.-13.X.(2014), aFE, 8 F a.L.; phänologische Verspätung 12d gegenüber bis 2013. Auch 2016 und 2018 wurden F der 2. Gen. registriert.

Ein Fund am 29.VIII.2018, ein F a.L. leg. Schwabe, gehört offenbar der 2. Gen. an, die in günstigen Jahren bereits E VIII (9d früher als in RicherT 2014 angegeben) zu fliegen beginnt.

Neue Nachweise von bekannten Fundorten:

c 3147/I Zerpenschleuse/Ortsgebiet: Berliner Straße, im Garten (2014, 2015, 2016, 2017, 2018: Busse)

3148/III Ebw. OT Finow/Stadtgebiet: Altenhofer Straße, im Garten $(2014,2015,2016,2017,2018)$

g 3050/II Stolpe a.O./Trockenhänge am Stadtweg (2018)

Fundorte (Ergänzung):

b 3247/II Biesenthal Umg./NSG Biesenthaler Becken: Weg nördlich der Pfauenwiesen (2014: Ockruck)

c 3147/I Marienwerder Umg./Oder-Havel-Kanal nordwestlich des Ortes (2014, 2016: Busse)

3147/IV Finowfurt Umg./Finowfließniederung: Neue Brücke an der Biesenthaler Straße (2014); Kesselmoor an der Finowfließniederung nordöstlich des Lehnssees (2016: Richert, Schwabe)

3148/IV Ebw./Stadtgebiet: Kleingartenanlage im Schwärzetal nördlich des Forstbotanischen Gartens (2014, 2016, 2017, 2018: Schwabe) 
e 3149/II Liepe Umg./Forst Chorin: NSG Plagefenn - LedumMoor (2018: Richert \& Schwabe)

h 3150/II Hohensaaten/Deponiegelände an der Straße nach der Siedlung (2014: Kleße)

\section{9 (10477) Cybosia mesomella (LinNaEus, 1758)}

Phänologie/Voltinismus: 31.VIII.2015 aFE, phänologische Verspätung 16d gegenüber bis 2013, offenbar ein „Nachzügler“ in diesem witterungsmäßig wechselhaften Sommer.

\section{0 (10479) Pelosia muscerda (Hufnagel, 1766)}

Phänologie/Voltinismus: Univoltin; nach einer durchgehenden Flugperiode vom 15. bis 31.VII. wurden M IX erneut $3 \mathrm{~F}$ a.L. an verschiedenen Plätzen im Gebiet registriert: 14.IX.2014, 15.IX.2014, 20.IX.(2014) aFE, je ein F; phänologische Verspätung 13d gegenüber bis 2013.

Wahrscheinlich handelt es sich um eine partielle 2. Gen. In der Literatur wird allgemein nur eine (langgestreckte) Generation angegeben, aus der Schweiz wird aber von einer partiellen 2. Gen. berichtet, die auch bei Zuchten beobachtet wurde (Pro NAtura - Schweizerischer BUND FÜR NATURSCHUTZ, 2000).

Neue Nachweise von bekannten Fundorten:

b 3247/II Biesenthal Umg./NSG Biesenthaler Becken: Langerönner Weg (2014: Ockruck), Weg nördlich der Pfauenwiesen (2014: Ockruck)

c 3147/I Zerpenschleuse/Ortsgebiet: Berliner Straße, im Garten (2014, 2015: Busse)

3147/II Finowfurt/Hirtenweg - Besters Fließ (1991, 1997: Kittel)

3148/III Ebw. OT Finow/Stadtgebiet: Altenhofer Straße, im Garten $(2014,2015,2016,2018)$

e 3049/I Groß Ziethen Umg./Steingruben an den Ihlowbergen (2014)

3149/II Liepe Umg./Forst Chorin: NSG Plagefenn - LedumMoor am Fuß der Plageberge (2018: Richert \& Schwabe)

g 3149/IV Liepe (1976: Kittel)

3150/I Oderberg/Teufelsberg (1973, 1975: Kittel)

Fundorte (Ergänzung):

c 3147/I Marienwerder Umg./Oder-Havel-Kanal nordwestlich des Ortes (2014: Busse)

3147/IV Finowfurt Umg./Finowfließniederung Neue Brücke an der Biesenthaler Straße (2014); Kesselmoor an der Finowfließniederung nordöstlich des Lehnssees (2016, 2017: Richert, Schwabe)

3148/IV Ebw./Stadtgebiet: Kleingartenanlage im Schwärzetal nördlich Forstbotanischer Garten (2017: Schwabe); Ebw.-Nordend/Gelände der Landesklinik: Ehemalige Gehörlosen-Hilfsschule (1983: Kittel)

e 3049/IV Chorin Umg./Forst Chorin: Teerbrennerberge - Am Olberg (2013: Müller)

f 3049/IV Brodowin/Krugberg (2014)

\section{1 (10480) Pelosia obtusa (HeRrich-SCHÄFFER,} 1847)

- RL Land Brandenburg: Kategorie 3 (gefährdet): NM;

- RL Deutschland (2011): Kategorie 3 (gefährdet).

Neue Nachweise von bekannten Fundorten:

c 3147/I Zerpenschleuse/Ortsgebiet: Berliner Straße, im Garten (2014: Busse)

3147/IV Finowfurt Umg./Kesselmoor an der Finowfließniederung nordöstlich des Lehnssees (2017: Schwabe)

\section{2 (10483) Atolmis rubricollis (LinNaEus, 1758)}

- RL Land Brandenburg: Kategorie G (Gefährdung anzunehmen): Habitatbindung ungeklärt.

Die Art wird im Gebiet zunehmend häufiger und verbreitet gefunden. Allein am 08.VI.2014 registrierte Busse bei Marienwerder 15 F a.L.; im Jahre 2016 am 24.VI. 15 F auf dem ehemaligen TÜP Trampe.

Phänologie/Voltinismus: Univoltin; 23.V.(2014), aFA, phänologische Verfrühung 5d bis gegenüber 2013.

Neue Nachweise von bekannten Fundorten:

c 3147/I Zerpenschleuse/Ortsgebiet: Berliner Straße, im Garten (2014, 2015, 2016, 2018: Busse)

3148/III Ebw. OT Finow/Stadtgebiet: Altenhofer Straße, im Garten $(2014,2015,2016,2018)$

d 3047/III Gr. Schönebeck/Ortsgebiet: Friedenstraße, im Garten (2016: Haase)

Fundorte (Ergänzung):

b 3247/II Biesenthal Umg./NSG Biesenthaler Becken: Langerönner Weg (2014: Ockruck)

3248/II Trampe/ehemaliger TÜP (2016: Busse \& Müller)

c 3146/IV Kreuzbruch Umg./ehemalige Römerwegbrücke am Oder-Havel-Kanal östlich des Ortes (2014: Busse) (westlich angrenzendes Nachbargebiet)

3147/I Marienwerder Umg./Oder-Havel-Kanal nordwestlich des Ortes (2014, 2016: Busse)

3147/IV Finowfurt Umg./Kesselmoor an der Finowfließniederung nordöstlich des Lehnssees $(2016,2017)$

3148/IV Ebw./Stadtgebiet: Kleingartenanlage im Schwärzetal nördlich des Forstbotanischen Gartens (2014, 2016 2018: Schwabe)

e 3149/II Liepe Umg./Forst Chorin: NSG Plagefenn - LedumMoor am Fuß der Plageberge (2018: Richert \& Schwabe)

g 3150/I Oderberg/Geistberg (2017, 2018: Schwabe)

\section{3 (10485) Lithosia quadra (LiNNAEUs, 1758)}

- RL Land Brandenburg: Kategorie G (Gefährdung anzunehmen): Nadelwäder;

- RL Deutschland (2011): Kategorie 3 (gefährdet).

Haase berichtete über einen misslungenen Zuchtversuch: Die Eiablage wurde von einem beim LF eingetragenen Weibchen erhalten. Die R fraßen ausschließlich Flechten, daneben gereichtes Blattwerk wurde nicht angenommen. Sie überwinterten zweimal, gingen dann aber ein. 
Phänologie/Voltinismus: Univoltin; nach einer durchgehenden Flugzeit vom 05.VII. bis 31.VII.2014 wurde am 18.X.(2014), aFE, erneut ein Männchen a.L. gefangen, phänologische Verspätung 32d gegenüber bis 2013. Die Vermutung liegt nahe, dass es sich bei den September-/Oktoberfunden um F einer partiellen 2. Gen. handelte. In der Literatur wird in der Regel nur eine Gen. angegeben. Lediglich FreinA \& Witt 1987 bemerken abweichend „... in geeigneten Regionen zuweilen 2. Generation Mitte September" (p.69). Der aktuelle Spätfund macht das Funddatum aus älterer Zeit glaubhaft, das mir bisher zweifelhaft erschien: 16.IX.1981, $1 \mathrm{~F}$ Ebw./Paschenberg. leg. Kruel.

\section{Neue Nachweise von bekannten Fundorten}

c 3147/I Zerpenschleuse/Ortsgebiet: Berliner Straße, im Garten (2014, 2015, 2018: Busse)

3148/III Ebw. OT Finow/Stadtgebiet: Altenhofer Straße, im Garten $(2014,2015,2016,2017,2018)$

d 3047/III Groß Schönebeck/Ortsgebiet: Am Friedhof (2014: Haase)

e 3049/I Groß Ziethen Umg./Steingruben an den Ihlowbergen (2014)

3149/II Liepe Umg./Forst Chorin: NSG Plagefenn - LedumMoor am Fuß der Plageberge (2018: Richert \& Schwabe)

\section{Fundorte (Ergänzung):}

b 3247/II Biesenthal Umg./NSG Biesenthaler Becken: Weg nördlich der Pfauenwiesen (2014: Ockruck)

3249/I Gersdorf Umg./Gamengrund südlich Teufelssee (2017: Richert, Schwabe)

c 3147/IV Finowfurt Umg./Finowfließniederung Neue Brücke an der Biesenthaler Straße (2014); Kesselmoor an der Finowfließniederung nordöstlich des Lehnssees (2016, 2017: Richert, Schwabe)

3148/IV Ebw./Stadtgebiet: Kleingartenanlage im Schwärzetal nördlich des Forstbotanischen Gartens (2014, 2017, 2018: Schwabe)

\section{4 (10487) Eilema depressa (ESPER, 1787) = deplana $($ EsPER, 1787)}

- RL Land Brandenburg: Vorwarnliste: Nadelwälder. Am 15.VIII.2017 wurde die Art im Forst Finowtal auf der Einflugschneise des Flugplatzes Finow (Calluna-Heide mit Sukzessionsstadien - Kiefer, Birke, Espe) i.M. a.L. beobachtet, ein F auch a.K.

\section{Neue Nachweise von bekannten Fundorten:}

b 3247/II Biesenthal Umg./NSG Biesenthaler Becken: Nördlich der Pfauenwiesen (2015: Ockruck)

c 3148/III Ebw. OT Finow/Stadtgebiet: Altenhofer Straße, im Garten (2014, 2015, 2016, 2017, 2018)

e 3149/II Liepe Umg./Forst Chorin: NSG Plagefenn - LedumMoor am Fuß der Plageberge (2018: Richert \& Schwabe)

g 3150/IV Altglietzen/Gabower Berge (2015)

\section{Fundorte (Ergänzung):}

a 3249/II Falkenberg Umg./Cöthener Forst: Am Klingenden Fließ unterhalb des Bismarck-Turmes (2014)

b 3249/I Gersdorf Umg./Gamengrund südlich Teufelssee (2017: Schwabe)

c 3147/IV Finowfurt Umg./Finowfließniederung Neue Brücke an der Biesenthaler Straße (2014); Kesselmoor an der Finowfließniederung nordöstlich des Lehnssees (2016, 2017: Richert, Schwabe)

3148/III Ebw. OT Finow Umg./Forst Finowtal: Einflugschneise Flugplatz (2017)

3148/IV Ebw./Stadtgebiet: Kleingartenanlage im Schwärzetal nördlich des Forstbotanischen Gartens (2014, 2016, 2017, 2018: Schwabe)

e 3048/II Joachimsthal (1976: Kittel)

g 3149/IV Niederfinow Umg./Oderhänge - ehemalige Kiesgruben südwestlich des Schiffshebewerks (2015, 2016: Schwabe)

3150/I Oderberg/Geistberg (2016: Schwabe)

h 3150/II Hohensaaten/Deponiegelände an der Straße nach der Siedlung (2014: Kleße)

\section{5 (10488) Eilema griseola (HÜBNER, 1803)}

- RL Land Brandenburg: Kategorie 3 (gefährdet): MB.

Neue Nachweise von bekannten Fundorten:

c 3147/I Zerpenschleuse/Ortsgebiet: Berliner Straße, im Garten (2014: Busse)

3147/II Finowfurt/Besters Fließ: Hirtenweg (1983, 1991: Kittel)

3148/III Ebw. OT Finow/Stadtgebiet: Altenhofer Straße, im Garten (2014, 2015, 2016, 2017)

e 3149/II Liepe Umg./Forst Chorin: NSG Plagefenn - LedumMoor am Fuß der Plageberge (2018: Richert \& Schwabe)

Fundorte (Ergänzung):

b 3247/II Biesenthal Umg./NSG Biesenthaler Becken: Weg nördlich der Pfauenwiesen (2014: Ockruck)

c 3147/IV Finowfurt Umg./Finowfließniederung: Neue Brücke an der Biesenthaler Straße (2014), Kesselmoor an der Finowfließniederung nordöstlich des Lehnssees (2016, 2017: Richert, Schwabe)

\section{7 (10490) Eilema complana (LINNAEUs, 1758)}

Ich beobachtete den F am Tag beim Saugen an Heideblüten (Calluna vulgaris).

In coll. Kittel fand ich in einer Serie von Eilema complana ein mir bisher unbekanntes Belegexemplar mit folgenden Daten: 09.VIII.1972, LF (Ebw. OT) Finow, Gartenstraße, leg. Duckert. Dieser F, ein Männchen, fiel durch seinen silbrig-seidigen Glanz auf. Die Gelbzeichnung an der Costa ist schmal und wenig intensiv, auch Thorax und Hinterleibsende-Ende sind nur schwach gelblich (siehe Fig. 9).

Meine Vermutung, dass es sich um E. paliatella handeln könnte, bestätigte sich nicht: Auf der Vorderflügelunterseite befinden sich in der Costalmitte abstehende Duftschuppen (Androkonien), ein Merkmal von L. complana, 
welches bei L. paliatella fehlt (vgl. Lepiforum). Im Genitalpräparat zeigt der bandartige cornutus einwärts eine Einschnürung und wird schmaler - ein Merkmal von complana, während für palliatella eine gleichbleibende Breite in der Literatur angegeben wird. Somit handelt es sich um eine besondere Form von complana.

Herrn M. Falkenberg, Staatliches Museum für Naturkunde Karlsruhe, danke ich auch an dieser Stelle für seine Hilfe bei der Determination.

Phänologie/Voltinismus: 06.VI.2015, phänologische Verfrühung 9d gegenüber bis 2013; 04.VI.(2016) aFA, erneute phänologische Verfrühung 2d, insgesamt also 11d gegenüber bis 2013 .

\section{9 (10495) Eilema pygmaeola (DoubLEDAY, 1847) = pallifrons ZELLER, 1847}

- RL Land Brandenburg: Kategorie 3 (gefährdet): TR;

- RL Deutschland (2011): Vorwarnliste.

Neue Nachweise von bekannten Fundorten:

c 3148/III Ebw. OT Finow/Stadtgebiet: Altenhofer Straße, im Garten $(2016,2017,2018)$

g 3150/III Gabow/Oderhänge: Granitberggebiet (2014)

Fundorte (Ergänzung):

e 3049/I Groß Ziethen Umg./Steingruben an den Ihlowbergen (2014)

\section{0 (10497) Eilema lutarella (LinNAEUs, 1758)}

- RL Brandenburg: Vorwarnliste: TR;

- RL Deutschland (2011): Vorwarnliste.

Ich beobachtete den F am Tag beim Saugen an Blüten von Origanum vulgare, Dost; Helichrysum arenarium, SandStrohblume, und Centaurea scabiosa, Skabiosen-Flockenblume.

\section{Neue Nachweise von bekannten Fundorten:}

c 3147/I Zerpenschleuse/Ortsgebiet: Berliner Straße, im Garten (2014, 2016: Busse)

3148/III Ebw. OT Finow/Stadtgebiet: Altenhofer Straße, im Garten $(2014,2015,2017)$ und Umg./Forst Finowtal: Einflugschneise Flugplatz (2017)

g 3150/IV Altglietzen Umg./Gabower Berge (2015)

Fundorte (Ergänzung):

c 3148/IV Ebw./Stadtgebiet: Kleingartenanlage im Schwärzetal nördlich Forstbotanischer Garten (2017, 2018: Schwabe)

3149/III Stecherschleuse Umg./Hänge zum Finowbruch westlich des Ortes (2017: Schwabe); ehemaliges Kiesgrubengelände nördlich des Ortes (2017: Richert)

d 3047/III Groß Schönebeck Umg./Liebenthaler Weg (2014: Haase)

f 3049/II Klein Ziethen Umg./Debritzberg (2015)

h 3150/II Hohensaaten/Deponiegelände an der Straße nach der Siedlung (2014: Kleße)

\section{1 (10499) Eilema sororcula (HUfNAGEL, 1766)}

- RL Land Brandenburg: Kategorie 2 (stark gefährdet): FW.

Phänologie/Voltinismus: In der Regel univoltin: Nach einer durchgehenden Flugperiode vom 28.IV. bis 09.VI. 2014 wurde am 18.VII.2014 erneut ein F a.L. registriert. 2. Gen.?

Neue Nachweise von bekannten Fundorten:

b 3247/II Biesenthal Umg./NSG Biesenthaler Becken: Langerönner Weg (2014: Ockruck)

3248/II Trampe/ehemaliger TÜP (2016: Busse \& Müller)

c 3146/IV Kreuzbruch Umg./ehemalige Römerwegbrücke am Oder-Havel-Kanal (2014: Busse) (westlich angrenzendes Nachbargebiet)

3147/I Zerpenschleuse/Ortsgebiet: Berliner Straße, im Garten (2014, 2015, 2016, 2018: Busse); Marienwerder Umg./Oder-Havel-Kanal nordwestlich des Ortes (2014, 2015, 2016: Busse)

3147/IV Marienwerder/Steinlaake (2015), Kiesgrube südwestlich des Sportplatzes (2015)

3148/III Ebw. OT Finow/Stadtgebiet: Altenhofer Straße, im Garten $(2014$, 2015, 2016, 2017, 2018)

e 3149/II Liepe Umg./Forst Chorin: NSG Plagefenn - LedumMoor am Fuß der Plageberge (2018: Richert \& Schwabe)

Fundorte (Ergänzung):

c 3147/IV Finowfurt Umg./Kesselmoor an der Finowfließniederung nordöstlich des Lehnssees (2017: Richert, Schwabe)

3148/IV Ebw./Stadtgebiet: Kleingartenanlage im Schwärzetal nördlich des Forstbotanischen Gartens (2014, 2016 2017, 2018: Schwabe)

d 3047/III Groß Schönebeck/Ortsgebiet: Friedenstraße, im Garten (2016, 2017: Haase) und Umg./Liebenthaler Weg $(2014,2015$ : Haase $)$

3147/I Groß Schönebeck Umg./Mülldeponie am Weg nach Klandorf östlich der Bahnlinie (2018: Haase)

g 3050/II Stolpe a.O./Trockenhänge am Stadtweg (2018)

3149/IV Niederfinow Umg./Oderhänge - ehemalige Kiesgruben südwestlich des Schiffshebewerks (2015: Schwabe)

3150/I Oderberg/Geistberg (2015, 2017, 2018: Schwabe)

3150/IV Altglietzen Umg./Gabower Berge (2015)

\section{2 (10509) Setina irrorella (LinNAEUs, 1758)}

- RL Land Brandenburg: Kategorie 2 (stark gefährdet): TR;

- RL Deutschland (2011): Vorwarnliste.

Neue Nachweise von bekannten Fundorten:

c 3149/III Ebw. Umg./Finowbruch östlich des Eichwerder: An der Bahnlinie (2016: Schwabe)

f 3050/III Parstein Umg./Sonnenberge südlich des Ortes (2015)

Fundorte (Ergänzung):

c 3149/III Stecherschleuse Umg./Finowtal westlich des Ortes Richtung F. Kahlenberg (2017: Schwabe).

e 3049/I Groß Ziethen Umg./Steingruben an den Ihlowbergen (2014) 


\section{Syntominae}

\section{4 (10521) Dysauxes ancilla (LinNAEus, 1767)}

Phänologie/Voltinismus: 14.VI.(2018) aFA, phänologische Verfrühung 3d gegenüber bis 2013.

\section{Neue Nachweise von bekannten Fundorten:}

g 3050/II Stolpe a.O./Trockenhänge am Stadtweg (2018)

3150/III Gabow/Granitberggebiet (2015: Schwabe)

3150/IV Altglietzen Umg./Gabower Berge (2015)

Fundorte (Ergänzung):

h 3150/II Hohensaaten/Deponiegelände an der Straße nach der Siedlung (2014: Kleße)

\section{Arctiinae}

\section{5 (10526) Spiris striata (Linnaeus, 1758)}

- RL Land Brandenburg: Kategorie 3 (gefährdet): TR;

- RL Deutschland (2011): Vorwarnliste.

Neue Nachweise von bekannten Fundorten:

b 3247/II Biesenthal Umg./NSG Biesenthaler Becken: Langerönner Weg (2014: Ockruck)

3248/II Trampe/ehemaliger TÜP (2018)

c 3148/III Ebw. OT Finow/Stadtgebiet: Altenhofer Straße, an der Straße am Tag schwärmend (2014)

3148/IV Ebw. OT Finow Umg./Forst Finowtal: Stromtrasse südlich des Ortes (2014)

g 3050/II Stolpe a.O./Trockenhänge am Stadtweg (2018; Richert \& Schwabe)

Fundorte (Ergänzung):

b 3248/II Schönholz Umg./Stromtrasse nordwestlich des Ortes (2018)

c 3149/I Niederfinow Umg./Oder-Havel-Kanal östlich der Klosterbrücke (2018: Barthelme)

e 3049/I Groß Ziethen Umg./Steingruben an den Ihlowbergen (2014)

\section{6 (10528) Coscinia cribraria (LINNAEUs, 1758)}

- RL Deutschland (2011): Vorwarnliste.

Neue Nachweise von bekannten Fundorten:

c 3147/II Finowfurt Umg./Üdersee - Südwestende (1971: Kittel)

3148/III Ebw. OT Finow/Stadtgebiet: Altenhofer Straße, im Garten (2015) und Umg./Forst Finowtal: Einflugschneise Flugplatz (2017: Richert, Schwabe; 2018: Richert)

3148/IV Ebw. OT Finow Umg./Forst Finowtal: Stromtrasse südlich des Ortes (2015)

g 3050/II Stolpe a.O./Trockenhänge am Stadtweg (2018: Richert) 3150/III Gabow/Granitberggebiet (2015: Schwabe)

Fundorte (Ergänzung):

c 3147/IV Finowfurt Umg./Kessselmoor an der Finowfließniederung nördlich des Lehnssees (2016: Schwabe)
3148/IV Ebw.-Westend/Unterheide: Wildparkstraße (1970: Kittel)

3149/I Niederfinow Umg./Oder-Havel-Kanal östlich der Klosterbrücke (2018: Barthelme)

d 3047/III Groß Schönebeck/Ortsgebiet: Friedenstraße, im Garten (2000, 2014: Haase) und Umg./Forst Gr. Schönebeck: Revier Rehluch Abt. 35 (2017: Haase)

\section{7 (10550) Phragmatobia fuliginosa (Linnaeus, 1758)}

Phänologie/Voltinismus: 22.IV.(2018) aFA, phänologische Verfrühung 4d gegenüber bis 2013.

Bivoltin; während mir Beobachtungen der 1. Gen. für 2014 nicht aus dem Gebiet vorlagen, wurde die seit den 1990er Jahren zunehmend regelmäßig beobachtete partielle 3. Gen. erneut 2014 E (23.)VIII bis M (15.)IX beobachtet. Auch im Jahre 2018 wurde vom 29.VIII.04.IX. eine 3. Gen. beobachtet.

\section{0 (10567) Spilosoma lubricipeda (LINNAEUS, 1758) = menthastri DENIS \& SCHIFFERMÜLLER, 1775}

Eine bemerkenswerte Form hatte Schwabe am 23.V.2016 in seinem Garten in Eberswalde a.L.: Der F hatte neben einigen schwarzen Punkten im Saumbereich schwarze Striemen an Costal- und Hinterrand der Vorderflügel sowie entlang der Discoidaladern der Zelle.

Phänologie/Voltinismus: Univoltin; 04.IX.(2014), aFE, phänologische Verspätung 5d gegenüber bis 2013. Hierbei handelt es sich mit Sicherheit um eine partielle 2. Gen., die in Jahren mit zeitigem warmem Frühjahr ab E VIII im Gebiet beobachtet wurde. Bereits SCHMIDT 1991 führte derartige Spätfunde für die ehemalige DDR an und rechnete sie einer partiellen 2. Gen. zu. Im südlichen Mitteleuropa tritt lubricipeda regulär bivoltin auf (Forster \& Wohlfahrt 1984; Freina \& Witt 1987; vgl. auch Ratzel \& Ratzel 1997 und Pro Natura SCHWEIZERISCher Bund FÜr NATURSChUtZ 2000).

\section{1 (10568) Spilosoma urticae (ESPER, 1789)}

- RL Deutschland (2011): Vorwarnliste.

Neue Nachweise von bekannten Fundorten:

c 3147/I Zerpenschleuse/Ortsgebiet: Berliner Straße (2018: Busse)

b 3247/II Biesenthal Umg./NSG Biesenthaler Becken: Langerönner Weg (2011: Ockruck)

g 3150/IV Altglietzen Umg./Gabower Berge (2015)

\section{3 (10579) Rhyparia purpurata (HüBNER, 1820)}

- Verordnung zur Neufassung der BArtschV von 2005: Besonders geschützte Art.

- RL Land Brandenburg: Kategorie 3 (gefährdet): TR, $\mathrm{CB}$;

- RL Deutschland (2011): Kategorie 3 (gefährdet). 
Die Art wird neuerdings zunehmend häufiger und verbreitet im Gebiet beobachtet. Am 11.VI. 2015 hatte ich auf den Oderhängen bei Gabow 12 F a.L. Busse registrierte in seinem Garten in Zerpenschleuse im Jahre 2015 vom 06.VI. bis 28.VI. insgesamt $26 \mathrm{~F}$ a.L.

Neue Nachweise von bekannten Fundorten:

b 3248/II Trampe/ehemaliger TÜP (2016: Busse \& Müller; 2018: Barthelme)

c 3147/I Zerpenschleuse/Ortsgebiet: Berliner Straße, im Garten (2014, 2015, 2016, 2018: Busse)

3148/III Ebw. OT Finow/Stadtgebiet: Altenhofer Straße, im Garten $(2015,2018)$

3149/III Stecherschleuse Umg./ehemaliges Kiesgrubengelände nördlich des Ortes (2018: Barthelme)

3149/IV Stecherschleuse Umg./ehemaliges Kiesgrubengelände nordöstlich des Ortes (2014: Schwabe)

g 3050/II Stolpe/Trockenhänge am Stadtweg (2018)

3150/III Gabow/Granitberggebiet (2015)

Fundorte (Ergänzung):

c 3147/I Marienwerder Umg./Oder-Havel-Kanal nordwestlich des Ortes (2015: Busse)

g 3149/IV Niederfinow Umg./Oderhänge - ehemalige Kiesgruben südwestlich des Schiffshebewerks (2015: Schwabe)

3150/I Oderberg/Geistberg (2017, 2018: Schwabe)

\section{4 (10583) Diacrisia sannio (Linnaeus, 1758)}

Phänologie Voltinismus: M (17.)VIII 2015 und A (07.) VIII 2018 wurde im Gebiet erneut die 2. Gen. beobachtet (vgl. Richert 2014).

Neue Nachweise von bekannten Fundorten:

b 3247/II Biesenthal Umg./NSG Biesenthaler Becken: Langerönner Weg (2014: Ockruck), nördlich der Pfauenwiesen (2015: Ockruck)

3248/II Trampe/ehemaliger TÜP (2014: Richert; 2016: Busse \& Müller)

c 3147/I Zerpenschleuse/Ortsgebiet: Berliner Straße, im Garten (2014, 2018: Busse)

3148/III Ebw. OT Finow/Stadtgebiet: Altenhofer Straße, im Garten (2014, 2015, 2016, 2018) und Umg./Forst Finowtal: Einflugschneise Flugplatz (2016)

3149/III Ebw. Umg./Finowbruch östlich des Eichwerder, an der Bahnlinie (2015)

3149/IV Stecherschleuse Umg./ehemaliges Kiesgrubengelände nordöstlich des Ortes (2015: Schwabe; 2018: Barthelme)

Fundorte (Ergänzung):

c 3146/IV Kreuzbruch Umg./ehemalige Römerwegbrücke am Oder-Havel-Kanal östlich des Ortes (2014: Busse) (westlich angrenzendes Nachbargebiet)

3149/III Stecherschleuse Umg./ehemaliges Kiesgrubengelände nördlich des Ortes (2017)

e 3049/I Groß Ziethen Umg./Steingruben an den Ihlowbergen $(2014,2016)$

\section{6 (10598) Arctia caja (LinnAeUs, 1758)}

- Verordnung zur Neufassung der BArtschV von 2005: Besonders geschützte Art.

- RL Land Brandenburg: Vorwarnliste: WS, GL, NM;

- RL Deutschland (2011): Vorwarnliste.

Neue Nachweise von bekannten Fundorten:

c 3147/I Zerpenschleuse/Ortsgebiet: Berliner Straße, im Garten (2014, 2015, 2016: Busse)

3148/III Ebw. OT Finow/Stadtgebiet: Altenhofer Straße, im Garten (2014, 2015, 2016, 2018)

g 3150/IV Altglietzen Umg./Gabower Berge (2015)

Fundorte (Ergänzung):

b 3247/II Biesenthal Umg./NSG Biesenthaler Becken: Nördlich der Pfauenwiesen (2015: Ockruck)

c 3147/IV Finowfurt Umg./Kesselmoor an der Finowfließniederung nördlich des Lehnssees (2016: Richert, Schwabe)

3148/IV Ebw./Stadtgebiet: Kleingartennalage im Schwärzetal nördlich Forstbotanischer Garten (2017: Schwabe)

e 3040/III Chorin Umg./Forst Chorin: Teerbrennerberge - Am Olberg (2013: MÜLLER 2015)

g 3050/II Gellmersdorf Umg./Gellmersdorfer Forst: Mühlenberggebiet (1994/1995: Borkowski)

3050/IV Lunow Umg./Gebiet Lunower Hölzchen (1994/1995 Borkowski)

h 3050/II Stolzenhagen Umg./Trockenpolder nördlich Pomeranzengraben (1994/1995: Borkowski)

\section{7 (10600) Arctia villica (Linnaeus, 1758)}

- Verordnung zur Neufassung der BArtschV von 2005: Streng geschützte Art.

- RL Land Brandenburg: Kategorie 1 (vom Aussterben bedroht); TR, HE;

- RL Deutschland (2011): Kategorie 2 (stark gefährdet).

Zwei neue Funde aus dem Ortsgebiet von Liepe wurden bekannt. Dort wurde der F 2013 und 2014 in einem Garten gefunden und gefilmt (Video: JusT, M. (2015): Die Lieper Tierwelt. Beobachtungen in einem märkischen Dorf).

Schwabe hatte am 10.VI.2017 2 Männchen in Oderberg a.L.

Neue Nachweise von bekannten Fundorten:

g 3149/II Liepe/Ortsgebiet: Im Garten (2013, 2014: Just)

Fundorte (Ergänzung):

g 3150/I Oderberg/Geistberg (2017: Schwabe)

\section{9 (10603) Callimorpha dominula (LinNAEUs,} 1758)

Auf dem ehemaligen TÜP Trampe wurde am 24.VI.2016 ein Massenanflug von $50 \mathrm{~F}$ a.L. registriert (Busse \& Müller i.l.).

Phänologie/Voltinismus: 31.V.(2018) aFA, phänologische Verfrühung 2d gegenüber bis 2013. 


\section{Neue Nachweise von bekannten Fundorten: \\ b 3248/II Trampe/ehemaliger TÜP (2016: Busse \& Müller; 2018: Barthelme) \\ c 3147/I Zerpenschleuse/Ortsgebiet: Berliner Straße, im Garten (2014, 2015, 2018: Busse) \\ 3148/III Ebw. OT Finow/Stadtgebiet: Altenhofer Straße, im Garten $(2014,2016,2018)$}

Fundorte (Ergänzung):

c 3147/IV Finowfurt Umg./Kesselmoor an der Finowfließniederung nordöstlich des Lehnssees (2017: Richert, Schwabe)

3148/IV Ebw./Stadtgebiet: Kleingartenanlage im Schwärzetal nördlich Forstbotanischer Garten (2016: Schwabe)

\section{0 (10607) Tyria jacobaeae (LinNAEUs, 1758)}

- RL Land Brandenburg: Kategorie 2 (stark gefährdet): TR.

Phänologie: Die R wurde im Gebiet in der Zeit vom 12.VI.(2018) bis 12.VIII.(2005) beobachtet.

\section{Neue Nachweise von bekannten Fundorten:}

b 3248/II Trampe/ehemaliger TÜP (2018: Barthelme)

e 3049/I Groß Ziethen Umg./Steingruben an den Ihlowbergen $(2014,2016)$

c 3149/I-II Stecherschleuse Umg./ehemaliges Kiesgrubengelände nördlich des Ortes: „Weidenbruch“ südlich der Tierversuchsstation am Oder-Havel-Kanal (2018: Kretschmer, Richert \& Seidel)

3149/III Stecherschleuse Umg./ehemaliges Kiesgrubengelände nördlich des Ortes (2017: Richert, 2018: Barthelme)

g 3150/I Oderberg/Geistberg (2015, 2017: Schwabe)

3150/III Gabow/Granitberggebiet (2015)

\section{Zu Teil III: Eulenfalter (Noctuidae et Pantheidae) (RICHERT 2003)}

\section{Acronictinae}

\section{1 (8772) Moma alpium (OsBeck, 1778)}

- RL Land Brandenburg: Kategorie 3 (gefährdet): EW. Phänologie/Voltinismus: In der Regel bisher univoltin; 13.VIII.(2014), aFE, ein noch relativ gut erhaltener F; phänologische Verspätung 20d gegenüber bis 2013. Berücksichtigt man die phänologische Verfrühung, die neuerdings bei M. alpium beobachtet wurde (RICHERT 2014), könnte dieser Fund als partielle 2. Gen. gedeutet werden.

Neue Nachweise von bekannten Fundorten:

c 3147/IV Marienwerder/Steinlaake (2015)

3147/II Finowfurt/Hirtenweg - Besters Fließ (1988, 1996, 1997: Kittel)

3148/III Ebw. OT Finow/Stadtgebiet: Altenhofer Straße, im Garten $(2014,2018)$

d 3047/III Groß Schönebeck/Ortsgebiet: Friedenstraße, im Garten (2006, 2014: Haase)

g 3149/II Liepe (1972: Kittel)

3150/I Oderberg/Teufelsberg (1975: Kittel)
Fundorte (Ergänzung):

c 3146/IV Kreuzbruch Umg./ehemalige Römerwegbrücke am Oder-Havel-Kanal östlich des Ortes (2014: Busse)

3147/I Zerpenschleuse/Ortsgebiet: Berliner Straße, im Garten (2018: Busse)

3147/IV Finowfurt Umg./Kesselmoor an der Finowfließniederung nördlich des Lehnssees (2016)

3148/IV Ebw./Stadtgebiet: Kleingartenanlage im Schwärzetal nördlich Forstbotanischer Garten (2016: Schwabe)

g 3150/I Oderberg/Geistberg (2017: Schwabe)

\section{2 (8774) Acronicta alni (Linnaeus, 1767)}

Neue Nachweise von bekannten Fundorten:

c 3147/I Zerpenschleuse/Ortsgebiet: Berliner Straße, im Garten (2015, 2017: Busse)

3147/II Finowfurt/Hirtenweg - Besters Fließ (1988, 19911993, 1995, 1996: Kittel)

3148/III Ebw. OT Finow/Stadtgebiet: Altenhofer Straße, im Garten $(2014,2015,2016,2017,2018)$

Fundorte (Ergänzung):

c 3147/IV Finowfurt Umg./Kesselmoor an der Finowfließniederung nordöstlich des Lehnssees (2017: Richert \& Schwabe)

3148/IV Ebw./Stadtgebiet: Kleingartenanlage im Schwärzetal nördlich Forstbotanischer Garten (2016: Schwabe)

g 3150/IV Altglietzen/Gabower Berge (2015)

\section{3 (8775) Acronicta cuspis (HüBNER, 1813)}

- RL Land Brandenburg: Kategorie 3 (gefährdet): MB;

- RL Deutschland (2011): Kategorie 3 (gefährdet).

Neue Nachweise von bekannten Fundorten:

c 3147/I Zerpenschleuse/Ortsgebiet: Berliner Straße, im Garten (2018: Busse)

3147/II Finowfurt/Hirtenweg - Besters Fließ (1986, 1989, 1990, 1991, 1994, 1995, 1997: Kittel)

3148/III Ebw. OT Finow/Stadtgebiet: Altenhofer Straße, im Garten $(2014,2015)$

d 3149/I Britz Umg./F. Britz (1973, 1974: Kittel)

g 3150/I Oderberg/Teufelsberg (1974: Kittel)

Fundorte (Ergänzung):

c $\quad 3147 / \mathrm{IV} \quad$ Finowfurt Umg./Kesselmoor an der Finowfließniederung nordöstlich des Lehnssees (2017: Schwabe)

\section{5 (8777) Acronicta psi (Linnaeus, 1758)}

Die R fand ich M (16.)VII.2014 in meinem Garten an Salix daphnoides, Reif-Weide. Weiterhin wurden $\mathrm{R}$ im Gebiet E (21.)VI.2017 an Prunus spinosa, Schlehe, und A (03.)X.2017 an Betula pendula, Hänge-Birke, gefunden (Foto Schwabe).

\section{6 (8778) Acronicta aceris (LinNAEUs, 1758)}

Acronicta aceris wurde im Gebiet nie in größerer Zahl beobachtet. Alle mir bekannten Fundorte werden nachgetragen. 
Tab. 1: Jährliche Anflugzahlen von Acronicta aceris beim regelmäßigen Hauslichtfang im Stadtgebiet von Finow von 19621974 (nach Aufzeichnungen von Duckert).

\begin{tabular}{|l|c|c|c|c|c|c|c|}
\hline Jahr & 1962 & 1963 & 1964 & 1965 & 1966 & 1967 & 1968 \\
\hline Anzahl & 6 & 9 & 1 & 7 & 9 & 15 & 6 \\
\hline Jahr & 1969 & 1970 & 1971 & 1972 & 1973 & 1974 & \\
Anzahl & 11 & - & 16 & 11 & 4 & 3 & \\
\cline { 1 - 6 }
\end{tabular}

Phänologie/Voltinismus: In der Regel wohl univoltin; Einzelfunde M August bis A September gehören offenbar $\mathrm{zu}$ einer partiellen 2. Gen., welche in günstigen Jahren mit warmem Frühjahr ausgebildet wird.

Im Jahre 2014 war das wieder der Fall: Der erste F im Jahr trat A (5.) V auf, der letzte wurde am 04.IX.(2014), aFE, registriert, phänologische Verspätung $13 \mathrm{~d}$ gegenüber bis 2013.

\section{Fundorte:}

a 3249/II Falkenberg Umg./Cöthener Forst: Ehemalige F. Tobbenberge (1971)

b 3148/IV Ebw. Umg./Oberheide an der Danckelmann-Str. (1962), F. Leuenberger Wiesen (1969)

3149/III Sommerfelde Umg./ehemaliger TÜP Panzerbahn (2003) (Richert 2006), Nordostrand des Hohenfinower Waldes (2003, 2004: Ockruck), Tornow Umg./Barnimhänge nördlich des Ortes (2010)

3247/II Biesenthal Umg./NSG Biesenthaler Becken: Langerönner Weg (2010: Ockruck)

3248/II Trampe/ehemaliger TÜP (2001: Ockruck; 2001, 2005: Richert) (RICHeRT 2006) und Umg./Tramper Forst: Bornemanns Pfuhl (2011: Schwabe)

c 3146/IV Kreuzbruch Umg./ehemalige Römerwegbrücke am Oder-Havel-Kanal östlich des Ortes (2012: Busse) (westlich angrenzendes Nachbargebiet)

3147/I Zerpenschleuse/Ortsgebiet (1980, 1981, 19871989: Ockruck; 1986, 2013, 2014, 2016, 2017, 2018: Busse) (Busse \& OCKRUCK 1991); Marienwerder Umg./Oder-Havel-Kanal nordwestlich des Ortes (2015: Busse)

3147/II Finowfurt Umg./Besters Fließ: Hirtenweg (1988, 1990, 1991, 1992, 1995: Kittel)

3148/I Finowfurt OT Steinfurt Umg./Oder-Havel-Kanal nordöstlich des Ortes (2000)

3148/II Lichterfelde Umg./Stromtrasse zwischen Wassertorbrücke am Oder-Havel-Kanal und Britzer Straße (2002)

3148/III Ebw. OT Finow/Stadtgebiet: Altenhofer Straße (1954), im Garten (1953, 1955, 1960-1962, 1966, $1969,1971,1974,1999,2000,2002,2003,2005-$ 2007, 2009-2012, 2014-2018: Richert); Siedlung Freie Scholle: Gartenstraße (1960, 1962-1969, 1971-1974: Duckert)

3148/IV Ebw. OT Finow/nordöstlicher Stadtrand zwischen Wolfswinkler Straße und dem ehemaligen Kraftwerk (1964), Ebw. Brandenburgisches Viertel (1997, 2000: Brauner) und Umg./Forst Finowtal: Stromtrasse südlich des Ortes am Forstort Sechsärmel (1998); Ebw.-Westend/am Kranbau (1954) und Unterheide: Wildparkstraße (1970, 1971, 1973: Kittel); Ebw./Stadtgebiet (1970: Kruel)
Groß Schönebeck/Ortsgebiet: Friedenstraße (1999, 2017: Haase)
3047/IV Groß Schönebeck Umg./Schorfheide: Pinnowseengebiet: Meelake (1991: Ockruck), Sarnow: Grahsee (1991: Ockruck) (NSG Kienhorst/Köllnseen/Eichheide, RicHERT 2010)

3048/III Jagdschloss Hubertusstock Umg./Engere Schorfheide: Kl. Kaisergrund (2006), Gr. Kaisergrund (2007) (NSG Kienhorst/Köllnseen/Eichheide, RICHERT 2010)

3147/II Eichhorst Umg./Üderheide am Weg nach Werbellin (2003)

3148/I Lichterfelde Umg./Straße nach Altenhof bei Gut Buckow (1979)

3148/II Britz/Ortsgebiet (1971: Kittel)

e 2948/IV Glambeck Umg./Straße nach Joachimsthal westlich F. Bärendikte (2004)

g 3050/II Stolpe/Trockenhänge (1987: Schottstädt); Gellmersdorf Umg./NSG Gellmersdorfer Forst: Buchsmühle (1990: Schottstädt); Stolzenhagen/ NSG Krähen- und Jungfernberge (1978, 1979, 1980, 2005) (RICHERT 1979)

3149/II Liepe (1987: Schottstädt)/Oderhänge: Sandberg (1973)

3150/I Oderberg (1962: Steinig)/NSG Pimpinellenberg (1968, 1988, 1989) (RICHERT 1994), Teufelsberg (1961, 1973, 1975, 1977, 1993: Richert; 1974. Kittel) (Richert 1994)

3150/III Gabow/Oderhänge (1991, 1992, 2004: Kleße), Granitberggebiet

3150/IV Altglietzen Umg./Oderhänge: Gabower Berge (1999: Busse \& Ockruck, 2003: Ockruck)

\section{8 (8780) Acronicta megacephala (DENIs \& SCHIFFERMÜLLER, 1775)}

Phänologie/Voltinismus: Die Art ist im Gebiet überwiegend univoltin, bildet aber jw eine partielle 2. Gen. aus, so auch im Jahre 2014: Nachdem der F vom 27.IV. bis 07.VIII. fast durchgängig registriert worden war, wurde nach einer Lücke von ca. fünf Wochen am 13.IX.(2014), aFE, ein F a.L. beobachtet, phänologische Verspätung 16d gegenüber bis 2013 .

\section{9 (8781) Acronicta strigosa (DENIS \& SCHIFFER- MÜLLER, 1775)}

- RL Land Brandenburg: Kategorie 1 (vom Aussterben bedroht): $\mathrm{HE}, \mathrm{MB}$;

- RL Deutschland (2011): Kategorie 2 (stark gefährdet).

Ältere Funde (Belege in coll. Kittel) werden ergänzend nachgetragen.

Neue Nachweise von bekannten Fundorten:

g 3149/II Liepe/Ortsgebiet (1973, 1975, 1977: Kittel)

3150/I Oderberg/Teufelsberg (1975, 1976: Kittel)

Fundorte (Ergänzung):

c 3148/IV Ebw.-Westend/Unterheide: Wildparkstraße (1973: Kittel) 


\section{1 (8783) Acronicta auricoma (DeNIs \& SCHIF- FERMÜLLER, 1775)}

\author{
Neue Nachweise von bekannten Fundorten: \\ c 3147/II Finowfurt/Hirtenweg - Besters Fließ (1991: Kittel) \\ 3148/III Ebw. OT Finow/Stadtgebiet: Altenhofer Straße, im \\ Garten (2014) \\ 3149/IV Stecherschleuse Umg./am Rande des ehemaligen \\ Kiesgrubengeländes nordöstlich des Ortes (2014: \\ Schwabe) \\ Fundorte (Ergänzung): \\ c 3146/IV Kreuzbruch Umg./ehemalige Römerwegbrücke am \\ Oder-Havel-Kanal östlich des Ortes (2014: Busse) \\ 3147/IV Finowfurt Umg./Kesselmoor an der Finowfließnie- \\ derung nordöstlich des Lehnssees (2016: Schwabe) \\ d 3047/III Groß Schönebeck Umg./Schorfheide: Revier Reh- \\ luch, Abt. 35 (2015: Haase) \\ g 3149/IV Niederfinow/Oderhänge - ehemalige Kiesgruben \\ südwestlich des Schiffshebewerks (1995: Kittel)
}

\section{3 (8787) Acronicta rumicis (Linnaeus, 1758)}

Als weitere Raupennahrungspflanze im Gebiet wurde Quercus petraea, Traubeneiche, festgestellt (Foto Schwabe).

Phänologie/Voltinismus: In der Regel bivoltin; im Jahre 2014 wurden von Mitte September bis Anfang Oktober $4 \mathrm{~F}$ a.L. registriert, die einer partiellen 3. Gen. zuzuordnen sind: 09.X.(2014), aFE, phänologische Verspätung $16 \mathrm{~d}$ gegenüber bis 2013 .

\section{4 (8789) Craniophora ligustri (DeNIs \& SCHIF- FERMÜLLER, 1775)}

Phänologie/Voltinismus: Bivoltin; 2. Gen.: 01.IX.(2014), aFE, phänologische Verspätung 6 d gegenüber bis 2013 .

Neue Nachweise von bekannten Fundorten:

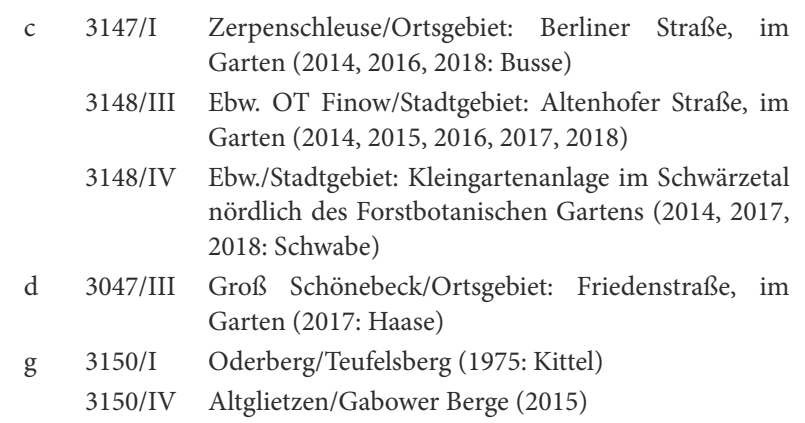

Fundorte (Ergänzung):

g 3150/I Oderberg/Geistberg (2015, 2017, 2018: Schwabe)

\section{6 (8793) Simyra albovenosa (GoEZE, 1781)}

- RL Land Brandenburg: Kategorie 3 (gefährdet): NM, $\mathrm{MB}, \mathrm{SM}$.
Neue Nachweise von bekannten Fundorten:

b 3247/II Biesenthal Umg./NSG Biesenthaler Becken: Weg nördlich der Pfauenwiesen (2014: Ockruck)

3248/II Trampe/ehemaliger TÜP (2016: Busse \& Müller)

c 3147/I Zerpenschleuse/Ortsgebiet: Berliner Straße, im Garten (2014, 2018: Busse)

3147/II Finowfurt/Hirtenweg - Besters Fließ (1991, 1995: Kittel)

3148/III Ebw. OT Finow/Stadtgebiet: Altenhofer Straße, im Garten (2014)

e 3049/I Groß Ziethen Umg./Steingruben an den Ihlowbergen (2014)

g 3149/II Liepe/Ortsgebiet (1974, 1976: Kittel)

3150/I Oderberg/Teufelsberg (1975: Kittel)

Fundorte (Ergänzung):

d 3047/III Groß Schönebeck/Ortsgebiet: Friedenstraße, im Garten (2014: Haase)

3150/I Oderberg/Geistberg (2016: Schwabe)

\section{Bryophilinae}

\section{8 (8801) Cryphia algae (FABRICIUS, 1775)}

Der F wurde am Tag auf einer Feuchtwiese aufgescheucht (Haase, pers. Mitt.).

Phänologie/Voltinismus: Univoltin; 24.VI.(2014), aFE, phänologische Verfrühung 17d gegenüber bis 2013.

Neue Nachweise von bekannten Fundorten:

c 3147/I Zerpenschleuse/Ortsgebiet: Berliner Straße, im Garten (2014, 2015: Busse)

3147/II Finowfurt/Hirtenweg - Besters Fließ (1993: Kittel)

3148/III Ebw. OT Finow/Stadtgebiet: Altenhofer Straße, im Garten $(2014,2015,2016,2017,2018)$

3148/IV Ebw.-Westend/Unterheide: Wildparkstraße (1973, 1974: Kittel)

g 3149/II Liepe/Ortsgebiet (1974: Kittel)

3149/IV Niederfinow/Oderhänge - ehemalige Kiesgruben südwestlich des Schiffshebewerks (2016: Schwabe)

3150/IV Altglietzen/Gabower Berge (2015)

Fundorte (Ergänzung):

c 3148/IV Ebw./Stadtgebiet: Kleingartenanlage im Schwärzetal nördlich Forstbotanischer Garten (2016: Schwabe)

d 3047/III Groß Schönebeck/Ortsgebiet: Friedenstraße, im Garten (2018: Haase) und Umg./Treptowsee (2015: Haase)

\section{9 (8810) Cryphia raptricula (DENIs \& SCHIF- FERMÜLLER, 1775) = divisa ESPER, 1791}

- RL Deutschland (2011): Vorwarnliste.

Ältere Funde (Belege in coll. Kittel) werden ergänzend nachgetragen.

Fundorte (Ergänzung):

c 3148/IV Ebw.-Westend/Unterheide: Wildparkstraße (1972, 1973: Kittel) 


\section{Herminiinae}

\section{2 (8839) Paracolax tristalis (FABRICIUS, 1794) = derivalis HüBNER, 1796}

- RL Land Brandenburg: Vorwarnliste: EW, WS. Phänologie/Voltinismus: 14.VI.(2018) aFA, phänologische Verfrühung 13d gegenüber bis 2013.

Neue Nachweise von bekannten Fundorten:

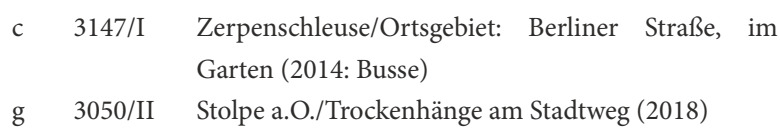

Fundorte (Ergänzung):

b 3247/II Biesenthal Umg./NSG Biesenthaler Becken: Weg nördlich der Pfauenwiesen (2014: Ockruck)

c 3147/IV Finowfurt Umg./Kesselmoor an der Finowfließniederung nordöstlich des Lehnssees (2016, 2017: Richert \& Schwabe)

d 3047/III Groß Schönebeck/Ortsgebiet: Friedenstraße, im Garten (2017: Haase)

3147/II Klandorf Umg./Forst Pechteich: Abt. 3118 (2016: Haase)

e 3049/I Groß Ziethen Umg./Steingruben an den Ihlowbergen (2014)

3149/II Liepe Umg./Forst Chorin: NSG Plagefenn - LedumMoor am Fuße der Plageberge (2018: Richert \& Schwabe)

g 3150/I Oderberg/Teufelsberg (1975: Kittel)

\section{3 (8843) Macrochilo cribrumalis (HüBNER, 1793)}

- RL Land Brandenburg: Kategorie 3 (gefährdet): NM, $\mathrm{MB}$.

Neue Nachweise von bekannten Fundorten:

c 3147/I Zerpenschleuse/Ortsgebiet: Berliner Straße, im Garten (2016: Busse)

3148/IV Ebw.-Westend/Unterheide: Wildparksstraße (1972: Kittel)

g 3149/II Liepe (1976, 1977: Kittel)

3150(I Oderberg/Teufelsberg (1974: Kittel)

Fundorte (Ergänzung):

c 3147/IV Finowfurt Umg./Kesselmoor an der Finowfließniederung nordöstlich des Lehnssees (2017: Schwabe)

3148/IV Ebw./Stadtgebiet: Kleingartenanlage im Schwärzetal nördlich Forstbotanischer Garten (2016: Schwabe)

\section{5 (8846) Herminia grisealis (DENIS \& SCHIF- FERMÜLLER, 1775) = nemoralis FABRICIUS, 1775}

Die Art kommt im Gebiet zwar verbreitet vor, wurde jedoch meist v, nur selten auch i.A. gefunden. Die mir bekannten Fundorte werden nachgetragen.

Phänologie/Voltinismus: In der Regel univoltin; in einzelnen Jahren, nach der Jahrtausendwende zunehmend regelmäßig, traten nach einer Lücke von etwa vier Wochen wieder frische $\mathrm{F}$ auf, die als partielle 2. Gen. zu deuten sind. Dies trifft auch auf das Jahr $2014 \mathrm{zu}$ : 05.VIII.2014 $1 \mathrm{~F}$ a.L.

Tab. 2: Jährliche Anflugzahlen von Herminia grisealis beim regelmäßigen Hauslichtfang im Stadtgebiet von Finow von 1962-1974 (nach Aufzeichnungen von Duckert).

\begin{tabular}{|l|c|c|c|c|c|c|c|}
\hline Jahr & 1962 & 1963 & 1964 & 1965 & 1966 & 1967 & 1968 \\
\hline Anzahl & - & 2 & 1 & - & 5 & 3 & 1 \\
\hline Jahr & 1969 & 1970 & 1971 & 1972 & 1973 & 1974 & \\
\cline { 1 - 6 } Anzahl & 4 & - & 2 & - & 3 & - & \\
\cline { 1 - 5 }
\end{tabular}

Fundorte:

a 3249/II Falkenberg Umg./Cöthener Forst: Ehemalige F. Tobbenberge (1970, 1971), F. Panekel (1985)

3250/I Bad Freienwalde Umg./Cöthener Forst: Hammertal nahe der Jugendherberge (2003: Kleße)

b 3148/IV Ebw./Oberheide: Institut für Waldschutz (1956, 1957: Kruel), Wiebecke-Damm am Möllergrab (2011: Schwabe)

3149/III Sommerfelde Umg./ehemaliger TÜP Panzerbahn (2001) (Richert 2006); Tornow Umg./Barnimhänge nördlich des Ortes: Abt. 630 (2008: Schwabe), Abt. 629 (2011: Schwabe)

3149/IV Struwenberg Umg./am Weg nach Karlswerk (1974)

3247/I Prenden Umg./Strehlesee: Südufer (2010: Ockruck, Rosenbauer)

3247/II Biesenthal Umg./NSG Biesenthaler Becken: Langerönner Weg (2014: Ockruck)

c 3147/I Zerpenschleuse/nordöstlicher Ortsrand an der Chaussee Liebenwalde-Ebw. (1981: Ockruck), Berliner Straße: Garten (2013, 2014, 2018: Busse) (Busse \& OCKRUCK 1992)

3147/II Finowfurt Umg./Besters Fließ: Hirtenweg (1991, 1992: Kittel)

3147/III Zerpenschleuse Umg./Nahe der Lottscher Brücke am Oder-Havel-Kanal (2000: Ockruck)

3147/IV Finowfurt Umg./Kesselmoor an der Finowfließniederung nordöstlich des Lehnssees (2016, 2017; Richert \& Schwabe)

3148/III Ebw. OT Finow/Stadtgebiet: Altenhofer Straße, im Garten (1961-1964, 1967, 1999, 2002, 2008, 2009, 2016, 2017, 2018: Richert); Gartenstraße in der Siedlung Freie Scholle (1963, 1964, 1966, 1967, 1968, 1969, 1971, 1973: Duckert) und Umg./Forst Finowtal: F. Schwärze (1963), 1. Melchower Weg nordöstlich vom Kl. Postluch (2004)

3148/IV Ebw.-Westend/Unterheide: Wildparkstraße (1971, 1975: Kittel), Ebw./Stadtgebiet (1960er Jahre: Friese; 1969: Kruel), Kleingartenanlage im Schwärzetal nördlich des Forstbotanischen Gartens (2014, 2016 Schwabe)

3149/I Ebw. Umg./Ragösetal bei Macherslust (1986); Britz Umg./Stadtseerinne westlich des Gr. Stadtsees (2013)

3149/III Ebw. Umg./Eichwerder (1979: Kruel); Sommerfelde Umg./Finowbruch nördlich des Ortes an der Bahnlinie (2012: Schwabe)

d 3047/IV Eichhorst Umg./Üderheide: Süßer Winkel am Werbellinsee (1980, 1982, 1985)

3048/I Joachimsthal (Schorfheide)/Werbellinsee (1999: Rosenbauer)

3048/III Hubertusstock Umg./Engere Schorfheide: Kl. Kaisergrund (2009) (NSG Kienhorst/Köllnseen/Eichheide, Richert 2010); Altenhof am Werbellinsee (1987: Schottstädt) 
3147/II Eichhorst Umg./Üderheide: Am Weg nach Werbellin (2003)

3148/I Werbellin Umg./NSG Buckowseerinne: Südlich der Autobahnanschlussstelle (2011); Lichterfelde Umg./ NSG Buckowseerinne: Streuobstwiesen südöstlich des Gr. Buckowsees (2012), Moorniederung nordöstlich vom Gr. Buckowsee (2010) (RICHERT 2012)

e 3049/I Grumsin Umg./NSG Grumsiner Forst: Waldstraße von Grumsin nach Louisenfelde westlich Langer Berg (2010)

3049/III Senftenhütte Umg./Forst Chorin: F. Senftenthal (1967)

3049/IV Brodowin Umg./Forst Chorin: Am Weg nach dem Plagefenn südlich der Mooskuten (2010)

3149/II Liepe Umg./Forst Chorin: F. Liepe (1964) (STÖCKEL 1955), NSG Plagefenn (2001, 2002: Richert; 2018: Richert \& Schwabe) (STÖCKel 1955; RicherT 2005);

f 3149/II Brodowin Umg./Vorwerk Zaun (1964)

g 3149/II Liepe (1973: Kittel; 1987: Schottstädt)

3149/IV Struwenberg Umg./NSG Kanonen- und Schlossberg (1998)

3050/II Stolpe/Trockenhänge (1987: Schottstädt); Gellmersdorf Umg./NSG Gellmersdorfer Forst: Buchsmühle (1990: Schottstädt); Stolzenhagen/NSG Krähenund Jungfernberge (1978, 1979) (RICHERT 1979)

3150/I Oderberg (1960, 1961: Steinig)/NSG Pimpinellenberg (1961: Urbahn; 1988: Richert) (RICHERT 1994), Teufelsberg (1975: Kittel; 1993: Richert) (RICHERT 1994), Geistberg (2018: Schwabe)

3150/III Gabow/Oderhänge (1991: Kleße)

\section{9 (8858) Zanclognatha tarsipennalis (TREITSCHKe, 1835)}

Phänologie/Voltinismus: Im Jahre 2014 wurde am 04.IX. ein F einer partiellen 2. Gen. beobachtet (vgl. RicherT 2014). Auch 2016 wurden F M (13.) bis E (23.) VIII beobachtet.

\section{Strepsimaninae (Hypenodinae)}

\section{0 (8863) Hypenodes humidalis (DoubledAY, 1850) = turfosalis WOCKE, 1850}

- RL Land Brandenburg: Kategorie 3 (gefährdet): SM, NM;

- RL Deutschland 2011: Kategorie 3 (gefährdet).

Ein vermutetes Vorkommen in einem Kesselmoor an der Finowfließniederung nördlich des Lehnssees konnte trotz gezielter zweijähriger Untersuchungen dieses Habitats nicht bestätigt werden.

Dagegen liegen neue Nachweise aus dem NSG Plagefenn im Forst Chorin vor.

\section{Neue Nachweise von bekannten Fundorten:}

e 3149/II Liepe Umg./Forst Chorin: NSG Plagefenn - LedumMoor am Fuße der Plageberge (2018: Richert \& Schwabe)

\section{1 (8866) Schrankia costaestrigalis (STEPHENS, 1834)}

- RL. Land Brandenburg: Kategorie 3 (gefährdet): NM, SM.

Die Beobachtungslücke im Naturraum a (Oberbarnimer Waldhügel, vgl. RICHERT 2003) wurde geschlossen: Ein F wurde bei Falkenberg im Cöthener Forst am Rand eines Quellmoores am Klingenden Fließ a.K. gefangen. Ein weiterer Nachweis gelang im Schwärzetal im Eberswalder Stadtgebiet: 2 F a.L. leg. Schwabe.

Phänologie/Voltinismus: Bisher als univoltin beurteilt; 24.VI.(2016), aFA, 1 F leg. Schwabe: Damit liegt erstmals ein Beleg für ein bivoltines Auftreten der Art im Gebiet vor. Der Fund ist der 1. Gen. zuzurechnen; alle übrigen bisher bekannten Funde gehören demnach zur 2. Gen., die im Gebiet offensichtlich häufiger ist als die 1. Gen.

2. Gen.: 06.X.(2014), aFE, phänologische Verspätung 21d gegenüber bis 2013 .

Fundorte (Ergänzung):

a 3249/II Falkenberg Umg./Cöthener Forst: Klingendes Fließ unterhalb des Bismarck-Turmes (2014)

c 3148/IV Ebw./Stadtgebiet: Kleingartenanlage im Schwärzetal nördlich des Forstbotanischen Gartens (2014, 2016 : Schwabe)

\section{Catocalinae}

\section{3 (8871) Catocala sponsa (LinnAEUs, 1767)}

Phänologie/Voltinismus: 14.X.2016 aFE, phänologische Verspätung 15d gegenüber bis 2013.

Neue Nachweise von bekannten Fundorten:

c 3147/I Zerpenschleuse/Ortsgebiet: Berliner Straße, im Garten (2014, 2016: Busse)

3148/III Ebw. OT Finow/Stadtgebiet: Altenhofer Straße, im Garten $(2016,2018)$

3148/IV Ebw.-Westend/Unterheide: Wildparkstraße (1974: Kittel)

\section{4 (8873) Catocala fraxini (Linnaeus, 1758)}

- Verordnung zur Neufassung der BArtschV von 2005: Besonders geschützte Art.

- RL Deutschland (2011): Vorwarnliste.

Neue Nachweise von bekannten Fundorten:

c 3147/I Zerpenschleuse/Ortsgebiet: Berliner Straße, im Garten (2015, 2016, 2018: Busse); Marienwerder Umg./Oder-Havel-Kanal nordwestlich des Ortes (2014: Busse)

Fundorte (Ergänzung):

c 3147/II Finowfurt/Hirtenweg - Besters Fließ (1986: Kittel)

3148/IV Ebw./Stadtgebiet: Kleingartenanlage im Schwärzetal nördlich Forstbotanischer Garten (2017: Schwabe) 
d 3047/III Groß Schönebeck/Ortsgebiet: Friedenstraße, im Garten (2017: Haase)

g 3149/IV Niederfinow/Oderhänge - ehemalige Kiesgruben südwestlich des Schiffshebewerks (2015: Schwabe)

\section{5 (8874) Catocala nupta (LinnAeus, 1967)}

- Verordnung zur Neufassung der BArtschV von 2005: Besonders geschützte Art.

Die Art ist in allen Naturräumen des Gebietes nachgewiesen. Die mir bekannten Fundorte werden nachgetragen (vgl. Richert 2003 und 2014).

Die Daten von Duckert spiegeln zwar sogenannte „Häufigkeitsjahre" wider, vermitteln jedoch insofern ein einseitiges Bild, da sie nur den Lichtanflug berücksichtigen. Die Art ist oft häufiger a.K. zu beobachten.

Tab. 3: Jährliche Anflugzahlen von Catocala nupta beim regelmäßigen Hauslichtfang im Stadtgebiet von Finow von 19621974 (nach Aufzeichnungen von Duckert).

\begin{tabular}{|c|c|c|c|c|c|c|c|}
\hline Jahr & 1962 & 1963 & 1964 & 1965 & 1966 & 1967 & 1968 \\
\hline Anzahl & 1 & 2 & 9 & 7 & 6 & 6 & 22 \\
\hline Jahr & 1969 & 1970 & 1971 & 1972 & 1973 & 1974 & \\
\hline Anzahl & 21 & 32 & 6 & 5 & 12 & 3 & \\
\hline
\end{tabular}

Fundorte:

a 3249/II Falkenberg Umg./Cöthener Forst: Ehemalige F. Tobbenberge (2012)

b 3148/IV Ebw.-Ostende/Bruno H. Bürgel-Schule $(1985,1986)$

3149/III Sommerfelde Umg. (2004: Ockruck)/ehemaliger TÜP Panzerbahn (2003) (RICHERT 2006), Hohenfinower Wald: Nordostrand (2004: Ockruck); Tornow Umg./Barnimhänge nördlich des Ortes (2000: Ockruck), Abt.: 629 (2011: Schwabe), Bachtal (2011: Schwabe)

3149/IV Hohenfinow/Ortsgebiet (1986; RICHERT \& HÜBERT 1991) und Umg./Karlswerker Straße oberhalb (südlich) Karlswerk (2011)

3247/I Prenden Umg./Strehle-See: Östlicher Uferweg (2010: Ockruck)

3247/II Biesenthal Umg./NSG Biesenthaler Becken: Langerönner Weg (2009: Ockruck)

3249/I Gerdorf Umg./Gamengrund (2017)

c 3147/I Zerpenschleuse/Ortsgebiet (1980, 1983-1985, 1987: Ockruck; 2011, 2014, 2016, 2017: Busse) und Umg./ Straße nach Liebenwalde (1997: Ockruck) (Busse \& OCKRUCK 1991)

3147/II Finowfurt/Hirtenweg - Besters Fließ (1986, 1987, 1993, 1995: Kittel); Marienwerder Umg./F. Pechteich (1950-1959: Haeger)

3147/III Zerpenschleuse Umg./Oder-Havel-Kanal nahe der Lottscher Brücke (1997: Ockruck); Marienwerder/ Auwinkel zum Oder-Havel-Kanal (2010: Ockruck); Ruhlsdorf Umg. (1999: Ockruck)

3147/IV Finowfurt Umg./Kesselmoor an der Finowfließniederung nördlich des Lehnssees (2016)

3148/I Finowfurt OT Steinfurt Umg./Kiesgrube westlich der Barschgrube $(1995,1997)$
3148/III Ebw. OT Finow/Stadtgebiet (1983, 1995): Altenhofer Straße, im Garten (1953, 1956, 1958-1964, 1967 1968, 1972, 1975, 1978, 1980, 1983-1986, 1995, 1999 , 2000, 2003-2005, 2007, 2011-2015, 2017), Finowkanal (2008), Gartenstraße in der Siedlung Freie Scholle (1962-1974: Duckert)

3148/IV Ebw.-Westend/Unterheide: Wildparkstraße (1968, 1973, 1977: Kittel), Ebw./Stadtgebiet (1905: Jonas; 1983: Rinnhofer), Kleingartenanlage im Schwärzetal nördlich Forstbotanischer Garten (2016, 2017 Schwabe); Ebw.-Nordend (1983: Kittel)

3149/II Niederfinow Umg./ehemaliges Kiesgrubengelände östlich der Klosterbrücke (2001: Riegel)

3149/III Ebw./Eichwerder (1986: Riegel)

d 3047/III Groß Schönebeck Umg./Schorfheide: Joachimsthaler Damm westlich der Pinnowseen (2004) (NSG Kienhorst/Köllnseen/Eichheide; RICHERT 2010a)

3047/IV Jagdschloss Hubertusstock/Engere Schorfheide: Westliche nahe Umgebung (2003) (NSG Kienhorst/ Köllnseen/Eichheide; RICHERT 2010a)

3147/II Eichhorst Umg./Moospfuhl östlich der Straße nach Finowfurt (1969)

3148/I Werbellin Umg./NSG Buckowseerinne: Südlich der Autobahnanschlussstelle (2011); Lichterfelde Umg./ NSG Buckowseerinne: Moorniederung nordöstlich des Gr. Buckowsees (2010) (RicherT 2012)

3148/II Lichterfelde Umg./am Weg nach Gut Blütenberg (1954)

3149/I Britz Umg./F. Britz (1973: Kittel)

e 2948/IV Glambeck Umg./Waldstraße nach Joachimsthal westlich F. Bärendikte (2004)

3049/I Grumsin (1973: Kittel); Groß Ziethen Umg./zwischen Schulzensee und Kl. Kagelpfuhl nordwestlich des Ortes bei Sperlingsherberge (2008: Rödel \& Schulze)

3049/IV Brodowin Umg./Forst Chorin: Große Mooskute am Dengler-Weg (1989) (Richert 2005)

3149/II Liepe Umg./Forst Chorin: F. Liepe (1964: Richert; 1986: Riegel), NSG Plagefenn (2003) (RICHERT 2005)

f 3049/IV Brodowin Umg./Kl. Rummelsberg (1986)

g 3050/II Stolzenhagen/NSG Krähen- und Jungfernberge (1978: Kruel; 1979, 1980, 1983, 2003: Richert) (RICHERT 1979)

3149/IV Struwenberg Umg./NSG Kanonen- und Schlossberg (1999)

3150/I Oderberg/NSG Pimpinellenberg (1968, 2011) (RICHERT 1994)

3150/III Gabow/Gabower Berge (2008: Rosenbauer)

3150/IV Altglietzen Umg./Gabower Berge (2007)

h 3149/II Liepe Umg./Niederoderbruch: Schöpfwerk Liepe (1973)

3150/III Bralitz/stillgelegte Kiesgrube (2009); Neuenhagen Umg./ehemaliges Kiesgrubengelände östlich der Straße nach Oderberg (2011: Ockruck)

\section{6 (8877) Catocala elocata (VIEWEg, 1790)}

- Verordnung zur Neufassung der BArtschV von 2005: Besonders geschützte Art.

- RL Land Brandenburg: Kategorie 2 (stark gefährdet): GL, FA, SO;

- RL Deutschland (2011): Kategorie 2 (stark gefährdet). 
Neue Nachweise von bekannten Fundorten:

c 3148/III Ebw. OT Finow/Stadtgebiet: Altenhofer Straße, im Garten (2018)

3148/IV Ebw./Stadtgebiet: Ruhlaer Straße (2015: Schwabe)

\section{0 (8932) Lygephila pastinum (TREITSCHKE, 1826)}

Phänologie/Voltinismus: Univoltin; im Jahre 2014 wurde nach einer regulären Flugzeit von E VI bis E VII ungewöhnlich spät ein sehr abgeflogener $\mathrm{F}$ am 20.IX a.L. beobachtet. Auch 2016 wurde bei einem Flugzeitbeginn E VI noch am 10.IX. ein F a.L. registriert. Unklar ist, ob es sich um Nachzügler handelt. Diese und ältere derartige Funde (vgl. Richert 2014) weisen wohl eher auf eine partielle 2. Gen. hin.

07.VI.(2018) aFA, phänologische Verfrühung 4d gegenüber bis 2013 .

\section{Neue Nachweise von bekannten Fundorten:}

c 3147/I Zerpenschleuse/Ortsgebiet: Berliner Straße, im Garten (2014, 2015: Busse)

3147/II Finowfurt/Hirtenweg - Besters Fließ (1995: Kittel)

3148/III Ebw. OT Finow/Stadtgebiet: Altenhofer Straße, im Garten $(2014,2017)$

3149/IV Stecherschleuse Umg./ehemaliges Kiesgrubengelände nordöstlich des Ortes (2015: Schwabe)

d 3047/III Groß Schönebeck/Ortsgebiet: Friedenstraße, im Garten (1999, 2018: Haase)

g 3050/II Stolpe a.O./Trockenhänge am Stadtweg (2018)

3150/I Oderberg/Teufelsberg (1975: Kittel)

3150/III Gabow/Granitberggebiet (2015: Schwabe)

Fundorte (Ergänzung):

c 3147/I Marienwerder Umg./Oder-Havel-Kanal nordwestlich des Ortes (2016: Busse)

3148/IV Ebw.-Nordend/Gelände der Landesklinik (1983: Kittel)

3149/III Stecherschleuse Umg./ehemaliges Kiesgrubengelände nördlich des Ortes (2017)

\section{1 (8933) Lygephila viciae (HüBNER, 1822)}

- RL Land Brandenburg: Kategorie 2 (stark gefährdet): WS;

- RL Deutschland (2011): Kategorie 3 (gefährdet).

Neue Nachweise von bekannten Fundorten:

c 3147/II Finowfurt/Hirtenweg - Besters Fließ (1986, 1988: Kittel)

3148/III Ebw. OT Finow/Stadtgebiet: Altenhofer Straße, im Garten $(2014,2017)$

g 3149/II Liepe/Ortsgebiet: Schule (1973: Kittel)

3149/IV Niederfinow/Oderhänge - ehemalige Kiesgruben südwestlich des Schiffshebewerks (2015: Schwabe)

3150/I Oderberg/Teufelsberg (1974, 1975, 1976, 1978: Kittel)

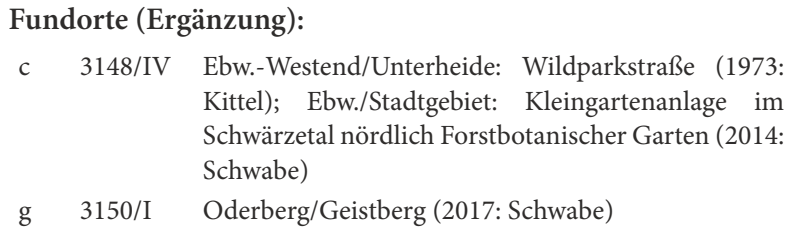

\section{2 (8934) Lygephila craccae (DeNis \& SCHIFFER- MÜLLER, 1775)}

- RL Land Brandenburg: Kategorie 1 (vom Aussterben bedroht): WS - TR;

- RL Deutschland (2011): Kategorie 3 (gefährdet).

Neue Nachweise von bekannten Fundorten:

g 3149/IV Niederfinow/Oderhänge - ehemalige Kiesgruben südwestlich des Schiffshebewerks (2015: Schwabe)

3150/I Oderberg/Teufelsberg (1973, 1974: Kittel)

Fundorte (Ergänzung):

g 3150/IV Altglietzen/Gabower Berge (2015)

\section{4 (8958) Aedia funesta (ESPER, 1786)}

- RL Land Brandenburg: Kategorie R (extrem seltene Art/Art mit geographischer Restriktion): GL, FA.

A (05.) VIII 2017 klopfte ich in meinem Garten in Finow nachts $5 \mathrm{R}$ in unterschiedlichen Stadien aus einem Geschlinge von Calystegia sepium, Gewöhnliche Zaunwinde. Klopfversuche am Tag waren zunächst vergeblich. Wenige Tage später klopfte ich dort aber auch bei Tage zwei weitere R. Bei der mit diesen Tieren durchgeführten Zucht schlüften nur zwei F A VI 2018. Die Puppen waren offenbar zu trocken aufbewahrt worden.

Tab. 4: Anflugzahlen und -zeiten von Aedia funesta beim Hauslichtfang in meinem Garten in Finow ab 2014.

\begin{tabular}{|c|c|c|c|c|c|}
\hline Jahr & 2014 & 2015 & 2016 & 2017 & 2018 \\
\hline Anzahl & 44 & 28 & 14 & 19 & 33 \\
\hline Flugzeit & $\begin{array}{c}25 . \mathrm{V} \\
-20 . \mathrm{VII} .\end{array}$ & $\begin{array}{c}25 . \mathrm{V} . \\
-07 . \mathrm{VIII} .\end{array}$ & $\begin{array}{c}\text { 14.VI. } \\
-10 . \text { VII. }\end{array}$ & $\begin{array}{c}\text { 18.V. } \\
-19 . \text { VII. }\end{array}$ & $\begin{array}{c}\text { 28.V. } \\
-13 . \mathrm{VII}\end{array}$ \\
\hline
\end{tabular}

Phänologie/Voltinismus: In coll. Kittel fand ich einen F mit den Daten 26.VIII.1972, Oderberg/Teufelsberg, leg. Kittel. Damit liegt für den Zeitraum vor 2000 ein extrem spätes Datum für das absolute Flugzeitende vor (vgl. Richert 2014). Die Flugzeit begann 1972 nach meinen Daten A (07.)VI und endete E (22.)VII. Ob der zeitlich separate Spätfund als Nachzügler einzuordnen ist oder $\mathrm{ob}$ es sich damals bereits um einen $\mathrm{F}$ einer partiellen 2. Gen. handelte, lässt sich nicht sicher entscheiden.

Neue Nachweise von bekannten Fundorten:

\footnotetext{
c 3147/I Zerpenschleuse/Ortsgebiet: Berliner Straße, im Garten 50 (2014-2018: Busse)

3148/III Ebw. OT Finow/Stadtgebiet: Altenhofer Straße, im Garten (2014-2018)

d 3047/III Groß Schönebeck/Ortsgebiet: Friedenstraße, im Garten (2015, 2017: Haase)
} 
g 3149/II Liepe (1972, 1974: Kittel)

3150/I Oderberg/Teufelsberg (1971, 1972, 1975: Kittel)

3150/IV Altglietzen/Gabower Berge (2015: Richert, Schwabe)

Fundorte (Ergänzung):

c 3148/IV Ebw.-Westend/Unterheide: Wildparkstraße (1970, 1973, 1975, 1976: Kittel), Ebw./Stadtgebiet: Kleingartenanlage im Schwärzetal nördlich Forstbotanischer Garten (2014, 2016: Schwabe)

g 3150/I Oderberg/Geistberg $(2017,2018:$ Schwabe $)$

335 (8965) Tyta luctuosa (DENIS \& SCHIFFERMüLLER, 1775)

- RL Land Brandenburg: Vorwarnliste; TR.

Neue Nachweise von bekannten Fundorten:

g 3149/II Liepe/Ortsgebiet: Schule (1973, 1974, 1976, 1977: Kittel)

3150/I Oderberg/Teufelsberg (1973: Kittel), Geistberg (2018: Schwabe)

3150/III Gabow/Granitberggebiet (2015: Richert, Schwabe)

3150/IV Altglietzen/Gabower Berge (2015: Richert, Schwabe)

Fundorte (Ergänzung):

c 3147/II Finowfurt/Hirtenweg - Besters Fließ (1993: Kittel)

3148/IV Ebw.-Westend/Unterheide: Wildparkstraße (1976: Kittel),

\section{6 (8967) Callistege mi (CLERCK, 1759)}

Der Falter wurde am Tag beim Saugen an Blüten von Senecio vernalis, Frühlings-Greiskraut, beobachtet.

Raupen der 1. Gen. wurden E VIII. gefunden: Ich fand eine $\mathrm{R}$ am 29.VIII.2017 nachts in einem Moor, wo sie in einem Pfeifengrasbestand an einem Grasstängel ruhte. Schwabe fotografierte eine im Gras ruhende $\mathrm{R}$ am 25.VIII. am Kanaldamm des Oder-Havel-Kanals.

Phänologie/Voltinismus: In der Regel univoltin: 22.IV. (2014), aFA, phänologische Verfrühung $8 d$ gegenüber bis 2013.

\section{7 (8969) Euclicia glyphica (LinNAEus, 1758)}

Als weitere Nektarsaugpflanzen im Gebiet wurden Leucanthemum vulgare, Wiesen-Margerite; Ligustrum vulgare, Gewöhnlicher Liguster; und Trifolium repens, Weiß-Klee, festgestellt.

\section{8 (8975) Laspeyria flexula (DeNis \& SCHIFFER- MÜLLER, 1775)}

Schwabe hatte am 07.IX.2016 eine bemerkenswerte melanistische Form mit schwarzem Wurzel- und Saumfeld in seinem Garten in Ebw. a.L.

Phänologie/Voltinismus: Seit 1999 zunehmend regelmäBig bivoltin, so auch 2014-2018.

2. Gen: 16.X.2014, 1 F a.L., phänologische Verspätung 7d
20.X.(2017), aFE, $1 \mathrm{~F}$ a.L. (Busse i.l.) erneute phänologische Verspätung 4d, insgesamt also 11d gegenüber bis 2013.

\section{Calpinae}

\section{9 (8984) Scoliopteryx libatrix (LinnAEUS, 1758)}

Phänologie/Voltinismus: Bivoltin; im Jahre 2014 wurden erste aktive F bereits Mitte März a.L. beobachtet: 13.III. (2014), aFA, phänologische Verfrühung 12d gegenüber bis 2013 .

\section{Hypeninae}

340 (8994) Hypena proboscidalis (LINNAEUs, 1758)

Der F wurde am Tag aus der Saumvegetation an Gebüschund Waldrändern sowie auf Sumpfwiesen und in Gärten aufgescheucht. Nachts erscheint er regelmäßig a.L., oft auch a.K.

Blütenbesuch wurde an Impatiens parviflora, Kleines Springkraut, beobachtet (MüLLER 2015).

\section{2 (9002) Hypena crassalis (FABRICIUS, 1787) = fontis THUNBERG, 1788}

Neue Nachweise von bekannten Fundorten:

b 3248/II Trampe/ehemaliger TÜP (2016: Busse \& Müller)

c 3146/IV Kreuzbruch Umg./ehemalige Römerweg-Brücke am Oder-Havel-Kanal östlich des Ortes (2014: Busse) (westlich angrenzendes Nachbargebiet)

3148/III Ebw. OT Finow/Stadtgebiet: Altenhofer Straße, im Garten $(2014,2015)$

e 3149/II Liepe Umg./Forst Chorin: NSG Plagefenn - LedumMoor am Fuß der Plageberge (2018: Richert \& Schwabe)

Fundorte (Ergänzung):

c 3147/IV Finowfurt Umg./Kesselmoor an der Finowfließniederung nordöstlich des Lehnssees $(2016,2017)$

3148/IV Ebw.-Westend/Unterheide: Wildparkstraße (1973, 1975, 1976: Kittel)

d 3047/III Groß Schönebeck/Ortsgebiet: Friedenstraße, im Garten (2017: Haase)

g 3150/I Oderberg/Geistberg (2018: Schwabe)

\section{3 (9006) Phytometra viridaria (CLERCK, 1759)}

- RL Land Brandenburg: Kategorie 1 (vom Aussterben bedroht); TR, MW;

- RL Deutschland (1998): Vorwarnliste; (2011): Kategorie 3 (gefährdet).

Bisher nicht berücksichtigte ältere Funde (Belege befanden sich in coll. Kittel) werden ergänzend mitgeteilt. Zwei dieser F vom 08.V.1971 waren etikettiert mit der Fundortbezeichnung „Ebw. Wiese Schwärze“. Für eine Quadrantenzuordnung ist die Fundortangabe zu ungenau. Zwei weitere Exemplare (22.VII.1960; 31.V.1964) hatte Kittel aus coll. Duckert übernommen. 
Neue Nachweise von bekannten Fundorten:

c 3148/III Ebw. OT Finow/Stadtgebiet: Siedlung Freie Scholle (1960, 1964: Duckert; Belege in coll. Kittel)

Fundorte (Ergänzung):

c 3148/IV Ebw.-Westend/Unterheide: Wildparkstraße (1973: Kittel)

3148 „Ebw. Wiese Schwärze“ (1971: Kittel)

\section{5 (9016) Parascotia fuliginaria (Linnaeus, 1761)}

Phänologie/Voltinismus: 05.VI.(2018) aFA, phänologische Verfrühung 1d gegenüber bis 2013.

Neue Nachweise von bekannten Fundorten:

c 3147/I Zerpenschleuse/Ortsgebiet: Berliner Straße, im Garten (2014, 2015, 2018: Busse)

3148/III Ebw. OT Finow/Stadtgebiet: Altenhofer Straße, im Garten (2014)

Fundorte (Ergänzung):

b 3249/I Gersdorf Umg./Gamengrund südlich Teufelssee (2017: Schwabe)

c 3147/IV Finowfurt Umg./Kesselmoor an der Finowfließniederung nordöstlich des Lehnssees (2016)

3148/IV Ebw.-Westend/Wildparkstraße (1973，1974，1975, 1979: Kittel)

e 3049/I Groß Ziethen Umg./Steingruben an den Ihlowbergen (2014)

\section{6 (9018) Colobochyla salicalis (DENIs \& SCHIF-} FERMÜLLER, 1775)

Die Beobachtungslücke im Naturraum e (Uckermärker Kuppen- und Hügelland einschließlich Choriner Waldhügel, Richert 2003) konnte inzwischen geschlossen werden.

Neue Nachweise von bekannten Fundorten:

c 3147/I Zerpenschleuse/Ortsgebiet: Berliner Straße, im Garten (2014, 2018: Busse)

3147/II Finowfurt/Hirtenweg - Besters Fließ (1995: Kittel)

3148/III Ebw. OT Finow/Stadtgebiet: Altenhofer Straße, im Garten $(2015,2016,2018)$

3148/IV Ebw.-Westend/Unterheide: Wildparkstraße (19731976: Kittel)

3149/III Ebw./Eichwerder (1972, 1976: Kittel)

Fundorte (Ergänzung):

c 3147/I Marienwerder Umg./Oder-Havel-Kanal nordwestlich des Ortes (2015, 2016: Busse)

3148/IV Ebw.-Nordend/Gelände der Landesklinik (1983: Kittel); Ebw./Stadtgebiet: Kleingartenanlage im Schwärzetal nördlich Forstbotanischer Garten (2016, 2018: Schwabe)

d 3047/III Groß Schönebeck/Ortsgebiet: Friedenstraße, im Garten (2015: Haase)

e 3049/I Groß Ziethen Umg./Steingruben an den Ihlowbergen (2014)

g 3050/I Oderberg/Geistberg (2017, 2018: Schwabe)

3150/IV Altglietzen/Gabower Berge (2015)

\section{Plusiinae}

347 (9036) Polychrysia moneta (FABRICIUS, 1787)

- RL Land Brandenburg: Kategorie 3 (gefährdet): GL. Ein älterer Fund (Beleg in coll. Kittel) wird ergänzend nachgetragen.

Neue Nachweise von bekannten Fundorten:

c 3148/IV Ebw.-Westend/Unterheide: Wildparkstraße (1973: Kittel)

\section{9/350 Diachrysia chrysitis-stenochrysis-Komplex}

Der Untersuchungen zum Status der beiden „Schwester-Arten" sind noch in vollem Gange (STEINER et al. 2014). Ergebnisse genetischer und pheromonbiologischer Untersuchungen stehen (zum Teil? Anm. Verf.) im Widerspruch zu genitalmorphologisch und habituell festgestellten Unterschieden. Wahrscheinlich handelt es sich um Arten in statu nascendi.

In Ermangelung anderer Möglichkeiten wird die Sortierung nach habituellen Unterschieden (RICHERT 2010) hier fortgesetzt, obwohl fraglich erscheint, ob das sinnvoll ist.

\section{9 (9045) Diachrysia chrysitis (Linnaeus, 1758)}

(Die Angaben beziehen sich auf Exemplare mit vollständig getrennten Messingglanz-Feldern im Saum- und Wurzelfeld).

Ältere bisher nicht berücksichtigte Funde (Belege aus coll. Kittel lagen mir vor) werden ergänzend mitgeteilt.

Phänologie/Voltinismus: Bivoltin, Generationen in den einzelnen Jahren deutlich getrennt.

1. Gen.: 21.V.(2014), aFA, phänologische Verfrühung 1d gegenüber bis 2013;

2. Gen.: 15.IX.(2014), aFE, phänologische Verspätung 7d gegenüber bis 2013 .

Neue Nachweise von bekannten Fundorten:

c 3148/III Ebw. OT Finow/Stadtgebiet: Altenhofer Straße, im Garten (2014, 2015, 2016, 2017, 2018)

g 3150/IV Altglietzen Umg./Gabower Berge (2015)

Fundorte (Ergänzung):

c 3147/II Finowfurt/Hirtenweg - Besters Fließ (1986, 1995: Kittel)

3148/IV Ebw.-Westend/Wildparkstraße (1976: Kittel); Ebw./ Stadtgebiet/Kleingartenanlage im Schwärzetal nördlich Forstbotanischer Garten (2016: Schwabe)

e 3049/I Groß Ziethen Umg./Steingruben an den Ihlowbergen (2014)

f 3049/IV Brodowin/Krugberg (2014) und Umg./Karpatenweg östlich des Krugbergs (2015) 


\section{0 (9046) Diachrysia stenochrysis (WARREN, 1913)}

Übergangsformen zu chrysitis mit schmalem/strichförmigem Steg zwischen den messingfarbenen Bändern auf den Vdfl. wurden sowohl im Stadtgebiet von Finow (in meinem Garten) und Ebw. (Belege in coll. Kittel) als auch auf dem Krugberg in Brodowin sowie in der Steinlaake bei Marienwerder und in Liepe (Belege in coll. Kittel) a.L. beobachtet.

Hier werden nur Daten von Faltern mit ausgeprägtem Steg zwischen den Messingglanz-Feldern aufgeführt.

Von älteren, bisher nicht berücksichtigten Funden, welche ergänzend mitgeteilt werden, lagen mir die Belege aus coll. Haase und coll. Kittel vor.

\footnotetext{
Neue Nachweise von bekannten Fundorten:

c 3148/III Ebw. OT Finow/Stadtgebiet: Altenhofer Straße, im Garten (2014, 2015, 2016, 2017, 2018)

d 3047/III Groß Schönebeck/Ortsgebiet: Friedenstraße, im Garten (1999: Haase)

g 3150(III Gabow/Granitberggebiet (2015: Schwabe)

3150/IV Altglietzen Umg./Gabower Berge (2015)
}

Fundorte (Ergänzung):

a 3249/II Falkenberg Umg./Cöthener Forst: Am Klingenden Fließ unterhalb des Bismarck-Turmes (2014)

c 3147/II Finowfurt/Hirtenweg - Besters Fließ (1991: Kittel)

3148/III Ebw. OT Finow Umg./Forst Finowtal: Einflugschneise Flugplatz (2017)

3148/IV Ebw.-Westend/Wildparkstraße (1969, 1971: Kittel); Ebw./Stadtgebiet: Kleingartenanlage im Schwärzetal nördlich Forstbotanischer Garten (2016, 2017: Schwabe)

e 3049/I Groß Ziethen Umg./Steingruben an den Ihlowbergen (2014)

3149/II Liepe Umg./Forst Chorin: NSG Plagefenn - LedumMoor am Fuß der Plageberge (2018: Richert \& Schwabe)

g 3149/IV Niederfinow Umg./Oderhänge - ehemalige Kiesgruben südwestlich des Schiffshebewerks (2015, 2016 : Schwabe)

3150/I Oderberg/am Friedhof (2016: Schwabe), Geistberg (2017: Schwabe)

\section{1 (9051) Macdunnoughia confusa (STEPHENS, 1850)}

Der F wurde am Tag beim Saugen an Armeria maritima, Gewöhnliche Grasnelke, und Eupatorium cannabium, Wasserdost, zwei weiteren Nektarsaugpflanzen im Gebiet, beobachtet (vgl. RicherT 2003).

\section{2 (9053) Plusia festucae (Linnaeus, 1758)}

- RL Land Brandenburg: Kategorie 3 (gefährdet): NM, SM;

- RL Deutschland (1998) und (2011): Vorwarnliste.
Neue Nachweise von bekannten Fundorten:

c 3148/III Ebw. OT Finow/Stadtgebiet: Altenhofer Straße, im Garten (2014)

g 3149/IV Liepe/Ortsgebiet: Schule (1973: Kittel)

3150/IV Altglietzen Umg./Gabower Berge (2015)

Fundort (Ergänzung):

b 3149/III Ebw.-Ostende (1983: Kittel)

d 3047/III Gr. Schönebeck/Ortsgebiet: Friedenstraße, im Garten (2016: Haase)

\section{3 (9054) Plusia putnami gracilis (LEMPKe, 1966)}

- RL Land Brandenburg: Kategorie 3 (gefährdet): NM, SM.

Ältere Funde (Belege in coll. Kittel) werden ergänzend nachgetragen.

Neue Nachweise von bekannten Fundorten:

c 3148/IV Ebw.-Westend/Unterheide: Wildparkstraße (1973, 1975, 1976: Kittel)

\section{4 (9056) Autographa gamma (LinNAeus, 1758)}

Weitere Nektarsaugpflanzen (vgl. Richert 2003, 2014) wurden im Gebiet dokumentiert: Arctium spec., Klette; Armeria maritima, Gewöhnliche Grasnelke; Aster novibelgii agg., Neubelgische Herbstaster; Aster novaeangliae, Neuengland-Herbstaster oder Raublattaster; Borago officinalis, Boretsch; Calluna vulgaris, Besenheide; Centaurea jacea, Wiesen-Flockenblume; Centaurea stoebe, Rispen-Flockenblume; Cirsium palustre, SumpfKratzdistel; Cirsium vulgare, Gemeine Kratzdistel; Dahlia pinnata, Garten-Dahlien; Echium vulgare, Natterkopf; Eupatorium cannabium, Wasserdost; Lavandula angustifolia, Echter Lavendel; Lythrum salicaria, Blut-Weiderich; Mentha aquatica, Wasser-Minze; Phlox paniculata, Stauden-Phlox; Saponaria spec., Seifenkraut; Silene latifolia (= Melandrium album), Weiße Lichtnelke; Solidago canadensis, Kanadische Goldrute; Tagetes patula, Studentenblume; Trifolium pratense, Wiesen-Klee.

Eine weitere R-Futterpflanzen wurden im Gebiet festgestellt (vgl. RICHERT 2003, 2010 und 2014): A (08.)VIII: R an Anethum graveolens, Dill, die Blätter fressend.

\section{5 (9059) Autographa pulchrina (HAwORTH, 1809)}

Neue Nachweise von bekannten Fundorten:

b 3247/II Biesenthal Umg./NSG Biesenthaler Becken: Langerönner Weg (2014: Ockruck)

c 3147/II Finowfurt/Hirtenweg - Besters Fließ (1986, 1993: Kittel)

3148/III Ebw. OT Finow/Stadtgebiet: Altenhofer Straße, im Garten (2014)

3149/III Ebw./Eichwerder (1970: Kittel)

d 3149/I Britz Umg./F. Britz (1971, 1972: Kittel) 
Fundorte (Ergänzung):

c $\quad 3147 / \mathrm{IV} \quad$ Finowfurt Umg./Kesselmoor an der Finowfließniederung nordöstlich des Lehnssees $(2016,2017)$

3148/IV Ebw.-Westend/Unterheide: Wildparkstraße (1973: Kittel); Ebw./Stadtgebiet: Kleingartenanlage im Schwärzetal nördlich Forstbotanischer Garten (2016: Schwabe)

\section{9 (9062) Abrostola triplasia (Linnaeus, 1758) = trigemina WERNEBURG, 1864}

Ich beobachtete den F E IX nachts beim Saugen an Blüten von Buddleja davidii, Chinesischer Sommerflieder, in meinem Garten.

Phänologie/Voltinismus: 23.IV.(2018) aFA, phänologische Verfrühung 4d gegenüber bis 2013.

\section{Acontiinae}

\section{2 (9097) Emmelia trabealis (ScOPOLI, 1763)}

Als weitere Nektarsaugpflanze (vgl. RICHERT 2003) wurde im Gebiet Centaurea scabiosa, Skabiosen-Flockenblume, festgetellt.

Phänologie/Voltinismus: Bivoltin; 2. Gen: 06.IX.(2014), aFE, phänologische Verspätung 7d gegenüber bis 2013.

\section{Neue Nachweise von bekannten Fundorten:}

b 3247/II Biesenthal Umg./NSG Biesenthaler Becken: Langerönner Weg (2014: Ockruck)

3248/II Trampe/ehemaliger TÜP (2016: Busse \& Müller)

c 3147/I Zerpenschleuse/Ortsgebiet: Berliner Straße, im Garten (2015, 2018: Busse)

3147/II Finowfurt/Hirtenweg - Besters Fließ (1991, 1993: Kittel)

3148/III Ebw. OT Finow/Stadtgebiet: Altenhofer Straße, im Garten $(2014,2018)$

g 3149/II Liepe (1972: Kittel)

3150/IV Altglietzen/Gabower Berge (2015)

Fundorte (Ergänzung):

c 3147/II Finowfurt Umg./Üdersee - SW-Ende (1974: Kittel)

3148/IV Ebw.-Westend/Unterheide: Wildparkstraße (1972: Kittel)

3149/I Ebw.-Nordend Umg./Oder-Havel-Kanal zwischen Wassertor und Ragöser Damm (2015: Schwabe)

d 3149/I Britz Umg./F. Britz (1971: Kittel)

f 3050/I Herzsprung Umg./Bunkerberg an der Straße nach Bölkendorf (2015: Schwabe)

g 3150/I Oderberg/Geistberg (2018: Schwabe)

\section{Eustrotiinae}

\section{3 (9114) Protodeltote pygarga (HufnageL,} 1766) = fasciana auct.

Phänologie/Voltinismus: In den Jahren 2014 und 2016 wurde eine partielle 2. Gen. vom 08.IX.-02.X. beobachtet.

\section{5 (9117) Deltote uncula (CLERCK, 1759)}

- RL Land Brandenburg: Kategorie 3 (gefährdet): NM.

Neue Nachweise von bekannten Fundorten:

c 3147/I Zerpenschleuse/Ortsgebiet: Berliner Straße, im Garten (2016, 2018: Busse)

Fundorte (Ergänzung):

c 3148/IV Ebw.-Westend/Unterheide: Wildparkstraße (1970, 1972: Kittel)

d 3149/I Britz Umg./F. Britz (1971, 1973: Kittel)

g 3150/I Oderberg/Geistberg (2018: Schwabe)

3150/IV Altglietzen Umg./Gabower Berge (2015)

\section{7 (9122) Pseudeustrotia candidula (Denis \& SCHIFFERMÜLLER, 1775)}

- RL Land Brandenburg: Kategorie 3 (gefährdet): Habitatbindung ungeklärt.

Im Jahre 2014 registrierte Busse die ungewöhnliche hohe Zahl von $268 \mathrm{~F}$ in seiner Lichtfalle in Zerpenschleuse. Dagegen beobachtete ich die Art 2014 an anderen Plätzen im Gebiet weniger häufig als in anderen Jahren. Die Zahl neuer Fundorte zeigt die derzeit weite Verbreitung der ehemals sehr lokal und selten gefundenen Art.

Neue Nachweise von bekannten Fundorten:

b 3247/II Biesenthal Umg./NSG Biesenthaler Becken: Langerönner Weg (2014: Ockruck), Weg nördlich der Pfauenwiesen (2014, 2015: Ockruck)

c 3147/I Zerpenschleuse Umg./Ortsgebiet: Berliner Straße, im Garten (2014-2018: Busse)

3148/III Ebw. OT Finow/Stadtgebiet: Altenhofer Straße, im Garten (2014-2018)

g 3150/IV Altglietzen Umg./Gabower Berge (2015: Richert; 2018: Schwabe)

Fundorte (Ergänzung):

b 3249/I Gersdorf Umg./Gamengrund südlich Teufelssee (2017: Richert \& Schwabe)

c 3147/I Marienwerder Umg./Oder-Havel-Kanal nordwestlich des Ortes (2014, 2015: Busse)

3147/IV Finowfurt Umg./Finowfließniederung: Neue Brücke an der Biesenthaler Straße (2014: Schwabe); Kesselmoor an der Finowfließniederung nordöstlich des Lehnssees (2016: Schwabe)

3148/IV Ebw./Stadtgebiet: Kleingartenanlage im Schwärzetal nördlich Forstbotanischer Garten (2016, 2017, 2018 Schwabe)

e 3049/I Groß Ziethen Umg./Steingruben an den Ihlowbergen (2014)

3149/II Liepe Umg./Forst Chorin: NSG Plagefenn - LedumMoor am Fuß der Plageberge (2018: Richert \& Schwabe)

g 3149/IV Niederfinow Umg./Oderhänge - ehemalige Kies gruben südwestlich des Schiffshebewerks (2015: Schwabe)

h 3150/II Hohensaaten/Deponiegelände an der Straße nach der Siedlung (2014: Kleße) 


\section{8 (9134) Eublemma minutata (FABRICIUs, 1894)} = noctualis HÜBNER 1796

- RL Land Brandenburg: Kategorie 3 (gefährdet): TR;

- RL Deutschland (2011): Kategorie 3 (gefährdet).

Neue Nachweise von bekannten Fundorten:

b 3248/II Trampe/ehemaliger TÜP (2016: Busse \& Müller)

c 3148/III Ebw. OT Finow/Stadtgebiet, im Garten (2015, 2016, je 1 F a.L.)

g 3150/IV Altglietzen/Gabower Berge (2017: Schwabe)

Fundorte (Ergänzung):

c 3148/IV Ebw.-Westend/Unterheide: Wildparkstraße (1975: Kittel)

3147/IV Finowfurt Umg./Biesenthaler Straße an der Einflugschneise Flugplatz (Solarfeld) (2014)

3148/IV Ebw./Stadtgebiet: Kleingartenanlage im Schwärzetal nördlich Forstbotanischer Garten (2017: Schwabe)

3149/I Britz Umg./F. Britz (1973: Kittel)

f 3049/IV Brodowin Umg./Gr. Rummelsberg (2016)

369 (9169) Trisateles emortualis (DENIS \& SCHIFFERMÜLLER, 1775)

Phänologie/Voltinismus: 24.V.(2018) aFA, phänologische Verfrühung 4d gegenüber bis 2013.

Im Jahre 2016 wurde ein F einer partiellen 2. Gen. am 13.IX. a.L. registriert.

Neue Nachweise von bekannten Fundorten:

b 3248/II Trampe/ehemaliger TÜP (2016: Busse \& Müller)

c 3148/III Ebw. OT Finow/Stadtgebiet: Altenhofer Straße, im Garten $(2016,2018)$

3148/IV Ebw.-Westend/Unterheide: Wildparkstraße (1976: Kittel)

d 3047/III Groß Schönebeck/Ortsgebiet: Friedenstraße, im Garten (2014: Haase)

e 3149/II Liepe Umg./Forst Chorin: NSG Plagefenn - LedumMoor am Fuß der Plageberge (2018: Richert \& Schwabe)

g 3150/I Oderberg/Teufelsberg (1975: Kittel)

Fundorte (Ergänzung):

c 3147/I Marienwerder Umg./Oder-Havel-Kanal nordwestlich des Ortes (2016: Busse)

3147/II Finowfurt/Hirtenweg - Besters Fließ (1992, 1995, 1997: Kittel)

3147/IV Finowfurt Umg./Kesselmoor an der Finowfließniederung nordöstlich des Lehnssees (2016: Schwabe; 2017: Richert)

3148/IV Ebw./Stadtgebiet: Kleingartenanlage im Schwärzetal nördlich des Forstbotanischen Gartens (2014, 2016, 2018: Schwabe)

\section{Cuculliinae}

\section{0 (9181) Cucullia fraudatrix (EversmanN, 1837)}

- Verordnung zur Neufassung der BArtschV von 2005: Besonders geschützte Art.

Phänologie/Voltinismus: Univoltin; 21.V.(2014), aFA, phänologische Verfrühung 29d gegenüber bis 2013.

Neue Nachweise von bekannten Fundorten:

c 3148/III Ebw. OT Finow/Stadtgebiet: Altenhofer Straße, im Garten (2014)

g 3150/I Oderberg/Teufelsberg (1974: Kittel)

Fundorte (Ergänzung):

c 3147/II Finowfurt/Hirtenweg - Besters Fließ (1998: Kittel)

d 3047/III Groß Schönebeck/Ortsgebiet: Friedenstraße, im Garten (1999: Haase)

3149/I Britz Umg./F. Britz (1974: Kittel)

\section{2 (9184) Cucullia argentea (HufNAGEL, 1766)}

- Verordnung zur Neufassung der BArtschV von 2005: Besonders geschützte Art.

- RL Land Brandenburg: Kategorie 2 (stark gefährdet): TR;

- RL Deutschland (2011): Kategorie 2 (stark gefährdet).

Ältere Funde (Belege in coll. Kittel) werden ergänzend nachgetragen.

Neue Nachweise von bekannten Fundorten:

g 3149/II Liepe (1973, 1975: Kittel)

\section{3 (9188) Cucullia artemisiae (HufNAGEL, 1766)}

- Verordnung zur Neufassung der BArtschV von 2005: Besonders geschützte Art.

Neue Nachweise von bekannten Fundorten:

c 3148/III Ebw. OT Finow/Stadtgebiet: Altenhofer Straße, im Garten (2014)

g 3150/ Gabow - Altglietzen: Oderhänge (Gabower Berge) III-IV (2016: Schnee)

\section{5 (9199) Cucullia umbratica (LinnaEus, 1758)}

- Verordnung zur Neufassung der BArtschV von 2005: Besonders geschützte Art.

Phänologie/Voltinismus: In der Regel univoltin; im Jahre 2014 registrierte Busse a.L. nach einer durchgehenden Flugperiode von A (08.)VI bis E (27.)VII je ein F am 12. IX. und am 02.X.(2014), aFE, phänologische Verspätung 21d gegenüber bis 2013. Es handelte sich wahrscheinlich um Tiere einer partiellen 2. Gen. Auch 2016 wurde nach einer Flugperiode E VI bis E VII am 10.IX. 1 F a.L. registriert. 


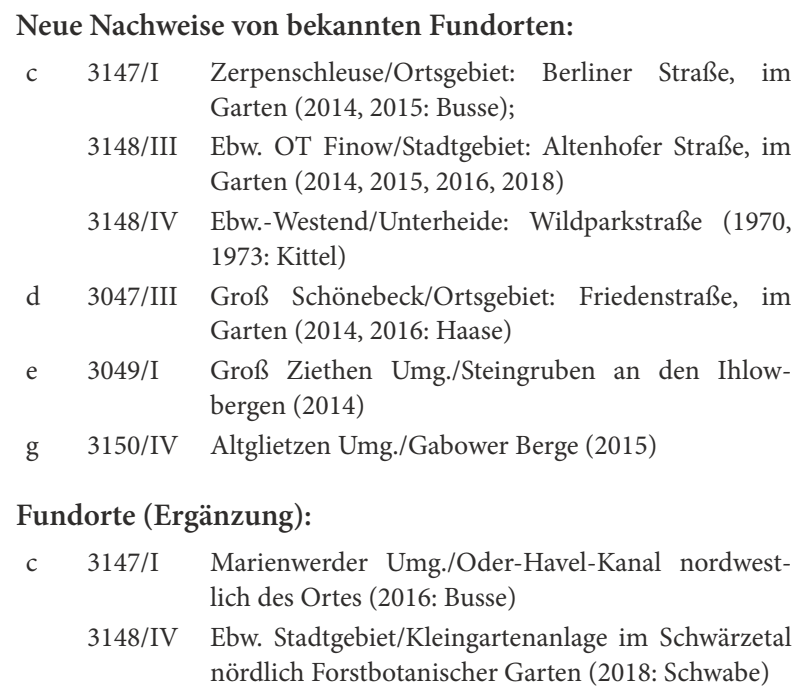

\section{7 (9217) Cucullia tanaceti (DENIS \& SCHIFFER-} MÜLLER, 1775)

- Verordnung zur Neufassung der BArtschV von 2005: Besonders geschützte Art.

- RL Land Brandenburg: Vorwarnliste; TR;

- RL Deutschland (2011): Kategorie 2 (stark gefährdet).

Ältere Funde (Belege in coll. Kittel) werden ergänzend nachgetragen.

Phänologie/Voltinismus: 17.VIII.(1974), aFE, (korrigiert nach Beleg in coll. Kittel; in RICHERT 2014 war der 02.VIII.(1972) als aFE angegeben). Damit wird ein bisher als unsicher geltendes Datum bestätigt.

Neue Nachweise von bekannten Fundorten:

g 3150/I Oderberg/Teufelsberg (1973, 1974: Kittel)

Fundorte (Ergänzung):

c 3148/IV Ebw-Westend/Unterheide: Wildparkstraße (1975: Kittel)

\section{8 (9221) Cucullia asteris (DENIS \& SCHIFFER- MÜLLER, 1775)}

- Verordnung zur Neufassung der BArtschV von 2005: Besonders geschützte Art.

- RL Land Brandenburg: Kategorie 3 (gefährdet): TR;

- RL Deutschland (2011): Kategorie 3 (gefährdet).

Ältere Funde (Belege in coll. Kittel) werden ergänzend nachgetragen.

Neue Nachweise von bekannten Fundorten:

g 3150/I Oderberg/Teufelsberg (1973, 1974, 1975: Kittel)

\section{9 (9229) Shargacucullia scrophulariae (DENIs \& SCHIFFERMÜLLER, 1775)}

- Verordnung zur Neufassung der BArtschV von 2005: Besonders geschützte Art.
- RL Land Brandenburg: Kategorie 2 (stark gefährdet): WS.

Die Beobachtungslücke im Naturraum e (Uckermärker Kuppen- und Hügelland einschließlich Choriner Waldhügel, Richert 2003) wurde geschlossen: Am Rande des Forstes Grumsin an den Steingruben bei Sperlingsherberge fand ich die R i.A an Scrophularia nudosa, Knotiger Braunwurz.

Phänologie/Voltinismus: Univoltin; 24.V.(2014), aFA, phänologische Verfrühung 7d gegenüber bis 2013.

Neue Nachweise von bekannten Fundorten:

c 3148/III Ebw. OT Finow/Stadtgebiet: Altenhofer Straße, im Garten (2014)

Fundorte (Ergänzung):

d 3047/III Groß Schönebeck/Ortsgebiet: Friedenstraße (2006: Haase)

e 3049/I Groß Ziethen Umg./Steingruben an den Ihlowbergen (2014)

\section{0 (9232) Shargacucullia lychnitis (RAMBUR,} 1833)

- Verordnung zur Neufassung der BArtschV von 2005: Besonders geschützte Art.

Neue Nachweise von bekannten Fundorten:

d 3047/III Groß Schönebeck/Ortsgebiet: Friedenstraße, im Garten (2014, 2017: Haase)

g 3150/III Gabow Umg./Granitberggebiet (2014: Richert; 2015: Schwabe)

Fundorte (Ergänzung):

c $3147 / \mathrm{IV} \quad$ Finowfurt Umg./Biesenthaler Straße an der Einflugschneise Flugplatz (Solarfeld) (2014)

\section{1 (9233) Shargacucullia verbasci (LINNAEUS, 1758)}

- Verordnung zur Neufassung der BArtschV von 2005: Besonders geschützte Art.

- RL Land Brandenburg: Kategorie 3 (gefährdet): TR. Am 03.VI.2017 fand ich zwei Jungraupen auf einer Ackerbrache bei Stecherschleuse an den Blättern von Verbascum thapsus, Kleinblütige Königskerze.

Fundorte (Ergänzung):

c 3149/III Stecherschleuse Umg./Hochfläche nordwestlich der Waldstraße (2017)

\section{2 (9240) Calophasia lunula (HufNAgel, 1766)}

- RL Land Brandenburg: Vorwarnliste: TR.

Der F wurde am Tag beim Saugen an Silene vulgaris, Taubenkropf-Leimkraut, beobachtet (Foto Schwabe). 
Neue Nachweise von bekannten Fundorten:

d 3047/III Groß Schönebeck/Ortsgebiet: Friedenstraße, im Garten (2015: Haase)

g 3149/II Liepe (1972, 1973, 1975: Kittel)

3150/IV Altglietzen Umg./Gabower Berge (2015)

Fundorte (Ergänzung):

c 3147/II Finowfurt/Hirtenweg - Besters Fließ (1996: Kittel)

3148/IV Ebw.-Westend/Unterheide - Wildparkstraße (1971, 1973, 1975, 1977: Kittel); Ebw./Stadtgebiet: Kleingartenanlage im Schwärzetal nördlich Forstbotanischer Garten (2016: Schwabe)

3148/II Britz (1972: Kittel)

g 3150/I Oderberg/Teufelsberg (1974: Kittel), Geistberg (2016: Schwabe)

\section{Amphipyrinae}

383 (9307) Amphipyra pyramidea (Linnaeus, 1758)

Als weitere Raupennahrungspflanze im Gebiet (vgl. Richert 2014) wurde Tilia spec., Linde, festgestellt (Schwabe).

\section{4 (9308) Amphipyra berbera Rungs, 1949}

Neue Nachweise von bekannten Fundorten:

c 3148/III Ebw. OT Finow/Stadtgebiet: Altenhofer Straße, im Garten $(2014,2017,2018)$

Fundorte (Ergänzung):

b 3249/I Gersdorf Umg./Gamengrund bis zum Teufelssee (2017)

c 3147/II Finowfurt/Hirtenweg - Besters Fließ (1991, 1993, 1995: Kittel)

3147/IV Finowfurt Umg./Finowfließniederung an der Neuen Brücke - Biesenthaler Straße (2014), Kesselmoor an der Finowfließniederung nördlich des Lehnssees (2016)

3148/IV Ebw.-Stadtgebiet/Kleingartenanlage im Schwärzetal nördlich Forstbotanischer Garten (2014: Schwabe)

386 (9311) Amphipyra tragopoginis (CLERCK, 1759)

Neue Nachweise von bekannten Fundorten:

c 3147/I Zerpenschleuse/Ortsgebiet: Berliner Straße, im Garten (2014: Busse)

3148/III Ebw. OT Finow/Stadtgebiet: Altenhofer Straße, im Garten $(2014,2016)$

Fundorte (Ergänzung):

b 3247/II Biesenthal Umg./NSG Biesenthaler Becken: Weg nördlich der Pfauenwiesen (2014: Ockruck), Langerönner Weg (2014: Ockruck)

3249/I Gersdorf Umg./Gamengrund südwärts bis Teufelssee (2017)

c 3148/IV Ebw.-Westend/Unterheide: Wildparkstraße (1968, 1969, 1975: Kittel)

d 3047/III Groß Schönebeck/Ortsgebiet: Friedenstraße, im Garten (2014: Haase)
3048/IV Golzow Umg. (= Althüttendorf Umg.)/Tiefer Bugsinsee (1983: Kittel)

3149/I Britz Umg./F. Britz (1973: Kittel)

3049/IV Brodowin/Krugberg (2014)

h 3150/II Hohensaaten/Deponiegelände an der Straße nach der Siedlung (2014: Kleße)

\section{Noctuidae: Psaphidinae}

387 (9320) Asteroscopus sphinx (Hufnagel, 1766)

Neue Nachweise von bekannten Fundorten:

b 3148/IV Ebw-Ostende/Breite Straße am Waldfriedhof (2014: Schwabe)

3147/I Zerpenschleuse/Ortsgebiet: Berliner Straße, im Garten (2014, 2017: Busse)

3148/IV Ebw.-Westend/Unterheide: Wildparkstraße (1973 1975: Kittel)

\section{8 (9323) Brachionycha nubeculosa (ESPER, 1785)}

- RL Deutschland (2011): Kategorie G (Gefährdung unbekannten Ausmaßes).

In coll. Kittel befand sich eine Serie von insgesamt $17 \mathrm{~F}$ aus der Eberswalder Unterheide, $9 \mathrm{~F}$ allein aus dem Jahre 1974. Die Daten zeigen, dass die Art jahrweise im Gebiet lokal nicht selten auftrat.

Neue Nachweise von bekannten Fundorten:

c 3147/I Zerpenschleuse/Ortsgebiet: Berliner Straße, im Garten (2014, 2015, 2016, 2018: Busse)

3148/III Ebw. OT Finow/Stadtgebiet: Altenhofer Straße, im Garten (2014)

3148/IV Ebw.-Westend/Unterheide: Wildparkstraße (1970, 1972, 1973, 1974, 1982, 1983, 1984: Kittel)

Fundorte (Ergänzung):

c 3148/IV Ebw./Stadtgebiet: Kleingartenanlage im Schwärzetal nördlich Forstbotanischer Garten (2015: Schwabe)

Noctuidae: Dilobinae

389 (9331) Diloba caeruleocephala (LINNAEUS, 1758)

Neue Nachweise von bekannten Fundorten:

c 3147/I Zerpenschleuse/Ortsgebiet: Berliner Straße, im Garten (2014, 2015: Busse)

\section{Noctuidae: Stiriinae}

390 (9338) Panemeria tenebrata (Scopoli, 1763)

- RL Land Brandenburg: Kategorie 3 (gefährdet).

Der tagaktive $\mathrm{F}$ wurde mehrfach beim Saugen an Cerastium arvense, Acker-Hornkraut, beobachtet. 
Neue Nachweise von bekannten Fundorten:

g 3050/II Stolzenhagen/NSG Krähen- und Jungfernberge (2015)

Fundorte (Ergänzung):

d 3148/I Lichterfelde Umg./NSG Buckowseerinne: Hänge südlich Margaretenhof (2015)

\section{Noctudae: Heliothinae}

\section{1 (9358) Schinia scutosa (DeNIS \& SCHIFFER-} MÜLLER, 1775)

Siehe wieder entdeckte Arten.

392 (9364) Heliothis viriplaca (HufNAgeL, 1766) = dipsacea LINNAEUs, 1767

Als weitere Nektarsaugpflanze im Gebiet wurde Helichrysum arenarium, Sand-Strohblume, festgestellt.

\section{3 (9395) Heliothis maritima (GrASLIN, 1855) - Heliothis adaucta BUTLER, 1878}

Heliothis maritima und Heliothis adaucta wurden früher als conspezifisch angesehen und deshalb nicht unterschieden. Inzwischen gilt Heliothis adaucta als allopatrische Schwesterart von Heliothis maritima (FIBIGER et al. 2009). Letztere kommt nur sehr lokal in nördlichen und nordwestlichen Teilen Deutschlands vor. Bei Funden in anderen Teilen Deutschlands handelt es sich um die in Südosteuropa heimische Heliothis adaucta, die als Einwanderer bis Süd-, Mittel- und Ostdeutschland vordringt (Segerer \& Hausmann 2011; STeiner et al.2014). Somit sind die Angaben zu H. maritima in RICHERT 2003, 2010 und 2014 dem Taxon Heliothis adaucta zuzuordnen.

\section{3a (9370) Helicoverpa armigera (Hü BNER, 1808)}

Dieser im Jahre 2002 erstmals im Gebiet nachgewiesene „Irrgast“ (Richert 2004) wurde in den Jahren 2015 (mehrfach), 2016, 2017 und 2018 (Einzelfunde) erneut bei uns a.L. beobachtet (Belegfoto Schwabe, 18.VIII.2015, Niederfinow).

Neue Nachweise von bekannten Fundorten:

c 3148/III Ebw. OT Finow/Stadtgebiet: Altenhofer Straße, im Garten $(2015,2016,2018)$

Fundorte (Ergänzung):

c 3148/III Ebw. OT Finow Umg./Forst Finowtal: Einflugschneise Flugplatz (2017)

g 3149/IV Niederfinow/Oderhänge - ehemalige Kiesgruben südwestlich des Schiffshebewerks (2015: Schwabe)
394 (9372) Pyrrhia umbra (HufNAgEL, 1766)

Neue Nachweise von bekannten Fundorten:

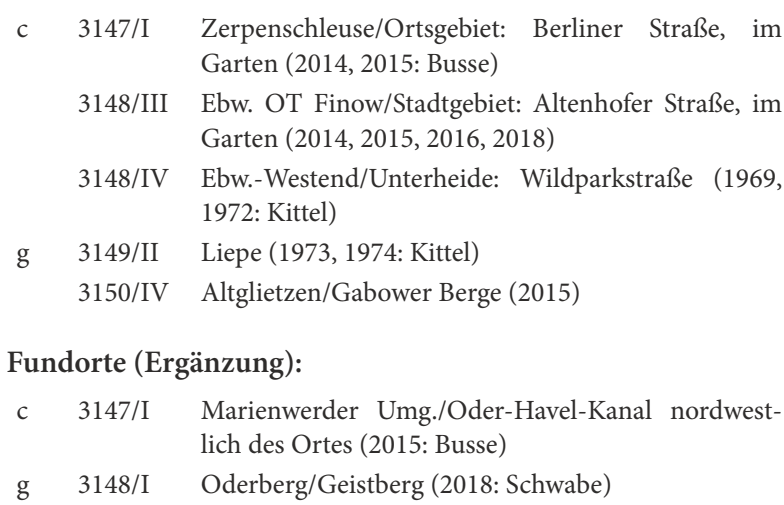

\section{Unterfamilie Hadeninae}

395 (9396) Elaphria venustula (HÜBNER, 1790)

Phänologie/Voltinismus: Univoltin: 06.V.(2014), aFA, phänologische Verfrühung 4d bis gegenüber 2013.

398 (9433) Paradrina clavipalpis (ScopolI, 1763)

Neue Nachweise von bekannten Fundorten:

c 3147/I Zerpenschleuse/Ortsgebiet: Berliner Straße, im Garten (2014: Busse)

3148/III Ebw. OT Finow/Stadtgebiet: Altenhofer Straße, im Garten $(2014,2015,2018)$

Fundorte (Ergänzung):

c 3148/IV Ebw.-Westend/Unterheide: Wildparkstraße (1968, 1971-1973, 1993: Kittel), Ebw./Stadtgebiet: Kleingartenanlage im Schwärzetal nördlich Forstbotanischer Garten (2018: Schwabe)

e 3049/IV Chorin Umg./Forst Chorin: Teerbrennerberge - Am Olberg (2013: Müller)

397 (9430) Paradrina selini (BoIsduval, 1840)

Neue Nachweise von bekannten Fundorten:

c 3147/II Finowfurt/Hirtenweg - Besters Fließ (1990, 1993 1995: Kittel)

g 3149/II Liepe (1973: Kittel)

3150/IV Altglietzen/Oderhänge: Gabower Berge (2015)

Fundorte (Ergänzung):

c 3148/IV Ebw.-Westend/Unterheide: Wildparkstraße (19701975 alljährlich: Kittel)

400 (9450) Hoplodrina blanda (DENIS \& SCHIFFERMÜLLER, 1775)

Phänologie/Voltinismus: Der bisher früheste Nachweis der Art im Gebiet erfolgte 2018 auf den Oderhängen bei 
Stolpe: 14.VI.2018 aFA, phänologische Verfrühung 8d gegenüber bis 2013 .

\section{1 (9453) Hoplodrina respersa (DENIS \& SCHIF- FERMÜLLER, 1775)}

- RL Land Brandenburg: Kategorie 3 (gefährdet): CB. TR;

- RL Deutschland (2011): Vorwarnliste.

Phänologie/Voltinismus: 03.VI.2018 aFA, phänologische Verfrühung $3 \mathrm{~d}$ gegenüber bis 2013 .

Neue Nachweise von bekannten Fundorten:

c 3147/I Zerpenschleuse/Ortsgebiet: Berliner Straße, im Garten (2014: Busse)

3148/III Ebw. OT Finow/Stadtgebiet: Altenhofer Straße, im Garten $(2014,2015,2017,2018)$

3148/IV Ebw.-Westend/Unterheide: Wildparkstraße (1970: Kittel)

Fundorte (Ergänzung):

c 3148/IV Ebw./Stadtgebiet: Kleingartenanlage im Schwärzetal nördlich Forstbotanischer Garten (2016: Schwabe)

g 3149/II Liepe (1973: Kittel)

3150/I Oderberg/Teufelsberg (1973-1975: Kittel)

\section{3 (9456) Charanyca trigrammica (HufNAGEL, 1766)}

Neue Nachweise von bekannten Fundorten:

b 3247/II Biesenthal Umg./NSG Biesenthaler Becken: Langerönner Weg (2014: Ockruck)

c 3147/I Zerpenschleuse/Ortsgebiet: Berliner Straße, im Garten (2014, 2015, 2016, 2017: Busse); Marienwerder Umg./Oder-Havel-Kanal nordwestlich des Ortes (2015: Busse)

3147/II Finowfurt/Hirtenweg - Besters Fließ (1987, 1990, 1991: Kittel)

3147/IV Marienwerder Umg./Steinlaake (2015), Kiesgrube südwestlich des Sportplatzes (2015)

3148/III Ebw. OT Finow/Stadtgebiet: Altenhofer Straße, im Garten $(2014,2015,2016,2018)$

g 3150/III Gabow/Granitberggebiet (2015)

3150/IV Altglietzen/Oderhänge: Gabower Berge (2015)

Fundorte (Ergänzung):

b 3149/III Ebw.-Ostende (1983: Kittel)

c 3148/IV Ebw.-Westend/Unterheide: Wildparkstraße (1977: Kittel); Ebw./Stadtgebiet: Kleingartenanlage im Schwärzetal nördlich Forstbotanischer Garten (2016, 2017, 2018: Schwabe)

d 3149/I Britz Umg./F. Britz (1971, 1972: Kittel)

e 3049/I Groß Ziethen Umg./Steingruben an den Ihlowbergen (2014)

g 3050/II Stolpe a.O./Trockenhänge am Stadtweg (2018)

3149/IV Niederfinow/Oderhänge - ehemalige Kiesgruben südwestlich des Schiffshebewerks (2015: Schwabe)

3150/I Oderberg/Geistberg (2017, 2018: Schwabe)

\section{4 (9471) Chilodes maritima (TAUSCHER, 1806)}

- RL Land Brandenburg: Kategorie 3 (gefährdet): NM.

Neue Nachweise von bekannten Fundorten:

c 3148/III Ebw. OT Finow/Stadtgebiet: Altenhofer Straße, im Garten (2016)

Fundorte (Ergänzung):

b 3149/II Liepe (1976: Kittel)

\section{5 (9476) Athetis pallustris (HüBNER, 1808)}

- RL Land Brandenburg: Kategorie 1 (vom Aussterben bedroht): MW (?);

- RL Deutschland 2011: Kategorie 2 (stark gefährdet). Im Gebiet verschollen. Der letzte Fund erfolgte 1999. Seitdem ist die A. palustris nicht mehr gefunden worden, obwohl ehemalige Fundplätze weiterhin faunistisch untersucht wurden.

Ältere Funde (Belege in coll. Kittel) werden nachgetragen.

Neue Nachweise von bekannten Fundorten:

c 3148/III Ebw. OT Finow/Gartenstraße (1971: Kittel)

g 3149/II Liepe (1976: Kittel)

Fundorte (Ergänzung):

c 3148/IV Ebw.-Westend/Unterheide: Wildparkstraße (1970: Kittel)

\section{6 (9481) Dipterygia scabriuscula (Linnaeus,} 1758)

Phänologie/Voltinismus: 29.IV.(2014), aFA; phänologische Verfrühung 12d gegenüber bis 2013.

\section{8 (9496) Talpophila matura (HUfNAgeL, 1766)}

Die Beobachtungslücke im Naturraum f (Parsteiner Becken, RICHERT 2003) konnte inzwischen geschlossen werden.

Alle mir aus dem Gebiet bekannten Fundorte werden nachgetragen.

Phänologie: Univoltin; 25.IX.(2017), aFE, phänologische Verspätung $25 \mathrm{~d}$ gegenüber bis 2013. Offensichtlich handelt es sich hier um einen „Nachzügler“.

Fundorte:

a 3250/I Bad Freienwalde Umg./Cöthener Forst: Hammertal nahe der Jugendherberge (2008: Kleße)

b 3148/IV Ebw./Oberheide: Institut für Waldschutz (1965: Kruel)

3149/III Sommerfelde Umg./Nordostrand des Hohenfinower Waldes (2005: Ockruck); Tornow Umg./Barnimhänge nördlich des Ortes (1991: Ockruck)

3247/II Biesenthal Umg./NSG Biesenthaler Becken: Langerönner Weg (2009: Ockruck), nördlich der Pfauenwiesen (2015: Ockruck) 
3248/II Trampe/ehemaliger TÜP $(1999,2005)$ (RICHERT 2006)

c 3147/I Zerpenschleuse/Ortsgebiet, im Garten (1984, 1988, 1989: Ockruck; 2013, 2014, 2015, 2017: Busse) (Busse \& OCKRUCK 1991)

3147/II Marienwerder Umg./F. Pechteich (1950-1959: Haeger); Finowfurt Umg./Besters Fließ - Hirtenweg (1989-1993: Kittel)

3147/III Marienwerder Umg./Oder-Havel-Kanal (1996: Ockruck)

3148/III Ebw. OT Finow/Stadtgebiet: Altenhofer Straße, im Garten (1952, 1958, 1961, 1967, 1987, 1990, 1991, 1994, 1995, 1997, 1999, 2001-2005, 2007-2009, 2012-2018: Richert); Gartenstraße in der Siedlung Freie Scholle (1962, 1963, 1966, 1967, 1968: Duckert) und Umg./Forst Finowtal: 1. Melchower Weg nordöstlich vom Kleinen Postluch (2004), Einflugschneise Flugplatz $(2016,2017)$

3148/IV Ebw. OT Finow Umg./Forst Finowtal: Stromtrasse südlich des Ortes (2009); Ebw.-Westend/Unterheide: Wildparkstraße (1969, 1973-1974, 1975: Kittel); Ebw./Stadtgebiet: Kleingartenanlage im Schwärzetal nördlich Forstbotanischer Garten (2016: Schwabe)

3149/II Niederfinow Umg./Oder-Havel-Kanal östlich der Klosterbrücke (2008: Schwabe)

3149/III Stecherschleuse/,Südhänge“ (1974: Kruel)

3149/IV Stecherschleuse Umg./am Rande des ehemaligen Kiesgrubengeländes nordöstlich des Ortes (2009: Schwabe)

3047/III Groß Schönebeck/Ortsgebiet: Friedenstraße, im Garten (2015: Haase)

3047/IV Groß Schönebeck Umg./Schorfheide: Pinnowseengebiet - NSG Meelake (1991: Ockruck) (NSG Kienhorst/Köllnseen/Eichheide, RICHERT 2010; Eichhorst Umg./Üderheide: Süßer Winkel am Werbellinsee $(1958,1960,1961)$

3048/III Jagdschloss Hubertusstock Umg. (1960)/Engere Schorfheide: Kl. Kaisergrund (2006, 2009) (NSG Kienhorst/Köllnseen/Eichheide, Richert 2010

3147/II Eichhorst Umg./Üderheide: F. Grünenberg (1961, 1962), Moospfuhl östlich der Straße nach Finowfurt (1969)

3148/I „Werbellin“ (1917: ohne Namen; Belege in coll. Deutschlandsammlung Naturkundemuseum Berlin); Lichterfelde Umg./NSG Buckowseerinne: Trockenhänge südwestlich Margaretenhof (2010) (RICHERT 2012)

3148/II Britz (1973: Kittel)

3149/I Britz Umg./F. Britz (1971: Kittel)

e 3049/I Neugrimnitz Umg./Forst Grumsin: Grumsin (1972); Groß Ziethen Umg./Feldsoll nördlich des Ortes (1994: DEI, Sommer)

f 3049/IV Brodowin Umg./Karpatenweg östlich des Krugbergs (2015)

g 3050/II Stolzenhagen/NSG Krähen- und Jungfernberge (1976, 1978: Kruel) (RICHERT 1979)

3149/II Liepe (STÖCKEL 1955)

3149/IV Niederfinow Umg./Oderhänge - ehemalige Kiesgruben südwestlich des Schiffshebewerks (1995: Richert; 2015, 2016: Schwabe)

3150/I Oderberg (1961: Steinig)/Teufelsberg (1991) (RICHERT 1994)

3150/III Gabow/Granitberg (1982, 1984: Richert; 1991, 2007: Kleße) (RICHERT 1984)

3150/IV Altglietzen Umg./Gabower Berge (2015) h 3150/III Bralitz/stillgelegte Kiesgrube (1999, 2000, 2009); Neuenhagen Umg./ehemaliges Kiesgrubengelände östlich der Straße nach Oderberg (2011: Ockruck), ehemaliger Altglietzener Segelflugplatz nordöstlich des Ortes (2009: Rosenbauer)

\section{9 (9501) Trachea atriplicis (LinnaEus, 1758)}

Phänologie/Voltinismus: In der Regel univoltin; im Jahre 2014 wurde nach einer regulären Flugzeit vom 01.VI. bis 27.VII. ein $\mathrm{F}$ am 05.IX. a.L. registriert, der einer partiellen 2. Gen. zuzuordnen ist.

\section{1 (9505) Phlogophora meticulosa (Linnaeus, 1758)}

Phänologie/Voltinismus: 20.XI.(2014), aFE, phänologische Verspätung $5 \mathrm{~d}$ gegenüber bis 2013.

\section{3 (9515) Actinotia polyodon (ClerCK, 1759)}

R der 1. Gen. fand Schwabe A (09.) IX 2016 an Hypericum spec., Johanniskraut.

Phänologie/Voltinismus: 2. Gen.: 04.IX.(2018) aFE, phänologische Verspätung 9d gegenüber bis 2013 .

\section{Neue Nachweise von bekannten Fundorten:}

c 3147/I Zerpenschleuse/Ortsgebiet: Berliner Straße, im Garten (2014: Busse)

3147/II Finowfurt/Hirtenweg - Besters Fließ (1990, 1991, 1995, 1997: Kittel)

3148/III Ebw. OT Finow/Stadtgebiet: Altenhofer Straße, im Garten (2014, 2015, 2016, 2017, 2018)

g 3149/IV Niederfinow/Oderhänge - ehemalige Kiesgruben südwestlich des Schiffshebewerks (2015: Schwabe)

3150/I Oderberg/Teufelsberg (1973, 1974: Kittel)

3150/IV Altglietzen/Gabower Berge (2015: Richert, Schwabe; 2018: Schwabe)

Fundorte (Ergänzung):

b 3148/IV Spechthausen Umg./Fischteiche nordöstlich des Ortes (2016: Schwabe)

c 3148/III Ebw. OT Finow Umg./Forst Finowtal: Einflugschneise Flugplatz (2017: Richert \& Schwabe)

d 3047/III Groß Schönebeck/Ortsgebiet: Friedenstraße, im Garten (1999, 2017: Haase)

e 3049/I Groß Ziethen Umg./Steingruben an den Ihlowbergen (2014)

g 3150/I Oderberg/Geistberg (2015, 2017: Schwabe),

\section{4 (9520) Callopistria juventina (STOLL, 1782)}

Busse registrierte in seiner Lichtfalle im Ortsgebiet von Zerpenschleuse bemerkenswerte Anflugzahlen: Bereits 2013 wurden vom 2.VI. bis 21.VII. insgesamt $136 \mathrm{~F}$ registriert; 2014 vom 04.VII. bis 26.VII. sogar 411 F. Allein am 10.VII. flogen $100 \mathrm{~F}$ an das Licht.

\section{Neue Nachweise von bekannten Fundorten:}

b 3248/II Trampe/ehemaliger TÜP (2016: Busse \& Müller) 
c 3147/I Zerpenschleuse/Ortsgebiet: Berliner Straße, im Garten (2014, 2015, 2016, 2017, 2018: Busse)

3147/II Finowfurt/Hirtenweg - Besters Fließ (1990-1992: Kittel)

3148/III Ebw. OT Finow/Stadtgebiet: Altenhofer Straße, im Garten $(2014,2015)$

d 3047/III Groß Schönebeck/Ortsgebiet: Friedenstraße, im Garten (1999, 2015, 2016: Haase)

e 3149/II Liepe Umg./Forst Chorin: NSG Plagefenn - LedumMoor am Fuß der Plageberge (2018: Richert \& Schwabe)

Fundorte (Ergänzung):

c 3147/IV Finowfurt Umg./Kesselmoor an der Finowfließniederung nordöstlich des Lehnssees $(2016,2017)$

3148/IV Ebw.-Westend/Unterheide: Wildparkstraße (1975: Kittel); Ebw./Stadtgebiet: Kleingartenanlage im Schwärzetal nördlich Forstbotanischer Garten (2016, 2017: Schwabe)

d 3047/III Groß Schönebeck Umg./Liebenthaler Weg (2016: Haase)

\section{4a (9525) Eucarta virgo (TreitsChKe, 1835)}

Die R klopfte Schnee M (18.)VIII (2016) von Artemisia vulgaris, Gewöhnlicher Beifuß.

Neue Nachweise von bekannten Fundorten:

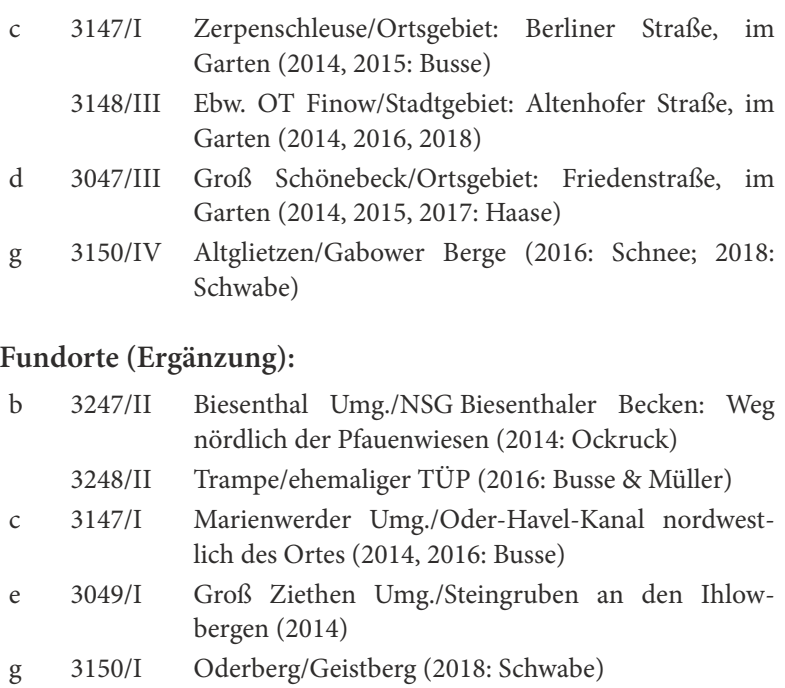

\section{5 (9527) Ipimorpha retusa (LinNAEUs, 1761)}

Phänologie/Voltinismus: Univoltin; 06.VII.(2014), aFA, phänologische Verfrühung 2d gegenüber bis 2013.

Neue Nachweise von bekannten Fundorten:

c 3147/II Finowfurt/Hirtenweg - Besters Fließ (1994: Kittel)

Fundorte (Ergänzung):

b 3247/II Biesenthal Umg./NSG Biesenthaler Becken: Weg nördlich der Pfauenwiesen (2014, 2015: Ockruck)

c 3148/IV Ebw./Stadtgebiet: Kleingartenanlage im Schwärzetal nördlich des Forstbotanischen Gartens (2014: Schwabe)

3149/I Neuehütte (1974: Kittel) d 3047/III Groß Schönebeck Ortsgebiet: Friedenstraße, im Garten (2014: Haase)

e 3049/I Groß Ziethen Umg./Steingruben an den Ihlowbergen (2014)

\section{6 (9528) Ipimorpha subtusa (DENIS \& SCHIF- FERMÜLLER, 1775)}

Neue Nachweise von bekannten Fundorten:

c 3147/II Finowfurt/Hirtenweg - Besters Fließ (1991, 1993, 1994, 1995: Kittel)

3148/III Ebw. OT Finow/Stadtgebiet: Altenhofer Straße, im Garten (2014)

e 3049/I Groß Ziethen Umg./Steingruben an den Ihlowbergen (2014)

Fundorte (Ergänzung):

e 3149/II Liepe Umg./Forst Chorin: NSG Plagefenn - LedumMoor am Fuß der Plageberge (2018: Richert \& Schwabe)

g 3149/II Liepe (1974: Kittel)

\section{7 (9531) Enargia paleacea (ESPER, 1788)}

Die Beobachtungslücke im Naturraum h (Odertal, RICHERT 2003) wurde inzwischen geschlossen (vgl. RICHERT 2014).

Phänologie: In coll. Kittel befanden sich zwei Belegexemplare mit den Funddaten 25.IX.1987 und 17.X.1987, Finowfurt/Hirtenweg - Besters Fließ. Beides sind Extremdaten. Es handelt sich offenbar um Nachzügler nach dem kühlen Sommer des Jahres 1987, was auch bei anderen Arten in diesem Jahr beobachtet worden war. 17.X.(1987), aFE (korrigiert nach Beleg in coll. Kittel; in Richert 2014 war der 29.VIII. $(1972,2002)$ als aFE angegeben).

Neue Nachweise von bekannten Fundorten:

c 3147/I Zerpenschleuse/Ortsgebiet: Berliner Straße, im Garten (2014: Busse)

3147/II Finowfurt/Hirtenweg - Besters Fließ (1987, 1993 1995: Kittel)

3148/III Ebw. OT Finow/Stadtgebiet: Altenhofer Straße, im Garten $(2014,2017)$

3148/IV Ebw.-Westend/Unterheide: Wildparkstraße (1969: Kittel)

d 3047/III Groß Schönebeck/Ortsgebiet: Friedenstraße, im Garten (2014, 2015: Haase)

g 3150/IV Altglietzen/Oderhänge: Gabower Berge (2015: Richert, Schwabe)

Fundorte (Ergänzung):

b 3247/II Biesenthal Umg./NSG Biesenthaler Becken: Weg nördlich der Pfauenwiesen (2014: Ockruck)

3249/I Gersdorf Umg./Gamengrund südwärts bis Teufelssee (2017)

e 3049/I Groß Ziethen Umg./Steingruben an den Ihlowbergen (2014)

h 3150/II Hohensaaten/Deponiegelände an der Straße nach der Siedlung (2014: Kleße) 


\section{8 (9536) Parastichtis suspecta (HüBNER, 1817) = iners TREITSCHKE, 1825}

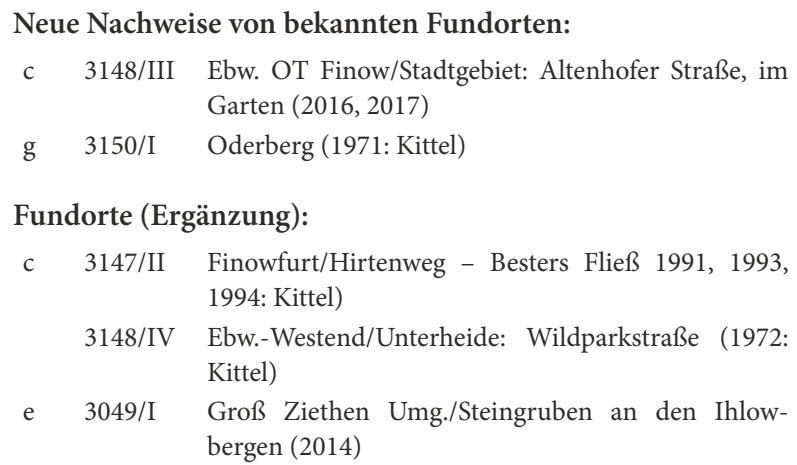

\section{9 (9537) Parastichtis ypsillon (DeNNIs \& SCHIF-} FERMÜLLER, 1775) = fissipuncta HAWORTH, 1809

Ein älterer Fund (Beleg in coll. Kittel) wird ergänzend mitgeteilt.

Phänologie/Voltinismus: 01.VI.(2018) aFA, phänologische Verfrühung 10d gegenüber bis 2013.

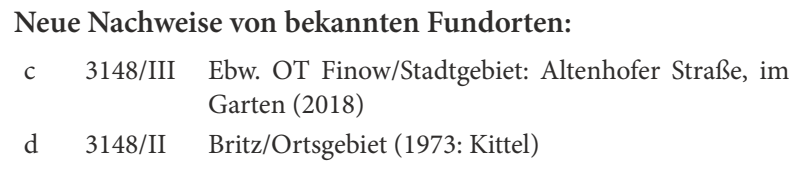

\section{0 (9540) Mesogona oxalina (HüBnER, 1803)}

- RL Land Brandenburg: Kategorie 1 (vom Aussterben bedroht): WA, SO;

- RL Deutschland 2011: Kategorie 3 (gefährdet).

Ein älterer, bisher unberücksichtigter Fund aus der Datenbank InsectIS Bbg wird ergänzend mitgeteilt.

Fundorte (Ergänzung):

g 3150/III Gabow (1993: Torsten Schermer)

\section{1 (9548) Cosmia affinis (Linnaeus, 1761)}

- RL Land Brandenburg: Kategorie 3 (gefährdet): UW, HA.

Neue Nachweise von bekannten Fundorten:

c 3147/I Zerpenschleuse/Ortsgebiet: Berliner Straße, im Garten (2014: Busse)

3148/III Ebw. OT Finow/Stadtgebiet: Altenhofer Straße, im Garten (2014)

Fundorte (Ergänzung):

c 3147/IV Finowfurt Umg./Kesselmoor an der Finowfließniederung nordöstlich des Lehnssees (2016: Schwabe)
422 (9549) Cosmia pyralina (DeNis \& SCHIFFERMÜLLER, 1775)

Neue Nachweise von bekannten Fundorten:

c 3147/I Zerpenschleuse/Ortsgebiet: Berliner Straße, im Garten (2014, 2015: Busse)

3148/III Ebw. OT Finow/Stadtgebiet: Altenhofer Straße, im Garten $(2014,2016)$

g 3150/I Oderberg/Teufelsberg (1974: Kittel)

3150/IV Altglietzen Umg./Gabower Berge (2015)

Fundorte (Ergänzung):

c 3147/II Finowfurt/Hirtenweg - Besters Fließ (1993, 1994 1995: Kittel)

3148/IV Ebw.-Westend/Unterheide: Wildparkstraße (1970: Kittel); Ebw./Stadtgebiet: Kleingartenanlage im Schwärzetal nördlich Forstbotanischer Garten (2015 2018: Schwabe)

d 3148/II Britz (1971: Kittel)

\section{3 (9550) Cosmia trapezina (Linnaeus, 1758)}

Phänologie/Voltinismus: 14.VI.(2018) aFA, phänologische Verfrühung 12d gegenüber bis 2013 .

\section{4 (9552) Atethmia centrago (HAWORTH, 1809)}

\section{= xerampelina auct. nec. ESPER, 1794}

- RL Land Brandenburg: Kategorie 3 (gefährdet): MB, HA.

Die Art breitet sich offenbar im Gebiet weiter aus. Nachdem sie 2013 erstmals in Zerpenschleuse nachgewiesen wurde (RICHERT 2014), wurde sie nun auch im Stadtgebiet von Ebw. gefunden. 2017 fing ich erstmals einen $\mathrm{F}$ in meinem Garten in Finow a.L. Außerdem liegt ein Erstnachweis für den Naturraum f (Parsteiner Becken; RICHERT 2003) von Brodowin vor.

Phänologie/Voltinismus: 20.VIII.(2018) aFA, phänologische Verfrühung 2d gegenüber bis 2013.

Fundorte (Ergänzung):

c 3147/I Zerpenschleuse/Ortsgebiet: Berliner Straße, im Garten (2014, 2018: Busse)

3148/III Ebw. OT Finow/Stadtgebiet: Altenhofer Straße, im Garten $(2017,2018)$

3148/IV Ebw./Stadtgebiet: Kleingartenanlage im Schwärzetal nördlich des Forstbotanischen Gartens (2014, 2016 Schwabe)

f 3049/IV Brodowin Umg./Karpatenweg östlich des Krugbergs (2015)

g 3150/I Oderberg/am Friedhof (2016: Richert \& Schwabe)

425 (9556) Xanthia togata (ESPER, 1788) = lutea STRÖM, 1783

Die Beobachtungslücken im Naturraum a (Oberbarnimer Waldhügelland) und $\mathrm{f}$ (Parsteiner Becken) (RICHERT 2003) wurden inzwischen geschlossen. 
Neue Nachweise von bekannten Fundorten:

c 3147/II Finowfurt/Hirtenweg - Besters Fließ (1986, 1993, 1995: Kittel)

Fundorte (Ergänzung):

c 3147/II Marienwerder Umg./Oder-Havel-Kanal nordwestlich des Ortes (2014, 2016, 2017: Busse)

3148/IV Ebw.-Westend/Unterheide: Wildparkstraße (1973, 1974, 1976, 1977: Kittel), Ebw./Stadtgebiet: Kleingartenanlage im Schwärzetal nördlich des Forstbotanischen Gartens (2014: Schwabe)

d 3047/III Groß Schönebeck/Ortsgebiet: Friedenstraße, im Garten (2014, 2017: Haase)

f 3049/IV Brodowin/Krugberg (2014)

\section{6 (9557) Xanthia aurago (DENIS \& SCHIFFER- MÜLLER, 1775)}

Neue Nachweise von bekannten Fundorten:

c 3147/I Zerpenschleuse/Ortsgebiet: Berliner Straße, im Garten (2015, 2016: Busse)

3147/II Finowfurt/Hirtenweg - Besters Fließ (1986, 1987, 1991, 1995: Kittel)

c 3148/III Ebw. OT Finow/Stadtgebiet: Altenhofer Straße, im Garten $(2014,2016,2017)$

Fundorte (Ergänzung):

c 3147/I Marienwerder Umg./Oder-Havel-Kanal nordwestlich des Ortes (2016: Busse)

3148/IV Ebw.-Westend/Unterheide: Wildparkstraße (1974, 1975, 1976: Kittel), Ebw./Stadtgebiet: Kleingartenanlage im Schwärzetal nördlich des Forstbotanischen Gartens (2014: Schwabe)

d 3047/III Groß Schönebeck/Ortsgebiet: Friedenstraße, im Garten (2018: Haase)

\section{7 (9559) Xanthia icteritia (HufNAGEL, 1766) = fulvago auct. neC. CLERCK, 1759}

Fehlende Nachweise im Naturraum f (Parsteiner Becken, Richert 2004) zeigen eine Beobachtungslücke an. Alle mir bekannten Fundorte im Gebiet werden nachgetragen.

\section{Fundorte:}

a 3249/II Falkenberg Umg./Cöthener Forst: ehemalige F. Tobbenberge (1970, 1982); Uchtenhagen (1974)

3250/I Bad Freienwalde Umg./Cöthener Forst: Hammertal nahe der Jugendherberge (2004: Kleße)

b 3148/IV Ebw.-Ostende/Hausberg: Bruno-H.-Bürgel-Schule (1987); Ebw./Oberheide (2011: Schwabe): Institut für Waldschutz (1960: Kruel), F. Leuenberger Wiesen (1975)

3149/III Sommerfelde Umg./ehemaliger TÜP Panzerbahn (2005) (RICHERT 2006), Bachtal nördlich des Ortes „am Wiesengraben“ (2011: Schwabe); Tornow Umg./ Barnimhänge nördlich des Ortes: Bachtal (2011)

3149/IV Hohenfinow/Ortsgebiet (1986: Hülbert) (RICHERT \& HÜLBERT 1991)

3247 Biesenthal Umg. (1980: Fichtner) c 3147/I Zerpenschleuse/Ortsgebiet (1980, 1984, 1986, 1987, 1988: Ockruck; 2013, 2014: Busse) (Busse \& OckRUCK 1991); Marienwerder Umg./Oder-HavelKanal nordwestlich des Ortes (2016: Busse \& Müller)

3147/II Marienwerder Umg./Pechteich (1995: Ockruck); Finowfurt Umg./Besters Fließ - Hirtenweg (1986, 1987, 1990, 1991, 1993: Kittel)

3147/III Marienwerder Umg./Oder-Havel-Kanal (1997: Ockruck)

3147/? Sophienstadt (2016: Busse \& Müller)

3148/I Finowfurt OT Steinfurt Umg./Kiesgrube westlich der Barschgrube (1995)

3148/III Ebw. OT Finow/Stadtgebiet: Im Garten (1962-1974: Duckert; 1954, 1960-1964, 1967, 1972, 1985, 1987 1990, 1992, 2000, 2001, 2004, 2009, 2011, 2014, 2016 Richert) und Umg./Forst Finowtal: Samithseemoor (1993)

3148/IV Ebw. (1904: Jonas); Ebw.-Westend/Unterheide: Wildparkstraße (1971, 1975: Kittel)

3149/I Neuehütte (1974: Kittel)

3149/III Ebw./Eichwerder (2010: Schwabe)

3149/IV Stecherschleuse Umg./ehemaliges Kiesgrubengelände nordöstlich des Ortes (2009: Schwabe)

d 3047/IV Groß Schönebeck Umg./Schorfheide: Joachimsthaler Damm westlich der Pinnowseen (2004) (NSG Kienhorst/Köllnseen/Eichheide, RicherT 2010)

3147/II Eichhorst Umg./Üderheide: F. Grünenberg (1963)

3148/I Lichterfelde Umg./Waldrand oberhalb südöstlich des Gr. Buckowsees (1960) (heute NSG Buckowseerinne), NSG Buckowseerinne: Trockenhänge südwestlich Margaretenhof (2011) (RICHERT 2012)

3149/I Britz Umg./F. Britz (1970er Jahre: Riegel)

e 3049/I Groß Ziethen Umg./Feldsoll nördlich des Ortes (1993: Sommer, DEI)

3049/IV Chorin Umg./Forst Chorin: Teerbrennerberge - Am Olberg (2013: MÜLlER 2015), Kreuzung Denglerweg - Olbergstraße (2013: MüLler 2015)

g 3149/IV Niederfinow/Oderhänge - ehemalige Kiesgruben südwestlich des Schiffshebewerks (2016: Schwabe)

3050/II Stolzenhagen/NSG Krähen- und Jungfernberge (1979, 2003) (RICHERT 1982)

3150/I Oderberg/Teufelsberg (1993) (RICHERT 1994)

3150/III Gabow/Granitberggebiet (1991, 1992: Kleße)

h 3149/II Liepe Umg./Niederoderbruch (1965: Kruel): Schöpfwerk Liepe (1973)

3150/III Bralitz/stillgelegte Kiesgrube (2009); Neuenhagen Umg./ehemaliges Kiesgrubengelände östlich der Straße nach Oderberg (2007: Rosenbauer; 2010: Ockruck)

\section{9 (9561) Xanthia ocellaris (BORKHAUSEN, 1792)}

Die Beobachtungslücke im Naturraum f (Parsteiner Becken, RICHERT 2003) konnte inzwischen geschlossen werden.

Neue Nachweise von bekannten Fundorten:

c 3148/III Ebw. OT Finow/Stadtgebiet: Altenhofer Straße, im Garten (2014)

Fundorte (Ergänzung):

c 3147/I Marienwerder Umg./Oder-Havel-Kanal nordwestlich des Ortes (2016: Busse \& Müller)

f 3049/IV Brodowin/Krugberg (2014: Schwabe) 


\section{0 (9562) Xanthia citrago (LinNAEUs, 1758)}

Aus dem Naturraum a (Oberbarnimer Waldhügel, RicheRT 2003) liegt inzwischen ein aktueller Nachweis vor. Die Beobachtungslücke im Naturraum e (Uckermärker Kuppen- und Hügelland einschließlich Choriner Waldhügel, RicherT 2003) konnte inzwischen geschlossen werden (MüLlER 2015).

Phänologie/Voltinismus: Univoltin; 30.VIII.(2013), aFA, phänologische Verfrühung 4d (korrigiert nach MüLLER 2015; in Richert 2014 03.IX.(1972, 2004) als aFA angegeben).

Neue Nachweise von bekannten Fundorten:

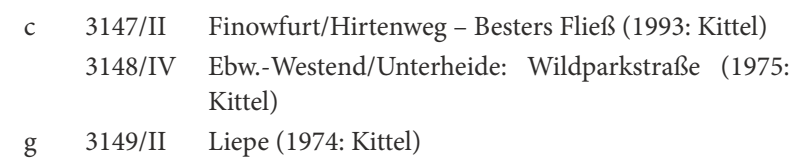

Fundorte (Ergänzung):

a 3249/II Falkenberg Umg./Cöthener Forst: Am Klingenden Fließ unterhalb des Bismarck-Turmes (2014)

e 3049/IV Chorin Umg./Forst Chorin: Teerbrennerberge - Am Olberg (2013: MüLler 2015), Kreuzung Denglerweg - Olbergstraße (2013: MüLler 2015)

d 3047/III Groß Schönebeck/Ortsgebiet: Friedenstraße, im Garten (2018: Haase)

\section{1 (9565) Agrochola lychnidis (DENIs \& SCHIF- FERMÜLLER, 1775)}

Ältere Funde (Belege in coll. Kittel) werden ergänzend nachgetragen.

Phänologie: 13.IX.(1974), aFA, (korrigiert nach Beleg in coll. Kittel; in Richert 2014 war der 15.IX.(2003) als aFA angegeben).

Neue Nachweise von bekannten Fundorten:

d 3149/I Britz Umg./F. Britz (1972: Kittel)

g 3149/II Liepe (1974: Kittel)

Fundorte (Ergänzung):

b 3149/III Ebw.-Ostende (1982, 1983: Kittel)

\section{2 (9566) Agrochola circellaris (HufNAGEL, 1766)}

Die Beobachtungslücke im Naturraum f (Parsteiner Becken, RICHERT 2003) konnte inzwischen geschlossen werden.

\section{3 (9569) Agrochola lota (ClerCK, 1759)}

Neue Nachweise von bekannten Fundorten:

\footnotetext{
c 3147/I Zerpenschleuse/Ortsgebiet: Berliner Straße, im Garten (2014, 2015: Busse)

3147/II Finowfurt/Hirtenweg - Besters Fließ (1995: Kittel)

3148/IV Ebw.-Westend/Unterheide: Wildparkstraße (1973, 1976, 1978: Kittel)
}

\author{
Fundorte (Ergänzung): \\ d 3047/III Groß Schönebeck/Ortsgebiet: Friedenstraße, im \\ Garten (2015: Haase)
}

\section{4 (9571) Agrochola macilenta (HüBNER, 1809)}

Neue Nachweise von bekannten Fundorten:

c 3147/I Zerpenschleuse/Ortsgebiet: Berliner Straße, im Garten (2014: Busse)

3147/II Finowfurt/Hirtenweg - Besters Fließ (1986, 1987, 1988, 1990: Kittel)

3148/III Ebw. OT Finow/Stadtgebiet: Altenhofer Straße, im Garten $(2014,2017)$

Fundorte (Ergänzung):

b 3149/III Sommerfelde Umg./ehemaliges Militärgelände südlich der Straße nach Ebw.(2017: Richert \& Schwabe)

d 3047/III Groß Schönebeck/Ortsgebiet: Friedenstraße, im Garten (2015, 2017: Haase)

\section{5 (9673) Agrochola nitida (DENIS \& SCHIF- FERMÜLLER, 1775) = lucida HUFNAGEL, 1766}

- RL Land Brandenburg: Kategorie 3 (gefährdet), EW, WS;

- RL Deutschland (2011): Kategorie 3 (gefährdet).

Fundorte (Ergänzung):

g 3150/I Oderberg/am Friedhof (2016)

\section{6 (9575) Agrochola helvola (Linnaeus, 1758)}

Die Beobachtungslücke im Naturraum f (Parsteiner Becken, Richert 2003) konnte inzwischen geschlossen werden.

Alle mir bekannten Fundorte im Gebiet werden nachgetragen.

Phänologie/Voltinismus: Univoltin. Die Art beginnt im Gebiet früher zu fliegen als bisher bekannt: Nach Datenreihen aus dem Choriner Endmoränenbogen von 2013 wurde der F ohne Unterbrechung beobachtet seit dem 27.VIII.(2013), aFA, phänologische Verfrühung 21d (korrigiert nach MüLler 2015; in RiCHERT 2014 war der 17.IX.(1963) u. 2009 als aFA angegeben).

\section{Fundorte:}

a 3249/II Falkenberg Umg./Cöthener Forst: ehemalige F. Tobbenberge (1982)

b 3148/IV Ebw.-Ostende/Hausberg: Bruno-H.-Bürgel-Schule (1989), am Waldfriedhof (1922: Jonas); Ebw./Oberheide: Institut für Waldschutz (1960: Kruel)

3149/III Sommerfelde Umg.ehemaliger TÜP Panzerbahn (2005) (RICHERT 2006), Barnimhänge nördlich des Ortes (2011, 2012: Schwabe), Nordostrand des Hohenfinower Waldes (2005: Ockruck)

3247/I Prenden/westlicher Ortsrand (2003: Gelbrecht \& Noack)

3248/II Trampe Umg./ehemaliger TÜP (2005) (RICHERT 2006) 
c 3147/I Zerpenschleuse/Ortsgebiet (1984, 1985, 1986, 1987, 1988, 1989: Ockruck; 2012, 2014, 2017: Busse) (Busse \& OCKRUCK 1991); Marienwerder Umg./ Oder-Havel-Kanal nordwestlich des Ortes (2014: Busse)

3147/II Finowfurt Umg./Besters Fließ - Hirtenweg (1985, 1986, 1989-1992: Kittel)

3147/III Zerpenschleuse Umg./Forst Liebenwalde nahe der Lottscher Brücke (2010: Ockruck)

3148/I Finowfurt OT Steinfurt Umg./Kiesgrube westlich der Barschgrube (1995); Ebw. OT Finow Umg./ Mäckersee $(1963,2001)$

3148/III Ebw. OT Finow/Stadtgebiet: Im Garten (1964, 19661971, 1973: Duckert; 1961-1963, 1967, 1998, 2001, 2009, 2011: Richert) und Umg./westlicher Stadtrand: Schwarzer Adler (1963), Forst Finowtal: F. Schwärze (1963), 1. Melchower Weg nordöstlich vom Kl. Postluch (2004), südlich vom Flugplatz (1975), Gr. Postluch (1990), Samithseemoor (2006)

3148/IV Ebw./Stadtgebiet (1961: Jarisch), Ebw.-Westend/ Unterheide: Wildparkstraße (1973, 1974, 1975, 1976: Kittel)

3149/III Stecherschleuse Umg./ehemaliges Kiesgrubengelände nördlich des Ortes (1997)

d 3048/III Hubertusstock/Engere Schorfheide: Parkplatz an der Ostseite (2000) und Umg./Kl. Kaisergrund (2006, 2009), Gr. Kaisergrund (2006, 2007); Joachimsthal Umg./Forst Grimnitz: Revier Michen, Abt. 40/41 (Schorfheide) (2006) (NSG Kienhorst/Köllnseen/ Eichheide, RICHERT 2010)

3147/I Groß Schönebeck Umg./„Sumpf bei Gardix“ (1997: Ockruck)

3147/II Eichhorst Umg./Üderheide: F. Grünenberg (1963, 1994), Moospfuhl östlich der Straße nach Finowfurt (1969)

3148/I Werbellin Umg./NSG Buckowseerinne: Südlich der Autobahnanschlussstelle (2011) (RICHERT 2012)

3149/I Britz Umg./F. Britz (1972: Kittel, um 1980: Riegel)

e 2948/IV Glambeck Umg./Forst Grumsin: Waldstraße nach Joachimsthal westlich F. Bärendikte (2004)

3049/III Chorin Umg./Forst Chorin: Südöstlich Pferdeberg (2013: MÜLLER 2015)

3049/IV Chorin Umg./Forst Chorin: Teerbrennerberge - Am Olberg (2013: MüLler 2015), Kreuzung Denglerweg - Olbergstraße (2013: MÜLler 2015)

3149/II Liepe Umg./Forst Chorin: F. Liepe (1964)

f 3049/IV Brodowin/Krugberg (2014)

g 3050/II Stolzenhagen/NSG Krähen- und Jungfernberge (1979) (RICHERT 1982)

3149/IV Niederfinow Umg./Oderhänge - ehemaliges Kiesgrubengelände südwestlich des Schiffshebewerks $(1995,1997)$

3150/I Oderberg/NSG Pimpinellenberg (1988, 2011) (Richert 1994), Teufelsberg (1990, 1993) (RICHERT 1994)

3150/III Gabow/Granitberggebiet (1985: Richert; 1991: Kleße)

g 3150/III Neuenhagen Umg./ehemaliges Kiesgrubengelände östlich der Straße nach Oderberg (2010: Ockruck)

\section{7 (9586) Agrochola litura (Linnaeus, 1758)}

Alle mir bekannten Fundorte im Gebiet werden nachgetragen.
Phänologie: Univoltin, 21.VIII.(1989), aFA (korrigiert nach Beleg in coll. Kittel; in RicherT 2014 war der 09.IX. (1989) als aFA angegeben).

\section{Fundorte:}

b 3148/IV Ebw.-Ostende/Hausberg: Bruno-H.-Bürgel-Schule (1989); Ebw. Umg./Oberheide: Bernauer Heerstraße (1906: Jonas); Spechthausen Umg./,Wiese an der Schwärze“ (1921: Jonas)

3149/IV Hohenfinow/Ortsgebiet (1987: Hülbert) (RICHERT \& HüLBERT 1991)

3247/I Prenden/westlicher Ortsrand (2003: Gelbrecht \& Noack)

3247 Biesenthal Umg. (1980: Fichtner)

c 3146/II Zerpenschleuse Umg./Hammerbruch (1998: Ockruck) (westlich angrenzendes Nachbargebiet)

3147/I Zerpenschleuse/Ortsgebiet (1981, 1987, 1988, 1997: Ockruck; 2013, 2014, 2015, 2016: Busse) und Umg./Straße nach Liebenwalde (1981, 1988), Weg nach Böhmerheide (1980,1995: Ockruck) (Busse \& OCKRUCK 1991)

3147/II Finowfurt Umg./Besters Fließ - Hirtenweg (1986, 1989-1991, 1993: Kittel)

3148/I Finowfurt OT Steinfurt Umg./Kiesgrube westlich der Barschgrube (1995, 1997); Ebw. OT Finow Umg./ Mäckersee (1963)

3148/III Ebw. OT Finow/Stadtgebiet: Im Garten (1964-1967, 1969-1971, 1973, 1974: Duckert; 1961, 1962, 1967, 1987, 2010, 2017: Richert) und Umg./Forst Finowtal: F. Schwärze (1963), Gr. Postluch westlich der Biesenthaler Straße (1990)

3149/I Sandkrug Umg. (1921: Jonas)

d 3048/III Jagdschloss Hubertusstock/Engere Schorfheide: Parkplatz an der Ostseite (2000) und Umg./ Gr. Kaisergrund (2006), Kl. Kaisergrund (2009); Joachimsthal Umg./Forst Grimnitz: Revier Michen (Schorfheide), Abt. 40/41 (2006) (NSG Kienhorst/ Köllnseen/Eichheide, RicherT 2010

3048/IV Golzow Umg./nördlich des Ortes (1994: Sommer DEI)

3147/I Groß Schönebeck Umg./,Sumpf bei Gardix“ (1997: Ockruck)

3147/II Eichhorst Umg./Üderheide: F. Grünenberg (1963, 1994), Moospfuhl östlich der Straße nach Finowfurt (1969)

3148/I Werbellin Umg./NSG Buckowseerinne: Südlich der Autobahnanschlussstelle (2010) (RICHERT 2012)

3149/I Britz Umg./F. Britz (1980-83: Riegel)

e 2948/IV Glambeck Umg./Autobahn (1982: Weidlich), Forst Grumsin: An der Staße nach Joachimsthal westlich F. Bärendikte (2004)

3149/II Liepe Umg./Forst Chorin: F. Liepe (1964)

f 3049/IV Brodowin Umg./Kl. Rummelsberg (1992: Sommer DEI), Krugberg (2014)

g 3050/II Stolzenhagen/NSG Krähen- und Jungfernberge (1979) (RICHERT 1982)

3149/IV Niederfinow Umg./Oderhänge - ehemalige Kiesgruben südwestlich des Schiffshebewerks (1995)

3150/I Oderberg/NSG Pimpinellenberg (2011), Teufelsberg (1988) (RICHERT 1994)

3150/III Gabow/Granitberggebiet (1985: Richert; 1991: Kleße) 


\section{8 (9588) Agrochola laevis (HÜBNER, 1803)}

Für die bei uns seltene Art liegen zwei neue Einzelfunde vor: 04.IX.2013, Forst Chorin; der F wurde auf einer Windwurffläche an den Teerbrennerbergen a.L. gefangen (Müller 2015, Beleg lag mir vor). Damit ist die Art auch im Naturraum e (Uckermärker Kuppen- und Hügelland einschließlich Choriner Waldhügel (RICHERT 2003)) nachgewiesen. 11.XI.2015, Groß Schönebeck/Ortsgebiet: Friedenstraße ein F im Garten am Köder leg. Haase; Beleg lag mir vor. Dieser Fund zeigt, dass die Art bedeutend länger fliegt als bisher bekannt, er kennzeichnet zugleich das absolute Flugzeitende im Gebiet.

Phänologie/Voltinismus: Univoltin; die Flugzeitangabe ( $\mathrm{n}=6$ datumsgenaue Falterfunde) kann wie folgt präzisiert werden: 04.IX.(2013) - 11.XI.(2015).

\section{Fundorte (Ergänzung):}

e 3049/IV Chorin Umg./Forst Chorin: Teerbrennerberge - Am Olberg (2013: MÜLLER 2015)

d 3047/III Groß Schönebeck/Ortsgebiet: Friedenstraße. Im Garten (2015: Haase)

\section{9 (9596) Eupsilia transversa (HufNAGEL, 1766) = satellitia LINNAEUs, 1767}

Die F der überwinternden Art sind bei günstiger Witterung jahrweise bis E XI aktiv: Am 21.XI.2003 wurden drei F a.L., am 24.XI.2017 zwei F a.K. beobachtet.

\section{2 (9603) Conistra rubiginosa (SCOPOLI, 1763) = vau-punctatum ESPER, 1786}

Neue Nachweise von bekannten Fundorten:

c 3147/I Zerpenschleuse/Ortsgebiet: Berliner Straße, im Garten (2014, 2015, 2017, 2018: Busse)

3148/III Ebw. OT Finow/Stadtgebiet: Altenhofer Straße, im Garten (2017)

Fundorte (Ergänzung):

c 3147/II Finowfurt/Hirtenweg - Besters Fließ (1987, 1996; Kittel)

3148/IV Ebw.-Westend/Unterheide: Wildparkstraße (19731975, 1977: Kittel); Ebw./Stadtgebiet: Kleingartenanlage im Schwärzetal nördlich Forstbotanischer Garten (2017, 2018: Schwabe)

d 3047/III Groß Schönebeck/Ortsgebiet: Friedenstraße, im Garten (2014, 2017: Haase)

\section{3 (9609) Conistra rubiginea (DenIs \& SCHIF-} FERMÜLLER, 1775)

Neue Nachweise von bekannten Fundorten:

c 3148/III Ebw. OT Finow/Stadtgebiet: Altenhofer Straße, im Garten (2014)

Fundorte (Ergänzung):

c 3148/IV Ebw./Stadtgebiet: Kleingartenanlage im Schwärzetal nördlich Forstbotanischer Garten (2015, 2016, 2017, 2018: Schwabe) g 3150/I Oderberg/Geistberg (2016: Schwabe)

\section{4 (9611) Conistra erythrocephala (DeNis \& SCHIFFERMÜLLER, 1775)}

Neue Nachweise von bekannten Fundorten:

c 3147/I Zerpenschleuse/Ortsgebiet: Berliner Straße, im Garten (2014, 2015, 2016: Busse)

Fundorte (Ergänzung):

b 3149/III Sommerfelde Umg./ehemaliges Militärgelände südlich der Straße nach Ebw. (2017)

c 3147/I Finowfurt Umg./Kesselmoor an der Finowfließniederung nordöstlich des Lehnssees (2016)

3148/IV Ebw./Stadtgebiet: Kleingartenanlage im Schwärzetal nördlich Forstbotanischer Garten (2018: Schwabe)

d 3047/III Groß Schönebeck/Ortsgebiet: Friedenstraße, im Garten (2015: Haase)

e 3149/II Liepe Umg./Forst Chorin: NSG Plagefenn südlich Gr. Plagesee (2018: Schwabe)

\section{5 (9649) Aporophila lutulenta (DENIS \& SCHIF-} FERMÜLLER, 1775)

- RL Land Brandenburg: Kategorie 3 (gefährdet): CB, TR.

Die Beobachtungslücke im Naturraum f (Parsteiner Becken, RICHERT 2003) wurde inzwischen geschlossen.

Neue Nachweise von bekannten Fundorten:

c 3147/I Zerpenschleuse/Ortsgebiet: Berliner Straße, im Garten (2014, 2015, 2016, 2017: Busse)

Fundorte (Ergänzung)

f 3049/IV Brodowin/Krugberg (2014)

446 (9655) Lithomoia solidaginis (HÜBNER, 1803)

Neue Nachweise von bekannten Fundorten:

c 3147/II Finowfurt/Hirtenweg - Besters Fließ (1990: Kittel)

Fundorte (Ergänzung):

c 3148/III Ebw. OT Finow Umg./Forst Finowtal: Einflug schneise Flugplatz (2017: Schwabe)

\section{7 (9658) Lithophane socia (HUFNAGEL, 1766)}

Neue Nachweise von bekannten Fundorten:

c 3147/I Zerpenschleuse/Ortsgebiet: Berliner Straße, im Garten (2015: Busse)

Fundorte (Ergänzung):

c 3147/IV Marienwerder Umg./Kiesgrube südwestlich des Sportplatzes (2015)

3148/IV Ebw./Stadtgebiet: Kleingartenanlage im Schwärzetal nördlich Forstbotanischer Garten (2016, 2017, 2018 Schwabe) 


\section{8 (9660) Lithophane ornitopus (HUFNAGEL, 1766)}

Phänologie/Voltinismus: Univoltin; 19.XII.2014, 1 F a.K. leg. Haase in Groß Schönebeck. Der Fund zeigt, dass die überwinternde Art bei günstigem Wetter auch im Winter aktiv ist. Schwabe beobachtete bereits am 03.IV.2016 in Eberswalde einen F a.L. (drei Tage früher nach der Überwinterung als bisher bekannt (vgl. RiCHERT 2014).

\section{Neue Nachweise von bekannten Fundorten:}

c 3147/I Zerpenschleuse/Ortsgebiet: Berliner Straße, im Garten (2015, 2017: Busse)

3147/II Finowfurt/Hirtenweg - Besters Fließ (1989, 1993, 1994, 1995: Kittel)

Fundorte (Ergänzung):

b 3149/III Sommerfelde Umg./ehemaliges Militärgelände südlich der Straße nach Eberswalde (2017)

d 3047/III Groß Schönebeck/Ortsgebiet: Friedenstraße, im Garten (2014: Haase)

\section{9 (9661) Lithophane furcifera (HuFNAGEL, 1766)}

- RL Deutschland (2011): Kategorie 3 (gefährdet). Die Beobachtungslücke im Naturraum e (Uckermärker Kuppen- und Hügelland einschließlich Choriner Waldhügel, vgl. RicherT 2003) wurde inzwischen geschlossen: Im Forst Chorin wurden vom 01.-27.IX.2013 $3 \mathrm{~F}$ auf einer Windwurffläche im Buchenwald an den Teerbrennerbergen registriert (MÜLLER 2015).

Neue Nachweise von bekannten Fundorten:

a 3149/IV Falkenberg (1973: Kittel)

c 3147/I Zerpenschleuse/Ortsgebiet: Berliner Straße, im Garten (2014, 2016: Busse)

3148/III Ebw. OT Finow/Stadtgebiet: Altenhofer Straße, im Garten (2017)

3148/IV Ebw.-Westend/Unterheide: Wildparkstraße: 1969_ 1974, 1976, 1984: Kittel)

Fundorte (Ergänzung):

c 3148/IV Ebw./Stadtgebiet Kleingartenanlage im Schwärzetal nördlich des Forstbotanischen Gartens (2015, 2016: Schwabe)

d 3047/III Groß Schönebeck/Ortsgebiet: Friedenstraße, im Garten (2014: Haase)

e 3049/IV Chorin Umg./Forst Chorin: Teerbrennerberge - Am Olberg (2013: MüLLER 2015)

\section{3 (9682) Allophyes oxyacanthae (LINNAEus, 1758)}

Phänologie/Voltinismus: Aus dem Jahre 2013 liegen kontinuierliche Beobachtungsdaten aus dem Forst Chorin vor, vom 26.VIII. bis 29.X. (Ende der Untersuchungen am Ort, nicht der Flugzeit). Insgesamt wurden $97 \mathrm{~F}$ gezählt. 26.VIII.(2013), aFA, phänologische Verfrühung 22d (korrigiert nach MüLler 2015; in RICHERT 2014 war der17. IX.(2010) als aFA angegeben).
Neue Nachweise von bekannten Fundorten:

c 3147/I Zerpenschleuse/Ortsgebiet: Berliner Straße, im Garten (2014: Busse)

3148/III Ebw. OT Finow/Stadtgebiet: Altenhofer Straße, im Garten $(2014,2017)$

Fundorte (Ergänzung):

b 3149/III Ebw.-Ostende (1972: Kittel); Sommerfelde Umg./ ehemaliges Militärgelände südlich der Straße nach Ebw. (2017: Schwabe)

c 3147/II Finowfurt/Hirtenweg - Bester Fließ (1986, 1987, 1995: Kittel)

3148/IV Ebw.-Westend/Unterheide: Wildparkstraße (1973: Kittel)

d 3047/III Groß Schönebeck/Ortsgebiet: Friedenstraße, im Garten (2015, 2017: Haase)

e 3049/III Chorin Umg./Forst Chorin: Südöstlich des Pferdebergs (2013: MüLlER 2015)

3049/IV Chorin Umg./Forst Chorin: Teerbrennerberge - Am Olberg (2013: MüLlER 2015), Kreuzung Denglerweg - Olbergstraße (2013: MÜLler 2015)

\section{4 (9694) Dichonia aprilina (Linnaeus, 1758)}

- RL Land Brandenburg: Vorwarnliste: EW;

- RL Deutschland (2011): Vorwarnliste.

Neue Nachweise von bekannten Fundorten:

c 3147/I Zerpenschleuse/Ortsgebiet: Berliner Straße, im Garten (2014, 2017: Busse)

3148/III Ebw. OT Finow/Stadtgebiet: Altenhofer Straße, im Garten (2014)

456 (9699) Dryobotodes eremita (FABRICIUS, $1775)=$ protea DENIS \& SCHIFFERMÜLLER, 1775

Die Beobachtungslücke im Naturraum f (Parsteiner Becken, RICHERT 2003) wurde inzwischen geschlossen.

Neue Nachweise von bekannten Fundorten:

c 3147/II Finowfurt/Hirtenweg - Besters Fließ (1990, 1995 Kittel)

Fundorte (Ergänzung):

f 3049/IV Brodowin/Krugberg (2014: Schwabe)

\section{7 (9699) Antitype chi (Linnaeus, 1758)}

Neue Nachweise von bekannten Fundorten:

c 3147/II Finowfurt/Hirtenweg - Besters Fließ (1986, 1987, 1995, 1997: Kittel)

3148/III Ebw. OT Finow/Stadtgebiet: Altenhofer Straße, im Garten (2014)

Fundorte (Ergänzung):

b 3247/II Biesenthal Umg./NSG Biesenthaler Becken: Langerönner Weg (2014: Ockruck)

d 3047/III Groß Schönebeck Umg./Liebenthaler Weg (2015: Haase) 


\section{8 (9710) Ammoconia caecimacula (DENIS \& SCHIFFERMÜLLER, 1775)}

Phänologie/Voltinismus: Univoltin; 04.IX.(2013), aFA, phänologische Verfrühung 4d (korrigiert nach MüLLER 2015; in Richert 2014 war der 08.IX.(1960) als aFA angegeben).

\section{Neue Nachweise von bekannten Fundorten:}

$$
\begin{aligned}
& \text { c 3147/I Zerpenschleuse/Ortsgebiet: Berliner Straße, im } \\
& \text { Garten (2014, 2016, 2017: Busse) }
\end{aligned}
$$

\section{Fundorte (Ergänzung):}

b 3247/II Biesenthal Umg./NSG Biesenthaler Becken: Langerönner Weg (2014: Ockruck)

c 3147/I Marienwerder Umg./Oder-Havel-Kanal nordwestlich des Ortes (2014: Busse)

d 3047/III Groß Schönebeck/Ortsgebiet: Friedenstraße, im Garten (2018: Haase)

e 3049/III Chorin Umg./südöstlich des Pferdebergs (2013: MÜLler 2015)

3049/IV Chorin Umg./Teerbrennerberge - Am Olberg (2013: Müller 2015), Kreuzung Denglerweg - Olbergstraße (2013: MÜLLER 2015)

\section{9 (9734) Polymixis gemmea (TreitschKe,} 1825)

- Verordnung zur Neufassung der BArtschV von 2005: Besonders geschützte Art.

Neue Nachweise von bekannten Fundorten:

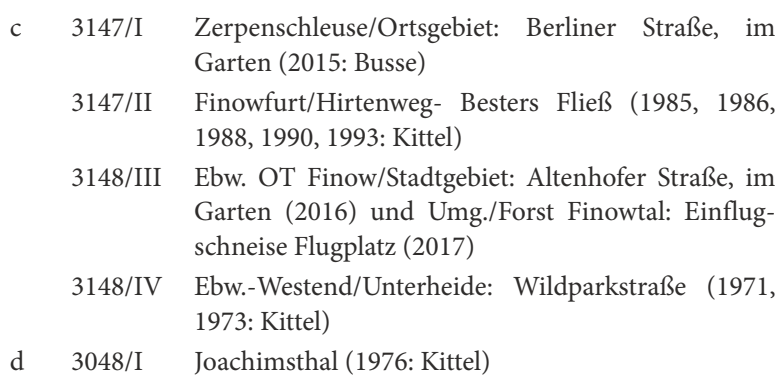

\section{0 (9738) Blepharita satura (DeNIS \& SCHIFFER- MÜLLER, 1775)}

Die Beobachtungslücke im Naturraum f (Parsteiner Becken, RicherT 2003) konnte inzwischen geschlossen werden. Damit ist die Art in allen Naturräumen des Gebietes nachgewiesen. STöckel (1955) bezeichnet Blepharita satura als „ausgesprochenes Waldtier“.
Aktuelle Funddaten aus dem Forst Chorin bestätigen diese Einschätzung: Vom 26.VIII.2013 - 29.X.2013 wurden $132 \mathrm{~F}$ a.L. und a.K. registriert (MüLLER 2015). Bemerkenswert ist in diesem Zusammenhang, dass Duckert die Art in 13 Jahren seiner umfangreichen Lichtfangpraxis im Siedlungsraum nur dreimal in Einzelexemplaren a.L. registrierte. Allerdings lassen LF-Ergebnisse nur eingeschränkte Schlussfolgerungen zu: Der F wurde vielfach a.K. nachgewiesen. Alle mir bekannten Funde werden nachfolgend mitgeteilt.

Phänologie/Voltinismus: Univoltin; die Art fliegt im Gebiet länger als bisher bekannt: 29.X.(2013), aFE, phänologische Verspätung 15d (korrigiert nach MüLleR 2015; in Richert 2014 war der 14.X.(2010) als aFE angegeben).

\section{Fundorte:}

a 3250/II Bad Freienwalde/Stadtgebiet (2010: Kleße)

b 3148/IV Ebw.-Ostende/Paschenberg (1981: Kruel), Breite Straße am Waldfriedhof (2013: Schwabe) und Umg./ Oberheide: Bernauer Heerstraße (1908: Jonas), F. Leuenberger Wiesen (1965: Kruel; 1975: Richert), Wiebecke-Damm am Möllergrab (2011, 2012: Schwabe); Spechthausen (1964: Rinnhofer) und Umg./Fischteiche nordöstlich des Ortes am Leuenberger Graben (2008, 2009: Schwabe)

3149/III Sommerfelde Umg./Barnimhänge nördlich des Ortes: Bachtal „am Wiesengraben“ (2008, 2011, 2012: Schwabe), Nordostrand des Hohenfinower Waldes (2004, 2005, 2007: Ockruck); Tornow Umg./ Barnimhänge nördlich des Ortes: Bachtal (2011), Abt. 629 (2011: Schwabe), am Weg nach Karlswerk (2012: Schwabe)

3149/IV Karlswerk/östlicher Ortsausgang (2005) und Umg./ NSG Tongrube (1973: Kruel), Hohenfinow/Ortsgebiet (1986: Hülbert) (RicherT \& HüLbERT 1991) und Umg./Karlswerker Straße oberhalb/südlich Karlswerk (2011)

3247/I Prenden/westlicher Ortsrand (2003: Gelbrecht \& Noack)

3247/II Biesenthal Umg. (1980: Fichtner)/NSG Biesenthaler Becken: Langerönner Weg (2014: Ockruck)

3248/II Trampe Umg./Tramper Forst: Bornemanns Pfuhl (2013: Schwabe)

c 3147/I Zerpenschleuse/Ortsgebiet (1980, 1981, 1983, 1984 1987, 1997: Ockruck; 2012-2014: Busse) und Umg./ an der Straße nach Liebenwalde (1981, 1984, 1999: Ockruck), Weg nach Böhmerheide (1980, 1987, 1995: Ockruck) (Busse \& OCKRUCK 1991); Marienwerder Umg./Oder-Havel-Kanal nordwestlich des Ortes (2016: Busse \& Müller)

3147/II Finowfurt/Besters Fließ: Hirtenweg (1986, 1987, 1989, 1991, 1995: Kittel)

3147/III Zerpenschleuse Umg./Oder-Havel-Kanal nahe der Lottscher Brücke (201: Ockruck); Ruhlsdorf Umg./ Bernsteinsee (1998: Ockruck)

3147/IV Finowfurt Umg./Kesselmoor an der Finowfließniederung nordöstlich des Lehnssees (2017: Schwabe)

3148/I Finowfurt OT Steinfurt Umg./Kiesgrube westlich der Barschgrube $(1995,1997)$ 
3148/III Ebw. OT Finow/Stadtgebiet: Altenhofer Straße, im Garten (1961-1963, 1967, 1970, 1972, 1974, 1997, 2006, 2007, 2011, 2012, 2016: Richert), Gartenstraße in der Siedlung Freie Scholle (1970, 1973, 1974: Duckert) und Umg./Forst Finowtal: Kalkofenbrück im Schwärzetal (1985), 1. Melchower Weg nordöstlich Kl. Postluch (2004), Gr. Postluch (1964, 1983), Samithseemoor $(1993,2006)$

3148/IV Ebw. OT Finow Umg./Forst Finowtal; Stromtrasse südlich des Ortes (2009); Ebw./Stadtgebiet (1923, 1939: Jonas), Schwappachweg am Pflanzgarten des Forstbotanischen Gartens (2009: Schwabe), Forstbotanischer Garten (2010: Schwabe), Kleingartenanlage im Schwärzetal nördlich des Forstbotanischen Gartens (2014, 2016, 2017: Schwabe), Ebw.-Westend: Radweg Richtung Familiengarten (2008: Ockruck), Unterheide: Wildparkstraße (1972-1974, 1976, 1980, 1981: Kittel)

d 3047/III Groß Schönebeck/Ortsgebiet: Friedenstraße, im Garten (2012, 2014: Haase)

3047/IV Groß Schönebeck Umg./Joachimsthaler Damm westlich der Pinnowseen (2004) (NSG Kienhorst/ Köllnsee/Eichheide, RichERT 2010)

3048/III Jagdschloss Hubertusstock/Engere Schorfheide: Parkplatz an der Ostseite (2000) und Umg./ Kl. Kaisergrund (2006, 2007), Gr. Kaisergrund (2006); Joachimsthal Umg./Schorfheide: Forst Grimnitz, Revier Michen, Abt. 40/41 (2006) (NSG Kienhorst/Köllnsee/Eichheide, RICHERT 2010)

3048/IV Golzow Umg./nördlich des Ortes (1994: DEI), Forst Grimnitz: Flacher Bugsinsee (1970: Kruel)

3147/II Eichhorst Umg./Forst Pechteich: An der Straße nach Gr. Schönebeck, Abt. 3381 (2011)

3148/I Werbellin Umg./NSG Buckowseerinne: Südlich der Autobahnanschlussstelle (2010, 2011); Lichterfelde Umg./NSG Buckowseerinne: Moorniederung nordöstlich des Gr. Buckowsees (2010) (Richert 2012)

3149/I Britz Umg./F. Britz (1982: Riegel)

e 2948/IV Glambeck Umg./Forst Grumsin: Straße nach Joachimsthal westlich F. Bärendikte (2004)

3049/I Groß Ziethen Umg./NSG Forst Grumsin: Langer Berg an der Waldstraße Louisenfelde-Grumsin (1994: Sommer, DEI), Schulzensee - Südufer und zwischen Schulzensee und Kl. Kagelpfuhl bei Sperlingsherberge nordwestlich des Ortes (2008: Rödel \& Schulze)

3049/III Chorin Umg./Forst Chorin: Südöstlich des Pferdebergs (2013: MüLLER 2015)

3049/IV Brodowin Umg./Forst Chorin: Mooskuten (2003), (Richert 2005) Teerbrennerberge - Am Olberg (2013: MÜLler 2015), Kreuzung Denglerweg Olbergstraße (2013: MüLLER 2015)

3149/II Liepe Umg./Forst Chorin: NSG Plagefenn (2003) (RICHERT 2005)

3150/I Liepe Umg./Forst Freienwalde: Abt. 172 ca. $1.1 \mathrm{~km}$ nördlich des Ortsausganges Richtung Oderberg (2013: Schwabe)

f 3049/IV Brodowin/Krugberg (2014)

g 3050/II Stolzenhagen/NSG Krähen- und Jungfernberge (1979, 2003) (RICHERT 1982)

3149/IV Struwenberg Umg./NSG Kanonen- und Schlossberg (1999)

3150/I Oderberg/NSG Pimpinellenberg (1988, 2011) (RICHERT 1994)

3150/III Gabow/Oderhänge (2004: Kleße)

h 3150/II Hohensaaten/Denponiegelände an der Straße nach der Siedlung (2014: Kleße)
3150/III Neuenhagen Umg./ehemaliges Kiesgrubengelände östlich der Straße nach Oderberg (2009, 2010: Ockruck)

\section{3 (9752) Apamea lithoxylaea (DENIS \& SCHIF- FERMÜLLER, 1775)}

Neue Nachweise von bekannten Fundorten:

c 3147/I Zerpenschleuse/Ortsgebiet: Berliner Straße, im Garten (2014: Busse)

3147/II Finowfurt/Hirtenweg - Besters Fließ (1991: Kittel)

3148/III Ebw. OT Finow/Stadtgebiet: Altenhofer Straße, im Garten (2014, 2017)

3148/IV Ebw.-Westend/Unterheide: Wildparkstraße (1973, 1975: Kittel)

g 3150/I Oderberg/Teufelsberg (1974: Kittel)

3150/III Gabow/Granitberggebiet (2016)

3150/IV Altglietzen/Gabower Berge (2016)

Fundorte (Ergänzung):

c $3147 / \mathrm{IV}$ Finowfurt Umg./Kesselmoor an der Finowfließniederung nordöstlich des Lehnssees(2016)

\section{4 (9753) Apamea sublustris (ESPER, 1788)}

Neue Nachweise von bekannten Fundorten:

c 3147/I Zerpenschleuse/Ortsgebiet: Berliner Straße, im Garten (2015: Busse)

3148/III Ebw. OT Finow/Stadtgebiet: Altenhofer Straße, im Garten $(2014,2017)$

g 3050/II Stolpe a.O./Trockenhänge am Stadtweg (2018)

3150/III Gabow/Granitberggebiet (2016)

3159/IV Altglietzen/Gabower Berge (2016)

Fundorte (Ergänzung):

c 3148/IV Ebw.-Westend/Unterheide: Wildparkstraße (1970, 1973: Kittel)

d 3149/I Britz Umg./F. Britz (1971, 1973: Kittel)

\section{5 (9755) Apamea crenata (HufNAGEL, 1766) = rurea FABRICIUS 1775}

Neue Nachweise von bekannten Fundorten:

b 3247/II Biesenthal Umg./NSG Biesenthaler Becken: Langerönner Weg (2014: Ockruck)

c 3147/II Finowfurt/Hirtenweg - Besters Fließ (1992, 1993, 1996, 1997: Kittel)

3148/III Ebw. OT Finow/Stadtgebiet: Altenhofer Straße, im Garten $(2014,2018)$

Fundorte (Ergänzung):

c 3147/IV Finowfurt Umg./Kesselmoor an der Finowfließniederung nordöstlich des Lehnssees $(2016,2017)$

3148/IV Ebw.-Westend/Unterheide: Wildparkstraße (1970, 1976, 1981: Kittel); Ebw./Stadtgebiet: Kleingartenanlage im Schwärzetal nördlich Forstbotanischer Garten (2016, 2018: Schwabe)

d 3149/I Britz Umg./F. Britz (1972: Kittel)

g 3149/II Liepe (1972: Kittel) 
466 (9756) Apamea epomidion (HAWORTH, 1809) $=$ hepatica auct.

Neue Nachweise von bekannten Fundorten:

c 3147/II Finowfurt/Hirtenweg - Besters Fließ (1991: Kittel)

3148/IV Ebw.-Westend/Unterheide: Wildparkstraße (1973: Kittel)

3150/I Oderberg/Teufelsberg $(1973,1975,1976:$ Kittel $)$

Fundorte (Ergänzung):

c 3148/IV Ebw./Stadtgebiet: Kleingartenanlage im Schwärzetal nördlich Forstbotanischer Garten (2016: Schwabe)

d 3047/III Groß Schönebeck/Ortsgebiet: Friedenstraße, im Garten (2018: Haase)

\section{7 (9758) Apamea lateritia (Hufnagel, 1766)}

Neue Nachweise von bekannten Fundorten:

c 3147/II Finowfurt/Hirtenweg - Besters Fließ (1996: Kittel)

3148/III Ebw. OT Finow/Stadtgebiet: Altenhofer Straße, im Garten (2014)

d 3149/I Britz Umg./F. Britz (1971: Kittel)

Fundorte (Ergänzung):

c 3148/IV Ebw.-Westend/Unterheide: Wildparkstraße (1970: Kittel)

d 3047/III Groß Schönebeck/Ortsgebiet: Friedenstraße, im Garten (1999: Haase)

\section{0 (9766) Apamea remissa (HüBNER, 1809 = obscura HAWORTH, 1809}

Neue Nachweise von bekannten Fundorten:

c 3148/III Ebw. OT Finow/Stadtgebiet: Altenhofer Straße, im Garten $(2014,2015)$

g 3149/II Liepe (1973, 1977: Kittel)

3150/I Oderberg/Teufelsberg (1975: Kittel)

Fundorte (Ergänzung):

b 3247/II Biesenthal Umg./NSG Biesenthaler Becken: Weg nördlich der Pfauenwiesen (2014: Ockruck)

c 3148/IV Ebw.-Westend/Unterheide: Wildparkstraße (1969, 1970, 1973, 1975: Kittel)

e 3049/I Groß Ziethen Umg./Steingruben an den Ihlowbergen (2014)

g 3150/I Oderberg/Geistberg (2017: Schwabe)

\section{1 (9767) Apamea unanimis (HüBNER, 1813)}

Neue Nachweise von bekannten Fundorten:

c 3148/III Ebw. OT Finow/Stadtgebiet: Altenhofer Straße, im Garten (2015)

g 3149/II Liepe (1973: Kittel)

3150/III Gabow/Granitberggebiet (2015: Schwabe)

Fundorte (Ergänzung):

c 3147/II Finowfurt/Hirtenweg - Besters Fließ (1991: Kittel)

3147/IV Marienwerder/Steinlaake (2015)
3148/IV Ebw./Stadtgebiet: Kleingartenanlage im Schwärzetal nördlich des Forstbotanischen Gartens (2014: Schwabe)

\section{4 (9774) Apamea scolopacina (ESPER, 1788)}

Neue Nachweise von bekannten Fundorten:

c 3147/I Zerpenschleuse/Ortsgebiet: Berliner Straße, im Garten (2014: Busse)

3147/II Finowfurt/Hirtenweg - Besters Fließ (1987, 1993, 1995: Kittel)

3148/III Ebw. OT Finow/Stadtgebiet: Altenhofer Straße, im Garten $(2015,2017)$

3148/IV Ebw.-Westend/Unterheide: Wildparkstraße (1970, 1973, 1981: Kittel)

g 3050/II Stolpe a.O./Trockenhänge am Stadtweg (2018)

3150/I Oderberg/Teufelsberg (1975: Kittel)

Fundorte (Ergänzung):

b 3247/II Biesenthal Umg./NSG Biesenthaler Becken: Weg nördlich der Pfauenwiesen (2014: Ockruck)

c 3147/IV Finowfurt Umg./Kesselmoor an der Finowfließniederung nordöstlich des Lehnssees $(2016,2017)$

3148/IV Ebw./Stadtgebiet: Kleingartenanlage nördlich Forstbotanischer Garten (2017: Schwabe)

e 3049/I Groß Ziethen Umg./Steingruben an den Ihlowbergen (2014)

h 3150/II Hohensaaten/Deponiegelände an der Straße nach der Siedlung (2014: Kleße)

\section{5 (9775) Apamea ophiogramma (EsPER, 1788)}

Die Beobachtungslücke im Naturraum h (Odertal, RICHERT 2003) ist inzwischen geschlossen worden (vgl. RICHERT 2014).

Neue Nachweise von bekannten Fundorten:

c 3147/II Finowfurt/Hirtenweg - Besters Fließ (1995: Kittel)

3148/III Ebw. OT Finow/Stadtgebiet: Altenhofer Straße, im Garten (2015)

d 3047/III Groß Schönebeck/Ortsgebiet: Friedenstraße, im Garten (2014: Haase)

g 3149/II Liepe (1974: Kittel)

3150/I Oderberg/Teufelsberg (1974, 1975: Kittel)

3150/III Gabow/Granitberggebiet (2015: Schwabe)

Fundorte (Ergänzung):

c 3148/IV Ebw./Stadtgebiet: Kleingartenanlage im Schwärzetal nördlich des Forstbotanischen Gartens (2014: Schwabe)

e 3049/I Groß Ziethen Umg./Steingruben an den Ihlowbergen (2014)

\section{7 (9781) Oligia versicolor (BORKHAUSEN, 1793)}

Neue Nachweise von bekannten Fundorten:

c 3147/I Zerpenschleuse/Ortsgebiet: Berliner Straße, im Garten (2014: Busse)

3147/II Finowfurt/Hirtenweg - Besters Fließ (1993, 1994: Kittel) 
3148/III Ebw. OT Finow/Stadtgebiet: Altenhofer Straße, im Garten $(2014,2015,2018)$

g 3149/II Liepe (1976: Kittel)

Fundorte (Ergänzung):

c 3147/IV Finowfurt Umg./Kesselmoor an der Finowfließniederung nordöstlich des Lehnssees (2017: Richert \& Schwabe)

3148/IV Ebw.-Westend/Unterheide: Wildparkstraße (1973, 1975: Kittel)

d 3148/II Britz (1979: Kittel)

e 3049/I Groß Ziethen Umg./Steingruben an den Ihlowbergen (2014)

\section{9 (9784) Oligia fasciuncula (HAwOrTH, 1809)}

Phänologie/Voltinismus: Univoltin; 23.VII.(1970), aFE (korrigiert nach Beleg in coll. Kittel; in RICHERT 2014 war der 17.VII.(1962) als aFE angegeben).

Neue Nachweise von bekannten Fundorten:

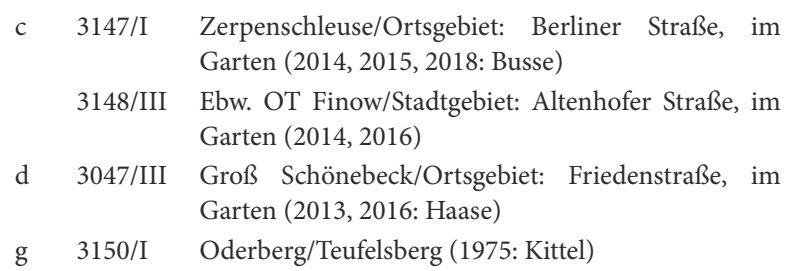

Fundorte (Ergänzung):

b 3247/II Biesenthal Umg./NSG Biesenthaler Becken: Langerönner Weg (2014: Ockruck)

c 3147/IV Finowfurt Umg./Kesselmoor an der Finowfließniederung nordöstlich des Lehnssees (2016)

3148/III Finowfurt (1972: Kittel)

3148/IV Ebw.-Westend/Unterheide: Wildparkstraße (1969, 1970, 1975: Kittel)

d 3149/I Britz Umg./F. Britz (1971, 1972: Kittel)

\section{3 (9790) Mesapamea didyma (EsPER, 1788) = secalella REMM, 1983}

Die Beobachtungslücke im Naturraum h (Odertal, RICHERT 2003) wurde inzwischen geschlossen.

Fundorte (Ergänzung):

g 3150/III Gabow/Granitberggebiet (2013, det. genit. Schwabe)

h 3150/II Hohensaaten/Deponiegelände an der Straße nach der Siedlung (2014, det. genit. Kleße)

\section{5 (9797) Eremobia ochroleuca (DENIS \& SCHIF- FERMÜLLER, 1775)}

Siehe wieder entdeckte Arten.

\section{6 (9801) Luperina testacea (DENIS \& SCHIFFER- MÜLLER, 1775)}

Alle mir aus dem Gebiet bekannten Funde werden nachfolgend mitgeteilt.
Phänologie/Voltinismus: Univoltin; 30.VII.(2014), aFA, phänologische Verfrühung 7d gegenüber bis 2013.

Fundorte:

a 3249/II Falkenberg Umg./Cöthener Forst: Ehemalige F. Tobbenberge (1970)

b 3148/IV Spechthausen (1965: Rinnhofer)

3149/III Sommerfelde Umg./ehemaliger TÜP Panzerbahn (2005) (RICHERT 2006); Tornow Umg./Barnimhänge nördlich des Ortes (2000, 2008: Ockruck), Abt. 629 (2010: Schwabe)

3149/IV Struwenberg Umg./Am Weg nach Karlswerk (1974); Hohenfinow/Ortsgebiet (1987: Hülbert) (RICHERT \& HüLBERT 1991)

3247/I Prenden Umf./Kiesgrube zwischen Prenden und Lanke (2000: Ockruck)

3247/II Biesenthal Umg. (1982: Fichtner)/NSG Biesenthaler Becken: Nördlich der Pfauenwiesen (2015: Ockruck)

3248/II Trampe/Ortsgebiet (2005) und Umg./ehemaliger TÜP $(1999,2000)$ (RICHERT 2006)

c 3146/IV Zerpenschleuse Umg./Hammerbruch (1998: Ockruck) (westlich angrenzendes Nachbargebiet)

3147/I Zerpenschleuse/Ortsgebiet (1980, 1981, 19831988: Ockruck; 2011, 2013, 2014: Busse) (Busse \& OCKRUCK 1991)

3147/II Finowfurt Umg./Besters Fließ - Hirtenweg (19901992: Kittel)

3147/III Klosterfelde Umg./Lottscheseegebiet: NSG Torfstich (1998: Ockruck); Marienwerder Umg./Oder-HavelKanal (1997: Ockruck)

3147/IV Marienwerder Umg./Grafenbrück (2000: Ockruck)

3148/III Ebw. OT Finow/Stadtgebiet: Im Garten (1961-1964, 1967, 1968, 2002, 2009, 2010, 2015: Richert; 1961: Duckert) und Umg./Forst Finowtal: F. Schwärze (1963), Samithseemoor (1993)

3148/IV Ebw. OT Finow Umg./Forst Finowtal: Stromtrasse südlich des Ortes (2007, 2009); Ebw.-Westend/ Unterheide: Wildparkstraße (1969, 1976: Kittel)

3149/III Sommerfelde Umg./Finowbruch nördlich des Ortes an der Bahnlinie (2008: Schwabe)

3149/IV Stecherschleuse Umg./am Rande des ehemaligen Kiesgrubengeländes nordöstlich des Ortes (2009: Schwabe)

d 3047/IV Groß Schönebeck Umg./Schorfheide: Pinnowseengebiet - Meelake (1991: Ockruck), Sarnow: Grahsee (1999: Ockruck) (NSG Kienhorst/Köllnseen/Eichheide; RICHERT 2010)

3048/III Jagdschloss Hubertusstock Umg./Engere Schorfheide: Kleiner Kaisergrund (2009) (NSG Kienhorst/ Köllnseen/Eichheide; RicherT 2010)

3048/IV Golzow Umg./nördlich des Ortes (1992, 1994: DEI, Sommer); Britz Umg./nördlich des Ortes (1993: DEI, Sommer)

3147/I Groß Schönebeck Umg./Sumpf bei Gardix (1998: Ockruck)

3147/II Eichhorst Umg./Üderheide: F. Grünenberg (1962), Moospfuhl östlich der Straße nach Finowfurt (1969)

3148/I Werbellin Umg./NSG Buckowseerinne: Südlich der Autobahnanschlussstelle (2011); Lichterfelde Umg./ NSG Buckowseerinne: Trockenhänge südwestlich Margaretenhof (2011) (RICHERT 2012)

3049/I Neugrimnitz Umg./Forst Grumsin: Grumsin (1972); Groß Ziethen Umg./Soll nördlich des Ortes (1993: DEI, Sommer) 
f 3049/II Serwest Umg./Serwester See am Fuß der Kernberge (1992: DEI, Sommer)

3049/IV Brodowin Umg./Kl. Rummelsberg (1992, 1994: DEI, Sommer), Schiefer Berg (1994), Karpatenweg östlich Krugberg (2015)

g 3050/II Stolzenhagen/NSG Krähen- und Jungfernberge $(1979,1983)$ (RICHERT 1982)

3149/IV Niederfinow Umg./Oderhänge - ehemalige Kiesgruben südwestlich des Schiffshebewerks (1995: Richert; 2015, 2016: Schwabe); Struwenberg Umg./ NSG Kanonen- und Schlossberg (1999)

3150/I Oderberg/NSG Pimpinellenberg (1968: Richert; 2011: Salpeter) (RICHERT 1994), am Friedhof (2016)

3150/III Gabow/Oderhänge: Granitberggebiet (1982: Richert: 1991, 2004, 2005, 2007: Kleße) (Richert 1984)

3150/IV Altglietzen Umg./Gabower Berge $(2007,2015)$

h 3149/II Liepe Umg./NSG Niederoderbruch: Schöpfwerk Liepe (1973)

3150/III Bralitz/stillgelegte Kiesgrube (1999)

\section{8 (9814) Rhizedra lutosa (HüBNER, 1803)}

Neue Nachweise von bekannten Fundorten:

c 3147/I Zerpenschleuse/Ortsgebiet: Berliner Straße, im Garten (2014-2017: Busse)

3147/II Finowfurt/Hirtenweg - Besters Fließ (1985-1987: Kittel)

3148/III Ebw. OT Finow/Stadtgebiet: Altenhofer Straße, im Garten $(2014,2016,2017)$

Fundorte (Ergänzung):

3148/IV Ebw.-Westend/Unterheide: Wildparkstraße (1977: Kittel)

\section{9 (9828) Amphipoea oculea (Linnaeus, 1761)}

Der F wurde am Tag beim Saugen an Jasione montana, Berg-Sandglöckchen, beobachtet.

Neue Nachweise von bekannten Fundorten:

b 3248/II Trampe/ehemaliger TÜP (2016)

c 3147/II Finowfurt/Hirtenweg - Besters Fließ (1997: Kittel)

d 3048/I Joachimsthal (1976: Kittel)

e 3049/I Groß Ziethen Umg./Steingruben an den Ihlowbergen (2014)

Fundorte (Ergänzung):

c 3148/IV Ebw./Stadtgebiet: Kleingartenanlage im Schwärzetal nördlich des Forstbotanischen Gartens (2014, 2016, 2017: Schwabe)

\section{0 (9829) Amphipoea fucosa (Freyer, 1830)}

Phänologie/Voltinismus: 14.VI.(2018) aFA, phänologische Verfrühung 3d gegenüber bis 2013.

\section{1 (9831) Amphipoea lucens (Freyer, 1845)}

- RL Land Brandenburg: Kategorie 1 (vom Aussterben bedroht): SM;
- RL Deutschland (2011): Kategorie 3 (gefährdet). In coll. Kittel steckte unerkannt ein F mit den Funddaten 22.VIII.1987, Finowfurt/Hirtenweg, leg. Kittel, in einer Serie von A. fucosa. Die Genitalpräparation bestätigte die Zugehörigkeit zur Art A. lucens. Der Fundort befindet sich in unmittelbarer Nähe der Möllenwiesen, einem Moorgebiet an der Einmündung von Besters Fließ in den Finowkanal. Damit liegt ein weiteres Belegexemplar dieser bei uns sehr seltenen Art vor. Dieser Fund zeigt, dass die Art länger im Gebiet vorkam als bisher bekannt war. Trotz gezielter Suche an bekannten und potenziellen Fundorten (z. B. im Kesselmoor an der Finowfließniederung nordöstlich des Lehnssees in den Jahren 2016-2017) ist die Art nicht wieder gefunden worden.

Fundorte (Ergänzung):

c 3147/II Finowfurt/Hirtenweg: Besters Fließ (1987: Kittel)

\section{2 (9834) Hydraecia micacea (ESPER, 1789)}

Der F wurde nachts beim Saugen an Blüten von Eupatorium cannabium, Wasserdost, beobachtet (Schwabe). Alle mir aus dem Gebiet bekannten Fundorte werden nachgetragen.

Tab. 5: Anflugzahlen von Hydraecia micacea beim regelmäßigen Hauslichtfang im Stadtgebiet von Finow (nach Aufzeichnungen von Duckert).

\begin{tabular}{|l|c|c|c|c|c|c|c|}
\hline Jahr & 1962 & 1963 & 1964 & 1965 & 1966 & 1967 & 1968 \\
\hline Anzahl & 1 & 26 & 20 & 8 & 3 & 12 & 16 \\
\hline Jahr & 1969 & 1970 & 1971 & 1972 & 1973 & 1974 & \\
\cline { 1 - 7 } Anzahl & 4 & 9 & 3 & 4 & 2 & 2 & \\
\cline { 1 - 6 }
\end{tabular}

Fundorte:

a 3249/II Falkenberg Umg./Cöthener Forst: Ehemalige F. Tobbenberge (1970), Uchtenhagen (1974)

b 3149/III Tornow Umg./Barnimhänge nördlich des Ortes (2000: Ockruck), Abt. 629 (2010, 2011: Schwabe)

3149/IV Karlswerk Umg./Am Weg nach Struwenberg (1974); Hohenfinow/Ortsgebiet (1986: Hülbert; RICHERT \& Hülbert, 1999)

3247/II Biesenthal Umg./NSG Biesenthaler Becken: Weg nördlich der Pfauenwiesen (2007, 2014, 2016: Ockruck), Langerönner Weg (2009: Ockruck)

3248/II Trampe/ehemaliger TÜP (2005; RICHERT 2006)

c 3147/I Zerpenschleuse/Ortsgebiet (1979, 1981, 1984, 1987, 1989: Ockruck; 2011, 2013, 2014: Busse; Busse \& OCKRUCK 1991); Marienwerder Umg./Oder-HavelKanal nordwestlich des Ortes (2014: Busse; 2016 Busse \& Müller)

3147/II Finowfurt/Hirtenweg - Besters Fließ (1987: Kittel)

3147/III Klosterfelde Umg./Lottscheseegebiet: Torfstich (1996, 1998: Ockruck); Marienwerder Umg./OderHavel-Kanal (1997: Ockruck)

3148/I Ebw. OT Finow Umg./Mäckersee (1963)

3148/III Ebw. OT Finow/Stadtgebiet: Im Garten (1962-1974: Duckert; 1961-1964, 1967, 1987, 1989, 1995, 1996, 2000，2003，2007-2009，2012，2014，2015，2017: RICHERT) 
3148/IV Ebw. OT Finow Umg./Forst Finowtal: Stromtrasse südlich des Ortes (2012); Ebw.-Westend/Unterheide: Wildparkstraße (1973: Kittel); Ebw./Stadtgebiet: Kleingartenanlage im Schwärzetal nördlich Forstbotanischer Garten (2017: Schwabe)

3149/III Ebw. Umg./Finowbruch an der Ragöser Schleuse (2009: Schwabe); Klärwerk am Eichwerder (2013: Schwabe)

3149/IV Niederfinow Umg./ca. 750 m nordöstlich des Ortszentrums (2013: Schwabe)

d 3047/IV Groß Schönebeck Umg./Schorfheide; Sarnow: Grahsee (1998, 1999: Ockruck)

3048/III Jagdschloss Hubertusstock Umg./Engere Schorfheide: Kl. Kaisergrund (2006, 2009; RICHERT 2010)

3148/I Werbellin Umg./NSG Buckowseerinne (RICHERT 2012): Südlich der Autobahnanschlussstelle (2011); Lichterfelde Umg./NSG Buckowseerinne: Alte Rüsternallee nordöstlich Gut Buckow (2007), Moorniederung nordöstlich des Gr. Buckowsees (2010), Schleiseegebiet bei Margaretenhof (2010)

e 2948/IV Parlow Umg./Mellnseegebiet an der Lindenalle nach Glambeck (2005)

3049/I Neugrimnitz Umg./Forst Grumsin: Grumsin (1972); Groß Ziethen Umg./Steingruben an den Ihlowbergen (1997)

3149/II Liepe Umg./Forst Chorin: F. Liepe (1964)

f 3149/II Brodowin Umg./Vorwerk Zaun (1964)

g 3050/II Stolzenhagen/NSG Krähen- und Jungfernberge (1978, 1979, 1980, 1982, 2005, 2013; RICHERT 1979)

3149/II Liepe (STöckel 1955; 1975: Kittel)

3149/IV Struwenberg Umg./NSG Kanonen- und Schlossberg (1999), Falkenberg (2005: Kleße)

3150/I Oderberg (1961: Steinig)/NSG Pimpinellenberg (1987, 1989; RICHERT 1993-1994), am Friedhof (2016: Richert \& Schwabe)

3150/III Gabow/Oderhänge 2008: Rosenbauer): Granitberggebiet (1984)

3150/IV Altglietzen Umg./Oderhänge: Gabower Berge (2004: Ockruck; 2007: Richert; 2015: Schwabe)

h 3149/II Liepe Umg./NSG Niederoderbruch: Schöpfwerk Liepe (2009)

\section{4 (9841) Gortyna flavago (DENIS \& SCHIFFER- MÜLLER, 1775)}

Siehe 3.3. wieder entdeckte Arten.

\section{5 (9848) Calamia tridens (HufNAgEL, 1766) = virens LINNAEUs, 1767}

Der F wurde am Tag beim Saugen an Armeria maritima, Gewöhnliche Grasnelke, einer weiteren Nektarsaugpflanze, beobachtet (Foto Seidel; vgl. Richert 2003 und 2014).

Phänologie/Voltinismus: Univoltin; 03.VII.(2014), phänologische Verfrühung 4d; 01.VII.(2015), aFE, erneute phänologische Verfrühung 2d, also insgesamt $6 \mathrm{~d}$ gegenüber bis 2013 .

Neue Nachweise von bekannten Fundorten:

c 3147/I Zerpenschleuse/Ortsgebiet: Berliner Straße, im

3148/III Ebw. OT Finow/Stadtgebiet: Altenhofer Straße, im Garten (2014) $\begin{array}{lll}\mathrm{d} & \text { 3149/I } & \text { Britz Umg./F. Britz (1973: Kittel) } \\ \mathrm{g} & 3150 / \mathrm{I} & \text { Oderberg/Teufelsberg (1974: Kittel) } \\ & 3150 / \mathrm{IV} & \begin{array}{l}\text { Altglietzen/Gabower Berge (2016: Richert; 2018: } \\ \text { Schwabe) }\end{array}\end{array}$

Fundorte (Ergänzung):

b 3149/III Sommerfelde Umg./ehemaliger TÜP Panzerbahn (2016: Foto Seidel)

c 3147/II Finowfurt/Hirtenweg - Besters Fließ (1994, 1995 Kittel)

3148/IV Ebw.-Westend/Unterheide: Wildparkstraße (1968, 1972: Kittel)

d 3048/I Joachimsthal (1976: Kittel)

\section{6 (9852) Staurophora celsia (LINNAEUs, 1758)}

Phänologie: In coll. Kittel befand sich eine Serie von Faltern aus dem Zeitraum 1985-1994. Das späteste mir bisher nicht bekannte Funddatum war der 16.X. Das ist das absolute bisher im Gebiet festgestellte Flugzeitende (in Richert 2014 war der 12.X.(1987) als aFE angegeben).

Neue Nachweise von bekannten Fundorten:

c 3147/I Zerpenschleuse/Ortsgebiet: Beerliner Straße, im Garten (2015, 2016: Busse)

3147/II Finowfurt/Hirtenweg - Besters Fließ (1985, 1986, 1989, 1992, 1994: Kittel)

3148/III Ebw. OT Finow/Stadtgebiet: Altenhofer Straße, im Garten (2016)

Fundorte (Ergänzung):

d 3047/III Groß Schönebeck/Ortsgebiet: Friedenstraße, im Garten (2017: Haase) und Umg./Forst Groß Schönebeck (Schorfheide), Revier Rehluch, Abt. 32 (2014: Haase)

\section{7 (9856) Celaena haworthii (CURTIs, 1829)}

- RL Land Brandenburg: Kategorie 3 (gefährdet): SM, NM;

- RL Deutschland (2011): Kategorie 2 (stark gefährdet).

Neue Nachweise von bekannten Fundorten:

c 3147/II Finowfurt/Hirtenweg - Besters Fließ (1993: Kittel)

Fundorte (Ergänzung):

c 3147/IV Finowfurt Umg./Kesselmoor an der Finowfließniederung nordöstlich des Lehnssees (2016: Richert \& Schwabe)

\section{8 (9857) Celaena leucostigma (HüBNER, 1808)}

Neue Nachweise von bekannten Fundorten:

c 3147/II Finowfurt/Hirtenweg - Besters Fließ (1987: Kittel)

3148/III Ebw. OT Finow/Stadtgebiet: Altenhofer Straße, im Garten (2014) 


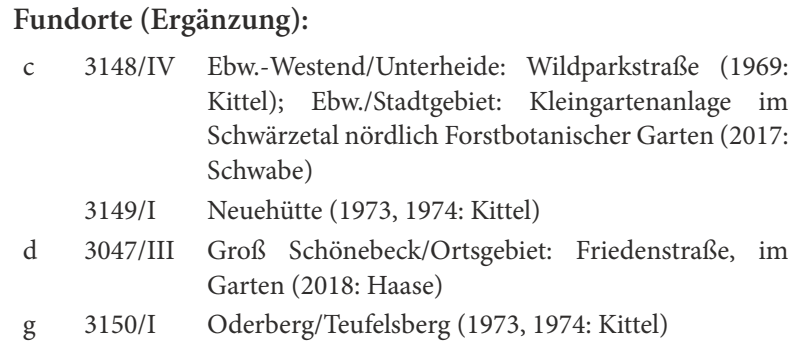

\section{9 (9859) Nonagria typhae (ThUnBerg, 1784)}

Neue Nachweise von bekannten Fundorten:

c 3147/II Finowfurt/Hirtenweg - Besters Fließ (1985, 1997: Kittel)

Fundorte (Ergänzung):

f 3049/IV Brodowin Umg./Karpatenweg östlich des Krugbergs (2015)

g 3149/IV Niederfinow/Oderhänge - ehemalige Kiesgruben südwestlich des Schiffshebewerks (2015: Schwabe)

\section{0 (9864) Phragmatiphila nexa (HüBNER, 1808)}

- RL Land Brandenburg: Kategorie 3 (gefährdet): NM. Die Beobachtungslücken in den Naturräumen a und f (Oberbarnimer Waldhügel und Parsteiner Becken, RicherT 2003) wurden inzwischen geschlossen. Phänologie/Voltinismus: Univoltin; 31.VII.(2014), aFA, phänologische Verfrühung 24d gegenüber bis 2013.

Neue Nachweise von bekannten Fundorten:

$\begin{array}{cl}\text { c 3147/I } & \begin{array}{l}\text { Zerpenschleuse/Ortsgebiet: Berliner Straße, im } \\ \text { Garten (2014: Busse) }\end{array} \\ & \text { Finowfurt/Hirtenweg - Besters Fließ (1993: Kittel) }\end{array}$

Fundorte (Ergänzung):

a 3249/II Falkenberg Umg./Cöthener Forst: Uchtenhagen (1974: Kittel)

c 3147/II Marienwerder Umg./Oder-Havel-Kanal nordwestlich des Ortes (2014: Busse);

3147/IV Finowfurt Umg./Finowfließniederung: Neue Brücke an der Biesenthaler Straße (2014)

3147/IV Sophienstadt/Pregnitzfließniederung (2016: Busse \& Müller)

3148/IV Ebw.-Westend/Unterheide: Wildparkstraße (1974, 1975, 1976: Kittel); Ebw./Stadtgebiet: Kleingartenanlage im Schwärzetal nördlich Forstbotanischer Garten (2016: Schwabe)

e 3049/IV Chorin Umg./Forst Chorin: Teerbrennerberge - Am Olberg (2013: MÜLler 2015), Kreuzung Denglerweg - Olbergstraße (2013: MÜLleR 2015)

f 3047/IV Brodowin/Krugberg (2014: Schwabe)

501 (9864) Archanara geminipuncta (HAwORTH, 1809)

Ältere Funde (Belege in coll. Kittel) werden ergänzend nachgetragen.
Fundorte (Ergänzung):

c 3147/II Finowfurt/Hirtenweg - Besters Fließ (1995: Kittel)

3148/IV Ebw.-Westend/Unterheide: Wildparkstraße (1975: Kittel)

\section{2 (9866) Archanara dissoluta (TreitschKe, 1825)}

- RL Land Brandenburg: Kategorie 2 (stark gefährdet): NM.

Fundorte (Ergänzung):

c 3148/IV Ebw./Stadtgebiet: Kleingartenanlage im Schwärzetal nördlich des Forstbotanischen Gartens (2014, 2017 2018: Schwabe)

\section{3 (9867) Archanara sparganii (ESPER, 1790)}

Die Beobachtungslücke im Naturraum f (Parsteiner Becken, RICHERT 2003) wurde inzwischen geschlossen.

Neue Nachweise von bekannten Fundorten:

c 3148/IV Ebw.-Westend/Unterheide: Wildparkstraße (1975: Kittel)

Fundorte (Ergänzung):

c 3147/IV Finowfurt Umg./Kesselmoor an der Finowfließniederung nordöstlich des Lehnssees $(2016,2017)$

3148/IV Ebw./Stadtgebiet: Kleingartenanlage im Schwärzetal nördlich Forstbotanischer Garten (2016: Schwabe)

f 3149/II Brodowin Umg./Feldsoll am Karpatenweg nördlich Rosinsee (2015)

\section{5 (9870) Sedina buettneri (H. C. W. HeRING, 1858)}

- RL Land Brandenburg: Kategorie 3 (gefährdet): NM.

Neue Nachweise von bekannten Fundorten:

c 3147/I Zerpenschleuse/Ortsgebiet: Berliner Straße, im Garten (2014: Busse)

3147/II Finowfurt/Hirtenweg - Besters Fließ (1984-1991, 1993, 1995: Kittel)

3148/III Ebw. OT Finow/Stadtgebiet: Altenhofer Straße, im Garten (2014)

Fundorte (Ergänzung):

c 3148/IV Ebw.-Westend/Unterheide: Wildparkstraße (1973, 1975, 1978, 1980: Kittel), Ebw./Stadtgebiet: Kleingartenanlage im Schwärzetal nördlich des Forstbotanischen Gartens (2014: Schwabe)

e 3049/IV Chorin Umg./Forst Chorin: Teerbrennerberge - Am Olberg (2013: MüLLER 2015)

\section{6 (9872) Arenostola phragmitidis (HÜBNER, 1803)}

Neue Nachweise von bekannten Fundorten:

c 3147/I Zerpenschleuse/Ortsgebiet: Berliner Straße, im Garten (2014: Busse) 
3148/III Ebw. OT Finow/Stadtgebiet: Altenhofer Straße, im Garten $(2016,2017)$

Fundorte (Ergänzung):

b 3247/II Biesenthal Umg./NSG Biesenthaler Becken: Weg nördlich der Pfauenwiesen (2014, 2015: Ockruck)

c 3147/II Finowfurt/Hirtenweg - Besters Fließ (1987, 19911993, 1995: Kittel)

3147/IV Finowfurt Umg./Kesselmoor an der Finowfließniederung nordöstlich Lehnssee (2016)

3148/IV Ebw./Stadtgebiet: Kleingartenanlage im Schwärzetal nördlich Forstbotanischer Garten (2017: Schwabe)

d 3047/III Groß Schönebeck/Ortsgebiet: Friedenstraße, im Garten (2018: Haase)

3148/II Britz (1973: Kittel)

g 3149/II Liepe (1975: Kittel)

\section{8 (9876) Chortodes pygmina (HüBNER, 1809)}

- RL Land Brandenburg: Kategorie 3 (gefährdet): NM.

Neue Nachweise von bekannten Fundorten:

c 3147/I Zerpenschleuse/Ortsgebiet: Berliner Straße 50. Im Garten (2014: Busse)

3147/II Finowfurt/Hirtenweg - Besters Fließ (1991: Kittel)

Fundorte (Ergänzung):

e 3049/IV Chorin Umg./Forst Chorin: Teerbrennerberge - Am Olberg (2013: MÜLLER 2015)

\section{9 (9890) Coenobia rufa (HAWORTH, 1809)}

- RL Land Brandenburg: Kategorie 2 (stark gefährdet): NM.

Am 31.VII.2017 hatte Schwabe einen F der bei uns seltenen Art im Stadtgebiet von Ebw. in seinem Garten im a.L.

Fundorte (Ergänzung):

c 3148/IV Ebw./Stadtgebiet: Kleingartenanlage im Schwärzetal nördlich Forstbotanischer Garten (2017: Schwabe)

\section{0 (9895) Hadula (Discestra) trifolii (HufNAGEL, 1766)}

Phänologie/Voltinismus: Bivoltin; 06.X.(2013), aFE, phänologische Verspätung 12d (korrigiert nach MüLLER 2015; in RicherT 2014 war der 24.IX.(1986) als aFE angegeben).

\section{1 (9907) Anarta myrtilli (LinNaEus, 1758)}

- RL Deutschland 2011: Vorwarnliste.

Neue Nachweise von bekannten Fundorten

c 3148/III Ebw. OT Finow Umg./Forst Finowtal: Einflugschneise Flugplatz (2017: Richert \& Schwabe)
Fundorte (Ergänzung):

c 3148/III Finowfurt (1978: Kittel)

b 3148/IV Spechthausen Umg./Barnimer Heide: Stromtrasse südwestlich des Ortes (2015, 2017: Schwabe)

3248/II Schönholz Umg./Dünengelände an Stromtrasse nordwestlich des Ortes (2018)

d 3047/III Groß Schönebeck Umg./Schorfheide: Revier Rehluch, Abt. 36 (2015, 2016: Haase)

\section{3 (9912) Lacanobia w-latinum (HuFNAGEL, 1766) = genistae BORKHAUSEN, 1792}

Die Beobachtungslücke im Naturraum h (Odertal, RICHERT 2003) wurde inzwischen geschlossen.

Alle mir aus dem Gebiet bekannten Fundorte werden nachgetragen.

Tab. 6: Anflugzahlen von Lacanobia w-latinum beim regelmäßigen Hauslichtfang im Stadtgebiet von Finow (nach Aufzeichnungen von Duckert).

\begin{tabular}{|l|c|c|c|c|c|c|c|}
\hline Jahr & 1962 & 1963 & 1964 & 1965 & 1966 & 1967 & 1968 \\
\hline Anzahl & 1 & 59 & 44 & 4 & 33 & 102 & 64 \\
\hline yahr & 1969 & 1970 & 1971 & 1972 & 1973 & 1974 & \\
\cline { 1 - 7 } Anzahl & 76 & 13 & 20 & 30 & 45 & 19 & \\
\cline { 1 - 6 }
\end{tabular}

Phänologie/Voltinismus: Univoltin. Im Jahre 2015 wurde nach einer Flugperiode im Mai/Juni ein frischer F am 23.VIII.(2015), aFE, phänologische Verspätung $8 \mathrm{~d}$ gegenüber bis 2013, beobachtet. Wahrscheinlich handelt es sich bei im August beobachteten Faltern um Tiere einer partiellen 2. Generation.

Fundorte:

a 3249/II Falkenberg Umg./Cöthener Forst: Ehemalige F. Tobbenberge (1978)

b 3148/IV Ebw. Umg./Oberheide: Bernauer Heerstraße nahe der Tramper Chaussee (1924: Jonas)

3149/III Sommerfelde Umg./ehemaliger TÜP Panzerbahn (2001) (RICHERT 2006), Barnimhänge nördlich des Ortes: Bachtal (2009: Ockruck)

3149/IV Hohenfinow/Ortsgebiet (1987: Hülbert) (RICHERT \& HÜLBERT 1991)

3248/II Trampe (ehemaliger TÜP (2005) (RICHERT 2006)

c 3146/II Zerpenschleuse Umg./Hammerbruch (1998: Ockruck) (westlich angrenzendes Nachbargebiet)

3147/I Zerpenschleuse/Ortsgebiet (1981, 1984, 1987-1990: Ockruck; 2013, 2014, 2018: Busse) und Umg./an der Chaussee nach Groß Schönebeck (1983: Ockruck); Groß Schönebeck Umg./ehemaliges Objekt Umformtechnik südlich des Ortes (1991: Ockruck) (Busse \& OCKRUCK 1991)

3147/I Marienwerder Umg./Oder-Havel-Kanal nordwestlich des Ortes (2013: Busse); Finowfurt Umg./Besters Fließ - Hirtenweg (1987, 1991, 1993: Kittel)

3147/III Klosterfelde Umg./Lottscheseegebiet: Bungalowsiedlung (2000: Ockruck)

3147/IV Finowfurt Umg./Möllenwiesen - Südostrand (1966) 
3148/III Ebw. OT Finow/Stadtgebiet: Im Garten (1962-1974: Duckert; 1959, 1961-1964, 1966-1969, 1986, 2001, 2003, 2005-2012, 2014, 2015: Richert) und Umg./ Finowbruch westlich des Durchstich (2003), nördlicher Stadtrand zwischen Wolfswinkler Straße und dem (ehemaligen) Kraftwerk (1964), Forst Finowtal: Einflugschneise Flugplatz (2016), F. Schwärze (1963, 1964), 1. Melchower Weg nordöstlich vom Kl. Postluch (2004)

3148/IV Ebw.-Westend/Unterheide: Wildparkstraße (1969: Kittel)

3149/III Ebw. Umg./Eichwerderberge (1964, 1967); Stecherschleuse Umg./Hochfläche oberhalb des Ortes (1997), ehemaliges Kiesgrubengelände nördlich des Ortes (1997)

d 3147/II Eichhorst Umg./Üderheide: Am Weg nach Werbellin (2003)

3148/I Werbellin Umg. (NSG Buckowseerinne: Südlich der Autobahnanschlussstelle (2011); Lichterfelde Umg./ NSG Buckowseerinne: Streuobstwiesen - Moospfuhlgebiet (2011), Moorniederung nordöstlich des Ortes (2010) (RICHERT 2012)

3148/II Lichterfelde Umg./NSG Buckowseerinne: Hänge westlich von Gut Blütenberg (2011) (RICHERT 2012)

3149/I Britz Umg./F. Britz (1972: Kittel)

e 2948/IV Parlow Umg./Mellngebiet an der Lindenallee nach Glambeck (2007)

3049/I Neugrimnitz Umg./Forst Grumsin: Grumsin (1973)

3149/II Liepe Umg./Forst Chorin: F. Liepe (1961), Sandberg (1974)

g 3050/II Stolpe a.O. (1990: Schottstädt)/Trockenhänge am Stadtweg (2018); Stolzenhagen/NSG Krähen- und Jungfernberge (1978, 1980, 1982) (RICHERT 1979)

3149/II Liepe (SтöcKel 1955)/Oderhänge (1961: Richert; 1973, 1974: Kittel)

3149/IV Falkenberg Umg./NSG Schäfergrund (1992: Kleße) Liebenstein bei Hohenfinow (2000: Ockruck); Struwenberg/NSG Kanonen- und Schlossberg (1998)

3150/I Oderberg (1961, 1962: Steinig)/NSG Pimpinellenberg (1968, 1988, 1989) (RICHERT 1994), Teufelsberg (1973, 1975, 1993: Richert, 1973, 1974: Kittel) (Richert 1994); Geistberg (2015, 2017, 2018: Schwabe)

3150/III Gabow/Oderhänge (1992, 2008: Kleße): Granitberggebiet (1983)

3150/IV Altglietzen Umg./Oderhänge: Gabower Berge (1999: Ockruck \& Busse, 2000, 2003: Ockruck; 2015: Richert)

h 3150/III Neuenhagen Umg./ehemaliger Altglietzener Segelflugplatz 1,5 km nordöstlich des Ortes (2009: Rosenbauer)

\section{4 (9914) Lacanobia splendens (HüBNER, 1808)}

- RL Land Brandenburg: Kategorie 3 (gefährdet): NM, $\mathrm{MB}$;

- RL Deutschland (2011): Kategorie 3 (gefährdet).

\section{Neue Nachweise von bekannten Fundorten:}

c 3147/II Finowfurt/Hirtenweg - Besters Fließ (1987, 1991, 1992, 1994, 1996: Kittel)

e 3149/II Liepe Umg./Forst Chorin: NSG Plagefenn - LedumMoor am Fuß der Plageberge (2018: Richert \& Schwabe)

g 3149/II Liepe/Ortsgebiet: Schule (1973, 1975, 1976: Kittel)
Fundorte (Ergänzung):

c $3147 / \mathrm{IV}$ Finowfurt Umg./Kesselmoor an der Finowfließniederung nordöstlich des Lehnssees (2016)

g 3150/I Oderberg/Geistberg (2018: Schwabe)

\section{5 (9917) Lacanobia oleracea (LINNAEus, 1758)}

Phänologie/Voltinismus: Bivoltin, 1. Gen.: 30.IV.(2014), aFA, phänologische Verfrühung 15d gegenüber bis 2013. 2. Gen: 29.IX.(2014), aFE, phänologische Verspätung 5d gegenüber bis 2013 .

\section{7 (9919) Lacanobia contigua (Denis \& SCHIF- FERMÜLLER, 1775)}

Alle mir aus dem Gebiet bekannten Fundorte werden nachgetragen.

Tab. 7: Anflugzahlen von Lacanobia contigua beim regelmäßigen Hauslichtfang im Stadtgebiet von Finow (nach Aufzeichnungen von Duckert).

\begin{tabular}{|l|c|c|c|c|c|c|c|}
\hline Jahr & 1962 & 1963 & 1964 & 1965 & 1966 & 1967 & 1968 \\
\hline Anzahl & 2 & 32 & 4 & 9 & 56 & 82 & 50 \\
\hline Jahr & 1969 & 1970 & 1971 & 1972 & 1973 & 1974 & \\
\cline { 1 - 6 } Anzahl & 50 & 3 & 12 & 20 & 27 & 23 \\
\hline
\end{tabular}

\section{Fundorte:}

a 3149/IV Falkenberg (STÖCKEL 1955)

b 3148/IV Ebw./Oberheide: Turnplatz (1910, 1921: Jonas) und Umg: F. Leuenberger Wiesen (1923: Jonas; 1969: Richert)

3149/III Sommerfelde Umg./ehemaliger TÜP Panzerbahn (2001) (RICHERT 2006)

3247/II Biesenthal Umg./NSG Biesenthaler Becken: Weg nördlich der Pfauenwiesen (2007: Ockruck)

3248/II Trampe/ehemaliger TÜP (2005) (RICHERT 2006)

c 3147/I Zerpenschleuse/Ortsgebiet (1979-1981, 1983-1985, 1987-1990: Ockruck; 2013, 2014: Busse) (Busse \& OCKRUCK 1991)

3147/II Finowfurt Umg./Besters Fließ: Hirtenweg (19901992, 1995: Kittel)

3147/IV Sophienstädt Umg./Pregnitzfließniederung nordöstlich des Mittelprenden-Sees (2012)

3148/III Ebw. OT Finow/Stadtgebiet: Im Garten (1961-1964, 1966, 1967, 1969, 1974, 1997, 1999, 2002, 2003, 2006, 2007, 2010-2015, 2018: Richert; 1962-1974: Duckert) und Umg. (1953) /Forst Finowtal: Einflugschneise Flugplatz (1997), F. Schwärze $(1963,1974)$

3148/IV Ebw./Stadtgebiet (1960er Jahre: Friese); Ebw.-Westend/Unterheide: Wildparkstraße (1972-1975: Kittel)

3149/III Ebw./Eichwerderberge (1964); Stecherschleuse Umg./ehemaliges Kiesgrubengelände nördlich des Ortes (1997)

d 3047/III Groß Schönebeck/Ortsgebiet: Friedenstraße, im Garten (2012, 2013, 2014, 2015, 2017: Haase)

3047/IV Eichhorst OT Wildau am Werbellinsee/Straßenhang an der Seerandstraße (1962) und Umg./Engere Schorfheide: F. Eichheide (1962) (NSG Kienhorst/ Köllnseen/Eichheide; RICHERT 2010); Eichhorst Umg./Üderheide: Süßer Winkel am Werbellinsee (1980) 
3048/III Jagdschloss Hubertusstock Umg./Engere Schorfheide: Gr. Kaisergrund (2006); Joachimsthal Umg./ Schorfheide: Forst Grimnitz, Revier Michen, Abt. 40/41 (2006) (NSG Kienhorst/Köllnseen/Eichheide; RICHERT 2010)

3147/II Eichhorst Umg./Üderheide: F. Grünenberg (1962, 1963)

3148/II Britz (1972: Kittel)

e 3049/I Groß Ziethen Umg./Gr. Kagelpfuhl nordwestlich des Ortes bei Sperlingsherberge (2008: Roedel \& Schulze), Forst Grimnitz: F. Gr. Ziethen (1967)

3049/III Senftenhütte Umg./Forst Chorin: F. Senftenthal (1967)

3149/II Liepe Umg./Forst Chorin: F. Liepe (1964)

f 3049/III Buchholz bei Serwest (1967)

3049/IV Brodowin Umg./Schiefer Berg (1994)

g 3050/II Stolpe/Trockenhänge (1987: Schottstädt); Stolzenhagen/NSG Krähen- und Jungfernberge (1979) (RICHERT 1982)

3149/II Liepe (1973-1975: Kittel; 1987: Schottstädt), (STöCKel 1955)/Oderhänge (1961), Sandberg (1973)

3149/IV Struwenberg Umg./NSG Kanonen- und Schlossberg (1998)

3150/I Oderberg (1960-1962: Steinig)/NSG Pimpinellenberg (1968, 1989) (RICHERT 1994), Teufelsberg (1961, 1973, 1975, 1977) (RICHERT 1994)

3150/III Gabow/Oderhänge (1982: Richert; 1987: Schottstädt; 1991, 1992, 2003: Kleße) (Richert 1984)

3150/IV Altglietzen Umg./Gabower Berge (2007)

h 3150/III Bralitz/stillgelegte Kiesgrube (2010: Rosenbauer)

\section{8 (9920) Lacanobia suasa (DENIS \& SCHIFFER-} MÜLLER, 1775)

Phänologie/Voltinismus: 2. Gen.: 15.IX.(2017), aFE phänologische Verspätung 5d gegenüber bis 2013

\section{9 (9925) Hada plebeja (LINNAEUs, 1761) = nana HUFNAGEL, 1766}

Phänologie/Voltinismus: Bivoltin; 1. Gen.: Ein sehr abgeflogener F (Nachzügler) am 25.VII.(2014), aFE, phänologische Verspätung 8d gegenüber bis 2013.

2. Gen.: 23.VIII.(2014), aFE, phänologische Verspätung 1d gegenüber bis 2013 .

Neue Nachweise von bekannten Fundorten:

c 3147/I Zerpenschleuse/Ortgebiet: Berliner Straße, im Garten (2014-2016, 2018: Busse)

3147/II Finowfurt/Hirtenweg: Besters Fließ (1989, 1993, 1995, 1996: Kittel)

3148/III Ebw. OT Finow/Stadtgebiet: Altenhofer Straße, im Garten (2014, 2015, 2016, 2017, 2018)

d 3047/III Groß Schönebeck/Ortsgebiet: Friedenstraße, im Garten (2014: Haase)

Fundorte (Ergänzung):

c 3147/IV Finowfurt Umg./Kesselmoor an der Finowfließniederung nordöstlich des Lehnssees (2016)
3148/IV Ebw./Stadtgebiet: Kleingartenanlage im Schwärzetal nördlich des Forstbotanischen Gartens (2014, 2016 Schwabe); Ebw.-Westend/Unterheide: Wildparkstraße (1973: Kittel)

g 3149/II Niederfinow/Oderhänge - ehemalige Kiesgruben südwestlich des Schiffshebewerks (2015: Schwabe)

3150/I Oderberg/Geistberg (2015, 2017: Schwabe)

520 (9927) Hecatera dysodea (DENIS \& SCHIFFERMÜLLER, 1775) = spinaciae VIEWEG, 1790

Die Beobachtungslücke im Naturraum d (Britzer Platte und Schorfheide, vgl. Richert 2003) konnte inzwischen geschlossen werden.

Fundorte (Ergänzung):

c 3148/III Ebw. OT Finow/Stadtgebiet (2015: 1 R auf einem Parkplatz an Stachellattich)

d 3047/III Groß Schönebeck/Ortsgebiet: Friedenstraße, im Garten (2000, 1 F a.L.: Haase)

\section{1 (9928) Hecatera bicolorata (HufNAGEL, 1766)} = serena DENIS \& SCHIFFERMÜLLER, 1775

Siehe wieder entdeckte Arten.

Hier werden ältere Funde ergänzend nachgetragen.

Die Beobachtungslücke im Naturraum b (Barnimplatte, vgl. RicherT 2003) konnte geschlossen werden.

Fundorte (Ergänzung):

b 3247 Biesenthal Umg. (1982, 1 F leg. Fichtner, Datei InsectIS Bbg.)

c 3148/IV Ebw.-Westend/Unterheide: Wildparkstraße (1973, 1975, 1976, 1977: Kittel)

\section{3 (9939) Hadena compta (Denis \& SCHIFFER-} MÜLLER, 1775)

- RL Deutschland (2011): Vorwarnliste.

Neue Nachweise von bekannten Fundorten:

c 3148/III Ebw. OT Finow/Stadtgebiet: Altenhofer Straße, im Garten (2017)

3148/IV Ebw.-Westend/Unterheide: Wildparkstraße (1969, 1975, 1976: Kittel), Ebw.-Nordend/ehemalige Gehörlosen-Hilfsschule auf dem Gelände der Landesklinik (1983: Kittel)

g 3150/IV Altglietzen/Gabower Berge (2015)

Fundorte (Ergänzung):

c 3147/II Finowfurt/Hirtenweg: Besters Fließ (1986: Kittel)

d 3148/II Britz (1971, 1972: Kittel)

524 (9940) Hadena confusa (HufNAGEL, 1766)

= nana ROTTEMBURG, 1776

- RL Land Brandenburg: Kategorie 2 (stark gefährdet): TR;

- RL Deutschland (2011): Kategorie 3 (gefährdet). 
Ältere Funde (Belege in coll. Kittel) werden ergänzend nachgetragen.

Neue Nachweise von bekannten Fundorten:

g 3150/I Oderberg/Teufelsberg (1971, 1974, 1975: Kittel)

\section{7 (9955) Hadena rivularis (FABRICIUS, 1775)}

Neue Nachweise von bekannten Fundorten:

$\begin{array}{ccl}\text { c } & 3147 / \mathrm{II} & \text { Finowfurt/Hirtenweg - Besters Fließ (1996: Kittel) } \\ & 3148 / \mathrm{III} & \begin{array}{l}\text { Ebw. OT Finow/Stadtgebiet, im Garten (2015, 2016, } \\ \text { 2018) }\end{array} \\ \text { g } & 3149 / \mathrm{IV} & \begin{array}{l}\text { Niederfinow/Oderhänge - ehemalige Kiesgruben } \\ \text { südwestlich des Schiffshebewerks (2016: Schwabe) } \\ \end{array} \\ 3150 / \mathrm{I} & \text { Oderberg/Teufelsberg (1974, 1975: Kittel) }\end{array}$

Fundorte (Ergänzung):

c 3148/IV Ebw.-Westend/Unterheide: Wildparkstraße (1969: Kittel), Ebw./Stadtgebiet: Kleingartenanlage im Schwärzetal nördlich des Forstbotanischen Gartens (2014, 2016, 2018: Schwabe)

d 3047/III Groß Schönebeck/Ortsgebiet: Friedenstraße, im Garten (2017: Haase)

3149/I Britz Umg./F. Britz (1971: Kittel)

g 3150/I Oderberg/Geistberg (2015, 2017, 2018: Schwabe)

3150/IV Altglietzen/Gabower Berge (2015: Richert; 2018: Schwabe)

\section{8 (9957) Hadena perplexa (DENIS \& SCHIFFER- MÜLLER, 1775) = lepida ESPER, 1790}

- RL Land Brandenburg: Kategorie 3 (gefährdet): TR. Ältere Funde (Belege in coll. Kittel) werden ergänzend nachgetragen.

\section{Neue Nachweise von bekannten Fundorten:}

c 3148/III Ebw. OT Finow/Stadtgebiet: Gartenstraße in der Siedlung Freie Scholle, im Garten (1963, 1972: Duckert; Belegexempl. in coll. Kittel)

g 3150/I Oderberg/Teufelsberg (1973, 1974, 1975, 1977: Kittel)

Fundorte (Ergänzung):

g 3149/II Liepe (1973: Kittel)

\section{9 (9964) Hadena irregularis (HufNAgeL, 1766)}

- Verordnung zur Neufassung der BArtschV von 2005: Streng geschützte Art.

- RL Land Brandenburg: Kategorie 1 (vom Aussterben bedroht): TR;

- RL Deutschland (2011): Kategorie 1 (vom Aussterben bedroht).

Neue Nachweise von bekannten Fundorten:

g 3150/III Gabow/Granitberggebiet (2015: Richert, Schwabe) 3150/IV Altglietzen/Gabower Berge (2015)

\section{0 (9969) Sideridis turbida (ESPER, 1790)}

\section{= albicolon $($ HÜBNER, 1813)}

- RL Land Brandenburg: Kategorie 3 (gefährdet): TR;

- RL Deutschland (2011): Kategorie 3 (gefährdet).

Neue Nachweise von bekannten Fundorten:

c 3147/II Finowfurt/Hirtenweg - Besters Fließ (1989, 1991, 1996: Kittel)

3148/III Ebw. OT Finow/Stadtgebiet: Altenhofer Straße, im Garten $(2014,2015)$

3148/IV Ebw.-Westend/Unterheide: Wildparkstraße (1973: Kittel)

g 3149/II Liepe (1974: Kittel)

3150/IV Altglietzen/Gabower Berge (2015)

\section{Fundorte:}

g 3149/II Niederfinow/Oderhänge - ehemalige Kiesgruben südwestlich des Schiffshebewerks (2015: Schwabe)

\section{1 (9972) Heliophobus reticulata (GoEZE, 1981)}

Alle mir aus dem Gebiet bekannten Fundorte werden nachgetragen.

Tab. 8: Anflugzahlen von Heliophobus reticulata beim regelmäßigen Hauslichtfang im Stadtgebiet von Finow (nach Aufzeichnungen von Duckert).

\begin{tabular}{|l|c|c|c|c|c|c|c|}
\hline Jahr & 1962 & 1963 & 1964 & 1965 & 1966 & 1967 & 1968 \\
\hline Anzahl & 18 & 55 & 50 & 11 & 23 & 58 & 37 \\
\hline
\end{tabular}

\begin{tabular}{|l|c|c|c|c|c|c|}
\hline Jahr & 1969 & 1970 & 1971 & 1972 & 1973 & 1974 \\
\hline Anzahl & 51 & 7 & 52 & 52 & 23 & 63 \\
\hline
\end{tabular}

\section{Fundorte:}

b 3148/IV Spechthausen (1960er Jahre: Friese)

3149/III Sommerfelde Umg./ehemaliger TÜP Panzerbahn (2001) (RICHERT 2006)

3149/IV Hohenfinow/Ortsgebiet (1986: Hülbert) (RICHERT \& HüLBERT 1991)

3247/II Biesenthal Umg./Pfauenfließ westlich Strehsee (Clemens \& Scheibe), Langerönner Weg (2011: Ockruck)

3248/II Trampe/ehemaliger TÜP (2000) (RICHERT 2006)

c 3147/I Zerpenschleuse/Ortsgebiet: Im Garten (1983-1990 Ockruck; 2013, 2014, 2015, 2018: Busse) (Busse \& OCKRUCK 1991)

3147/II Finowfurt Umg./Besters Fließ: Hirtenweg (1988, 1990-1992, 1995: Kittel)

3147/IV Finowfurt Umg./Finowbruch westlich des Ortes: Möllenwiesen - Südostrand (1966); Sophienstädt Umg./Pregnitzfließniederung nordöstlich des Mittel-Prendensees (2012)

3148/II Britz Umg./Stadtseerinne westlich des Gr. Stadtsees (2013)

3148/III Ebw. OT Finow/Stadtgebiet: Im Garten (1962-1974: Duckert); 1955, 1956, 1960-1964, 1966, 1967, 1969, 1992, 2003, 2004, 2006-2016), nordöstlicher Ortsrand zwischen Wolfswinkler Straße und dem (ehemaligen) Kraftwerk (1964) und Umg./Forst Finowtal: F. Schwärze (1963, 1964, 1974), Samithseemoor (1993); Menningsfließgebiet westlich des Ortes (1993) 
3148/IV Ebw.-Westend/Unterheide: Wildparkstraße (1970: Kittel); Ebw./Stadtgebiet (1960er Jahre: Friese), Forstbotanischer Garten (2010: Schwabe), Kleingartenanlage im Schwärzetal nördlich Forstbotanischer Garten (2016, 2018: Schwabe);

3149/III Stecherschleuse Umg./Hochfläche und ehemaliges Kiesgrubengelände nördlich des Ortes (1996)

d 3047/III Groß Schönebeck/Ortsgebiet: Friedenstraße, im Garten (2013, 2013, 2015, 2016: Haase)

3147/I Groß Schönebeck Umg./am Objekt für Umformtechnik südlich des Ortes (1991: Ockruck)

3147/II Eichhorst Umg./Üderheide: F. Grünenberg (1972, 1995)

3148/I Werbellin Umg./NSG Buckowseerinne: Südlich der Autobahnanschlussstelle (2011); Lichterfelde Umg./ NSG Buckowseerinne/Streuobstwiesen südöstlich des Gr. Buckowsees (2012) (Richert 2012)

g 3050/II Stolpe a.O. (1990: Schottstädt)/Trockenhänge am Stadtweg (2018); Stolzenhagen/NSG Krähen- und Jungfernberge (1979) (RICHERT 1982)

3149/II Liepe (STÖcKEL 1955)/Ortgebiet: Schulhaus (1974)/ Oderhänge (1961), Sandberg $(1973,1974)$

3150/I Oderberg (1960, 1962: Steinig)/NSG Pimpinellenberg (1961: Urbahn; 1988, 1989: Richert) (RICHERT 1994), Teufelsberg (1973, 1975) (RICHERT 1994), Geistberg (2018: Schwabe)

3150/III Gabow/Oderhänge (1987: Schottstädt; 1991, 1992, 2003-2005: Kleße): Granitberggebiet (1983: Richert; 2015: Schwabe)

3150/IV Altglietzen Umg./Gabower Berge (1998: Rosenbauer; 1999: Ockruck \& Busse; 2003, 2004, 2013: Ockruck; 2007, 2015: Richert)

h 3150/III Bralitz/stillgelegte Kiesgrube (2009: Richert; 2010: Rosenbauer), Neuenhagen Umg./ehemaliger Altglietzener Segelflugplatz nordöstlich des Ortes (2010)

\section{4 (9987) Mamestra brassicae (Linnaeus, 1758)}

Phänologie/Voltinismus: In der Regel bivoltin, jw. offenbar eine partielle 3. Gen.: 06.X.(2013), aFE, phänologische Verspätung 5d (korrigiert nach MüLleR 2015; in RICHERT 2014 war der 01.X.(1962) als aFE angegeben).

\section{6 (9991) Polia bombycina (Hufnagel, 1766) = advena DENIS \& SCHIFFERMÜLLER, 1775}

Neue Nachweise von bekannten Fundorten:

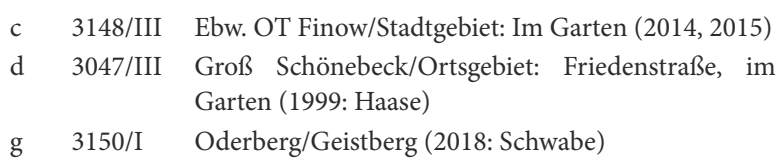

\section{7 (9992) Polia hepatica (Clerck, 1759) = tincta} ВRАНM, 1791)

- RL Land Brandenburg: Vorwarnliste: NW;

- RL Deutschland (2011): Vorwarnliste.

Neue Nachweise von bekannten Fundorten:

c 3147/I Zerpenschleuse/Ortsgebiet: Berliner Straße, im Garten (2014: Busse)
Fundorte (Ergänzung):

c 3147/IV Finowfurt Umg./Kesselmoor an der Finowfließniederung nordöstlich des Lehnssees (2017)

\section{9 (9999) Mythimna turca (LinnAeus, 1761)}

Neue Nachweise von bekannten Fundorten:

c 3147/I Zerpenschleuse/Ortsgebiet: Berliner Straße, im Garten (2014-2016: Busse)

3147/II Finowfurt/Hirtenweg - Besters Fließ (1991: Kittel)

3148/III Ebw. OT Finow/Stadtgebiet: Altenhofer Straße, im Garten $(2014,2015,2017)$

d 3047/III Groß Schönebeck/Ortsgebiet: Friedenstraße, im Garten (2015: Haase)

3148/II Britz (1971: Kittel)

Fundorte (Ergänzung):

c 3148/IV Ebw./Stadtgebiet: Kleingartenanlage im Schwärzetal nördlich des Forstbotanischen Gartens (2014, 2016 2018: Schwabe); Ebw.-Westend/Unterheide: Wildparkstraße 1970, 1972, 1973: Kittel)

e 3049/I Groß Ziethen Umg./Steingruben an den Ihlowbergen (2014)

\section{0 (10000) Mythimna conigera (DENIs \& SCHIF-} FERMÜLLER, 1775)

Phänologie/Voltinismus: 24.V.(2016), aFA, phänologische Verfrühung 14d gegenüber bis 2013.

541 (10001) Mythimna ferrago (FABRICIUs, 1787) = lithargyria auct.

Der F wurde am Tag beim Saugen an Origanum vulgare, Dost, beobachtet.

\section{3 (1004) Mythimna pudorina (DeNIS \& SCHIF- FERMÜLLER, 1775)}

Phänologie/Voltinismus: Univoltin; 09.VI.(2014), phänologische Verfrühung 1d; 08.VI.(2018), aFA, erneute phänologische Verfrühung $1 \mathrm{~d}$ (insgesamt $2 \mathrm{~d}$ gegenüber bis 2013).

Neue Nachweise von bekannten Fundorten:

b 3247/II Biesenthal Umg./NSG Biesenthaler Becken: Langerönner Weg (2014: Ockruck)

c 3147/I Zerpenschleuse/Ortsgebiet: Berliner Straße, im Garten (2014, 2018: Busse)

3147/II Finowfurt/Hirtenweg - Besters Fließ (1995: Kittel)

3148/III Ebw. OT Finow/Stadtgebiet: Altenhofer Straße, im Garten (2014)

3148/IV Ebw.-Westend/Unterheide: Wildparkstraße (19731975: Kittel)

g 3150/III Gabow/Granitberggebiet (2015)

3150/IV Altglietzen Umg./Gabower Berge (2015)

Fundorte (Ergänzung):

d 3148/II Britz (1971: Kittel) 
e 3049/I Groß Ziethen Umg./Steingruben an den Ihlowbergen (2014)

\section{4 (10005) Mythimna straminea (TREITSCHKE, 1825)}

- RL Land Brandenburg: Vorwarnliste: NM. Phänologie/Voltinismus: Univoltin; ein relativ frischer F am 02.X.(2014), aFE, phänologische Verspätung 18d gegenüber bis 2013. Hier handelt es sich wiederum um einen F der partiellen 2. Gen. (vgl. Richert 2014).

\section{Neue Nachweise von bekannten Fundorten:}

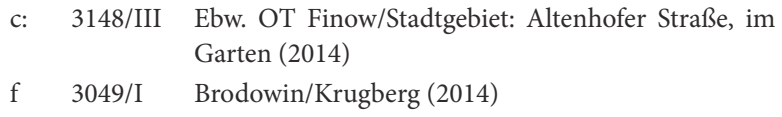

\section{7 (10010) Mythimna obsoleta (HüBNER, 1803)}

Alle mir aus dem Gebiet bekannten Fundorte werden nachgetragen.

Tab. 9: Anflugzahlen von Mythimna obsoleta beim regelmäßigen Hauslichtfang im Stadtgebiet von Finow (nach Aufzeichnungen von Duckert).

\begin{tabular}{|l|c|c|c|c|c|c|c|}
\hline Jahr & 1962 & 1963 & 1964 & 1965 & 1966 & 1967 & 1968 \\
\hline Anzahl & 1 & 6 & 16 & 3 & 9 & 3 & 9 \\
\hline Jahr & 1969 & 1970 & 1971 & 1972 & 1973 & 1974 & \\
\cline { 1 - 7 } Anzahl & 5 & - & 16 & 15 & 11 & 4 \\
\cline { 1 - 6 }
\end{tabular}

Phänologie/Voltinismus: Univoltin; 15.IX.(2014), aFE, phänologische Verspätung 27d gegenüber bis 2013. Der bisher späteste F-Fund vom 19.VIII.2010 (vgl. RicheRT 2014) war als Nachzügler beurteilt worden. Der neue extrem späte Fund ist mit Sicherheit als partielle 2. Gen. $\mathrm{zu}$ deuten. Bereits in Richert 2003 war als ExtremDatum für das Flugzeitende der 10.IX. angegeben. Da sich die Herkunft der Angabe nicht mehr ermitteln ließ, schätzte ich sie als unsicher ein und führte sie im 3. Nachtrag nicht mehr auf. Der erneute Spätfund bestätigt auch dieses Datum als richtig.

\section{Fundorte:}

a 3249/II Falkenberg Umg./Cöthener Forst: Ehemalige F. Tobbenberge (1971)

b 3148/IV Ebw. Umg./Oberheide: F. Leuenberger Wiesen (1969)

3149/III Sommerfelde Umg./ehemaliger TÜP Panzerbahn (2005) (Richert 2006); Tornow Umg./Barnimhänge nördlich des Ortes: Abt. 629 (2011: Schwabe)

3149/IV Hohenfinow/Ortsgebiet (1986, 1987: Hülbert) (Richert \& HülberT 1991)

3247/I Prenden Umg./Strehlesee - Südufer (2010: Rosenbauer)

3247/II Biesenthal Umg./NSG Biesenthaler Becken: Langerönner Weg (2014: Ockruck)

3248/II Trampe/ehemaliger TÜP (2005) (RICHERT 2006)

c 3147/I Zerpenschleuse/Ortsgebiet: Im Garten (1981, 1983, 1984, 1985, 1986, 1989: Ockruck) (BUSSE \& OCKRUCK 1991)
3147/II Marienwerder Umg./Pechteich (1991: Ockruck); Finowfurt Umg./Besters Fließ - Hirtenweg (1986, 1989, 1990, 1991, 1992: Kittel), Möllenwiesen Nordwestrand $(2012,2013)$

3147/III Klosterfelde Umg./Lottscheseegebiet: Bungalowsiedlung (1987: Ockruck)

3147/IV Marienwerder Umg./Steinlaake (2015), Schilfgebiet südwestllich des Sportplatzes (2009: Rosenbauer); Finowfurt Umg./Möllenwiesen - Südostrand (1966), Kesselmoor an der Finowfließniederung nordöstlich des Lehnssees $(2016,2017)$

3148/II Britz Umg./Stadtseerinne westlich des Gr. Stadtsees (2013)

3148/III Ebw. OT Finow/Stadtgebiet: Im Garten (1962-1969, 1971-1974: Duckert; 1960-1964, 1966-1969, 1977, 1986, 2001-2011, 2013-2016, 2017, 2018: Richert) und Umg./Stadtrand zwischen Wolfswinkler Straße und dem (ehemaligen) Kraftwerk (1964), Forst Finowtal: F. Schwärze (1964, 1974), Einflugschneise Flugplatz (1998, 2016); Menningsfließgebiet (1993)

3148/IV Ebw.-Westend/Unterheide: Wildparkstraße (1970, 1972: Kittel); Ebw./Stadtgebiet (1960er Jahre: Friese); Ebw./Stadtgebiet: Kleingartenanlage im Schwärzetal nördlich Forstbotanischer Garten (2016, 2018: Schwabe)

3149/III Ebw. Umg./Eichwerderberge (1968), Finowbruch am Eichwerder: Kläranlage (2011, 2013: Schwabe); Stecherschleuse Umg./Hochfläche nördlich/oberhalb des Ortes (1997), Finowtalhänge am westlichen Ortsausgang (1997), an der Benzintrasse (2009: Schwabe): Sommerfelde Umg./Finowbruch nördlich des Ortes (2009: Schwabe)

d 3047/III Größ Schönebeck/Ortgebiet: Friedenstraße, im Garten (2015: Haase)

3047/IV Groß Schönebeck Umg./Pinnowseengebiet NSG Meelake (1991: Ockruck), Sarnow: Grahsee (1991: Ockruck) (NSG Kienhorst/Köllnseen/Eichheide; RICHERT 2010)

3048/II Joachimsthal Umg./Forst Grimnitz: F. Voigtswiese (1987: Schottstädt)

3147/I Groß Schönebeck Umg./ehemaliges Objekt Umformtechnik südlich des Ortes (1991: Ockruck)

3147/II Eichhorst Umg./Üderheide: F. Grünenberg (1963), am Weg nach Werbellin (1995)

3148/I Werbellin Umg./NSG Buckowseerinne: Südlich der Autobahnanschlussstelle (2011) (Richert 2012); Lichterfelde Umg./NSG Buckowseerinne: Schleiseegebiet bei Margaretenhof (2010) (Richert 2012)

e 2948/IV Parlow Umg./Mellnseegebiet an der Lindenallee nach Glambeck (2007)

3049/I Groß Ziethen Umg./Gr. Kagelpfuhl-Südufer sowie zwischen Schulzensee und Kl. Kagelpfuhl nordwestlich des Ortes bei Sperlingsherberge (2008: Roedel \& Schulze), Steingruben an den Ihlowbergen (2014), Forst Grimnitz: F. Gr. Ziethen (1967);

3049/III Senftenhütte Umg./Forst Chorin: F. Senftenthal (1967)

3149/II Liepe Umg./Forst Chorin: F. Liepe (1964); NSG Plagefenn - Ledum-Moor am Fuß der Plageberge (2018: Richert \& Schwabe)

3150/I Neuendorf bei Oderberg/Ortsgebiet: Wehrkirche (2010: Schwabe)

f 3049/II Buchholz bei Serwest (1967); Brodowin Umg./Vor werk Zaun (1964)

3049/IV Brodowin/Krugberg (2014) 
g 3050/II Stolpe/Trockenhänge (1987, 1990: Schottstädt); Stolzenhagen/NSG Krähen- und Jungfernberge (1978, 1979) (RICHERT 1979)

3149/II Liepe (1973, 1974: Kittel; 1987: Schottstädt) (STöckel 1955)/Ortsgebiet: Schule (1974, 1975), Oderhänge: Sandberg $(1973,1974)$

3149/IV Struwenberg Umg./NSG Kanonen- und Schlossberg (1999)

3150/I Oderberg (1960, 1961: Steinig)/NSG Pipinellenberg (1968, 1989) (RICHERT 1994), Teufelsberg (1961, 1973, 1975, 1993) (RICHERT 1994)

3150/III Gabow/Oderhänge (1991, 1992, 2003, 2004, 2008: Kleße): Granitberggebiet (1983, 2015: Richert)

3150/IV Altglietzen Umg./Oderhänge: Gabower Berge (1999: Ockruck \& Busse; 2003, 2004, 2013: Ockruck; 2007, 2015: Richert)

h 3149/II Liepe Umg./NSG Niederoderbruch: Schöpfwerk Liepe $(1973,1974,2009)$

3150/II Hohensaaten Umg./Kiesgrube an der Straße nach Lunow (2009: Rosenbauer)

3150/III Bralitz/stillgelegte Kiesgrube (2009: Richert; 2010, 2012: Rosenbauer \& Theimer); Neuenhagen Umg./ ehemaliger Altglietzener Segelflugplatz nordöstlich des Ortes (2010)

\section{8 (10011) Mythimna comma (LinnAeUs, 1761)}

Der F wurde überwiegend a.L, aber auch a.K. nachgewiesen, woran er auf sumpfigem Gelände gelegentlich i.M. erschien (Ockruck).

Die Nachweise zeigen aber an vielen Fundorten im Laufe der Zeit erhebliche Lücken. Von potentiellen Fundorten fehlen Nachweise, obwohl dort faunistische Untersuchungen erfolgten. In der Regel werden neuerdings Einzelfunde gemeldet, nur selten wurde der F i.A. beobachtet. Alle mir aus dem Gebiet bekannten Fundorte werden nachgetragen.

Tab. 10: Anflugzahlen von Mythimna comma beim regelmäBigen Hauslichtfang im Stadtgebiet von Finow (nach Aufzeichnungen von Duckert).

\begin{tabular}{|l|c|c|c|c|c|c|c|}
\hline Jahr & 1962 & 1963 & 1964 & 1965 & 1966 & 1967 & 1968 \\
\hline Anzahl & 3 & 51 & 24 & 10 & 33 & 31 & 59 \\
\hline Jahr & 1969 & 1970 & 1971 & 1972 & 1973 & 1974 & \\
\cline { 1 - 6 } Anzahl & 58 & 3 & 13 & 11 & 16 & 4 & \\
\end{tabular}

\section{Fundorte:}

b 3148/IV Ebw.-Südend Umg./F. Leuenberger Wiesen (1969)

3149/III Sommerfelde Umg./ehemaliger TÜP Panzerbahn (2001)

3149/IV Hohenfinow/Ortsgebiet (1986: Hülbert; RICHERT \& HÜLBERT 1991)

3248/II Trampe/ehemaliger TÜP (2000) (RICHERT 2006)

c 3147/I Zerpenschleuse/Ortsgebiet: Im Garten (19831985, 1987, 1988, 1989, 1990: Ockruck) (Busse \& OCKRUCK 1991)

3147/II Marienwerder Umg./Pechteich (1950 bis 1959: Haeger; 1991: Ockruck); Finowfurt/Besters Fließ Hirtenweg (1986, 1988-1993: Kittel)

3147/III Klosterfelde Umg./Lottscheseegebiet: Bungalowsiedlung (1991: Ockruck); Marienwerder Umg./OderHavel-Kanal (1999: Ockruck)
3147/IV Marienwerder Umg./Schleuse Grafenbrück (1967); Finowfurt/Möllenwiesen am Finowkanal - Südrand (1966)

3148/III Ebw. OT Finow/Stadtgebiet: Gartenstraße in der Siedlung Freie Scholle (1962-1975: Duckert); nördlicher Stadtrand nahe dem ehemaligen Kraftwerk Finow (1964); Altenhofer Straße: Im Garten (1955, 1960-1964, 1966-1969, 2006, 2007, 2009: Richert) und Umg./Forst Finowtal: F. Schwärze (1963, 1964), Einflugschneise Flugplatz (1997, 2016), 1. Melchower Weg nordöstlich Kleines Postluch (2004); Menningsfließgebiet (1993)

3148/IV Ebw.-Westend/Wildparkstraße (1968, 1969, 1976: Kittel)

3149/III Ebw./Eichwerderberge - Ostrand (1964, 1968); Stecherschleuse Umg./ehemaliges Kiesgrubengelände nördlich des Ortes (1997)

d 3047/III Groß Schönebeck/Ortsgebiet: Friedenstraße (2015: Haase)

3047/IV Eichhorst Umg./Engere Schorfheide: F. Eichheide (1962), Sarnow (1991: Ockruck; Richert 2009), Süßer Winkel am Werbellinsee (1977)

3048/II Joachimsthal Umg./F. Voigtswiese (1987: Schottstädt)

3048/III Altenhof (1987, 1990: Schottstädt); Jagdschloss Hubertusstock Umg./Engere Schorfheide: Kl. Kaisergrund (2006, 2009; RICHERT 2009); Joachimsthal Umg./Forst Griminitz: Revier Michen, Abt. 40/41 (2006; Richert 2009)

3147/I Groß Schönebeck Umg./ehemaliges Objekt Umformtechnik (1991: Ockruck), Sumpf bei Gardix (1991: Ockruck)

3147/II Eichhorst Umg./Üderheide: F. Grünenberg (1963, 1972) und Umg./am Weg nach Werbellin (1995)

3148/I Lichterfelde Umg./NSG Buckowseerinne: Rüsternallee nordöstlich Gut Buckow (2007)

e 3049/IV Chorin Umg. (1962: Kames)

3149/II Liepe Umg./Forst Chorin: F. Liepe (19649

f 3049/III Buchholz bei Serwest (1967)

3049/IV Brodowin Umg./Schiefer Berg (1994)

3149/II Brodowin Umg./Vorwerk Zaun (1964)

g 3050/II Stolzenhagen/NSG Krähen- und Jungfernberge (1979; Richert 1982); Gellmersdorf Umg./NSG Gellmersdorfer Forst: Buchsmühle (1990: Schottstädt)

3149/II Liepe/Oderhänge (1961: Richert; 1978: Kittel)

3149/IV Struwenberg (NSG Kanonen- und Schlossberg (1997)

3150/I Oderberg (1960, 1962: Steinig)/NSG Pimpinellenberg (1961: Urbahn; 1968, 1989: Richert, RICHERT 1994), Teufelsberg (1973, 1975, 1977 Richert; 1974: Kittel, RicherT 1994)

3150/III Gabow/Oderhänge: Granitberggebiet (1983: Richert; 1991, 1992: Kleße)

3150/IV Altglietzen/Oderhänge: Gabower Berge (1999: Busse \& Ockruck)

\section{9 (10017) Mythimna flammea (CuRTIS, 1828)}

- RL Land Brandenburg: Kategorie 3 (gefährdet): NM. Die Art tritt im Gebiet zwar sehr lokal auf (vgl. RicHERT 2003, 2010, 2014), wird jedoch an den Fundplätzen gelegentlich in größerer Zahl beobachtet: So hatten Gelbrecht \& Salpeter am 23.V.2010 bei Prenden 16 F a.L., überwiegend an superaktinischen Röhren; Ockruck registrierte am 22.V.2014 bei Biesenthal $10 \mathrm{~F}$ a.L. 


\author{
Neue Nachweise von bekannten Fundorten: \\ b 3247/II Biesenthal Umg./NSG Biesenthaler Becken: Lange- \\ rönner Weg (2014: Ockruck) \\ c 3147/I Zerpenschleuse/Ortsgebiet: Berliner Straße, im \\ Garten (2014: Busse)
}

\section{0 (10022) Mythimna l-album (LinNAeus, 1767)}

Die Beobachtungslücke im Naturraum h (Odertal, RICHERT 2004) wurde inzwischen geschlossen (vgl. Richert 2014). Damit ist die Art in allen Naturräumen des Gebietes nachgewiesen.

Neue Nachweise von bekannten Fundorten:

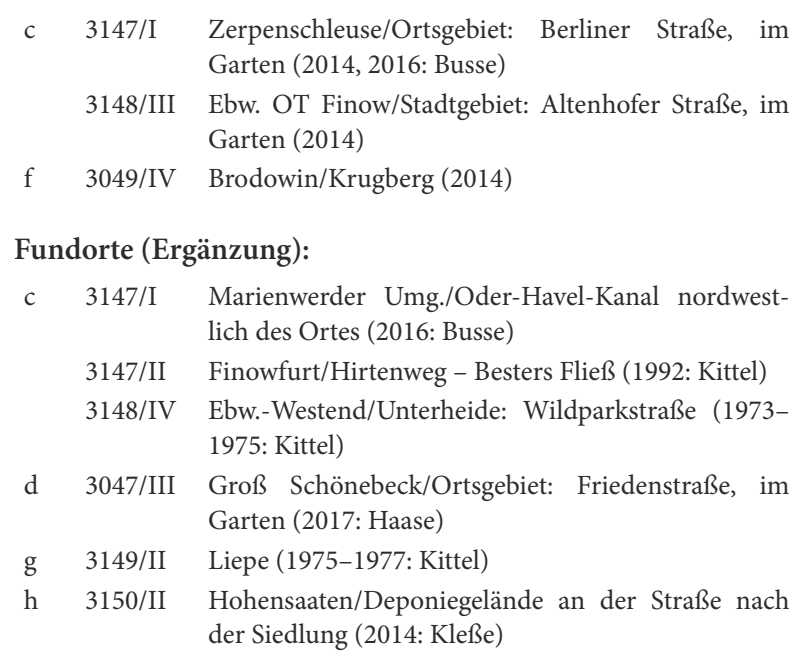

\section{2 (10038) Orthosia gothica (LinnAEus, 1758)}

Phänologie/Voltinismus: Univoltin; 14.III.(2015), aFA, phänologische Verfrühung 2d gegenüber bis 2013.

\section{4 (10041) Orthosia miniosa (DENIS \& SCHIFFER- MÜLLER, 1775)}

- RL Land Brandenburg: Vorwarnliste: EW;

- RL Deutschland (2011): Vorwarnliste.

Neue Nachweise von bekannten Fundorten:

c 3148/III Ebw. OT Finow/Stadtgebiet: Altenhofer Straße, im Garten (2014)

Fundorte (Ergänzung):

d 3047/III Groß Schönebeck/Ortsgebiet: Friedenstraße, im Garten (2014: Haase)

\section{5 (10042) Orthosia opima (HüBNER, 1809)}

- RL Land Brandenburg: Kategorie 3 (gefährdet): HW;

- RL Deutschland (2011): Kategorie 3 (gefährdet).

Phänologie/Voltinismus: Univoltin; 18.III.(2014), aFA, phänologische Verfrühung 13d gegenüber bis 2013.
Neue Nachweise von bekannten Fundorten:

c 3147/I Zerpenschleuse/Ortsgebiet: Berliner Straße, im Garten (2014, 2016: Busse)

3148/IV Ebw.-Westend/Unterheide: Wildparkstraße (1994: Kittel)

Fundorte (Ergänzung):

b 3148/IV Spechthausen Umg./Barnimer Heide: Stromtrasse südwestlich des Ortes (2015)

c 3148/IV Ebw./Stadtgebiet: Kleingartenanlage im Schwärzetal nördlich des Forstbotanischen Gartens (2016, 2018 Schwabe)

556 (10043) Orthosia populeti (FABRICIUs, 1775) = populi STRöm, 1783

Phänologie/Voltinismus: Univoltin; 09.III.(2014), aFA, phänologische Verfrühung 11d gegenüber bis 2013.

Neue Nachweise von bekannten Fundorten:

c 3147/I Zerpenschleuse/Ortsgebiet: Berliner Straße (2014, 2015: Busse)

\section{7 (10044) Orthosia cerasi (FABRICIUs, 1775) = stabilis (DENIS \& SCHIFFERMÜLLER, 1775)}

Haase trug die R bei Groß Schönebeck mit Populus $x$ canadensis, Kanadische Pappel, ein.

\section{8 (10048) Orthosia gracilis (DENIS \& SCHIFFER-} MÜLLER, 1775)

Phänologie/Voltinismus: Univoltin; $1 \mathrm{~F}$ a.L. am 28.III. (2014), aFA, phänologische Verfrühung $8 \mathrm{~d}$ gegenüber bis 2013.

Neue Nachweise von bekannten Fundorten:

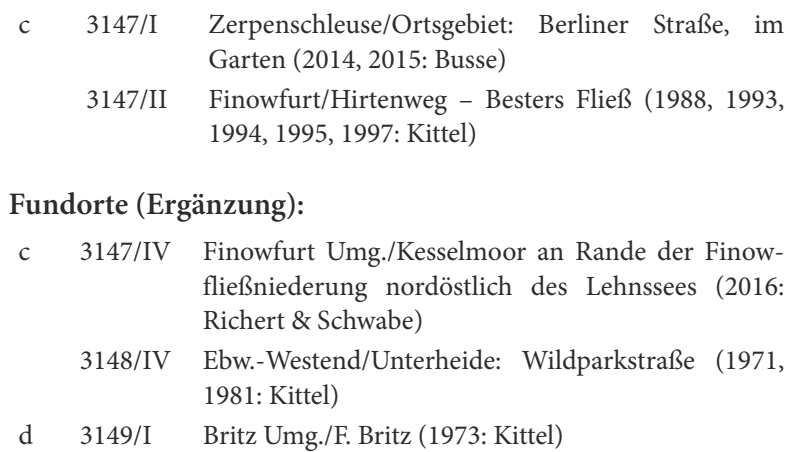

559 (10050) Orthosia munda (DENIS \& SCHIFFERMÜLLER, 1775)

Phänologie/Voltinismus: Univoltin; 30.V.(1987), aFE, (korrigiert nach Beleg in coll. Kittel; in RicherT 2014 war der 12.V.(1969) als aFE angegeben).

Neue Nachweise von bekannten Fundorten:

c 3147/I Zerpenschleuse/Ortsgebiet: Berliner Straße, im Garten (2014, 2017: Busse) 
3148/III Ebw. OT Finow/Stadtgebiet: Altenhofer Straße, im Garten (2014)

Fundorte (Ergänzung):

c 3147/II Finowfurt/Hirtenweg - Besters Fließ (1987: Kittel)

3148/IV Ebw.-Westend/Unterheide: Wildparkstraße (1974, 1982, 1984: Kittel); Ebw./Stadtgebiet: Kleingartenanlage im Schwärzetal nördlich Forstbotanischer Garten (2015, 2016: Schwabe)

d 3047/III Groß Schönebeck/Ortsgebiet: Friedenstraße, im Garten (2015: Haase)

g 3150/I Oderberg/Teufelsberg (1979: Kittel)

560 (10062) Panolis flammea (Denis \& SCHIFFERMÜLLER, 1775)

Im Jahr 2018 wurde die Art im Gebiet lokal ungewöhnlich häufig beobachtet: Am 10.IV. hatte Schwabe in seinem Garten in Eberswalde $>600 \mathrm{~F}$ a.L. (genaue Zählung bis 500 Expl.; danach erfolgte weiterer starker Anflug). Solche Mengen hatte er weder vorher noch an den Folgetagen beobachtet. Bei im gleichen Zeitraum an anderen Plätzen im Gebiet durchgeführten Lichtfängen erschien die Art zwar häufig a.L., jedoch nicht in solchen Massen.

\section{1 (10054) Egira conspicillaris (LINNAEUs, 1758)}

Neue Nachweise von bekannten Fundorten:

c 3147/II Finowfurt/Hirtenweg - Besters Fließ (1993, 1994: Kittel)

3148/IV Ebw.-Westend/Unterheide: Wildparkstraße (1974, 1977: Kittel)

g 3149/II Liepe (1974, 1975: Kittel)

3150/I Oderberg/Teufelsberg (1973, 1974: Kittel)

Fundorte (Ergänzung):

g 3150/I Oderberg/Geistberg $(2015,2016,2017$ : Schwabe)

562 (10062) Cerapteryx graminis (LinNaEus, 1758)

Der F wurde am Tag beim Saugen an Solidago canadensis, Kanadische Goldrute, beobachtet (Foto Schwabe).

\section{3 (10064) Tholera cespitis (DENIs \& SCHIFFER- MÜLLER, 1775)}

Die Art wird in neuerer Zeit im Gebiet nur noch sehr sporadisch gefunden.

Neue Nachweise von bekannten Fundorten:

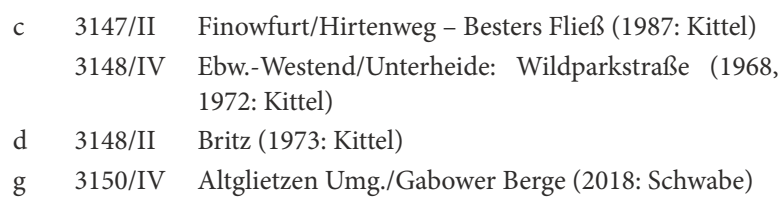

Fundorte (Ergänzung):

d 3048/I Joachimsthal (1976: Kittel)

h 3150/II Hohensaaten/Deponiegelände an der Straße nach der Siedlung (2014: Kleße)
564 (10065) Tholera decimalis (PoDA, 1761) = popularis FABRICIUS, 1775

Die Beobachtungslücken in den Naturräumen e (vgl. RicherT 2014) und f (Uckermärker Kuppen- und Hügelland einschließlich Choriner Waldhügel sowie Parsteiner Becken, vgl. RicherT 2003) konnten inzwischen geschlossen werden. Damit ist die Art in allen Naturräumen des Gebietes nachgewiesen.

Neue Nachweise von bekannten Fundorten:

c 3147/I Zerpenschleuse/Ortsgebiet: Berliner Straße, im Garten (2014, 2015: Busse)

3148/III Ebw. OT Finow/Stadtgebiet: Altenhofer Straße, im Garten $(2014,2015,2016,2018)$

Fundorte (Ergänzung):

a 3249/II Falkenberg Umg./Cöthener Forst: Am Klingenden Fließ unterhalb des Bismarck-Turmes (2014)

c 3147/I Marienwerder Umg./Oder-Havel-Kanal nordwestlich des Ortes (2016: Busse)

3148/IV Ebw.-Westend/Unterheide: Wildparkstraße (1968, 1969, 1973: Kittel); Ebw./Stadtgebiet: Kleingartenanlage im Schwärzetal nördlich des Forstbotanischen Gartens (2014, 2015, 2017: Schwabe)

d 3047/III Groß Schönebeck/Ortsgebiet: Friedenstraße, im Garten (2017: Haase)

e 3049/IV Chorin Umg./Forst Chorin: Teerbrennerberge - Am Olberg (2013: MüLLER 2015), Kreuzung Denglerweg - Olbergstraße (2013: MüLler 2015)

f 3049/IV Brodowin/Krugberg (2014), Karpatenweg östlich Krugberg (2015)

g 3149/II Niederfinow/Oderhänge - ehemalige Kiesgruben südwestlich des Schiffshebewerks (2016: Schwabe)

3150/I Oderberg/am Friedhof (2016: Schwabe)

3150/IV Altglietzen Umg./Gabower Berge (2018: Schwabe)

h 3150/II Hohensaaten/Deponiegelände an der Straße nach der Siedlung (2014: Kleße)

565 (10068) Pachetra sagittigera (HufNagel, 1766) = fulminea FABRICIUS, 1777

Neue Nachweise von bekannten Fundorten:

c 3147/I Zerpenschleuse/Ortsgebiet: Berliner Straße, im Garten (2015: Busse)

3147/II Finowfurt/Hirtenweg - Besters Fließ (1986: Kittel)

3148/III Ebw. OT Finow/Stadtgebiet: Altenhofer Straße, im Garten $(2016,2017,2018)$ und Umg./Forst Finowtal: Einflugschneise Flugplatz (2016)

Fundorte (Ergänzung):

c $3147 / \mathrm{IV}$ Finowfurt Umg./Kesselmoor an der Finowfließniederung nordöstlich des Lehnssees (2016)

3148/IV Ebw.-Westend/Unterheide: Wildparkstraße (1971, 1973, 1997: Kittel) 


\section{Unterfamilie Noctuinae}

568 (10089) Diarsia mendica (FABRICIUS, 1775) = festiva DENIS \& SCHIFFERMÜLLER, 1775

\section{Neue Nachweise von bekannten Fundorten:}

c 3147/II Finowfurt/Hirtenweg - Besters Fließ (1991, 1995, 1997: Kittel)

g $\quad 3149 / \mathrm{II} \quad$ Liepe (1973: Kittel)

Fundorte (Ergänzung):

c 3147/IV Finowfurt Umg./Kesselmoor an der Finowfließniederung nordöstlich des Lehnssees $(2016,2017)$

3148/III Ebw. OT Finow Umg./Forst Finowtal: Einflugschneise Flugplatz (2016)

3148/IV Ebw.-Westend/Unterheide: Wildparkstraße (1973, 1976, 1977: Kittel); Ebw./Stadtgebiet: Kleingartenanlage im Schwärzetal nördlich des Forstbotanischen Gartens (2014: Schwabe)

d 3047/III Groß Schönebeck/Ortsgebiet: Friedenstraße, im Garten (2016: Haase)

3148/II Britz (1979: Kittel)

\section{9 (10092) Diarsia brunnea (DeNIS \& SCHIFFER- MÜLLER, 1775)}

Alle mir aus dem Gebiet bekannten Fundorte der Art werden nachgetragen.

Die von Duckert beim regelmäßigen Hauslichtfang im Siedlungsraum von Finow im Zeitraum 1962-1974 registrierten Anflugzahlen spiegeln die Häufigkeit der Art im Gebiet nicht wider.

Tab. 11: Anflugzahlen von Diarsia brunnea beim regelmäßigen Hauslichtfang im Stadtgebiet von Finow (nach Aufzeichnungen von Duckert).

\begin{tabular}{|l|c|c|c|c|c|c|c|}
\hline Jahr & 1962 & 1963 & 1964 & 1965 & 1966 & 1967 & 1968 \\
\hline Anzahl & 2 & 1 & 1 & - & 1 & - & 1 \\
\hline Jahr & 1969 & 1970 & 1971 & 1972 & 1973 & 1974 & \\
\cline { 1 - 7 } Anzahl & - & 5 & - & 2 & 3 & - & \\
\cline { 1 - 6 }
\end{tabular}

\section{Fundorte:}

a 3249/II Falkenberg Umg./Cöthener Forst: Ehemalige F. Tobbenberge (1970, 2011, 2012), F. Panekel (1985)

b 3148/IV Ebw. Umg./Oberheide: F. Leuenberger Wiesen (1969), Waldschneise am Leuenberger Graben Richtung Wiebecke-Damm (2011: Schwabe), WiebeckeDamm am Möllergrab (2011: Schwabe)

3149/III Sommerfelde Umg./ehemaliger TÜP Panzerbahn (2001, 2005) (RICHERT 2006), Nordostrand des Hohenfinower Waldes (2003: Ockruck); Tornow Umg./Barnimhänge nördlich des Ortes: Abt. 630 (2008, 2009: Schwabe), Abt. 629 (2011, 2012, 2013: Schwabe)

3149/IV Hohenfinow/Ortsgebiet (1988: Hülbert) (RicherT \& HÜLBERT 1991)

3247/I Prenden Umg./Strehlesee - Südufer (2008: Rosenbauer)

3247/II Biesenthal Umg./NSG Biesenthaler Becken: Weg nördlich der Pfauenwiesen (2007: Ockruck), Langerönner Weg (2010, 2014: Ockruck)
3248/II Trampe/ehemaliger TÜP (2000: Gelbrecht, Kallies \& Noack; 2000, 2005: Richert) (RicherT 2006) und Umg./Tramper Forst: Bornemannspfuhl (2011: Schwabe)

c 3146/II Zerpenschleuse Umg./Hammerbruch (1998, 2000 Ockruck) (westlich angrenzendes Nachbargebiet)

3146/IV Kreuzbruch Umg./ehemalige Römerwegbrücke am Oder-Havel-Kanal östlich des Ortes (2014: Busse)

3147/I Zerpenschleuse/Ortsgebiet (1980, 1981, 1987, 1988: Ockruck; 2013, 2014, 2018: Busse) (Busse \& OCKRUCK 1991)

3147/II Marienwerder Umg./F. Pechteich (1950-1959: Haeger); Finowfurt Umg./Besters Fließ: Hirtenweg (1988, 1990-1992: Kittel), Möllenwiesen im Finowbruch westlich des Ortes, Nordwestrand $(2011,2012)$

3147/III Zerpenschleuse Umg./Oder-Havel-Kanal nahe der Lottscher Brücke (1999: Ockruck)

3147/IV Marienwerder/Schilfgebiet südwestlich des Sportplatzes (2000: Rosenbauer) und Umg./Grafenbrück (1985: Ockruck); Sophienstädt Umg./Pregnitzfließniederung nordöstlich des Mittel-Prendensees (2011, 2012); Finowfurt Umg./Kesselmoor an der Finowfließniederung nordöstlich des Lehnssees $(2016,2017)$

3148/II Britz Umg./Stadtseerinne westlich des Gr. Stadtsees (2013)

3148/III Ebw. OT Finow/Stadtgebiet: Im Garten (1963, 1966, 1967, 1968, 1969, 2002, 2003, 2006, 2008, 2009, 2011, 2013-2016) und Umg./Forst Finowtal: Einflugschneise Flugplatz (1995), F. Schwärze (1963, 1974, 1986 [siehe auch Daten von Duckert]), 1. Melchower Weg nordöstlich Kl. Postluch (2004), Gr. Postluch (2007), Samithseemoor (2006)

3148/IV Ebw.-Westend/Unterheide: Drehnitzwiesen (2011), Wildparkstraße (1969, Anfang der 1970er Jahre, 1975: Kittel); Ebw./Stadtgebiet (1936: Jonas), Kleingartenanlage im Schwärzetal nördlich des Forstbotanischen Gartens (2014, 2016, 2018: Schwabe)

3149/I Ebw. Umg./Ragösetal bei Mönchsbrück (2003); Stecherschleuse Umg./Benzintrasse nordwestlich des Ortes (2013: Schwabe)

3149/III Ebw./Eichwerder $(1964,1968)$

3149/IV Stecherschleuse Umg./am Rande des ehemaligen Kiesgrubengeländes nordöstlich des Ortes (2013: Schwabe)

d 3047/IV Groß Schönebeck Umg./Schorfheide: Joachimsthaler Damm westlich der Pinnowseen (2004) (NSG Kienhorst/Köllnseen/Eichheide; RICHERT 2010); Eichhorst Umg./Üderheide: Süßer Winkel am Werbellinsee (1977, 1980)

3048/I Joachimsthal/Schorfheide: Werbellinsee (1999: Rosenbauer)

3048/II Joachimsthal Umg./Forst Grimnitz: F. Voigtswiese (1987: Schottstädt)

3048/III Jagdschloss Hubertusstock/Engere Schorfheide Kl. Kaisergrund (2006, 2007, 2009); Joachimsthal Umg./Schorfheide: Forst Grimnitz, Revier Michen, Abt. 40/41 (2006) (NSG Kienhorst/Köllnseen/Eichheide; Richert 2010); Altenhof/Werbellinsee (1987: Schottstädt)

3147/II Eichhorst Umg./Üderheide: F. Grünenberg (1962)

3148/I Werbellin Umg./NSG Buckowseerinne: Südlich der Autobahnanschlussstelle (2011); Lichterfelde Umg./ NSG Buckowseerinne: Moorniederung nordöstlich des Gr. Buckowsees (2010), Streuobstwiesen südöstlich des Gr. Buckowsees $(2011,2012)$ (Richert 2012)

3148/II Britz/Ortsgebiet (1979: Kittel)

3149/I Britz Umg./F. Britz (1982: Riegel) 
e 2948/IV Glambeck Umg./Forst Grumsin: Waldstraße nach Joachimsthal westlich F. Bärendikte (2004)

3049/I Groß Ziethen Umg./Gr. Kagelpfuhl - Ostufer sowie zwischen Schulzensee und Kl. Kagelpfuhl nordwestlich des Ortes bei Sperlingsherberge (2008: Rödel \& Schulze), Steingruben an den Ihlowbergen (2014)

3049/III Senftenhütte Umg./Forst Chorin: F. Senftenthal (1967); Chorin Umg./Forst Chorin: F. Teerofen (1964)

3149/II Liepe Umg./Forst Chorin: F. Liepe (1964), NSG Plagefenn (2002: Richert; 2018: Richert \& Schwabe)

3150/I Oderberg Umg./Forst Freienwalde: F. Maienpfuhl (1964)

f 3049/III Buchholz bei Serwest (1967)

3049/IV Brodowin/Ortsgebiet: Mühle (1964)

3149/II Brodowin Umg./Vorwerk Zaun (1964)

g 3050/II Stolpe/Trockenhänge (1987: Schottstädt); Stolzenhagen/NSG Krähen- und Jungfernberge (1978, 1979, 1981, 2013) (RICHERT 1979)

3149/II Liepe (1987: Schottstädt)/Oderhänge: Sandberg (1973)

3149/IV Niederfinow Umg./Oderhänge - ehemalige Kiesgruben südwestlich des Schiffshebewerks (1995)

3150/I Oderberg/Pimpinellenberg (1968, 1988, 1989) (Richert 1994), Teufelsberg (1962: Haeger; 1977: Richert; 1983: Kittel) (RICHERT 1994)

3150/III Gabow/Oderhänge (1987, 1989: Schottstädt; 1991, 2003: Kleße), Granitberggebiet (2015: Schwabe)

3150/IV Altglietzen Umg./Gabower Berge (2003: Ockruck)

h 3150/III Bralitz/stillgelegte Kiesgrube (2012: Rosenbauer \& Theimer)

\section{0 (10093) Diarsia rubi (VIEWEg, 1790)}

Neue Nachweise von bekannten Fundorten:

c 3147/I Zerpenschleuse/Ortsgebiet: Berliner Straße, im Garten (2014, 2015: Busse)

3147/II Finowfurt/Hirtenweg - Besters Fließ (1987, 1995, 1997: Kittel)

3147/IV Marienwerder/Finowbruch: Steinlaake (2015) und Umg./Kiesgrube südwestlich des Sportplatzes (2015)

3148/III Ebw. OT Finow/Stadtgebiet: Altenhofer Straße, im Garten (2014)

g 3150/IV Altglietzen Umg./Gabower Berge (2015: Schwabe)

Fundorte (Ergänzung):

b 3247/II Biesenthal Umg./NSG Biesenthaler Becken: Langerönner Weg (2014: Ockruck)

c 3147/IV Finowfurt Umg./Finowfließniederung: Neue Brücke an der Biesenthaler Straße (2014), Kesselmoor an der Finowfließniederung nordöstlich des Lehnssees (2016)

3148/IV Ebw.-Westend/Unterheide: Wildparkstraße (Anfang der 1970er Jahre: Kittel); Ebw./Stadtgebiet: Kleingartenanlage im Schwärzetal nördlich des Forstbotanischen Gartens (2014: Schwabe)

d 3149/I Britz Umg./F. Britz (1973: Kittel)

\section{1 (10094) Diarsia florida (F. SснмIDT, 1859)}

- RL Deutschland (2011): Kategorie 3 (gefährdet). Ein älterer Fund (Beleg in coll. Kittel) wird ergänzend nachgetragen.
Neue Nachweise von bekannten Fundorten:

c 3148/IV Ebw.-Westend/Unterheide: Wildparkstraße (10.VI. 1977: Kittel)

\section{2 (10096) Noctua pronuba (LinNAEUs, 1758)}

Am 30.VIII.2014 registrierte Busse bei Marienwerder am Oder-Havel-Kanal einen Massenanflug von $200 \mathrm{~F}$ a.L. Ich beobachtete den $\mathrm{F}$ mehrfach nachts beim Saugen an Blüten von Buddleja davidii, Chinesischer Sommerflieder.

\section{3 (10097) Noctua orbona (HUfNAGEL, 1766)}

Neue Nachweise von bekannten Fundorten:

c 3147/I Zerpenschleuse/Ortsgebiet: Berliner Straße, im Garten (2016: Busse)

3147/II Finowfurt/Hirtenweg - Besters Fließ (1991: Kittel)

3148/III Ebw. OT Finow/Stadtgebiet: Altenhofer Straße, im Garten (2018)

g 3150/I Oderberg/Teufelsberg (1973: Kittel)

Fundorte (Ergänzung):

c 3148/IV Ebw.-Westend/Unterheide: Wildparkstraße (1973, 1976: Kittel)

d 3047/III Groß Schönebeck/Ortsgebiet: Friedenstraße, im Garten (2017: Haase)

f 3049/IV Brodowin/Krugberg (2014)

g 3149/II Liepe (1975: Kittel)

\section{3a (10098) Noctua interposita (HüBNER, 1790)}

Die Art ist inzwischen wohl im gesamten Gebiet verbreitet: Im Jahre 2014 wurde sie im Naturraum e (Uckermärker Kuppen- und Hügelland, RicherT 2003) erstmals nachgewiesen.

Phänologie/Voltinismus: Univoltin; mit zunehmender Datenmenge können die Flugzeitangaben präzisiert werden: 08.VI.2014, phänologische Verfrühung 24d gegenüber bis 2013; 31.V.(2016), aFA, erneute phänologische Verfrühung 7d, (insges. 31d gegenüber bis 2013)-14. IX.(2014), aFE, phänologische Verspätung 4d gegenüber bis 2013.

Neue Nachweise von bekannten Fundorten:

c 3147/I Zerpenschleuse/Ortsgebiet: Berliner Straße, im Garten (2014, 2016: Busse)

3148/III Ebw. OT Finow/Stadtgebiet: Altenhofer Straße, im Garten (2014, 2016, 2017, 2018)

d 3047/III Groß Schönebeck/Ortsgebiet: Friedenstraße, im Garten (2014, 2016, 2017: Haase)

g 3150/III Gabow/Granitberggebiet (2015: Schwabe)

3150/IV Altglietzen Umg./Gasbower Berge (2018: Schwabe)

Fundorte (Ergänzung):

b 3247/II Biesenthal Umg./NSG Biesenthaler Becken: Langerönner Weg (2014: Ockruck)

3249/I Gersdorf Umg./Gamengrund südlich Teufelssee (2017: Richert \& Schwabe) 
c 3147/I Marienwerder Umg./Oder-Havel-Kanal nordwestlich des Ortes (2014: Busse)

3147/IV Finowfurt Umg./Kesselmoor an der Finowfließniederung nordöstlich des Lehnssees $(2016,2017)$

3148/III Ebw. OT Finow Umg./Forst Finowtal: Einflugschneise Flugplatz (2017)

3148/IV Ebw./Stadtgebiet: Kleingartenanlage im Schwärzetal nördlich des Forstbotanischen Gartens (2014, 2016, 2017: Schwabe)

e 3049/I Groß Ziethen Umg./Steingruben an den Ihlowbergen (2014)

3149/II Liepe Umg./Forst Chorin: NSG Plagefenn - LedumMoor am Fuß der Plagebege (2018: Richert \& Schwabe)

g 3149/IV Niederfinow/Oderhänge - ehemalige Kiesgruben südwestlich des Schiffshebewerks (2016: Schwabe)

3150/I Oderberg/am Friedhof (2016: Richert \& Schwabe)

\section{4 (10099) Noctua comes (HüBNER, 1813)}

Alle mir aus dem Gebiet bekannten Fundorte der Art werden nachgetragen.

Die von Duckert beim regelmäßigen Hauslichtfang im Siedlungsraum von Finow im Zeitraum 1962-1974 registrierten Anflugzahlen zeigen erhebliche Abundanzschwankungen. Ehemalige Maximalwerte werden neuerdings aber nicht mehr erreicht.

Den F beobachtete ich nachts beim Saugen an Blüten von Buddleja davidii, Chinesischer Sommerflieder.

Tab. 12: Anflugzahlen von Noctua comes beim regelmäßigen Hauslichtfang im Stadtgebiet von Finow (nach Aufzeichnungen von Duckert).

\begin{tabular}{|l|c|c|c|c|c|c|c|}
\hline Jahr & 1962 & 1963 & 1964 & 1965 & 1966 & 1967 & 1968 \\
\hline Anzahl & 15 & 2 & 9 & 20 & 30 & 34 & 90 \\
\hline Jahr & 1969 & 1970 & 1971 & 1972 & 1973 & 1974 & \\
\cline { 1 - 8 } Anzahl & 57 & 52 & 12 & 32 & 95 & 71 & \\
\cline { 1 - 6 }
\end{tabular}

Phänologie/Voltinismus: Univoltin; 07.X.(2013), aFE, phänologische Verspätung 13d (korrigiert nach MüLLER 2015; in RICHERT 2014 war der 24.IX.(2013) als aFE angegeben).

\section{Fundorte:}

a 3249/II Falkenberg Umg./Cöthener Forst: Uchtenhagen (1974), F. Panekel (1985)

b 3148/IV Ebw./Oberheide: Institut für Waldschutz (1959: Kruel), F. Leuenberger Wiesen (1975)

3149/III Sommerfelde Umg./ehemaliger TÜP Panzerbahn (2001, 2002, 2009) (RICHERT 2006), Nordostrand des Hohenfinower Waldes (2005: Ockruck); Tornow Umg./Barnimhänge nördlich des Ortes (2008: Ockruck)

3149/IV Karlswerk Umg./am Weg nach Struwenberg (1974); Hohenfinow/Ortsgebiet (1986: Hülbert) (RicherT \& HüLBERT 1991) und Umg./Karswerker Straße oberhalb/südlich Karlswerk (2011)

3247/I Prenden/Strehlesee: Südufer (2010: Ockruck)

3247/II Biesenthal Umg./NSG Biesenthaler Becken: Weg nördlich der Pfauenwiesen (2014: Ockruck), Langerönner Weg (2009: Ockruck)

3248/II Trampe/ehemaliger TÜP (2005) (RICHERT 2006)

c 3147/I Zerpenschleuse/Ortsgebiet (1979-1981, 1983-1990: Ockruck; 2013, 2014: Busse) (BUsse \& OCKRUCK 1991)
3147/II Finowfurt Umg./Besters Fließ: Hirtenweg (1990, 1991, 1992, 1994: Kittel)

3147/IV Finowfurt Umg./Kesselmoor an der Finowfließniederung nordöstlich des Lehnssees (2016)

3148/III Ebw. OT Finow/Stadtgebiet: Im Garten (1960, 1962-1974: Duckert; 1961, 1962, 1964, 1966-1968, 1974, 1977, 1987, 1990, 1991, 1995, 1999-2016, 2018: Richert) und Umg./Forst Finowtal: 1. Melchower Weg nordöstlich vom Kl. Postluch (2004), Samithseemoor (1993)

3148/IV Ebw./Stadtgebiet: Im Garten (1936: Jonas), Kleingartenanlage im Schwärzetal nördlich Forstbotanischer Garten (2016: Schwabe); Ebw.-Kupferhammer/ Güterbahnhof (2010: Schwabe); Ebw.-Westend/ Unterheide: Wildparkstraße (1969, 1974, 1975, 1976, 1986: Kittel)

3149/II Niederfinow Umg./Oder-Havel-Kanal östlich der Klosterbrücke (2008: Schwabe)

d 3047/III Groß Schönebeck/Ortsgebiet (1999: Haase), Friedenstraße, im Garten (2017: Haase)

3047/IV Groß Schönebek Umg./Schorfheide: Pinnowseengebiet - NSG Meelake (1991: Ockruck) (NSG Kienhorst/Köllnseen/Eichheide; RICHERT 2010); Eichhorst Umg./Üderheide: Nahe dem Süßen Winkels am Werbellinsee (1964)

3048/I Joachimsthal Umg./Schorfheide: Werbellinsee (1999: Rosenbauer)

3048/II Joachimsthal Umg./Forst Grimnitz: F. Voigtswiese (1987: Schottstädt)

3048/III Jagdschloss Hubertusstock Umg./Engere Schorfheide: Kl. Kaisergrund (2009) (NSG Kienhorst/ Köllnseen/Eichheide; RICHERT 2010)

3147/II Eichhorst Umg./Fenn am Fennberg östlich der Straße nach Finowfurt (1989), Moospfuhl östlich der Straße nach Finowfurt (1969)

3148/I Werbellin Umg./NSG Buckowseerinne: Südlich der Autobahnanschlussstelle (2011) (RICHERT 2012)

3149/I Britz Umg./F. Britz (1973: Kittel)

e 3049/I Neugrimnitz Umg./Forst Grumsin: Grumsin (1972); Groß Ziethen Umg./Forst Grimnitz: F. Gr. Ziethen (1967), Soll nördlich des Ortes (1994: DEI, Sommer), Steingruben an den Ihlowbergen $(1997,2014)$

3049/III Chorin Umg./Forst Chorin: F. Teerofen (1964), südöstlich des Pferdebergs (2013: MüLler 2015)

3049/IV Brodowin Umg./Forst Chorin: Mooskuten (1994: DEI, Sommer); Teerbrennerberge - Am Olberg (2013: MüLler 2015), Kreuzung Denglerweg Olbergstraße (2013: MüLleR 2015)

3149/II Liepe Umg./Forst Chorin: F. Liepe (1964), NSG Plagefenn (2002)

3150/I Oderberg Umg./Forst Freienwalde: F. Maienpfuhl (1964)

f 3049/II Serwest Umg./Serwester See am Fuß der Kernberge (1992, 1994: DEI, Sommer)

3049/IV Brodowin Umg./Kl. Rummelsberg (1992: DEI, Sommer), Schiefer Berg (1994), Krugberg (2013, 2014), Karpatenweg östlich Krugberg (2015)

3149/II Brodowin Umg./Vorwerk Zaun (1964)

g 3050/II Stolpe/Trockenhänge (1987: Schottstädt); Stolzenhagen/NSG Krähen- und Jungfernberge (1978: Kruel; 1979, 2002, 2005, 2013: Richert) (RICHERT 1979, dort als orbona verzeichnet)

3149/II Liepe/Oderhänge: Sandberg (1973)

3149/IV Niederfinow Umg./Oderhänge - ehemalige Kiesgruben südwestlich des Schiffshebewerks (1995: Richert; 2015: Schwabe); Struwenberg Umg./NSG Kanonenund Schlossberg (1999) 
3150/I Oderberg (1961, 1965: Steinig)/NSG Pimpinellenberg (1961: Urbahn; 1968, 1987, 1988, 1989: Richert) (Richert 1994), am Friedhof (2016), Teufelsberg (1962: Haeger; 1973, 1974: Kittel; 1977, 1991: Richert) (RICHERT 1994)

3150/III Gabow/Oderhänge (1987, 1989: Schottstädt; 1991, 1992, 2003-2005: Kleße): Granitberggebiet (1983, 1984: Richert) (RICHERT 1984)

3150/IV Altglietzen Umg./Oderhänge: Gabower Berge (2004, 2008: Ockruck; 2015: Richert)

h 3149/II Liepe Umg./NSG Niederoderbruch: Schöpfwerk Liepe $(1973,2009)$

3150/III Bralitz/Stillgelegte Kiesgrube (2009)

\section{7 (10105) Noctua interjecta (HüBNER, 1803)}

Phänologie/Voltinismus: Univoltin; 12.IX.(2014), aFE, phänologische Verspätung 13d gegenüber bis 2013.

Neue Nachweise von bekannten Fundorten:

b 3247/II Biesenthal Umg./NSG Biesenthaler Becken: Langerönner Weg (2014: Ockruck), Weg nördlich der Pfauenwiesen (2014: Ockruck)

c 3147/I Zerpenschleuse/Ortsgebiet: Berliner Straße, im Garten (2014, 2015, 2015: Busse)

3147/II Finowfurt/Hirtenweg - Besters Fließ (1989-1993: Kittel)

3148/III Ebw. OT Finow/Stadtgebiet: Altenhofer Straße, im Garten (2014, 2015, 2016, 2017)

d 3047/III Groß Schönebeck/Ortsgebiet: Friedenstraße, im Garten $(1999,2014,2015$ : Haase)

Fundorte (Ergänzung):

e 3049/I Groß Ziethen Umg./Steingruben an den Ihlowbergen (2014)

c 3147/IV Finowfurt Umg./Finowfließniederung: Neue Brücke an der Biesenthaler Straße (2014)

3148/IV Ebw./Stadtgebiet: Kleingartenanlage im Schwärzetal nördlich des Forstbotanischen Gartens (2014, 2015, 2017: Schwabe)

h 3150/II Hohensaaten/Deponie an der Straße nach der Siedlung (2014: Kleße)

578 (10108) Epilecta linogrisea (DeNIs \& SCHIFFERMÜLLER, 1775)

- RL Land Brandenburg: Kategorie 3 (gefährdet): TR, $\mathrm{CB}$;

- RL Deutschland (2011): Vorwarnliste.

Neue Nachweise von bekannten Fundorten:

c 3147/II Finowfurt/Hirtenweg - Besters Fließ (1986, 1990, 1991, 1992, 1993, 1995, 1995: Kittel)

3148/III Ebw. OT Finow/Stadtgebiet: Im Garten (2015)

3148/IV Ebw.-Westend/Unterheide: Wildparkstraße (1973, 1976, 1978: Kittel)

d 3149/I Britz Umg./F. Britz (1973: Kittel)

Fundorte (Ergänzung):

c 3148/III Ebw. OT Finow Umg./Forst Finowtal; Einflugschneise Flugplatz (2017: Schwabe)
579 (10113) Lycophotia porphyrea (DENIS \& SCHIFFERMÜLLER, 1775)

Neue Nachweise von bekannten Fundorten:

c 3147/I Zerpenschleuse/Ortsgebiet: Berliner Straße, im Garten (2014, 2015: Busse)

3147/II Finowfurt/Hirtenweg - Besters Fließ (1994, 1997: Kittel)

3148/III Ebw. OT Finow/Stadtgebiet: Im Garten (2015, 2017, 2018) und Umg./Forst Finowtal: Einflugschneise Flugplatz (2017: Richert \& Schwabe)

3148/IV Ebw.-Westend/Unterheide: Wildparkstraße (1969, 1970, 1972, 1977: Kittel)

g 3150/I Oderberg/Teufelsberg (1973: Kittel)

Fundorte (Ergänzung):

b 32477 Biesenthal Umg./NSG Biesenthaler Becken: Nördlich der Pfauenwiesen (2015: Ockruck)

c 3147IV Finowfurt Umg./Kesselmoor an der Finowfließniederung nordöstlich des Lehnssees (2016)

3148/IV Ebw./Stadtgebiet: Kleingartenanlage im Schwärzetal nördlich Forstbotanischer Garten (2016: Schwabe)

d 3047/III Groß Schönebeck/Ortsgebiet: Friedenstraße, im Garten (1999, 2014, 2015, 2018: Haase)

\section{0 (10139) Rhyacia simulans (HÜBNER, 1766)}

- RL Land Brandenburg: Kategorie 3 (gefährdet): TR.

- RL Deutschland (2011): Vorwarnliste.

Phänologie/Voltinismus: Univoltin; 01.X.(1977), aFE (korrigiert nach Beleg in coll. Kittel; in RichERT 2014 war der 25.IX.(1987) als aFE angegeben).

Neue Nachweise von bekannten Fundorten:

c 3147/I Zerpenschleuse/Ortsgebiet: Berliner Straße, im Garten (2014: Busse)

3147/II Finowfurt/Hirtenweg - Besters Fließ (1990: Kittel)

3148/IV Ebw.-Westend/Unterheide: Wildparkstraße (1968, 1977: Kittel)

g 3150/I Oderberg/Teufelsberg (1974: Kittel)

Fundorte (Ergänzung):

h 3150/II Hohensaaten/Deponiegelände an der Staße nach der Siedlung (2014: Kleße)

\section{1 (10161) Eurois occulta (LinNAEUs, 1758)}

- RL Land Brandenburg: Kategorie 3 (gefährdet): HW, NW;

- RL Deutschland (2011): Vorwarnliste.

Neue Nachweise von bekannten Fundorten:

c 3147/I Zerpenschleuse/Ortsgebiet: Berliner Straße, im Garten (2016: Busse)

Fundorte (Ergänzung):

c 3148/IV Ebw.-Westend/Unterheide: Wildparkstraße (1972: Kittel) 


\section{2 (10163) Spaelotes ravida (DENIS \& SCHIFFER- MÜLLER, 1775)}

- RL Land Brandenburg: Kategorie 3 (gefährdet): TR;

- RL Deutschland (2011): Kategorie 2 (stark gefährdet). Ältere Funde (Belege in coll. Kittel) werden ergänzend nachgetragen.

Neue Nachweise von bekannten Fundorten:

g 3149/II Liepe (1974: Kittel)

Fundorte (Ergänzung):

c 3147/II Finowfurt/Hirtenweg - Besters Fließ (1995: Kittel)

d 3048/I Joachimsthal (1976: Kittel)

\section{3 (10169) Opigena polygona (DENIS \& SCHIF- FERMÜLLER, 1775)}

Im Gebiet verschollen. Die letzten mir bekannten Funde erfolgten im Jahre 1999. O. polygona ist für langfristige Populationsschwankungen bekannt, sie kann gebietsweise für längere Zeit völlig verschwinden; (siehe dazu die Ausführungen in RicherT 2003).

Ältere Funde (Belege in coll. Kittel) werden ergänzend nachgetragen.

Neue Nachweise von bekannten Fundorten:

c 3147/II Finowfurt/Hirtenweg - Besters Fließ (1990, 1995:

$\begin{array}{lll} & \text { Kittel) } \\ \text { g } 3149 / \mathrm{II} & \text { Liepe (1974: Kittel) }\end{array}$

3150/I Oderberg/Teufelsberg (1973: Kittel)

Fundorte (Ergänzung):

c 3148/IV Ebw.-Westend/Unterheide: Wildparkstraße (1972, 1974: Kittel)

d 3149/I Britz Umg./F. Britz (1974: Kittel)

\section{4 (10171) Graphiphora augur (FABRICIUs, 1775)}

Siehe 3.3. Wieder entdeckte Arten.

\section{6 (10201) Xestia triangulum (Hufnagel, 1766)}

Phänologie/Voltinismus: Die Auswertung aktueller und bisher nicht bekannter Daten aus dem Gebiet macht folgende Ergänzung notwendig: 22.V.(2016), aFA, phänologische Verfrühung 11d gegenüber bis 2013, - 04.IX. (2008) aFE, phänologische Verspätung 3d gegenüber bis 2013. Damit zeigt auch diese Art phänologische Veränderungen in neuerer Zeit (in RICHERT 2014 war 01.IX. (1963) als aFE angegeben).

\section{8 (10204) Xestia stigmatica (HÜBNER, 1813)} $=$ rhomboidea auct.

- RL Land Brandenburg: Kategorie 3 (gefährdet): BW, WS.
Neue Nachweise von bekannten Fundorten:

c 3148/IV Ebw.-Westend/Unterheide: Wildparkstraße (1971, 1974, 1975: Kittel)

Fundorte (Ergänzung):

b 3249/I Gersdorf Umg./Gamengrund südlich Teufelssee (2017)

\section{9 (10211) Xestia sexstrigata (HAwORTH, 1809) = umbrosa HüBNER, 1813}

Die Beobachtungslücke in den Naturräumen e und $\mathrm{f}$ (Ückermärkisches Kuppen- und Hügelland einschließlich Choriner Waldhügel, Parsteiner Becken; vgl. RicherT 2003) konnte inzwischen geschlossen werden.

Phänologie/Voltinismus: Univoltin; 12.IX.(2013), aFE, phänologische Verspätung 1d (korrigiert nach MÜLLER 2015; in Richert 2014 war der 11.IX.(1993) als aFE angegeben).

Neue Nachweise von bekannten Fundorten:

b 3247/II Biesenthal Umg./NSG Biesenthaler Becken: Nördlich der Pfauenwiesen (2015: Ockruck)

c 3147/I Zerpenschleuse/Ortsgebiet: Berliner Straße, im Garten (2014: Busse)

3147/II Finowfurt/Hirtenweg - Besters Fließ (1993-1995: Kittel)

3148/III Ebw. OT Finow/Stadtgebiet: Altenhofer Straße, im Garten (2014)

g 3150/I Oderberg/Teufelsberg (1973, 1974: Kittel)

3150/IV Altglietzen Umg./Gabower Berge (2015)

Fundorte (Ergänzung):

c 3147/I Marienwerder Umg./Oder-Havel-Kanal nordwestlich des Ortes (2014: Busse)

3148/IV Ebw.-Westend/Unterheide: Wildparkstraße (1971, 1973, 1974: Kittel); Ebw./Stadtgebiet: Kleingartenanlage im Schwärzetal nördlich Forstbotanischer Garten (2017: Schwabe)

e 3049/IV Chorin Umg./Forst Chorin: Teerbrennerberge Am Olberg nahe dem Fischerbruch (2013: MüLLER 2015), Kreuzung Denglerweg - Olbergstraße (2013: MÜLLER 2015)

f 3049/IV Brodowin Umg./Karpatenweg östlich des Krugbergs (2015)

g 3150/I Oderberg/am Friedhof (2016: Schwabe)

\section{2 (10225) Cerastis leucographa (DENIs \& SCHIFFERMÜLLER, 1775)}

- RL Land Brandenburg: Vorwarnliste.

Fundorte (Ergänzung):

c 3148/IV Ebw./Stadtgebiet: Kleingartenanlage im Schwärzetal nördlich Forstbotanischer Garten (2016: Schwabe)

593 (10228) Naenia typica (LinNAEUs, 1758)

- RL Land Brandenburg: Vorwarnliste: FA, WS, GL

- RL Deutschland (2011): Vorwarnliste. 
Die Art wird an vielen ehemaligen Fundplätzen vermisst. Gezielte Suchen an solchen Orten blieben erfolglos. Die letzten mir vorliegenden Funddaten stammen aus dem Jahre 2005.

Ältere Funde (Belege in coll. Kittel) werden ergänzend nachgetragen.

Neue Nachweise von bekannten Fundorten:

g 3149/II Liepe (1975: Kittel)

Fundorte (Ergänzung):

c 3148/IV Ebw.-Westend/Unterheide: Wildparkstraße (1969, 1979: Kittel)

\section{4 (10232) Anaplectoides prasina (DENIS \& SCHIFFERMÜLLER, 1775)}

Neue Nachweise von bekannten Fundorten:

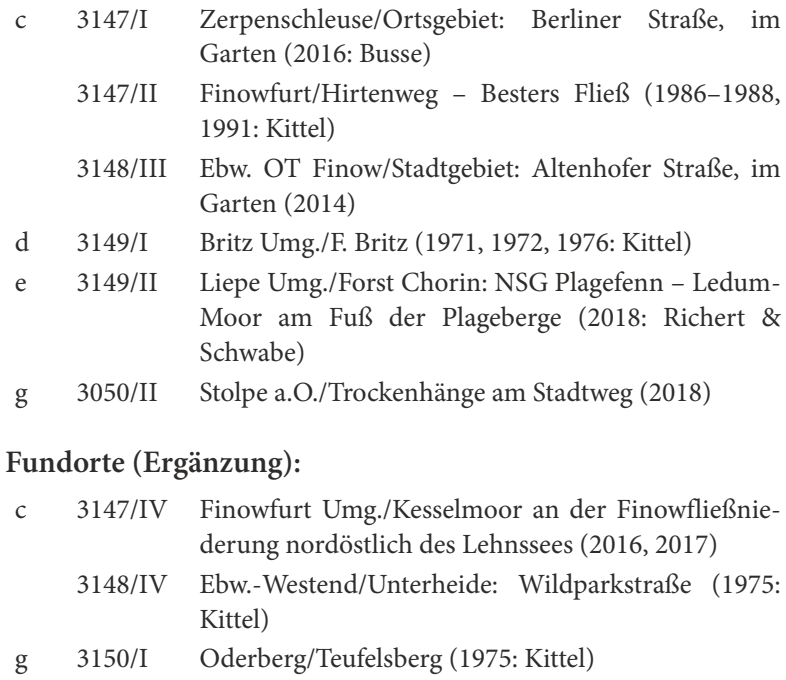

\section{6 (10244) Actebia praecox (LinNAEUs, 1758)}

- RL Land Brandenburg: Kategorie 1 (vom Aussterben bedroht): TR;

- RL Deutschland (2011): Kategorie 1 (vom Aussterben bedroht).

Ältere Funde (Belege in coll. Kittel) der im Gebiet seit 1995 verschollenen Art werden ergänzend nachgetragen (korrigiert; in RICHERT 2010 war für den letzten Nachweis das Jahr 1991 angegeben).

Neue Nachweise von bekannten Fundorten:

g 3150/I Oderberg/Teufelsberg (1974, 1975, 1977: Kittel)

Fundorte (Ergänzung):

c 3147/II Finowfurt/Hirtenweg - Besters Fließ (1987, 1995: Kittel)

3148/IV Ebw.-Westend/Unterheide: Wildparkstraße (1980: Kittel)

3149/I Neuehütte (1974: Kittel)

g 3149/II Liepe (1973: Kittel)
597 (10266) Euxoa aquilina (DeNis \& SCHIFFERMÜLLER, 1775)

- RL Land Brandenburg: Kategorie 3 (gefährdet): TR.

Neue Nachweise von bekannten Fundorten:

c 3148/III Ebw. OT Finow/Stadtgebiet: Altenhofer Straße, im Garten (2014, 2018)

g 3150/III Gabow/Granitberggebiet (2015: Schwabe)

3150/IV Altglietzen Umg./Gabower Berge (2015)

Fundorte (Ergänzung):

c 3147/II Finowfurt/Hirtenweg - Besters Fließ (1994: Kittel)

3148/IV Ebw.-Westend/Unterheide: Wildparkstraße (1975 Kittel)

\section{8 (10275) Euxoa nigricans (LiNNAEUs, 1758)}

- RL Deutschland (2011): Kategorie 3 (gefährdet).

Neue Nachweise von bekannten Fundorten:

c: $\quad 3147 / \mathrm{II} \quad$ Finowfurt/Hirtenweg - Besters Fließ (1989: Kittel)

3148/III Ebw. OT Finow/Stadtgebiet: Altenhofer Straße, im Garten $(2014,2015,2016,2018)$

3150/I Oderberg/Teufelsberg (1971: Kittel)

Fundorte (Ergänzung):

c 3148/IV Ebw.-Westend/Unterheide/Wildparkstraße (1969, 1974: Kittel)

3148/IV Ebw./Stadtgebiet: Kleingartenanlage im Schwärzetal nördlich des Forstbotanischen Gartens (2014: Schwabe)

\section{Euxoa tritici-nigrofusca-eruta-Komplex}

Im neuen Beobachtungszeitraum liegen seit 2014 aus dem Gebiet >17 Datensätze über Falterfunde aus diesem schwierigen Artenkomplex vor. Eine Artzuordnung ist problematisch: Nach Meinung von Segerer \& HAUSMANN (2011) „... erlauben weder äußere noch genitalmorphologische Merkmale die zweifelsfreie Unterscheidung der Taxa ... Auch vorläufige Ergebnisse des „DNA-Barcoding“ sprechen nicht für Unterschiede auf Artniveau ... “ (S. 92-93).

Bei den jährlichen Treffen märkischer Entomologen in Dannenreich, die auch der Bestimmung schwieriger Arten dienten, wurden drei der vorgelegten Falter dem Habitus nach den Taxa nigrofusca bzw. eruta durch J. Gelbrecht und A. Steiner zugeordnet. Die Daten dieser Funde werden nachfolgend aufgeführt (keine Genitalpräparation).

600 (10282) Euxoa nigrofusca (ESPER, 1788)

$=$ tritici auct., nec. LINNAEUS, 1761

- RL Deutschland (2011): Kategorie 3 (gefährdet). Phänologie: Falterfunde am 29.VI.(2016), 24.VII.(2016). 
Neue Nachweise von bekannten Fundorten:

c: 3148/III Ebw. OT Finow/Stadtgebiet: Altenhofer Straße, im Garten, 2 F leg. Richert, Artzuordnung: Gelbrecht \& Steiner

\section{1 (10280a) Euxoa eruta (HÜBNER, 1827)}

- RL Deutschland (2011): Kategorie D (Daten unzureichend).

Phänologie: Falterfund am 10.VII.(2016).

Neue Nachweise von bekannten Fundorten:

c: 3148/III Ebw. OT Finow/Stadtgebiet: Altenhofer Straße, im Garten, 1 F leg. Richert, Artzuordnung: Gelbrecht \& Steiner

\section{2 (10282) Euxoa obelisca (Denis \& SCHIFFER- MÜLLER, 1775)}

- RL Deutschland (2011): Vorwarnliste.

Die Beobachtungslücke im Naturraum f (Parsteiner Becken; vgl. RICHERT 2003) konnte inzwischen geschlossen werden.

\section{Neue Nachweise von bekannten Fundorten:}

c: 3148/III Ebw. OT Finow/Stadtgebiet: Altenhofer Straße, im Garten $(2014,2015)$

Fundorte (Ergänzung):

c 3147/II Finowfurt/Hirtenweg - Besters Fließ (1995: Kittel)

3148/III Ebw. OT Finow Umg./Forst Finowtal: Einflugschneise Flugplatz (2017: Richert \& Schwabe)

3148/IV Ebw.-Westend/Unterheide: Wildparkstraße (1969, 1972, 1974: Kittel)

f 3040/IV Brodowin/Schiefer Berg (1994: Kittel)

g 3150/I Oderberg/Teufelsberg (1973: Kittel)

h 3150/II Hohensaaten/Deponiegelände an der Straße nach der Siedlung (2014: Kleße)

\section{4 (10336) Agrotis bigramma (ESPER, 1790)}

\section{= crassa HüBNER, 1803}

- RL Land Brandenburg: Vorwarnliste: TR;

- RL Deutschland (2011): Vorwarnliste.

Die erwachsene R fand ich am 07.V.2015 auf Sandtrockenrasengelände am Rande einer Photovoltaikanlage, als sie einen Weg überquerte (Suche nach Verpuppungsplatz?). Der F schlüpfte am 09.VIII.(2015).

\section{Neue Nachweise von bekannten Fundorten:}

d 3148/I Werbellin Umg./NSG Buckowseerinne (2014: Clemens)

Fundorte (Ergänzung):

c 3148/II Ebw. OT Finow Umg./Forst Finowtal: Nordrand des Flugplatzgeländes zwischen Finow und Finowfurt (2015)

\section{4a (10343) Agrotis puta (HüBNER, 1803)}

Nachdem die Art 2013 erstmals im Gebiet gefunden wurde (RICHERT 2014), kann nun ein weiterer Fund gemeldet werden. Am 26.VI.2015 wurde auf den Gabower Bergen bei Altglietzen ein Männchen a.L. gefangen. Damit ist die Art auch im Naturraum g (Odertalhänge, vgl. Richert 2003) nachgewiesen.

Fundorte (Ergänzung):

g 3150/IV Altglietzen Umg./Oderhänge: Gabower Berge (2015)

\section{6 (10348) Agotis exclamationis (Linnaeus, 1758)}

Phänologie/Voltinismus: In den Jahren 2015 und 2016 wurden E VIII bis A IX erneut F einer partiellen 2. Gen. beobachtet.

\section{7 (10350) Agrotis clavis (HufNAgEL, 1766) = corticea DENIS \& SCHIFFERMüLLER, 1775}

Während die Art jahrelang nur vereinzelt im Gebiet beobachtet worden war, registrierte Busse im Jahre 2014 vom 03.VI.-10.VII. den $\mathrm{F}$ in seiner Lichtfalle in Zerpenschleuse in ungewöhnlich hoher Zahl (insgesamt $>50 \mathrm{~F}$ a.L.). Ähnliche Ergebnisse erzielte er auch 2013. Beim gleichzeitig von ihm durchgeführten regulären Lichtfang am gleichen Platz flog kein $\mathrm{F}$ an. Als mögliche Erklärung könnte eine im Verlauf der Nacht späte Aktivität der $\mathrm{F}$ angenommen werden (während die Lichtfalle bis zur Morgendämmerung betrieben wird, wurde der reguläre LF früher abgebrochen), aber auch Unterschiede bei den eingesetzten Leuchtmitteln (Lichtfalle mit superaktin. Röhre; Lichtfangstelle dto., jedoch zusätzlich $250 \mathrm{~W}$ Mischlichtlampe).

Neue Nachweise von bekannten Fundorten:

c 3147/I Zerpenschleuse/Ortsgebiet: Berliner Straße, im Garten (2014: Busse)

3147/II Finowfurt/Hirtenweg - Besters Fließ (1995: Kittel)

3148/III Ebw. OT Finow/Stadtgebiet: Altenhofer Straße, im Garten (2014, 2018)

d 3047/III Groß Schönebeck/Ortsgebiet: Friedenstraße, im Garten (2015, 2017: Haase)

g 3149/II Liepe (1975: Kittel)

Fundorte (Ergänzung):

c 3147/I Marienwerder Umg./Oder-Havel-Kanal nordwestlich des Ortes (2014: Busse)

3148/IV Ebw.-Westend/Unterheide: Wildparkstraße (1969, 1970, 1975: Kittel); Ebw./Stadtgebiet: Kleingartenanlage im Schwärzetal nördlich Forstbotanischer Garten (2016: Schwabe) 
608 (10351) Agrotis segetum (DENIS \& SCHIFFERMÜLLER, 1775) = segitis auct.

Phänologie/Voltinismus: In der Regel bivoltin; im Jahre 2014 wurden erneut M (15.)IX und E (23.)X F einer partiellen 3. Gen. registriert.

609 (10356) Agrotis vestigialis (HufNagel, 1766)

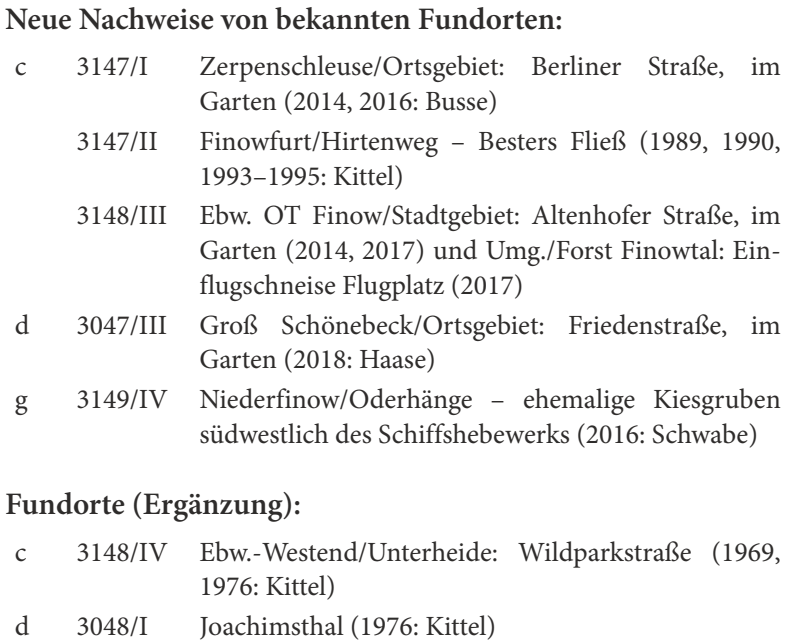

\section{Pantheidae}

\section{1 (10368) Panthea coenobita (ESPER, 1785)}

Panthea coenobita gehört zu den Arten, die (etwa seit den 1990er Jahren) häufiger und mit größerer Verbreitung im Gebiet gefunden werden.

Phänologie/Voltinismus: Univoltin; 21.V.(2014), aFA, phänologische Verfrühung 3d gegenüber bis 2013.

Besonders bemerkenwert sind folgende Beobachtungen: Ein frischer F a.L. am15.IX.(2014), aFE, phänologische Verspätung 26d gegenüber bis 2013. Ein weiterer $\mathrm{F}$ wurde am 13.IX.2014 a.L. registriert. Bei diesen extrem späten Funden handelt es sich wahrscheinlich um eine in diesem Jahr ausgebildete partielle 2. Gen. Die Art gilt als univoltin. Eine 2. Gen. wurde bisher in der Literatur weder für Deutschland (EсKSTEIN 1920, Косн 1984) noch für Mitteleuropa (FAJČIK, 1998, FORSTER \& WOHLFAHRT 1980) verzeichnet. STEINER 1997 beurteilt den Fund eines frischen Falters A (3.) IX in Baden Württemberg allerdings vorsichtig folgendermaßen: , ... ob es sich hier eventuell um ein Tier einer zweiten Generation handelt, ist unklar; auch aus dem Mittelmeergebiet sind späte Falter vom September bekannt ....“(1.c.: 529).

Neue Nachweise von bekannten Fundorten:

\footnotetext{
c 3147/I Zerpenschleuse/Ortsgebiet: Berliner Straße, im Garten (2014-2018: Busse)

3147/II Finowfurt/Hirtenweg - Besters Fließ (1988, 1991, 1992, 1996, 1997: Kittel)

3148/III Ebw. OT Finow/Stadtgebiet: Altenhofer Straße, im Garten (2014-2018)

g 3149/II Liepe (1975: Kittel)
}

Fundorte (Ergänzung):

b 3247/II Biesenthal Umg./NSG Biesenthaler Becken: Langerönner Weg (2014: Ockruck), Weg nördlich der Pfauenwiesen (2014, 2015: Ockruck)

c 3147/I Marienwerder Umg./Oder-Havel-Kanal nordwestlich des Ortes (2016: Busse)

3147/IV Marienwerder/Finowbruch: Steinlaake (2015); Finowfurt Umg./Finowfließniederung: Neue Brücke an der Biesenthaler Straße (2014), Kesselmoor an der Finowfließniederung nordöstlich des Lehnssees (2016)

3148/IV Ebw.-Westend/Unterheide: Wildparkstraße (1969, 1971, 1972, 1975, 1976: Kittel); Ebw./Stadtgebiet: Kleingartenanlage im Schwärzetal nördlich Forstbotanischer Garten (2017, 2018: Schwabe)

d 3148/II Britz (1976: Kittel)

f 3049/IV Brodowin/Krugberg (2014)

h 3150/II Hohensaaten/Deponiegelände an der Straße nach der Siedlung (2014: Kleße)

\section{2 (10372) Colocasia coryli (LinnAeus, 1758)}

Phänologie/Voltinismus: Bivoltin; 07.IV.2014, phänologische Verfrühung 4d; 01.IV.(2017), aFA, erneute phänologische Verfrühung 6d gegenüber bis 2013 .

Zu Teil IV: Spanner (Geometridae) (RICHERT 2004)

\section{Unterfamilie Archiearinae}

612 (7517) Archiearis parthenias (Linnaeus, 1761)

Ältere Funde (Belege in coll. Kittel) werden ergänzend mitgeteilt.

Neue Nachweise von bekannten Fundorten:

d 3148/I Lichterfelde Umg./NSG Buckowseerinne: Moospfuhlgebiet (1978, 1980: Kittel)

Fundorte (Ergänzung):

c 3147/II Finowfurt/Hirtenweg - Besters Fließ (1997: Kittel)

\section{3 (7518) Archiearis notha (HüBNER, 1803)}

Ältere Funde (Belege in coll. Kittel) werden ergänzend mitgeteilt.

Neue Nachweise von bekannten Fundorten:

d 3148/I Lichterfelde Umg./NSG Buckowseerinne: Moospfuhlgebiet (1978, 1979, 1981, 1982, 1984: Kittel)

\section{Unterfamilie Ennominae}

\section{5 (7524) Abraxas sylvata (SCOPOLI, 1763)}

Phänologie/Voltinismus: 08.VI.(2018) aFA, phänologische Verfrühung 3d gegenüber bis 2013. 


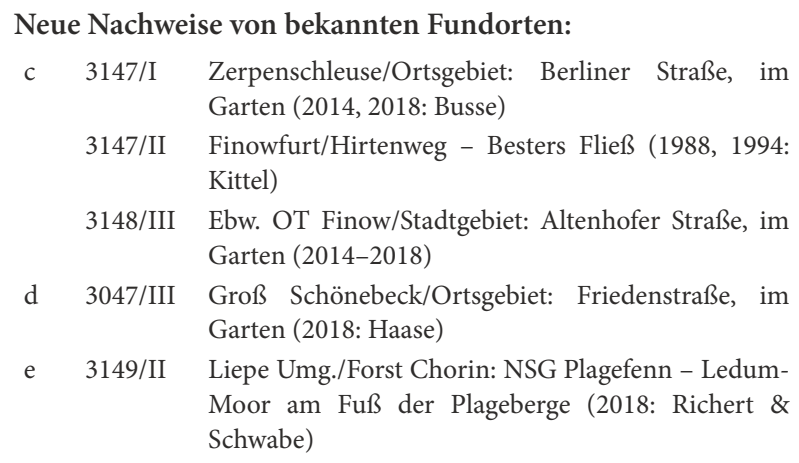

Fundorte (Ergänzung):

c 3147/II Finowfurt Umg./Üdersee: Südwestende (1974: Kittel)

3147/IV Finowfurt Umg./Finowfließniederung: Neue Brücke an der Biesenthaler Straße (2014)

3148/IV Ebw./Stadtgebiet: Kleingartenanlage im Schwärzetal nördlich des Forstbotanischen Gartens (2014, 2016, 2017: Schwabe)

\section{7a (7533) Stegania trimaculata (DE VILLERS, 1789)}

Die Art wurde inzwischen auch im Naturraum d (Schorfheide - Britzer Platte, Richert 2010, 2014) nachgewiesen.

Phänologie/Voltinismus: 1. Gen.: 06.V.(2015), aFA, phänologische Verfrühung 7d gegenüber bis 2013.

Neue Nachweise von bekannten Fundorten:

c 3148/III Ebw. OT Finow/Stadtgebiet: Altenhofer Straße, im Garten $(2014$, 2015, 2016, 2017, 2018)

g 3149/IV Niederfinow/Oderhänge - ehemalige Kiesgruben südwestlich des Schiffshebewerks (2015, 2016: Schwabe)

3150/III Gabow/Granitberggebiet (2015)

3150/IV Altglietzen/Gabower Berge (2015: Richert; 2018: Schwabe)

Fundorte (Ergänzung):

c 3147/I Zerpenschleuse/Ortsgebiet: Berliner Straße, im Garten (2016, 2018: Busse)

3148/III Ebw. OT Finow Umg./Forst Finowtal: Einflugschneise Flugplatz (2017)

d 3047/III Groß Schönebeck/Ortsgebiet: Friedenstraße, im Garten (2014, 2017: Haase)

g 3150/I Oderberg/Geistberg (2017: Schwabe)

\section{8 (7537) Heliomata glarearia (Denis \& SCHIF- FERMÜLLER, 1775)}

- RL Land Brandenburg: Kategorie 3 (gefährdet): TR;

- RL Deutschland (2011): Vorwarnliste.

Neue Nachweise von bekannten Fundorten:

g 3050/II Stolpe a.O./Trockenhänge am Stadtweg (2018); Stolzenhagen (1971: Kittel)/NSG Krähen- und Jungfernberge (2015: Richert)

3149/III Stecherschleuse Umg./Hochfläche nordwestlich der Waldstraße (2017)
3149/IV Niederfinow/Oderhänge - ehemalige Kiesgruben südwestlich des Schiffshebewerks (2015: Schwabe)

3150/I Oderberg/Geistberg (2014, 2015, 2017, 2018: Schwabe), NSG Pimpinellenberg (1971: Kittel), Teufelsberg (1973, 1976: Kittel)

3150/III Gabow/Granitberggebiet (2015)

3150/IV Altglietzen/Gabower Berge (2015)

Fundorte (Ergänzung):

f 3049/IV Brodowin/Krugberg (2015)

\section{1 (7541) Macaria signaria (HüBNER, 1809)}

Im Jahre 2014 hatte ich den F erstmals in meinem Garten in Finow a.L.

Phänologie/Voltinismus: Univoltin, 08.VI.(2014), phänologische Verfrühung 8d gegenüber bis 2013; 07.VI.(2018) aFA, erneute phänologische Verfrühung 1d (insgesamt 9d gegenüber bis 2013).

\section{Neue Nachweise von bekannten Fundorten:}

b: 3148/IV Ebw. Umg./Oberheide: Waldschneise am Leuenberger Graben zwischen Möllergrab (am WiebeckeDamm) und Leuenberger Wiesen (2014: Schwabe)

c 3147/II Finowfurt/Hirtenweg - Besters Fließ (1986, 1990: Kittel)

e 3149/II Liepe Umg./Forst Chorin: NSG Plagefenn - LedumMoor am Fuß der Plageberge (2018: Richert \& Schwabe)

Fundorte (Ergänzung):

c 3148/III Ebw. OT Finow/Stadtgebiet: Altenhofer Straße, im Garten (2014)

3148/IV Ebw./Stadtgebiet: Kleingartenanlage im Schwärzetal nördlich Forstbotanischer Garten (2016: Schwabe)

g 3159/I Oderberg/Teufelsberg (1975: Kittel)

\section{3 (7543) Macaria wauaria (LiNNAEUs, 1758)}

Neue Nachweise von bekannten Fundorten:

c 3147/I Zerpenschleuse/Ortgebiet: Berliner Straße, im Garten (2015: Busse)

3147/II Finowfurt/Hirtenweg - Besters Fließ (1990: Kittel)

3148/III Ebw. OT Finow/Stadtgebiet: Altenhofer Straße, im Garten $(2014,2015)$

3148/IV Ebw.-Westend/Unterheide: Wildparkstraße (1991, 19973: Kittel); Ebw./Stadtgebiet: Kleingartenanlage im Schwärzetal nördlich des Forstbotanischen Gartens (2014: Schwabe)

g 3050/II Stolpe a.O./Trockenhänge am Stadtweg (2018)

3150/I Oderberg/Teufelsberg (1975: Kittel)

3150/III Gabow/Granitberggebiet (2015)

Fundorte (Ergänzung):

c 3148/III Finowfurt (1970, 1972, 1976, 1979: Kittel)

g 3150/I Oderberg/Geistberg (2018: Schwabe) 


\section{5 (7547) Chiasmia clathrata (Linnaeus, 1748)}

Der F wurde am Tag beim Saugen an Blüten von Origanum vulgare, Dost; Chrysanthemum (= Tanacetum) vulgare, Rainfarn; und Daucus carota, Wilder Möhre, beobachtet.

\section{6 (7556) Narraga fasciolaria (HufNAGEL, 1767)}

- RL Land Brandenburg: Kategorie 3 (gefährdet): TR;

- RL Deutschland (2011): Kategorie 3 (gefährdet).

Neue Nachweise von bekannten Fundorten:

d 3149/I Britz Umg./F. Britz: 1973: Kittel)

g 3149/II Liepe (1975, 1978: Kittel)

3149/IV Niederfinow/Oderhänge - ehemalige Kiesgruben südwestlich des Schiffshebewerks (2015: Schwabe)

3150/I Oderberg/Teufelsberg (1976: Kittel)

Fundorte (Ergänzung):

d 3047/III Groß Schönebeck/Ortsgebiet: Friedenstraße, im Garten (2015: Haase)

\section{7 (7567) Itame brunneata (THUNBERG, 1784) = fulvaria De VILLIERS, 1789}

Phänologie/Voltinismus: 29.V.(2018) aFA, phänologische Verfrühung 2d gegenäber bis 2013 .

Neue Nachweise von bekannten Fundorten:

b 3247/II Biesenthal Umg./NSG Biesenthaler Becken: Langerönner Weg (2014: Ockruck)

c 3146/IV Kreuzbruch Umg./ehemalige Römerwegbrücke am Oder-Havel-Kanal östlich des Ortes (2014: Busse) (westlich angrenzendes Nachbargebiet)

3147/I Zerpenschleuse/Ortsgebiet: Berliner Straße, im Garten (2014, 2016, 2018: Busse)

3147/II Finowfurt/Hirtenweg - Besters Fließ (1995: Kittel)

3148/III Finowfurt (1976: Kittel); Ebw. OT Finow/Stadtgebiet: Altenhofer Straße, im Garten $(2014,2015,2018)$

3148/IV Ebw.-Westend/Unterheide: Wildparkstraße (1970, 1974: Kittel), Ebw./Stadtgebiet: Kleingartenanlage im Schwärzetal nördlich des Forstbotanischen Gartens (2014: Schwabe)

g 3150/IV Altglietzen/Gabower Berge (2015)

Fundorte (Ergänzung):

c $3147 / \mathrm{IV}$ Finowfurt Umg./Kesselmoor an der Finowfließniederung nordöstlich des Lehnssees $(2016,2017)$

d 3047/III Groß Schönebeck/Ortsgebiet: Friedenstraße, im Garten (2015: Haase)

g 3050/II Stolpe a.O./Trockenhänge am Stadtweg (2018)

3150/I Oderberg/Geistberg (2018: Schwabe)

\section{8 (7594) Cepphis advenaria (HüBNER, 1790)}

Phänologie/Voltinismus: 28.V.(2018) aFA, phänologische Verfrühung 1d gegenäber bis 2013 .
Neue Nachweise von bekannten Fundorten:

c 3147/I Zerpenschleuse/Ortsgebiet: Berliner Straße, im Garten (2018: Busse)

3148/III Ebw. OT Finow/Stadtgebiet: Altenhofer Straße, im Garten $(2014,2017)$

3148/IV Ebw.-Westend/Unterheide: Wildparkstraße (1974 1995: Kittel)

Fundorte (Ergänzung):

c 3146/IV Kreuzbruch Umg./ehemalige Römerwegbrücke am Oder-Havel-Kanal östlich des Ortes (2014: Busse) (westlich angrenzendes Nachbargebiet)

3147/I Marienwerder Umg./Oder-Havel-Kanal nordwestlich des Ortes (2014: Busse)

3147/IV Finowfurt Umg./Kesselmoor an der Finowfließniederung nordöstlich des Lehnssees $(2016,2017)$

3148/IV Ebw./Stadtgebiet: Kleingartenanlage im Schwärzetal nördlich des Forstbotanischen Gartens (2014, 2016: Schwabe)

3149/III Ebw. Umg./Finowbruch östlich des Eichwerder (2015: Schwabe)

d 3047/III Groß Schönebeck/Ortsgebiet: Friedenstraße, im Garten (2014: Haase)

g 3150/I Oderberg/Geistberg (2018: Schwabe)

\section{9 (7596) Petrophora chlorosata (ScOPOLI, 1763)}

Neue Nachweise von bekannten Fundorten:

b 3148/IV Ebw. Umg./Oberheide: Wiebecke-Damm am Möllergrab (2016: Schwabe); Spechthausen Umg./Fischteiche nordöstlich des Ortes (1974: Kittel)

c 3146/IV Kreuzbruch Umg./ehemalige Römerwegbrücke am Oder-Havel-Kanal östlich des Ortes (2014: Busse) (westlich angrenzendes Nachbargebiet)

3147/I Zerpenschleuse/Ortsgebiet: Berliner Straße, im Garten (2014, 2015, 2016, 2018: Busse)

3147/II Finowfurt/Hirtenweg - Besters Fließ (1995: Kittel)

3148/III Ebw. OT Finow/Stadtgebiet: Altenhofer Straße, im Garten $(2014,2015$, 2016, 2017, 2018)

3148/IV Ebw.-Westend/Unterheide: Wildparkstraße (1972, 1973: Kittel)

d 3047/III Groß Schönebeck/Ortsgebiet: Friedenstraße, im Garten (2014, 2016, 2017: Haase)

Fundorte (Ergänzung):

b 3248/II Trampe Umg./Tramper Forst bei Bornemanns Pfuhl (2018: Schwabe)

c 3147/IV Finowfurt Umg./Kesselmoor an der Finowfließniederung nordöstlich des Lehnssees $(2016,2017)$

3148/III Ebw. OT Finow Umg./Forst Finowtal: Einflugschneise Flugplatz (2016)

3148/IV Ebw./Stadtgebiet: Kleingartenanlage im Schwärzetal nördlich des Forstbotanischen Gartens (2014, 2016, 2017: Schwabe)

g 3149/II Liepe (1976: Kittel)

630 (7606) Plagodis pulveraria (Linnaeus, 1758)

Siehe 3.3. Wieder entdeckte Arten. 


\section{1 (7607) Plagodis dolabraria (Linnaeus, 1767)}

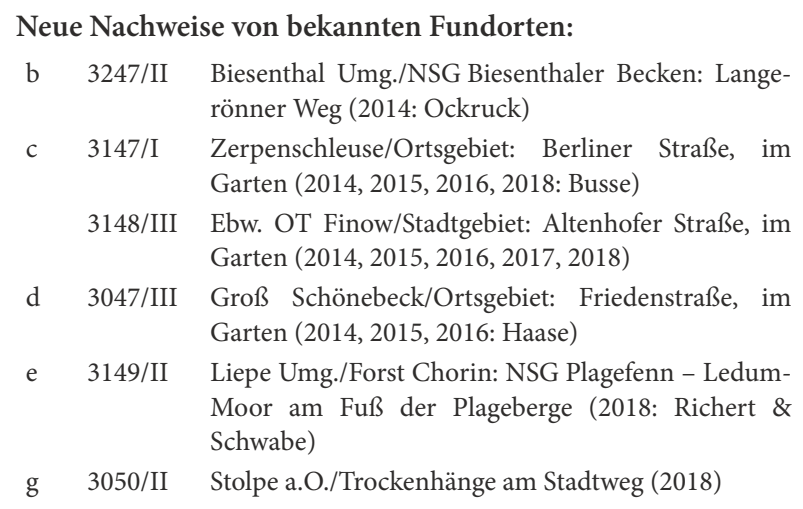

Fundorte (Ergänzung):

c 3147/I Marienwerder Umg./Oder-Havel-Kanal nordwestlich des Ortes (2014: Busse)

3147/IV Marienwerder Umg./Kiesgrube südwestlich des Sportplatzes (2015); Finowfurt Umg./Moor an der Finowfließniederung nordöstlich des Lehnssees $(2016,2017)$

3148/IV Ebw.-Westend/Unterheide: Wildparkstraße (1969, 1971, 1974: Kittel); Ebw./Stadtgebiet: Kleingartenanlage im Schwärzetal nördlich des Forstbotanischen Gartens (2014, 2016, 2017, 2018: Schwabe)

g 3150/I Oderberg/Geistberg (2017, 2018: Schwabe)

\section{2 (7613) Opisthograptis luteolata (LinNAEus,} 1758)

Neue Nachweise von bekannten Fundorten:

$\begin{array}{lll}\text { c 3147/I } & \begin{array}{l}\text { Zerpenschleuse/Ortsgebiet: Berliner Straße, im } \\ \text { Garten }(2014,2015,2016,2018: \text { Busse })\end{array} \\ & 3148 / \mathrm{III} & \begin{array}{l}\text { Ebw. OT Finow/Stadtgebiet: Altenhofer Straße, im } \\ \text { Garten }(2014,2016,2017,2018)\end{array} \\ & & \text { Oderberg (1969: Kittel) } \\ \text { g } 3150 / \mathrm{I} & \text { Altglietzen Umg./Gabower Berge (2015) }\end{array}$

Fundorte (Ergänzung):

c 3147/I Marienwerder Umg./Oder-Havel-Kanal nordwestlich des Ortes (2014: Busse)

3147/IV Marienwerder/Steinlaake (2015); Finowfurt Umg./ Kesselmoor an der Finowfließniederung nordöstlich des Lehnssees (2017)

3148/IV Ebw.-Westend/Unterheide: Wildparkstraße (1970, 1973, 1974: Kittel); Ebw./Stadtgebiet: Kleingartenanlage im Schwärzetal nördlich Forstbotanischer Garten (2016, 2018: Schwabe)

g 3149/IV Niederfinow/Oderhänge - ehemalige Kiesgruben südwestlich des Schiffshebewerks (2015: Schwabe)

3150/I Oderberg/Geistberg (2017, 2018: Schwabe)

\section{3 (7615) Epione repandaria (HUFNAGEL, 1767)}

Die Art wurde im Naturraum e (Uckermärker Kuppenund Hügelland einschließlich Choriner Waldhügel, vgl. RICHERT 2004) erstmals nachgewiesen.

Den F beobachtete ich nachts beim Saugen an Blüten von Buddleja davidii, Chinesischer Sommerflieder.
Phänologie/Voltinismus: Bivoltin, 1. Gen.: 09.VI.(2014), phänologische Verfrühung $2 \mathrm{~d}$ gegenüber bis 2013; 07.VI. (2018) aFA, erneute phänologische Verfrühung, insgesamt $4 \mathrm{~d}$ gegenüber bis 2013

Neue Nachweise von bekannten Fundorten:

c 3147/I Zerpenschleuse/Ortsgebiet: Berliner Straße, im Garten (2014: Busse)

3148/III Ebw. OT Finow/Stadtgebiet: Altenhofer Straße, im Garten (2014)

3148/IV Ebw.-Westend/Unterheide: Wildparkstraße (1973, 1975, 1976, 1981: Kittel)

g 3149/II Liepe (1974: Kittel)

Fundorte (Ergänzung):

c 3147/I Marienwerder Umg./Oder-Havel-Kanal nordwestlich des Ortes (2014: Busse)

3148/IV Ebw.-Nordende/Gehörlosen-Hilfsschule auf dem Gelände der Landesklinik (1983: Kittel); Ebw./Stadtgebiet: Kleingartenanlage im Schwärzetal nördlich des Forstbotanischen Gartens (2014, 2017: Schwabe)

e $\quad 3149 /$ II $\quad$ Liepe Umg./Forst Chorin: NSG Plagefenn - LedumMoor am Fuß der Plageberge (2018: Richert \& Schwabe)

\section{4 (7620) Pseudopanthera macularia (Linnaeus, 1758)}

Phänologie/Voltinismus: Univoltin; ein bisher übersehenes Funddatum: 06.V.(2011), aFA, phänologische Verfrühung 8d (korrigiert; in RICHERT 2014 war der 14.V.(1975) als aFA angegeben).

\section{Neue Nachweise von bekannten Fundorten:}

b 3247/II Biesenthal Umg./NSG Biesenthaler Becken: Langerönner Weg (2014: Ockruck)

g 3149/II Liepe (1973, 1974: Kittel)

3150/I Oderberg/Teufelsberg (1973: Kittel)

\section{5 (7630) Apeira syringaria (Linnaeus, 1748)}

Neben neuen Daten werden ältere (Belege in coll. Kittel) ergänzend nachgetragen.

Phänologie/Voltinismus: 06.VI.(2018) aFA, phänologische Verfrühung 3d gegenüber bis 2013.

Neue Nachweise von bekannten Fundorten:

c 3148/III Ebw. OT Finow/Stadtgebiet: Altenhofer Straße, im Garten (2018)

3148/IV Ebw.-Westend/Unterheide: Wildparkstraße (1970: Kittel)

d 3047/III Groß Schönebeck/Ortsgebiet: Friedenstraße, im Garten (2018: Haase)

Fundorte (Ergänzung):

d 3149/I Britz Umg./F. Britz (1971: Kittel) 
636 (7632) Ennomos autumnaria (WERNEBURG, 1859)

- RL Deutschland (2011): Vorwarnliste.

Neue Nachweise von bekannten Fundorten:

c 3147/I Zerpenschleuse/Ortsgebiet: Berliner Straße, im Garten (2014, 2105: Busse)

3148/III Ebw. OT Finow/Stadtgebiet: Altenhofer Straße, im Garten (2016)

3947/III Groß Schönebeck/Ortsgebiet: Friedenstraße, im Garten (2017: Haase)

Fundorte (Ergänzung):

c 3147/IV Finowfurt Umg./Kesselmoor an der Finowfließniederung nordöstlich des Lehnssees (2016)

g 3150/IV Altglietzen Umg./Gabower Berge (2015)

\section{7 (7833) Ennomos quercinaria (LinNAEus, 1758)}

Phänologie/Voltinismus: Univoltin; die Flugzeit dauert im Gebiet länger als bisher bekannt: In coll. Kittel fand ich ein Weibchen mit den Daten Finowfurt/Hirtenweg, 19.IX.(1986), aFE, phänologische Verspätung 16d (korrigiert; in RICHERT 2014 war der 13.IX.(2013) angegeben). Weitere Funde sind vom 24.IX. - 06.X.2013 gemeldet (MÜLler 2015). Diese Daten sind allerdings unsicher: In dem mir vorgelegten, nicht datumsgenau sortierten Belegmaterial dieser Art befand sich auch die Art E. erosaria, sodass nicht auszuschließen ist, ob sich die späten Daten auf die 2. Gen. dieser Art beziehen.

Neue Nachweise von bekannten Fundorten:

c 3148/IV Ebw.-Westend/Unterheide: Wildparkstraße (1978: Kittel)

Fundorte (Ergänzung):

e 3049/III Chorin Umg./Forst Chorin: Südöstlich des Pferdebergs (2013: MÜLLER 2015)

3049/IV Chorin Umg./Forst Chorin: Teerbrennerberge - Am Olberg (2013: MüLLER 2015), Kreuzung Denglerweg - Olbergstraße (2013: MÜLLER 2015)

\section{8 (7634) Ennomos alniaria (LINNAEUs, 1758)}

Neue Nachweise von bekannten Fundorten:

c 3147/I Zerpenschleuse/Ortsgebiet: Berliner Straße, im Garten (2014, 2016, 2017: Busse)

3147/II Finowfurt/Hirtenweg - Besters Fließ (1985, 1987, 1990: Kittel)

3148/III Ebw. OT Finow/Stadtgebiet: Altenhofer Straße, im Garten $(2014,2016,2017)$ und Umg./Forst Finowtal: Einflugschneise Flugplatz (2017)

3148/IV Ebw.-Westend/Unterheide: Wildparkstraße (1977: Kittel)

g 3150/IV Altglietzen Umg./Gabower Berge (2015)

Fundorte (Ergänzung):

a 3249/II Falkenberg Umg./Cöthener Forst: Klingendes Fließ unterhalb des Bismarck-Turmes (2014: Schwabe) b 3249/I Gersdorf Umg./Gamengrund südlich Teufelssee (2017: Richert \& Schwabe)

c 3148/IV Ebw./Stadtgebiet: Kleingartenanlage im Schwärzetal nördlich Forstbotanischer Garten (2016: Schwabe)

f 3049/IV Brodowin/Krugberg (2014: Schwabe), Karpatenweg östlich des Krugbergs (2015: Richert)

g 3149/II Liepe (1974, 1975: Kittel)

\section{9 (7635) Ennomos fuscantaria (HAwORTH, 1809)}

Die Beobachtungslücke im Naturraum f (Parsteiner Becken; RICHERT 2004) wurde inzwischen geschlossen.

Phänologie/Voltinismus: In Richert 2014 S. 179 wurde der 07.IX.(1966) als absolutes Flugzeitende im Gebiet angegeben. Hier liegt ein Druckfehler vor, richtig ist 07.X.(1966).

Neue Nachweise von bekannten Fundorten:

c 3147/I Zerpenschleuse/Ortsgebiet: Berliner Straße, im Garten (2014-2018: Busse)

3148/III Ebw. OT Finow/Stadtgebiet: Altenhofer Straße, im Garten (2015-2018)

g 3149/II Liepe (1974: Kittel)

Fundorte (Ergänzung):

c 3147/II Finowfurt/Hirtenweg - Besters Fließ (1986: Kittel)

d 3047/III Groß Schönebeck/Ortsgebiet: Friedenstraße, im Garten (2017: Haase)

f 3049/IV Brodowin Umg./Karpatenweg östlich Krugberg (2015)

g 3150/I Oderberg/am Friedhof (2016)

\section{0 (7636) Ennomos erosaria (DENIS \& SCHIFFER- MÜLLER, 1775)}

Phänologie/Voltinismus: Bivoltin; 1. Gen.: 03.VI.(2018) aFA, phänologische Verfrühung 16d gegenüber bis 2013; 2. Gen.: 24.X.(2013), aFE, phänologische Verspätung 8d gegenüber bis 2013 .

Neue Nachweise von bekannten Fundorten:

c 3147/I Zerpenschleuse/Ortsgebiet: Berliner Straße, im Garten (2014, 2015, 2017, 2018: Busse)

3147/II Finowfurt/Hirtenweg - Besters Fließ (1986, 1988, 1989: Kittel)

3148/III Ebw. OT Finow/Stadtgebiet: Altenhofer Straße, im Garten (2014-2018)

3148/IV Ebw.-Westend/Unterheide: Wildparkstraße (1970, 1973: Kittel)

g 3149/II Liepe (1974: Kittel)

Fundorte (Ergänzung):

b 3247/II Biesenthal Umg./NSG Biesenthaler Becken: Langerönner Weg (2014: Ockruck)

c 3147/I Marienwerder Umg./Oder-Havel-Kanal nordwestlich des Ortes (2014, 2016: Busse)

3147/IV Finowfurt Umg./Moor an der Finowfließniederung nordöstlich des Lehnssees (2016)

3148/IV Ebw./Stadtgebiet: Kleingartenanlage im Schwärzetal nördlich Forstbotanischer Garten (2017: Schwabe) 
e 3049/III Chorin Umg./Forst Chorin: Südöstlich des Pferdebergs (2013: MÜLLER 2015)

3049/IV Chorin Umg./Forst Chorin: Teerbrennerberge - Am Olberg (2013: MüLLER 2015), Kreuzung Denglerweg - Olbergstraße (2013: MülLER 2015)

g 3050/II Stolpe a.O./Trockenhänge am Stadtweg (2018)

3150/IV Altglietzen/Gabower Berge (2015: Schwabe)

641 (7641) Selenia dentaria (FABRICIUS, 1775) = bilunaria ESPER, 1801

Neue Nachweise von bekannten Fundorten:

c 3147/I Zerpenschleuse/Ortsgebiet: Berliner Straße, im Garten (2014, 2015, 2018: Busse)

3147/II Finowfurt/Hirtenweg - Besters Fließ (1987, 1994, 1995, 1997: Kittel)

3148/III Ebw. OT Finow/Stadtgebiet: Altenhofer Straße, im Garten $(2014,2015,2018)$

g 3150/I Oderberg/Teufelsberg (1974: Kittel)

Fundorte (Ergänzung):

c 3147/IV Finowfurt Umg./Finowfließniederung: Neue Brücke an der Biesenthaler Straße (2014), Kesselmoor an der Finowfließniederung nordöstlich des Lehnssees (2016, 2017: Richert \& Schwabe)

d 3149/I Britz Umg./F. Britz (1972: Kittel)

e 3049/I Groß Ziethen Umg./Steingruben an den Ihlowbergen (2014)

3149/II Liepe Umg./Forst Chorin: NSG Plagefenn - LedumMoor am Fuß der Plageberge (2018: Richert \& Schwabe)

\section{2 (7643) Selenia tetralunaria (HUFNAGEL, 1767)}

Die Beobachtungslücke im Naturraum h (Odertal, vgl. Richert 2004), wurde geschlossen. Damit ist die Art in allen Naturräumen des Gebietes nachgewiesen.

Phänologie/Voltinismus: Bivoltin; 2. Gen.: 04.IX.(2014), aFE, phänologische Verspätung 22d gegenüber bis 2013.

Neue Nachweise von bekannten Fundorten:

c 3147/I Zerpenschleuse/Ortsgebiet: Berliner Straße, im Garten (2017, 2018: Busse)

3148/III Ebw. OT Finow/Stadtgebiet: Altenhofer Straße, im Garten $(2014,2015,2018)$

Fundorte (Ergänzung):

a 3249/II Falkenberg Umg./Cöthener Forst: Klingendes Fließ unterhalb des Bismarck-Turmes (2014: Schwabe)

b 3148/IV Spechthausen Umg./Fischteiche nordöstlich des Ortes (1974: Kittel)

c 3147/II Finowfurt/Hirtenweg - Besters Fließ (1986, 1987, 1993, 1994, 1995: Kittel)

3148/IV Ebw./Stadtgebiet: Kleingartenanlage im Schwärzetal nördlich des Forstbotanischen Gartens (2014-2018: Schwabe)

e 3149/II Liepe Umg./Forst Chorin: NSG Plagefenn - LedumMoor am Fuß der Plageberge (2018: Richert \& Schwabe) und südlich Gr. Plagesee (2018: Richert, Schwabe \& Theimer)

h 3150/II Hohensaaten/Deponie an der Straße nach der Siedlung (2014: Kleße)
644 (7647) Odontopera bidentata (CLERCK, 1759)

Neue Nachweise von bekannten Fundorten:

c 3147/II Finowfurt/Hirtenweg - Besters Fließ (1989, 1991, 1997: Kittel)

g 3150/I Oderberg/Teufelsberg (1973: Kittel)

Fundorte (Ergänzung):

c 3147/IV Marienwerder Umg./Kiesgrube südwestlich des Sportplatzes (2015)

3148/IV Ebw./Stadtgebiet: Kleingartenanlage im Schwärzetal nördlich Forstbotanischer Garten (2015: Schwabe)

\section{5 (7654) Crocallis elinguaria (Linnaeus, 1758)}

Neue Nachweise von bekannten Fundorten:

c 3148/III Ebw. OT Finow/Stadtgebiet: Altenhofer Straße, im Garten $(2014,2017)$

\section{6 (7659) Ourapteryx sambucaria (Linnaeus, 1758)}

Neue Nachweise von bekannten Fundorten:

c 3147/I Zerpenschleuse/Ortsgebiet: Berliner Straße, im Garten (2014-2016, 2018: Busse)

3148/III Ebw. OT Finow/Stadtgebiet: Altenhofer Straße, im Garten $(2014,2016,2018)$

g 3050/II Stolpe a.O./Trockenhänge am Stadtweg (2018)

Fundorte (Ergänzung):

c 3148/IV Ebw.-Westend/Unterheide: Wildparkstraße (1971, 1976: Kittel); Ebw./Stadtgebiet: Kleingartenanlage im Schwärzetal nördlich des Forstbotanischen Gartens (2014: Schwabe)

g 3150/I Oderberg/Geistberg (2018: Schwabe)

\section{7 (7663) Colotois pennaria (Linnaeus, 1761)}

Neue Nachweise von bekannten Fundorten:

c 3147/I Zerpenschleuse/Ortsgebiet: Berliner Straße, im Garten (2014: Busse)

3148/III Ebw. OT Finow/Stadtgebiet: Altenhofer Straße, im Garten (2016)

3148/IV Ebw.-Westend/Unterheide: Wildparkstraße (19721974, 1976-1978: Kittel)

Fundorte (Ergänzung):

c 3147/II Finowfurt/Hirtenweg - Besters Fließ (1987, 1991: Kittel)

e 3049/IV Chorin Umg./Forst Chorin: Teerbrennerberge, Kreuzung Dengler-Weg - Olbergstraße; Umg. Pferdeberg (2013: MüLLER 2015)

\section{8 (7665) Angerona prunaria (LinNAeUs, 1758)}

Phänologie/Voltinismus: 29.V.(2018) aFA, phänologische Verfrühung 1d gegenüber bis 2013. 
Neue Nachweise von bekannten Fundorten:

b 3248/II Trampe/ehemaliger TÜP (2016: Busse \& Müller)

c 3147/I Zerpenschleuse/Ortsgebiet: Berliner Straße, im Garten (2014, 2015, 2018: Busse)

3147/II Finowfurt/Hirtenweg - Besters Fließ (1996: Kittel)

3148/III Ebw. OT Finow/Stadtgebiet: Altenhofer Straße, im Garten (2018)

3148/IV Ebw.-Westend/Unterheide: Wildparkstraße (1969: Kittel)

e 3149/II Liepe Umg./Forst Chorin: NSG Plagefenn - LedumMoor am Fuß der Plageberge (2018: Richert \& Schwabe)

g 3149/II Liepe (1973, 1974: Kittel)

Fundorte (Ergänzung):

c 3146/IV Kreuzbruch Umg./ehemalige Römerwegbrücke am Oder-Havel-Kanal östlich des Ortes (2014: Busse) (westlich angrenzendes Nachbargebiet)

3147/I Marienwerder Umg./Oder-Havel-Kanal nordwestlich des Ortes (2016: Busse)

3147/IV Finowfurt Umg./Kesselmoor an der Finowfließniederung nordöstlich des Lehnssees $(2016,2017$ : Richert \& Schwabe)

3148/IV Ebw./Stadtgebiet: Kleingartenanlage im Schwärzetal nördlich des Forstbotanischen Gartens (2014, 2016: Schwabe)

g 3150/I Oderberg/Geistberg (2018: Schwabe)

\section{9 (7671) Apocheima hispidaria (DenIs \& SCHIFFERMÜLLER, 1775)}

Neue Nachweise von bekannten Fundorten:

c 3147/I Zerpenschleuse/Ortsgebiet: Berliner Straße, im Garten (2014, 2016, 2018: Busse)

3148/I Werbellin Umg./NSG Buckowseerinne nahe der Autobahnanschlussstelle (2017: Clemens)

Fundorte (Ergänzung):

c 3148/IV Ebw.-Westend/Unterheide: Wildparkstraße (1982: Kittel); Ebw./Stadtgebiet: Kleingartenanlage im Schwärzetal nördlich des Forstbotanischen Gartens (2015: Schwabe)

\section{0 (7672) Apocheima pilosaria (DenIs \& SCHIF- FERMÜLLER, 1775) = pedaria FABRICIUS, 1787}

Ältere Daten (Belege in coll. Kittel) werden ergänzend mitgeteilt.

Neue Nachweise von bekannten Fundorten:

c 3147/I Zerpenschleuse/Ortsgebiet: Berliner Straße, im Garten (2018: Busse)

3148/IV Ebw.-Westend/Unterheide: Wildparkstraße (1973, 1974, 1977, 1978, 1980, 1981: Kittel)

Fundorte (Ergänzung):

c 3147/II Finowfurt/Hirtenweg - Besters Fließ (1991: Kittel)

\section{1 (7674) Lycia hirtaria (CLERCK, 1759)}

Als Raupennahrungspflanze wurde im Gebiet Syringa vulgaris, Gemeiner Flieder, festgestellt (Foto Schwabe).

Neue Nachweise von bekannten Fundorten:

c 3147/I Zerpenschleuse/Ortsgebiet: Berliner Straße, im Garten (2014, 2018: Busse)

3147/II Finowfurt/Hirtenweg - Besters Fließ (1988, 1995: Kittel)

3148/III Ebw. OT Finow/Stadtgebiet: Altenhofer Straße, im Garten $(2015,2016)$

Fundorte (Ergänzung):

c 3148/IV Ebw./Stadtgebiet: Kleingartenanlage im Schwärzetal nördlich des Forstbotanischen Gartens $(2014,2017$ Schwabe)

g 3150/I Oderberg/Geistberg (2017: Schwabe)

\section{2 (7685) Biston stratarius (HufNAGEL, 1767)}

Neue Nachweise von bekannten Fundorten:

c 3147/I Zerpenschleuse/Ortsgebiet: Berliner Straße, im Garten (2014-2018: Busse)

3147/II Finowfurt/Hirtenweg - Besters Fließ (1991, 1992: Kittel)

3148/III Ebw. OT Finow/Stadtgebiet: Altenhofer Straße, im Garten $(2014,2016)$

3148/IV Ebw.-Westend/Unterheide: Wildparkstraße (1973 1974, 1977-1979, 1990: Kittel)

d 3047/III Groß Schönebeck/Ortsgebiet: Friedenstraße, im Garten (2018: Haase)

Fundorte (Ergänzung):

c 3148/IV Ebw./Stadtgebiet: Kleingartenanlage im Schwärzetal nördlich des Forstbotanischen Gartens (2015-2018: Schwabe)

e 3149/II Liepe Umg./Forst Chorin: NSG Plagefenn - LedumMoor am Fuß der Plageberge (2018: Richert, Schwabe \& Theimer)

\section{4 (7693) Agriopis leucophaearia (DeNIs \& SCHIFFERMÜLLER, 1775)}

Neue Nachweise von bekannten Fundorten:

c 3147/I Zerpenschleuse/Ortsgebiet: Berliner Straße, im Garten (2014, 2018: Busse)

3147/II Finowfurt/Hirtenweg - Besters Fließ (1991: Kittel)

3148/I Werbellin Umg./NSG Buckowseerinne nahe der Autobahnanschlussstelle (2017: Clemens)

3148/III Ebw. OT Finow/Stadtgebiet: Altenhofer Straße, im Garten (2014)

3148/IV Ebw.-Westend/Unterheide: Wildparkstraße (1971, 1977,1991, 1993: Kittel)

Fundorte (Ergänzung):

c 3148/IV Ebw./Stadtgebiet: Kleingartenanlage im Schwärzetal nördlich des Forstbotanischen Gartens (2015: Schwabe) 


\section{5 (7695) Agriopis aurantiaria (HüBNER, 1799)}

Phänologie/Voltinismus: Univoltin; die Flugzeit beginnt im Gebiet bereits früher als bisher bekannt: 24.IX.(2013), aFA, phänologische Verfrühung 16d (korrigiert nach Müller 2015; in RiCherT 2014 war der 10.X.(1904) als aFA angegeben).

Fundorte (Ergänzung):

e 3049/III Chorin Umg./Forst Chorin: Südöstlich des Pferdebergs (2013: MüLLER 2015)

3049/IV Chorin Umg./Forst Chorin: Teerbrennerberge - Am Olberg (2013: MüLLER 2015), Kreuzung Denglerweg - Olbergstraße (2013: MüLLER 2015)

\section{6 (7696) Agriopis marginaria (FABRICIUS, 1775)}

Neue Nachweise von bekannten Fundorten:

c 3147/I Zerpenschleuse/Ortsgebiet: Berliner Straße, im Garten (2016: Busse)

3148/IV Ebw.-Westend/Unterheide: Wildparkstraße (1973, 1974, 1993: Kittel)

Fundorte (Ergänzung):

c 3148/IV Ebw./Stadtgebiet: Kleingartenanlage im Schwärzetal nördlich des Forstbotanischen Gartens (2015: Schwabe)

g 3150/I Oderberg/Teufelsberg (1979: Kittel)

\section{7 (7699) Erannis defoliaria (CLERCK, 1759)}

Als weitere Raupennahrungspflanze im Gebiet (vgl. Richert 2004, 2014) wurde Acer pseudoplatanus, BergAhorn, festgestellt (Foto Schwabe).

Neue Nachweise von bekannten Fundorten:

c 3149/III Ebw. Umg./Finowbruch westlich des Eichwerder (2017: Schwabe)

Fundorte (Ergänzung):

g 3150/I Oderberg/Geistberg (2014: Schwabe)

\section{8 (7754) Peribatodes rhomboidaria (Denis \& SCHIFFERMÜLLER, 1775)}

Ich beobachtete den F E VIII nachts beim Saugen an Blüten von Buddleja davidii, Chinesischer Sommerflieder.

Phänologie/Voltinismus: 16.V.(2018) aFA, phänologische Verfrühung 3d gegenüber bis 2013.

\section{9 (7762) Peribatodes secundaria (ESPER, 1794)}

Während der F bisher überwiegend a.L. nachgewiesen wurde, liegen nun auch Funde a.K. vor.
Neue Nachweise von bekannten Fundorten:

c 3147/I Zerpenschleuse/Ortsgebiet: Berliner Straße, im Garten (2014, 2016: Busse)

3147/II Finowfurt/Hirtenweg - Besters Fließ (1987, 1991, 1994-1996: Kittel)

3148/III Ebw. OT Finow/Stadtgebiet: Altenhofer Straße, im Garten (2014-2016)

3148/IV Ebw.-Westend/Unterheide: Wildparkstraße (1972, 1974, 1975: Kittel);

d 3047/III Groß Schönebeck/Ortsgebiet: Friedenstraße, im Garten (2017: Haase)

Fundorte (Ergänzung):

b 3249/I Gersdorf Umg./Gamengrund südlich Teufelssee (2017: Richert \& Schwabe)

c 3147/IV Finowfurt Umg./Finowfließniederung: Neue Brücke an der Biesenthaler Straße (2014), Kesselmoor an der Finowfließniederung nordöstlich des Lehnssees (2016, 2017)

3148/IV Ebw./Stadtgebiet: Kleingartenanlage im Schwärzetal nördlich des Forstbotanischen Gartens (2014 Schwabe)

g 3150/IV Oderberg/Teufelsberg (1974: Kittel)

\section{0 (7773) Cleora cinctaria (DENIS \& SCHIFFER- MÜLLER, 1775)}

- RL Land Brandenburg: Kategorie 3 (gefährdet): CB, WS;

- RL Deutschland (2011): Kategorie 3 (gefährdet).

Fundorte (Ergänzung):

b 3148/IV Spechthausen Umg./Barnimer Heide: Stromtrasse südwestlich des Ortes (2015)

c 3146/IV Kreuzbruch Umg./ehemalige Römerwegbrücke am Oder-Havel-Kanal östlich des Ortes (2014: Busse) (westlich angrenzendes Nachbargebiet)

3147/II Finowfurt/Hirtenweg - Besters Fließ (1995: Kittel)

d 3148/II Britz (1973: Kittel)

g 3149/II Liepe (1974, 1979: Kittel)

\section{1 (7777) Alcis repandata (LinNAEUs, 1758)}

Neue Nachweise von bekannten Fundorten:

c 3146/IV Kreuzbruch Umg./ehemalige Römerwegbrücke am Oder-Havel-Kanal östlich des Ortes (2014: Busse) (westlich angrenzendes Nachbargebiet)

3147/I Zerpenschleuse/Ortsgebiet: Berliner Straße, im Garten (2014, 2015, 2018: Busse)

3147/II Finowfurt/Hirtenweg - Besters Fließ (1990, 1991, 1995, 1997: Kittel)

d 3047/III Groß Schönebeck/Ortsgebiet: Friedenstraße, im Garten (2016: Haase)

Fundorte (Ergänzung):

b 3148/IV Spechthausen Umg./Barnimer Heide: Stromtrasse südwestlich des Ortes (2018)

c 3147/IV Finowfurt Umg./Kesselmoor an der Finowfließniederung nordöstlich des Lehnssees (2017: Richert \& Schwabe) 


\footnotetext{
3148/IV Ebw. OT Finow Umg./Forst Finowtal: Stromtrasse am Forstort Sechsärmel (2018); Ebw./Stadtgebiet: Kleingartenanlage im Schwärzetal nördlich Forstbotanischer Garten (2018: Schwabe)

g 3150/IV Altglietzen/Gabower Berge (2015)
}

\section{2 (7781) Arichanna melanaria (Linnaeus, 1758)}

- RL Land Brandenburg: Kategorie 2 (stark gefährdet): SM;

- RL Deutschland: Kategorie 2 (stark gefährdet).

Unsere Hoffnung, ein aktuelles Vorkommen in einem Kesselmoor mit Restbeständen an Sumpfporst am Rande der Finowfließniederung nordöstlich des Lehnssees zu finden, hat sich trotz zweijähriger gezielter Suche nicht erfüllt.

Ältere Daten (Belege in coll. Kittel) werden ergänzend nachgetragen.

Nach Päparation älterer Lichtfangausbeuten aus Groß Schönebeck konnte Haase einen F aus dem Jahre 1999 aus dem Ortsgebiet vorlegen. Der Fund ist ein weiterer Beleg für die Dispersionsfähigkeit der Art (vgl. RicherT 2004, 2014).

Neue Nachweise von bekannten Fundorten:

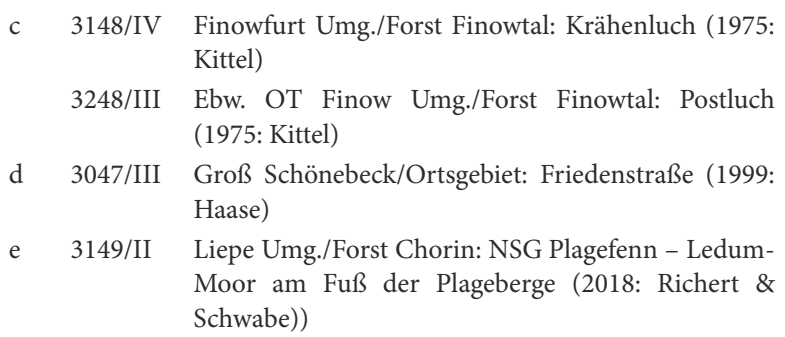

663 (7783) Hypomecis roboraria (DENIS \& SCHIFFERMÜLLER, 1775)

Phänologie/Voltinismus: Nach der Jahrtausendwende wurden in einzelnen Jahren F einer partiellen 2. Gen. beobachtet (RICHERT 2010). Das trifft auch für den neuen Beobachtungszeitraum zu: 04.IX.2014, 1 F leg. Richert; 30.IX.2014, 03.X.2014 je $1 \mathrm{~F}$ leg. Busse; 13.VIII.2015 $1 \mathrm{~F}$ leg. Ockruck.

03.X.(2014), aFE, phänologische Verspätung $8 \mathrm{~d}$ gegenüber bis 2013 .

Neue Nachweise von bekannten Fundorten:

b 3247/II Biesenthal Umg./NSG Biesenthaler Becken: Langerönner Weg (2014: Ockruck)

c 3147/I Zerpenschleuse/Ortsgebiet: Berliner Straße, im Garten (2014-2018: Busse)

3147/II Finowfurt/Hirtenweg - Besters Fließ (1986, 1988, 1991: Kittel)

3148/III Ebw. OT Finow/Stadtgebiet: Altenhofer Straße, im Garten $(2014,2017,2018)$

e 3149/II Liepe Umg./Forst Chorin: NSG Plagefenn - LedumMoor am Fuß der Plageberge (2018: Richert \& Schwabe)

g 3149/II Liepe (1974, 1978: Kittel)

\section{0/I Oderberg/Teufelsberg (1975: Kittel) \\ 3150/III Gabow/Granitberggebiet (2015)}

Fundorte (Ergänzung):

a 3249/II Falkenberg Umg./Cöthener Forst: Klingendes Fließ unterhalb des Bismarck-Turmes (2014)

b 3249/I Trampe (1970: Kittel)

c 3147/I Marienwerder Umg./Oder-Havel-Kanal nordwestlich des Ortes (2015, 2016: Busse)

3147/IV Finowfurt Umg./Kesselmoor an der Finowfließniederung nordöstlich des Lehnssees (2016)

3148/IV Ebw./Stadtgebiet: Kleingartenanlage im Schwärzetal nördlich des Forstbotanischen Gartens (2014, 2018 : Schwabe)

d 3148/II Britz (1978: Kittel)

g 3150/I Oderberg/Geistberg (2018: Schwabe)

\section{5 (7794) Ascotis selenaria (DENIS \& SCHIFFER- MÜLLER, 1775)}

Die Art wird im Gebiet zunehmend häufiger und verbreitet beobachtet.

Phänologie/Voltinismus: 31.V.2016, phänologische Verfrühung 6d; 29.V.(2017), aFA, erneute phänologische Verfrühung 2d, insgesamt $8 \mathrm{~d}$ gegenüber bis 2013 .

Neue Nachweise von bekannten Fundorten:

c 3148/III Ebw. OT Finow/Stadtgebiet: Altenhofer Straße, im Garten $(2016,2017,2018)$

Fundorte (Ergänzung):

c $3147 / \mathrm{IV}$ Finowfurt Umg./Kesselmoor an der Finowfließniederung nordöstlich des Lehnssees $(2016,2017)$

3148/III Ebw. OT Finow Umg./Forst Finowtal: Einflugschneise Flugplatz (2016)

g 3150/III Gabow/Granitberggebiet (2015)

\section{6 (7796) Ectropis crepuscularia (DENIs \& SCHIF- FERMÜLLER, 1775) = bistortata GOEZE, 1781}

Phänologie/Voltinismus: Die in der Regel bei uns bivoltine Art bildete 2014 erneut drei Gen.: aus: M III, E VI-E VII, A IX, wobei die 1. und 3. Gen. nur sehr individuenschwach ausfielen. Inzwischen liegt umfangreicheres Datenmaterial für die 3. Gen. vor: 2013 wurden die F lückenlos vom 28.VIII. - 13.IX. a.L. beobachtet.

\section{7 (7798) Paradarisa consonaria (HüBNER, 1799)}

Ein älterer Fund (Beleg in coll. Kittel) wird ergänzend nachgetragen.

Fundorte (Ergänzung):

c 3148/IV Ebw.-Westend/Unterheide: Wildparkstraße (1973: Kittel); Ebw./Stadtgebiet: Kleingartenanlage im Schwärzetal nördlich Forstbotanischer Garten (2018: Schwabe) 
668 (7800) Parectropis similaria (HufNAGEL, 1767) = extersaria HÜBNER, 1799

Phänologie/Voltinismus: 11.V.(2018) aFA, phänologische Verfrühung $6 \mathrm{~d}$ gegenüber bis 2013.

Neue Nachweise von bekannten Fundorten:

c 3147/I Zerpenschleuse/Ortsgebiet: Berliner Straße, im Garten (2015: Busse)

3147/II Finowfurt/Hirtenweg - Besters Fließ (1992, 1995: Kittel)

3148/III Ebw. OT Finow/Stadtgebiet: Altenhofer Straße, im Garten $(2014,2016,2017,2018)$

Fundorte (Ergänzung):

c 3147/I Marienwerder Umg./Oder-Havel-Kanal nordwestlich des Ortes (2016: Busse)

3147/IV Finowfurt Umg./Kesselmoor an der Finowfließniederung nordöstlich des Lehnssees (2017)

3148/IV Ebw./Stadtgebiet: Kleingartenanlage im Schwärzetal nördlich des Forstbotanischen Gartens (2014, 2016, 2018: Schwabe)

d 3047/III Groß Schönebeck/Ortsgebiet: Friedenstraße, im Garten (2014: Haase)

e 3149/II Liepe Umg./Forst Chorin: NSG Plagefenn - LedumMoor am Fuß der Plageberge (2018: Richert \& Schwabe)

g 3150/I Oderberg/Geistberg (2018: Schwabe)

669 (7802) Aethalura punctulata (DENIS \& SCHIFFERMÜLLER, 1775)

Neue Nachweise von bekannten Fundorten:

b 3247/II Biesenthal Umg./NSG Biesenthaler Becken: Langerönner Weg (2014: Ockruck)

3248/II Trampe/ehemaliger TÜP (2016: Busse \& Müller)

c 3146/IV Kreuzbruch Umg./ehemalige Römerwegbrücke am Oder-Havel-Kanal östlich des Ortes (2014: Busse) (westlich angrenzendes Nachbargebiet)

3147/I Zerpenschleuse/Ortsgebiet: Berliner Straße, im Garten (2014, 2017, 2018: Busse); Marienwerder Umg./Oder-Havel-Kanal nordwestlich des Ortes (2014, 2016: Busse)

3147/II Finowfurt/Hirtenweg - Besters Fließ (1993-1997: Kittel)

3148/III Ebw. OT Finow/Stadtgebiet: Altenhofer Straße, im Garten (2018) und Umg./Forst Finowtal: Einflugschneise Flugplatz (2016)

e $\quad 3149 /$ II $\quad$ Liepe Umg./Forst Chorin: NSG Plagefenn - LedumMoor am Fuß der Plageberge (2018: Richert \& Schwabe)

Fundorte (Ergänzung):

c 3147/IV Finowfurt Umg./Kesselmoor an der Finowfließniederung nordöstlich des Lehnssees $(2016,2017)$

3148/IV Ebw./Stadtgebiet: Kleingartenanlage im Schwärzetal nördlich Forstbotanischer Garten (2018: Schwabe)

d 3148/II Britz/Ortsgebiet (1978: Kittel)

g 3149/II Liepe (1971: Kittel)
671 (7822) Bupalus piniarius (LinNAEUs, 1758)

Phänologie/Voltinismus: Univoltin, 29.IV.(2014), aFA, phänologische Verfrühung 6d gegenüber bis 2013.

\section{2 (7824) Cabera pusaria (LinnaEus, 1758)}

Phänologie/Voltinismus: Univoltin, jw bivoltin: 2. Gen.: 28.IX.(2013), aFE, phänologische Verspätung 19d (korrigiert nach MüLLER 2015; in RICHERT 2014 war der 09.IX. (2005) als aFE angegeben).

\section{3 (7826) Cabera exanthemata (SCOPOLI, 1763)}

Phänologie/Voltinismus: Univoltin, jw bivoltin: 2. Gen.: 12.IX.(2013), aFE, phänologische Verspätung 12d (korrigiert nach MüLler 2015; in RICHERT 2014 war der 31.VIII.(1994) als aFE angegeben).

\section{4 (7828) Lomographa bimaculata (FABRICIUS, 1775)}

Neue Nachweise von bekannten Fundorten:

c 3148/III Ebw. OT Finow/Stadtgebiet: Altenhofer Straße, im Garten $(2014,2016,2018)$

g 3150/I Oderberg/Teufelsberg (1975: Kittel)

Fundorte (Ergänzung):

c 3148/IV Ebw.-Westend/Unterheide: Drehnitzwiesen (1974 Kittel); Ebw./Stadtgebiet: Kleingartenanlage im Schwärzetal nördlich des Forstbotanischen Gartens (2014, 2016: Schwabe)

d 3047/III Groß Schönebeck/Ortsgebiet: Friedenstraße, im Garten (2014: Haase)

\section{5 (7829) Lomographa temerata (DENIS \& SCHIFFERMÜLLER, 1775)}

Neue Nachweise von bekannten Fundorten:

c 3146/IV Kreuzbruch Umg./ehemalige Römerwegbrücke am Oder-Havel-Kanal östlich des Ortes (2014: Busse) (westlich angrenzendes Nachbargebiet)

3147/I Zerpenschleuse/Ortsgebiet: Berliner Straße, im Garten (2014-2018)

3147/II Finowfurt/Hirtenweg - Besters Fließ (1987, 1991, 1994, 1995: Kittel)

3148/III Ebw. OT Finow/Stadtgebiet: Altenhofer Straße, im Garten $(2014,2015,2016,2017,2018)$

e 3149/II Liepe Umg./Forst Chorin: NSG Plagefenn - LedumMoor am Fuß der Plageberge (2018: Richert \& Schwabe)

g 3149/II Liepe/Ortsgebiet: Schule (1973: Kittel)

3150/IV Altglietzen/Gabower Berge (2015)

Fundorte (Ergänzung):

c 3147/I Marienwerder Umg./Oder-Havel-Kanal nordwest lich des Ortes (2014, 2015. 2016: Busse)

3147/IV Finowfurt Umg./Kesselmoor an der Finowfließniederung nordöstlich des Lehnssees $(2016,2017)$ 


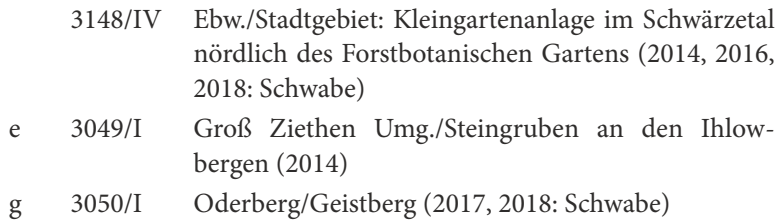

676 (7831) Aleucis distinctata (HERRICH-SCHÄFFER, 1839)

- RL Land Brandenburg: Kategorie 3 (gefährdet): HE. Fundorte (Ergänzung):

g 3150/I Oderberg/Geistberg (2016: Schwabe)

\section{7 (7833) Theria rupicapraria (DenIs \& SCHIF- FERMÜLLER, 1775)}

- RL Brandenburg: Vorwarnliste: HE.

Fundorte (Ergänzung):

d 3148/I Werbellin Umg./NSG Buckowseerinne: Südlich der g 3150/I Oderberg/Geistberg (2015: Schwabe)

\section{8 (7836) Campaea margaritata (LinnAEus, 1767)}

Im Gebiet festgestellte Nektarsaugpflanzen: Der F wurde nachts beim Saugen an Blüten von Buddleja davidii, Chinesischer Sommerflieder, beobachtet.

Phänologie/Voltinismus: Bivoltin, beide Generationen deutlich getrennt.

1. Gen.: 13.V.(2018) aFA, phänologische Verfrühung 1d gegenüber bis 2013 .

Die Flugzeit der 2. Gen. ist ausgedehnter als bisher bekannt:

31.VII.(2014), aFA, phänologische Verfrühung 2d gegenüber bis 2013;

23.X.(2013), aFE, phänologische Verspätung 15d (korrigiert nach MüLLER 2015; in RICHERT 2014 war der 08.X.1987 als aFE angegeben).

\section{9 (7839) Hylaea fasciaria (LiNNAEUs, 1758)}

Neue Nachweise von bekannten Fundorten:

c 3147/I Zerpenschleuse/Ortsgebiet: Berliner Straße, im Garten (2014-2018: Busse)

3147/II Finowfurt/Hirtenweg - Besters Fließ (1990, 1993, 1995: Kittel)

3148/III Ebw. OT Finow/Stadtgebiet: Altenhofer Straße, im Garten $(2014,2015,2017)$

g 3149/II Liepe (1975: Kittel)

3150/IV Altglietzen Umg./Gabower Berge (2015)

Fundorte (Ergänzung):

c 3146/IV Kreuzbruch Umg./ehemalige Römerwegbrücke am Oder-Havel-Kanal östlich des Ortes (2014: Busse) (westlich angrenzendes Nachbargebiet)
3147/I Marienwerder Umg./Oder-Havel-Kanal nordwestlich des Ortes (2014: Busse)

3147/IV Finowfurt Umg./Kesselmoor an der Finowfließniederung nordöstlich des Lehnssees $(2016,2017)$

3148/III Ebw. OT Finow Umg./Forst Finowtal: Einflugschneise Flugplatz (2016)

3148/IV Ebw./Stadtgebiet: Kleingartenanlage im Schwärzetal nördlich Forstbotanischer Garten (2017: Schwabe)

e 3049/IV Chorin Umg./Forst Chorin: Teerbrennerberge - Am Olberg (2013: MüLLER 2015), Kreuzung Denglerweg - Olbergstraße (2013: MÜLler 2015)

f 3049/IV Brodowin/Krugberg (2014) und Umg./Karpatenweg östlich des Krugbergs (2015)

g 3050/II Stolpe a.O./Trockenhänge am Stadtweg (2018)

3149/IV Niederfinow/Oderhänge - ehemalige Kiesgruben südwestlich des Schiffshebewerks (2015, 2016: Schwabe)

h 3150/II Hohensaaten/Deponiegelände an der Straße nach der Siedlung (2014: Kleße)

\section{0 (7857) Charissa obscurata (DENIS \& SCHIF- FERMÜLLER, 1775)}

- RL Deutschland (2011): Vorwarnliste.

Im Gebiet verschollen. Seit 2002 liegen keine Beobachtungen vor, obwohl bis dahin von ihr besiedelte Gebiete weiter faunistisch untersucht wurden.

Ein älterer Fund (Beleg in coll. Kittel) wird ergänzend nachgetragen.

Neue Nachweise von bekannten Fundorten:

c 3147/II Finowfurt/Hirtenweg - Besters Fließ (1995: Kittel)

\section{2 (7916) Siona lineata (Scopoli, 1763)}

- RL Land Brandenburg: Kategorie 2 (stark gefährdet): TR.

Die Art breitet sich weiter im Gebiet aus: 2014 hatte ich sie erstmals in meinem Garten im Stadtgebiet von Finow a.L.; Busse beobachtete sie 2016 ertmals in seinem Garten in Zerpenschleuse a.L.

Der F wurde am Tag beim Saugen an Gewöhnlicher Ochsenzunge (Anchusa officinalis) beobachtet (Schwabe, Fotobeleg).

Schwabe beobachtete am 31.V.2014 auf dem Geistberg bei Oderberg die Eiablage an Wiesen-Kerbel (Anthriscus sylvestris) und Glatthafer (Arenatherum elatius). Die Eier wurden im Blütenstand einzeln und $\mathrm{zu}$ mehreren an Stielen von Blüten/Früchten im Blütenstand bzw. am Rande von Blattspreiten abgelegt.

Neue Nachweise von bekannten Fundorten:

b 3247/II Biesenthal Umg./NSG Biesenthaler Becken: Langerönner Weg (2014: Ockruck)

3248/II Trampe/ehemaliger TÜP (2014)

c 3147/I Marienwerder Umg./Oder-Havel-Kanal nordwestlich des Ortes (2015: Busse);

3147/II Finowfurt/Hirtenweg - Besters Fließ (1987, 1988: Kittel)

3149/III Ebw. Umg./Finowbruch östlich des Ortes (2015: Richert, Schwabe) 
d 3148/I Lichterfelde Umg./NSG Buckowseerinne: Trockenhänge südwestlich Margaretenhof (2014)

f 3049/IV Brodowin Umg./Kl. Rummelsberg; Biodeponie an der Straße nach Pehlitz nordwestlich des Kl. Rummelsberges (2014)

g 3150/I Oderberg/Geistberg (2014-2018: Schwabe)

3150/III Gabow/Granitberggebiet (2015)

Fundorte (Ergänzung):

c 3146/IV Kreuzbruch Umg./ehemalige Römerwegbrücke am Oder-Havel-Kanal östlich des Ortes (2014: Busse) (westlich angrenzendes Nachbargebiet)

3147/I Zerpenschleuse/Ortgebiet: Berliner Straße, im Garten (2016, 2018: Busse)

3148/III Ebw. OT Finow/Stadtgebiet: Altenhofer Straße, im Garten $(2014,2016)$

3149/III Stecherschleuse Umg./ehemaliges Kiesgrubengelände nördlich des Ortes (2017), Hochfläche nordwestlich der Waldstraße (2017)

d 3047/III Groß Schönebeck Umg./Liebenthaler Weg (2014: Haase)

e 3049/I Groß Ziethen Umg./Steingruben an den Ihlowbergen (2014); Grumsin/Wildacker und Wiesengelände am Ortsrand (2015) und Umg./Forst Grumsin: Stromtrasse westsüdwestlich des Ortes (2015)

3149/II Liepe Umg./Vorwerk Liepe an der neuen Parsteiner Straße: Energietrasse (2016)

g 3050/II Stolpe a.O./Trockenhänge am Stadtweg (2018); Stolzenhagen Umg./NSG Krähen- und Jungfernberge $(2015,2016)$

\section{3 (7939) Perconia strigillaria (HÜBNER, 1787)}

- RL Land Brandenburg: Kategorie 3 (gefährdet): CB;

- RL Deutschland (2011): Kategorie 3 (gefährdet).

Neue Nachweise von bekannten Fundorten:

c 3148/III Ebw. OT Finow Umg./Forst Finowtal: Einflugschneise Flugplatz (2016)

\section{Unterfamilie Oenochrominae}

\section{4 (7953) Alsophila aescularia (DENIS \& SCHIF- FERMÜLLER, 1775)}

Neue Nachweise von bekannten Fundorten:

c 3147/I Zerpenschleuse/Ortsgebiet: Berliner Straße, im Garten (2014, 2016, 2017, 2018: Busse)

b 3148/IV Ebw. Umg./Oberheide: Brunnenberge (2014)

Fundorte (Ergänzung):

c 3148/IV Ebw./Stadtgebiet: Kleingartenkolonie im Schwärzetal nördlich Forstbotanischer Garten $(2015,2016$ : Schwabe)

685 (7954) Alsophla aceraria (DENIS \& SCHIFFERMÜLLER, 1775) = quadripunctata EsP.

Ältere Funde (Belege in coll. Kittel) werden ergänzend nachgetragen.
Fundorte (Ergänzung):

b 3149/III Ebw.-Ostende (1982: Kittel)

c 3147/II Finowfurt/Hirtenweg - Besters Fließ (1988: Kittel)

\section{Unterfamilie Geometrinae}

\section{6 (7961) Aplasta ononaria (FuessLy, 1783)}

- RL Land Brandenburg: Kategorie 3 (gefährdet): TR;

- RL Deutschland (2011): Kategorie 3 (gefährdet).

Neue Nachweise von bekannten Fundorten:

g 3150/III Gabow/Granitberggebiet (2015: Schwabe)

3150/IV Altglietzen Umg./Gabower Berge (2015)

Fundorte (Ergänzung):

c 3147/I Zerpenschleuse/Ortgebiet: Berliner Straße, im Garten (2015: Busse)

3148/III Ebw. OT Finow Umg./Forst Finowtal: Einflugschneise Flugplatz (2017: Schwabe)

\section{7 (7965) Pseudoterpna pruinata (HufNAgeL, 1767)}

- RL Deutschland (2011): Kategorie 3 (gefährdet).

Neue Nachweise von bekannten Fundorten:

c 3147/II Finowfurt/Hirtenweg - Besters Fließ (1988, 1992, 1993: Kittel)

Fundorte (Ergänzung):

d 3047/III Groß Schönebeck/Ortsgebiet: Friedenstraße, im Garten (2014: Haase)

\section{8 (7969) Geometra papilionaria (LinNAEUs,} 1758)

Neue Nachweise von bekannten Fundorten:

b 3247/II Biesenthal Umg./NSG Bisenthaler Becken: Langerönner Weg (2014: Ockruck)

3248/II Trampe/ehemalger TÜP (2016: Busse \& Müller)

c 3147/I Zerpenschleuse/Ortsgebiet: Berliner Straße, im Garten (2014, 2015, 2016: Busse)

3147/IV Finowfurt Umg./Finowfließniederung: Neue Brücke an der Biesenthaler Str. (2014)

3148/III Ebw. OT Finow/Stadtgebiet: Altenhofer Straße, im Garten $(2014,2015,2016)$

3149/III Ebw./Eichwerder (1976: Kittel)

e 3149/II Liepe Umg./Forst Chorin: NSG Plagefenn - LedumMoor am Fuß der Plageberge (2018: Richert \& Schwabe)

g 3050/II Stolpe a.O./Trockenhänge am Stadtweg (2018)

3150/III Gabow/Granitberggebiet (2015: Schwabe)

3150/IV Altglietzen Umg./Gabower Berge (2015)

Fundorte (Ergänzung):

b 3247/II Biesenthal Umg./NSG Biesenthaler Becken: Nördlich der Pfauenwiesen (2014: Ockruck)

3249/I Gersdorf Umg./Gamengrund südlich Teufelssee (2017: Schwabe) 
c 3147/II Finowfurt/Hirtenweg - Besters Fließ (1988, 1990: Kittel)

3147/IV Finowfurt Umg./Kesselmoor an der Finowfließniederung nordöstlich des Lehnssees $(2016,2017)$

3148/IV Ebw./Stadtgebiet: Kleingartenanlage im Schwärzetal nördlich Forstbotanischer Garten (2014, 2016, 2018 : Schwabe)

h 3150/II Hohensaaten/Deponie an der Straße nach der Siedlung (2014: Kleße)

\section{9 (7971) Comibaena bajularia (DENIS \& SCHIF-} FERMÜLLER, 1775) = pustulata HUFNAGEL, 1767

Phänologie/Voltinismus: In coll. Kittel befand sich ein F mit den bisher unberücksichtigten Daten 13.IX.1995, Finowfurt/Hirtenweg. Hier handelte es sich offenbar um einen $\mathrm{F}$ der partiellen 2. Gen., die somit bereits früher als bisher angenommen erstmals im Gebiet auftrat (vgl. RICHERT 2014).

Neue Nachweise von bekannten Fundorten:

b 3247/II Biesenthal Umg./NSG Biesenthaler Becken: Langerönner Weg (2014: Ockruck)

c 3146/IV Kreuzbruch Umg./ehemalige Römerwegbrücke am Oder-Havel-Kanal östlich des Ortes (2014: Busse) (westlich angrenzendes Nachbargebiet)

3147/I Zerpenschleuse/Ortsgebiet: Berliner Straße, im Garten (2014-2016, 2018: Busse)

3147/II Finowfurt/Hirtenweg - Besters Fließ (1991, 1993, 1994, 1996: Kittel)

3148/III Ebw. OT Finow/Stadtgebiet: Altenhofer Straße, im Garten $(2017,2018)$

e 3149/II Liepe Umg./Forst Chorin: NSG Plagefenn - LedumMoor am Fuß der Plageberge (2018: Richert \& Schwabe)

g 3150/I Oderberg/Teufelsberg (1973: Kittel)

3150/IV Altglietzen Umg./Gabower Berge (2015)

Fundorte (Ergänzung):

c 3147/I Marienwerder Umg./Oder-Havel-Kanal nordwestlich des Ortes (2015, 2016: Busse)

3147/IV Finowfurt Umg./Kesselmoor an der Finowfließniederung nordöstlich des Lehnssees (2017)

3148/IV Ebw./Stadtgebiet: Kleingartenanlage im Schwärzetal nördlich des Forstbotanischen Gartens (2014: Schwabe)

e 3049/I Groß Ziethen Umg./Steingruben an den Ihlowbergen (2014)

g 3050/I Oderberg/Geistberg (2017, 2018: Schwabe)

\section{0 (7980) Hemithea aestivaria (HüBNER, 1789)}

Phänologie/Voltinismus: 30.V.(2018); aFA, phänologische Verfrühung 5d gegenüber bis 2013.

In coll. Kittel befanden sich zwei F mit den bisher unberücksichtigten Daten 10.VIII.1995 und 13.IX.1995, Finowfurt/Hirtenweg. Diese Daten bestätigen die Annahme, dass die Art in günstigen Jahren im Gebiet eine partielle 2. Gen. hervorbringt (vgl. RicherT 2014).
Neue Nachweise von bekannten Fundorten:

c 3147/I Zerpenschleuse/Ortsgebiet: Berliner Straße, im Garten (2014, 2016, 2018: Busse); Marienwerder Umg./Oder-Havel-Kanal nordwestlich des Ortes (2014: Busse)

3147/II Finowfurt/Hirtenweg - Besters Fließ (1991, 1995 Kittel)

3148/III Ebw. OT Finow/Stadtgebiet: Altenhofer Straße, im Garten $(2014$, 2015, 2016, 2017, 2018)

e 3149/II Liepe Umg./Forst Chorin: NSG Plagefenn - LedumMoor am Fuß der Plageberge (2018: Richert \& Schwabe)

g 3050/II Stolpe a.O./Trockenhänge am Stadtweg (2018)

3150/IV Altglietzen Umg./Gabower Berge (2015)

Fundorte (Ergänzung):

c 3147/I Marienwerder Umg./Oder-Havel-Kanal nordwestlich des Ortes (2016: Busse)

3148/IV Ebw.-Westend/Unterheide: Wildparkstraße (1975, 1977: Kittel); Ebw. - Nordend/Gehörlosenhilfsschule im Gelände der Landesklinik (1983: Kittel); Ebw./ Stadtgebiet: Kleingartenanlage im Schwärzetal nördlich des Forstbotanischen Gartens (2014, 2016, 2018 : Schwabe)

d 3149/I Britz Umg./F. Britz (1973: Kittel)

g 3150/I Oderberg/Geistberg (2016, 2017, 2018: Schwabe)

\section{2 (7998) Thalera fimbrialis (ScOPOLI, 1763)}

Die R fand ich am 04.VI.(2015) auf einer Stromtrasse an Calluna vulgaris, Besenheide, F e.l. 16.VII.(2015).

Neue Nachweise von bekannten Fundorten:

b 3247/II Biesenthal Umg./NSG Biesenthaler Becken: Weg nördlich der Pfauenwiesen (2014: Ockruck)

g 3149/IV Niederfinow Umg./Oderhänge - ehemalige Kiesgruben südwestlich des Schiffshebewerks (2014: Schwabe)

Fundorte (Ergänzung):

b 3248/II Schönholz Umg./Barnimer Heide: Stromtrasse nordwestlich des Ortes (2015)

e 3049/I Groß Ziethen Umg./Steingruben an den Ihlowbergen (2014)

\section{3 (8000) Hemistola chrysoprasaria (ESPER, 1794)}

Ältere Funde (Belege in coll. Kittel) werden ergänzend nachgetragen.

Neue Nachweise von bekannten Fundorten:

g 3149/II Liepe (1976: Kittel)

3150/I Oderberg/Teufelsberg (1975: Kittel)

Fundorte (Ergänzung):

c 3147/II Finowfurt/Hirtenweg - Besters Fließ (1995: Kittel)

3148/IV Ebw.-Westend/Unterheide: Wildparkstraße (1975: Kittel) 


\section{4 (8002) Jodis lactearia (Linnaeus, 1758)}

Die R fand Schwabe M (19.)IX 2016 bei Spechthausen an Acer pseudoplatanus, Bergahorn. Diese R-Nahrungspflanze wird in der Literatur nicht genannt (EcKsTeIN 1923, Косh 1984; Forster \& Wohlfahrt 1981, BergMANN 1955, SKou 1986, BARTSCH 2001 in Ebert 2001, HaUsmann 2001).

Fundorte (Ergänzung):

b 3148/IV Spechthausen Umg./Fischteiche nordöstlich des Ortes (2016: Schwabe)

\section{5 (8002) Jodis putata (LinNAEUs, 1758)}

- RL Land Brandenburg: Vorwarnliste: HW, NW. Phänologie/Voltinismus: Am 31.X.2014 fing ich beim Pilzesuchen im Forst Finowtal einen $\mathrm{F}$ dieser Art; phänologische Verspätung 122d gegenüber bis 2013. Dieser ungewöhnliche Fund kann nur als partielle 2. Gen. gedeutet werden, die erstmalig im Gebiet beobachtet wurde.

In der Literatur wird die Art sowohl für Deutschland (BARTSCH 2001, EcKstein 1923, КосH 1984) als auch Mitteleuropa (Forster \& Wohlfahrt 1981, FajČIK 2003) und Europa (Hausmann 2001) ausschließlich als univoltin angegeben.

\section{Neue Nachweise von bekannten Fundorten:}

e 3149/II Liepe Umg./Forst Chorin: NSG Plagefenn - LedumMoor am Fuß der Plageberge (2018: Richert \& Schwabe)

Fundorte (Ergänzung):

b 3247/II Biesenthal Umg./Forst Biesenthal: Stellweg nach dem Lehnssee (2017)

c 3148/III Ebw. OT Finow Umg./Forst Finowtal südlich vom Flugplatz (2014)

d 3047/III Groß Schönebeck Umg./Schorfheide, Revier Rehluch: Alte Döllner Straße (2014: Haase), Liebenthaler Weg $(2017,2018$ : Haase $)$

\section{Unterfamilie Sterrhinae}

696 (8012) Cyclophora pendularia (CLERCK, 1798) = orbicularia HÜBNER, 1799

- RL Land Brandenburg: Kategorie 3 (gefährdet): MW;

- RL Deutschland (2011): Kategorie 2 (stark gefährdet).

Neue Nachweise von bekannten Fundorten:

c 3147/I Zerpenschleuse/Ortsgebiet: Berliner Straße, im Garten (2014: Busse)

3147/II Finowfurt/Hirtenweg - Besters Fließ (1990, 1994: Kittel)

Fundorte (Ergänzung):

c 3147/IV Marienwerder/Steinlaake (2015: Schwabe) e 3049/I Groß Ziethen Umg./Steingruben an den Ihlowbergen (2014)

\section{7 (8014) Cyclophora annularia (FABRICIUS,} 1775) = annulata SCHULZE, 1775

- RL Land Brandenburg: Vorwarnliste: WS, GL. Phänologie/Voltinismus: Bivoltin: 1. Gen. 26.IV.(2014), aFA, phänologische Verfrühung 14d gegenüber bis 2013.

Neue Nachweise von bekannten Fundorten:

c 3148/III Ebw. OT Finow/Stadtgebiet: Altenhofer Straße, im Garten (2014)

Fundorte (Ergänzung):

c 3148/IV Ebw./Stadtgebiet: Kleingartenanlage im Schwärzetal nördlich des Forstbotanischen Gartens $(2014,2015$, 2016: Schwabe)

g 3149/IV Niederfinow/Oderhänge - ehemalige Kiesgruben südwestlich des Schiffshebewerks (2015: Schwabe)

\section{8 (8016) Cyclophora albipunctata (HUFNAGEL, 1767) = pendularia ClERCK, 1759}

Phänologie/Voltinismus: Bivoltin; 1. Gen.: 29.IV.(2014); aFA, phänologische Verfrühung 6d gegenüber bis 2013. Im Jahre 2014 wurden nach einer regulären Flugperiode der 2. Gen. bis A (7.)VIII je ein F am 05.IX. und am 01.X. a.L. beobachtet;

01.X.(2014), aFE, phänologische Verspätung 12d gegenüber bis 2013. Bei den September- und Oktoberfunden handelt es sich offensichtlich um F einer partiellen 3. Gen., die somit bisher in den Jahren 1999, 2003, 2006 und 2014 nachgewiesen wurde (vgl. RICHERT 2014).

\section{9 (8019) Cyclophora porata (LinnAEus, 1767)}

Neue Nachweise von bekannten Fundorten:

c 3147/I Zerpenschleuse/Ortsgebiet: Berliner Straße, im Garten (2017: Busse)

g 3150/IV Altglietzen Umg./Gabower Berge (2015)

Fundorte (Ergänzung):

c 3147/IV Finowfurt Umg./Kesselmoor an der Finowfließniederung nordöstlich des Lehnssees (2017: Schwabe)

\section{0 (8020) Cyclophora quercimontaria (BASTEL- BERGER, 1897)}

- RL Land Brandenburg: Kategorie 3 (gefährdet): WS;

- RL Deutschland (2011): Kategorie 3 (gefährdet).

Neue Nachweise von bekannten Fundorten:

c 3147/I Zerpenschleuse/Ortsgebiet: Berliner Straße, im Garten (2014, 2015, 2017: Busse)

3148/III Ebw. OT Finow/Stadtgebiet: Altenhofer Straße, im Garten $(2014,2015,2018)$ 
Fundorte (Ergänzung):

b 3249/I Gersdorf Umg./Gamengrund südlich Teufelssee (2017: Schwabe)

c 3146/IV Kreuzbruch Umg./ehemalige Römerwegbrücke am Oder-Havel-Kanal östlich des Ortes (2014: Busse) (westlich angrenzendes Nachbargebiet)

g 3150/IV Altglietzen Umg./Gabower Berge (2015)

\section{1 (8022) Cyclophora punctaria (LinNAEus, 1758)}

Phänologie/Voltinismus: Bivoltin: 1. Gen.: 25.IV.(2014), aFA, phänologische Verfrühung 4d gegenüber bis 2013 . Nach einer regulären Flugzeit der 2. Gen., die diesem Jahr A (04.)VIII endete, wurden nach einer mehrwöchigen Pause E (30.)VIII und M(15.)IX erneut F beobachtet, offenbar eine partielle 3. Gen. Im Sonnensommer 2018 wurde am 10.IX. ein sehr abgeflogener F a.L. registriert, in diesem Jahr sicher ein Tier der 3. Gen.

\section{3 (8027) Timandra comae SCHмIDT, 1931}

Während der F bisher ausschließlich bei Tage in der Vegetation bzw. nachts a.L. gefunden wurde, beobachtete ich ihn in meinem Garten nunmehr auch nachts beim Saugen am Köder.

\section{4 (8036) Scopula immorata (LINNAEUs, 1758)}

Der F wurde am Tag beim Saugen an Armeria maritima, Gewöhnliche Grasnelke, beobachtet (Foto Seidel).

\section{6 (8042) Scopula nigropunctata (DeNIS \& SCHIFFERMÜLLER, 1775)}

Phänologie/Voltinismus: Nachdem 2006 erstmals eine partielle 2. Gen. der bis dahin univoltinen Art beobachtet worden war, konnte dies seitdem wiederholt registriert werden (RICHERT 2008, 2010, 2014). Am 15.IX.2014 wurde je ein F in Zerpenschleuse und Finow a.L. beobachtet. Am 12.X.2014 scheuchte ich im Blaubeer-Kiefernwald südlich vom Flugplatz Finow einen frischen F der partiellen 2. Gen. aus der Krautschicht. Am 20.IX.2018 hatte Haase in Groß Schönebeck einen F der 2. Gen. a.L.

\section{8 (8045) Scopula ornata (ScopolI, 1763)}

- RL Land Brandenburg: Kategorie 2 (stark gefährdet): TR.

- RL Deutschland (2011): Vorwarnliste.

Die Beobachtungslücken in den Naturräumen b, e und h (Barnimplatte, Uckermärker Kuppen- und Hügelland einschließlich Choriner Waldhügel, Odertal) konnten inzwischen geschlossen werden (vgl. dazu RicherT 2004, 2014). Im Forst Chorin wurden $2 \mathrm{~F}$ auf einer Windwurffläche inmitten eines Buchenwaldgebietes a.L. beobachtet (MüLleR 2015; Beleg lag mir vor). Der
Fund an diesem ungewöhnlichen Ort weist auf ausgedehnte Dispersionsflüge hin.

Phänologie/Voltinismus: In der Regel bisher bivoltin; neuerdings wurden $\mathrm{jw}$. frische F im IX beobachtet, welche einer partiellen 3. Gen. zuzurechnen sind; so auch 2014: Am 15.IX. wurden $4 \mathrm{~F}$ auf dem Krugberg bei Brodowin a.L. beobachtet.

Neue Nachweise von bekannten Fundorten:

d 3148/I Lichterfelde Umg./NSG Buckowseerinne: Trockenhänge südwestlich von Margaretenhof (2015)

3149/IV Stecherschleuse Umg./am Rande des ehemaligen Kiesgrubengeländes nordöstlich des Ortes (2017)

g 3149/II Liepe/Ortsgebiet: Schule (1973, 1975: Kittel)

3050/II Stolzenhagen/NSG Krähen- und Jungfernberge (2015, 2016: Richert)

3150/I Oderberg/NSG Pimpinellenberg (1976: Kittel), Teufelsberg (1973, 1974, 1976: Kittel)

Fundorte (Ergänzung):

c 3147/II Finowfurt/Hirtenweg - Besters Fließ (1987: Kittel)

3148/IV Ebw.-Westend/Unterheide: Wildparkstraße (1974, 1975: Kittel)

d 3047/IV Wildau am Werbellinsee/Ablage Spring (1969: Kittel)

e 3049/I Groß Ziethen Umg./Steingruben an den Ihlowbergen (2014)

3049/IV Chorin Umg./Forst Chorin: Teerbrennerberge - Am Olberg (2013: Müller 2015)

f 3049/IV Brodowin/Krugberg (2014), Gr. Rummelsberg (2015: Richert)

g 3050/II Stolpe a.O./Trockenhänge am Stadtweg (2018)

3150/I Oderberg/Geistberg (2015, 2018: Schwabe), am Friedhof (2016: Schwabe)

3150/IV Altglietzen Umg./Gabower Berge (2018: Schwabe)

h 3150/II Hohensaaten/Deponie an der Straße nach der Siedlung (2014: Kleße)

\section{0 (8054) Scopula rubiginata (HufNAGEL, 1767)}

- RL Deutschland (2011): Vorwarnliste.

Phänologie/Voltinismus: Bivoltin, 2. Gen: 12.IX.(2014), aFE, phänologische Verspätung 2d gegenüber bis 2013.

\section{Neue Nachweise von bekannten Fundorten:}

b 3248/II Trampe/ehemaliger TÜP (2016: Busse \& Müller; 2018: Richert)

c 3147/I Zerpenschleuse/Ortsgebiet: Berliner Straße, im Garten (2014, 2015, 2016, 2017, 2018: Busse)

3148/III Ebw. OT Finow/Stadtgebiet: Altenhofer Straße, im Garten $(2014,2017,2018)$

3149/IV Stecherschleuse Umg./ehemaliges Kiesgrubengelände nordöstlich des Ortes (2015: Schwabe)

d 3047/III Groß Schönebeck/Ortsgebiet: Friedenstraße, im Garten (2018: Haase)

g 3149/IV Niederfinow/Oderhänge - ehemalige Kiesgruben südwestlich des Schiffshebewerks (2016: Schwabe)

3150/IV Altglietzen Umg./Gabower Berge (2015: Richert; 2018: Schwabe) 
Fundorte (Ergänzung):

b 3247/II Biesenthal Umg./NSG Biesenthaler Becken: Langerönner Weg (2014: Ockruck), Weg nördlich der Pfauenwiesen (2014: Ockruck)

c 3146/IV Kreuzbruch Umg./ehemalige Römerwegbrücke am Oder-Havel-Kanal östlich des Ortes (2014: Busse; westlich angrenzendes Nachbargebiet)

3147/I Marienwerder Umg./Oder-Havel-Kanal nordwestlich des Ortes (2014, 2015: Busse)

3148/III Ebw. OT Rinow Umg./Forst Finowtal: Einflugschneise Flugplatz (2017)

3148/IV Ebw./Stadtgebiet: Kleingartenanlage im Schwärzetal nördlich des Forstbotanischen Gartens (2014, 2016, 2017: Schwabe)

g 3150/I Oderberg/Geistberg (2017: Schwabe)

\section{1 (8059) Scopula marginepunctata (GoEZE, 1781)}

Die Beobachtungslücke im Naturraum d (Britzer Platte, vgl. Richert 2004) konnte geschlossen werden.

\section{Neue Nachweise von bekannten Fundorten:}

c 3148/IV Ebw.-Westend/Unterheide: Wildparkstraße 1971, 1972, 1976: Kittel)

g 3149/II Liepe (1973, 1974, 1982: Kittel)

3150/III Gabow/Granitberggebiet (2015)

3150/IV Altglietzen Umg./Gabower Berge (2015)

Fundorte (Ergänzung):

c 3147/II Finowfurt/Hirtenweg - Besters Fließ (1990, 1994: Kittel)

d 3148/II Britz (1972: Kittel)

\section{2 (8064) Scopula immutata (Linnaeus, 1758)}

Phänologie/Voltinismus: Univoltin; neuerdings in Jahren mit zeitigem Frühjahr infolge deutlicher Flugzeitvorverlagerung zunehmend regelmäßig eine partielle 2. Gen. im VIII: 04.IX.(2014), aFE, phänologische Verspätung 9d gegenüber bis 2013.

\section{3 (8067) Scopula ternata (SCHRANK, 1802)}

Neue Nachweise von bekannten Fundorten:

c 3148/III Ebw. OT Finow/Stadtgebiet: Altenhofer Straße, im Garten (2014)

Fundorte (Ergänzung):

c 3147/IV Finowfurt Umg./Kesselmoor an der Finowfließniederung nordöstlich des Lehnssees $(2016,2017)$

\section{4 (8069) Scopula floslactata (HAWORTH, 1809)}

Fundorte (Ergänzung):

c 3148/IV Ebw./Stadtgebiet: Kleingartenanlage im Schwärzetal nördlich Forstbotanischer Garten (2018: Schwabe)

\section{5 (8099) Idaea ochrata (ScOPOLI, 1763)}

Neue Nachweise von bekannten Fundorten:

b 3248/II Trampe/ehemaliger TÜP (2016: Busse \& Müller)

c 3147/I Zerpenschleuse/Ortsgebiet: Berliner Straße, im Garten (2014, 2015, 2016: Busse)

3147/II Finowfurt/Hirtenweg - Besters Fließ (1995: Kittel)

3148/III Ebw. OT Finow/Stadtgebiet: Altenhofer Straße, im Garten $(2014,2015,2016,2017)$

3149/I Ebw.-Nordend Umg./Oder-Havel-Kanal Richtung Ragöser Damm (2015: Schwabe)

3149/IV Stecherschleuse Umg./ehemaliges Kiesgrubengelände nordöstlich des Ortes (2015: Schwabe)

e 3049/II Klein Ziethen Umg./Debritzberg $(2015,2017)$

f 3049/IV Brodowin Umg./Kl. Rummelsberg (2015)

3050/III Parstein Umg./Sonnenberge südlich des Ortes (2016)

g 3050/II Stolpe a.O./Trockenhänge am Stadtweg (2018); Stolzenhagen/NSG Krähen- und Jungfernberge (2015)

3150/I Oderberg/Geistberg (2015: Schwabe)

3150/III Gabow/Granitberggebiet (1983: Kittel; 2015: Richert)

3150/IV Altglietzen Umg./Gabower Berge (2015: Richert; 2017, 2018: Schwabe)

Fundorte (Ergänzung):

b 3247/II Biesenthal Umg./NSG Biesenthaler Becken: Weg nördlich der Pfauenwiesen (2014: Ockruck)

c 3148/IV Ebw.-Westend/Unterheide: Wildparkstraße (1972, 1974, 1976: Kittel); Ebw./Stadtgebiet: Kleingartenanlage im Schwärzetal nördlich Forstbotanischer Garten (2016: Schwabe)

d 3047/III Groß Schönebeck/Ortsgebiet: Friedenstraße, im Garten (2015: Haase)

e 3049/I Grumsin (1980: Kittel); Groß Ziethen Umg./Steingruben an den Ihlowbergen (2014)

3050/I Oderberg Umg./Feldflur nördlich NSG Pimpinellenberg (2016)

f 3050/I Herzsprung Umg./Bunkerberg an der Straße nach Bölkendorf (2015)

g 3050/II Stolpe a.O./Trockenhänge an der Straße nach Crussow: Stadtweg (2017)

\section{6 (8100) Idaea serpentata (HuFNAGEL, 1767)}

- RL Land Brandenburg: Kategorie 2 (stark gefährdet): TR;

- RL Deutschland (2011): Vorwarnliste.

Phänologie: Am 24.VI.(2015), phänologische Verfrühung $2 \mathrm{~d}$ gegenüber bis 2013, $3 \mathrm{~F}$ leg. R. Busse \& B. Müller auf dem ehemaligen TÜP Trampe; 12.VI.(2018) aFA, erneute phänologische Verfrühung 12d, insgesamt 14d gegenüber bis 2013.

Neue Nachweise von bekannten Fundorten:

b 3248/II Trampe/ehemaliger TÜP (2016, Busse \& Müller, Richert; 2017: Richert)

c 3149/III Stecherschleuse Umg./ehemaliges Kiesgrubengelände nördlich des Ortes $(2017,2018)$

d 3148/I Lichterfelde Umg./NSG Buckowseerinne: Streuobstwiesen südöstlich Gr. Buckowsee - Moospfuhlgebiet (2018) 
Fundorte (Ergänzung):

g 3050/II Stolpe a.O./Trockenhänge am Stadtweg (2018)

\section{7 (8102) Idaea aureolaria (DENIS \& SCHIFFER- MÜLLER, 1775)}

- RL Land Brandenburg: Kategorie 1 (vom Aussterben bedroht): TR;

- RL Deutschland (2011): Kategorie 1 (vom Aussterben bedroht).

Neue Nachweise von bekannten Fundorten:

g 3150/I Oderberg/Teufelsberg (1974, 1976: Kittel), Geistberg (2017: Schwabe)

3150/III Gabow/Granitberggebiet (2015: Richert, Schwabe; 2018: Kleße)

3150/IV Altglietzen Umg./Gabower Berge (2015: Richert, Schwabe)

\section{8 (8104) Idaea muricata (HUFNAGEL, 1767)}

Neue Nachweise von bekannten Fundorten:

c 3147/I Zerpenschleuse/Ortsgebiet: Berliner Straße, im Garten (2014, 2015: Busse)

3147/II Finowfurt/Hirtenweg - Besters Fließ (1986, 1991, 1995: Kittel)

3148/III Ebw. OT Finow/Stadtgebiet: Altenhofer Straße, im Garten $(2014,2015,2016$, 2017)

Fundorte (Ergänzung):

b 3247/II Biesenthal Umg./NSG Biesenthaler Becken: Weg nördlich der Pfauenwiesen (2014: Ockruck)

c 3147/IV Finowfurt Umg./Kesselmoor an der Finowfließniederung nordöstlich des Lehnssees (2016, 2017: Richert \& Schwabe)

3148/IV Ebw./Stadtgebiet: Kleingartenanlage im Schwärzetal nördlich des Forstbotanischen Gartens (2014, 2017: Schwabe)

e 3049/I Groß Ziethen Umg./Steingruben an den Ihlowbergen (2014)

h 3150/II Hohensaaten/Deponiegelände an der Straße nach der Siedlung (2014: Kleße)

\section{0 (8123) Idaea sylvestraria (HÜBNER, 1798)}

Neue Nachweise von bekannten Fundorten:

c 3148/III Ebw. OT Finow/Stadtgebiet: Altenhofer Straße, im Garten (2014)

Fundorte (Ergänzung):

c 3147/II Finowfurt/Hirtenweg - Besters Fließ (1991: Kittel)

3148/III Ebw. OT Finow Umg./Forst Finowtal: Einflugschneise Flugplatz (2017)

3148/IV Ebw./Stadtgebiet: Kleingartenanlage im Schwärzetal nördlich des Forstbotanischen Gartens (2014: Schwabe)

e 3049/I Groß Ziethen Umg./Steingruben an den Ihlowbergen (2014)

d 3047/III Groß Schönebeck/Ortsgebiet: Friedenstraße, im Garten (2015, 2016, 2018: Haase)
3149/I Britz Umg./F. Britz (1973: Kittel)

\section{1 (8132) Idaea biselata (HufNAGEL, 1767)}

Phänologie/Voltinismus: 14.VI.(2018) aFA, phänologische Verfrühung 5d gegenüber bis 2013.

\section{3 (8137) Idaea fuscovenosa (GoEZE, 1781)}

Neue Nachweise von bekannten Fundorten:

c 3147/I Zerpenschleuse/Ortsgebiet: Berliner Straße, im Garten (2014, 2015: Busse)

3147/II Finowfurt/Hirtenweg - Besters Fließ (1993: Kittel)

3148/III Ebw. OT Finow/Stadtgebiet: Altenhofer Straße, im Garten $(2014,2017,2018)$

g 3150/III Gabow/Granitberggebiet (2015)

Fundorte (Ergänzung):

g 3150/IV Altglietzen/Gabower Berge (2017: Schwabe)

\section{4 (8140) Idaea humiliata (HufNAGEL, 1767)}

Phänologie/Voltinismus: 04.VIII.(2014), aFE, phänologische Verspätung 3d gegenüber bis 2013 .

Neue Nachweise von bekannten Fundorten:

b 3248/II Trampe/ehemaliger TÜP (2016: Busse \& Müller)

c 3147/I Zerpenschleuse/Ortsgebiet: Berliner Straße, im Garten (2014-2016: Busse)

3148/III Ebw. OT Finow/Stadtgebiet: Altenhofer Straße, im Garten $(2014,2016)$

g 3050/II Stolpe a.O./Trockenhänge am Stadtweg (2018)

3150/IV Altglietzen Umg./Gabower Berge (2015)

Fundorte (Ergänzung):

f 3049/IV Brodowin Umg./Gr. Rummelsberg (2015)

e 3049/I Groß Ziethen Umg./Steingruben an den Ihlowbergen (2014)

\section{5 (8155) Idaea seriata (SCHRANK, 1802)}

= virgularia HüBNER, 1799

Phänologie: 2. Gen.: Am 27.IX.(2017), aFE, phänologische Verspätung 3d gegenüber bis 2013, $1 \mathrm{~F}$ leg. Busse in Zerpenschleuse a.L.

Neue Nachweise von bekannten Fundorten:

c 3147/I Zerpenschleuse/Ortsgebiet: Berliner Straße, im Garten (2014-2018: Busse)

3148/III Ebw. OT Finow/Stadtgebiet: Altenhofer Straße, im Garten (2014, 2018)

d 3047/III Groß Schönebeck/Ortsgebiet: Friedenstraße, im Garten (2014, 2015, 2017, 2018: Haase)

Fundorte (Ergänzung):

c 3148/IV Ebw./Stadtgebiet: Kleingartenanlage im Schwärzetal nördlich Forstbotanischer Garten (2016: Schwabe)

g 3150/I Oderberg/am Friedhof (2016: Schwabe)

3150/IV Altglietzen Umg./Gabower Berge (2015) 


\section{6 (8161) Idaea dimidiata (HufNAGEL, 1767)}

Der F wurde im Gebiet nur selten auch a.K. beobachtet (>114 Datensätzen von Nachweisen a. L. stehen nur 2 Datensätze für Nachweise a.K. gegenüber). Er besucht nachts auch Blüten: Schwabe fotografierte einen saugenden F auf einem Blütenstand von Chrysanthemum (Tanacetum) vulgare, Rainfarn.

Phänologie/Voltinismus: Univoltin, 09.VI.(2014), phänologische Verfrühung 4d gegenüber bis 2013; 07.VI.(2018) aFA, erneute phänologische Verfrühung $2 \mathrm{~d}$, insgesamt $6 \mathrm{~d}$ gegenüber bis 2013 .

Neue Nachweise von bekannten Fundorten:

c 3147/I Zerpenschleuse/Ortsgebiet: Berliner Straße, im Garten (2014, 2015, 2016: Busse)

3148/III Ebw. OT Finow/Stadtgebiet: Altenhofer Straße, im Garten $(2015,2017)$

e $\quad 3149 /$ II Liepe Umg./Forst Chorin: NSG Plagefenn - LedumMoor am Fuß der Plageberge (2018: Richert \& Schwabe)

Fundorte (Ergänzung):

b 3247/II Biesenthal Umg./NSG Biesenthaler Becken: Weg nördlich der Pfauenwiesen (2014, 2015: Ockruck)

3249/I Gersdorf Umg./Gamengrund südwärts bis Teufelssee (2017: Richert \& Schwabe)

c 3147/I Marienwerder Umg./Oder-Havel-Kanal nordwestlich des Ortes (2014: Busse)

3147/IV Finowfurt Umg./Finowfließniederung an der Neuen Brücke - Biesenthaler Str. (2014); Kesselmoor an der Finowfließniederung nordöstlich des Lehnssees (2016, 2017: Richert \& Schwabe)

3148/IV Ebw./Stadtgebiet: Kleingartenanlage im Schwärzetal nördlich des Forstbotanischen Gartens (2014, 2015, 2016, 2017: Schwabe)

g 3149/IV Niederfinow/Oderhänge-ehemalige Kiesgruben südwestlich des Schiffshebewerks (2015, 2016: Schwabe)

\section{7 (8183) Idaea emarginata (LINNAEUs, 1758)}

Neue Nachweise von bekannten Fundorten:

b 3248/II Trampe/ehemaliger TÜP (2016: Busse \& Müller, Seidel)

c 3147/I Zerpenschleuse/Ortsgebiet: Berliner Straße, im Garten (2014: Busse)

3147/II Finowfurt/Hirtenweg - Besters Fließ (1993: Kittel)

3148/III Ebw. OT Finow/Stadtgebiet: Altenhofer Straße, im Garten $(2016,2018)$

3148/IV Ebw.-Westend/Unterheide: Wildparkstraße (1976: Kittel)

Fundorte (Ergänzung):

c 3148/IV Ebw./Stadtgebiet: Kleingartenanlage im Schwärzetal nördlich Forstbotanischer Garten (2016: Schwabe)

\section{8 (8184) Idaea aversata (LiNNAEUs, 1758)}

Phänologie/Voltinismus: Nach der Jahrtausendwende zunehmend regelmäßig bivoltin: 2. Gen.: 29.X.(2013),
aFE, phänologische Verspätung 3d (korrigiert nach MüLLER 2015: in RicheRT 2014 war der 26.X.(2013) als aFE angegeben). Auch in den Jahren 2014, 2016 und 2018 wurden F einer (partiellen) 2. Gen. vom 06.-28.IX. beobachtet.

\section{9 (8187) Idaea straminata (BORKHAUSEN, 1794) = inornata HAWORTH, 1809}

Phänologie/Voltinismus: 08.VI.2015, phänologische Verfrühung 2d gegenüber bis 2013; 02.VI.(2016), erneute phänologische Verfrühung 6d, 01.VI.(2018) aFA, erneute phänologische Verfrühung 1d (insgesamt $9 \mathrm{~d}$ gegenüber bis 2013).

In der Regel bisher univoltin, neuerdings zunehmend bivoltin, so auch 2014:

02.X.(2014) ein frischer F a.L., aFE, phänologische Verspätung 10d gegenüber bis 2013.

\section{Neue Nachweise von bekannten Fundorten:}

b 3247/II Biesenthal/NSG Biesenthaler Becken: Nördlich der Pfauenwiesen (2015: Ockruck)

c 3147/I Zerpenschleuse/Ortsgebiet: Berliner Straße, im Garten (2014: Busse)

3147/II Finowfurt/Hirtenweg - Besters Fließ (1989, 1991: Kittel)

3148/III Ebw. OT Finow/Stadtgebiet: Altenhofer Straße, im Garten (2014-2018) und Umg./Forst Finowtal: Einflugschneise Flugplatz (2017)

g 3149/IV Niederfinow/Oderhänge - ehemalige Kiesgruben südwestlich des Schiffshebewerks $(2015,2016$ : Schwabe)

3150/III Gabow/Granitberggebiet (2015: Richert; 2018: Schwabe)

3150/IV Altglietzen Umg./Gabower Berge (2015)

Fundorte (Ergänzung):

b 3249/I Gersdorf Umg./Gamengrund südlich Teufelssee (2017: Schwabe)

c 3147/I Marienwerder Umg./Oder-Havel-Kanal nordwestlich des Ortes (2016: Busse \& Müller)

3147/IV Finowfurt Umg./Kesselmoor an der Finowfließniederung nordöstlich des Lehnssees $(2016,2017)$

3148/IV Ebw./Stadtgebiet: Kleingartenanlage im Schwärzetal nördlich des Forstbotanischen Gartens $(2014,2016$, 2017: Schwabe)

d 3047/III Groß Schönebeck/Ortsgebiet: Friedenstraße, im Garten (2018: Haase)

3147/II Finowfurt Umg./Üdersee - Südwestende (1973: Kittel)

e 3049/I Groß Ziethen Umg./Steingruben an den Ihlowbergen (2014)

f 3049/IV Brodowin/Krugberg (2014)

g 3149/II Liepe (1976, 1988: Kittel)

730 (8188) Idaea deversaria (HERRICH-SCHÄFFER, 1847)

Neue Nachweise von bekannten Fundorten:

c 3148/III Ebw. OT Finow/Stadtgebiet: Altenhofer Straße, im Garten $(2014,2015,2016)$ 
g 3150/III Gabow/Granitberggebiet (2015)

3150/IV Altglietzen Umg./Gabower Berge (2015)

Fundorte (Ergänzung):

c 3147/IV Finowfurt Umg./Kesselmoor an der Finowfließniederung nordöstlich des Lehnssees (2017)

e 3049/I Groß Ziethen Umg./Steingruben an den Ihlowbergen (2014)

\section{1 (8205) Rhodostrophia vibicaria (CLERCK, 1759)}

- RL Land Brandenburg: Vorwarnliste: TR;

- RL Deutschland (2011): Vorwarnliste.

Neue Nachweise von bekannten Fundorten:

c 3148/III Ebw. OT Finow/Stadtgebiet: Altenhofer Straße, im Garten (2016)

Fundorte (Ergänzung):

d 3047/III Groß Schönebeck Umg./Forst Groß Schönebeck: Revier Rehluch, Priesterberge (2017: Haase)

\section{Unterfamilie Larentiinae}

\section{2 (8221) Lythria purpuraria (LINNAEUs, 1758)}

- RL Land Brandenburg: Kategorie 2 (stark gefährdet): TR;

- RL Deutschland (2011): Vorwarnliste.

Phänologie/Voltinismus: Bivoltin; inzwischen liegt ein neuer Fund der bei uns nur wenig beobachteten 1. Gen. vor: 04.VI.(2014), aFA, phänologische Verfrühung 25d gegenüber bis 2013 .

Neue Nachweise von bekannten Fundorten:

c 3147/I Zerpenschleuse/Ortsgebiet: Berliner Straße (2014: Busse)

Fundorte (Ergänzung):

c 3147/II Marienwerder Umg./Langer Grund an der Straße nach Finowfurt (2014: Busse)

733 (8222) Lythria cruentaria (HUFNAGEL, 1767) = purpurata LiNNAEUs, 1761, nec. 1758

Neue Nachweise von bekannten Fundorten:

b 3148/II Trampe/ehemaliger TÜP $(2016,2018)$

c 3148/II? Eberswalde-Nordend/Oder-Havel-Kanal nördlich des Ortes (2015: Schwabe)

g 3150/I Oderberg/Geistberg (2014, 2016: Schwabe)

3150/III Gabow/Granitberggebiet (2014: Richert; 2015: Richert \& Schwabe)

3150/IV Altglietzen Umg./Gabower Berge (2017, 2018: Schwabe)

Fundorte (Ergänzung):

b 3148/IV Spechthausen Umg./Barnimer Heide: Stromtrasse südwestlich des Ortes (2018)
734 (8227) Phibalapteryx virgata (HufnAGEL, 1767)

- RL Land Brandenburg: Kategorie 2 (stark gefährdet): TR

- RL Deutschland (2011): Kategorie 2 (stark gefährdet). Phänologie/Voltinismus: Bivoltin, 1. Gen.: 22.IV.(2014), aFA, phänologische Verfrühung 6d gegenüber bis 2013. 04.IX.(2018) aFE, phänologische Verspätung 8d gegenüber bis 2013 .

Neue Nachweise von bekannten Fundorten:

g 3150/III Gabow/Oderhänge: Granitberggebiet (2014: Richert; 2015: Richert, Schwabe)

3150/IV Altglietzen Umg./Gabower Berge (2014: Richert; 2105: Richert, Schwabe, 2018: Schwabe)

737 (8240) Scotopteryx mucronata (Scopoli, 1763)

- RL Land Brandenburg: Kategorie 3 (gefährdet): CB;

- RL Deutschland 2011: Kategorie 2 (stark gefährdet). Im Gebiet verschollen. Letzte Nachweise 2003.

\section{1 (8248) Xanthorhoe biriviata (BORKHAUSEN,} 1794)

- RL Land Brandenburg: Kategorie 3 (gefährdet): MB. Phänologie/Voltinismus: 2. Gen.: 30.VIII.(2016), aFE, phänologische Verspätung 25d gegenüber bis 2013.

Neue Nachweise von bekannten Fundorten:

c 3148/III Ebw. OT Finow/Stadtgebiet: Altenhofer Straße, im Garten $(2016,2018)$

Fundorte (Ergänzung):

c $3147 / \mathrm{IV} \quad$ Finowfurt Umg./Finowfließniederung an der Neuen Brücke - Biesenthaler Str. (2014), Kesselmoor an der Finowfließniederung nordöstlich des Lehnssees (2016, 2017: Schwabe)

g 3150/I Oderberg/am Friedhof (2016: Schwabe)

742 (8249) Xanthorhoe designata (HufNAGEL, 1767)

Phänologie/Voltinismus: Bivoltin, 1. Gen.: 24.IV.(2014), aFA, phänologische Verfrühung 5d gegenüber bis 2013.

Neue Nachweise von bekannten Fundorten:

c 3146/ Kreuzbruch Umg./ehemalige Römerwegbrücke am Oder-Havel-Kanal östlich des Ortes (2014: Busse)

3147/I Zerpenschleuse/Ortsgebiet: Berliner Straße, im Garten (2014, 2016: Busse)

3147/II Finowfurt/Hirtenweg - Besters Fließ (1987, 1989, 1990, 1993, 1994: Kittel)

3148/III Ebw. OT Finow/Stadtgebiet: Altenhofer Straße, im Garten (2014)

Fundorte (Ergänzung):

b 3249/I Gersdorf Umg./Gamengrund südlich Teufelssee (2017: Richert \& Schwabe) 
c 3147/IV Marienwerder Umg./Kiesgrube südwestlich des Sportplatzes (2015); Finowfurt Umg./Finowfließniederung an der Neuen Brücke - Biesenthaler Str. (2014), Kesselmoor an der Finowfließniederung nordöstlich des Lehnssees (2016)

3148/IV Ebw./Stadtgebiet: Kleingartenanlage im Schwärzetal nördlich des Forstbotanischen Gartens (2014-2017: Schwabe)

g 3149/IV Niederfinow/Oderhänge - ehemalige Kiesgruben südwestlich des Schiffshebewerks (2016: Schwabe)

3150/IV Altglietzen Umg./Gabower Berge (2015)

\section{4 (8253) Xanthorhoe ferrugata (CLERCK, 1759)}

Phänologie/Voltinismus: In der Regel bivoltin; die Daten aus dem Jahre 2014 bestätigen die Vermutung, dass in günstigen Jahren (warmes Frühjahr) eine partielle 3. Gen. auftritt (vgl. RICHERT 2014): 02.-14.IX.2014 >20 F, nachdem am 09.VIII. letzte abgefl. F der 2. Gen. beobachtet worden waren.

\section{6 (8255) Xanthorhoe montanata (DENIS \& SCHIFFERMÜLLER, 1775)}

Neue Nachweise von bekannten Fundorten:

b 3247/II Biesenthal Umg./NSG Biesenthaler Becken: Langerönner Weg (2014: Ockruck)

3248/II Trampe/ehemaliger TÜP (2016: Busse \& Müller)

c 3147/I Zerpenschleuse/Ortsgebiet: Berliner Straße, im Garten (2014: Busse)

3147/II Finowfurt/Hirtenweg - Besters Fließ (1993: Kittel)

3149/III Ebw. Umg./Finowbruch östlich des Eichwerder (2015)

e 3149/II Liepe Umg./Forst Chorin: NSG Plagefenn - LedumMoor am Fuß der Plageberge (2018: Richert \& Schwabe)

Fundorte (Ergänzung):

c 3147/IV Finowfurt Umg./Kesselmoor an der Finowfließniederung nordöstlich des Lehnssees (2016)

3148/IV Ebw.-Westend/Unterheide: Drehnitzwiesen (1974: Kittel); Ebw./Stadtgebiet: Kleingartenanlage im Schwärzetal nördlich Forstbotanischer Garten (2016: Schwabe)

3149/III Stecherschleuse Umg./ehemaliges Kiesgrubengelände nördlich des Ortes (2017)

e 3049/II Albrechtshöhe Umg./Forst Grumsin: Lichtung nördlich der Waldstraße nach Grumsin (2014)

g 3149/IV Niederfinow/Oderhänge - ehemalige Kiesgruben südwestlich des Schiffshebewerks (2015: Schwabe)

3150/I Oderberg/Geistberg (2018: Schwabe)

3150/IV Altglietzen Umg./Gabower Berge (2015)

\section{7 (8256) Xanthorhoe fluctuata (LinNAeus, 1758)}

Phänologie/Voltinismus: Bivoltin; Flugzeitende 2. Gen.: 07.X.(2013), aFE, phänologische Verspätung 2d (korrigiert nach MüLler 2015; in Richert 2014 war der 05.X.(1966) als aFE angegeben).
Neue Nachweise von bekannten Fundorten:

c 3146/IV Kreuzbruch Umg./ehemalige Römerwegbrücke am Oder-Havel-Kanal östlich des Ortes (2014: Busse) (westlich angrenzendes Nachbargebiet)

3147/I Zerpenschleuse/Ortsgebiet: Berliner Straße, im Garten (2014-2017: Busse); Marienwerder Umg./ Oder-Havel-Kanal nordwestlich des Ortes (2014, 2016, 2018: Busse)

3148/III Ebw. OT Finow/Stadtgebiet: Altenhofer Straße, im Garten (2014, 2016, 2017, 2018)

3148/IV Ebw.-Westend/Unterheide: Wildparkstraße (1968, 1969, 1971, 1972, 1975: Kittel)

g 3149/II Liepe (1982: Kittel)

Fundorte (Ergänzung):

b 3247/II Biesenthal Umg./NSG Biesenthaler Becken: Langerönner Weg (2014: Ockruck)

3249/I Gersdorf Umg./Gamengrund südlich Teufelssee (2017: Richert \& Schwabe)

c 3148/IV Ebw./Stadtgebiet: Kleingartenanlage im Schwärzetal nördlich des Forstbotanischen Gartens (2014-2018: Schwabe)

d 3149/I Britz Umg./F. Britz: 1973: Kittel)

e 3049/III Chorin Umg./Forst Chorin: Südöstlich des Pferdebergs (MÜLLER 2015)

3049/IV Chorin Umg./Forst Chorin: Teerbrennerberge - Am Olberg (MüLler 2015), Kreuzung Denglerweg Olbergstraße (MüLler 2015)

g 3150/I Oderberg/Geistberg (2015, 2016: Schwabe)

\section{8 (8268) Catarrhoe rubidata (DENIs \& SCHIF- FERMÜLLER, 1775)}

- RL Land Brandenburg: Kategorie 3 (gefährdet): WS, NM, TR;

- RL Deutschland (2011): Vorwarnliste.

Phänologie/Voltinismus: Univoltin. Das angezweifelte Datum „08.VI.? die Herkunft des Datums in Richert 2004 ist nicht mehr zu ermitteln“; RICHERT 2014: 213, konnte durch ein Belegexemplar vom 08.VI.1973 in coll. Kittel und einen weiteren Falterfund am 08.VI.2014 bestätigt werden. In coll. Kittel befand sich ein weiterer Beleg vom 05.VI.1973. Dieses Funddatum wurde durch einen bisher übersehenen Falterfund am 05.VI.2008 erneut bestätigt. Somit ist der 05.VI.(1973, 2008) aFA, (korrigiert; in RICHERT 2014 war der 11.VI.(1998) als aFA angegeben).

Neue Nachweise von bekannten Fundorten:

c 3147/I Zerpenschleuse/Ortsgebiet: Berliner Straße, im Garten (2016: Busse)

3147/II Finowfurt/Hirtenweg - Besters Fließ (1995: Kittel)

3148/III Ebw. OT Finow/Stadtgebiet: Altenhofer Straße, im Garten (2014)

Fundorte (Ergänzung):

d 3149/I Britz Umg./F. Britz (1973: Kittel)

g 3150/I Oderberg/Geistberg (2017: Schwabe) 
749 (8269) Catarrhoe cuculata (DENIS \& SCHIFFERMÜLLER, 1775)

Am 24.VI.2016 registrierten Busse und Müller auf dem ehemaligen TÜP Trampe die ungewöhnlich hohe Zahl von $25 \mathrm{~F}$ a.L.

Neue Nachweise von bekannten Fundorten:

b 3248/II Trampe/ehemaliger TÜP (2016: Busse \& Müller)

c 3147/II Finowfurt/Hirtenweg - Besters Fließ (1989, 1991, 1995: Kittel)

3148/III Ebw. OT Finow/Stadtgebiet: Altenhofer Straße, im Garten (2014-2018)

g 3149/II Liepe (1973, 1978: Kittel)

3149/IV Niederfinow/Oderhänge - ehemalige Kiesgruben südwestlich des Schiffshebewerks (2016: Schwabe)

3150/I Oderberg/Teufelsberg (1973-1975, 1978: Kittel)

3150/III Gabow/Granitberggebiet (2015)

3150/IV Altglietzen Umg./Gabower Berge (2015)

Fundorte (Ergänzung):

c 3148/III Ebw. OT Finow Umg./Forst Finowtal: Einflugschneise Flugplatz (2017)

e 3049/I Groß Ziethen Umg./Steingruben an den Ihlowbergen (2014)

g 3150/I Oderberg/Geistberg (2017, 2018: Schwabe)

\section{1 (8275) Epirrhoe alternata (MüLLER, 1764)}

Ich beobachtete den F am Tag an Origanum vulgare, Dost, und mehrfach auch an Chrysanthemum (Tanacetum) vulgare, Rainfarn, saugend.

\section{2 (8277) Epirrhoe rivata (HüBNER, 1813)}

Neue Nachweise von bekannten Fundorten:

b 3248/II Trampe/ehemaliger TÜP (2016: Busse \& Müller)

Fundorte (Ergänzung):

c 3147/II Finowfurt/Hirtenweg - Besters Fließ 1995: Kittel)

3148/IV Ebw.-Westend/Unterheide: Wildparkstraße (1972, 1975, 1976: Kittel)

\section{4 (8257) Costaconvexa polygrammata (BoRK- HAUSEN, 1794)}

- RL Land Brandenburg: Kategorie 3 (gefährdet): TR NM, HF;

- RL Deuschland (2011): Vorwarnliste.

Am 11.VI. und 13.VIII.2015 hatte Schwabe je einen F der in unserem Gebiet nur sporadisch nachgewiesenen Art a.L.

Neue Nachweise von bekannten Fundorten:

g 3150/III Gabow/Granitberggebiet (2015: Schwabe) 3150/IV Altglietzen Umg./Gabower Berge (2015: Schwabe)
755 (8289) Camptogramma bilineata (LinNAEus, 1758)

Phänologie/Voltinismus: Im Jahre 2014 wurde der F von 27.V.-15.IX. beobachtet, 2015 vom 19.V.-14.IX., 2016 vom 25.V.-12.IX.: Die Art trat in diesen Jahren offensichtlich bivoltin auf.

\section{7a (8310) Anticlea derivata (DENIS \& SCHIF- FERMÜLLER, 1775)}

- RL Land Brandenburg: Kategorie R (extrem seltene Art/Art mit geographischer Restriktion): HE, GL, WS. Nach dem Erstnachweis im Gebiet im Jahre 2011 (RICHERT 2014) folgten drei Jahre, in denen mir keine weiteren Funde von hier bekannt wurden. Im Jahre 2015 wurde der F erneut beobachtet, u. a. im Stadtgebiet von Eberswalde OT Finow. Damit liegt ein Erstfund aus dem Naturraum Eberswalder Tal vor. 2016 und 2017 wurde der F auch in Groß Schönebeck beobachtet (Haase). Damit liegen Erstfunde aus dem Naturraum d (Schorfheide) vor.

Phänologie/Voltinismus: Die Angaben zur Flugzeit können präzisiert werden: Univoltin: 10.IV.(2011)19.V.(2017).

Fundorte (Ergänzung):

b 3148/IV Ebw.-Ostende/Freienwalder Straße am Waldfriedhof (2015: Schwabe)

c 3148/III Ebw. OT Finow/Stadtgebiet: Altenhofer Straße, im Garten (2015)

d 3047/III Groß Schönebeck/Ortsgebiet: Friedenstraße, im Garten (2016, 2017: Haase)

g 3150/I Oderberg/Geistberg (2015, 2016: Schwabe)

\section{8 (8312) Mesoleuca albicillata (LinnAeus, 1758)}

Phänologie/Voltinismus: Die neuerdings ziemlich regelmäßig beobachtete partielle 2. Gen. fliegt länger als bisher bekannt: 06.IX.(2013), aFE, phänologische Verspätung 17d (korrigiert nach MÜLLER 2015; in RICHERT 2014 war der 18.VIII.(2005) als aFE angegeben).

Neue Nachweise von bekannten Fundorten:

c 3147/II Finowfurt/Hirtenweg - Besters Fließ (1992: Kittel)

Fundorte (Ergänzung):

b 3249/I Gersdorf Umg./Gamengrund südlich Teufelssee (2017: Richert \& Schwabe)

c 3147/IV Finowfurt Umg./Finowfließniederung an der Neuen Brücke - Biesenthaler Str. (2014), Kesselmoor an der Finowfließniederung nordöstlich des Lehnssees (2016, 2017: Richert \& Schwabe)

3148/IV Ebw./Stadtgebiet: Kleingartenanlage im Schwärzetal nördlich Forstbotanischer Garten (2017: Schwabe)

d 3047/III Groß Schönebeck/Ortsgebiet: Friedenstraße, im Garten (2017: Haase)

e 3049/IV Chorin Umg./Forst Chorin: Teerbrennerberge - Am Olberg (2013: MÜLLER 2015), Kreuzung Denglerweg - Olbergstraße (2013: MÜLLER 2015)

g 3150/I Oderberg/Geistberg (2018: Schwabe) 


\section{9 (8314) Pelurga comitata (Linnaeus, 1758)}

Neue Nachweise von bekannten Fundorten:

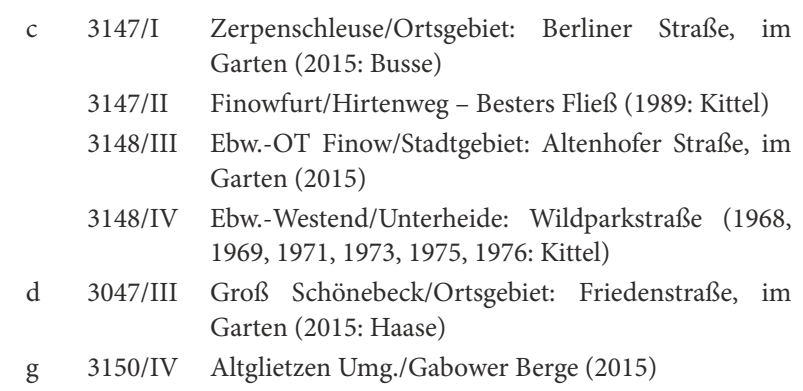

Fundorte (Ergänzung):

e 3049/I Groß Ziethen Umg./Steingruben an den Ihlowbergen (2014)

\section{0 (8316) Lampropteryx suffumata (DENIs \& SCHIFFERMÜLLER, 1775)}

\section{Neue Nachweise von bekannten Fundorten:}

c 3147/I Zerpenschleuse/Ortsgebiet: Berliner Straße, im Garten (2015: Busse)

Fundorte (Ergänzung):

c 3147/IV Finowfurt Umg./Kesselmoor an der Finowfließniederung nordöstlich des Lehnssees (2016)

3148/IV Ebw./Stadtgebiet: Kleingartenanlage im Schwärzetal nördlich Forstbotanischer Garten $(2016,2018$ : Schwabe)

e 3149/II Liepe Umg./Forst Chorin: NSG Plagefenn südlich Gr. Plagesee (2018: Richert, Schwabe \& Theimer)

\section{1 (8319) Cosmorhoe ocellata (LinNAEUs, 1758)}

Neue Nachweise von bekannten Fundorten:

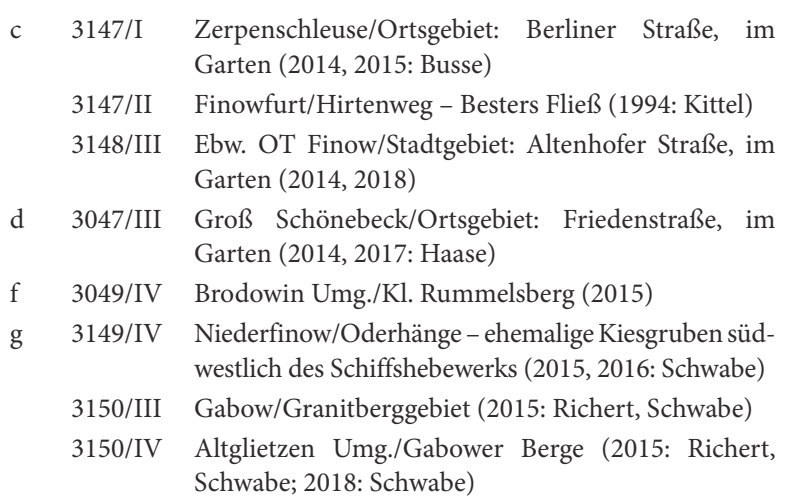

Fundorte (Ergänzung):

c 3147/IV Finowfurt Umg./Kesselmoor an der Finowfließniederung nordöstlich des Lehnssees $(2016,2017)$

3148/IV Ebw./Stadtgebiet: Kleingartenanlage im Schwärzetal nördlich Forstbotanischer Garten (2016: Schwabe)

e 3049/I Groß Ziethen Umg./Steingruben an den Ihlowbergen (2014)

g 3150/I Oderberg/Geistberg (2015, 2016, 2017, 2018: Schwabe)

\section{2 (8330) Eulithis prunata (LinNAEUs, 1758)}

Neue Nachweise von bekannten Fundorten:

c 3147/I Zerpenschleuse/Ortsgebiet: Berliner Straße, im Garten (2014, 2015: Busse)

3148/III Ebw. OT Finow/Stadtgebiet: Altenhofer Straße, im Garten $(2014,2016,2017,2018)$

g 3050/II Stolpe a.O./Trockenhänge am Stadtweg (2018)

3150/I Oderberg/Teufelsberg (1983: Kittel)

Fundorte (Ergänzung):

b 3247/II Biesenthal Umg./NSG Biesenthaler Becken: Weg nördlich der Pfauenwiesen (2014: Ockruck)

3248/II Trampe/ehemaliger TÜP (2016: Busse \& Müller)

c 3147/I Marienwerder Umg./Oder-Havel-Kanal nordwestlich des Ortes (2015: Busse)

3148/IV Ebw./Stadtgebiet: Kleingartenanlage im Schwärzetal nördlich des Forstbotanischen Gartens (2014, 2016 : Schwabe)

d 3047/III Groß Schönebeck/Ortsgebiet: Friedenstraße, im Garten (2016: Haase)

e 3049/I Groß Ziethen Umg./Steingruben an den Ihlowbergen (2014)

g 3150/I Oderberg/Geistberg (2018: Schwabe)

\section{4 (8332) Eulithis populata (LiNNAEUs, 1758)}

Neue Nachweise von bekannten Fundorten:

c 3147/I Zerpenschleuse/Ortsgebiet: Berliner Straße, im Garten (2014, 2016: Busse)

\section{5 (8334) Eulithis mellinata (FABRICIUS, 1787)}

Die Beobachtungslücke im Naturraum e (Uckermärker Kuppen- und Hügelland einschließlich Choriner Waldhügel, vgl. Richert 2004) wurde geschlossen.

Neue Nachweise von bekannten Fundorten:

c 3147/I Zerpenschleuse/Ortsgebiet: Berliner Straße, im Garten (2014: Busse)

3147/II Finowfurt/Hirtenweg - Besters Fließ (1992, 1995, 1997: Kittel)

3148/III Ebw. OT Finow/Stadtgebiet: Altenhofer Straße, im Garten (2014-2018)

d 3047/III Groß Schönebeck/Ortsgebiet: Friedenstraße, im Garten (2016: Haase)

g 3149/II Liepe (1973, 1975, 1977: Kittel)

3150/IV Altglietzen Umg./Gabower Berge (2015: Richert, Schwabe)

Fundorte (Ergänzung):

c 3148/IV Ebw.-Westend/Unterheide: Wildparkstraße (1971, 1972: Kittel)

d 3148/II Britz (1971, 1972: Kittel)

e 3049/I Groß Ziethen Umg./Steingruben an den Ihlowbergen (2014) 
766 (8335) Eulithis pyraliata (DENIS \& SCHIFFERMÜLLER, 1775)

Neue Nachweise von bekannten Fundorten:

b 3248/II Trampe/ehemaliger TÜP (2016: Busse \& Müller; 2018: Richert)

c 3147/I Zerpenschleuse/Ortsgebiet: Berliner Straße, im Garten (2014-2016: Busse)

3148/III Ebw. OT Finow/Stadtgebiet: Altenhofer Straße, im Garten (2015)

g 3050/II Stolpe a.O./Trockenhänge am Stadtweg (2018)

3149/II Liepe (1970: Kittel)

3150/I Oderberg/Teufelsberg (1970: Kittel)

3150/III Gabow/Granitberggebiet (2015: Schwabe)

3150/IV Altglietzen Umg./Gabower Berge (2015: Richert, Schwabe)

Fundorte (Ergänzung):

c 3147/II Finowfurt/Hirtenweg - Besters Fließ (1993, 1994: Kittel)

3148/IV Ebw.-Westend: Unterheide: Wildparkstraße (1975, 1976: Kittel)

d 3148/II Britz (1979: Kittel)

e 3049/I Groß Ziethen Umg./Steingruben an den Ihlowbergen (2014)

f 3050/III Bölkendorf Umg./NSG Tiefer See (2016)

g 3150/I Oderberg/Geistberg (2017, 2018: Schwabe)

\section{7 (8338) Ecliptopera silaceata (DENIS \& SCHIF- FERMÜLLER, 1775)}

Neue Nachweise von bekannten Fundorten:

c 3147/I Zerpenschleuse/Ortsgebiet: Berliner Straße, im Garten (2014, 2016, 2018: Busse)

3147/II Finowfurt/Hirtenweg - Besters Fließ (1983, 1987, 1991, 1995: Kittel)

3148/III Ebw.-OT Finow/Stadtgebiet: Altenhofer Straße, im Garten (2015-2018)

g 3149/II Liepe (1974, 1978, 1979: Kittel)

3149/IV Niederfinow/Oderhänge - ehemalige Kiesgruben südwestlich des Schiffshebewerks (2015: Schwabe)

3150/I Oderberg/Teufelsberg (1995: Kittel)

3150/IV Altglietzen Umg./Gabower Berge (2015: Schwabe)

Fundorte (Ergänzung):

a 3249/II Falkenberg Umg./Cöthener Forst: Klingendes Fließ unterhalb des Bismarck-Turmes (2014)

b 3249/I Gersdorf Umg./Gamengrund südlich Teufelssee (2017: Richert \& Schwabe)

c 3147/I Marienwerder Umg./Oder-Havel-Kanal nordwestlich des Ortes (2016: Busse)

3147/IV Finowfurt Umg./Finowfließniederung an der Neuen Brücke - Biesenthaler Straße (2014), Kesselmoor an der Finowfließniederung nordöstlich des Lehnssees (2016, 2017)

3148/III Ebw. OT Finow Umg./Forst Finowtal: Einflugschneise Flugplatz (2017)

3148/IV Ebw./Stadtgebiet: Kleingartenanlage im Schwärzetal nördlich des Forstbotanischen Gartens (2014, 2016 2017, 2018: Schwabe)

d 3047/III Groß Schönebeck/Ortsgebiet: Friedenstraße, im Garten (1999, 2014: Haase) e 3049/I Groß Ziethen Umg./Steingruben an den Ihlowbergen (2014)

\section{8 (8339) Ecliptopera capitata (HERRICH- SCHÄFFER, 1839)}

- RL Land Brandenburg: Kategorie 3 (gefährdet): MB. Phänologie/Voltinismus: Offenbar trat die Art im Jahre 2014 erneut bivoltin auf (vgl. RiCHERT 2014): Am 31.VII. wurden $5 \mathrm{z}$. T. frische $\mathrm{F}$ in der Finowfließniederung südwestlich von Finowfurt a.L. beobachtet.

Fundorte (Ergänzung):

c 3147/IV Marienwerder Umg./Kiesgrube südwestlich des Sportplatzes (2015); Finowfurt Umg./Finowfließniederung an der Neuen Brücke - Biesenthaler Straße (2014: Richert, Schwabe), Kesselmoor an der Finowfließniederung nordöstlich des Lehnssees (2016)

e 3149/II Liepe Umg./Forst Chorin: NSG Plagefenn - LedumMoor am Fuß der Plageberge (2014: Richert \& Schwabe)

\section{9 (8341) Chloroclysta siterata (HufNAGEL, 1767)}

Phänologie/Voltinismus: Univoltin; 27.VIII.(2014), aFA, phänologische Verfrühung 11d gegenüber bis 2013.

Neue Nachweise von bekannten Fundorten:

c 3147/I Zerpenschleuse/Ortsgebiet: Berliner Straße, im Garten (2014-2018: Busse)

3147/II Finowfurt/Hirtenweg - Besters Fließ (1990, 1995, 1996, 1997: Kittel)

3148/III Ebw. OT Finow/Stadtgebiet: Altenhofer Straße, im Garten (2014, 2015); Poststraße (2015)

d 3047/III Groß Schönebeck/Ortsgebiet: Friedenstraße, im Garten $(2014,2015,2017$ : Haase $)$

Fundorte (Ergänzung):

b 3149/III Sommerfelde Umg./ehemaliges Militärgelände südlich der Straße nach Eberswalde (2017: Richert \& Schwabe)

3247/II Biesenthal Umg./NSG Biesenthaler Becken: Langerönner Weg (2014: Ockruck)

c 3146/II Kreuzbruch Umg./ehemalige Römerwegbrücke am Oder-Havel-Kanal östlich des Ortes (2014: Busse) (westlich angrenzendes Nachbargebiet)

3147/I Marienwerder Umg./Oder-Havel-Kanal nordwestlich des Ortes (2016: Busse)

3147/IV Finowfurt Umg./Kesselmoor an der Finowfließniederung nordöstlich des Lehnssees (2016) Sophienstädt/Pregnitzfließniederung (2016: Busse \& Müller)

3148/IV Ebw.-Westend/Unterheide: Wildparkstraße (1997: Kittel); Ebw./Stadtgebiet: Kleingartenanlage im Schwärzetal nördlich des Forstbotanischen Gartens (2014-2018: Schwabe)

e 3049/III Chorin Umg./Forst Chorin: Südöstlich des Pferdebergs (2013: Müller 2015)

3049/IV Chorin Umg./Forst Chorin: Teerbrennerberge - Am Olberg (2013: MüLLER 2015), Kreuzung Denglerweg - Olbergstraße (2013: MÜLlER 2015) 


\section{0 (8343) Chloroclysta citrata (LinNAEUs, 1761)}

Fundorte (Ergänzung):

b 3247/II Biesenthal Umg./NSG Biesenthaler Becken: Langerönner Weg (2014: Ockruck)

c 3147/IV Finowfurt Umg./Kesselmoor an der Finowfließniederung nordöstlich des Lehnssees (2017: Richert \& Schwabe)

3148/IV Ebw./Stadtgebiet: Kleingartenanlage im Schwärzetal nördlich Forstbotanischer Garten (2017: Schwabe)

\section{2 (8350) Cidaria fulvata (DENIs \& SCHIFFER- MÜLLER, 1775)}

Neue Nachweise von bekannten Fundorten:

c 3147/I Zerpenschleuse/Ortsgebiet: Berliner Straße, im Garten (2018: Busse)

3147/II Finowfurt/Hirtenweg - Besters Fließ (1993: Kittel)

3148/III Ebw. OT Finow/Stadtgebiet: Altenhofer Straße, im Garten (2014, 2015, 2018)

g 3050/II Stolpe a.O./Trockenhänge am Stadtweg (2018)

3150/III Gabow/Granitberggebiet (2015: Schwabe)

3150/IV Altgliezen Umg./Gabower Berge (2015: Richert, Schwabe)

Fundorte (Ergänzung):

c 3148/IV Ebw./Stadtgebiet: Kleingartenanlage im Schwärzetal nördlich des Forstbotanischen Gartens (2014, 2016: Schwabe)

e 3149/II Liepe Umg./Forst Chorin: NSG Plagefenn - LedumMoor am Fuß der Plageberge (2018: Richert \& Schwabe)

g 3150/I Oderberg/Geistberg $(2017,2018:$ Schwabe $)$

\section{3 (8352) Plemyria rubiginata (DeNIs \& SCHIF- FERMÜLLER, 1775)}

Neue Nachweise von bekannten Fundorten:

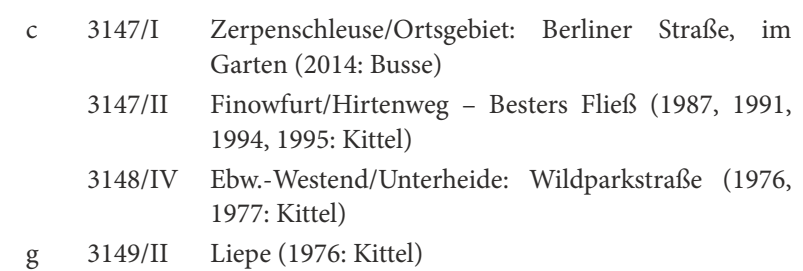

Fundorte (Ergänzung):

c 3147/IV Finowfurt Umg./Kesselmoor an der Finowfließniederung nordöstlich des Lehnssees $(2016,2017)$

3148/IV Ebw./Stadtgebiet: Stadtgebiet: Kleingartenanlage im Schwärzetal nördlich Forstbotanischer Garten (2016: Schwabe)

\section{4 (8354) Pennithera firmata (HüBNER, 1822)}

Neue Nachweise von bekannten Fundorten:

c 3147/I Zerpenschleuse/Ortsgebiet: Berliner Straße, im (2014-2017: Busse)

3147/II Finowfurt/Hirtenweg - Besters Fließ (1995: Kittel)
3148/III Ebw. OT Finow/Stadtgebiet: Altenhofer Straße, im Garten $(2014,2016,2017)$

d 3047/III Groß Schönebeck/Ortsgebiet: Friedenstraße, im Garten (2017, 2018: Haase)

f 3049/IV Brodowin/Krugberg (2014)

g 3149/IV Niederfinow/Oderhänge - ehemalige Kiesgruben südwestlich Schiffshebewerk (2016: Schwabe)

Fundorte (Ergänzung):

c 3147/II Marienwerder Umg./Oder-Havel-Kanal nordwestlich des Ortes (2014: Busse)

3147/IV Sophienstädt/Pregnitzfließniederung (2016: Busse \& Müller); Finowfurt Umg./Kesselmoor an der Finowfließniederung nordöstlich des Lehnssees (2017)

3148/IV Ebw./Stadtgebiet: Kleingartenanlage im Schwärzetal nördlich des Forstbotanischen Gartens (2014, 2017: Schwabe)

e 3049/IV Chorin Umg./Forst Chorin: Teerbrennerberge - Am Olberg (2013: MÜLlER 2015), Kreuzung Denglerweg - Olbergstraße (2013: MüLLER 2015)

\section{5 (8356) Thera obeliscata (HÜBNER, 1787)}

Die Beobachtungslücke im Naturraum h (Odertal, RICHERT 2004) wurde inzwischen geschlossen. Damit ist die häufige Art in allen Naturräumen des Gebietes nachgewiesen:

Phänologie/Voltinismus: Bivoltin; 1. Gen.: 29.IV.(2014), aFA, phänologische Verfrühung 5d gegenüber bis 2013.

\section{6 (8357) Thera variata (DENIs \& SCHIFFER- MÜLLER, 1775)}

Neue Nachweise von bekannten Fundorten:

c 3147/I Zerpenschleuse/Ortsgebiet: Berliner Straße, im Garten (2014, 2016, 2018: Busse)

3148/III Ebw. OT Finow/Stadtgebiet: Altenhofer Straße, im Garten $(2015,2016,2018)$

d 3047/III Groß Schönebeck/Ortsgebiet: Friedenstraße, im Garten (2017: Haase)

Fundorte (Ergänzung):

b 3247/II Biesenthal Umg./NSG Biesenthaler Becken: Langerönner Weg (2014: Ockruck)

c $3147 / \mathrm{IV}$ Finowfurt Umg./Kesselmoor an der Finowfließniederung nordöstlich des Lehnssees (2017: Schwabe)

3148/IV Ebw./Stadtgebiet: Kleingartenanlage im Schwärzetal nördlich des Forstbotanischen Gartens (2014, 2016 2017: Schwabe)

\section{8 (8362) Thera juniperata (LINNAEUs, 1758)}

Die Beobachtungslücke im Naturraum e (Uckermärker Kuppen- und Hügelland, RicherT 2004) wurde inzwischen geschlossen: Die Art wurde mehrfach im Gebiet der Teerbrennerberge im Forst Chorin auf Windwurfflächen nachgewiesen (MÜLLER 2015).

Phänologie/Voltinismus: Univoltin; 04.IX.(2013), aFA, phänologische Verfrühung 11d (korrigiert nach MüLLER 2015). Damit liegt auch für diese Art eine Veränderung 
der absoluten Flugzeit vor. In RICHERT 2014 war der 15.IX.(1967) als aFA angegeben.

Neue Nachweise von bekannten Fundorten:

c 3147/I Zerpenschleuse/Ortsgebiet: Berliner Straße, im Garten (2014; 2017: Busse)

3147/II Finowfurt/Hirtenweg - Besters Fließ (1986, 1988, 1990: Kittel)

3148/IV Ebw.-Westend/Unterheide: Wildparkstraße (1985, 1989, 1995, 1997: Kittel)

Fundorte (Ergänzung):

e 3049/IV Chorin Umg./Forst Chorin: Teerbrennerberge - am Olberg (2013: MüLLER 2015), Denglerweg - Kreuzung Olbergstraße (2013: MüLLER 2015)

\section{9 (8366) Eustroma reticulata (DeNIs \& SCHIF-} FERMÜLLER, 1775)

Siehe 3.3. Wieder entdeckte Arten.

\section{0 (8368) Electrophaes corylata (THUNBERG, 1792)}

Neue Nachweise von bekannten Fundorten:

c 3147/I Zerpenschleuse/Ortsgebiet: Berliner Straße, im Garten (2018: Busse)

3148/III Ebw. OT Finow/Stadtgebiet: Altenhofer Straße, im Garten $(2015,2016,2017,2018)$

3147/II Finowfurt/Hirtenweg - Besters Fließ (1986, 1990, 1991, 1995: Kittel)

e 3149/II Liepe Umg./Forst Chorin: NSG Plagefenn - LedumMoor am Fuß der Plageberge (2018; Richert \& Schwabe)

g 3150/I Oderberg/Teufelsberg $(1975,1978:$ Kittel $)$

Fundorte (Ergänzung):

c 3148/IV Ebw./Stadtgebiet: Kleingartenanlage im Schwärzetal nördlich des Forstbotanischen Gartens (2014: Schwabe)

d 3047/III Groß Schönebeck/Ortsgebiet: Friedenstraße, im Garten (2014: Haase)

\section{2 (8391) Hydriomena furcata (THUNBERG, 1784)}

Phänologie/Voltinismus: 22.V.(2014), aFA, phänologische Verfrühung 11d gegenüber bis 2013; 13.VIII.(1980), aFE, korrigiert nach Beleg in coll. Kittel; in RICHERT (2014) war der 11.VIII.(2010) als AFE angegeben.

Neue Nachweise von bekannten Fundorten:

\footnotetext{
b 3247/II Biesenthal Umg./NSG Biesenthaler Becken: Langerönner Weg (2014: Ockruck)

3248/II Trampe/ehemaliger TÜP (2016: Busse \& Müller)

c 3147/I Zerpenschleuse/Ortsgebiet: Berliner Straße, im Garten (2014, 2018: Busse)

3147/II Finowfurt/Hirtenweg - Besters Fließ (1988: Kittel)

d 3047/III Groß Schönebeck/Ortsgebiet: Friedenstraße (2014: Haase)
}

Fundorte (Ergänzung):

c 3147/I Marienwerder Umg./Oder-Havel-Kanal nordwestlich des Ortes (2015, 2016: Busse)

3148/IV Ebw.-Nordend/ehemalige Gehörlosenhilfsschule auf dem Gelände der Landesklinik (1983: Kittel)

e 3049/I Grumsin (1980: Kittel); Groß Ziethen Umg./Steingruben an den Ihlowbergen (2014)

783 (8392) Hydriomena impluviata (DENIs \& SCHIFFERMÜLLER, 1775) = coerulata $($ FABRICIUS 1777)

Neue Nachweise von bekannten Fundorten:

b 3248/II Trampe/ehemaliger TÜP (2016: Busse \& Müller)

c 3147/I Zerpenschleuse/Ortsgebiet: Berliner Straße, im Garten (2014, 2015, 2018: Busse)

3147/II Finowfurt/Hirtenweg - Besters Fließ (1990, 1991, 1994, 1995: Kittel)

3148/III Ebw. OT Finow/Stadtgebiet: Altenhofer Straße, im Garten $(2014,2016,2018)$

d 3148/II Britz (1978: Kittel)

e 3149/II Liepe Umg./Forst Chorin: NSG Plagefenn - LedumMoor am Fuß der Plageberge (2018: Richert \& Schwabe)

g 3149/II Liepe (1982: Kittel)

Fundorte (Ergänzung):

b 3249/I Trampe/Ortsgebiet (1970: Kittel)

3247/II Biesenthal Umg./NSG Biesenthaler Becken: Langerönner Weg (2014: Ockruck)

c 3147/I Marienwerder Umg./Oder-Havel-Kanal nordwestlich des Ortes (2014, 2015: Busse)

3147/IV Marienwerder/Steinlaake (2015) und Umg./Kiesgrube südwestlich des Sportplatzes (2015); Finowfurt Umg./Kesselmoor an der Finowfließniederung nordöstlich des Lehnssees $(2016,2017)$

3148/IV Ebw./Stadtgebiet: Kleingartenanlage im Schwärzetal nördlich Forstbotanischer Garten (2016: Schwabe)

\section{4 (8400) Horisme vitalbata (DENIS \& SCHIFFER- MÜLLER, 1775)}

- RL Land Brandenburg: Kategorie D (Daten defizitär: HE.

Im Gebiet verschollen (Einzelfund 2003). Trotz gezielter Suche in geeigneten potenziellen Habitaten wurde die Art nicht mehr gefunden. Wahrscheinlich gehörte sie nie zum Artenbestand des Gebietes.

\section{5 (8401) Horisme corticata (TREITSCHKE, 1835)}

Neue Nachweise von bekannten Fundorten:

c 3147/I Zerpenschleuse/Ortsgebiet: Berliner Straße, im Garten (2014: Busse)

3148/III Ebw. OT Finow/Stadtgebiet: Altenhofer Straße, im Garten (2014-2018)

3148/IV Ebw.-Westend/Unterheide: Wildparkstraße (1975: Kittel)

d 3047/III Groß Schönebeck/Ortsgebiet: Friedenstraße, im Garten (2014: Haase) 


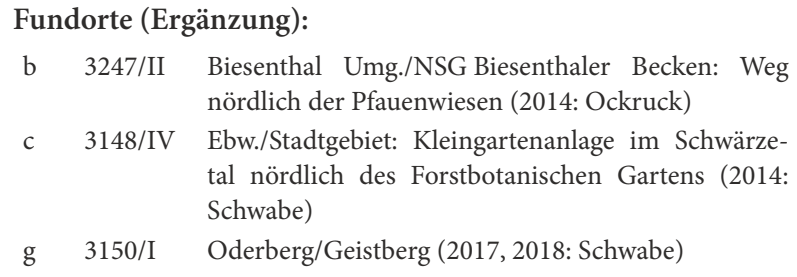

786 (8402) Horisme tersata (DENIS \& SCHIFFERMÜLLER, 1775)

- RL Land Brandenburg: Kategorie R (extrem seltene Art oder Art mit geographischer Restriktion): HE.

Am 19.V.2017 hatte Haase im Ortsgebiet von Groß Schönebeck in seinem Garten einen F a.L. Damit liegt ein erster Nachweis im Naturraum d (Schorfheide) vor. Die Ausbreitungstendenz der ehemals nur von den Oderhängen bekannten Art setzt sich offenbar fort (vgl. Richert 2010: 115).

Phänologie/Voltinismus: Die Art wird im Gebiet neuerdings bivoltin beobachtet. Es ist allerdings nicht sicher, ob dies durch Vorverlagerung der Flugzeit verursacht wird oder ob wegen der Seltenheit in früherer Zeit nicht genügend Daten für eine richtige Beurteilung des Voltinismus vorlagen: 19.V.(1917) phänologischer Verfrühung 31d gegenüber bis 2013; 11.V.(2018) aFA, erneute phänologischer Verfrühung 8d (insgesamt 39d gegenüber bis 2013).

Fundorte (Ergänzung):

c 3148/III Ebw. OT Finow/Stadtgebiet: Altenhofer Straße, im Garten (11.V., 25. und 31.VII.2018: det. genit., Richert)

3148/IV Ebw./Stadtgebiet: Kleingartenanlage im Schwärzetal nördlich Forstbotanischer Garten (2018: det. genit., Schwabe

d 3047/III Groß Schönebeck/Ortsgebiet: Friedenstraße, im Garten (11.V.2017: Haase)

\section{8 (8411) Melanthia procellata (DENIS \& SCHIF- FERMÜLLER, 1775)}

Neue Nachweise von bekannten Fundorten:

c 3148/III Ebw. OT Finow/Stadtgebiet: Altenhofer Straße, im Garten $(2014,2018)$

g 3150/I Oderberg/Teufelsberg (1975, 1977, 1978: Kittel)

Fundorte (Ergänzung):

a 3249/II Falkenberg Umg./Cöthener Forst: Klingendes Fließ unterhalb vom Bismarck-Turm (2014)

c 3148/IV Ebw./Stadtgebiet: Kleingartenanlage im Schwärzetal nördlich des Forstbotanischen Gartens (2014, 2016, 2018: Schwabe)

\section{9 (8414) Pareulype berberata (DenIs \& SCHIF-} FERMÜLLER, 1775)

- RL Land Brandenburg: Vorwarnliste: WS, HE, LW. Phänologie/Voltinismus: Bivoltin; 1. Gen.: 24.IV.(2014), aFA, phänologische Verfrühung 5d gegenüber bis 2013.
2. Gen.: 18.VII.(2014), phänologische Verfrühung 6d gegenüber bis 2013; 10.VII.(2016), aFA, erneute phänologische Verfrühung 2. Gen. insgesamt $8 \mathrm{~d}$ gegenüber bis 2013.

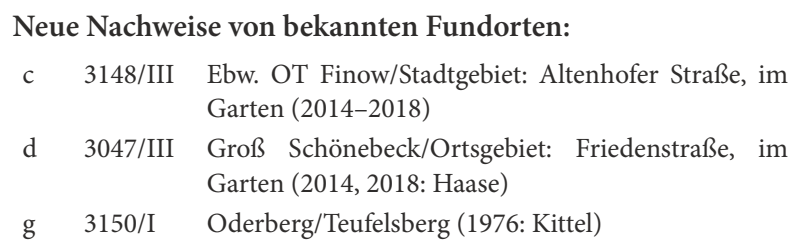

Fundorte (Ergänzung):

c 3148/IV Ebw./Stadtgebiet: Kleingartenanlage im Schwärzetal nördlich des Forstbotanischen Gartens (2014-2016: Schwabe)

g 3149/II Liepe (1979: Kittel)

\section{2 (8421) Rheumaptera cervinalis (SCOPOLI,} 1763)

- RL Land Brandenburg: Vorwarnliste: WS, HE, LW.

Neue Nachweise von bekannten Fundorten:

c 3148/IV Ebw./Stadtgebiet: Kleingartenanlage im Schwärzetal nördlich Forstbotanischer Garten (2015: Schwabe)

d 3047/III Groß Schönebeck/Ortsgebiet: Friedenstraße, im Garten (2014, 2017: Haase)

\section{3 (8423) Rheumaptera undulata (LiNNAEUs, 1758)}

Die R fand Schwabe A (09.) IX in der Eberswalder Oberheide auf einer breiten Schneise an Populus tremula, Espe.

\section{Neue Nachweise von bekannten Fundorten:}

b 3247/II Biesenthal Umg./NSG Biesenthaler Becken: Langerönner Weg (2014: Ockruck)

c 3147/I Zerpenschleuse/Ortsgebiet: Berliner Straße, im Garten (2018: Busse)

3147/II Finowfurt/Hirtenweg - Besters Fließ (1986, 1988, 1993, 1997: Kittel)

3148/III Ebw. OT Finow/Stadtgebiet: Altenhofer Straße, im Garten (2015) und Umg./Forst Finowtal: Einflugschneise Flugplatz (2016)

Fundorte (Ergänzung):

c 3147/IV Finowfurt Umg./Kesselmoor an der Finowfließniederung nordöstlich des Lehnssees (2016, 2017: Richert \& Schwabe)

3148/IV Ebw.-Westend/Unterheide: Wildparkstraße (1969, 1973: Kittel)

d 3147/II Finowfurt Umg./Üdersee: Südwest-Ende (1974: Kittel)

\section{5 (8432) Philereme vetulata (Denis \& SCHIF-} FERMÜLLER, 1775)

Phänologie/Voltinismus: 09.VI.(2014), phänologische Verfrühung 1d; 30.V.(2018) aFA, erneute phänologischer Verfrühung 10d, insgesamt 11d gegenüber bis 2013. 


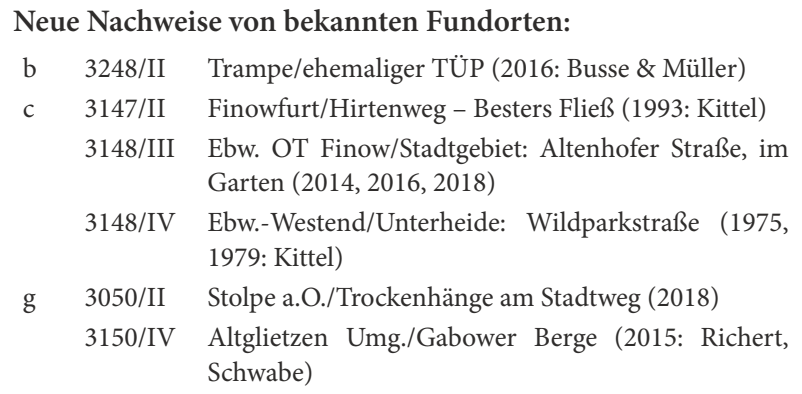

Fundorte (Ergänzung):

c 3148/IV Ebw./Stadtgebiet: Kleingartenanlage im Schwärzetal nördlich Forstbotanischer Garten (2016: Schwabe)

e 3049/I Groß Ziethen Umg./Steingruben an den Ihlowbergen (2014)

g 3150/I Oderberg/Geistberg (2018: Schwabe)

\section{6 (8433) Philereme transversata (HufNAgEL, 1767)}

Phänologie/Voltinismus: 03.VI.(2018), aFA, phänologische Verfrühung 7d gegenüber bis 2013.

Neue Nachweise von bekannten Fundorten:

b 3248/II Trampe/ehemaliger TÜP (2016: Busse \& Müller)

c 3147/II Finowfurt/Hirtenweg - Besters Fließ (1990, 1992, 1993: Kittel)

3148/III Ebw. OT Finow/Stadtgebiet: Altenhofer Straße, im Garten (2016)

g 3150/I Oderberg/Teufelsberg (1973, 1975, 1994: Kittel)

Fundorte (Ergänzung):

b 3249/I Gersdorf Umg./Gamengrund südlich Teufelssee (2017: Schwabe)

c 3147/IV Finowfurt Umg./Kesselmoor an der Finowfließniederung nordöstlich des Lehnssees (2017: Schwabe)

3148/IV Ebw.-Westend/Unterheide: Wildparkstraße (1971, 1974, 1977: Kittel); Ebw./Stadtgebiet: Kleingartenanlage im Schwärzetal nördlich Forstbotanischer Garten (2016: Schwabe)

d 3149/I Britz Umg./F. Britz (1971: Kittel)

g 3150/I Oderberg/Geistberg (2018: Schwabe)

\section{7 (8435) Euphyia biangulata (HAwORTH, 1809) = picata HÜBNER, 1813}

- RL Land Brandenburg: Kategorie 2 (stark gefährdet):

Habitatbindung ungeklärt.

Phänologie/Voltinismus: Die wenigen Daten aus dem Gebiet lassen zurzeit nur den Schluss zu, dass die Art bei uns univoltin ist; 04.IX.(2014), aFE, phänologische Verspätung 18d gegenüber bis 2013.

Neue Nachweise von bekannten Fundorten:

c 3148/III Ebw. OT Finow/Stadtgebiet: Altenhofer Straße, im Garten (2017)
Fundorte (Ergänzung):

c 3148/IV Ebw./Stadtgebiet: Kleingartenanlage im Schwärzetal nördlich des Forstbotanischen Gartens (2014, 2017: Schwabe)

\section{9 (8442) Epirrita dilutata (DENIS \& SCHIFFER- MÜLLER, 1775)}

Müller (2015) verzeichnete die Art in großer Zahl von Chorin Umg. aus Buchenwäldern im Forst Chorin, MTB 3049. Das von Müller vorgelegte Material enthielt mit Sicherheit E. christyi. Da durch Genitaldetermination sicher bestimmte Belege von dilutata fehlen, bleibt die Angabe für diese Art unsicher.

Neue Nachweise von bekannten Fundorten:

c 3148/III Ebw. OT Finow/Stadtgebiet: Altenhofer Straße, im Garten (2014)

Fundorte (Ergänzung):

c 3148/IV Ebw./Stadtgebiet: Kleingartenanlage im Schwärzetal nördlich des Forstbotanischen Gartens (2014: Schwabe)

\section{0 (8443) Epirrita christyi (ALlEN, 1906)}

Siehe die Ausführungen bei $E$. dilutata bezüglich der Angaben von MülLER 2015. Da aus dem MTB 3049 bereits sichere Nachweise vorliegen (RICHERT 2004) und eine detaillierte Fundortzuordnung des Materials von Müller nicht mehr möglich ist, wird hier auf eine Fundortangabe verzichtet.

\section{1 (8444) Epirrita autumnata (BORKHAUSEN 1794)}

Neue Nachweise von bekannten Fundorten:

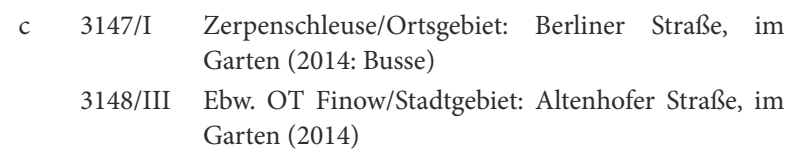

\section{2 (8447) Operophthera brumata (LINNAEUs, 1758)}

E XI 2017 beobachtete ich erstmals einen männlichen F am Rotweinköder. Unklar ist, ob der F dort zufällig war oder trotz rückgebildeten Rüssels vom Köder angezogen worden war.

803 (8448) Operophthera fagata (SCHARFENBERG, 1805)

Fundorte (Ergänzung):

c 3148/IV Ebw.-Westend/Unterheide: Wildparkstraße (1972, 1973: Kittel)

d 3047/III Groß Schönebeck/Ortsgebiet: Am Bahnhof (2016: Haase) 
e 3049/III Chorin Umg./Forst Chorin: Südwestlich des Pferdebergs (2013: MüLLER 2015)

3049/IV Chorin Umg./Forst Chorin: Teerbrennerberge - am Olberg (2013: MüLLER 2015), Denglerweg - Kreuzung Olbergstraße (2013: MüLLER 2015)

\section{4 (8456) Perizoma alchemillata (Linnaeus, 1775)}

Im Jahre 2014 trat der F ungewöhnlich h in Zerpenschleuse auf: Busse registrierte in seiner Lichtfalle in der Zeit vom 03.VII. - 14.VIII. insgesamt $342 \mathrm{~F}$, einen weiteren F noch sehr spät am 09.X.

Phänologie/Voltinismus: Die bisher in der Regel univoltine Art bildete offensichtlich auch 2014 eine partielle 2. Gen. im September/Oktober. Diese Tatsache wird zunehmend häufiger beobachtet: 13.IX.1967, 18.IX.2006， 11.IX.2012，22.X.2013，09.X.2014， 23.IX. 2016.

\section{6 (8462) Perizoma blandiata (Denis \& SCHIF- FERMÜLLER, 1775)}

- RL Land Brandenburg: Kategorie 1 (vom Aussterben bedroht): MW;

- RL Deutschland (2011): Kategorie 2 (stark gefährdet).

Ältere Funde (Belege in coll. Kittel) der im Gebiet seit 1977 verschollenen Art (korrigiert nach Beleg in coll. Kittel; in Richert 2010 war 1973 angegeben worden) werden ergänzend nachgetragen.

Neue Nachweise von bekannten Fundorten:

c 3148/IV Ebw.-Westend/Unterheide: Wildparkstraße (1975, 1976, 1977: Kittel)

g 3148/II Britz (1973: Kittel)

\section{7 (8463) Perizoma albulata (Denis \& SCHIF- FERMÜLLER, 1775)}

- RL Land Brandenburg: Kategorie 1 (vom Aussterben bedroht): MW;

- RL Deutschland (2011): Vorwarnliste.

Im Gebiet verschollen seit 2000. Gezielte Suche an ehemaligen Fundplätzen war vergeblich. Ältere Funde (Belege in coll. Kittel) werden ergänzend nachgetragen.

Phänologie/Voltinismus: 12.VIII.(1974), aFE (korrigiert nach Beleg in coll. Kittel; in RicherT 2014 war der 07.VIII.(1972) als aFE angegeben).

\section{Neue Nachweise von bekannten Fundorten:}

c 3148/IV Ebw.-Westend/Unterheide: Wildparkstraße (1974, 1975: Kittel)

g 3149/II Liepe (1973, 1975: Kittel)

\section{8 (8464) Perizoma flavofasciata (Thunberg,} 1792)

Neue Nachweise von bekannten Fundorten:

b 3248/II Trampe/ehemaliger TÜP (2016: Busse \& Müller)

c 3147/II Finowfurt/Hirtenweg-Besters Fließ (1994: Kittel)

3148/III Ebw. OT Finow/Stadtgebiet: Altenhofer Straße, im Garten $(2014,2015)$

g 3149/II Liepe (1974, 1975: Kittel)

3150/IV Altglietzen Umg./Gabower Berge (2015)

Fundorte (Ergänzung):

c 3148/IV Ebw./Stadtgebiet: Kleingartenanlage im Schwärzetal nördlich Forstbotanischer Garten (2016: Schwabe)

d 3047/III Groß Schönebeck/Ortsgebiet: Friedenstraße, im Garten (1999: Haase)

g 3050/II Stolpe a.O./Trockenhänge am Stadtweg (2018)

3159/I Oderberg/Geistberg (2018: Schwabe)

h 3150/II Hohensaaten/Deponiegelände an der Straße nach der Siedlung (2014: Kleße)

\section{9 (8465) Perizoma didymata (Linnaeus, 1758)}

Ein mir bisher nicht bekannter Fund im Hammerbruch bei Zerpenschleuse, leg. Haase, wird nachgetragen.

Phänologie/Voltinismus: Univoltin; 20.VI.(1999), aFA, (korrigiert nach Beleg in coll. Haase; in RicherT war der 28.VI.(1999) als aFA angegeben).

Neue Nachweise von bekannten Fundorten:

c 3148/IV Ebw.-Westend/Unterheide: Wildparkstraße (1972, 1974, 1975, 1976: Kittel)

Fundorte (Ergänzung):

c 3146/II Zerpenschleuse Umg./Hammerbruch (1999: Haase) (westlich angrenzendes Nachbargebiet)

\section{0 (8468) Perizoma sagittata (FABRICIUS, 1787)}

- RL Land Brandenburg: Kategorie 2 (stark gefährdet): NM, MW, FA-WS;

- RL Deutschland 2011: Kategorie 2 (stark gefährdet). Im Gebiet verschollen seit 2000. Gezielte Suche nach der Art verlief ergebnislos.

\section{2 (8475) Eupithecia tenuiata (HüBNER, 1913)}

Die Beobachtungslücke im Naturraum e (Uckermärker Kuppen- und Hügelland einschließlich Choriner Waldhügel, RicheRT 2004) wurde inzwischen geschlossen.

Neue Nachweise von bekannten Fundorten:

c 3148/III Ebw. OT Finow/Stadtgebiet: Altenhofer Straße, im Garten $(2015,2016)$

Fundorte (Ergänzung):

c 3148/IV Ebw.-Westend/Unterheide: Wildparkstraße (1974: Kittel) 
e 3049/I Groß Ziethen Umg./Steingruben an den Ihlowbergen (2014)

\section{3 (8476) Eupithecia inturbata (HüBNER, 1817)}

Die Art tritt zunehmend verbreitet im Gebiet auf. Sie wurde inzwischen auch im Naturraum e (Uckermärker Kuppen- und Hügelland) nachgewiesen (vgl. RicherT 2004, 2010, 2014).

Phänologie/Voltinismus: Univoltin; 06.VII.(2014), aFA, phänologische Verfrühung 3d gegenüber bis 2013.

Neue Nachweise von bekannten Fundorten:

c 3148/III Ebw. OT Finow/Stadtgebiet: Altenhofer Straße, im Garten $(2014,2015,2016,2017,2018)$

Fundorte (Ergänzung):

c 3148/IV Ebw./Stadtgebiet: Kleingartenanlage im Schwärzetal nördlich des Forstbotanischen Gartens (2014: Schwabe)

e 3149/II Liepe Umg./Forst Chorin: NSG Plagefenn - LedumMoor am Fuß der Plageberge (2018: Richert \& Schwabe)

\section{4 (8477) Eupithecia haworthiata (DouBLEDAY,} 1856)

Fundorte (Ergänzung):

c 3148/IV Ebw.-Westend/Unterheide: Wildparkstraße (1979: Kittel); Ebw./Stadtgebiet: Kleingartenanlage im Schwärzetal nördlich Forstbotanischer Garten (2015 1 Männchen a.L. leg. et det. genit. Schwabe, Päp. Nr. 26/2016)

g 3150/I Oderberg/Geistberg (2018: Schwabe)

\section{6 (8479) Eupithecia plumbeolata (HAWORTH, 1809)}

Neue Nachweise von bekannten Fundorten:

c 3147/II Finowfurt/Hirtenweg - Besters Fließ (1986, 1990, 1994: Kittel)

3148/III Ebw. OT Finow/Stadtgebiet: Altenhofer Straße, im Garten (2016, 2017, 2018)

g 3050/II Stolpe a.O./Trockenhänge am Stadtweg (2018)

3150/I Oderberg/Teufelsberg (1975: Kittel)

Fundorte (Ergänzung):

c 3147/IV Finowfurt Umg./Kesselmoor an der Finowfließniederung nordöstlich des Lehnssees (2017)

3148/IV Ebw./Stadtgebiet: Kleingartenanlage im Schwärzetal nördlich Forstbotanischer Garten (2016: Schwabe)

d 3249/I Britz Umg./F. Britz (1979, 1980: Kittel)

\section{7 (8481) Eupithecia abietaria (GoEZE, 1781)} = pini RETZIUS, 1783

Fundorte (Ergänzung):

c 3147/I Marienwerder Umg./Oder-Havel-Kanal nordwestlich des Ortes (2016: Busse)
3147/IV Finowfurt Umg./Kesselmoor an der Finowfließniederung nordöstlich des Lehnssees (2017)

3148/IV Ebw./Stadtgebiet: Kleingartenanlage im Schwärzetal nördlich des Forstbotanischen Gartens (2014, 2016 : Schwabe)

819 (8483) Eupithecia linariata (DENIS \& SCHIFFERMÜLLER, 1775)

Neue Nachweise von bekannten Fundorten:

c 3147/I Zerpenschleuse/Ortsgebiet: Berliner Straße, im Garten (2014-2016: Busse)

3148/III Ebw. OT Finow/Stadtgebiet: Altenhofer Straße, im Garten $(2014,2015,2017,2018)$

g 3150/IV Altglietzen Umg./Gabower Berge (2015: Richert, Schwabe)

Fundorte (Ergänzung):

c 3147/I Marienwerder Umg./Oder-Havel-Kanal nordwestlich des Ortes (2016: Busse \& Müller)

3147/IV Finowfurt Umg./Finowfließniederung an der Neuen Brücke - Biesenthaler Str. (2014)

3148/III Ebw. OT Finow Umg./Forst Finowtal: Einflugschneise Flugplatz (2017)

d 3047/III Groß Schönebeck/Ortsgebiet: Friedenstraße, im Garten (1999, 2014: Haase)

\section{1 (8491) Eupithecia exiguata (HüBNER, 1813)}

Fundorte (Ergänzung):

c 3148/IV Ebw./Stadtgebiet: Kleingartenanlage im Schwärzetal nördlich des Forstbotanischen Gartens (2018: Schwabe)

\section{2 (8494) Eupithecia valerianata (HÜBNER, 1813)}

- RL Land Brandenburg: Kategorie 2 (stark gefährdet): HF.

Phänologie/Voltinismus: Univoltin, 08.VI.(2014), aFA, phänologische Verfrühung 3d gegenüber bis 2013.

Neue Nachweise von bekannten Fundorten:

c 3148/III Ebw. OT Finow/Stadtgebiet: Altenhofer Straße, im Garten (2014)

Fundorte (Ergänzung):

c 3147/IV Finowfurt Umg./Kesselmoor an der Finowfließniederung nordöstlich des Lehnssees (2016: Schwabe)

e 3049/I Groß Ziethen Umg./Steingruben an den Ihlowbergen (2014)

g 3150/IV Altglietzen Umg./Gabower Berge (2015)

\section{4 (8502) Eupithecia venosata (FABRICIUS, 1787)}

- RL Land Brandenburg: Kategorie 3 (gefährdet): TR;

- RL Deutschland (2011): Vorwarnliste. 


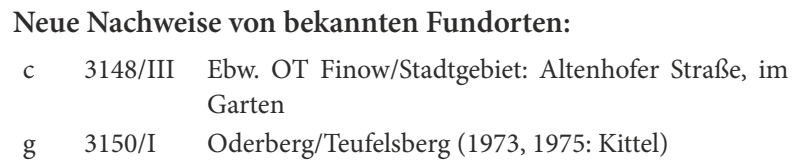

Fundorte (Ergänzung):

g 3159/I Oderberg/Geistberg (2018: Schwabe)

3150/IV Altglietzen Umg./Gabower Berge (2015)

\section{5 (8507) Eupithecia egenaria HERRICH-SCHÄF- FER, 1848}

Phänologie/Voltinismus: Univoltin, 06.V.(2014), aFA, phänologische Verfrühung 3d bis 2013.

Neue Nachweise von bekannten Fundorten:

c 3148/III Ebw. OT Finow/Stadtgebiet: Altenhofer Straße, im Garten $(2014,2016,2017,2018)$

Fundorte (Ergänzung):

c 3138/IV Ebw./Stadtgebiet: Kleingartenanlage im Schwärzetal nördlich Forstbotanischer Garten (2016, 2018: Schwabe)

\section{6 (8509) Eupithecia centaureata (DeNIs \& SCHIFFERMÜLLER, 1775)}

\section{Neue Nachweise von bekannten Fundorten:}

b 3247/II Biesenthal Umg./NSG Biesenthaler Becken: Langerönner Weg (2014: Ockruck)

c 3147/I Zerpenschleuse/Ortsgebiet: Berliner Straße, im Garten (2014: Busse)

3148/III Ebw. OT Finow/Stadtgebiet: Altenhofer Straße, im Garten (2014, 2015, 2017, 2018) und Umg./Forst Finowtal: Einflugschneise Flugplatz (2017)

g 3150/III Gabow/Granitberggebiet (2015: Richert, Schwabe)

3150/IV Altglietzen Umg./Gabower Berge (2015: Richert, Schwabe; 2018: Schwabe)

Fundorte (Ergänzung):

b 3249/I Gersdorf Umg./Gamengrund südlich Teufelssee (2017: Richert \& Schwabe)

c 3137/II Marienwerder Umg./Oder-Havel-Kanal nordwestlich des Ortes (2014: Busse)

3148/IV Ebw./Stadtgebiet: Kleingartenanlage im Schwärzetal nördlich Forstbotanischer Garten (2018: Schwabe)

g 3150/I Oderberg/Geistberg (2016, 2017, 2018: Schwabe)

\section{7 (8516) Eupithecia selinata HERRICH-SCHÄF- FER, 1848}

- RL Land Brandenburg: Vorwarnliste: MB, WS.

Phänologie/Voltinismus: 13.V.(2018) aFA, phänologische Verfrühung 6d gegenüber bis 2013.

Neue Nachweise von bekannten Fundorten:

c 3148/III Ebw. OT Finow/Stadtgebiet: Altenhofer Straße, im Garten $(2014,2018)$ e 3149/II Liepe Umg./Forst Chorin: NSG Plagefenn - LedumMoor am Fuß der Plageberge (2018: Richert \& Schwabe)

Fundorte (Ergänzung):

b 3249/I Gersdorf Umg./Gamengrund südlich Teufelssee (2017: Richert \& Schwabe)

c 3147/IV Finowfurt Umg./Finowfließniederung an der Neuen Brücke - Biesenthaler Str. (2014), Moor an der Finowfließniederung nordöstlich des Lehnssees (2016: Schwabe)

3148/III Ebw. OT Finow Umg./Forst Finowtal: Einflugschneise Flugplatz (2017)

3148/IV Ebw./Stadtgebiet: Ruhlaer Straße (2004: Schwabe) und Kleingartenanlage im Schwärzetal nördlich des Forstbotanischen Gartens (2014, 2016, 2018 : Schwabe)

g 3150/I Oderberg/Geistberg (2018: Schwabe)

\section{9 (8519) Eupithecia intricata (ZETTERSTEDT,} 1839)

Neue Nachweise von bekannten Fundorten:

c $3147 / \mathrm{IV} \quad$ Marienwerder/Steinlaake (2015)

3148/III Ebw. OT Finow/Stadtgebiet: Altenhofer Straße, im Garten $(2014,2015)$

3148/IV Ebw:-Westend/Unterheide: Wildparkstraße (19721974: Kittel)

d 3047/III Groß Schönebeck/Ortsgebiet: Friedenstraße, im Garten (2016: Haase)

Fundorte (Ergänzung):

c 3148/IV Ebw./Stadtgebiet: Kleingartenanlage im Schwärzetal nördlich des Forstbotanischen Gartens (2014 Schwabe)

\section{1 (8527) Eupithecia absinthiata (CLERCK, 1759)}

Neue Nachweise von bekannten Fundorten:

c 3147/II Finowfurt/Hirtenweg - Besters Fließ (1993: Kittel)

3148/III Ebw. OT Finow/Stadtgebiet: Altenhofer Straße, im Garten $(2016,2018)$

g 3150/I Oderberg/Teufelsberg (1975: Kittel)

3150/V Altglietzen Umg./Gabower Berge (2015: Richert \& Schwabe)

Fundorte (Ergänzung):

b 3247/II Biesentahl Umg./NSG Biesenthaler Becken: Weg nördlich der Pfauenwiesen (2014: Busse)

c 3147/I Marienwerder Umg./Oder-Havel-Kanal nordwestlich des Ortes (2016: Busse \& Müller)

3147/IV Finowfurt Umg./Kesselmoor an der Finowfließniederung nordöstlich des Lehnssees (2017)

3148/III Ebw. OT Finow Umg./Forst Finowtal: Einflugschneise Flugplatz (2017: Richert \& Schwabe)

3148/IV Ebw./Stadtgebiet: Kleingartenanlage im Schwärzetal nördlich des Forstbotanischen Gartens (2014, 2016 , 2017: Schwabe)

3149/III Sommerfelde Umg./Finowbruch nördlich des Ortes, am Bahnübergang (2008: Schwabe) 


\section{3 (8531) Eupithecia assimilata Doubleday, 1856}

Der F wurde überwiegend a.L. beobachtet (vgl. RICHERT 2004), in der 2. Gen. selten auch a.K.

Phänologie/Voltinismus: Bivoltin, 2. Gen.: 04.IX.(2014), aFE, phänologische Verspätung $4 \mathrm{~d}$.

Neue Nachweise von bekannten Fundorten:

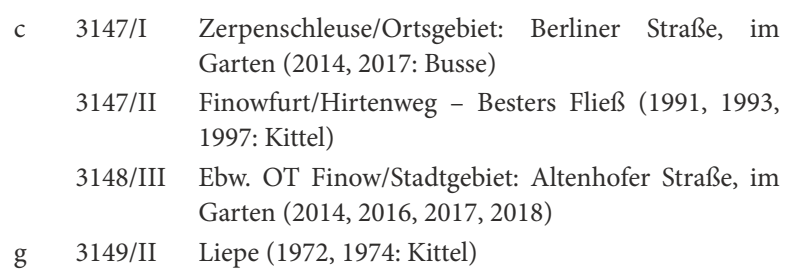

Fundorte (Ergänzung):

a 3249/II Falkenberg Umg./Cöthener Forst: Am Klingenden Fließ unterhalb des Bismarck-Turmes (2014)

c 3147/IV Marienwerder/Steinlaake (2015: Schwabe)

3148/III Ebw. OT Finow Umg./Forst Finowtal: Einflugschneise Flugplatz (2017)

3148/IV Ebw./Stadtgebiet: Kleingartenanlage im Schwärzetal nördlich des Forstbotanischen Gartens (2014, 2017: Schwabe)

3149/IV Niederfinow/Oderhänge - ehemalige Kiesgruben südwestlich des Schiffshebewerks (2015: Schwabe)

g 3150/I Oderberg/Teufelsberg (1974: Kittel), Geistberg (2017: Schwabe)

\section{4 (8534) Eupithecia vulgata (HAWORTH, 1809)}

Neue Nachweise von bekannten Fundorten:

c 3148/III Ebw. OT Finow/Stadtgebiet: Altenhofer Straße, im Garten (2014)

g 3150/I Oderberg/Teufelsberg (1975: Kittel)

Fundorte (Ergänzung):

c 3148/IV Ebw.-Westend/Unterheide: Wildparkstraße (19711973, 1975: Kittel)

g 3149/II Niederfinow/Oderhänge - ehemalige Kiesgruben südwestlich des Schiffshebewerks (2015: Schwabe)

\section{5 (8535) Eupithecia tripunctaria HERRICH- SCHÄFFER, 1852}

Neue Nachweise von bekannten Fundorten:

c 3148/III Ebw. OT Finow/Stadtgebiet: Altenhofer Straße, im Garten $(2014,2015)$

e 3149/II Liepe Umg./Forst Chorin: NSG Plagefenn - LedumMoor am Fuß der Plageberge (2018: Schwabe)

Fundorte (Ergänzung):

e 3049/I Groß Ziethen Umg./Steingruben an den Ihlowbergen (2014: Richert, Schwabe)

\section{6 (8536) Eupithecia denotata (HüBNER, 1813)}

- RL Land Brandenburg: Kategorie 1 (vom Aussterben bedroht): WS.

Gezielte Suche nach Raupen der seit 1991 im Gebiet verschollenen Art bei Oderberg im Jahre 2016 verlief wieder erfolglos (vgl. RICHERT 2014: 236).

\section{8 (8538) Eupithecia icterata (DE VILLERS 1789)}

Neue Nachweise von bekannten Fundorten:

b 3247/II Biesenthal Umg./NSG Biesenthaler Becken: Langerönner Weg (2014: Ockruck)

c 3147/I Zerpenschleuse/Ortsgebiet: Berliner Straße, im Garten (2014-2016: Busse)

3147/II Finowfurt/Hirtenweg - Besters Fließ (1989, 1991: Kittel)

3148/III Ebw. OT Finow/Stadtgebiet: Altenhofer Straße, im Garten (2014-2017) und Umg./Forst Finowtal: Einflugschneise Flugplatz (2017: Richert \& Schwabe)

d 3047/III Groß Schönebeck/Ortsgebiet: Friedenstraße, im Garten (2014, 2015: Haase)

g 3149/II Liepe (1975: Kittel)

3149/IV Niederfinow/Oderhänge - ehemalige Kiesgruben südwestlich des Schiffshebewerks (2015, 2016: Schwabe)

3150/IV Altglietzen Umg./Gabower Berge (2015)

Fundorte (Ergänzung):

b 3249/I Gersdorf Umg./Gamengrund südlich Teufelssee (2017: Richert \& Schwabe)

c 3147/II Marienwerder Umg./Oder-Havel-Kanal nordwestlich des Ortes (2014: Busse)

3147/IV Finowfurt Umg./Finowfließniederung an der Neuen Brücke - Biesenthaler Str. (2014)

3148/IV Ebw./Stadtgebiet: Kleingartenanlage im Schwärzetal nördlich Forstbotanischer Garten (2016, 2017: Schwabe)

d 3149/I Britz Umg./F. Britz (1973: Kittel)

f 4049/IV Brodowin/Krugberg (2014)

g 3150/I Oderberg/am Friedhof (2016: Schwabe)

\section{9 (8539) Eupithecia succenturiata (DENIs \&} SCHIFFERMÜLLER, 1775)

Die Beobachtungslücke im Naturraum a (Oberbarnimer Waldhügel, RicherT 2004) konnte inzwischen geschlossen werden. Damit ist die Art in allen Naturräumen des Gebietes nachgewiesen.

Neue Nachweise von bekannten Fundorten:

b 3248/II Trampe/ehemaliger TÜP (2016: Busse \& Müller)

c 3147/I Zerpenschleuse/Ortsgebiet: Berliner Straße, im Garten (2014: Busse)

3147/II Finowfurt/Hirtenweg - Besters Fließ (1994: Kittel)

3148/III Ebw. OT Finow/Stadtgebiet: Altenhofer Straße, im Garten (2014-2016, 2018) und Umg./Forst Finowtal: Einflugschneise Flugplatz (2017)

d 3047/III Groß Schönebeck/Ortsgebiet: Friedenstraße, im Garten (2014, 2015: Haase) 
3149/IV Niederfinow/Oderhänge - ehemalige Kiesgruben südwestlich des Schiffshebewerks (2015: Schwabe)

g 3149/II Liepe (1975: Kittel)

Fundorte (Ergänzung):

a 3249/II Falkenberg Umg./Cöthener Forst: Klingendes Fließ unterhalb des Bismarck-Turmes (2014)

d 3148/II Britz (1979: Kittel)

e 3049/I Groß Ziethen Umg./Steingruben an den Ihlowbergen (2014: Richert, Schwabe)

\section{0 (8546) Eupithecia subumbrata (DENIs \& SCHIFFERMÜLLER, 1775)}

Neue Nachweise von bekannten Fundorten:

b 3248/II Trampe/ehemaliger TÜP (2016: Busse \& Müller)

c 3147/I Zerpenschleuse/Ortsgebiet: Berliner Straße, im Garten (2014-2016: Busse)

3147/II Finowfurt/Hirtenweg - Besters Fließ (1986, 1991, 1995, 1997: Kittel)

g 3149/II Liepe (1975: Kittel)

3150/I Oderberg/Teufelsberg $(1978,1983:$ Kittel $)$

3150/III Gabow/Granitberggebiet (2015)

3150/IV Altglietzen Umg./Gabower Berge (2015)

Fundorte (Ergänzung):

c 3247/I Marienwerder Umg./Oder-Havel-Kanal nordwestlich des Ortes (2016: Busse)

g 3050/II Stolpe a.O./Trockenhänge am Stadtweg (2018)

3150/I Oderberg/Geistberg (2018: Schwabe)

\section{1 (8551) Eupithecia millefoliata RössLER, 1866}

- RL Deutschland (2011): Kategorie 3 (gefährdet). Ein älterer Fund (Beleg in coll. Kittel) wird ergänzend nachgetragen.

Fundorte (Ergänzung):

c 3147/II Finowfurt/Hirtenweg - Besters Fließ (1991: Kittel)

\section{2 (8553) Eupithecia simpliciata (HAWORTH, 1809) = subnotata HÜBNER, 1813}

Ein älterer Fund (Beleg in coll. Kittel) wird ergänzend nachgetragen.

Fundorte (Ergänzung):

c 3148/III Ebw. OT Finow/Stadtgebiet: Altenhofer Straße, im Garten (2018)

3148/IV Ebw.-Westend/Unterheide: Wildparkstraße (1993: Kittel)

\section{3 (8557) Eupithecia sinuosaria (EvERSMANN, 1848)}

Im Gebiet verschollen. Seit 2003 liegen keine Nachweise mehr vor, obwohl ehemals von ihr besiedelte Plätze weiter faunistisch untersucht wurden.

\section{4 (8565) Eupithecia indigata (HüBNER, 1813)}

Phänologie/Voltinismus: Univoltin, 21.IV.(2014), aFA, phänologische Verfrühung 2d gegenüber bis 2013.

Neue Nachweise von bekannten Fundorten:

c 3146/IV Kreuzbruch Umg./ehemalige Römerwegbrücke am Oder-Havel-Kanal östlich des Ortes (2012, 2013: Busse) (westlich angrenzendes Nachbargebiet)

3147/I Zerpenschleuse/Ortsgebiet: Berliner Straße, im Garten (2014, 2016, 2018: Busse)

3147/II Finowfurt/Hirtenweg - Besters Fließ (1988, 1992, 1993, 1995, 1996, 1997: Kittel)

3148/III Ebw. OT Finow/Stadtgebiet: Altenhofer Straße, im Garten $(2014,2016,2017,2018)$

3148/IV Ebw.-Westend/Unterheide: Wildparkstraße (1975, 1976, 1979: Kittel)

Fundorte (Ergänzung):

c 3148/IV Ebw./Stadtgebiet: Kleingartenanlage im Schwärzeta nördlich Forstbotanischer Garten (Schwabe: 2018)

\section{5 (8567) Eupithecia pimpinellata (HüBNER, 1813)}

- RL Deuschland (2011): Vorwarnliste.

Ältere Funde (Belege in coll. Kittel) werden ergänzend nachgetragen.

Fundorte (Ergänzung):

c 3147/II Finowfurt/Hirtenweg - Besters Fließ (1997: Kittel)

3148/IV Ebw.-Westend/Unterheide: Wildparkstraße (1993: Kittel)

\section{7 (8570) Eupithecia nanata (HÜBNER, 1813)}

Fundorte (Ergänzung):

c 3147/IV Finowfurt Umg./Finowfließniederung an der Neuen Brücke - Biesenthaler Str. (2014)

3148/III Ebw. OT Finow Umg./Forst Finowtal: Einflugschneise Flugplatz (2017: Richert \& Schwabe)

3148/IV Ebw.-Westend/Unterheide: Wildparkstraße (1975, 1976: Kittel)

g 3150/I Oderberg/Teufelsberg (1974: Kittel)

\section{8 (8573) Eupithecia innotata (HufNAGEL, 1767)}

Neue Nachweise von bekannten Fundorten:

c 3147/I Zerpenschleuse/Ortsgebiet: Berliner Straße, im Garten (2014-2016, 2018: Busse)

3147/II Finowfurt/Hirtenweg - Besters Fließ (1986, 1988 1990, 1992, 1995: Kittel)

3148/III Ebw. OT Finow/Stadtgebiet: Altenhofer Straße, im Garten (2014-2016, 2018)

g 3149/II Liepe (1974: Kittel)

3150/III Gabow/Granitberggebiet (2015)

3150/IV Altglietzen Umg./Gabower Berge (2015: Richert \& Schwabe; 2017: Schwabe) 
Fundorte (Ergänzung):

c 3147/IV Finowfurt Umg./Finowfließniederung an der Neuen Brücke - Biesenthaler Str. (2014); Kesselmoor an der Finowfließniederung nordöstlich des Lehnssees (2016)

3148/IV Ebw./Stadtgebiet: Kleingartenanlage im Schwärzetal nördlich Forstbotanischer Garten (2016, 2017: Schwabe)

g 3150/I Oderberg/Geistberg (2016, 2017: Schwabe)

\section{9 (8574) Eupithecia ochridata PINKER, 1968}

- RL Deutschland (2011): Kategorie D (Daten unzureichend).

Neue Nachweise erlauben eine weitere Präzisierung der phänologischen Daten.

Phänologie/Voltinismus: Bivoltin; 1. Gen.: 23.V.2014, aFE, phänologische Verspätung 3d gegenüber bis 2013.

Neue Nachweise von bekannten Fundorten:

b 3148/IV Ebw. Umg./Oberheide (2008: Schwabe. det. genit.)

c 3148/III Ebw. OT Finow/Stadtgebiet: Altenhofer Straße, im Garten (2014, det. genit.)

3148/IV Ebw./Stadtgebiet: Forstbotanischer Garten (2010: Schwabe, det. genit./Lepiforum), Kleingartenanlage im Schwärzetal nördlich Forstbotanischer Garten (2015: Schwabe, det. genit.)

\section{0 (8577) Eupithecia virgaureata DoubledAY,} 1861

Die Beobachtungslücke im Naturraum f (Parsteiner Becken, Richert 2004, 2010) wurde inzwischen geschlossen. Damit ist die Art in allen Naturräumen des Gebietes nachgewiesen.

Phänologie/Voltinismus: Bivoltin, 1. Gen.: 07.IV.(2014), aFA, phänologische Verfrühung 8d gegenüber bis 2013 .

2. Gen.: 15.IX.(2014), aFE, phänologische Verspätung $6 \mathrm{~d}$ gegenüber bis 2013 .

Neue Nachweise von bekannten Fundorten:

$\begin{array}{ccl}\text { c 3147/I } & \begin{array}{l}\text { Zerpenschleuse/Ortsgebiet: Berliner Straße, im } \\ \text { Garten }(2014,2018: \text { Busse })\end{array} \\ & 3148 / \mathrm{III} & \begin{array}{l}\text { Ebw. OT Finow/Stadtgebiet: Altenhofer Straße, im } \\ \text { Garten }(2014-2016,2018)\end{array} \\ & & \text { Oderberg/Geistberg (2016: Schwabe) }\end{array}$

Fundorte (Ergänzung):

c 3147/II Finowfurt/Hirtenweg - Besters Fließ (1989: Kittel)

3148/IV Ebw./Stadtgebiet: Kleingartenanlage im Schwärzetal nördlich Forstbotanischer Garten (2014, 2015, 2017: Schwabe)

d 3148/I Werbellin Umg./NSG Buckowseerinne: Kanzelberggebiet (2013: Ockruck)

f 3049/IV Brodowin/Krugberg (2014)

\section{1 (8578) Eupithecia abbreviata STEPHENs, 1831}

Die Art zeigt eine deutliche Häufigkeitszunahme im Gebiet. Bei einem LF im NSG Plagefenn am 19.IV.2018 flog die Art i.M. an: Ich zählte $>50 \mathrm{~F}$.

Neue Nachweise von bekannten Fundorten:

c 3146/IV Kreuzbruch Umg./ehemalige Römerwegbrücke am Oder-Havel-Kanal östlich des Ortes (2014: Busse) (westlich angrenzendes Nachbargebiet)

3147/I Zerpenschleuse/Ortsgebiet: Berliner Straße, im Garten (2014-2016, 2018: Busse)

3148/III Ebw. OT Finow/Stadtgebiet: Altenhofer Straße, im Garten (2014-2018: det. genit. Richert)

Fundorte (Ergänzung):

c 3148/IV Ebw./Stadtgebiet: Kleingartenanlage im Schwärzetal nördlich Forstbotanischer Garten (2014-2018: Schwabe)

e 3149/II Liepe Umg./Forst Chorin: NSG Plagefenn südlich Gr. Plagesee (2018: Richert, Schwabe, Theimer)

\section{2 (8579) Eupithecia dodoneata GuENEE, 1757}

Neue Nachweise von bekannten Fundorten:

c 3147/II Finowfurt/Hirtenweg - Besters Fließ (1993, 1994, 1995: Kittel)

3148/III Ebw. OT Finow/Stadtgebiet: Altenhofer Straße, im Garten (2015; 2017: det. genit. Richert, 2018)

Fundorte (Ergänzung):

c 3148/IV Ebw./Stadtgebiet: Kleingartenanlage im Schwärzetal nördlich Forstbotanischer Garten (2016: Schwabe: det. genit.)

g 3150/I Oderberg/Geistberg (2017: Schwabe)

\section{3 (8583) Eupithecia pusillata (DeNIs \& SCHIF-} FERMÜLLER, 1775) = sobrinata HÜBNER, 1817

Neue Nachweise von bekannten Fundorten:

c 3148/III Ebw. OT Finow/Stadtgebiet: Altenhofer Straße, im Garten $(2014,2018)$

3148/IV Ebw.-Westend/Unterheide: Wildparkstraße (1973: Kittel)

Fundorte (Ergänzung):

c 3148/IV Ebw./Stadtgebiet: Kleingartenanlage im Schwärzetal nördlich Forstbotanischer Garten (2017: Schwabe)

\section{4 (8592) Eupithecia lanceata (HüBNER, 1825)}

Phänologie/Voltinismus: 28.III.(2016), aFA, phänologische Verfrühung 1d gegenüber bis 2013.

Neue Nachweise von bekannten Fundorten:

c 3147/I Zerpenschleuse/Ortsgebiet: Berliner Straße, im Garten (2016: Busse) 
Fundorte (Ergänzung):

c $\quad 3147 / \mathrm{IV} \quad$ Finowfurt Umg./Kesselmoor an der Finowfließniederung nordöstlich des Lehnssees (2016)

3148/IV Ebw./Stadtgebiet: Kleingartenanlage im Schwärzetal nördlich Forstbotanischer Garten (2017, 2018: Schwabe)

b 3148/IV Spechthausen Umg./Barnimer Heide: Stromtrasse südwestlich des Ortes (2015)

e 3149/II Liepe Umg./Forst Chorin: NSG Plagefenn südlich Gr. Plagesee (2018)

\section{5 (8595) Eupithecia lariciata (FrEYeR, 1842)}

Phänologie/Voltinismus: Die Art gilt als univoltin (Forster \& Wohlfahrt 1981, Koch 1984, Mironov 2003); zumindest jw wird aber bei uns eine partielle 2. Gen. ausgebildet, die nach der Jahrtausendwende mit Sicherheit nachgewiesen wurde.

1. Gen.: 30.IV.(2014), aFA, phänologische Verfrühung $14 \mathrm{~d}$ gegenüber bis 2013 .

\section{Neue Nachweise von bekannten Fundorten:}

e 3149/II Liepe Umg./Forst Chorin: NSG Plagefenn - LedumMoor am Fuß der Plageberge (2018: Richert \& Schwabe)

Fundorte (Ergänzung):

c 3148/IV Ebw./Stadtgebiet: Kleingartenanlage im Schwärzetal nördlich Forstbotanischer Garten (2014: Schwabe)

g 3150/III Gabow/Granitberggebiet (2015: Richert, Schwabe)

\section{6 (8596) Eupithecia tantillaria BoISDUvAL, 1840}

Neue Nachweise von bekannten Fundorten:

b 3247/II Biesenthal Umg./NSG Biesenthaler Becken: Langerönner Weg (2014: Ockruck)

c 3147/I Zerpenschleuse/Ortsgebiet: Berliner Straße, im Garten (2015, 2018: Busse)

3147/II Finowfurt/Hirtenweg - Besters Fließ (1983, 1993., 1994, 1995: Kittel)

3147/IV Marienwerder/Steinlaake (2015), Kiesgrube südwestlich des Sportplatzes (2015)

3148/III Ebw. OT Finow/Stadtgebiet: Altenhofer Straße, im Garten (2014-2018)

Fundorte (Ergänzung):

b 3149/III Eberswalde-Südende Umg./Hohenfinower Wald: Östliche Leuenberger Wiese (2014)

c 3147/IV Finowurt Umg./Kesselmoor an der Finowfließniederung nordöstlich des Lehnssees (2016)

3148/IV Ebw./Stadtgebiet: Kleingartenanlage im Schwärzetal nördlich Forstbotanischer Garten (2014, 2016, 2017, 2018: Schwabe),

d 3148/II Britz (1978: Kittel)

g 3149/II Liepe (1976: Kittel)

\section{7 (8599) Gymnoscelis rufifasciata (HAWORTH,} 1809) = pumilata HÜBNER, 1813

Phänologie/Voltinismus: Bivoltin, 1. Gen.: 02.IV.(2014), aFA, phänologische Verfrühung 1d gegenüber bis 2013.

Neue Nachweise von bekannten Fundorten:

c 3147/I Zerpenschleuse/Ortsgebiet: Berliner Straße, im Garten (2014, 2015, 2016: Busse)

3148/III Ebw. OT Finow/Stadtgebiet: Altenhofer Straße, im Garten (2014-2018) und Umgeb./Forst Finowtal: Einflugschneise Flugplatz (2017)

d 3047/III Groß Schönebeck/Ortsgebiet: Friedenstraße, im Garten (2018: Haase)

e $\quad 3149 /$ II Liepe Umg./Forst Chorin: NSG Plagefenn - LedumMoor am Fuß der Plageberge (2018: Richert \& Schwabe)

g 3150/III Gabow/Granitberggebiet (2015: Schwabe)

3150/IV Altglietzen Umg./Gabower Berge (2015: Richert; 2018: Schwabe)

Fundorte (Ergänzung):

a 3249/II Falkenberg Umg./Cöthener Forst: Klingendes Fließ unterhalb des Bismarck-Turmes (2014)

b 3249/I Gersdorf Umg./Gamengrund südlich Teufelssee (2017: Richert \& Schwabe)

c 3147/II Finowfurt/Hirtenweg - Besters Fließ (1990, 1993: Kittel)

3147/IV Finofurt Umg./Kesselmoor an der Finowfließniederung nordöstlich des Lehnssees $(2016,2017)$

3148/IV Ebw./Stadtgebiet: Kleingartenanlage im Schwärzetal nördlich Forstbotanischer Garten (2014, 2015, 2017, 2018: Schwabe)

g 3150/I Oderberg/Geistberg (2015, 2018: Schwabe)

\section{8 (8601) Chloroclystis v-ata (HAWORTH, 1809) = coronata $\mathrm{HÜBNER}, 1813$}

Ich beobachtete den F nachts beim Saugen an Blüten von Buddleja davidii, Chinesischer Sommerflieder.

Die R trug Haase M (18.)VI 1999 mit Blüten von Sambucus niger, Schwarzer Holunder, ein; F e.l. 19.VII.1999. Phänologie/Voltinismus: Bivoltin, 1. Gen.: 20.IV.2014, aFA, phänologische Verfrühung 7d gegenüber bis 2013.

Neue Nachweise von bekannten Fundorten:

c: 3146/IV Kreuzbruch Umg./ehemalige Römerwegbrücke am Oder-Havel-Kanal östlich des Ortes (2014: Busse) (westlich angrenzendes Nachbargebiet)

3147/I Zerpenschleuse/Ortsgebiet: Berliner Straße, im Garten (2014-2016, 2018: Busse)

3147/II Finowfurt/Hirtenweg - Besters Fließ (1991, 1994, 1997: Kittel)

3148/III Ebw. OT Finow/Stadtgebiet: Altenhofer Straße, im Garten (2014-2018)

d 3047/III Groß Schönebeck/Ortsgebiet: Friedenstraße, im Garten (2018: Haase)

g 3150/I Oderberg/Teufelsberg (1973, 1975: Kittel)

3150/III Gabow (1983: Kittel) 
Fundorte (Ergänzung):

c 3147/I Zerpenschleuse Umg./Langer Trödel (1999: Haase)

3147/IV Marienwerder/Steinlaake (2015: Schwabe); Finowfurt Umg./Kesselmoor an der Finowfließniederung nordöstlich des Lehnssees (2017)

3148/IV Ebw./Stadtgebiet: Kleingartenanlage im Schwärzetal nördlich Forstbotanischer Garten $(2014,2016,2017$, 2018: Schwabe)

e 3049/I Groß Ziethen Umg./Steingruben an den Ihlowbergen (2014)

g 3150/I Oderberg/Geistberg (2017: Schwabe)

\section{0 (8604) Rhinoprora chloerata (MABILLE, 1870)}

- RL Land Brandenburg: Kategorie 3 (gefährdet): HE.

Neue Nachweise von bekannten Fundorten:

c 3148/III Ebw. OT Finow/Stadtgebiet: Altenhofer Straße, im Garten (2014)

Fundorte (Ergänzung):

c 3148/IV Ebw./Stadtgebiet: Kleingartenanlage im Schwärzetal nördlich Forstbotanischer Garten (2014: Schwabe)

e 3049/I Gr. Ziethen Umg./Steingruben an den Ihlowbergen (2015); Neugrimnitz Umg./am Weg nach Gr. Ziethen (2015)

\section{1 (8605) Rhinoprora debiliata (HüBNER, 1817)}

Phänologie/Voltinismus: Univoltin, 08.VI.(2014) aFA, phänologische Verfrühung 13d gegenüber bis 2013.

Neue Nachweise von bekannten Fundorten:

c 3146/IV Kreuzbruch Umg./ehemalige Römerwegbrücke am Oder-Havel-Kanal östlich des Ortes (2014: Busse) (westlich angrenzendes Nachbargebiet)

Fundorte (Ergänzung):

c 3148/IV Ebw./Stadtgebiet: Kleingartenanlage im Schwärzetal nördlich Forstbotanischer Garten (2018: Schwabe)

\section{2 (8607) Anticollix sparsata (TreitschKe,} 1818)

- RL Land Brandenburg: Kategorie 3 (gefährdet): NM, $\mathrm{MB}$

Fundorte (Ergänzung):

c 3147/IV Finowfurt Umg./Finowfließniederung an der Neuen Brücke - Biesenthaler Str. (2014: Richert, Schwabe); Kesselmoor an der Finowfließniederung nordöstlich des Lehnssees (2016, 2017: Richert \& Schwabe)

3148/IV Ebw./Stadtgebiet: Kleingartenanlage im Schwärzetal nördlich Forstbotanischer Garten (2016, 2018: Schwabe)

\section{3 (8609) Chesias legatella (DENIS \& SCHIFFER-} MÜLLER, 1775)

Neue Nachweise von bekannten Fundorten:

c 3147/I Zerpenschleuse/Ortsgebiet: Berliner Straße, im Garten (2014: Busse)

3147/II Finowfurt/Hirtenweg - Besters Fließ (1986, 1988, 1990: Kittel)

3148/IV Ebw.-Westend/Unterheide: Wildparkstraße (1978: Kittel)

\section{5 (8620) Aplocera plagiata (Linnaeus, 1758)}

Neue Nachweise von bekannten Fundorten:

c 3147/I Zerpenschleuse/Ortsgebiet: Berliner Straße (2014, 2015, 2018: Busse)

3148/III Ebw. OT Finow/Stadtgebiet: Altenhofer Straße, im Garten $(2016,2018)$

g 3149/IV Niederfinow/Oderhänge - ehemalige Kiesgruben südwestlich des Schiffshebewerks $(2015,2016$ : Schwabe)

Fundorte (Ergänzung):

c 3148/IV Ebw./Stadtgebiet: Kleingartenanlage im Schwärzetal nördlich Forstbotanischer Garten (2014, 2016 : Schwabe)

3148/III Ebw. OT Finow Umg./Forst Finowtal: Einflugschneise Flugplatz (2016)

d 3057/III Groß Schönebeck/Ortsgebiet: Friedenstraße, im Garten (2017: Haase)

f 3049/IV Brodowin/Krugberg (2014)

g 3150/I Oderberg/am Friedhof (2016)

\section{7 (8631) Odezia atrata (LinNAEus, 1758)}

Ich beobachtete den F am Tag beim Saugen an Trifolium repens, Weiß-Klee.

Fundorte (Ergänzung):

c 3148/I Ebw.-Westend/Unterheide: Wildparkstraße (1970: Kittel)

d 3048/II Althüttendorf Umg./Tiefer Bugsinsee (1977, 1978: Kittel)

e 3049/I Groß Ziethen Umg./Steingruben an den Ihlowbergen (2014); bei Albrechtshöhe: Waldlichtung nordöstlich der Waldstraße nach Grumsin (2014); Grumsin Umg./Forst Grumsin: Stromtrasse westsüdwestlich des Ortes (2015)

\section{8 (8638) Lithostege griseata (DENIS \& SCHIF- FERMÜLLER, 1775)}

- RL Land Brandenburg: Kategorie 2 (stark gefährdet): $\mathrm{TR}$

- RL Deutschland (2011): Kategorie 3 (gefährdet).

Neue Nachweise von bekannten Fundorten:

c 3147/I Zerpenschleuse/Ortsgebiet: Berliner Straße, im Garten (2014, 2015: Busse)

3148/III Ebw. OT Finow/Stadtgebiet: Altenhofer Straße, im Garten $(2015,2018)$ 
d 3047/III Groß Schönebeck/am Bahndamm (2015: Haase)

Fundorte (Ergänzung):

c 3147/I Marienwerder Umg./Oder-Havel-Kanal nordwestlich des Ortes (2016: Busse)

\section{9 (8639) Lithostege farinata (HUFNAGEL, 1766)}

- RL Land Brandenburg: Kategorie 2 (stark gefährdet): TR;

- RL Deutschland (2011): Kategorie 2 (stark gefährdet).

Neue Nachweise von bekannten Fundorten:

c 3147/I Zerpenschleuse/Ortsgebiet: Berliner Straße, im Garten (2015: Busse)

d 3047/III Groß Schönebeck/Bahndamm an der Rosenbecker Straße (2015: Haase)

\section{0 (8654) Euchoeca nebulata (Scopoli, 1763)}

Neue Nachweise von bekannten Fundorten:

b 3247/II Biesenthal Umg./NSG Biesenthaler Becken: Langerönner Weg (2014: Ockruck)

c 3146/II Zerpenschleuse Umg./Hammerbruch nordwestlich des Ortes (1999: Haase) (westlich angrenzendes Nachbargebiet)

3147/I Zerpenschleuse/Ortsgebiet: Berliner Straße, im Garten (2015: Busse)

3148/III Ebw. OT Finow/Stadtgebiet: Altenhofer Straße, im Garten $(2014,2018)$

e 3149/II Liepe Umg./Forst Chorin: NSG Plagefenn - LedumMoor am Fuß der Plageberge (2018: Richert \& Schwabe)

Fundorte (Ergänzung):

c 3147/IV Marienwerder/Steinlaake (2015: Schwabe), Kiesgrube südwestlich des Sportplatzes (2015); Finowfurt Umg./Finowfließniederung an der Neuen Brücke - Biesenthaler Str. (2014), Kesselmoor an der Finowfließniederung nordöstlich des Lehnssees (2016, 2017: Richert \& Schwabe)

3148/IV Ebw./Stadtgebiet: Kleingartenanlage im Schwärzetal nördlich Forstbotanischer Garten (2014, 2016, 2017, 2018: Schwabe)

g 3150/I Oderberg/Geistberg (2017: Schwabe)

\section{1 (8656) Asthena albulata (HufNAGEL, 1767)}

Neue Nachweise von bekannten Fundorten:

c 3147/I Zerpenschleuse/Ortsgebiet: Berliner Straße, im Garten (2014: Busse)

e 3149/II Liepe Umg./Forst Chorin: NSG Plagefenn - LedumMoor am Fuß der Plageberge (2018: Richert \& Schwabe)

g 3149/II Liepe (1975: Kittel)

3150/I Oderberg/Teufelsberg (1974: Kittel)

Fundorte (Ergänzung):

c 3147/II Finowfurt/Hirtenweg - Besters Fließ (1991: Kittel)
3148/IV Ebw./Stadtgebiet: Kleingartenanlage im Schwärzetal nördlich Forstbotanischer Garten (2014, 2016: Schwabe)

\section{2 (8658) Asthena anseraria (HERRICH-SCḦ̈F- FER, 1855)}

- RL Land Brandenburg: Kategorie 3 (gefährdet): WS;

- RL Deutschland (2011): Vorwarnliste.

Neue Nachweise von bekannten Fundorten:

e 3149/II Liepe Umg./Forst Chorin: NSG Plagefenn - LedumMoor am Fuß der Plageberge (2018: Richert \& Schwabe)

\section{3 (8660) Hydrelia flammeolaria (HUFNAGEL, 1767)}

Neue Nachweise von bekannten Fundorten:

b 3248/II Trampe/ehemaliger TÜP (2016: Busse \& Müller)

c 3147/I Zerpenschleuse/Ortsgebiet: Berliner Straße, im Garten (2015, 2018: Busse)

3148/III Ebw. OT Finow/Stadtgebiet: Altenhofer Straße, im Garten (2014-2016, 2018)

e 3149/II Liepe Umg./Forst Chorin: NSG Plagefenn - LedumMoor am Fuß der Plageberge (2018: Richert \& Schwabe)

Fundorte (Ergänzung):

b 3247/II Biesenthal Umg./NSG Biesenthaler Becken: Langerönner Weg (2014: Ockruck), Weg nördlich der Pfauenwiesen (2014: Ockruck)

c 3146/IV Kreuzbruch Umg./ehemalige Römerwegbrücke am Oder-Havel-Kanal östlich des Ortes (2014: Busse) (westlich angrenzendes Nachbargebiet)

347/IV Finowfurt Umg./Kesselmoor an der Finowfließniederung nordöstlich des Lehnssees (2016, 2017: Richert \& Schwabe)

3148/IV Ebw./Stadtgebiet: Kleingartenanlage im Schwärzetal nördlich Forstbotanischer Garten (2016: Schwabe)

e 3049/I Groß Ziethen Umg./Steingruben an den Ihlowbergen (2014)

g 3150/I Oderberg/Geistberg (2018: Schwabe)

\section{5 (8663) Minoa murinata (SCOPOLI, 1763)}

Phänologie/Voltinismus: Bivoltin, 1. Gen.: 22.IV.(2014), damit wird die historische Datumsangabe bei Heese (1936, in RicheRT 2014) bestätigt; 20.IV.(2018) aFA, phänologische Verfrühung 2d gegenüber bis 2013.

\section{6 (8665) Lobophora halterata (HufNAGEL, 1767)}

Neue Nachweise von bekannten Fundorten:

c 3146/IV Kreuzbruch Umg./ehemalige Römerwegbrücke am Oder-Havel-Kanal östlich des Ortes (2014: Busse) (westlich angrenzendes Nachbargebiet)

3147/I Zerpenschleuse/Ortsgebiet: Berliner Straße, im Garten (2014-2016, 2018: Busse) 
3148/III Ebw. OT Finow/Stadtgebiet: Altenhofer Straße, im Garten (2014-2018)

g 3150/I Oderberg/Teufelsberg (1975: Kittel); Geistberg (2016: Schwabe)

Fundorte (Ergänzung):

g 3149/II Liepe (1979: Kittel)

877 (8668) Trichopteryx carpinata (BORKHAUSEN, 1794)

- RL Land Brandenburg: Vorwarnliste: WS.

Die Beobachtungslücke im Naturraum g (Odertalhänge, vgl. Richert 2004, 2014) wurde inzwischen geschlossen.

\section{Neue Nachweise von bekannten Fundorten:}

c 3147/II Finowfurt/Besters Fließ - Hirtenweg (1987, 1995, 1997: Kittel)

3148/III Ebw. OT Finow/Stadtgebiet: Altenhofer Straße, im Garten $(2015,2016,2017)$

3149/III Ebw./Eichwerder (1974: Kittel)

Fundorte (Ergänzung):

c 3148/IV Ebw./Stadtgebiet: Kleingartenanlage im Schwärzetal nördlich Forstbotanischer Garten (2016, 2018: Schwabe)

e 3149/II Liepe Umg./Forst Chorin: NSG Plagefenn südlich Gr. Plagesee (2018: Richert, Schwabe \& Theimer)

\section{8 (8675) Pterapherapteryx sexalata (Retzius,} 1783)

\section{Neue Nachweise von bekannten Fundorten:}

b 3248/II Trampe/ehemaliger TÜP (2016: Busse \& Müller)

c 3147/I Zerpenschleuse/Ortsgebiet: Berliner Straße, im Garten (2014, 2015, 2017, 2018: Busse)

3147/I Marienwerder Umg./Oder-Havel-Kanal nordwestlich des Ortes (2014, 2016: Busse)

3147/II Finowfurt/Hirtenweg - Besters Fließ (1994: Kittel)

3147/IV Marienwerder/Steinlaake (2015)

3148/III Ebw. OT Finow/Stadtgebiet: Altenhofer Straße, im Garten (2014-2016, 2018)

3148/IV Ebw.-Westend/Unterheide: Wildparkstraße (1991: Kittel)

e 3149/II Liepe Umg./Forst Chorin: NSG Plagefenn - LedumMoor am Fuß der Plageberge (2018: Richert \& Schwabe)

g 3150/I Oderberg/Teufelsberg (1974: Kittel)

Fundorte (Ergänzung):

c $3147 / \mathrm{IV}$ Finowfurt Umg./Kesselmoor an der Finowfließniederung nordöstlich des Lehnssees (2017: Richert \& Schwabe)

3148/IV Ebw./Stadtgebiet: Kleingartenanlage im Schwärzetal nördlich Forstbotanischer Garten $(2014,2016,2018$ : Schwabe)

g 3150/I Oderberg/Geistberg (2017: Schwabe)

\section{9 (8681) Acasis viretata (HÜBNER, 1799)}

Neue Nachweise von bekannten Fundorten:

c 3146/IV Kreuzbruch Umg./ehemalige Römerwegbrücke am Oder-Havel-Kanal östlich des Ortes (2014: Busse) (westlich angrenzendes Nachbargebiet)

3147/I Zerpenschleuse/Ortsgebiet: Berliner Straße, im Garten (2014, 2015: Busse)

3147/II Finowfurt/Hirtenweg - Besters Fließ (1987, 1988, 1991, 1993, 1994, 1995: Kittel)

3148/III Ebw. OT Finow/Stadtgebiet: Altenhofer Straße, im Garten $(2015,2017)$

e 3149/II Liepe Umg./Forst Chorin: NSG Plagefenn - LedumMoor am Fuß der Plageberge (2018: Richert \& Schwabe)

Fundorte (Ergänzung):

c 3148/IV Ebw./Stadtgebiet: Kleingartenanlage im Schwärzetal nördlich Forstbotanischer Garten $(2015,2016,2018$ Schwabe)

e 3049/I Groß Ziethen Umg./Steingruben an den Ihlowbergen (2014)

d 3047/III Groß Schönebeck/Ortsgebiet: Friedenstraße, im Garten (1999, 2017: Haase)

g 3150/I Oderberg/Geistberg (2017: Schwabe)

\section{Ergebnisse - Diskussion}

\subsection{Phänologische Veränderungen im Unter- suchungszeitraum 2014 - 2018}

Die Beobachtungen phänologischer Veränderungen wurden im Untersuchungszeitraum fortgesetzt und führten zu folgenden Ergebnissen:

- Die Tendenz phänologischer Veränderungen (phänologischer Verfrühungen, phänologischer Verspätungen, Ausbildung zusätzlicher, in früherer Zeit nicht oder nur ausnahmsweise beobachteter zusätzlicher Generationen) setzte sich im Beobachtungszeitraum 2014-2018 fort. Sie erreichte in den Jahren 2014 und 2018 erneut Maxima, fiel in den Jahren dazwischen aber deutlich schwächer aus.

Ein Vergleich der phänologischen Veränderungen mit Wetterdaten der einzelnen Jahre für Ostbrandenburg bestätigte die nach 25-jähriger Beobachtungszeit in RICHERT (2014) getroffene Aussage, dass Witterungsextreme mit überdurchschnittlich hohen Temperaturen im 1. Halbjahr, vornehmlich im Monat April, und unterdurchschnittliche Niederschlagsmengen im April wesentlicher Auslöser für die phänologischen Veränderungen sind.

In Ostbrandenburg war das Frühjahr 2014 deutlich zu warm und zu trocken: Februar: 3,7 $7^{\circ}$ über Mittel, zu trocken; März 3,6 $6^{\circ}$ über Mittel, trocken; April 2,5 über Mittel, trocken (Quelle: Wetterkontor Frankfurt/ Oder - monatliche Grafik in Märkische Oderzeitung). Bemerkenswert ist, dass auch der Herbst bei uns überdurchschnittlich warm ausfiel. 
Nach Pressemitteilungen des DWD aus Offenbach war 2014 deutschlandweit das wärmste Jahr seit mehr als 130 Jahren mit einem überdurchschnittlich warmen Herbst: zweitwärmster seit 133 Jahren. Das Jahr 2014 zeichnete sich wieder durch ein Maximum an phänologischen Veränderungen aus (Tabelle 13, Tabelle 14).

Dies trifft für die Jahre 2015-2017 nicht zu. Nur einzelne Frühjahrsmonate waren zu warm, der April jedoch in diesen Jahren stets zu kühl. Das spiegelt sich in einer erheblich geringeren Zahl phänologischer Veränderungen wider (Tabellen 15 bis 20).

Charakteristisch war wechselhaftes Wetter (Hitzerekorde wechselten mit kühlen Phasen, Trockenheit mit lokalen Extremniederschlägen. Das gesamte Frühjahr 2017 war besonders trocken. Spätfröste Ende April und Anfang Mai hatten katastrophale Folgen für die Obstblüte. Die Herbstmonate der Jahre 2015-2017 zeigten positive und negative Abweichungen von den Mittelwerten (auffällige Abweichungen: 2015: November $+3,3^{\circ}$ über Mittel, zu nass; Dezember: $+5,8^{\circ}$ über Mittel, zu trocken; 2016: September $+4,0^{\circ}$ über Mittel, trocken; der Dezember war in allen Jahren zu warm (2015: sogar 5,8 ${ }^{\circ}$ über Mittel; 2017: $2.5^{\circ}$ über Mittel).

Das Jahr 2018 fiel in Nordostbrandenburg durch einen deutlich zu warmen April (4,7 über langjährigem Mittel) und Mai (3,7 $7^{\circ}$ über langjährigem Mittel) auf. Auch in allen folgenden Sommermonaten lagen die Mittelwerte der Temparaturen deutlich $\left(+2,0\right.$ bis $\left.+3,3^{\circ} \mathrm{C}\right)$ über den
Durchschnittswert. Außergewöhnlich hoch war die Anzahl der Sommertage (seit April) und der heißen Tage (seit Mai und bis in den September) in diesem Jahr. Gleichzeitig herrschte seit April bis zum Sommerende extreme Trockenheit. Mit Hitze und Trockenheit seit dem Frühling korrelierten 2018 erneut Maxima phänologischer Veränderungen (siehe Tabelle 21).

- Eine Verlängerung der Vegetationsperiode wurden in allen Jahren beobachtet, in den letzten Jahren vor allem im Herbst;

- Im Zeitraum 2014-2018 wurden phänologischen Veränderungen auch bei solchen Arten beobachtet, von denen bisher keine derartigen Beobachtungen im Gebiet bekannt waren. Sie sind in nachfolgenden Tabellen mit ${ }^{* *}$ gekennzeichnet.

Arten, die zwar phänologische Veränderungen in der Vergangenheit zeigten, bei denen jedoch erstmals entweder phänologische Verfrühungen oder phänologische Verspätungen registriert wurden, sind mit * gekennzeichnet.

Nachfolgend werden sämtliche (auch geringfügige) phänologischen Veränderungen, bezogen auf die in RICHERT (2014) publizierten Phänologiewerte, jahr- und artweise zusammengefasst und tabellarisch dargestellt. Korrekturen bereits publizierter Phänologiewerte ergeben sich durch Auswertung bisher nicht erschlossener Quellen (siehe 1. Einleitung).

\subsubsection{Phänologische Veränderungen im Beobachtungsjahr 2014}

Bei 59 Arten wurden (z. T. erneute) phänologische Verfrühungen beobachtet. Diese Arten erreichten im Jahre 2014 ihren bis dahin festgestellten absoluten Flugzeitanfang im Gebiet (Tabelle 13).

Tab. 13: Phänologische Verfrühungen 2014: Das Datum kennzeichnet den absoluten Flugzeitanfang (der 1. Gen. bei mehrbrütigen Arten) im Gebiet.

\begin{tabular}{|c|c|c|c|}
\hline & Art & Datum & phänol. Verfrühung gegenüber bis 2013 \\
\hline 01 & 005 Erynnis tages & 22.IV.2014 & 3d, bisher 25.IV.(2007) \\
\hline 02 & 019 Pieris brassicae ${ }^{*}$ & 30.III.2014 & 13d, bisher 12.IV.(1961) \\
\hline 03 & 021 Pieris napi & 31.III.2014 & 2d, bisher 02.IV.(1990) \\
\hline 04 & 023 Anthocharis cardamines & 30.III.2014 & 11d, bisher 10.IV.(2011) \\
\hline 05 & 027 Lycaena tityrus & 22.IV.2014 & 6d, bisher 28.IV.(2007) \\
\hline 06 & 066 Vanessa atalanta & 30.III.2014 & 11d, bisher 10.IV.(1998) \\
\hline 07 & 089 Coenonympha pamphilus & 22.IV.2014 & 1d, bisher 23.IV.(2007) \\
\hline 08 & 172 Mimas tiliae ${ }^{* *}$ & 19.IV.2014 & 4d, bisher 23.IV.(1961 u. 2009) \\
\hline 09 & 203 Thaumetopoea processionea & 16.VII.2014 & 1d, bisher 17.VII.(2013) \\
\hline 10 & 214 Notodonta dromedarius & 02.IV.2014 & 18d, bisher 20.IV.(1990) \\
\hline 11 & 218 Drymonia ruficornis $^{*}$ & 05.IV.2014 & 5d, bisher 10.IV.(1974 u. 1999) \\
\hline 12 & 222 Pheosia tremula & 20.IV.2014 & 6d, bisher 26.IV.(1998) \\
\hline 13 & 262 Atolmis rubricollix & 23.V.2014 & 5d, bisher 28.V.(2011) \\
\hline 14 & 308 Cryphia algae & 24.VI.2014 & 17d, bisher 11.VII.(1999) \\
\hline 15 & 336 Callistege $m i^{*}$ & 22.IV.2014 & 8d, bisher 30.IV.(1974) \\
\hline
\end{tabular}




\begin{tabular}{|c|c|c|c|}
\hline & Art & Datum & phänol. Verfrühung gegenüber bis 2013 \\
\hline 16 & 349 Diachrysia chrysitis & 21.V.2014 & 1d, bisher 22.V.(2007) \\
\hline 17 & 370 Cucullia fraudatrix ${ }^{* *}$ & 21.V.2014 & 29d, bisher 19.VI.(1964) \\
\hline 18 & 379 Shargacucullia scrophulariae & 24.V.2014 & 7d, bisher 31.V.(2009) \\
\hline 19 & 395 Elaphria venustula & 06.V.2014 & 4d, bisher 10.V.(2012) \\
\hline 20 & 406 Dipterygia scabriuscula* & 29.IV.2014 & 12d, bisher 11.V.(1971) \\
\hline 21 & 415 Ipimorpha retusa $^{* *}$ & 06.VII.2014 & 2d, bisher 08.VII.(1965) \\
\hline 22 & 430 Xanthia citrago $^{*}$ & 30.VIII.2014 & 4d, bisher 03.IX.(1972 u. 2004) \\
\hline 23 & 486 Luperina testacea ${ }^{* *}$ & 30.VII.2014 & 7d, bisher 06.VIII.(1982) \\
\hline 25 & 495 Calamia tridens & 03.VII.2014 & 4d, bisher 07.VII.(2003) \\
\hline 26 & 500 Phragmatiphila nexa* & 31.VII.2014 & 24d, bisher 24.VIII.(1964) \\
\hline 27 & 515 Lacanobia oleracea* & 30.IV.2014 & 15d, bisher 15.V.(1967) \\
\hline 28 & 543 Mythimna pudorina & 09.VI.2014 & 1d, bisher 10.VI.(2002) \\
\hline 29 & 555 Orthosia opima & 18.III.2014 & 13d, bisher 31.III.(2008) \\
\hline 30 & 556 Orthosia populeti & 09.III.2014 & 11d, bisher 20.III.(2012) \\
\hline 31 & 558 Orthosia gracilis ${ }^{* *}$ & 28.III.2014 & 8d, bisher 05.IV.(1961) \\
\hline 32 & 573a Noctua interposita & 08.VI.2014 & 24d, bisher 02.VII.(2009) \\
\hline 33 & 611 Panthea coenobita & 21.V.2014 & 3d, bisher 24.V.(1988) \\
\hline 34 & 612 Colocasia coryli & 07.IV.2014 & 4d, bisher 11.IV.(2011) \\
\hline 35 & 621 Macaria signaria ${ }^{* *}$ & 08.VI.2014 & 8d, bisher 16.VI.(1985) \\
\hline 36 & 633 Epione repandaria & 09.VI.2014 & 2d, bisher 11.VI.(2011) \\
\hline 37 & 671 Bupalus piniarius & 29.IV.2014 & 6d, bisher 05.V.(2007) \\
\hline 38 & 697 Cyclophora annularia & 26.IV.2014 & 14d, bisher 10.V.(2009) \\
\hline 39 & 698 Cyclophora albipunctata* & 29.IV.2014 & 6d, bisher 05.V.(1961) \\
\hline 40. & 701 Cyclophora punctaria & 25.IV.2014 & 4d, bisher 29.IV.(1968 u. 1999) \\
\hline 41 & 726 Idaea dimidiata & 09.VI.2014 & 4d, bisher 13.VI.(2009) \\
\hline 42 & 732 Lythria purpuraria & 04.VI.2014 & 25d, bisher 20.VI.(1993) \\
\hline 43 & 734 Phibalapteryx virgata ${ }^{* *}$ & 22.IV.2014 & 6d, bisher 28.VI.(1968) \\
\hline 44 & 742 Xanthorrhoe designata ${ }^{* *}$ & 24.IV.2014 & 5d, bisher 28.IV.(1966 u. 2012) \\
\hline 45 & 769 Chloroclysta siterata & 27.VIII.2014 & 11d, bisher 07.IX.(2008) \\
\hline 46 & 775 Thera obeliscata & 29.IV.2014 & 5d, bisher 04.V.(2007) \\
\hline 47 & 782 Hydriomena furcata & 22.V.2014 & 11d, bisher 02.VI.(1998) \\
\hline 48 & 789 Pareulype berberata & 24.IV.2014 & 5d, bisher 29.IV.(2011) \\
\hline 49 & 795 Philereme vetulata & 09.VI.2014 & 1d, bisher 10.VI.(1993) \\
\hline 50 & 813 Eupithecia inturbata & 06.VII.2014 & 3d, bisher 09.VII.(2006) \\
\hline 51 & 822 Eupithecia valerianata & 08.VI.2014 & 3d, bisher 11.VI.(1998) \\
\hline 52 & 825 Eupithecia egenaria & 06.V.2014 & 3d, bisher 09.V.(2000 u. 2012) \\
\hline 53 & 844 Eupithecia indigata & 21.IV.2014 & 2d, bisher 23.IV.(2011) \\
\hline 54 & 850 Eupithecia virgaureata ${ }^{*}$ & 07.IV.2014 & 8d, bisher 15.IV.(1959) \\
\hline 55 & 855 Eupithecia lariciata & 30.IV.2014 & 14d, bisher 14.V.(1994) \\
\hline 56 & 857 Gymnoscelis rufifasciata & 02.IV.2014 & 1d, bisher 03.IV.(1994) \\
\hline 57 & 858 Chloroclystis $v$-ata* & 20.IV.2014 & 7d, bisher 27.IV.(1968) \\
\hline 58 & 861 Rhinprora debiliata & 08.VI.2014 & 13d, bisher 21.VI.(2004) \\
\hline 59 & 875 Minoa murinata & 22.IV.2014 & 2d, bisher 24.IV.(2012) \\
\hline
\end{tabular}

Bei 47 Arten wurden (z. T. erneute) phänologische Verspätungen beobachtet. Diese Arten erreichten im Jahre 2014 ihr bis dahin festgestelltes absolutes Flugzeitende im Gebiet (Tabelle 14). 
Tab. 14: Phänologische Verspätungen 2014: Das Datum kennzeichnet das absolute Flugzeitende (bei mehrbrütigen Arten der letzten Generation) im Gebiet.

\begin{tabular}{|c|c|c|c|}
\hline & Art & Datum & phänol. Verspätung gegenüber bis 2013 \\
\hline 01 & 020 Pieris rapae & 18.X.2014 & 5d, bisher 13.X.(1984, 2012), part. 4. Gen.? \\
\hline 02 & 067 Vanessa cardui & 15.IX.2014 & 1d, bisher 14.IX.(2001) \\
\hline 03 & 069 Nymphalis antiopa & 12.VI.2014 & 9d, bisher 03.VI.(2003) \\
\hline 04 & 085 Pararge aegeria & 03.X.2014 & 5d, bisher 28.IX.(2008) \\
\hline 05 & 184 Hyles gallii $^{*}$ & 20.IX.2014 & 15d, bisher 05.IX.(1957), part. 2. Gen. \\
\hline 06 & 187 Thyatira batis & 14.IX.2014 & 8d, bisher 06.IX.(2013) \\
\hline 07 & 189 Tethea ocularis & 15.IX.2014 & 26d, bisher 20.VIII.(2007) \\
\hline 08 & 207 Clostera anachoreta & 26.VIII.2014 & 4d, bisher 22.VIII.(2011) \\
\hline 09 & 212 Furcula bicuspis* & 16.VIII.2014 & 1d, bisher 15.VIII.(1964 und 2005) \\
\hline 10 & 214 Notodonta dromedarius & 08.IX.2014 & 6d, bisher 02.IX.(2005) \\
\hline 11 & 216 Notodonta ziczac ${ }^{*}$ & 15.IX.2014 & 12d, bisher 03.IX.(1968) \\
\hline 12 & 221 Drymonia velitaris & 09.X.2014 & 21d, bisher 18.IX.(2006) \\
\hline 13 & 222 Pheosia tremula & 04.IX.2014 & 7d, bisher 27.VIII.(2008) \\
\hline 14 & 247 Meganola albula & 07.X.2014 & 47d, bisher 21.VIII.(2008), part. 2. Gen.? \\
\hline 15 & 253 Bena bicolorana & 02.X.2014 & 33d, bisher 30.VIII.(2013), part. 2. Gen.? \\
\hline 16 & 258 Miltochrista miniata & 13.X.2014 & 12d, bisher 01.X.(2011), part. 2. Gen. \\
\hline 17 & 260 Pelosia muscerda & 20.IX.2014 & 13d, bisher 07.IX.(2001), part. 2. Gen.? \\
\hline 18 & 263 Lithosia quadra** & 18.X.2014 & 32d, bisher 16.IX.(1981), part. 2. Gen. \\
\hline 19 & 280 Spilosoma lubricipeda ${ }^{* *}$ & 04.IX.2014 & 5d, bisher 30.VIII.(1974), part. 2. Gen. \\
\hline 20 & 291 Moma alpium* & 13.VIII.2014 & 20d, bisher 24.VII.(1987), part. 2. Gen.? \\
\hline 21 & 296 Acronicta aceris & 04.IX.2014 & 13d, bisher 22.VIII.(2010), part. 2. Gen. \\
\hline 22 & 298 Acronicta megacephala & 13.IX.2014 & 16d, bisher 28.VIII.(1973), part. 2. Gen. \\
\hline 23 & 303 Acronicta rumicis & 09.X.2014 & 17d, bisher 23.IX.(2013), part. 3. Gen. \\
\hline 24 & 304 Craniophora ligustri & 01.IX.2014 & 7d, bisher 25.VIII.(2012) \\
\hline 25 & 321 Schrankia costaestrigalis & 06.X.2014 & 21d, bisher 15.IX.(1991) \\
\hline 26 & 338 Laspeyria flexula & 16.X.2014 & 7d, bisher 09.X.(2011) \\
\hline 27 & 349 Diachrysia chrysitis & 15.IX.2014 & 7d, bisher 08.IX.(2005) \\
\hline 28 & 362 Emmelia trabealis * & 06.IX.2014 & 7d, bisher 30.VIII.(1968) \\
\hline 29 & 375 Cucullia umbratica & 02.X.2014 & 21d, bisher 11.IX.(2005), part. 2. Gen.? \\
\hline 30 & 411 Phlogophora meticulosa & 20.XI.2014 & 5d, bisher 15.XI.(2004) \\
\hline 31 & 515 Lacanobia oleracea & 29.IX.2014 & 5d, bisher 24.IX.(2013) \\
\hline 32 & 519 Hada plebeja & 23.VIII.2014 & 1d, bisher 22.VIII.(2010) \\
\hline 33 & 544 Mythimna straminea & 02.X.2014 & 18d, bisher 14.IX.(2006), part. 2. Gen. \\
\hline 34 & 547 Mythimna obsoleta & 15.IX.2014 & 27d, bisher 19.VIII.(2010), part. 2. Gen? \\
\hline 35 & 573a Noctua interposita & 14.IX.2014 & 4d, bisher 10.IX.(2010) \\
\hline 36 & 577 Noctua interjecta & 12.IX.2014 & 13d, bisher 29.VIII.(2002) \\
\hline 37 & 611 Panthea coenobita & 15.IX.2014 & 26d, bisher 20.VIII.(2007), part. 2. Gen? \\
\hline 38 & 642 Selenia tetralunaria* & 04.IX.2014 & 22d, bisher 13.VIII.(1987) \\
\hline 39 & 663 Hypomecis roboraria & 03.X.2014 & 8d, bisher 26.IX.(2006), part. 2. Gen. \\
\hline 40 & 695 Jodis putata* & 31.X.2014 & 122d bisher 01.VII.(1962), part. 2. Gen.? \\
\hline 41 & 698 Cyclophora albipunctata & 01.X.2014 & 12d, bisher 19.IX.(2003) \\
\hline 42 & 710 Scopula rubiginata & 12.IX.2014 & 2d, bisher 10.IX.(1999) \\
\hline 43 & 712 Scopula immutata & 04.IX.2014 & 9d, bisher 26.VIII.(2002) \\
\hline 44 & 724 Idaea humiliata & 04.VIII.2014 & 3d, bisher 01.VIII.(1991) \\
\hline 45 & 729 Idaea straminata & 02.X.2014 & 10d, bisher 22.IX.(1963, 2003), part. 2. Gen. \\
\hline
\end{tabular}




\begin{tabular}{|c|l|c|l|}
\hline & \multicolumn{1}{|c|}{ Art } & Datum & \multicolumn{1}{|c|}{ phänol. Verspätung gegenüber bis 2013 } \\
\hline 46 & 797 Euphyia biangulata ${ }^{* *}$ & 04.IX.2014 & 18d, bisher 17.VIII.(1994) \\
\hline 47 & 850 Eupithecia virgaureata & 15.IX.2014 & 6d, bisher 09.IX.(2007) \\
\hline
\end{tabular}

Eine Reihe von Arten, die ehemals in der Regel uni- (bzw. bivoltin) im Gebiet auftraten, bildeten 2014 (partielle) zusätzliche Generationen aus (u. a. Araschnia levana 3. Gen.; siehe dazu auch Tab. 14).

Bei folgenden Arten wurden von der regulären Flugzeit stark abweichende Spätfunde registriert, die als erstmals im Gebiet beobachtete zusätzliche Generation gedeutet wurden: Lithosia quadra M X; Bena bicolorana E IX und A X; Jodis putata E X.

\subsubsection{Phänologische Veränderungen im Beobachtungsjahr 2015}

Bei 12 Arten wurden (z. T. erneute) phänologische Verfrühungen beobachtet. Sie erreichten 2015 ihren bis dahin festgestellten absoluten Flugzeitbeginn im Gebiet (Tabelle 15).

Tab. 15: Phänologische Verfrühungen 2015: Das Datum kennzeichnet den absoluten Flugzeitbeginn (der 1. Gen. bei mehrbrütigen Arten) im Gebiet.

\begin{tabular}{|c|c|c|c|}
\hline & Art & Datum & phänol. Verfrühung gegenüber bis 2013 \\
\hline 01 & 005 Erynnis tages & 21.IV.2015 & 1d, bisher 22.IV.(2014) \\
\hline 02 & 066 Vanessa atalanta & 25.III.2015 & 5d, bisher 30.III(2014) \\
\hline 03 & 067 Vanessa cardui & 11.V.2015 & 6d, bisher 17.V.(2009) \\
\hline 04 & 187 Thyatira batis & 04.V.2015 & 1d, bisher 05.V.(2007) \\
\hline 05 & 224 Pterostoma palpina ${ }^{* *}$ & 11.IV.2015 & 7d, bisher 18.IV.(1961) \\
\hline 06 & 254 Pseudoips prasinana & 11.V.2015 & 8d, bisher 19.V.(2007) \\
\hline 07 & 267 Eilema complana & 06.VI.2015 & 9d, bisher 15.VI.(2003) \\
\hline 08 & 495 Calamia tridens & 01.VII.2015 & 6d, bisher 07.VII.(2003) \\
\hline 09 & 552 Orthosia gothica* & 14.III.2015 & 2d, bisher 16.III.(1961) \\
\hline 10 & 617a Stegania trimaculata & 06.V.2015 & 7d, bisher 13.V.(2011) \\
\hline 11 & 716 Idaea serpentata & 24.VI.2015 & 2d, bisher 26.VI.(1996) \\
\hline 12 & 729 Idaea straminata & 08.VI.2015 & 2d, bisher 10.VI.(2007) \\
\hline
\end{tabular}

Bei 7 Arten wurden (z. T. erneute) phänologische Verspätungen beobachtet. Sie erreichten 2015 ihr bis dahin festgestelltes absolutes Flugzeitende im Gebiet (Tabelle 16).

Tab. 16: Phänologische Verspätungen 2015: Das Datum kennzeichnet das absolute Flugzeitende (bei mehrbrütigen Arten der letzten Generation) im Gebiet.

\begin{tabular}{|c|c|c|c|}
\hline & Art & Datum & phänol. Verspätung gegenüber bis 2013 \\
\hline 01 & 005 Erynnis tages & 23.VIII.2015 & 18d, bisher 05.VIII.(2010) \\
\hline 02 & 155 Phragmataecia castaneae & 02.VIII.2015 & 12d, bisher 21.VII.(2009) \\
\hline 03 & 223 Pheosia gnoma & 18.IX.2015 & 12d, bisher 06.IX.(2013) \\
\hline 04 & 249 Nola confusalis* & 02.VI.2015 & 4d, bisher 29.V.(1980) \\
\hline 05 & 259 Cybosia mesomella* & 31.VIII.2015 & 16d, bisher 15.VIII.(1974) \\
\hline 06 & 438 Agrochola laevis & 11.XI.2015 & 45d, bisher 27.IX.(2011) \\
\hline 07 & 513 Lacanobia $w$-latinum $* *$ & 22.VIII.2015 & 8d, bisher 15.VIII.(1974) \\
\hline
\end{tabular}

Arten, die vormals in der Regel uni- oder bivoltin im Gebiet auftraten, bildeten 2015 (partielle) zusätzliche Generationen aus:

Lycaena dispar 2. Gen. M IX; Araschnia levana 3. Gen. E VIII; Pseudoips prasinana 2. Gen. M VIII; Diacrisia sannio 2. Gen. M VIII; Lacanobia w-latinum 2. Gen. E VIII; Gluphisia crenata 2. Gen. M VIII, Thyatira pyritoides 2. Gen. im IX. 


\subsubsection{Phänologische Veränderungen im Beobachtungsjahr 2016}

Bei 12 Arten wurden (z. T. erneute) phänologische Verfrühungen beobachtet (Tabelle 17).

Tab. 17: Phänologische Verfrühungen 2016: Das Datum kennzeichnet den neuen absoluten Flugzeitbeginn (der 1. Gen. bei mehrbrütigen Arten) im Gebiet.

\begin{tabular}{|c|c|c|c|}
\hline & Art & Datum & phänol. Verfrühung gegenüber bis 2013 \\
\hline 01 & 024 Lycaena dispar & 24.V.2016 & 5d, bisher 29.V. $(1999,2007,2012)$ \\
\hline 02 & 137 Zygaena lonicerae & 20.VI.2016 & 4d, bisher 24.VI.(2008) \\
\hline 03 & 177 Spinx ligustri & 08.V.2016 & 15d, bisher 23.V.(2011) \\
\hline 04 & 254 Pseudoips prasinana & 10.V.2016 & 9d, bisher 19.V.(2007) \\
\hline 05 & 267 Eilema complana & 04.VI.2016 & 2d, bisher 06.VI.(2015) \\
\hline 06 & 321 Schrankia costaestrigalis $^{* *}$ & 24.VI.2016 & 43d, bisher 06.VIII.(1973) 1. Gen. beob. \\
\hline 07 & 540 Mythimna conigera & 24.V.2016 & 14d, bisher 07.VI.(2011) \\
\hline 08 & 573a Noctua interposita & 31.V.2016 & 2d, bisher 02.VI.(2009) \\
\hline 09 & 586 Xestia triangulum ${ }^{* *}$ & 22.V.2016 & 11d, bisher 02.VI.(1978) \\
\hline 10 & 665 Ascotis selenaria & 31.V.2016 & 6d, bisher 06.VI.(2008) \\
\hline 11 & 729 Idaea straminata & 02.VI.2016 & 6d, bisher 08.VI.(2015) \\
\hline 12 & 854 Eupithecia lanceata & 28.III.2016 & 1d, bisher 29.III.(1998) \\
\hline
\end{tabular}

Bei 3 Arten wurden (z. T. erneute) phänologische Verspätungen beobachtet. Sie erreichten 2016 ihr bis dahin festgestelltes absolutes Flugzeitende im Gebiet (Tabelle 18).

Tab. 18: Phänologische Verspätungen 2016: Das Datum kennzeichnet das absolute Flugzeitende (bei mehrbrütigen Arten der letzten Generation) im Gebiet.

\begin{tabular}{|l|l|c|l|}
\hline & \multicolumn{1}{|c|}{ Art } & Datum & \multicolumn{1}{|c|}{ phänol. Verspätung gegenüber bis 2013} \\
\hline 01 & 005 Erynnis tages & 03.IX.2016 & 11d, bisher 23.VIII.(2015) \\
\hline 02 & 323 Catocala sponsa & 14.X.2016 & 15d, bisher 29.IX.(2003) \\
\hline 03 & 741 Xanthorhoe biriviata & 30.VIII.2016 & 25d, bisher 05.VIII.(2010) \\
\hline
\end{tabular}

Arten, die vormals in der Regel uni- oder bivoltin im Gebiet auftraten, bildeten 2016 (partielle) zusätzliche Generationen aus:

Lycaena dispar 2. Gen. E VIII; Araschnia levana 3. Gen. M VIII - A IX; Thyathira batis 2. Gen. VIII - A IX; Habrosyne pyritoides 2. Gen. M IX; Miltochrista miniata 2. Gen. A VIII; Pseudoips prasinana 2. Gen. M - E VIII; Toxocampa pastinum 2.(?) Gen. A X; Laspeyria flexula 2. Gen. im VIII - M IX; Lithacodia pygarga 2. Gen. A IX; Aethia emortualis 2. Gen. M IX; Agrotis exclamationis 2. Gen. E VIII - A IX; Idaea aversata 2. Gen. A IX; Perizoma alchemillata 2. Gen. E IX.

\subsubsection{Phänologische Veränderungen im Beobachtungsjahr 2017}

Bei fünf Arten wurden (z. T. erneute) phänologische Verfrühungen beobachtet (Tabelle 19).

Tab. 19: Phänologische Verfrühungen 2017: Das Datum kennzeichnet den neuen absoluten Flugzeitbeginn (der 1. Gen. bei mehrbrütigen Arten) im Gebiet.

\begin{tabular}{|l|l|c|l|}
\hline \multicolumn{1}{|c|}{ Art } & \multicolumn{1}{|c|}{ Datum } & \multicolumn{1}{|c|}{ phänol. Verfrühung gegenüber bis 2013 } \\
\hline 01 & 020 Pieris rapae & 31.III.2017 & 3d, bisher 03.IV.(2011) \\
\hline 02 & 066 Vanessa atalanta & $24 . I I I .2017$ & 1d, bisher 25.III.(2015) \\
\hline 03 & 612 Colocasia coryli & 01.IV.2017 & 6d, bisher 07.IV.(2014) \\
\hline 04 & 665 Ascotis selenaria & 29.V.2017 & 2d, bisher 31.V. (2016) \\
\hline 05 & 786 Horisme tersata & 19.V.2017 & 31d, bisher 19.VI.(1979) \\
\hline
\end{tabular}


Bei 5 Arten wurden (z. T. erneute) phänologische Verspätungen beobachtet (Tabelle 20).

Tab. 20: Phänologische Verspätungen 2017: Das Datum kennzeichnet das absolute Flugzeitende (bei mehrbrütigen Arten der letzten Generation) im Gebiet.

\begin{tabular}{|c|l|c|l|}
\hline \multicolumn{1}{|c|}{ Art } & \multicolumn{1}{|c|}{ Datum } & \multicolumn{1}{|c|}{ phänol. Verspätung gegenüber bis 2013 } \\
\hline 01 & 019 Pieris brassicae & 15.X.2017 & 5d, bisher 10.X.(2008) \\
\hline 02 & 338 Laspeyria flexula & $20 . X .2017$ & $4 d$, bisher 16.X.(2014) \\
\hline 03 & 408 Talpophila matura ${ }^{* *}$ & 25.IX.2017 & 25d, bisher 31.VIII.(1984) \\
\hline 04 & 518 Lacanobia suasa & 15.IX.2017 & 5d, bisher 10.IX.(1999) \\
\hline 05 & 725 Idaea seriata & 27.IX.2017 & 3d, bisher 24.IX.(2013) \\
\hline
\end{tabular}

Folgende Arten, die ehemals in der Regel uni- oder bivoltin im Gebiet auftraten, bildeten 2017 (partielle) zusätzliche Generationen aus: Lycaena dispar 2. Gen. E VIII; Inachis io 2. Gen. ab A IX; Araschnia levana 3. Gen. E VIII-A IX; Hyles euphorbiae 2. Gen. (nach R-Fund M X) Pseudoips prasinana 2. Gen. E VIII; Laspeyria flexula 2. Gen. VIII-MX.

\subsubsection{Phänologische Veränderungen im Beobachtungsjahr 2018:}

Bei 60 Arten wurden (z. T. erneute) phänologische Verfrühungen beobachtet (Tabelle 21).

Tab. 21: Phänologische Verfrühungen 2018: Das Datum kennzeichnet den neuen absoluten Flugzeitbeginn (der 1. Gen. bei mehrbrütigen Arten) im Gebiet.

\begin{tabular}{|c|c|c|c|}
\hline & Art & Datum & phänol. Verfrühung gegenüber bis 2013 \\
\hline 01 & Thymelicus sylvestris & 05.VI.2018 & 2d, bisher 07.VI. $(2007,2011)$ \\
\hline 02 & Neozephyrus quercus & 16.VI.2018 & 6d, bisher 22.VI.(2009) \\
\hline 03 & Satyrium spini & 12.VI.2018 & 7d, bisher 19.VI.(2006) \\
\hline 04 & Satyrium pruni & 20.V.2018 & 9d, bisher 29.V.(2012) \\
\hline 05 & Plebeius idas & 07.VI.2018 & 10d, bisher 17.VI.(2000) \\
\hline 06 & Vanessa cardui & 17.IV.2018 & 30d, bisher 17.V.(2009) (siehe auch 2015) \\
\hline 07 & Coenonympha arcania ${ }^{* *}$ & 05.VI.2018 & 2d, bisher 07.VI.(1961) \\
\hline 08 & Melanargia galathea & 05.VI.2018 & 2d, bisher 07.VI.(2007) \\
\hline 09 & Heterogenea asella* & 07.VI.2018 & 3d, bisher 10.VI.(1960) \\
\hline 10 & Zygaena minos & 05.VI.2018 & 1d, bisher 06.VI.(2011) \\
\hline 11 & Zygaena loti & 12.VI.2018 & 7d, bisher 19.VI.(2000) \\
\hline 12 & Zygaena filipendulae & 14.VI.2018 & 3d, bisher 17.VI.(2000) \\
\hline 13 & Zygaena lonicerae & 18.VI.2018 & 6d, bisher 24.VI.(2008) \\
\hline 14 & Cossus cossus & 30.V.2018 & 3d, bisher 02.VI.(2000) \\
\hline 15 & Zeuzera pyrina & 03.VI.2018 & 1d, bisher 04.VI. $(2000,2011)$ \\
\hline 16 & Malacosoma neustria & 05.VI.2018 & 9d, bisher 14.VI.(2011) \\
\hline 17 & Odonestis pruni ${ }^{* *}$ & 03.VI.2018 & 17d, bisher 20.VI.(1964) \\
\hline 18 & Aglia tau & 17.IV.2018 & 2d, bisher 19.IV. $(1957,2009)$ \\
\hline 19 & Sphinx ligustri & 08.V.2018 & 15d, bisher 23.V.(2000) \\
\hline 20 & Tethea ocularis & 13.V.2018 & 4d, bisher 17.V.(2003) \\
\hline 21 & Arctornis l-nigrum & 07.VI.2018 & 1d, bisher 08.VI.(2008) \\
\hline 22 & Nola aerugula & 10.VI.2018 & 8d, bisher 18.VI.(2013) \\
\hline 23 & Miltochrista miniata & 01.VI.2018 & 5d, bisher 06.VI.(2008) \\
\hline 24 & Phragmatobia fuliginosa & 22.IV.2018 & 4d, bisher 26.IV.(2012) \\
\hline 25 & Dysauxes ancilla & 14.VI.2018 & 3d, bisher 17.VI (2007) \\
\hline
\end{tabular}




\begin{tabular}{|c|c|c|c|}
\hline & Art & Datum & phänol. Verfrühung gegenüber bis 2013 \\
\hline 26 & Callimorpha dominula $^{* *}$ & 31.V.2018 & 2d, bisher 02.VI.(1981) \\
\hline 27 & Paracolax tristalis ${ }^{* *}$ & 14.VI.2018 & 13d, bisher 27.VI. $(1963,2003)$ \\
\hline 28 & Lygephila pastinum & 07.VI.2018 & 4d, bisher 11.VI.(1998) \\
\hline 29 & Parascotia fuliginaria* & 05.VI.2018 & 1d, bisher 06.VI.(1964) \\
\hline 30 & Abrostola triplasia ${ }^{* *}$ & 23.IV.2018 & 4d, bisher 27.IV.(1961) \\
\hline 31 & Trisateles emortualis & 24.V.2018 & 4d, bisher 28.V.(2005) \\
\hline 32 & Hoplodrina blanda ${ }^{* *}$ & 14.VI.2018 & 8d, bisher 22.VI.(1969) \\
\hline 33 & Hoplodrina respersa & 03.VI.2018 & 3d, bisher 06.VI.(1998) \\
\hline 34 & Parastichtis ypsillon & 01.VI.2018 & 10d, bisher 11.VI.(2012) \\
\hline 35 & Cosmia trapezina* & 14.VI.2018 & 12d, bisher 26.VI. $(1961,2011)$ \\
\hline 36 & Atethmia centrago & 20.VIII.2018 & 2d, bisher 22.VIII.(1983) \\
\hline 37 & Amphopoea fucosa & 14.VI.2018 & 3d, bisher 17.VI.(2007) \\
\hline 38 & Hecatera bicolorata ${ }^{* *}$ & 03.VI.2018 & 14d, bisher 17.VI.(1963) \\
\hline 39 & Mythimna pudorina & 08.VI.2018 & 2d, bisher 10.VI.(2007) (siehe auch 2014) \\
\hline 40 & Calospilos sylvata & 08.VI.2018 & 3d, bisher 11.VI.(1993) \\
\hline 41 & Macaria signaria & 07.VI.2018 & 9d, bisher 16.VI.(1985) (siehe auch 2014) \\
\hline 42 & Itame brunneata $^{* *}$ & 29.V.2018 & 2d, bisher 31.V.(1971) \\
\hline 43 & Cepphis advenaria & 28.V.2018 & 1d, bisher 29.V.(1998) \\
\hline 44 & Epione repandaria & 07.VI.2018 & 4d, bisher 11.VI.(2011) (siehe auch 2014) \\
\hline 45 & Phalaena syringaria & 06.VI.2018 & 3d, bisher 09.VI.(2012) \\
\hline 46 & Ennomos erosaria & 03.VI.2018 & 16d, bisher 19.VI. $(2003,2008)$ \\
\hline 47 & Angerona prunaria & 29.V.2018 & 1d, bisher 30.V.(2011) \\
\hline 48 & Peribatodes rhomboidaria & 16.V.2018 & 3d, bisher 19.V.(2007) \\
\hline 49 & Parectropis similaria & 11.V.2018 & 6d, bisher 17.V.(2009) \\
\hline 50 & Campaea margaritata & 13.V.2018 & 1d, bisher 14.V.(2000) \\
\hline 51 & Hemithea aestivaria & 30.V.2018 & 5d, bisher 04.VI. $(2000,2011)$ \\
\hline 52 & Idaea serpentata & 12.VI.2018 & 14d, bisher 26.VI.(1996) (siehe auch 2015) \\
\hline 53 & Idaea biselata & 14.VI.2018 & 5d, bisher 19.VI.(2008) \\
\hline 54 & Idaea dimidiata & 07.VI.2018 & 6d, bisher 13.VI.(2009) (siehe auch 2014) \\
\hline 55 & Idaea straminata & 01.VI.2018 & 9d, bisher 10.VI.(2007) (siehe auch 2015, 2016) \\
\hline 56 & Horisme tersata & 11.V.2018 & 39d, bisher 19.VI.(1979) (siehe auch 2017) \\
\hline 57 & Philereme vetulata & 30.V.2018 & 11d, bisher 10.VI.(1993) (siehe auch 2014) \\
\hline 58 & Philerem transversata & 03.VI.2018 & 7d, bisher 10.VI.(1993) \\
\hline 59 & Eupithecia selinata & 13.V.2018 & 6d, bisher 19.V.(2007) \\
\hline 60 & Minoa murinata ${ }^{* *}$ & 20.IV.2018 & 2d, bisher 22.IV.(Zeitraum 1922-1934) \\
\hline
\end{tabular}

Bei 6 Arten wurden phänologische Verspätungen beobachtet (Tabelle 22; Anmerkung: Die Angaben sind nicht repräsentativ, weil unvollständig, da durch Redaktionsschluss A IX die meisten zu erwartenden späten Daten noch nicht verfügbar waren).

Tab. 22: Phänologische Verspätungen 2018: Das Datum kennzeichnet das absolute Flugzeitende (bei mehrbrütigen Arten der letzten Generation) im Gebiet.

\begin{tabular}{|l|l|c|l|}
\hline \multicolumn{1}{|c|}{ Art } & \multicolumn{1}{|c|}{ Datum } & \multicolumn{1}{|c|}{ phänol. Verspätung gegenüber bis 2013} \\
\hline 01 & Carcharodus alceae & 08.IX.2018 & 16d, bisher 23.VIII.(2007, 2. Gen.) \\
\hline 02 & Hipparchia semele & 19.IX.2018 & 10d, bisher 09.IX.(2007) \\
\hline 03 & Smerinthus ocellata ${ }^{* *}$ & 16.VIII.2018 & 4d, bisher 12.VIII.(1962, 2. part. Gen.) \\
\hline
\end{tabular}




\begin{tabular}{|c|l|c|l|}
\hline \multicolumn{1}{|c|}{ Art } & Datum & \multicolumn{1}{|c|}{ phänol. Verspätung gegenüber bis 2013 } \\
\hline 04 & Drymonia querna & 25.VIII.2018 & 23d, bisher 02.VIII.(2003) \\
\hline 05 & Actinotia polyodon* & 04.IX.2018 & 9d, bisher 26.VIII.(1964, 2. Gen.) \\
\hline 06 & Phibalapteryx virgata & 04.IX.2018 & 8d, bisher 27.VIII.(1960, 2. Gen.) \\
\hline
\end{tabular}

Folgende Arten, die in der Regel uni- oder bivoltin im Gebiet auftraten, bildeten 2017 (partielle) zusätzliche Generationen aus (siehe auch Anmerkung zu Tabelle 22):

Ochlodes sylvanus 2. Gen. A IX; Araschnia levana 3. part. Gen. E VIII; Smerinthus ocellata 2. part. Gen. M VIII; Habrosyne pyritoides 2. part. Gen. A IX; Miltochrista miniata 2. part. Gen. E VIII; Diacrisia sannio 2. part. Gen. A VIII; Laspeyria flexula 2. Gen. E VIII - E IX; Peribatodes rhomboidaria 2. part. Gen. ab M VIII; Scopula nigropunctata 2. part. Gen. M IX; Idaea aversata 2. Gen. A IX; Agrotis exclamationis 2. Gen. M IX.

\subsection{Bestandsentwicklung seit 2014}

Im Beobachtungszeitraum 2014-2018 wurden vier Arten im Gebiet neu entdeckt (Tabelle 23). Damit steigt die Gesamtartenzahl aller im Gebiet nachgewiesenen Arten auf 901.

Tab. 23: Im Gebiet neu entdeckte Arten.

\begin{tabular}{|l|l|c|l|}
\hline \multicolumn{1}{|c|}{ Artengruppe } & \multicolumn{1}{|c|}{ Art } & Jahr des Erstnachweises & \multicolumn{1}{c|}{ Bemerkung } \\
\hline Tagfalter & Nymphalis xanthomelas & 2015 & $\begin{array}{l}\text { Fluktuation an der Areal- } \\
\text { Westgrenze }\end{array}$ \\
\hline Spinner \& Schwärmer & Malacosoma franconica & 2015 & \\
\hline Eulenfalter & Thysanoplura orichalcea & 2015 & Verschleppung \\
\cline { 2 - 4 } & Athetis lepigone & 2017 & \\
\hline
\end{tabular}

Sechs als verschollen gemeldete Arten (RICHERT 2010: 160-161, Richert 2014: 252) wurden im Gebiet wieder entdeckt (Tabelle 24):

Tab. 24: Im Gebiet wieder entdeckte Arten.

\begin{tabular}{|l|l|l|l|}
\hline \multicolumn{1}{|c|}{ Artengruppe } & \multicolumn{1}{|c|}{ Art } & verschollen seit & Wiederfund \\
\hline \multirow{5}{*}{ Eulenfalter } & Schinia scutosa & 1942 & 2014 Irrgast \\
\cline { 2 - 4 } & Eremobia ochroleuca & vor 1951 & 2014 \\
\cline { 2 - 4 } & Gortyna flavago & 1998 & 2015 \\
\cline { 2 - 4 } & Hecatera bicolorata & 1990 & 2016 \\
\cline { 2 - 4 } & Graphiphora augur & 1997 & 2014 \\
\hline \multirow{5}{*}{ Spanner } & Plagodis pulveraria & 1992 & 2014 \\
\cline { 2 - 4 } & Eustroma reticulata & 1985 & 2018 \\
\hline
\end{tabular}

Neun weitere Arten des bisherigen Bestandes müssen inzwischen als im Gebiet verschollen gemeldet werden (Tabelle 25):

Tab. 25: Im Gebiet im Beobachtungszeitraum als verschollen gemeldete Arten.

\begin{tabular}{|l|l|c|}
\hline \multicolumn{1}{|c|}{ Artengruppe } & \multicolumn{1}{|c|}{ Art } & verschollen seit \\
\hline Spinner \& Schwärmer & Dicallomera fascelina & 2000 \\
\hline \multirow{2}{*}{ Eulenfalter } & Athetis palustris & 1999 \\
\cline { 2 - 3 } & Opigena polygena & 1999 \\
\hline
\end{tabular}




\begin{tabular}{|l|l|c|}
\hline \multicolumn{1}{|c|}{ Artengruppe } & Art & verschollen seit \\
\hline Spanner & Charissa obscurata & 2002 \\
\cline { 2 - 3 } & Scotopteryx mucronata & 2003 \\
\cline { 2 - 3 } & Horisme vitalbata & 2003 \\
\cline { 2 - 3 } & Perizoma albulata & 2000 \\
\cline { 2 - 3 } & Perizoma sagittata & 2000 \\
\cline { 2 - 3 } & Eupithecia sinuosaria & 2003 \\
\hline
\end{tabular}

Tabelle 26 vermittelt eine Übersicht über die Veränderung des Artenbestandes im Beobachtungszeitraum 2014-2018.

Tab. 26: Übersicht über den Artenbestand.

\begin{tabular}{|c|c|c|c|c|c|}
\hline & Tagfalter & Spinner \& Schwärmer & Eulenfalter & Spanner & Summe \\
\hline $\begin{array}{l}\text { Gesamtartenzahl 3. Nachtrag } \\
\text { (RICHERT 2014) }\end{array}$ & 103 spec. & 195 spec. & 329 spec. & 270 spec. & 897 spec. \\
\hline $\begin{array}{l}\text { aktueller Bestand 3. Nachtrag } \\
\text { (Richert 2014) }\end{array}$ & 73 spec. & 179 spec. & 284 spec. & 247 spec. & 783 spec. \\
\hline Neufunde seit 2014 & 1 spec. $)^{1}$ & $1 \mathrm{spec}$. & 2 spec. $)^{2}$ & - & 4 spec. \\
\hline Gesamtartenzahl 4. Nachtrag & 104 spec. & 196 spec. & 331 spec. & 270 spec. & 901 spec. \\
\hline Wiederfunde seit 2014 & 1 spec. $)^{1}$ & - & 5 spec.) $)^{3}$ & 2 spec. & 8 spec. \\
\hline verschollen seit 2014 & - & 1 spec. & 2 spec. & 6 spec. & 9 spec. \\
\hline aktueller Bestand 2018 & 73 spec. & 179 spec. & 287 spec. & 243 spec. & $782 \mathrm{spec}$. \\
\hline \multicolumn{6}{|c|}{$\begin{array}{l})^{1} \text { : Der Neufund N. xanthomelas (zeitweise Fluktuation an der Arealwestgrenze) und der Wiederfund } \\
\text { (noch) nicht zum aktuellen Bestand im Gebiet gezählt. } \\
\text { ) }^{2} \text { davon eine Art (T. orichalcea) durch Verschleppung, zählt nicht zum aktuellen Bestand im Gebiet. } \\
)^{3} \text { : davon eine Art (S. scutosa) Wanderfalter/Irrgast, zählt nicht zum aktuellen Bestand im Gebiet. }\end{array}$} \\
\hline
\end{tabular}

Die Zunahme der im Gebiet insgesamt nachgewiesenen Arten kann nicht darüber hinwegtäuschen, dass gleichzeitig viele Arten verschwinden. Auffällig ist weiterhin der Rückgang der Populationsdichten vieler ehemals im Gebiet häufiger Arten.

\subsection{Analyse des Durchforschungsgrades der Naturräume des Gebietes}

Eine Übersicht über den Durchforschungsgrad der Naturräume des Gebietes vermittelt Tabelle 27 (siehe dazu auch 3.1. unter „Naturräumliche Zuordnung der Nachweise“):

Tab. 27: Durchforschungsgrad der Naturräume des Gebietes. ( $G=$ Gesamtartenzahl im Gebiet)

\begin{tabular}{|c|c|c|c|c|c|c|c|c|c|}
\hline Naturraum & a & $\mathrm{b}$ & c & $\mathrm{d}$ & $\mathrm{e}$ & f & $\mathrm{g}$ & $\mathrm{h}$ & G \\
\hline Tagfalter-Arten & 49 & 85 & 93 & 82 & 81 & 61 & 85 & 55 & 104 \\
\hline Spinner- \& Schwärmer-Arten & 86 & 160 & 184 & 162 & 141 & 100 & 158 & 105 & 196 \\
\hline Eulenfalter-Arten & 156 & 274 & 315 & 281 & 235 & 170 & 287 & 179 & 331 \\
\hline Spanner-Arten & 154 & 240 & 260 & 233 & 210 & 129 & 245 & 124 & 270 \\
\hline Summe Arten & 445 & 759 & 852 & 758 & 667 & 460 & 775 & 463 & 901 \\
\hline Anteil am Gesamtbestand & $49,4 \%$ & $84,2 \%$ & $94,6 \%$ & $84,1 \%$ & $74,0 \%$ & $51,1 \%$ & $86,0 \%$ & $51,4 \%$ & $100 \%$ \\
\hline
\end{tabular}

Trotz des Bemühens um eine gleichmäßige Durchforschung (siehe dazu auch Richert 2010: 125-126) weisen die Naturräume des Gebietes in dieser Hinsicht erhebliche Unterschiede auf. Der Naturraum c (Eberswalder Tal) hat den höchsten Anteil an der Gesamtzahl der im Gebiet festgestellten Arten. Das ist einerseits Ausdruck des Falterreichtums dieses Naturraumes, andererseits jedoch das Ergebnis des hohen Durchforschungsgrades: Hier sind (waren) aktive Entomologen beheimatet, die regelmäßig über längere Zeiträume kontinuierlich sowohl die Tag- als auch die Nachtfalterfauna (Licht- und Köderfänge) beobachtet haben. 
Es folgen die „Odertalhänge“(g), die keinen Naturraum im eigentlichen Sinne darstellen. Es handelt sich um klimatisch begünstigte und deshalb faunistisch besonders wertvolle Abhänge angrenzender Hochflächen. Sie wurden wegen ihrer faunistischen Bedeutung gesondert aufgeführt (vgl. Richert 1999: 7). Dort befinden sich mehrere über Jahre gut bearbeitete NSG (u. a. NSG Krähen- und Jungfernberge, NSG Pimpinellenberg). Der vermutete Falterreichtum hat sich bestätigt.

In den Naturräumen Barnimplatte (b), Britzer Platte und Schorfheide (d) und Uckermärker Kuppen und Hügelland einschließlich Choriner Waldhügel (e), die in der Rangordnung nach dem Durchforschungsgrad folgen, befinden sich einerseits zwei ehemalige TÜP (Refugien für viele gefährdete Arten), andererseits mehrere NSG, die alle gut bearbeitet sind.

Die Naturräume Odertal (h) (die Flussniederung), Parsteiner Becken (f) (weitgehend landwirtschaftlich genutzt) und Oberbarnimer Waldhügel (a) (mit nur kleinflächigem Anteil am bearbeiteten Gebiet) sind nur lückenhaft untersucht.

Somit bieten die Diluviallandschaften um Eberswalde auch weiterhin ein umfangreiches Potential für faunistische Forschung.

\section{Literatur}

BARTsCH, D. 2001: Geometrinae. - In: EBERT, W. (Hrsg.) (2001): Die Schmetterlinge Baden-Württembergs. Band 8 Nachtfalter VI. - Verlag Eugen Ulmer, Stuttgart 2001: 541 S.

Borkowski, A. 1996: Schmetterlinge (Großschmetterlinge - Macrolepidoptera). (Abschnitt 3.11 im Gewässerrandstreifenprogramm Unteres Odertal - Teil Fauna). - Unveröffentlichtes Manuskript. (Kopie im Literaturbestand Richert).

Brauner, O. 2014: Kartierung der FFH-Art Großer Feuerfalter (Lycaena dispar) im Rahmen der B 167 Ortsumgehung Eberswalde, Manuskript: 17 S. (PDFDatei).

Eckstein, K. 1923: Die Schmetterlinge Deutschlands. 4. Band. Die Spanner und die bärenartigen Falter. K. G. Lutz Verlag, Stuttgart: 88 S., 16 Tafeln.

Fibiger, M.; Ronkay, L.; Steiner, A. \& Zilli, A. 2009: Pantheinae - Bryophilinae. Noctuidae Europaeae, Vol. 11. - Entomological Press. Sorǿ: 504 pp.

Gaedike, R.; Nuss, M.; Steiner, A. \& Trusch, R. (Hrsg.) 2017: Verzeichnis der Schmetterlinge Deutschlands (Lepidoptera). (2. überarbeitete Auflage). - Entomologische Nachrichten und Berichte, Dresden, Beiheft 21: $1-362$.

Hausmann, A. 2001: The Geometrid Moth of Europe. Vol. 1. - Apollo Books, Stenstrup: 282 pp.

FAJČIK, J. (Hrsg.) 1998: Die Schmetterlinge Mittel- und Nordeuropas. II. Band Noctuidae, Bratislava: 170 S., 42 Tafeln.
FAJČıK, J. (Hrsg.) 2003: Die Schmetterlinge Mittel- und Nordeuropas. Drepanidae, Geometridae, Lasiocampidae, Endromidae, Lemoniidae, Saturniidae, Sphingidae, Notodontidae, Lymantriidae, Arctiidae. Bratislava: 172 S., 60 Tafeln.

Forster, W. \& Wohlfahrt, T. A. 1981: Die Schmetterlinge Mitteleuropas. Band V Spanner. - Franckh'sche Verlagsbuchhandlung Stuttgart: 312 S., 26 Tafeln.

Forster, W. \&Wohlfahrt, T. A. 1984:DieSchmetterlinge Mitteleuropas. Band 3 Spinner und Schwärmer (Bombyces und Sphinges). 2. Aufl. - Franckh'sche Verlagshandlung Stuttgart: 239 S., 28 Tafeln.

FreinA, J. J. DE \& WitT, T. 1987: Die Bombyces und Sphinges der Westpalaearktis Band 1. - Edition Forschung \& Wissenschaft GmbH, München: 708 S.

Gelbrecht, J. \& Seiger, G. 1999: Euxoa hastifera (Donzel, 1848) - neu für Deutschland und Spaelotes suecica (Aurivillius 1889) - Wiederfund für Deutschland sowie weitere neue und wiedergefundene Schmetterlinge in Berlin und Brandenburg (Lepidoptera). Zur Faunistik und Ökologie der Schmetterlinge in der Mark Brandenburg. XIV. Entomologische Nachrichten und Berichte, Dresden 43 (2): 101-108.

Gelbrecht, J. \& Kallies, A. 2001: Aktuelle Verbreitung von Malacosoma franconica (DENIs \& SChiffermüller, 1775) in Deutschland. - Märkische Entomologische Nachrichten, Potsdam 3 (1): 11-20.

Gelbrecht, J.; Göritz, U. \& OCKRUCK, F. 2011: Vorübergehende Arealerweiterung von Malacosoma franconica (DENIS \& SCHIFFERMÜLLER, 1775) im Norden Brandenburgs? - Märkische Entomologische Nachrichten, Potsdam 13 (1): 67-74.

Gelbrecht, J.; RÄmisch, F.; Reinhardt, R.; Richert, A. \& Schmiтt, T. 2015: Der Silbergrüne Bläuling Polyommatus coridon (PoDA, 1761). - Insekt des Jahres 2015 (Deutschland, Österreich, Schweiz): Einwanderungsgeschichte, aktuelle Verbreitung und Gefährdung in Brandenburg und Berlin (Lepidoptera, Lycaenidae). - Märkische Entomologische Nachrichten, Potsdam 17 (1): 37-51.

Gelbrecht, J.; Clemens, F.; Kretschmer, H.; LANDECK, I.; LANDECK, R.; ReINHARDT, R.; Richert, A.; Schmitz, O. \& Rämisch, F. 2017: Die Tagfalter von Brandenburg und Berlin (Lepidoptera: Rhopalocera und Hesperiidae). - Naturschutz und Landschaftspflege in Brandenburg, Potsdam 25 (3/4) (2016): 1-327

GotTwald, F. 2010: Tagfalter. - In: Stein-BACHINGer, K.; Fuchs, S.; Gottwald, F.; Helmecke, A.; Grimm, J.; Zander, P.; Schuler, J.; Bachinger, J. \& GotTschall, R.: Naturschutzfachliche Optimierung des ökologischen Landbaus „Naturschutzhof Brodowin“. Ergebnisse des E+E-Projektes „Naturschutzhof Brodowin“. - Naturschutz und biologische Vielfalt. - Bundesanstalt für Naturschutz BonnBad Godesberg, Heft 90: 409 S. 
Hausmann, A. 2001: The Geometrid Moths of Europe. Vol. 1. - Apollo Books, Stenstrup: 282 pp.

Jäger, E.; Ebel, F.; Hanelt, P. \& Müller, G. (Hrsg.) 2008: Rothmaler. Exkursionsflora von Deutschland. Band 5. Krautige Zier- und Nutzpflanzen. - SpringerVerlag, Berlin, Heidelberg: 874 S.

Косн, M. 1984: Wir bestimmen Schmetterlinge. Ausgabe in einem Band, bearb. von W. HeINICKE. -Neumann Verlag Leipzig, Radebeul: 790 S.

Lussi, H. G. 1997: Chloephorinae. - In: EBERT, W. (Hrsg.): Die Schmetterlinge Baden-Württembergs. Band 5 Nachtfalter III. - Verlag Eugen Ulmer, Stuttgart: 575 S.

MülleR, M. 2015: Vergleichende Untersuchungen der nachtaktiven Großschmetterlinge (Lepidoptera, Macroheterocera) auf Windwurfflächen in TieflandBuchenwäldern der Choriner Endmoräne. - Bachelorarbeit. Hochschule für Nachhaltige Entwicklung Eberswalde: $97 \mathrm{~S}$.

Ockruck, F. \& Gelbrecht, J. 2011: Weitere Arealerweiterung von Malacosoma franconica (Denis \& SChiffermüller, 1775) in Brandenburg im Jahre 2011 (Lepidoptera, Lasiocampidae). Märkische Entomologische Nachrichten, Potsdam 13 (2): 245-249.

Pro Natura- Schweizerischer Bund für Naturschutz (Hrsg.) 2000: Schmetterlinge und ihre Lebensräume. Arten, Gefährdung, Schutz. Band 3. - Verlag Neue Medien, Egg: 914 S.

Ratzel, U. \& Ratzel, M. 1997: Die Gattungen Spilosoma und Diaphora. - In: EBERT, W. (Hrsg.): Die Schmetterlinge Baden-Württembergs. Band 5 Nachtfalter III. - Verlag Eugen Ulmer, Stuttgart 1997: 575 S.

Richert, A. 1977: Ernst Duckert zum Gedenken (1901-1975). - Entomologische Berichte, Berlin (1): 69-70.

Richert, A. 1979: Die Schmetterlingsfauna des Naturschutzgebietes Krähen- und Jungfernberge im Kreis Eberswalde. 1. Beitrag. - Naturschutzarbeit in Berlin und Brandenburg, Potsdam 15: 82-89.

Richert, A. 1982: Die Schmetterlingsfauna des Naturschutzgebietes Krähen- und Jungfernberge im Kreis Eberswalde. 2. Beitrag. - Naturschutzarbeit in Berlin und Brandenburg, Potsdam 18: 20-23.

RicherT, A. 1984: Bemerkenswerte Schmetterlingsfunde auf den Oderhängen im Kreis Bad Freienwalde - ein neuer Fundort von Procris chloros Нв. - Entomologische Nachrichten und Berichte, Dresden 28: 57-62.

Richert, A. 1994: Die Schmetterlinge (Lepidoptera) des Pimpinellenberges und des Teufelsberges bei Oderberg (Mark), Teil 2. - Brandenburgische Entomologische Nachrichten, Potsdam 2 (1): 5-30.

RicherT, A. 1999: Die Großschmetterlinge (Macrolepidoptera) der Diluviallandschaften um Eberswalde. Teil I. Allgemeiner Teil und Tagfalter. - Deutsches Entomologisches Institut (Hrsg.), Eberswalde: $61 \mathrm{~S}$.
RIChert, A. 2001: Teil II Spinner und Schwärmer. Deutsches Entomologisches Institut, Zentrum für Agrarlandschafts- und Landnutzungsforschung Müncheberg (Hrsg.), Eberswalde: $80 \mathrm{~S}$.

Richert, A. 2003: Teil III Die Eulenfalter (Noctuidae et Pantheidae). - Deutsches Entomologisches Institut, Zentrum für Agrarlandschafts- und Landnutzungsforschung Müncheberg (Hrsg.), Eberswalde, $105 \mathrm{~S}$.

Richert, A. 2004: Teil IV Die Spanner (Geometridae). Erster Nachtrag zu den Teilen I-III. - Deutsches Entomologisches Institut, Leibniz-Zentrum für Agrarlandschafts- und Landnutzungsforschung Müncheberg (ZALF) e.V. (Hrsg.): $141 \mathrm{~S}$.

RicherT, A. 2005: Historische und aktuelle Untersuchungsergebnisse zur Schmetterlingsfauna des NSG Plagefenn und benachbarter Moore im Biosphärenreservat Schorfheide-Chorin (Nordostbrandenburg). - Märkische Entomologische Nachrichten, Potsdam 7 (2): 23-44.

Richert, A. 2006: Schmetterlinge (Lepidoptera) auf den ehemaligen Truppenübungsplätzen „Trampe“ und „Panzerbahn“ im Landkreis Barnim (Nordostbrandenburg). - Märkische Entomologische Nachrichten, Potsdam 8 (1): 1-36.

Richert, A. 2010: Die Großschmetterlinge (Macrolepidoptera) der Diluviallandschaften um Eberswalde. Zweiter Nachtrag 2010. - Senckenberg Deutsches Entomologisches Institut (Hrsg.), Müncheberg: $162 \mathrm{~S}$.

Richert, A. 2010a: Schmetterlinge (Lepidoptera) im NSG „Kienhorst/Köllnseen/Eichheide“ (Biosphärenreservat Schorfheide - Chorin), Nordostbrandenburg. - Märkische Entomologische Nachrichten, Potsdam 12 (1): 1-42.

Richert, A. 2012: Schmetterlinge (Lepidoptera) im NSG „Buckowseerinne“ (Biosphärenreservat Schorfheide-Chorin), Nordostbrandenburg. - Märkische Entomologische Nachrichten, Potsdam 14 (2): 251-280.

Richert, A. 2014: Die Großschmetterlinge (Macrolepidoptera) der Diluviallandschaften um Eberswalde. Dritter Nachtrag mit einer Darstellung phänologischer Veränderungen im Zeitraum 19892013 und einer Betrachtung über die Ursachen. - Nova Supplementa Entomologica, Senckenberg Deutsches Entomologisches Institut, Müncheberg 24: 1-284.

RicherT, A. 2015: Erstnachweis von Thysanoplura orichalcea (FABRICIUs, 1775) in Brandenburg (Lepidoptera, Noctuidae). - Märkische Entomologische Nachrichten, Potsdam 17 (1): 128.

Richert, A. 2016: Fred Kittel. - In mermoriam. Märkische Entomologische Nachrichten, Potsdam 18 (1/2): 187-188. 
RicheRT, A. 2017: Schmetterlinge (Lepidoptera) im NSG Krähen- und Jungfernberge bei Stolzenhagen (Barnim, Brandenburg) 1977-2017. - NationalparkJahrbuch Unteres Odertal, Verlag Nationalparkstiftung Unteres Odertal, Schloss Criewen, Schwedt/Oder 14: 46-67.

Richert, A. \& HülberT, D. 1991: Auswertung fünfjähriger Lichtfallenfänge (1984-1988) auf der Phänobasis Hohenfinow (Kreis Eberswalde-Finow) für die Lepidopterenfaunistik. - Beiträge zur Entomologie, Berlin 41 (1): 251-264. - https://www.contributions-to-entomology.org/article/view/1327/1326.

Richert, A. \& Brauner, O. 2018: Nektarpflanzen und andere Nahrungsquellen sowie Raupennahrungspflanzen der Tagfalter von Brandenburg und Berlin (Lepidoptera: Rhopalocera et Hesperiidae). Märkische Entomologische Nachrichten, Potsdam 20 (2): 155-240.

SCHMIDT, P. 1991: Beiträge zur Insektenfauna der DDR: Lepidoptera - Arctiidae, Nolidae, Ctenuchidae, Drepanidae, Cossidae, Hepialidae. - Beiträge zur Entomologie, Berlin 41 (1): 123-236. - https://www. contributions-to-entomology.org/article/view/ $1324 / 1323$.

Segerer, A. H. 2017: Schmetterlinge im Sturzflug - Erkenntnisse aus der Inventur der Lepidoptera Bayerns. - Entomologische Nachrichten und Berichte, Dresden 60 (3/4): 169-174.
Segerer, H. \& Hausmann, A. 2011: Die Großschmetterlinge Deutschlands. - Heterocera Press, Budapest: $308 \mathrm{~S}$.

Seyboldt, S. 2009: Schmeil - Fitschen Flora von Deutschland und angrenzender Länder, 94. Aufl. Quelle \& Meyer Verlag, Wiebelsheim: 863 S.

Steiner, A. 1994: Nolidae. - In: Ebert, W. (Hrsg.): (1994): Die Schmetterlinge Baden-Württembergs. Band 4. Nachtfalter II. - Verlag Eugen Ulmer, Stuttgart: 535 S.

Steiner, A. 1997: Pantheinae. - In: Ebert, W. (Hrsg.): Die Schmetterlinge Baden-Württembergs. Band 5 Nachtfalter III. - Verlag Eugen Ulmer, Stuttgart: 575 S.

Steiner, A. 1997: - In: Ebert, G. (Hrsg.): Die Schmetterlinge Baden-Württembergs. Band 6 Nachtfalter IV. - Eugen Ulmer, Stuttgart: 622 S.

Steiner, A.; Ratzel, U.; Top-Jensen, M. \& Fibiger, M. 2014: Die Nachtfalter Deutschlands. Ein Feldführer. Østermarie, Bugbook Publishing: 878 S., 76 Farbtafeln.

Vössing, A. 1998: Der Internationalpark Unteres Odertal. Ein Werk- und Wanderbuch. - Stapp Verlag, Berlin: 313 S.

Weisbach, P. \& Gelbrecht, J. 2013: Faunistische Notizen - Zweitnachweis von Athetis lepigone (Möschler, 1860) in Sachsen - wo kommt die Art in Brandenburg vor? - Märkische Entomologische Nachrichten, Potsdam 15 (1): 81-82. 


\section{Register}

A

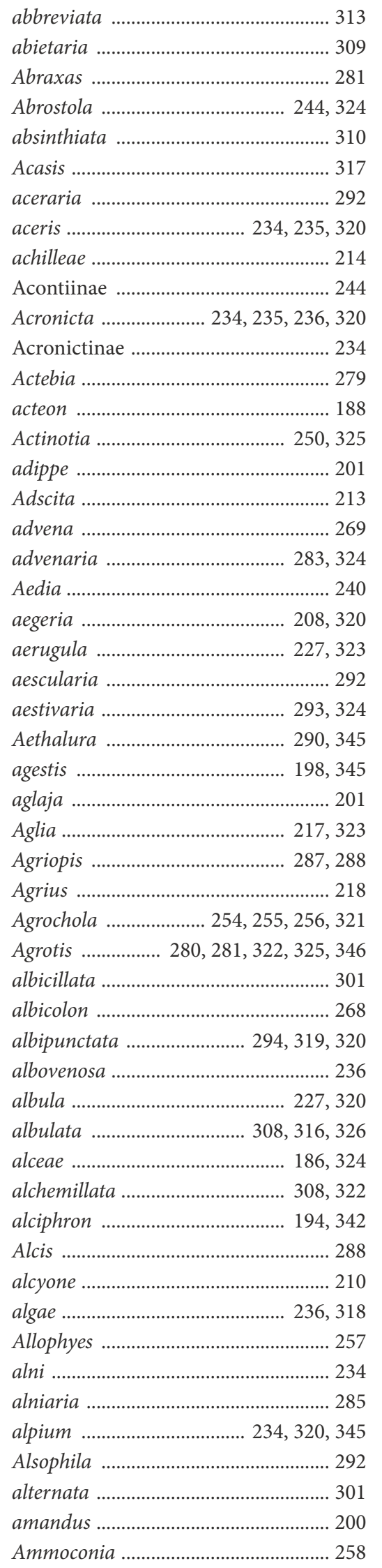

amphidamas ......................................... 195 Amphipoea ................................... 262, 341 Amphipyra ............................................ 247

Amphipyrinae …………………….... 247

anachoreta .................................. 223, 320

Anaplectoides ....................................... 279

Anarta ........................................ 265, 340

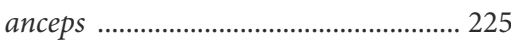

ancilla ....................................... 232, 324

Angerona ..................................... 286, 324

annularia ..................................... 294, 319

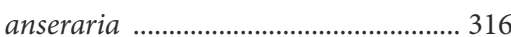

Anthocharis ................................. 192, 318

Anticlea ............................................. 301

Anticollix .............................................. 315

antiopa ..................................... 204, 320

Antitype ………………………….......... 257

Apamea ............................... 259, 260, 345

Apatura .................................................. 208

Apaturinae ............................................. 208

Apeira ..................................................... 284

Aphantopus .......................................... 210

aphirape ............................................... 202

Aplasta .................................................... 292

Aplocera …………………………..... 315

Apocheima …………………………......... 287

Aporia ..................................................... 191

Aporophila ........................................... 256

aprilina .................................................. 257

Araschnia ........... 205, 321, 322, 323, 325

arcania ................................. 209, 323, 343

Archanara ............................................ 264

Archiearis ................................................ 281

Arctia .......................................... 180, 233

Arctiidae ................................................ 228

Arctiinae ................................................. 232

Arctornis ................................... 226, 323

Arenostola .................................... 264, 345

argentea ...... 194, 198, 200, 209, 210, 245

argiades .............................................. 197

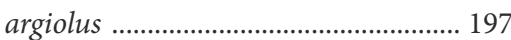

Argynnis ..................................... 200, 201

Arichanna ................................... 289, 341

Aricia ................................................... 345

armigera ................................................. 248

artemisiae ................................................. 245

Ascotis ....................................... 289, 322

asella .......................................... 213, 323

assimilata ............................................... 311

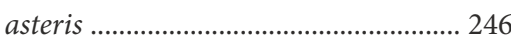

Asteroscopus .......................................... 247

Asthena ................................................... 316

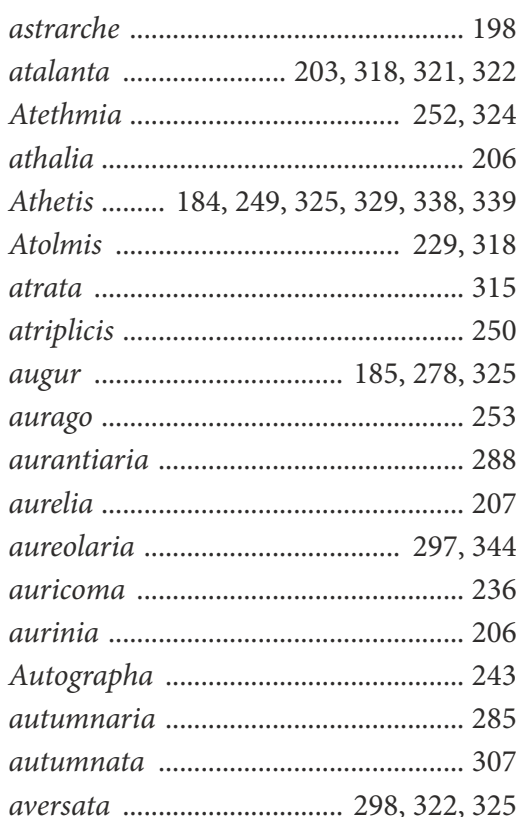

\section{B}

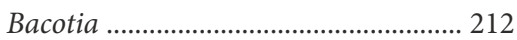

bajularia ................................................ 293

batis .......................... 220, 320, 321, 322

Bena ............................ 228, 320, 321, 345

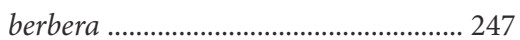

berberata ..................................... 306, 319

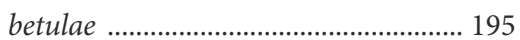

betulina ................................................... 212

biangulata ................................... 307, 321

bicolorana ................... 228, 320, 321, 345

bicolorata ............ 185, 267, 324, 325, 339

bicoloria ................................................. 225

bicuspis ........................................ 223, 320

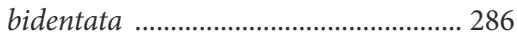

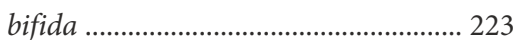

bigramma ............................................. 280

bilineata ..................................................... 301

bimaculata ............................................ 290

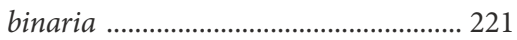

biriviata ...................................... 299, 322

biselata ..................................... 297, 324

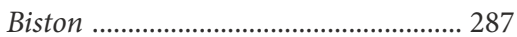

blanda .......................................... 248, 324

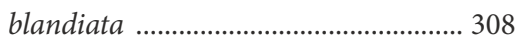

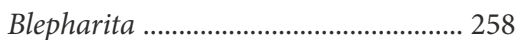

Boloria ......................................... 202, 203

bombycina ........................................... 269

Brachionycha ....................................... 247

brassicae ............ 191, 269, 318, 323, 343

Brenthis ................................................. 202

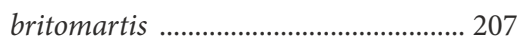




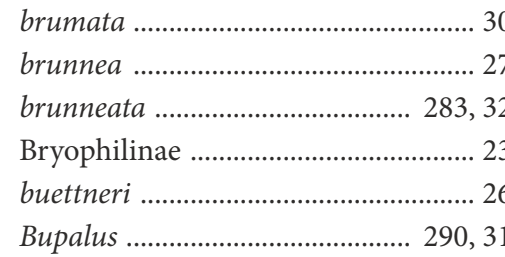

\section{C}

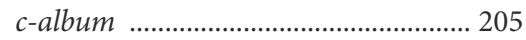

Cabera ................................................ 290

caecimacula ......................................... 258

caeruleocephala ..................................... 247

caja ...................................................... 233

Calamia ............................... 263, 319, 321

Callimorpha .............................. 233, 323

Callistege ...................................... 241, 318

Callophrys ............................................... 197

Callopistria ............................................ 250

Calophasia ............................................. 246

Calpinae ................................................ 241

camilla .................................................. 208

Campaea ..................................... 291, 324

Camptogramma .................................... 301

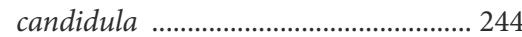

Canephora …........................................... 212

capitata ................................................. 303

Carcharodus ................................ 186, 324

cardamines .................................. 192, 318

cardui .......................... 204, 320, 321, 323

carmelita ................................................. 225

carpinata .................................................. 317

Carterocephalus ........................... 187, 341

castaneae .................................... 216, 321

Catarrhoe ................................... 300, 301

Catocala .............................. 238, 239, 322

Catocalinae ............................................ 238

Celaena ....................................... 263, 340

Celastrina ................................................. 197

celsia .................................................... 263

centaureata …………………………..... 310

centrago ........................................ 252, 324

Cepphis ...................................... 283, 324

Cerapteryx ............................................... 273

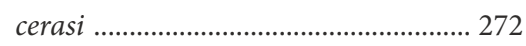

Cerastis ..................................................... 278

Cerura ................................................... 223

cervinalis ................................................. 306

cespitis ...................................................... 273

chaonia ................................................... 224

Charanyca ............................................... 249

Charissa ..................................... 291, 326

Chesias ..................................................... 315

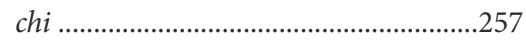

Chiasmia ................................................. 283
Chilodes

... 249

Chloephorinae

227,328

chloerata

.... 315

Chloroclysta

303, 304, 319

Chloroclystis

314,319

chlorosata ............................................... 283

Chortodes …….......................................... 265

christyi .................................................. 307

chrysitis ...................... 242, 243, 319, 320

chrysoprasaria ....................................... 293

Cidaria .................................................... 304

Cilix ......................................................... 222

cinctaria ................................................. 288

cinxia .................................................... 206

circellaris ............................................... 254

citrago .......................................... 254, 319

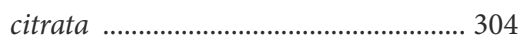

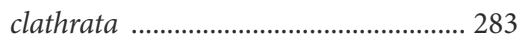

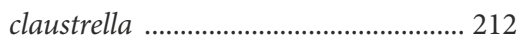

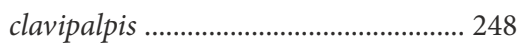

clavis ...................................................... 280

Cleora ........................................................ 288

Clostera .............................. 222, 223, 320

Coenobia ................................................ 265

coenobita ............................. 281, 319, 320

Coenonympha .... 208, 209, 318, 323, 343

Colias .......................................... 190, 345

Colobochyla ........................................... 242

Colocasia ............................ 281, 319, 322

Colotois ..................................................... 286

comes ...................................................... 276

Comibaena ................................................ 293

comitata ................................................... 302

comma ....................................... 188, 271

complana ........... 230, 231, 321, 322, 339

compta ............................................... 267

confusa ...................................... 243, 267

confusalis ..................................... 227, 321

conigera ..................................... 269, 322

Conistra ............................................. 256

consonaria ............................................... 289

conspicillaris .......................................... 273

contigua .................................................. 266

convolvuli .............................................. 218

coridon ................. 199, 327, 343, 345, 346

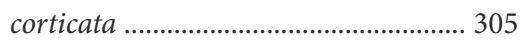

corticea ................................................. 280

corylata .................................................. 305

coryli .................................. 281, 319, 322

Coscinia .............................. 232, 340, 342

Cosmia ......................................... 252, 324

Cosmorhoe ............................................. 302

Cossidae ...................................... 216, 329

Cossus ....................................... 216, 323
Costaconvexa ........................................ 301

costaestrigalis ....................... 238, 320, 322

craccae .................................................. 240

Craniophora ................................. 236, 320

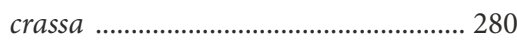

crassalis ................................................ 241

crassiorella .............................................. 212

crataegi .................................................. 191

crenata ................................ 225, 259, 321

crepuscularia ......................................... 289

cribraria .................................... 232, 340

cribrumalis ............................................ 237

Crocallis ................................................. 286

crocea ..................................................... 190

cruentaria ............................................... 299

Cryphia ...................................... 236, 318

cuculata .................................................... 301

cuculla .................................................... 225

cucullatella .............................................. 227

Cucullia ............. 245, 246, 319, 320, 342

Cuculliinae .............................................. 245

cucullina .................................................... 225

curtula .................................................... 222

curvatula .............................................. 221

Cybosia ......................................... 229, 321

Cyclophora ................. 294, 295, 319, 320

Cymatophorina ...................................... 221

\section{D}

Dahlica ..................................................... 212

daphne ...................................................... 202

debiliata ....................................... 315, 319,

decimalis .................................................. 273

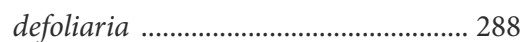

Deilephila ............................................... 220

Deltote ....................................................... 244

denotata .................................................. 311

dentaria .................................................. 286

deplana ..................................................... 230

depressa ..................................................... 230

derasa ..................................................... 220

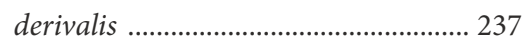

derivata ..................................................... 301

designata .................................... 299, 319

deversaria ................................................. 298

dia ......................................................... 203

Diacrisia .............................. 233, 321, 325

diamina .................................................... 206

Diaphora .............................................. 328

Diarsia ........................................... 274, 275

Dichonia ................................................ 257

dictaeoides ................................................. 225

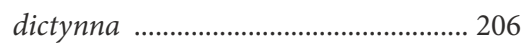

didyma ..................................................... 261 


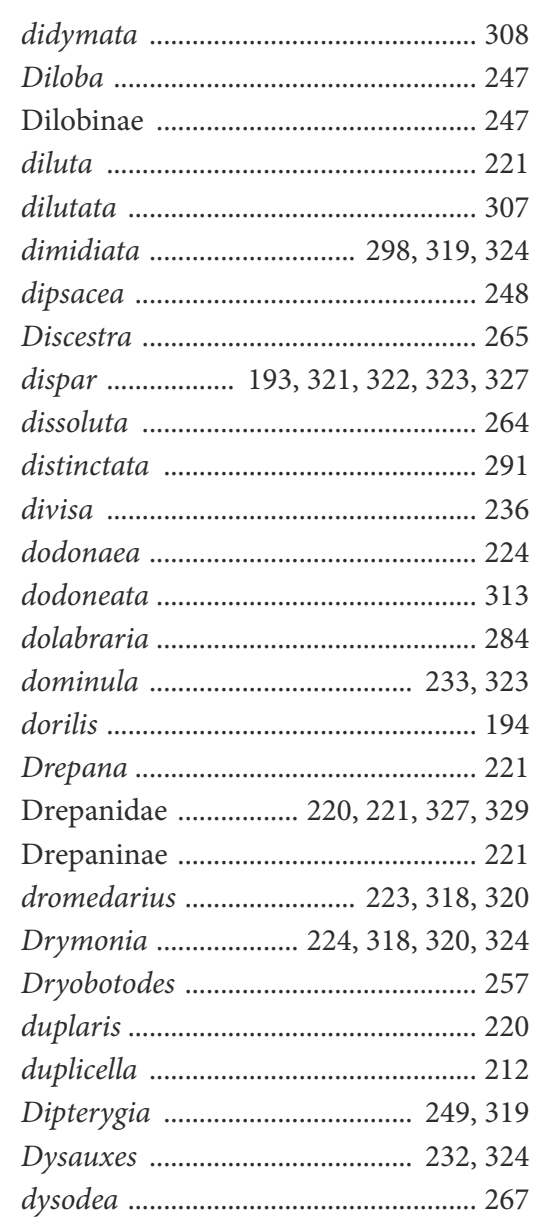

\section{$\mathrm{E}$}

Earias ………………………………..... 228

Ecliptopera ............................................. 303

Ectropis ................................................... 289

edusa .................................................... 192

egenaria ....................................... 310, 319

Egira …............................................... 273

Eilema ................ 230, 231, 321, 322, 339

Elaphria ....................................... 248, 319

Electrophaes ............................................. 305

elinguaria ................................................ 286

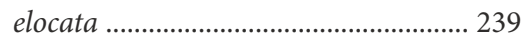

elpenor ................................................... 220

emarginata ............................................... 298

Emmelia ..................................... 244, 320

emortualis .......................... 245, 322, 324

Enargia .................................................. 251

Endromidae .................................. 217, 327

Endromis ................................................. 217

Ennomos ....................................... 285, 324

ephialtes .................................................. 215

Epichnopteryx ............................. 212, 340

Epilecta ................................................... 277

Epione ……………………... 284, 319, 324

Epirrhoe ……............................................ 301

Epirrita ..................................................... 307

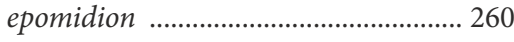

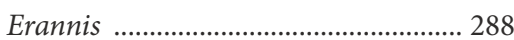

eremita .................................................. 257

Eremobia ............ 177, 184, 261, 325, 338

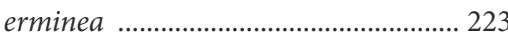

erosaria ..................................... 285, 324

eruta ......................................... 279, 280

Erynnis ....................... 186, 318, 321, 322

erythrocephala ..................................... 256

Eublemma ............................................ 245

Euchoeca ............................................... 316

Eulithis ......................................... 302, 303

eunomia ................................................. 202

euphorbiae ................................... 219, 323

euphrosyne ................................................. 203

Euphydryas ............................................ 206

Euphyia ....................................... 307, 321

Eupithecia ................ 308-314, 319, 321,

$322,324,326$

Euproctis .............................................. 226

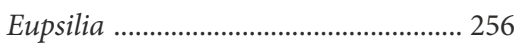

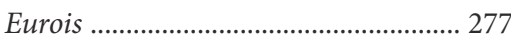

Eustroma .................... 177, 185, 305, 325

Eustrotiinae ............................................ 244

Euxoa ................................ 279, 280, 327

exanthemata .......................................... 290

exclamationis ....................... 280, 322, 325

exiguata ............................................... 309

\section{F}

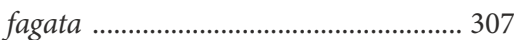

Falcaria ................................................ 205

farinata ................................................. 316

fasciana ................................................... 244

fasciaria ............................................... 291

fasciolaria ............................................ 283

fasciuncula ............................................. 261

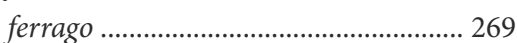

ferrugata .............................................. 300

festucae ................................................. 243

filipendulae ................................. 215, 323

fimbrialis ..................................... 293, 340

firmata ................................................. 304

fissipuncta ............................................ 252

flammea ..................................... 271, 273

flammeolaria ........................................ 316

flavago .................................. 185, 263, 325

flavofasciata ......................................... 308

flexula ......... 241, 320, 322, 323, 325, 339

florida .................................................... 275

floslactata ............................................... 296

fluctuata ............................................... 300

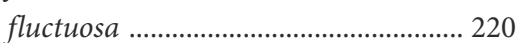

fontis ..................................................... 241

fraudatrix ..................................... 245, 319

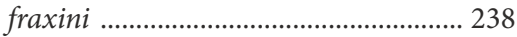

fuciformis ................................................ 218

fucosa ...................................... 262, 324

fuliginaria .................................. 242, 324

fuliginosa ............................ 232, 323, 346

fulminea ................................................. 273

fulvago .................................................... 253

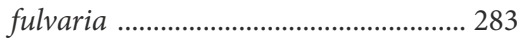

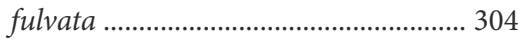

fumosella .............................................. 212

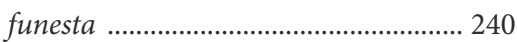

furcata .................................................. 319

furcifera .................................................. 257

Furcula .................................... 223, 320

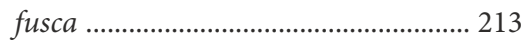

fuscantaria ........................................... 285

fusconebulosa ......................................... 211

fuscovenosa ............................................. 297

G

galathea ..................................... 211, 323

galii ........................................................... 219

gallii ......................................... 219, 320

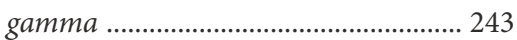

Gastropacha ............................... 217, 342

geminipuncta ........................................ 264

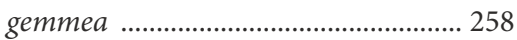

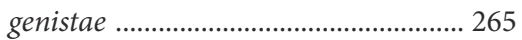

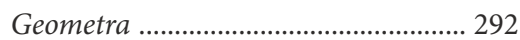

glarearia ..................................... 282, 343

glaucata ................................................... 222

Gluphisia ................................... 225, 321

glycerion .................................................. 209

glyphica ............................................... 241

gnoma ........................................ 225, 321

Gonepteryx .................................. 191, 343

Gortyna ............................. 185, 263, 325

gothica ........................................ 272, 321

gracilis .............................. 243, 272, 319

graminis ................................................. 273

Graphiphora ...................... 185, 278, 325

grisealis ................................................. 237

griseata ................................................ 315

Gymnoscelis ................................ 314, 319

H

Habrosyne ............................ 220, 322, 325

Hada ............................................. 267, 320

Hadena ........................................ 267, 268

Hadeninae ............................................... 248

Hadula....................................................... 265

halterata .................................................. 316

harpagula ………….................... 221, 342

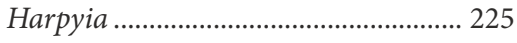

haworthiata............................................ 309 
haworthii 263,340

Hecatera .............. 185, 267, 324, 325, 339

$$
\text { hecta }
$$
$185,267,324,325,339$

Heliconiinae ……................................ 200

Helicoverpa ............................................ 248

Heliomata................................................ 282

Heliophobus ............................................ 268

Heliothinae ............................................. 248

Heliothis ..................................................... 248

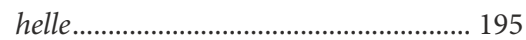

helvola ................................................... 254

Hemaris .................................................. 218

Hemistola ................................................ 293

Hemithea ..................................... 293, 324

hepatica ........................................ 260, 269

Hepialidae ................................... 211, 329

Hepialus ................................................... 211

Herminia ............................................. 237

Herminiinae .......................................... 237

hermione ……………………………..... 210

Hesperia ................................................. 188

Hesperiidae ................................... 186, 329

Heterogenea ............................... 213, 323

Heteropterus .......................................... 187

Hipparchia ........................... 210, 211, 324

hippothoe ............................................... 195

hirsuta ....................................... 190, 212

hirsutella ................................................. 213

hirtaria ................................................. 287

hispidaria .......................................... 287

Hoplodrina .......................... 248, 249, 324

Horisme ............. 305, 306, 322, 324, 326

humidalis ...................................... 238, 340

humiliata .................................... 297, 320

humuli .................................................... 211

hyale ............................................ 190, 345

Hydraecia ........................................... 262

Hydrelia ................................................... 316

Hydriomena ................................. 305, 319

Hylaea .................................................... 291

Hyles .................................. 219, 320, 323

Hyloicus .................................................. 218

Hypena ................................................... 241

Hypeninae ............................................... 241

Hypenodes ................................... 238, 340

Hypenodinae …………………………... 238

hyperantus .............................................. 210

Hypomecis .................................... 289, 320

I

icarus .......................................... 200, 345

icterata .................................................. 311

icteritia .................................................... 253

Idaea ................... 296-298, 319-325, 344

idas

$198,323,340$

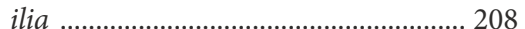

immorata 295

immutata .................................... 296, 320

impluviata .............................................. 305

indigata ..................................... 312, 319

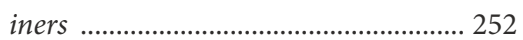

innotata ................................................. 312

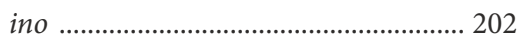

interjecta ..................................... 277, 320

intricata ……................................................ 310

inturbata ................................... 309, 319

io ............................................... 204, 323

iphis ......................................................... 209

Ipimorpha .................................. 251, 319

iris ............................................. 188, 208

irregularis ………………………............ 268

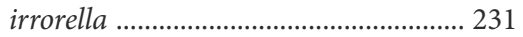

Issoria .................................................... 201

Itame .................................... 283, 324

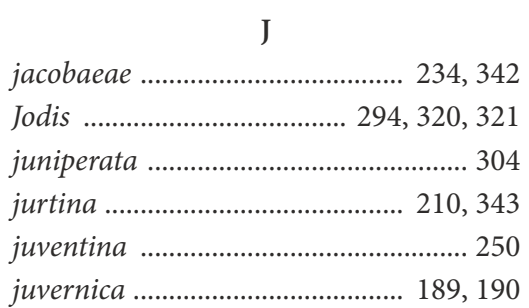

L

l-nigrum ....................................... 226, 323

Lacanobia ........... 265-267, 319-321, 323

lactearia ..................................................... 294

laevis ........................................... 256, 321

Lampropteryx ....................................... 302

lanceata ...................................... 313, 322

lariciata .................................... 314, 319

Lasiocampa ............................................ 217

Lasiocampidae ........... 183, 216, 327, 328

Lasiommata ............................................ 208

Laspeyria .... 241, 320, 322, 323, 325, 339

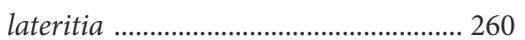

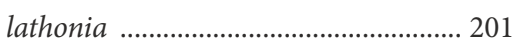

legatella ................................................... 315

lepida .................................................... 268

Leptidea ............................... 189, 190, 341

Leucodonta ................................................ 225

leucographa ............................................ 278

Leucoma ............................................... 226

leucophaearia .......................................... 287

leucostigma ............................................ 263

levana .......................... 205, 321-323, 325

libatrix .................................................. 241

ligustri ................ 218, 236, 320, 322, 323

Limacodidae ......

.... 213
Limenitinae .......................................... 207

Limenitis ...................................... 207, 208

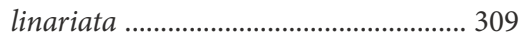

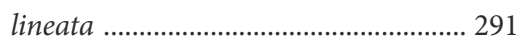

lineola .................................................... 188

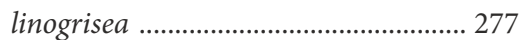

lithargyria ............................................... 269

Lithomoia .................................................. 256

Lithophane ................................. 256, 257

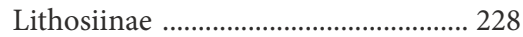

Lithostege .................................... 315, 316

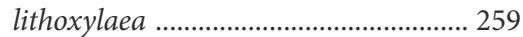

litura ....................................................... 255

Lobophora .................................................. 316

Lomographa ........................................... 290

lonicerae .............................. 215, 322, 323

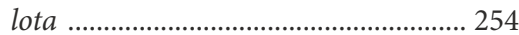

loti .......................................... 214, 323

lubricipeda .......................... 232, 320, 339

lucens .......................................... 262, 341

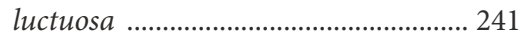

lunula ..................................................... 246

Luperina ....................................... 261, 319

lutarella .................................................. 231

lutea ....................................................... 252

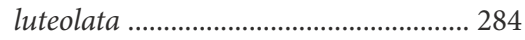

lutosa .................................................... 262

lutulenta ........................................... 256

Lycaena ................ 193-195, 318, 321-323

$327,342,345$

Lycaenidae ................................... 193, 327

lycaon .................................................... 210

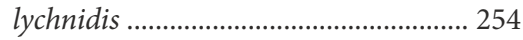

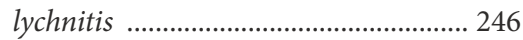

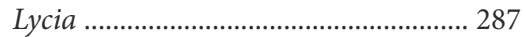

Lygephila .................................... 240, 324

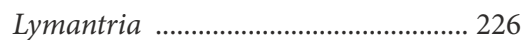

Lymantriidae .............................. 226, 327

Lythria .......................................... 299, 319

\section{M}

Macaria ............................... 282, 319, 324

machaon ........................................ 189, 343

macilenta ................................................ 254

Macrochilo ............................................... 237

Macroglossum ............................ 219, 345

macularia ............................................ 284

Malacosoma ............... 183, 216, 217, 323,

$325,327,328,338$

malvae ................................................... 186

Mamestra ............................................... 269

Maniola …................................... 210, 343

margaritata ................................. 291, 324

marginaria ............................................ 288

marginepunctata ................................... 296 
maritima .......... 188, 191, 192, 194, 200, 201, 204, 205, 207, 208, 210, 211, $214,243,248,249,263,295$

matura 249,323

megacephala 235,320

Meganola 227,320

megera .. 208

Melanargia

211,323

melanaria

289,341

Melanthia 306

meliloti ... 214

Melitaea ........................................ 206, 207

mellinata ............................................... 302

mendica ................................................. 274

menthastri ............................................. 323

Mesapamea ……………………………... 261

Mesogona ............................................... 252

Mesoleuca ................................................ 301

mesomella ................................... 229, 321

meticulosa .................................... 250, 320

mi ........................................................... 241

micacea ............................................... 262

milhauseri ................................................ 225

millefoliata .......................................... 312

Miltochrista ....... 228, 320, 322, 323, 325

Mimas ........................................ 218, 318

miniata .............. 228, 320, 322, 323, 325

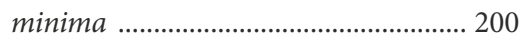

miniosa ................................................. 272

Minoa ............................... 316, 319, 324

minos .................................. 213, 323, 342

minutata ................................................ 245

Moma ................................ 234, 320, 345

monacha ................................................. 226

moneta .................................................. 242

montanata ................................................. 300

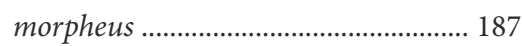

mucronata .................................... 299, 326

munda ................................................ 272

muricata .............................................. 297

murinata ........................... 316, 319, 324

muscerda .................................... 229, 320

myrtilli ......................................... 265, 340

Mythimna ................. 269-272, 319, 320,

322,324

\section{$\mathrm{N}$}

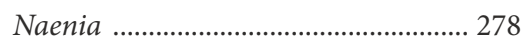

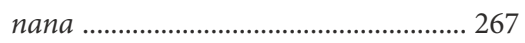

nanata ........................................................... 312

napi ............................................ 192, 318

Narraga .................................................... 283

Narycia .................................................. 212

nebulata .................................................. 316

nemoralis ................................................. 237
Neozephyrus ................................. 196, 323

neustria ......................................... 216, 323

nexa ...................................... 264, 316

nigricans ................................................ 279

nigrofusca .............................................. 279

nigropunctata .............................. 295, 325

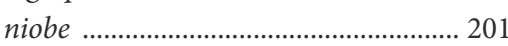

nitida .................................................... 254

Noctua ................. 275-277, 319, 320, 322

noctualis ............................................... 245

Noctuidae ................. 183, 184, 234, 247,

327,328

Noctuinae ............................................ 274

Nola ................................... 227, 321, 323

Nolidae ............................... 177, 227, 329

Nolinae ................................................... 227

Nonagria .............................................. 264

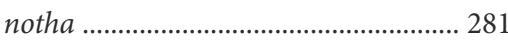

Notodonta ................... 223, 224, 318, 320

Notodontidae ............................. 222, 327

Notodontinae ........................................ 223

nubeculosa .............................................. 247

nupta .................................................... 239

Nycteola ................................................... 227

Nymphalidae ..... 183, 200, 203, 207, 208

Nymphalinae ....................................... 203

Nymphalis .......... 183, 204, 205, 320, 325

O

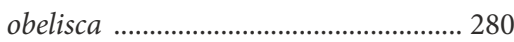

obeliscata ...................................... 304, 319

obscura .................................................... 260

obscurata .................................... 291, 326

obsoleta ....................................... 270, 320

obtusa ....................................... 229, 342

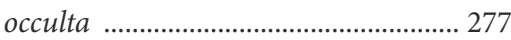

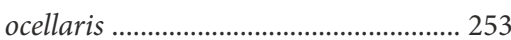

ocellata ......................... 218, 302, 324, 325

Ochlodes ...................................... 188, 325

ochrata .................................................... 296

ochridata .............................................. 313

ochroleuca .......... 177, 184, 261, 325, 338

Ochropacha .......................................... 220

ocularis ............................... 220, 320, 323

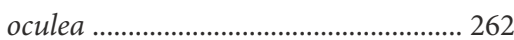

Odezia .................................................... 315

Odonestis ...................................... 217, 323

Odontopera ............................................ 286

Odontosia ............................................... 225

oleracea ......................... 191, 266, 319, 320

Oligia ........................................... 260, 261

ononaria ................................................ 292

Opigena ...................................... 278, 325

opima .................................. 272, 319, 340
Opisthograptis ............................................ 284

orbona ........................................ 275, 276

ornata ......................................... 295343

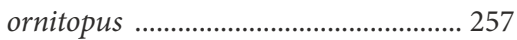

Orthosia ...................... 272, 319, 321, 340

Ourapteryx ......................................... 286

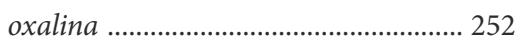

oxyacanthae .......................................... 257

\section{$\mathbf{P}$}

Pachetra ................................................... 273

Pachythelia ............................................ 212

palaemon .................................... 187, 341

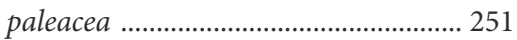

palliatella ................................................... 231

pallifrons ................................................... 231

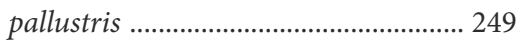

palpina ........................................ 225, 321

pamphilus ................................... 208, 318

Panemeria .............................................. 247

Panolis ................................................... 273

Panthea ............................... 281, 319, 320

Pantheidae ........ 183, 184, 234, 281, 328

paphia .................................................... 200

Papilio ........................................... 189, 343

papilionaria .......................................... 292

Paracolax ..................................... 237, 323

Paradarisa ........................................... 289

Paradrina .............................................. 248

Pararge ...................................... 208, 320

Parascotia .................................... 242, 324

Parastichtis ................................. 252, 324

Parectropis ................................... 290, 324

Pareulype ................................... 306, 319

parthenias .............................................. 281

pastinum ............................ 240, 322, 324

pavonia ...................................... 218, 340

Pelosia .................................. 229, 320, 342

Pelurga ................................................. 302

pendularia ............................................... 294

pennaria ..................................................... 286

Pennithera ............................................ 304

Perconia ................................................... 292

Peribatodes ........................ 288, 324, 325

Peridea ................................................ 225

Perizoma ..................... 308, 322, 326, 344

perplexa ................................................ 268

Petrophora .............................................. 283

Pharmacis ............................................... 211

Pheosia ....................... 225, 318, 320, 321

Phibalapteryx ..................... 299, 319, 325

Philereme .................... 306, 307, 317, 324

phlaeas ..................................................... 194

Phlogophora .............................. 250, 320 
phoebe

Phragmataecia

Phragmatiphila

216,321

Phragmatiphila

.264, 319

Phragmatobia

$232,323,346$

phragmitidis

264,345

Phyllodesma ......................................... 217

Phymatopus ........................................... 211

Phytometra ............................................. 241

Pieridae

189

Pieris

$191,192,318,320$,

$322,323,343,345$

pigra ....................................................... 222

pilosaria ................................................... 287

pimpinellata ........................................... 312

pinastri ................................................... 218

pini ............................................................. 309

piniarius ...................................... 290, 319

pinivora ................................................. 222

plagiata ................................................... 315

Plagodis ...................... 185, 283, 284, 325

Plebeius ............................... 198, 323, 340

plebeja ......................................... 267, 320

Plemyria ................................................. 304

plumbeolata ............................................ 309

plumella ................................................... 212

Plusia .................................................... 243

Plusiinae .................................................... 242

Poecilocampa ........................................ 216

Polia ......................................................... 269

polychloros ................................................ 205

Polychrysia ............................................. 242

polygona .................................................. 278

polygrammata ........................................ 301

Polymixis …………………………........... 258

polyodon ...................................... 250, 325

Polyommatus .............. 198-200, 327, 343

345,346

Polyploca ............................................... 221

Pontia ...................................................... 192

popularis ................................................. 273

populata ................................................ 302

populeti ....................................... 2272, 319

populi .................................... 207, 216, 272

porata ........................................................ 294

porphyrea ................................................ 277

praecox ................................................... 279

prasina .................................................. 279

proboscidalis ............................................ 241

procellata ................................................... 306

processionea ................................. 222, 318

pronuba ................................................. 275

proserpina …………………………........ 219

Proserpinus ............................................ 219

protea ...................................................... 257
Protodeltote .............................................. 244

Proutia .................................................... 212

pruinata ................................................. 292

prunaria ..................................... 286, 324

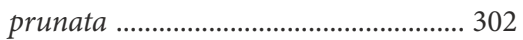

pruni ........................... 196, 213, 217, 323

Psaphidinae .......................................... 247

Pseudeustrotia ...................................... 244

Pseudoips ............................ 228, 321-323

Pseudopanthera ..................................... 284

Pseudoterpna ........................................ 292

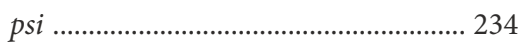

Psyche .................................................... 212

Psychidae ................................... 186, 212

Psychinae ................................................. 212

Pterapherapteryx ................................... 317

Pterostoma ................................... 225, 321

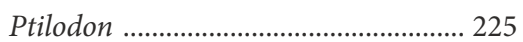

pudorina .............................. 269, 319, 324

pulchrina ................................................. 243

pulla ............................................. 212, 340

pulveraria ........................... 185, 283, 325

punctaria ..................................... 295, 319

punctulata ................................... 290, 345

purpuralis ................................................. 214

purpurata ................................... 232, 299

pusaria ............................................... 290

pusillata ............................................... 313

putata .................................. 294, 320, 321

putnami ................................................... 243

Pygaerinae ............................................ 222

pygarga ...................................... 244, 322

pygmaeola .................................................. 231

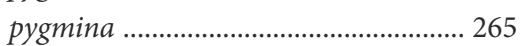

pyraliata ................................................. 303

pyralina .................................................. 252

pyramidea ............................................ 247

Pyrgus ........................................ 186, 343

pyrina ........................................ 216, 323

pyritoides .................... 220, 321, 322, 325

Pyrrhia .... 248

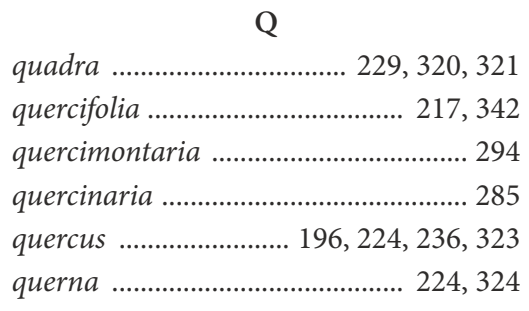

\section{R}

rapae .......................... 191, 320, 322, 345

raptricula .............................................. 236

ravida ................................................. 278 reali

189,190

remissa ................................................... 260

repandaria ........................... 284, 319, 324

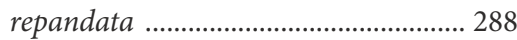

respersa ....................................... 249, 324

reticulata ............ 177, 185, 268, 305, 325

retusa .......................................... 251, 319

revayana .............................................. 227

Rhagades ............................................. 213

rhamni .......................................... 191, 343

Rheumaptera ....................................... 306

Rhinoprora ........................................... 315

Rhizedra ................................................. 262

Rhodostrophia ...................................... 299

rhomboidaria ...................... 288, 324, 325

rhomboidea ............................................ 278

Rhyacia ................................................. 277

Rhyparia ................................................ 232

ridens ...................................................... 221

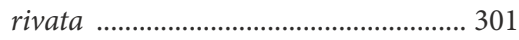

rivularis ................................................. 268

roboraria ...................................... 289, 320

rubi .............................................. 197, 275

rubiginata ........................... 295, 304, 320

rubiginea ................................................. 256

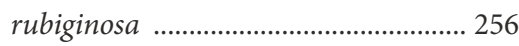

rubricollis ................................................ 229

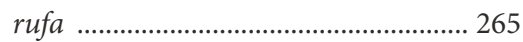

ruficornis .................................... 224, 318

rufifasciata ................................ 314, 319

rumicis ....................................... 236, 320

rupicapraria ............................................ 291

rurea …………………………………... 259

\section{S}

Sabra ......................................... 221, 342

sagittata .................................... 308, 326

sagittigera ……………………………... 273

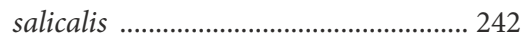

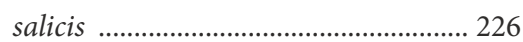

sambucaria ............................................... 286

sannio .................................. 233, 321, 325

satellitia .................................................... 256

satura .................................................... 258

Saturnia ..................................... 218, 340

Saturniidae ................................. 217, 327

Satyrinae ................................................ 208

Satyrium ................................... 196, 323

scabriuscula ................................ 249, 319

Schinia ........................ 184, 248, 325, 338

Schrankia ............................ 238, 320, 322

scoliaeformis ........................................... 216

Scoliopteryx ........................................... 241

scolopacina .................................. 260, 345 
Scopula .............. 295, 296, 320, 325, 343

Scotopteryx ................................. 299, 326

scrophulariae ..................... 246, 319, 342

scutosa ....................... 184, 248, 325, 338

secalella .................................................... 261

secundaria ............................................. 288

Sedina .................................................... 264

segetum ….................................... 281, 346

selenaria ..................................... 289, 322

selene ...................................................... 202

Selenia ....................................... 286, 320

selinata …...................................... 310, 324

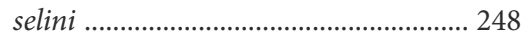

semele ......................................... 211, 324

semiargus ................................................ 198

senex ................................................... 228

sepium ............................... 191, 212, 240

serena …....................................... 185, 267

seriata .......................................... 297, 323

serpentata ........................... 296, 321, 324

Sesiidae ................................................. 216

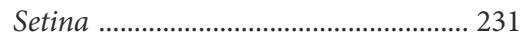

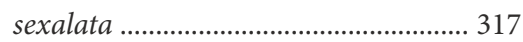

sexstrigata ............................................. 278

Shargacucullia ............................ 246, 319

Sideridis .................................................. 268

signaria .............................. 282, 319, 324

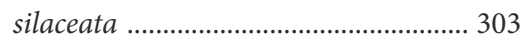

similaria ..................................... 290, 324

similis ..................................................... 226

simpliciata ............................................ 312

simulans ................................................ 277

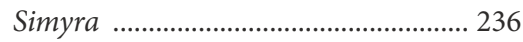

sinapis ........................ 189-192, 200, 202,

$205,206,341$

sinuosaria .................................... 312, 326

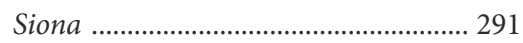

siterata ......................................... 303, 319

Smerinthus ......................... 218, 324, 325

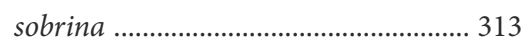

socia .................................................... 256

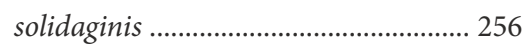

sororcula ................................................. 231

Spaelotes ................................... 278, 327

sparganii .................................................. 264

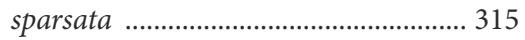

Sphingidae ................................. 218, 327

Sphinx ................................ 218, 247, 323

Spilosoma .................... 232, 320, 328, 339

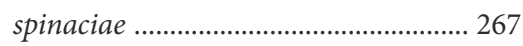

spini .......................................... 196, 323

Spiris ..................................................... 232

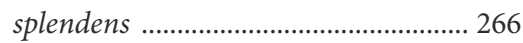

sponsa .......................................... 238, 322

stabilis ....... statices 213

Staurophora

... 263

Stegania

282,321

stellatarum

219,345

stigmatica

... 278

Stiriinae

.... 247

straminata

$298,320-322,324$

straminea

270,320

stratarius

... 287

Strepsimaninae

... 238

striata

232,342

strigillaria

... 292

strigosa

... 235

strigula

... 227

suasa .....

267,323

sublustris ............................................. 259

subtusa ............................................... 251

subumbrata .......................................... 312

succenturiata ........................................ 311

suffumata ............................................. 302

suspecta ................................................ 252

sylvanus ..................................... 188, 325

sylvata ........................................ 281, 324

sylvestraria ........................................... 297

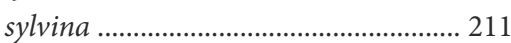

Synanthedon ......................................... 216

Syntominae ........................................... 232

syringaria

284,324

$\mathrm{T}$

tages

$186,318,321,322$

Talpophila

249,323

tanaceti

200, 246

tantillaria

314

tau

temerata

... 217

tenebrata

... 290

tenuiata

ternata

tersata

testacea

Tethea

Theteella

tetralunaria

Thalera

thaumas

Thaumetopoea

Thaumetopoeinae

Thecla

Thera

Theria

Tholera

Thumata

Thyatira

.... 247

. 308

296

$306,322,324$

261,319

$220,320,323$

\section{0}

286, 320

. 293,340

... 188

222,318

222

... 195

.304, 319

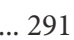

273

228

……..................... 220, 320,321
Thyatirinae .......................................... 220

Thymelicus .................................. 188, 323

tiliae ......................................... 218, 318

Timandra .............................................. 295

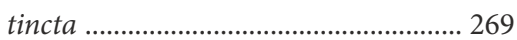

tityrus ................................ 194, 318, 345

Trisateles ...................................... 245, 324

togata ................................................... 252

trabealis .................................... 244, 320

Trachea ................................................. 250

tragopoginis ........................................... 247

transversa .............................................. 256

transversata ................................. 307, 324

trapezina .................................... 252, 324

tremula ........................ 225, 306, 318, 320

tremulifolia ............................................ 217

triangulum ……………............. 278, 322

Trichopteryx ........................................ 317

tridens ................................. 263, 319, 321

trifolii ................................... 215, 217, 265

trigemina ............................................ 244

trigrammica ………………………....... 249

trimacula ................................................ 224

trimaculata ................................. 282, 321

Triodia ................................................... 211

triplasia ...................................... 244, 324

tripunctaria .......................................... 311

triquetrella ............................................ 212

tristalis ……................................. 237, 323

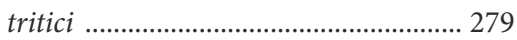

tritophus ................................................ 223

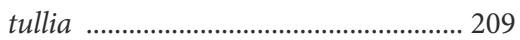

turbida ..................................................... 268

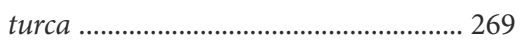

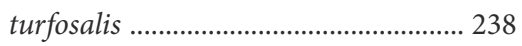

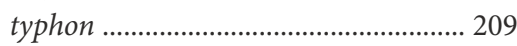

typhae .................................................... 264

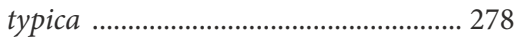

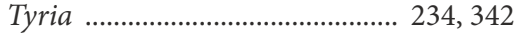

Tyta ...................................................... 241

$\mathrm{U}$

umbra

248

umbratica .................................. 245, 320

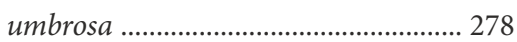

unanimis ................................................... 260

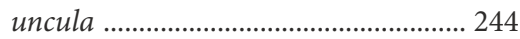

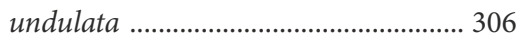

unicolor ................................................... 212

urticae ................................. 204, 205, 232

valerianata ................................... 309, 319

Vanessa ............... 203, 204, 318, 320-323

variata …………………………….... 304 


\begin{tabular}{|c|c|c|}
\hline 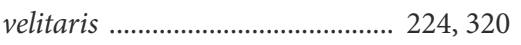 & 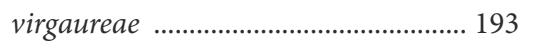 & 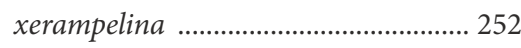 \\
\hline 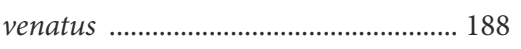 & virgaureata ........................... 313, 319, 321 & 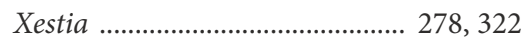 \\
\hline venosata & 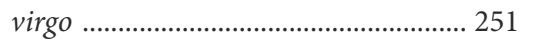 & \\
\hline 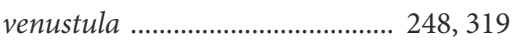 & 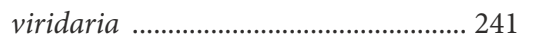 & $\mathrm{Y}$ \\
\hline 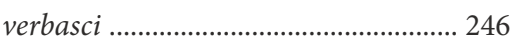 & 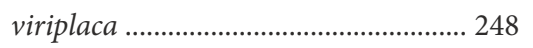 & 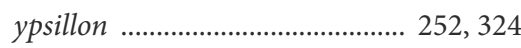 \\
\hline 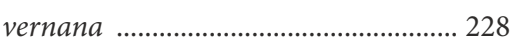 & vitalbata ............................................ 326 & \\
\hline versicolor & 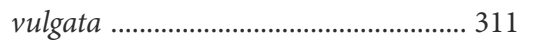 & $\mathrm{Z}$ \\
\hline 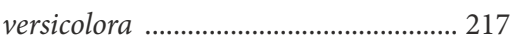 & & Zanclognatha ...................................... 238 \\
\hline 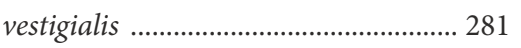 & $\mathrm{W}$ & Zeuzera ......................................... 216, 323 \\
\hline vetulata & 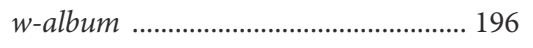 & 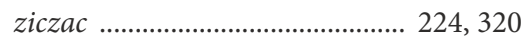 \\
\hline 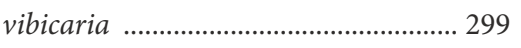 & Watsonalla ................................................. 221 & Zygaena ............... 213-215, 322, 323, 342 \\
\hline viciae …….................................. 240 & 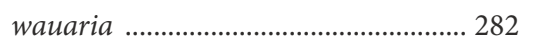 & 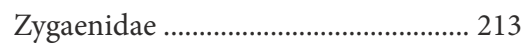 \\
\hline \multicolumn{3}{|l|}{ 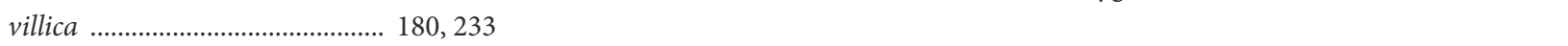 } \\
\hline 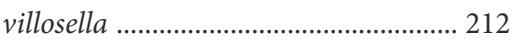 & $\mathrm{X}$ & \\
\hline vinula & xanthia ............................... 252-254, 319 & \\
\hline virens & Xanthorhoe …...................... 299, 300, 322 & \\
\hline 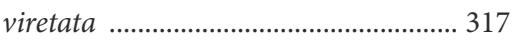 & & \\
\hline $99,319,325$ & & \\
\hline
\end{tabular}




\section{Farbfiguren}

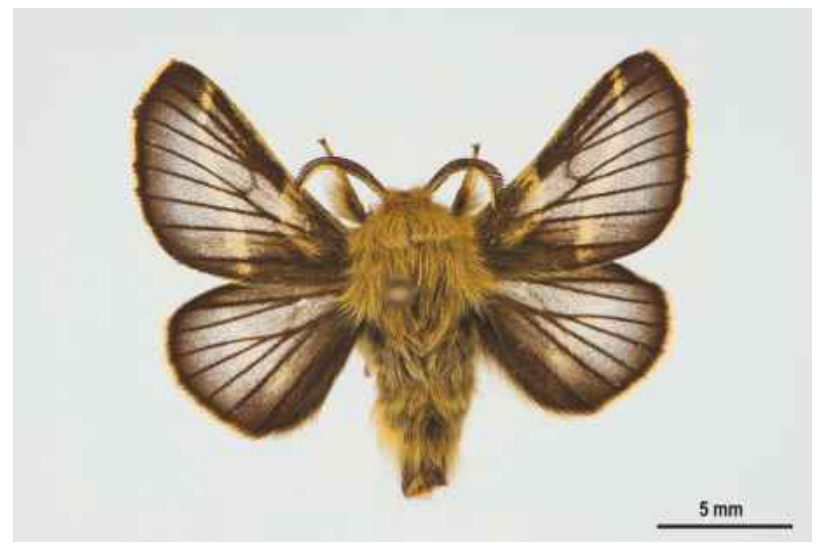

Fig. 1: Malacosoma franconica (DENIS \& SCHIFFeRmüLLER, 1775), neu entdeckte Art, Männchen, e.l. 09.VI.2018; Groß Schönebeck/ Revier Rehluch, leg. Haase, coll. Richert.

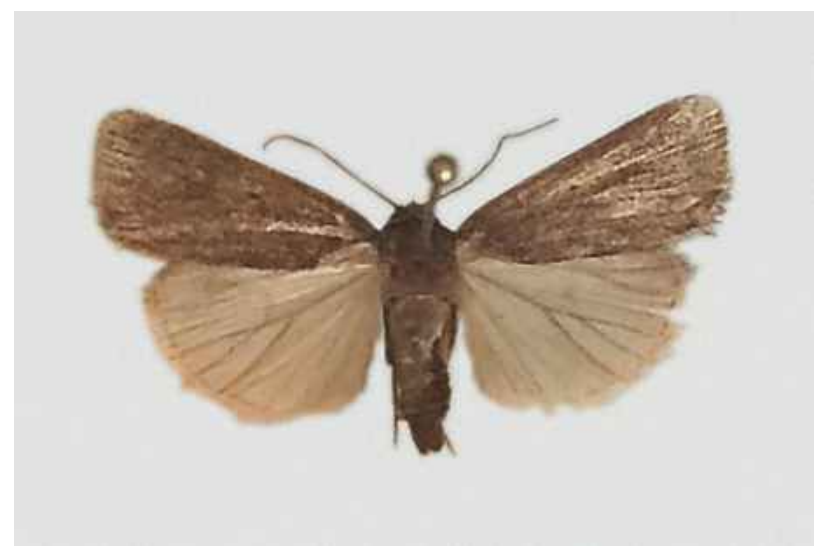

Fig. 3: Athetis lepigone (Möschler, 1860), neu entdeckte Art, LF 15.VIII.2017; Biesenthal, leg. et coll. Theimer.

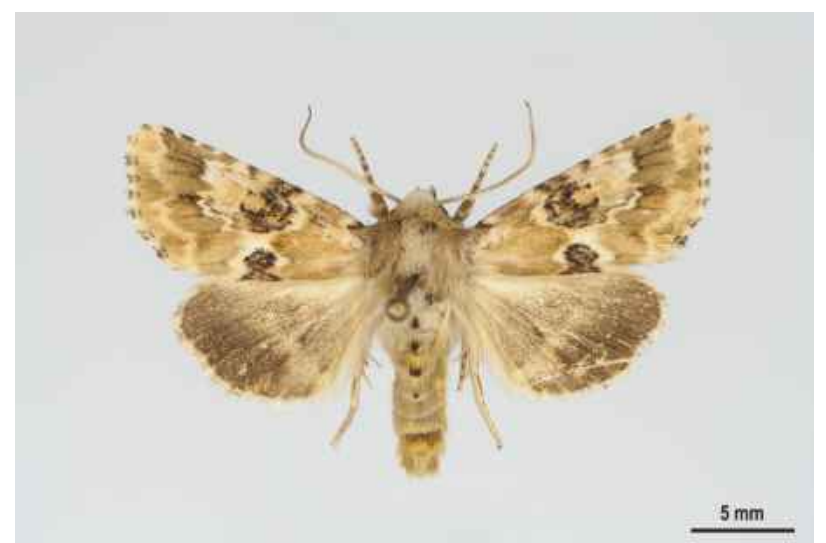

Fig. 5: Eremobia ochroleuca (DENIS \& SCHIFFERMÜLlER, 1775), wiederentdeckte Art, Männchen, 14.VII.2014, Neukünkendorf/ Straße nach Parstein, leg. et coll. Richert.

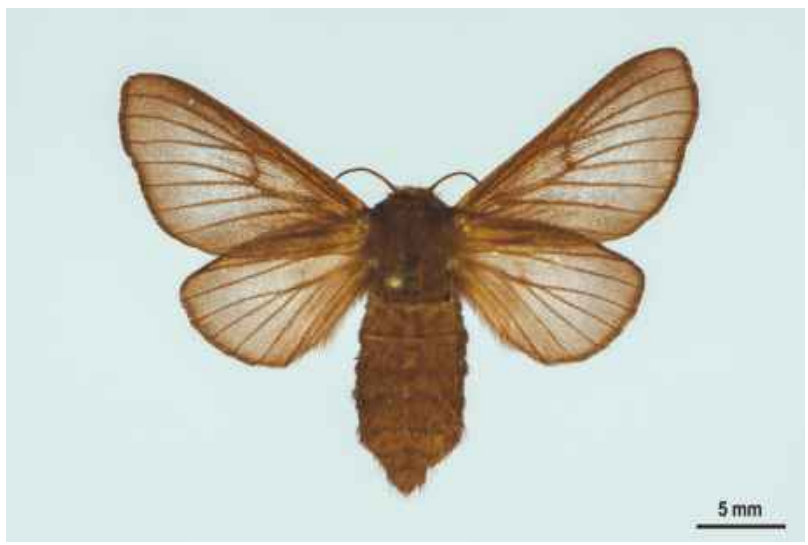

Fig. 2: Malacosoma franconica (DENIS \& SCHIFFERMÜLLER, 1775), neu entdeckte Art, Weibchen, e.l. 08.VI.2018; Groß Schönebeck/ Revier Rehluch, leg. Haase, coll. Richert.

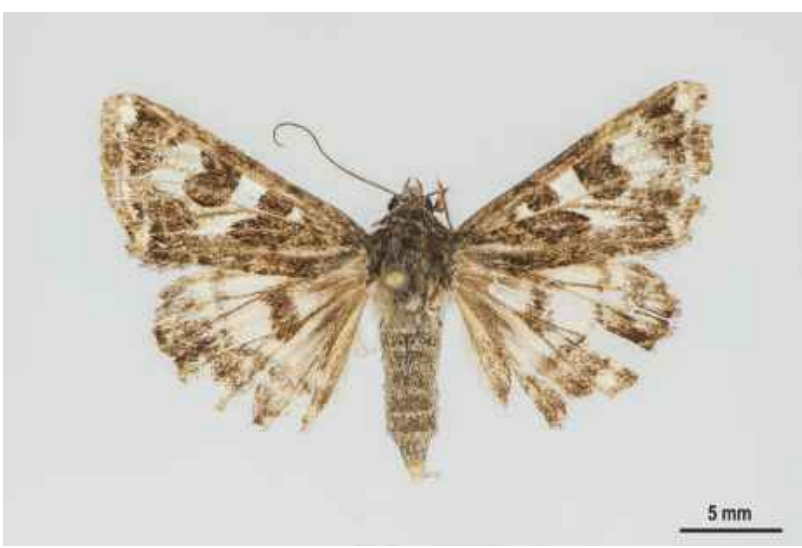

Fig. 4: Schinia scutosa (DENIS \& SCHIFFERMÜLLER, 1775), wiederentdeckte Art, LF 15.IX.2014, Brodowin/Krugberg, leg. et coll. Richert.

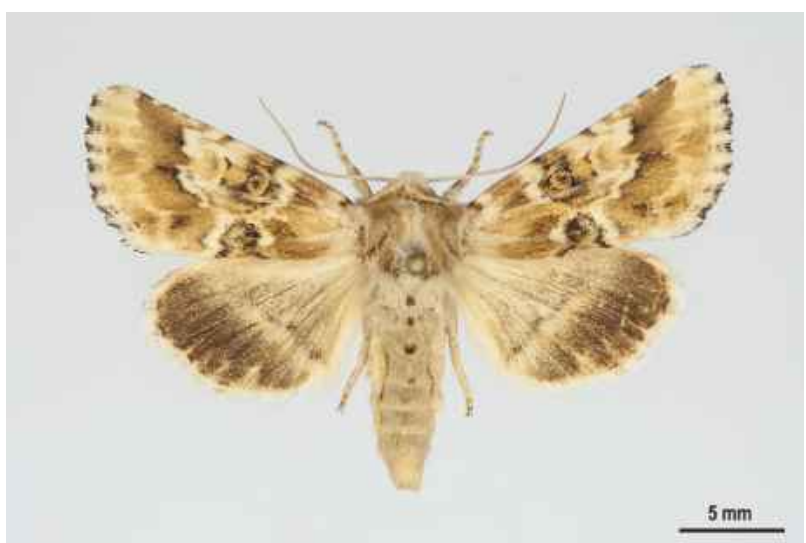

Fig. 6: Eremobia ochroleuca (DENIS \& SCHIFFERMÜLleR, 1775), wiederentdeckte Art, Weibchen, 14.VII.2014, Herzsprung/ Bunkerberg an der Straße nach Bölkendorf, leg. et coll. A. Richert. 


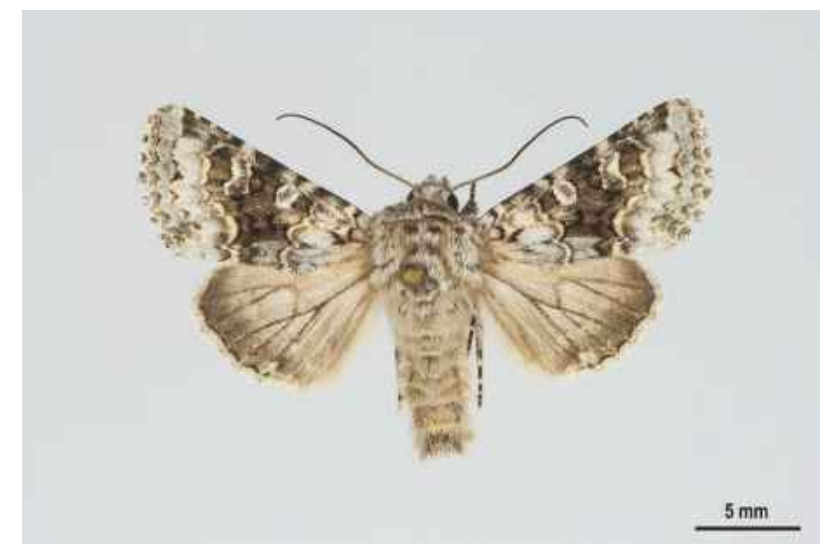

Fig. 7: Hecatera bicolorata (HufNAGEL, 1766), wiederentdeckte Art, LF 27.VI.2016, Niederfinow/Oderhänge südwestlich des Schiffshebewerks, leg. et coll. Schwabe.

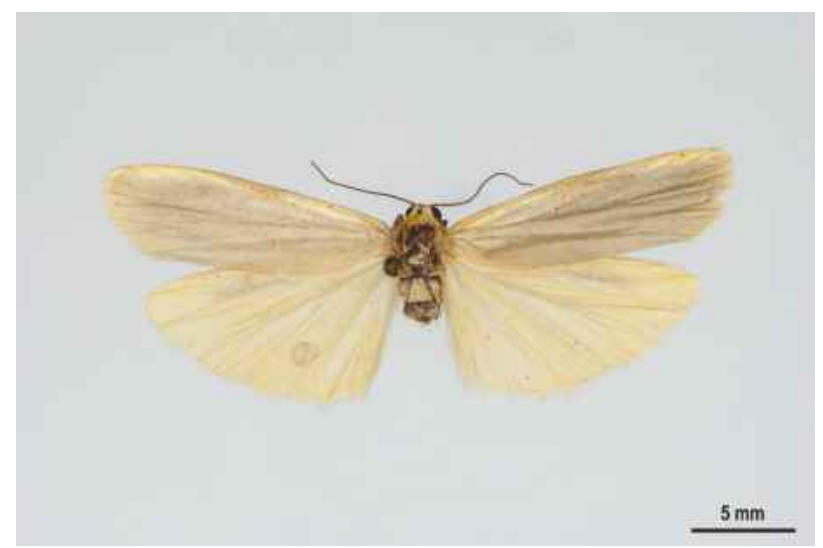

Fig. 9: Eilema complana (SCOPOLI, 1763), abberative Form, LF „9.8.1972 Finow“ (heute OT von Eberswalde), leg. Duckert, coll. Richert.

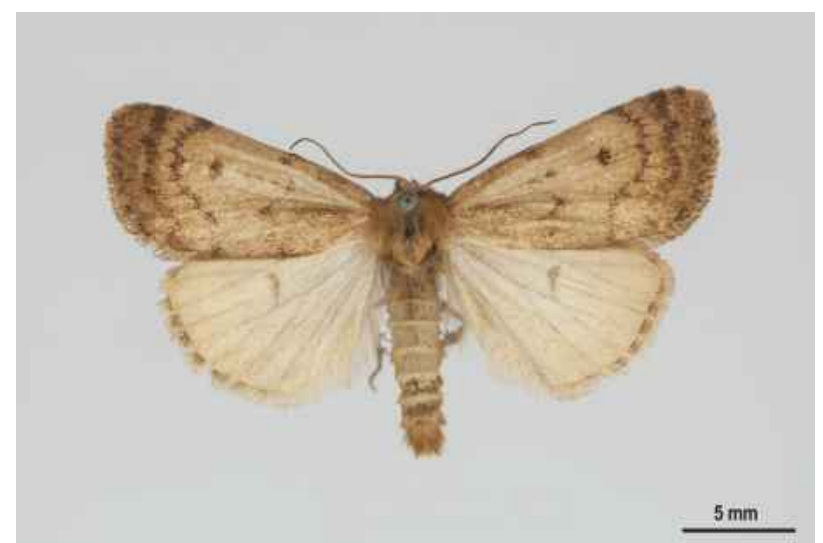

Fig. 11: Athetis palustris (HÜBNER, 1808), verschollene Art, Männchen: LF „20.VI.1962 Finow“ (heute OT von Eberswalde), leg. Duckert, coll. Richert.

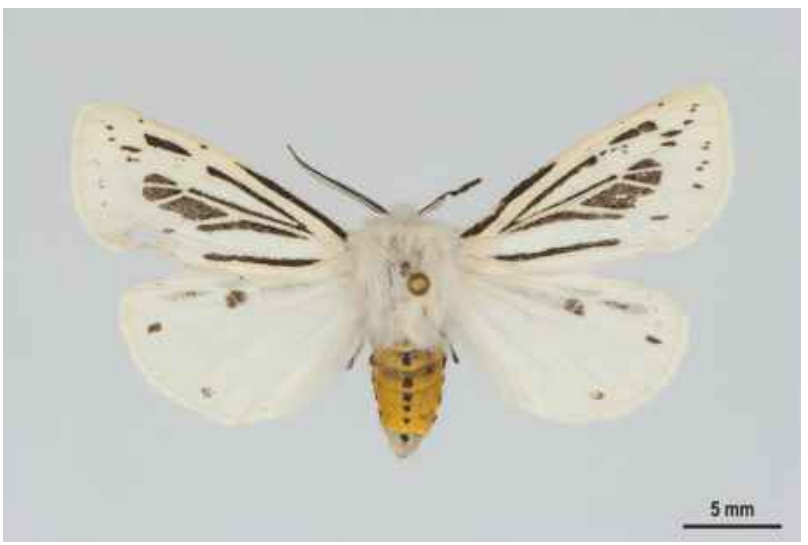

Fig. 8: Spilosoma lubricipeda (LinNAEus, 1758), aberrative Form, LF 23.V.2016, Eberswalde/Stadtgebiet, leg. et coll. Schwabe.

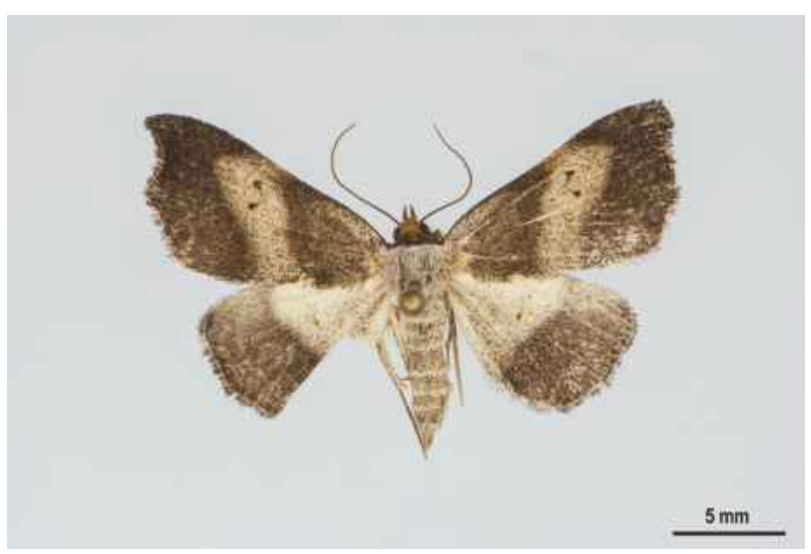

Fig. 10: Laspeyria flexula (Denis \& SCHIFFermüLler, 1775), aberrative Form, LF 07.IX.2016, Eberswalde/Stadtgebiet, leg. et coll. Schwabe.

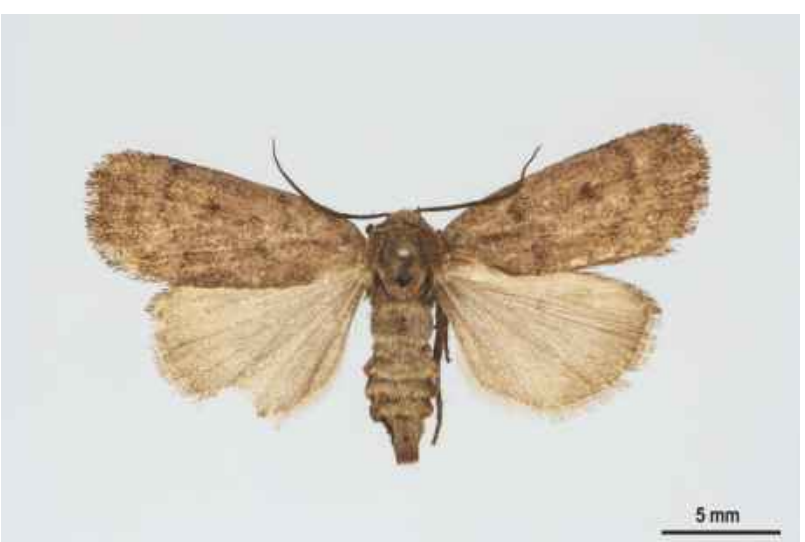

Fig. 12: Athetis palustris (HüBNER, 1808), verschollene Art, Weibchen: LF 05.VI.1964 Liepe/Forst Chorin: Försterei Liepe, leg. et coll. Richert. 

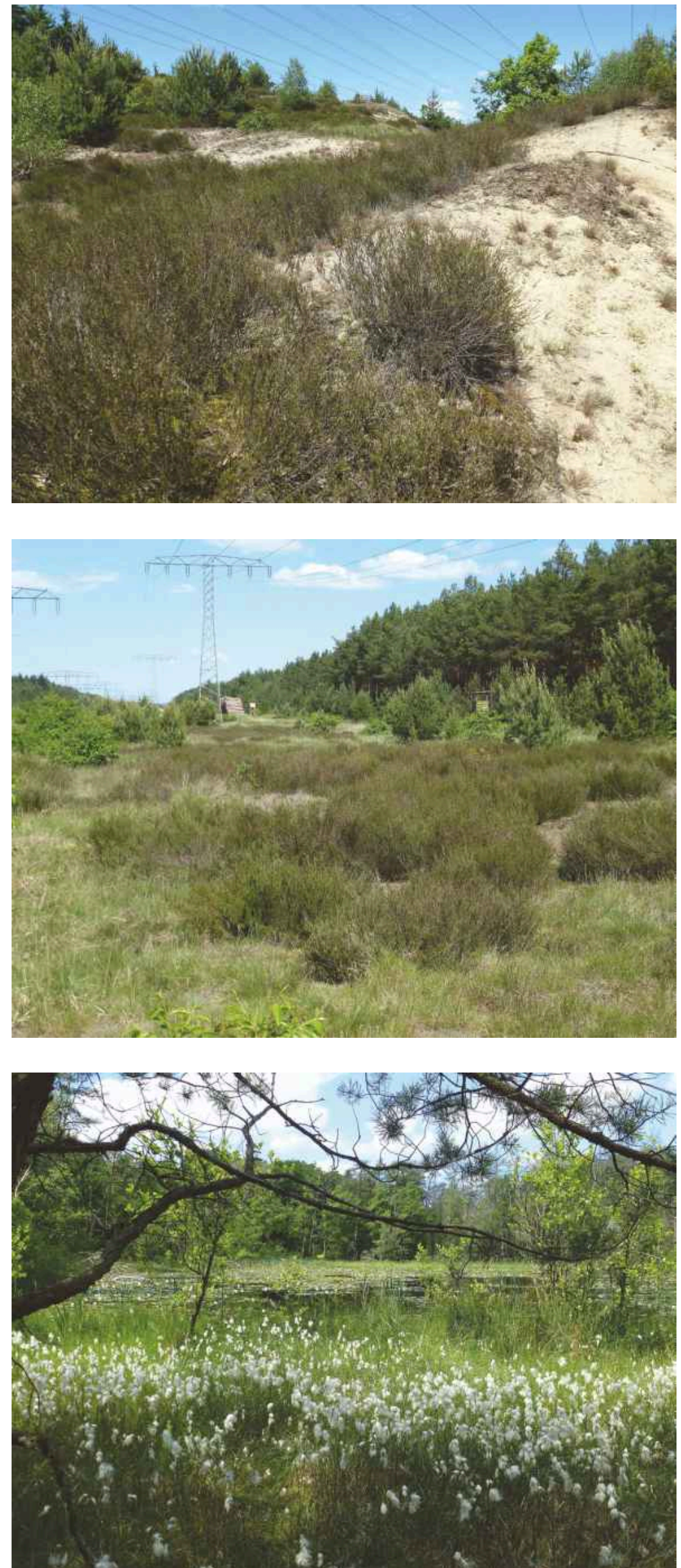

Fig. 13: Barnimplatte: Dünengelände an der Stromtrasse nordwestlich von Schönholz. Lebensraum von u. a. Coscinia cribraria, Anarta myrtilli, Thalera fimbrialis. Nach Plebeius idas suchte ich dort vergeblich. Aufn. Verfasser 04.06.2015.

Fig. 14: Barnimplatte: Stromtrasse in der Barnimer Heide südwestlich von Spechthausen. Lebensraum von Saturnia pavonia und Anarta myrtilli. Hier wurde auch Epichnopteryx pulla gefunden. Aus angrenzenden Blaubeer-Kiefernforsten flog u. a. Orthosia opima an das Licht. Aufn. Verfasser 04.06.2015.

Fig. 15: Eberswalder Tal: Kesselmoor an der Finowfließniederung nordöstlich des Lehnssees. Moorbereich mit Beständen des Schmalblättrigen und Scheidigen Wollgrases. Hier konnte u. a. Celaena haworthii nachgewiesen werden. Die erwartete Art Hypenodes humidalis suchten wir vergeblich. Aufn. Verfasser 20.06.2016. 
Fig. 16: Eberswalder Tal: Kesselmoor an der Finowfließniederung nordöstlich des Lehnssees. Lokal kommen dort noch Sumpfporst, Poleigränke, Moosbeere und Rundblättriger Sonnentau als Reste einer einst reichen Moorvegetation vor. Ein dort noch vermutetes Vorkommen von Arichanna melanaria, einer früher auf Mooren des Eberswalder Tales verbreitete Art, konnte nicht bestätigt werden. Aufn. Verfasser 14.06.2017.

Fig. 17: Eberswalder Tal: Kesselmoor an der Finowfließniederung nordöstlich des Lehnssees. Moorbereich mit reichem Pfeifengrasbestand. Hier flog Pelosia obtusa an das Licht. Amphipoea lucens, die dort zu erwarten war, konnte nicht nachgewiesen werden. Aufn. Verfasser 10.08.2017.
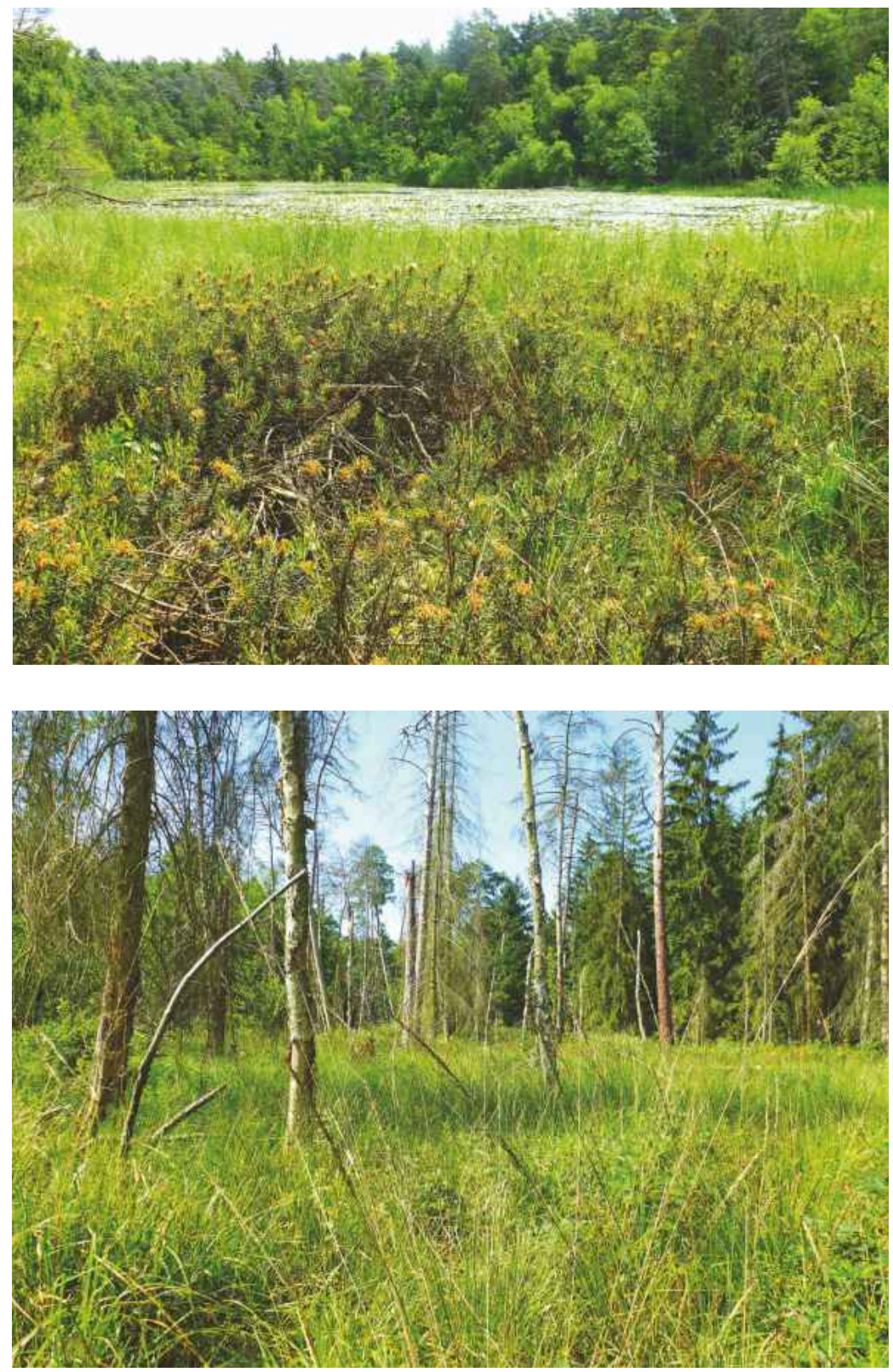

Fig. 18: Eberswalder Tal: Aufgeforstetes ehemaliges Kiesgrubengelände nördlich Stecherschleuse. Bestände der Feinblättrigen Wicke am Wegrand: Lebensraum von Leptidea sinapis. An dieser Wickenart wurde die Eiablage beobachtet. Hier wurde auch Carterocephalus palaemon gefunden. Aufn. Verfasser 03.06.2017.

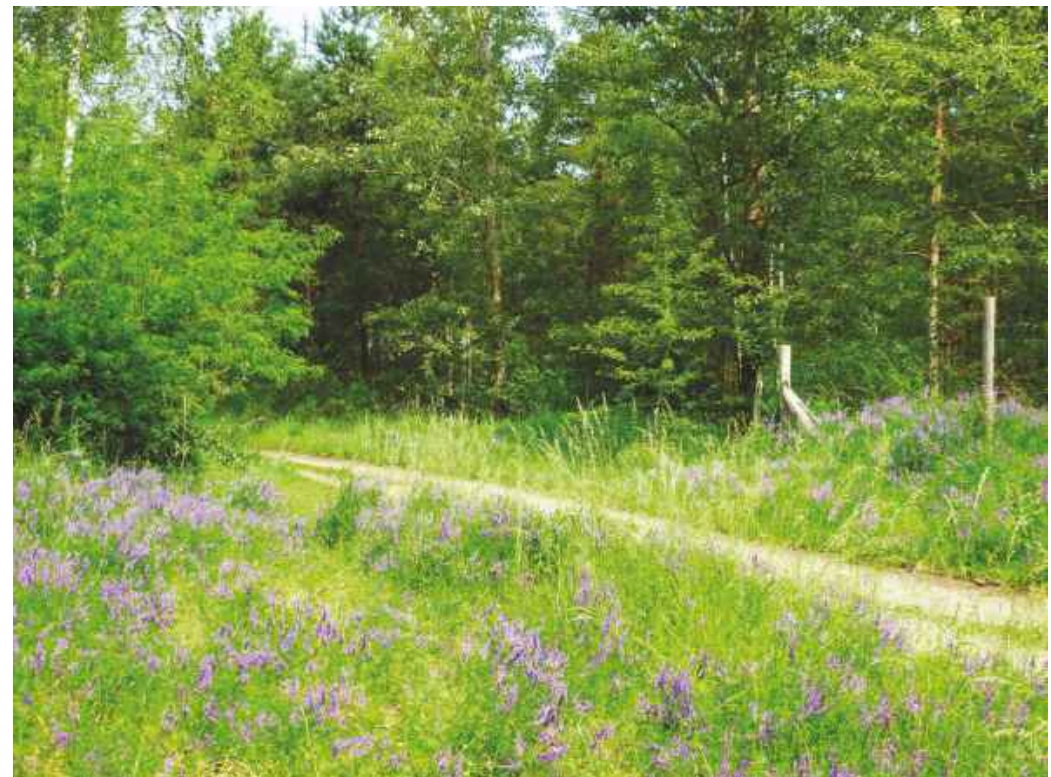



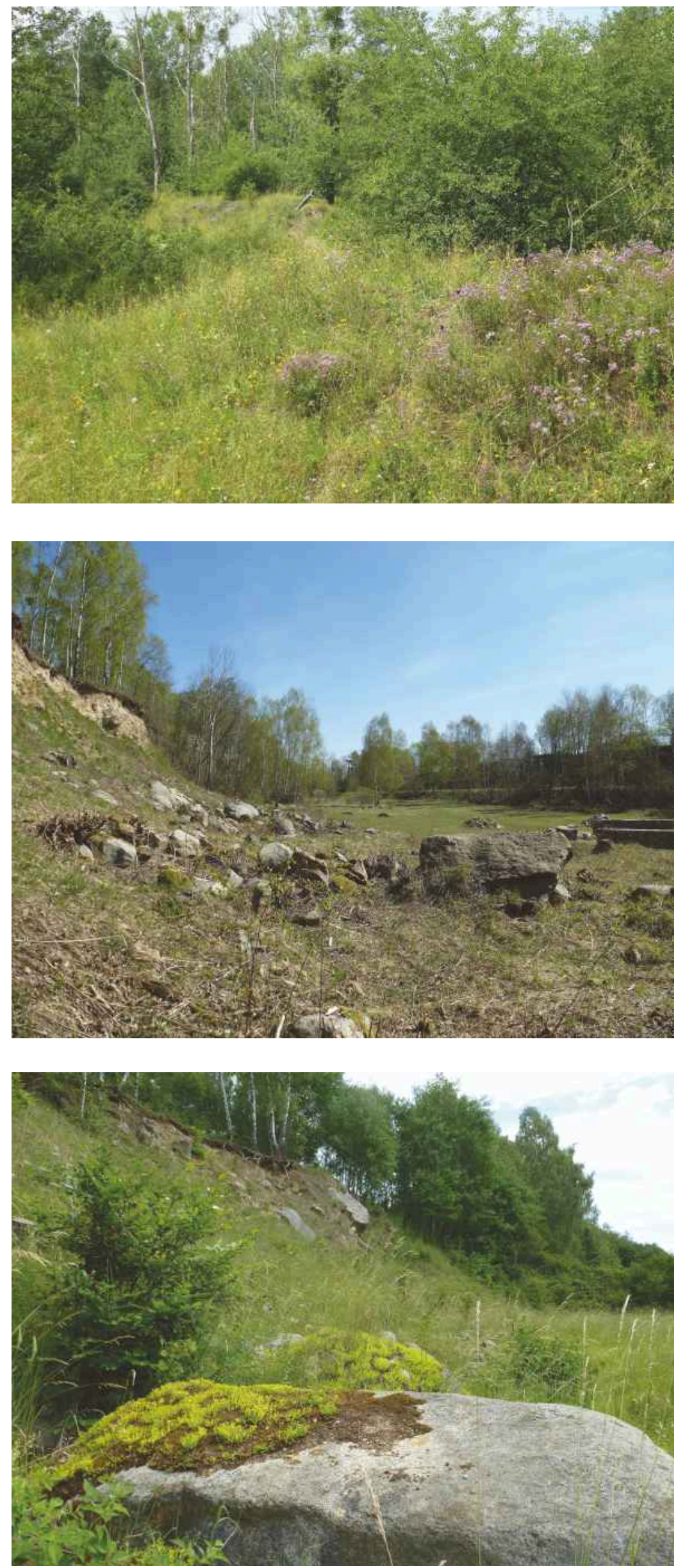

Fig. 19: Eberswalder Tal: Aufgeforstetes ehemaliges Kiesgrubengelände nördlich Stecherschleuse. Durch Abgrabungen entstand ein starkes Relief. Der arme Boden trägt auf lichten Stellen blütenreiche Trockenrasen mit Dost, Karthäuser-Nelken, Flocken- und Acker-Witwenblumen, Natternkopfblättrigem Habichtskraut, Färber-Hundskamille u. a. Hier wurde ein auffallend reiches Falterleben beobachtet. Aufn. Verfasser 20.07.2017.

Fig. 20: Uckermärker Kuppen- und Hügelland: Ehemalige Steingruben bei Groß Ziethen mit Geschieben der Pommerschen Endmoräne, Frühjahrsaspekt, Lebensraum zahlreicher gefährdeter Arten z. B. Lycaena alciphron, Zygaena minos, Gastropacha quercifolia, Coscinia striata, Tyria jacobaea. Aufn. Verfasser 23.04.2015.

Fig. 21: Uckermärker Kuppen- und Hügelland: Ehemalige Steingruben bei Groß Ziethen - Blockpackungen der Pommerschen Endmoräne; Frühsommeraspekt. Hier flog u. a. Sabra harpagula an das Licht. In den Saumstrukturen am oberen Grubenrand wurden die Raupen von Cucullia scrophulariae gefunden. Aufn. Verfasser 16.06.2014. 
Fig. 22: Odertalhänge: Stolpe a.O., Trockenhänge am Stadtweg mit Blick nach Südosten in das Untere Odertal. Auf den artenreichen Trockenrasen mit ihrer Blütenvielfalt fand ich einige weniger anspruchsvolle Falterarten in auffallend großer Populationsdichte u. a. Maniola jurtina, auch Polyommatus coridon. Die Säume und lichten Haine sind von einer vitalen Population von Coenonympha arcania besiedelt. Typische Trockenrasenarten wie Heliommata glarearia oder Scopula ornata traten unerwartet spärlich auf. Aufn. Verfasser 19.05.2018.

Fig. 23: Odertalhänge: Stolpe a.O., Steilhang am Stadtweg mit reichen Beständen von Wiesen-Salbei im Haar-Federgrasrasen. Dieser Xerothermstandort fiel durch zahlreiche Fingerkrautpolster auf und wurde als potentielles Habitat für Pyrgus carthami eingeschätzt, jedoch gelang kein Nachweis dieser Art. Aufn. Verfasser 08.05.2018.
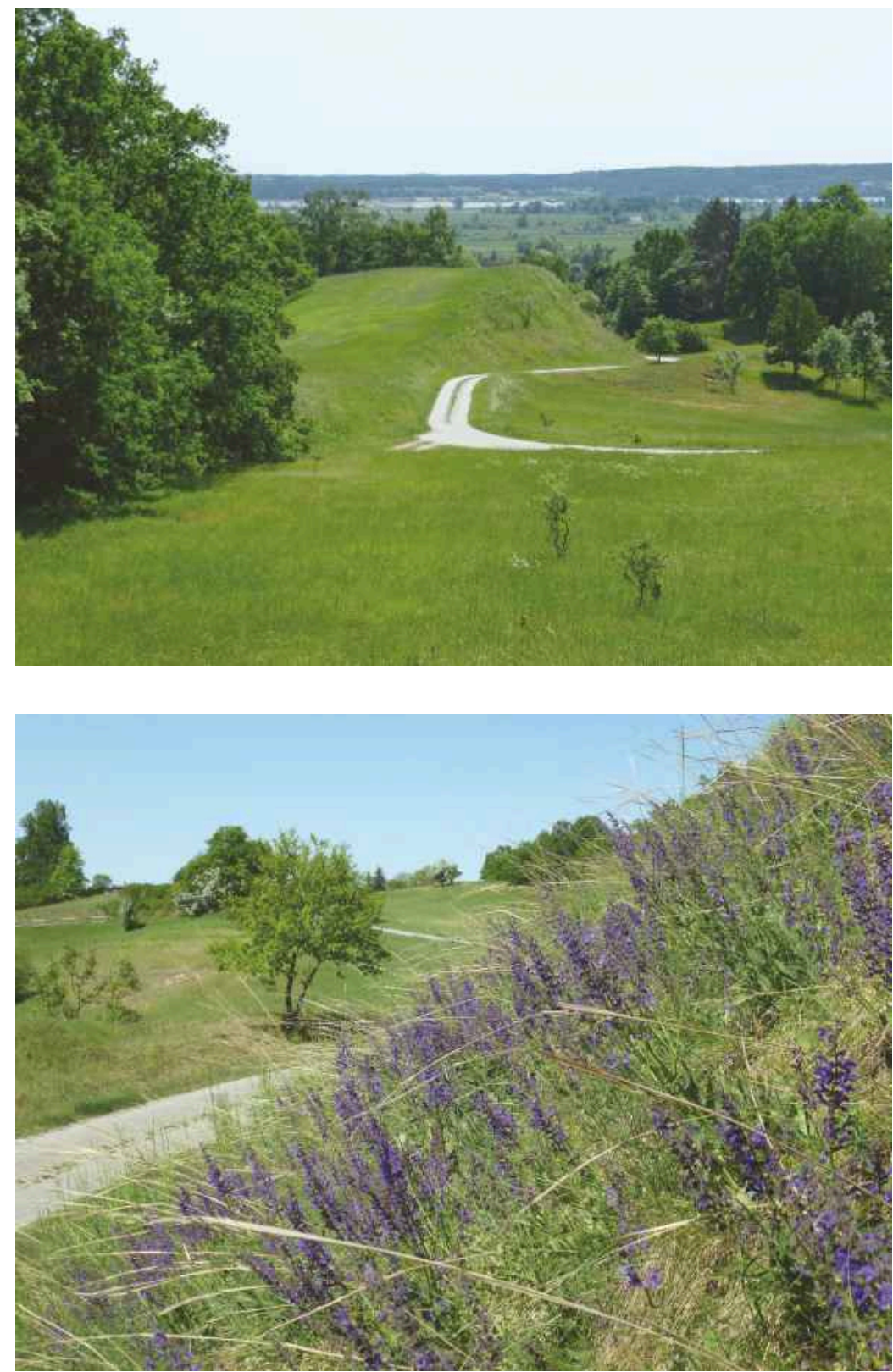

Fig. 24: Odertalhänge: Stolpe a.O., Massenbestände von Karthäuser-Nelken am Stadtweg; bevorzugte Nektarsaugpflanze u. a. von Papilio machaon, Gonepteryx rhamni und Pieris brassicae. Aufn. Verfasser 28.06.2018.

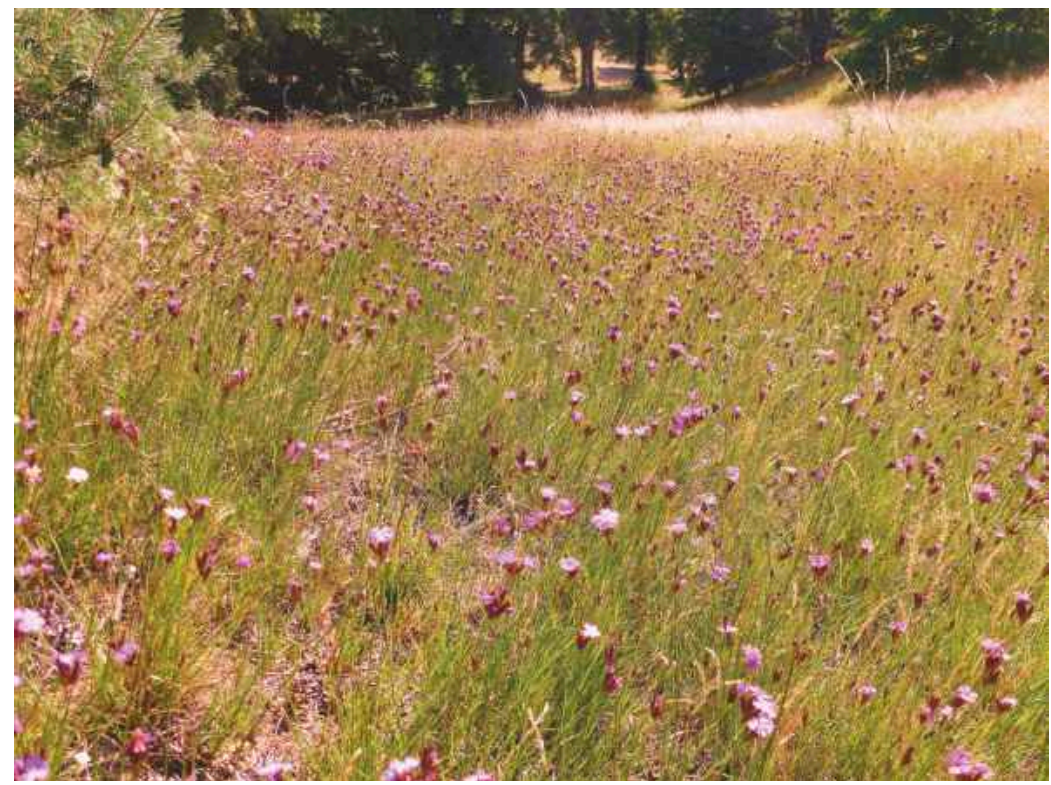



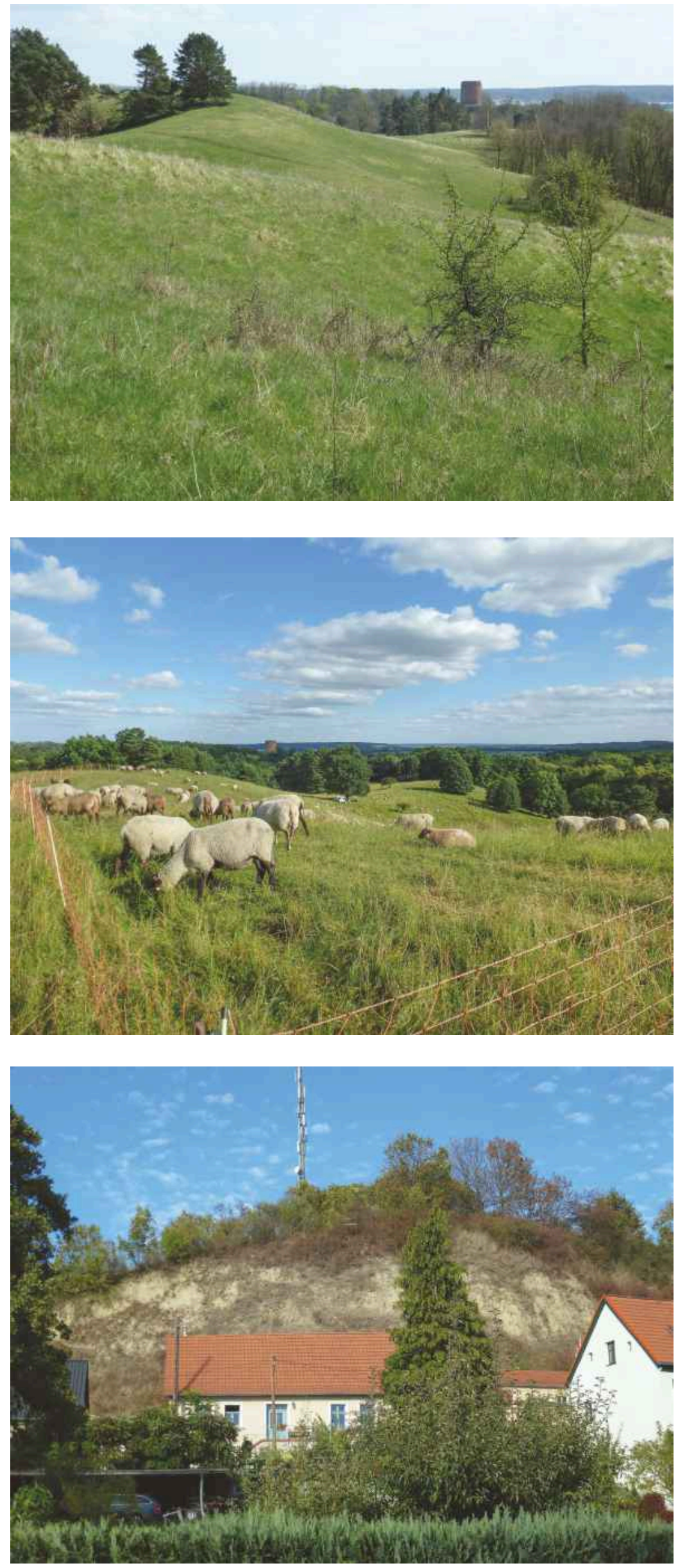

Fig. 25: Odertalhänge: Stolpe a.O., Trockenhänge östlich der Straße nach Crussow: Frühjahrsaspekt. Hier wurde "hilltopping“ des Schwalbenschwanzes beobachtet. Aufn. Verfasser 17.04.2018.

Fig. 26: Odertalhänge: Stolpe a.O., Trockenhänge östlich der Straße nach Crussow: Die Trockenrasenstandorte bei Stolpe wurden 2017 und 2018 während der Hauptblütezeit durch eine eingepferchte Schafherde weiträumig beweidet, was sich bei der extremen Trockenheit im Jahre 2018 für viele Schmetterlingsarten besonders nachteilig auswirkte. Aufn. Verfasser 17.07.2017.

Fig. 27: Odertalhänge: Der Geistberg in Oderberg; südexponierte Abbruchkante zur Alten Oder. Auf dem Geistberg wurden xerothermophile Arten nachgewiesen, u. a. Idaea aureolaria und Perizoma bifasciata. Aufn. Verfasser 30.09.2018. 
Fig. 28: Odertalhänge: Der Geistberg in Oderberg; Westflanke des Berges; Trockenrasen, artenreiche Waldgürtel und Feldgehölze sowie wärmeliebendes Gebüsch sind Lebensräume einer artenreichen Schmetterlingsfauna. Aufn. Verfasser 30.09.2018.

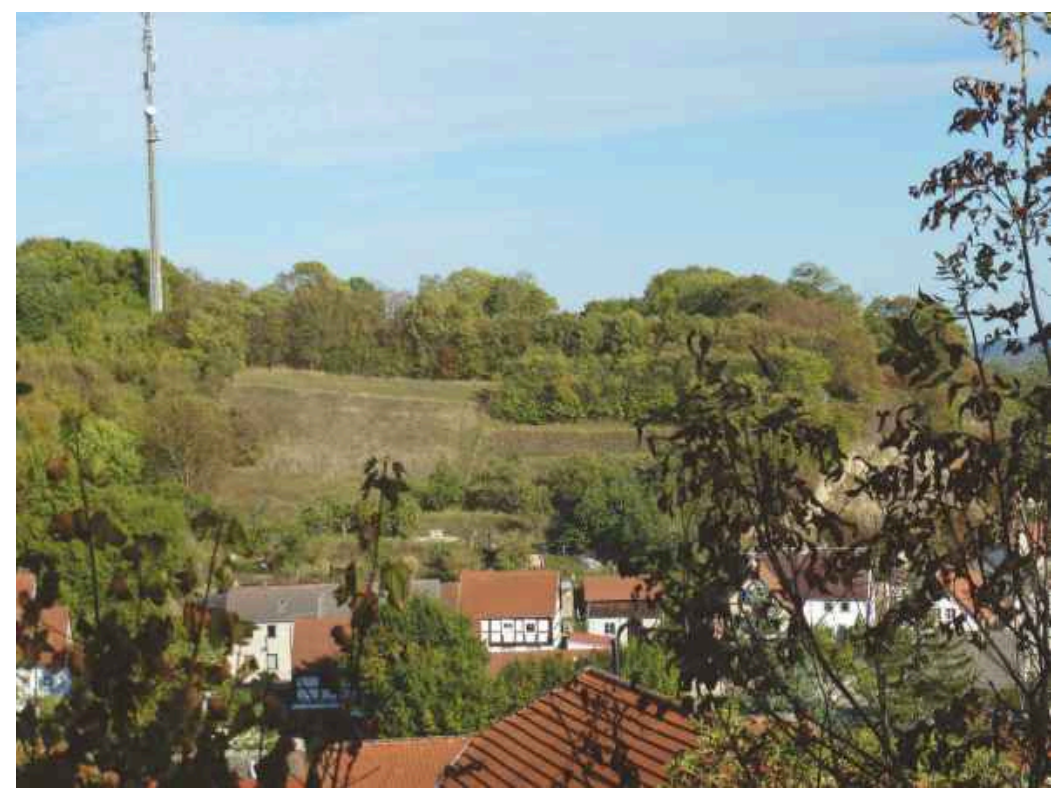

\section{Anhang}

\section{Einfügung Daten nach Redaktionsschluss:}

Datenauswertungen für das Jahr 2018 ergaben nach Redaktionsschluss bei einigen Arten weitere Änderungen in Phänologie und Voltinismus, die noch ergänzend nachgetragen werden:

Bei sechs weiteren Arten wurden phänologische Verfrühungen beobachtet (insgesamt im Jahre 2018 bei 66 Arten):

Ergänzung zu Tab. 21: Phänologische Verfrühungen 2018: Das Datum kennzeichnet den neuen absoluten Flugzeitbeginn (der 1. Gen. bei mehrbrütigen Arten) im Gebiet.

\begin{tabular}{|c|l|c|l|}
\hline & \multicolumn{1}{|c|}{ Art } & Datum & \multicolumn{1}{|c|}{ phän. Verfrühung gegenüber bis 2013 } \\
\hline 61 & Macroglossum stellatarum & $06 . V I .2018$ & 11d, bisher 17.VI.(2010) \\
\hline 62 & Bena bicolorana & 09.V.2018 & 10d, bisher 19.V.(2000) \\
\hline 63 & Moma alpium & 16.V.2018 & 3d, bisher 19.V.(1993) \\
\hline 64 & Apamea scolopacina & $14 . V I .2018$ & 6d, bisher 20.VI.(2000) \\
\hline 65 & Arenostola phragmitidis & 20.VI.2018 & 12d, bisher 02.VI.(2008) \\
\hline 66 & Aethalura punctulata & 17.IV.2018 & 1d, bisher 18.IV.(2000) \\
\hline
\end{tabular}

Bei sieben weiteren Arten wurden phänologische Verspätungen beobachtet, insgesamt also bei 13 Arten:

Ergänzung zu Tab. 22: Phänologische Verspätungen 2018: Das Datum kennzeichnet das absolute Flugzeitende (bei mehrbrütigen Arten der letzten Generation) im Gebiet.

\begin{tabular}{|c|c|c|c|}
\hline & Art & Datum & phän. Verspätung gegenüber bis 2013 \\
\hline 07 & Pieris rapae & 19.X.2018 & 6d, bisher 13.X.(1984, 2012) (siehe auch 2014) \\
\hline 08 & Lycaena tityrus ${ }^{* *}$ & 10.X.2018 & 8d, bisher 02.X.(1962) \\
\hline 09 & Colias hyale & 30.X.2018 & 11d, bisher 19.X.(2005) \\
\hline 10 & Aricia agestis ${ }^{*}$ & 12.X.2018 & 12d, bisher 01.X.(1983) \\
\hline 11 & Polyommatus coridon & $10 . X .2018$ & 5d, bisher 05.X.(1996) \\
\hline 12 & Polyommatus icarus ${ }^{\star *}$ & 12.X.2018 & 5d, bisher 07.X.(1985) \\
\hline 13 & Macroglossum stellatarum & 03.XI.2018 & 27d, bisher 07.X. $(1906,2006)$ \\
\hline
\end{tabular}


Folgende Arten, die in der Regel uni- oder bivoltin im Gebiet auftraten, bildeten 2018 (partielle) zusätzliche Generationen aus:

Polyommatus coridon 2. part. Gen. (?) A X;

Anmerkung: Art gilt allgemein als einbrütig (in Brandenburg „strikt einbrütig“!). Lediglich in der Slowakei wird sehr lokal eine 2. Gen. ausgebildet.

Der Falter flog bei uns im extrem heißen und Sonnenschein reichen Jahr 2018 bereits seit A VI. Die Flugzeit allgemein endet hier im IX. Aus einer regelmäßigen Beobachtungszeit im Gebiet von 64 Jahren liegen mir nur 2 Funde aus dem Oktober vor (01.X.1983; 05.X.1996). Dabei handelte es sich stets um sehr abgeflogene Exemplare. Der Fund eines fransenreinen Weibchens am 10.X.2018 auf den Oderhängen bei Liepe (vidit Brauner, Fotobelege) kann meines Erachtens nur als 2. partielle Generation gedeutet werden.

Phragmatobia fuliginosa 3. part. Gen. E VIII-A IX;

Agrotis segetum 3. Gen. M-E X.

\section{Berichtigungen zum 3. Nachtrag (RICHERT 2014)}

Trotz der engagierten Arbeit aller Beteiligten entstanden bei der redaktionellen Bearbeitung des Manuskripts für die Layoutgestaltung einige Fehler, die bedauerlicherweise vor der Drucklegung nicht erkannt wurden. Das betrifft einzelne Hinweise auf Grafiken im laufenden Text sowie einige Bildlegenden zu Habitatabbildungen, welche vertauscht wurden:

- $\quad$ S. 257, rechte Spalte, 1. Absatz: (Figur 5 und Figur 6) statt (Figur 5);

- $\quad$ S. 258, linke Spalte, letzter Absatz: (Figur 7 bzw. 8) statt (Figur 6);

- $\quad$ S. 259, linke Spalte, 2. Zeile: (Tabelle 45, Figuren 11 und 12) statt (Tabelle 45, Figuren 7-10);

- S. 260, linke Spalte, 1. Absatz: (Figuren 5, 8, 9; Tabelle 44) statt (Figuren 5, 6, 7; Tabelle 44);
- S. 281, oberes Bild: Bildlegende Figur 22 statt Bildlegende Figur 21;

- S. 281, mittleres Bild: Bildlegende Figur 21 statt Bildlegende Figur 22;

- $\quad$ S. 283, oberes Bild: Bildlegende Figur 28 statt Bildlegende Figur 27;

- S. 283, mittleres Bild: Bildlegende Figur 27 statt Bildlegende Figur 28;

- S. 283, unteres Bild: Bildlegende Figur 30 statt Bildlegende Figur 29 (S. 284);

- $\quad$ S. 284, oberes Bild: Bildlegende Figur 29 statt Bildlegende Figur 30 (S. 283). 